Progress Report

of the Air Force Project

Covering research period $3 / 01 / 01$ to $8 / 31 / 02$

Agency No: DOD/F49620-02-1-0138

$U$ of M No: 1613-189-6235

NRRI Technical Report No: NRRI/TR-2002/12

\title{
Use of Biodescriptors and Chemodescriptors in Predictive Toxicology: A Mathematical / Computational Approach
}

August 28, 2002

\author{
Submitted by: \\ Subhash C. Basak, Ph.D. \\ Principal Investigator \\ Natural Resources Research Institute \\ University of Minnesota, Duluth \\ 5013 Miller Trunk Highway \\ Duluth, MN 55811 \\ Tel: (218)720-4230 \\ Fax: (218)720-4328 \\ Email: sbasak@nrri.umn.edu
}




\section{Table of Contents}

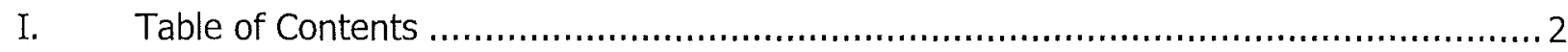

II. Manuscripts submitted/published during the current grant cycle $\ldots \ldots \ldots \ldots \ldots \ldots \ldots \ldots \ldots \ldots . \ldots \ldots$

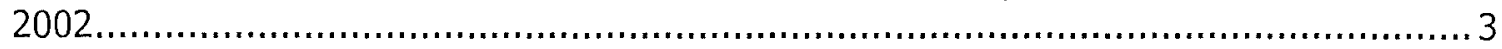

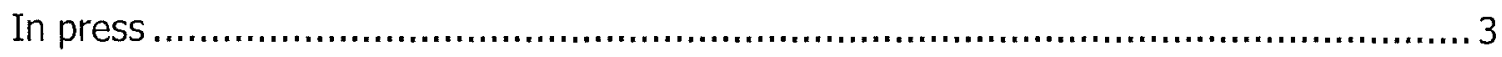

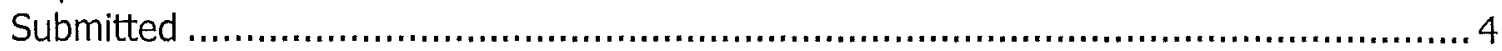

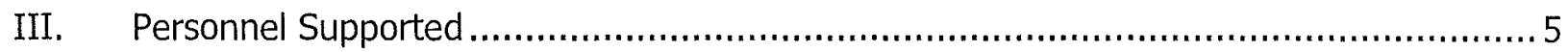

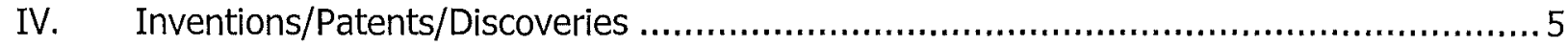

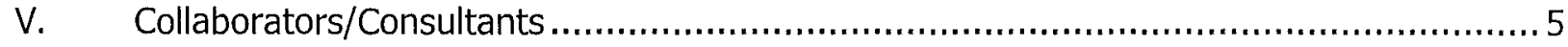

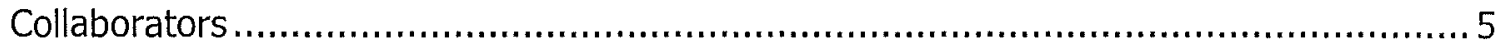

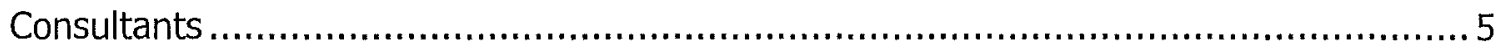

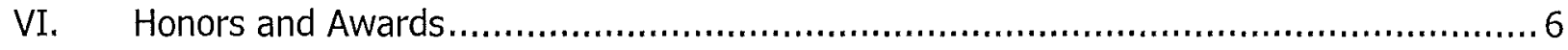

Advisory/Organizational Positions Held by Dr. Basak....................................... 6

Invited Lectures and Seminars Presented by Dr. Basak..................................... 6

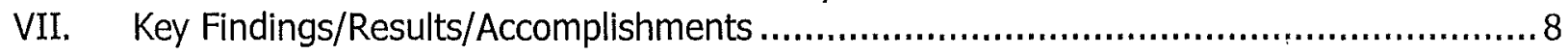

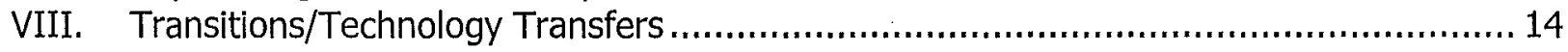

Appendix 1: $\quad$ Publications/Reprints .................................................................... 16 


\section{Manuscripts submitted/published during the current grant cycle}

The following peer-reviewed papers, which are currently either published, in press, or submitted, report results of research carried out between March 1, 2002 and August 31, 2002.

\section{2}

Alkane ordering as a criterion for similarity between topological indices: Index $\mathrm{J}$ as a "sharpened Wiener index", A.T. Balaban, D. Mills and S.C. Basak, MATCH (Commun. Math. Comput. Chem.), 45, 5-26 (2002).

Quantitative descriptor for SNP related gene sequences, A. Nandy, P. Nandy and S.C. Basak, Internet Electron. J. Mol. Des., 1, 367-373 (2002), http://www.biochempress.com.

Structure-water solubility modeling of aliphatic alcohols using the weighted path numbers, D. Amic, S.C. Basak, B. Lučić, S. Nikolić and N. Trinajstić, SAR QSAR Environ. Res., 13, 281-295 (2002).

\section{In press}

A comparative study of proteomics maps using graph theoretical biodescriptors, $M$. Randić and S. C. Basak, J. Chem. Inf. Comput. Sci.

Novel matrix invariants for characterization of changes of proteomics maps, M. Randić, J. Zupan, M. Novič, B.D. Gute and S.C. Basak, SAR QSAR Environ. Res.

Predicting blood:air partition coefficient of structurally diverse chemicals using theoretical molecular descriptors, S.C. Basak, D.M. Hawkins and D. Mills, in Advances in Molecular Similarity, R. Carbo-Dorca, P.G. Mezey, Eds., vol. 5.

Predicting mutagenicity of congeneric and diverse sets of chemicals using computed molecular descriptors: A hierarchical approach, S.C. Basak, D. Mills, B.D. Gute and D.M. Hawkins, in Quantitative Structure-Activity Relationship (QSAR) Models of Mutagens and Carcinogens, R. Benigni, Ed., CRC Press, Boca Raton, FL.

Prediction of tissue:air partition coefficients: A comparison of structure-based and property-based methods, S.C. Basak, D. Mills, D.M. Hawkins and H.A. El-Masri, SAR QSAR Environ. Res.

Quantitative molecular similarity analysis (QMSA) methods for property estimation: A comparison of property-based, arbitrary, and tailored similarity spaces, S.C. Basak, B.D. Gute and D. Mills, SAR QSAR Environ. Res.

Quantitative molecular similarity methods in the property/ toxicity estimation of chemicals: A comparison of arbitrary versus tailored similarity spaces, S.C. Basak, B.D. Gute, D. Mills and D.M. Hawkins, J. Mol. Struct. THEOCHEM. 
Tailored similarity spaces for the prediction of physicochemical properties, B.D. Gute, S.C. Basak, D. Mills and D.M. Hawkins, Internet Electron. J. Mol. Des., http://www.biochempress.com/.

Use of mathematical structural invariants in analysing combinatorial libraries: A case study with Psoralen derivatives, S.C. Basak, D. Mills, B.D. Gute, A.T. Balaban, K. Basak and G.D. Grunwald, In Some Aspects of Mathematical Chemistry, Eds. D.K. Sinha, S.C. Basak, R.K. Mohanty and I.N. Basumallick, Visva-Bharati University: Santiniketan, West Bengal, India.

Variable molecular descriptors, M. Randić and S.C. Basak, In Some Aspects of Mathematical Chemistry, Eds. D.K. Sinha, S.C. Basak, R.K. Mohanty and I.N. Basumallick, Visva-Bharati University: Santiniketan, West Bengal, India.

\section{Submitted}

Assessing model fit by cross-validation, D.M. Hawkins, S.C. Basak and D. Mills, J. Mol. Graphics and Modelling.

Chemometrical treatment of proteomic maps, M. Vračko and S.C. Basak, J. Proteom. Res.

Distance based molecular shape descriptors, M. Randić, S.C. Basak and D. Mills, Croat. Chem. Acta.

Exposure assessment of volatile organic chemicals (VOCs): Predicting blood:air partition coefficients of diverse chemicals using theoretical descriptors, S.C. Basak, H. El-Masri, D.M. Hawkins and D. Mills, J. Chem. Inf. Comput. Sci.

Interrelationship of major topological indices, S.C. Basak, A.T. Balaban and B.D. Gute, J. Chem. Inf. Comput. Sci..

On canonical labeling of proteins of proteomics maps, M. Randić and S.C. Basak, J. Chem. Inf. Comput. Sci.

On numerical characterization of DNA primary sequences, M. Randić, A. Nandy and S.C. Basak, J. Math. Chem.

Prediction of cellular toxicity of halocarbons from their computed chemodescriptors: A hierarchical QSAR approach, S.C. Basak, K. Balasubramanian, B.D. Gute, D. Mills, A. Gorczynska and S. Roszak, J. Chem. Inf. Comput. Sci.

Prediction of human blood:air partition coefficient: A comparison of structure-based and property-based methods, S.C. Basak, D. Mills, D.M. Hawkins and H. El-Masri, Risk Analysis.

Of the 22 papers listed above, copies of three of the manuscripts dealing with the development 
of biodescriptors have been attached as Appendices 1.1. The manuscript developed to examine the use of HiQSAR in modeling the toxicity of halocarbons has been attached as Appendix 1.2 and the three manuscripts detailing the development of the tailored similarity method have been attached as Appendix 1.3. Additionally, the four papers dealing with PBPK modeling have been attached as Appendix 1.4. Copies of the remaining manuscripts have been omitted for the sake of brevity.

\section{Personnel Supported}

Subhash C. Basak, Principal Investigator

Krishnan Balasubramanian, Consultant (Professor; Arizona State Univ., Tempe, AZ)

Brian Gute, Assistant Scientist

Douglas Hawkins, Co-principal Investigator (Professor; Dept. of Applied Statistics; Univ. of

Minnesota, St. Paul, MN)

Denise Mills, Senior Laboratory Technician

Milan Randić, Consultant (Distinguished Professor; Drake Univ., Des Moines, IA)

Marjan Vračko, Consultant (Professor; National Institute of Chemistry, Slovenia)

\section{Inventions/Patents/Discoveries}

None to report at this time.

\section{Collaborators/Consultants}

\section{Collaborators}

Douglas Hawkins, Ph.D., is a professor and former Chairman of the Department of Applied Statistics at the University of Minnesota Minneapolis campus. He has been involved in the statistical analysis of data for developing QSAR models as part of this project. Specifically, he has worked with our research group in the implementation of new statistical techniques, such as ridge regression (RR) and partial least squares (PLS) in QSAR analysis and in the development of the tailored QMSA method (Basak, Gute, Mills and Hawkins, J. Mol. Struct. THEOCHEM, in press; Gute, Basak, Mills and Hawkins, Internet Electron. J. Mol. Des., in press). A recent study with Dr. Hawkins examines the role of cross-validation techniques in QSAR studies, challenging some of the commonly held misconceptions in regards to cross-validation (Hawkins, Basak and Mills, $J$. Mol. Graphics and Modelling, submitted).

\section{Consultants}

Krishnan Balasubramanian, Ph.D., is currently a professor at both the University of California Davis and the Lawrence Livermore National Laboratory and Glenn T. Seaborg Center, Lawrence Berkeley National Lab, Berkeley, CA. Dr. Balasubramanian has been pivotal in our implementation of ab initio calculations using the Gaussian98W software package. He has trained our research team in the use of the software and has served as a resource for further troubleshooting of technical problems. More importantly, he has been advising our group in the selection of quantum chemical basis sets and proper molecular solvation states for calculations based on the electronic nature of the chemicals being studied. Under his guidance we have 
expanded our previous analysis of the set of 55 halocarbons to include even higher levels of quantum theoretic calculations including the 6-311G* and cc-pVTZ basis sets, all with geometric optimization (Basak, Balasubramanian, Gute, Mills, Gorczynska and Roszak, J. Chem. Inf. Comput. Sci, submitted). Dr. Balasubramanian is currently working on extremely intensive calculations for a small subset of the 55 halocarbons.

Milan Randić, Ph.D., is the Ellis \& Nelle Levitt Professor Emeritus for the Department of Mathematics \& Computer Science at Drake University, in Des Moines, Iowa. We have worked closely with Dr. Randić over the past six months to expand on the group of biodescriptors based on proteomics maps developed during the previous project. This effort has been successful and has been well received by the scientific community. Our continuing work on developing novel biodescriptors has been presented at five international conferences to-date and has resulted in 11 manuscripts in varying states of publication to date with several more currently in progress. Appendix 1.5 presents a complete bibliography of proteomics papers developed by our group and our collaborators.

Marjan Vračko, Ph.D., is a senior scientist at the National Institute of Chemistry, in Ljubljana, Slovenia. He has worked with the NRRI team in the development of spectrum-like descriptors for proteomics maps and has contributed to our previous work on the creation of biodescriptors to characterize proteomics maps (Randić, Witzmann, Vračko and Basak, Med. Chem. Res., 2001). A manuscript describing our work to-date on spectrum-like biodescriptors was recently submitted (Vračko and Basak, J. Proteom. Res., submitted).

Frank Witzmann, Ph.D., is a Professor of Cellular \& Integrative Physiology and Biochemistry \& Molecular Biology, at the Indiana University School of Medicine. Without Dr. Witzmann's assistance and specialized knowledge, this project would not be possible. He has provided proteomics data, in the form of standardized proteomics 'spot' maps and has served as an advisor in the use and interpretation of this data. None of the proteomics related manuscripts listed above would have been possible without the high-quality data that he has made available to our research team. We eagerly await more data from his laboratory.

\section{Honors and Awards}

\section{Advisory/Organizational Positions Held by Dr. Basak}

1. Co-chair of the Indo-US Workshop in Mathematical Chemistry, an on-going biennial conference series designed to promote an open exchange of scientific ideas between international scholars and with an emphasis on encouraging young Indian and American scientists (since 1997).

\section{Invited Lectures and Seminars Presented by Dr. Basak}

1. Estimation of Toxicological and Ecotoxicological Properties of Chemicals from Structure: $A$ Mathematical-cum-Computational Approach, presented to the faculty and students of the Toxicology Program of the University of Minnesota, broadcast from Duluth, MN (March 2002).

2. Numerical Graph Invariants: Development and Applications in Drug Discovery and Risk Assessment of Chemicals, presented to the Department of Mathematics and Statistics, University of Minnesota Duluth, Duluth, MN (March 2002). 
3. Interrelationship of Major Topological Indices, co-authored by B.D. Gute and A.T. Balaban, was presented at the Joint Annual Meeting of the Society of Environmental Toxicology and Chemistry (Midwest Chapter) and Society of Toxicology (Northland Chapter) organized at the USEPA Mid- Continental Ecology Division, in Duluth, MN (April 2002).

4. Development of New Tools for Quantitative Characterization of Proteomics Maps, at the symposium Development and "Application of Ecogenomics for Water Quality Assessment" organized jointly by the Council of State Governments and the United States Environmental Protection Agency, in Kansas City, MO (May 2002).

5. Chemodescriptors Versus Biodescriptors in Toxicity Prediction of Halocarbons, co-authored by B.D. Gute, D. Mills, K. Balasubramanian (UC - Davis), K. Geiss (Wright Patterson Air Force Base, Dayton, OH), D. Hawkins (U of MN, TC Campus), M. Randić (NRRI and National Institute of Chemistry, Ljubljana, Slovenia), F. Witzmann (Indiana University School of Medicine), and M. Vračko (National Institute of Chemistry, Ljubljana, Slovenia), was presented at the QSAR 2002 Conference, May 25-29, Ottawa, Canada.

6. Predicting Toxicity of Chemicals in the Post-Genomic Era: A Computational Approach, delivered to the Department of Molecular Biology and Genetics, at the University of Guelph, Guelph, Ontario, Canada (June 2002).

7. Predicting Bioactivity of Chemicals from Structural and Proteomics-Based Descriptors, presented at the University of Texas Medical Branch (UTMB) sponsored jointly by UTMB and Department of Marine Sciences, Texas A \& M University at Galveston, Galveston, TX (June 2002).

8. Dr. Basak gave the following invited seminar lectures on predictive toxicology, QSAR and Mathematical Chemistry during his recent trip to India:

a. Predicting therapeutic activity, health hazard, and ecotoxicity of chemicals using structural and and proteomics based descriptors: An integrated approach, July 26, 2002, at the Bose Institute, Kolkata, India;

b. A two part lecture on Predicting bioactivity and toxicity of chemicals from structure and proteomics based descriptors at the Department of Biochemistry, Calcutta University, Kolkata, August 2, 2002;

c. A two part lecture on Use of discrete mathematics in chemistry, drug design and ecotoxicology, "Part I: Molecular descriptors and QSAR," August 7, 2002; "Part II: Molecular similarity and integrated QSAR in predictive toxicology," August 8, 2002, at the Sivatosh Mookerjee Science Center, Kolkata, India;

d. An invited lecture on Integration of chemoinformatics and bioinformatics for drug discovery and environmental protection: An integrated approach in the post-genomic era, at the Indian Institute of Chemical Biology, Kolkata;

e. Use of mathematical invariants in drug discovery and toxicology in the post-genomic era: An in silico approach. 


\section{Key Findings/Results/Accomplishments}

\section{Biodescriptors}

Development and applications of biodescriptors for predictive toxicology by our NRRI team has been expanded to incorporate three major types of biodescriptors: a) global descriptors from invariants of matrices associated with proteomics maps, b) a set of local invariants describing various aspects of each map (instead of one global biodescriptor), and c) spectrum-like descriptors for the characterization of proteomics patterns.

\subsection{Global Biodescriptors}

Invariants of matrices (e.g. D/ D matrix) and their higher power Kronecker products have been used to develop profiles for proteomics patterns for hepatocytes exposed to eight halocarbons (Basak et al, QSAR 2002 Conference, presented). This data was obtained from Dr. Frank Witzmann and is derived from hepatocytes exposed to halocarbons at the WPAFB, Dayton, Ohio, by Frazier, Geiss and coworkers. The profile given in Figure 1 shows that, even for the structurally similar halocarbons, the profiles for the entire set of eigenvalues and their higher powers are non-overlapping. This indicates that these biodescriptors have no degeneracy for the set of eight compounds tested thus far. It would be interesting to see whether this pattern holds when the set of halocarbons is expanded by the future addition of data on an expanded set of halocarbons.

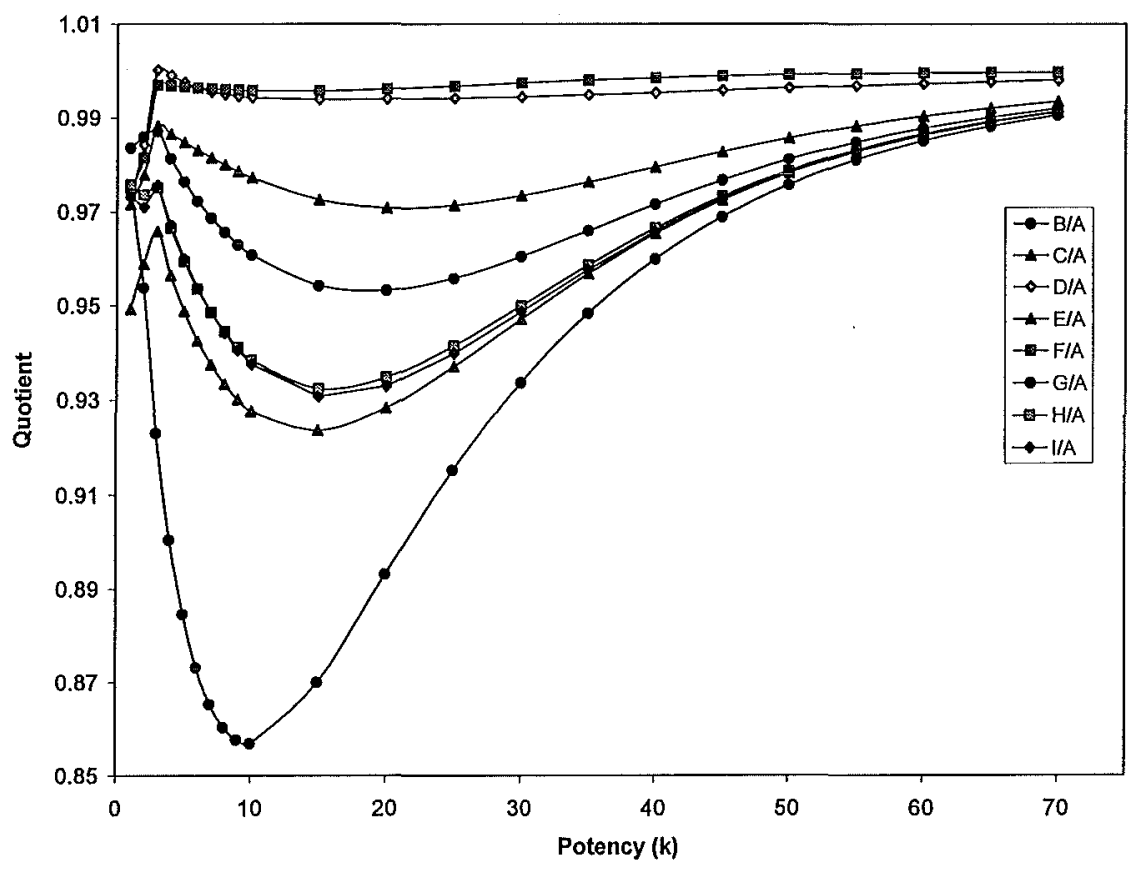

Figure 1. Patterns of the eigenvalues for matrices of eight halocarbons and their Kroenecker products.

\subsection{Local Biodescriptors}

Local biodescriptors are derived from the consideration of a smaller subset of spots than those used in formulating global descriptors. Smaller subsets of $m$ spots are used sequentially instead of using all $n$ spots simultaneously, $m<<n$ (Randić, Zupan, Novič, Gute and Basak, SAR \& QSAR Environ. Res., accepted). The importance of local 
invariants in contrast to global biodescriptors is that the former indices can be used to quantify effects of selected subsets of proteins related to a particular toxicological mode of action, e.g., peroxisome proliferation, apoptosis, etc.

\subsection{Spectrum like biodescriptors of proteomics maps}

For our purposes a proteomic map is considered as a set of points in 3D space where each point represents a spot in the map. The $x$ and $y$ coordinates determine the position of the spot while the $z$ coordinate coresponds to protein abundance (or intensity). Such a pattern of points in 3D space can be represented with three spectrum-like objects. A representation is constructed in three steps. First, the pattern is projected on the $x y, x z$, and yz planes, respectively (see Figure 2). In the second step, each projection (or figure) is treated separately. A figure is placed into a circle of arbitrary radius. A projection beam from the center of a circle produces a pattern of points on the circle where each point represents a particular point. In the third step, each point on the circle is taken as a center for a Lorentzian curve of the form:

$$
f_{i}\left(\varphi_{i}\right)=\frac{\rho_{i}}{\left(\varphi-\varphi_{i}\right)^{2}+\sigma_{i}^{2}}
$$

Here, $\rho_{l}$ and $\varphi_{i}$ are the distances between the origin of the coordinate system and the position of the $i$ th point and its polar angle, respectively. $\sigma_{l}$ is a free parameter, which can be associated with any property given to the point. If we consider only positions of proteomic peaks, the $\sigma_{i}$ 's are set to one. The spectrum related to the figure is the sum of all atomic Lorentzians and it is defined in the interval $(0,2 \pi)$. An example of three projections using a pattern comprised of five points is shown in Figure 3 . The complete pattern of points in the 3D space is represented with three spectra, each of them associated with the $x y, x z$, or $y z$ projection, respectively. By selecting $k$ equidistant points on the interval, one figure is represented by a $k$-dimensional vector. The value of $k$ determines the resolution of the representation. It is obvious that $k$ should be close to the number of points in the proteomic map, otherwise some information is lost. Finally, the three spectrum-like objects are compressed into a single vector $\vec{v}$.

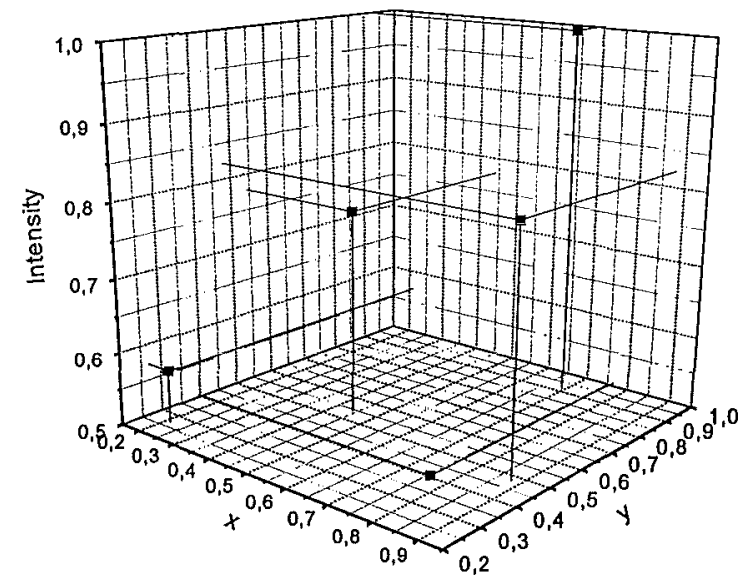

Figure 2. Five arbitrarily selected spots of reference proteomic map. The red, yellow and blue lines are projection lines on $x y, x z$ and $y z$ planes, respectively. 
A
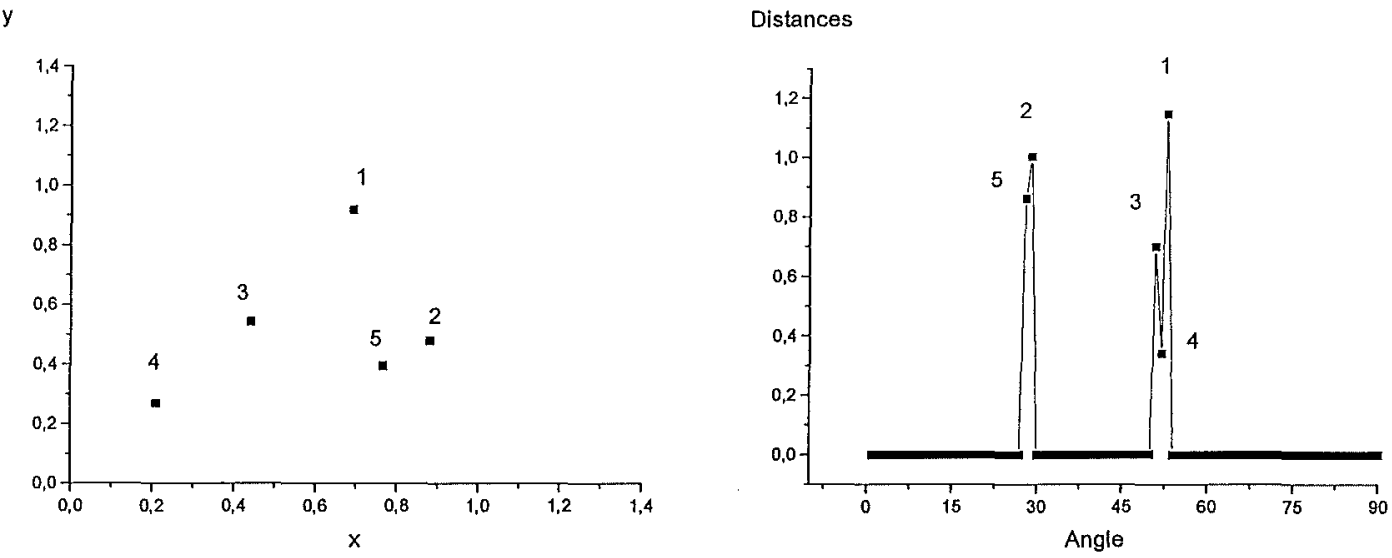

B

Intensity

Distances
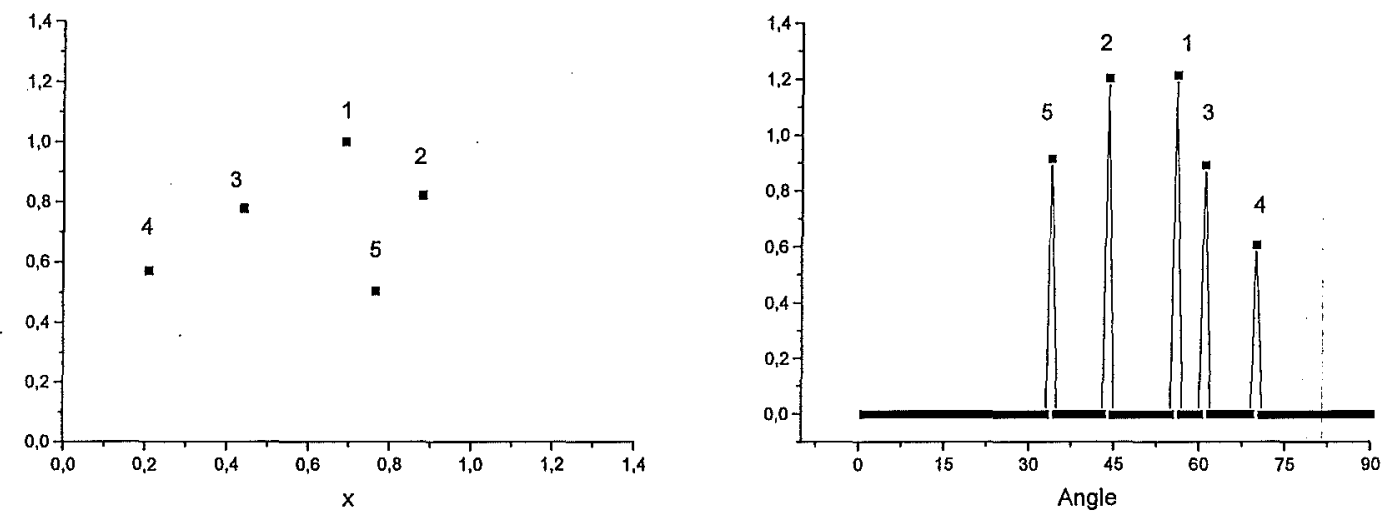

C
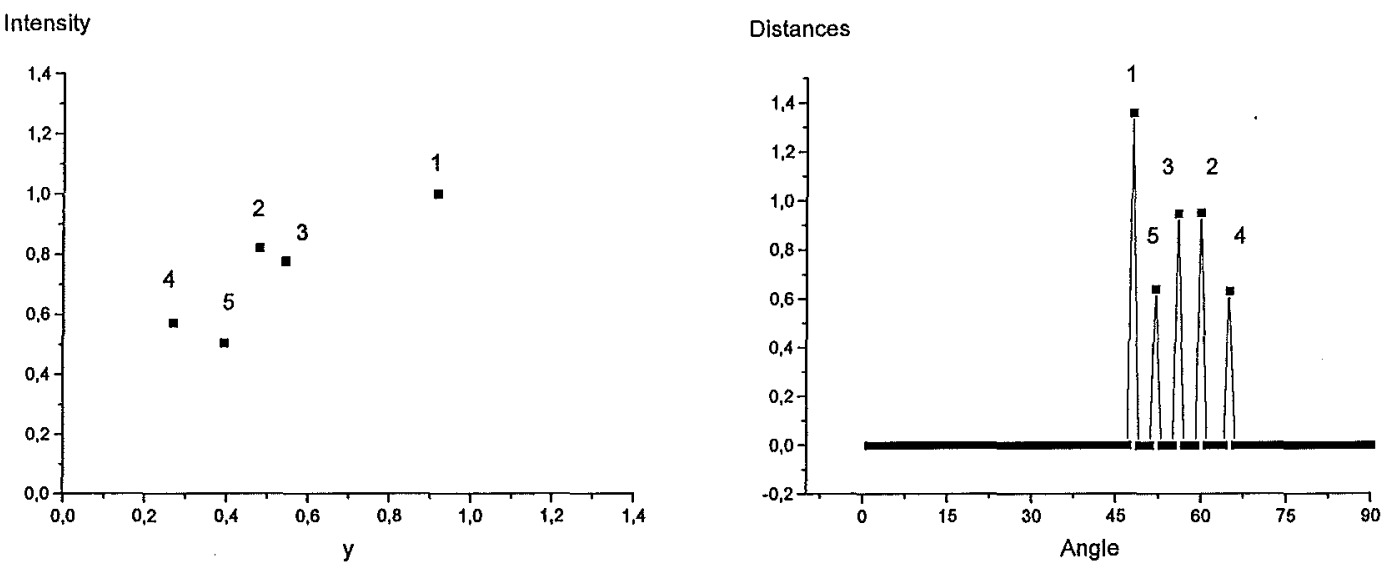

Figure 3: $\quad$ A: $x y$ projection and corresponding 'spectrum-like' object

B: $x z$ projection and corresponding 'spectrum-like' object

C: yz projection 


\section{Characterization of Macromolecular Structure}

It should be mentioned that our work on the development of biodescriptors for proteomics maps is a necessary extension of our research on the characterization of small molecules and biomolecules using discrete mathematics. We have continued ours efforts in this area in addition to our work on developing biodescriptors. In the area of DNA sequence characterization, we have developed methods for the characterization of single nucleotide polymorphism (SNP) using numerical sequence descriptors (Nandy, Nandy and Basak, Internet Electron. J. Mol. Des., 2002). This work has great potential for transition in view of the fact that more and more genomics data are being amassed and they need to be compared and stored efficiently by the scientific community.

Continuing this line of effort with the storage and retrieval of proteomics maps, a method for the efficient storage of these maps has been developed based on the partial ordering of the spots of maps and their unique canonical numbering (Randic and Basak, J. Chem. Inf. Comput. Sci., in press).

\section{Chemodescriptors and Computational Toxicology}

\subsection{Mechanism-based HiQSAR for Predictive Toxicology}

A successful HiQSAR model has been developed for a set of 55 halocarbons for which cellular level toxicity data is available. It may be noted that this is the "superset" of compounds from which the "subset" of twenty halocarbons, currently being tested by WPAFB and Dr. Witzmann using the DNA microarray and proteomics analysis, was selected. Selection of parameters for the HiQSAR was based on the mechanistic hypothesis that dissociate electron attachment and subsequent formation of free radicals, leading to lipid peroxidation, is a major factor in halocarbon toxicity. This conclusion was derived from Dr. Balasubramanian's previous research, based on high-level quantum chemical calculations. If the hypothesis is correct, calculated parameters such as vertical electron affinity (VEA) should be strongly related to a chemical's toxicity. Accordingly, we used the following classes of indices in a hierarchical manner to develop QSAR models for the toxicity of 55 halocarbons:

Topostructural (TS), topochemical (TC), geometrical (3-D), semi-empirical quantum chemical (AM1), and calculations using five ab initio basis sets (STO-3G, 6-31G(d), 6311G, 6-311G* and cc-pVTZ).

Results of this analysis are summarized below in Table 1 (Basak, Balasubramanian, Gute, Mills, Gorczynska and Roszak, J. Chem. Inf. Comput. Sci., submitted).

It is evident from these results that only higher level $a b$ initio indices (6-311G* and cc-pVTZ) made significant improvement in model quality over topological indices for the prediction of toxicity. The lower level $a b$ initio and semi-empirical indices did not make any improvement over the models developed by the topostructural and topochemical indices. It may be noted that even with the addition of these high-level $a b$ initio parameters, the easily calculable topological indices outperformed all other types of molecular descriptors in predicting toxicity from structure. This shows the utility and robustness of these simple descriptors. 
Table 1. Summary results for the HiQSAR modeling of $D_{37}$ in Aspergillus nidulans for 55 halogenated aliphatic hydrocarbons.

\begin{tabular}{lccccc} 
Model & $\begin{array}{c}\text { \# indep. } \\
\text { variables }\end{array}$ & $\boldsymbol{R}^{\boldsymbol{2}}$ & $\boldsymbol{R}_{c \boldsymbol{V}}{ }^{2}$ & $\boldsymbol{s} \boldsymbol{e}$ & $\boldsymbol{F}$ \\
\hline TSI only & 2 & 0.3659 & 0.2945 & 1.243 & 15.00 \\
TCI only & 8 & 0.8623 & 0.7749 & 0.6161 & 36.00 \\
3D only & 8 & 0.8838 & 0.6496 & 0.5660 & 43.72 \\
AM1 only & 3 & 0.4591 & 0.3008 & 1.159 & 14.43 \\
STO-3G only & 3 & 0.3055 & 0.1624 & 1.314 & 7.48 \\
6-31G(d) only & 4 & 0.4111 & 0.2458 & 1.222 & 8.73 \\
6-311G only & 4 & 0.5140 & 0.3853 & 1.110 & 13.22 \\
6-311G* only & 4 & 0.6318 & 0.5053 & 0.9663 & 21.45 \\
cC-pVTZ only & 1 & 0.5099 & 0.4787 & 1.083 & 55.14 \\
TSI + TCI & 7 & 0.8791 & 0.8074 & 0.5710 & 48.83 \\
TSI + TCI + 3D & Sam as previous model & & & \\
TSI + TCI + AM1 & 7 & 0.8840 & 0.8121 & 0.5594 & 51.17 \\
TSI + TCI + STO-3G & 7 & 0.8724 & 0.7923 & 0.5866 & 45.91 \\
TSI + TCI + 6-31G(d) & 7 & 0.8713 & 0.7926 & 0.5893 & 45.45 \\
TSI + TCI + 6-311G & 10 & 0.9015 & 0.8283 & 0.5328 & 40.26 \\
TSI + TCI + 6-311G(d) & 8 & 0.9056 & 0.8335 & 0.5100 & 55.17 \\
TSI + TCI + aug-cC-pVTZ & 8 & 0.9476 & 0.9236 & 0.3800 & 103.98 \\
\hline
\end{tabular}

\subsection{Hierarchical QSAR}

We have continued our work exploring the various levels of parameters in the development of QSARs for a wide variety of properties (Basak, Mills, Gute and Hawkins, in Quantitative Structure-Activity Relationship (QSAR) Models of Mutagens and Carcinogens, in press; Basak, Balasubramanian, Gute, Mills, Gorczynska and Roszak, J. Chem. Inf. Comput. Sci., submitted).

\subsection{Estimation of PBPK parameters}

Two distinct factors are involved in the causation of toxicity: a) toxicokinetics and b) toxicodynamics. While QSAR for toxicity endpoints can estimate the potential hazard of the pollutant, the extent of exposure determines the bioavailability of the chemical in the biophase and determines the actual risk from the chemical. Physiologically-based pharmacokinetic (PBPK) modeling is used routinely to estimate the availability of chemicals to various biotargets within the organism. These models need a variety of properties as input parameters. Blood:air and tissue:air partition coefficients fall into the category of parameters essential for PBPK modeling. We have developed HiQSARs to estimate blood:air and tissue:air partition coefficients of chemicals using structural parameters which can be calculated directly from the chemical structure without the need for the input of any other experimental data (Basak, Hawkins and Mills, in Advances in Molecular Similarity, in press; Basak, Mills, Hawkins and El-Masri, SAR QSAR Environ. Res., in press; Basak, El-Masri, Hawkins and Mills, J. Chem. Inf. Comput. Sci., submitted; Basak, Mills, Hawkins and El-Masri, Risk Analysis, submitted). Such models are expected 
to be very useful to the PBPK modeling community.

\subsection{Tailored Similaríty}

Our work on developing and refining quantitative molecular similarity analysis (QMSA) methods also continues. One recent addition to the field by our laboratory is the concept of tailored or property-specific QMSA methods (Basak, Gute and Mills, SAR QSAR Environ. Res, in press; Basak, Gute, Mills and Hawkins, J. Mol. Struct. THEOCHEM, in press; Gute, Basak, Mills and Hawkins, Internet Electron. J. Mol. Des., in press). Whereas ordinary similarity methods are based on an arbitrary set of descriptors or at best a set of parameters chosen subjectively based on previous experience of the scientist, in tailored QMSA we first choose a subset of indices strongly correlated with the property and then use them to created the $n$-dimensional space used to measure the structural similarity/ dissimilarity of molecules. Results show that tailored QMSA (T-QMSA) outperforms standard (or arbitrary) QMSA methods. It is expected that this novel methodology will find wide applications both in drug design and predictive toxicology and will lead to substantial technology transfer.

\subsection{Statistical Methods for QSAR Model Validation}

Development of useful quantitative structure-activity/ toxicity relationship (QSAR/QSTR) models has to be based on sound statistical methodology. Our group has been involved in the formulation of methods for the validation of QSAR models (Hawkins, Mills and Basak, submitted). It is expected that such methods will be used widely in pharmaceutical and toxicological QSAR development.

\section{Proposed Research Plan for 2002-2003}

\subsection{Global Biodescriptors}

4.1.1 The current set of global biodescriptors will be used to characterize an expanded set of halocarbons to be provided by Dr. Witzmann.

4.1.2 Now that we have established a set of biodescriptors, we will begin to study the utility of combining chemo- and biodescriptors in HiQSAR for toxicity prediction.

\subsection{Local Biodescriptors}

4.2.1 Local biodescriptors will be used to characterize the toxic effects of peroxisome proliferators on proteomics maps.

4.2.2 Local biodescriptors will be used to characterize the toxic effects of halocarbons on proteomics maps.

\subsection{Spectrum-like Biodescriptors}

4.3.1 Local biodescriptors will be used to characterize the toxic effects of peroxisome proliferators on proteomics maps.

4.3.2 Local biodescriptors will be used to characterize the toxic effects of halocarbons on proteomics maps.

\subsection{Development of Novel Matrix Invariants}

4.4.1 Novel matrix invariants for the characterization of proteomics maps will be developed based on the 3D-Wiener number.

4.4.2 Novel matrix invariants for the characterization of proteomics maps will be 
developed based on information theoretic indices.

\subsection{Characterization of the Similarity of Proteomics Maps}

An $n$-dimensional biodescriptor space will be developed using global, local, and spectrum-like biodescriptors. Attempts will be made to use standard similarity measures to characterize this space.

\subsection{Mechanism-based HiQSAR}

4.6.1 The greater success of the aug-cc-pVTZ indices indicates that more sophisticated or higher level quantum chemical indices might better reflect the electronic basis of the toxicity of these halocarbons. To this end, we will run $a b$ initio calculations at the $\operatorname{CCSD}(T)$ level for a subset of the 55 halocarbons, however this can only be a small subset since is it prohibitively time consuming to do so for the entire set. This work will allows us to investigate how far the success of the less expensive parameters can be vindicated using higher level parameters.

4.6.2 Calculations on solvated molecules, as opposed to the standard gas phase calculations, will be conducted to see whether such involved calculations give better models or shed some light in the toxic modes of action of halocarbons.

\subsection{Tailored Similarity}

4.7.1 Further work will be done to continue developing the tailored molecular similarity method and to examine its applications for the estimation of toxicologically- and pharmaceutically-relevant properties. In particular we will be examining the usefulness of the method for estimating halocarbon toxicity and for analog selection within a proprietary Astra-Zeneca pharmaceutical database.

4.7.2 We will examine the application of the tailored approach to dissimilarity analysis and chemical clustering. Specifically, we will use the JP-8 database as an interesting example for cluster analysis.

\subsection{Statistical Method Development}

We plan to explore the development and implementation of novel statistical methods for microarray and proteomics analysis.

\section{Transitions/Technology Transfers}

\section{Contribution to Computational Toxicology Program}

The hierarchical QSAR approach for modeling toxicity (HIQSTR) developed by our research team has been adopted into the curriculum of the Masters coursework for the Predictive Toxicology program of the Environmental Sciences Department of the University of Calcutta, Kolkata, India. In addition, they have incorporated various molecular similarity methods, and just recently as a result of Dr. Basak's most recent visit to India, they have decided to add our recently developed tailored approach to molecular similarity (T-QMSA) into the curriculum as well.

\section{Cooperative Work with Astra-Zeneca}

We have recently begun a collaborative work with Dr. Indira Ghosh of Astra-Zeneca. We 
will be applying our newly developed tailored quantitative molecular similarity analysis (T-QMSA) techniques to a large proprietary drug discovery or pharmaceutical database provided by Astra-Zeneca. 


\section{Appendix 1: Publications/Reprints}

Appendix 1.1: Manuscripts on the development of biodescriptors for proteomics maps

A comparative study of proteomics maps using graph theoretical biodescriptors, M. Randić and S. C. Basak, J. Chem. Inf. Comput. Sci.

Novel matrix invariants for characterization of changes of proteomics maps, $M$. Randić, J. Zupan, M. Novič, B.D. Gute and S.C. Basak, SAR QSAR Environ. ReS,

On canonical labeling of proteins of proteomics maps, M. Randić and S.C. Basak, J. Chem. Inf. Comput. Sci.

Appendix 1.2: Prediction of cellular toxicity of halocarbons from their computed chemodescriptors: A hierarchical QSAR approach, S.C. Basak, K. Balasubramanian, B.D. Gute, D. Mills, A. Gorczynska and S. Roszak, J. Chem. Inf. Comput. Sci.

Appendix 1.3: Manuscripts on tailored similarity (T-QMSA)

Quantitative molecular similarity analysis (QMSA) methods for property estimation: A comparison of property-based, arbitrary, and tailored similarity spaces, S.C. Basak, B.D. Gute and D. Mills, SAR QSAR Environ. Res.

Quantitative molecular similarity methods in the property/ toxicity estimation of chemicals: A comparison of arbitrary versus tailored similarity spaces, S.C. Basak, B.D. Gute, D. Mills and D.M. Hawkins, J. Mol. Struct. THEOCHEM.

Tailored similarity spaces for the prediction of physicochemical properties, B.D. Gute, S.C. Basak, D. Mills and D.M. Hawkins, Internet Electron. J. Mol. Des., http://www.biochempress.com/.

Appendix 1.4: Manuscripts on PBPK modeling

Predicting blood:air partition coefficient of structurally diverse chemicals using theoretical molecular descriptors, S.C. Basak, D.M. Hawkins and D. Mills, in Advances in Molecular Similarity, R. Carbo-Dorca, P.G. Mezey, Eds., vol. 5.

Prediction of tissue:air partition coefficients: A comparison of structure-based and property-based methods, S.C. Basak, D. Mills, D.M. Hawkins and H.A. El-Masri, SAR QSAR Environ. Res.

Exposure assessment of volatile organic chemicals (VOCs): Predicting blood:air partition coefficients of diverse chemicals using theoretical descriptors, S.C. Basak, H. El-Masri, D.M. Hawkins and D. Mills, J. Chem. Inf. Comput. SCi.

Prediction of human blood:air partition coefficient: A comparison of structurebased and property-based methods, S.C. Basak, D. Mills, D.M. Hawkins and H. El-Masri, Risk Analysis.

Appendix 1.5: Bibliography of papers on development of proteomics-based biodescriptors 
Appendix 1.1

Manuscripts on the development of biodescriptors for proteomics maps 


\title{
A Comparative Study of Proteomics Maps Using Graph Theoretical Biodescriptors
}

\author{
Milan Randić ${ }^{\dagger}$ and Subhash C. Basak ${ }^{*, \neq}$ \\ National Institute of Chemistry, Ljubljana, Slovenia, Department of Mathematics and Computer Science, \\ Drake University, Des Moines, Iowa 50311, and Natural Resources Research Institute, University of \\ Minnesota at Duluth, 5013 Miller Trunk Highway, Duluth, Minnesota 55811
}

Received August 10, 2001

\begin{abstract}
This paper reports the development of new methods for mathematical characterization of effects of different toxic agents on the cellular proteome. We describe numerical characterization of proteomics maps based on mathematical invariants. A graph is first associated with a proteomics map by considering partial ordering of spots on 2-D gels by ordering proteins with respect to the mass and the charge, the two properties by which proteins are separated. The graph is then embedded over the map, and several graph theoretical invariants have been constructed. In particular we consider invariants that can be extracted from the Euclidean distance-adjacency matrix of the embedded graph, in which only Euclidean distances between adjacent vertices of a graph are considered. The approach is illustrated using proteomics patterns of normal liver cells of rats and those derived from liver cells of animals exposed to four peroxisome proliferators. In contrast to direct comparison of spot abundance our approach incorporates information on spots locations. The difference between the two approaches is that in the first case only changes in abundances are considered as a measure of perturbation of the proteome map, but in the second case not only the charge but also the mass of proteins are used for ordering protein spots.
\end{abstract}

\section{INTRODUCTION}

In this paper we have attempted the development of novel numerical descriptors for characterization of proteomics maps, that could facilitate comparison of proteomics data, whether from a single laboratory or from different laboratories. A typical proteomics map may have 2000 and more separated proteins that appear as spots on a 2-D gel in which they are separated by charge ( $x$-axis) and mass ( $y$-axis). When an experimental animal is exposed to drugs or toxins, the protein content of cells usually change. As a consequence the relative abundance of various proteins that characterize a normal cell is perturbed resulting in an altered proteomics map. To facilitate comparison of such changes we want to characterize individual proteomics maps by a set of map descriptors, which are mathematical invariants, derived from proteomics patterns.

Information contained in a map can be (and often is) represented as a list of coordinates and abundance of individual spots, as illustrated in Table 1 for 20 most intensive spots from the liver of normal rats and those exposed to four toxic chemicals. Figure 1 shows the positions of the 20 most abundant protein spots labeled A-T based on information in the columns listing coordinates $x, y$ of Table 1. We consider the information in Table 1 as input information, and our goal is to arrive at some numerical descriptors that characterize a map as a whole so that they can be used for quantitative comparison of different proteomics maps. What makes our approach different from a direct comparison of spot abundance, as shown in different

* Corresponding author phone: (218)720-4230; fax: (218)720-4328; e-mail: sbasak@nrri.umn.edu.

$\uparrow$ National Institute of Chemistry and Drake University.

¥ University of Minnesota at Duluth.
Table 1. Gel Coordinates and Abundance for the 20 Most Intensive Protein Spots of Rat Liver Cells' of the Normal Cells and the Corresponding Abundance for Rat Liver Cells Exposed to Four Chemicals

\begin{tabular}{lrrrrrrr}
\hline$\#$ & \multicolumn{1}{c}{$x$} & \multicolumn{1}{c}{$y$} & control & PFOA & PFDA & clofibrate & DEHP \\
\hline A & 2111.7 & 2278.6 & 144357 & 108713 & 95028 & 147081 & 165886 \\
B & 2804.3 & 903.6 & 143630 & 155565 & 188582 & 159898 & 155055 \\
C & 1183.9 & 959.6 & 136653 & 113859 & 150253 & 163645 & 8111 \\
D & 2182.2 & 928.8 & 127195 & 99160 & 73071 & 76642 & 112096 \\
E & 2685.6 & 1196.1 & 118581 & 112790 & 49769 & 109856 & 138795 \\
F & 1527.9 & 825.5 & 114929 & 192437 & 221567 & 166080 & 180590 \\
G & 1346.0 & 1352.5 & 112251 & 58669 & 38915 & 73159 & 77075 \\
H & 2868.5 & 778.0 & 108883 & 26105 & 50735 & 45923 & 116849 \\
I & 1406.3 & 1118.1 & 98224 & 91147 & 82963 & 84196 & 92942 \\
J & 2450.2 & 409.2 & 93601 & 83172 & 62934 & 79870 & 109381 \\
K & 1474.0 & 665.1 & 90004 & 129340 & 112361 & 112655 & 119402 \\
L & 2974.9 & 772.8 & 86730 & 70746 & 78691 & 105760 & 116281 \\
M & 2068.4 & 823.1 & 84842 & 73814 & 45482 & 71911 & 97444 \\
N & 642.2 & 669.8 & 82492 & 73974 & 74466 & 84703 & 88545 \\
O & 2860.7 & 1649.9 & 81965 & 16137 & 16501 & 60077 & 148992 \\
P & 2032.7 & 902.8 & 80015 & 77314 & 80072 & 76027 & 100836 \\
Q & 2752.7 & 765.6 & 79847 & 20782 & 13103 & 38816 & 53830 \\
R & 2334.2 & 982.2 & 72791 & 76369 & 52749 & 55599 & 77432 \\
S & 1053.6 & 864.3 & 72173 & 77982 & 60376 & 46808 & 78121 \\
T & 2519.5 & 1365.9 & 69452 & 37838 & 16129 & 57167 & 71274 \\
\hline
\end{tabular}

columns of Table 1 , is that the approach incorporates besides the information on the abundance also the information on spot locations. The difference between the two approaches is that in the first case only changes in abundances are considered as a measure of perturbation of a proteomics map, but in the second case also locations of protein spots, i.e., the charge and mass of proteins, make an influence.

Before continuing with details we need to respond to the question: "Why the 20 most abundant proteins are chosen for analysis?" The answer is that for the development of a mathematical approach neither the number of selected points 


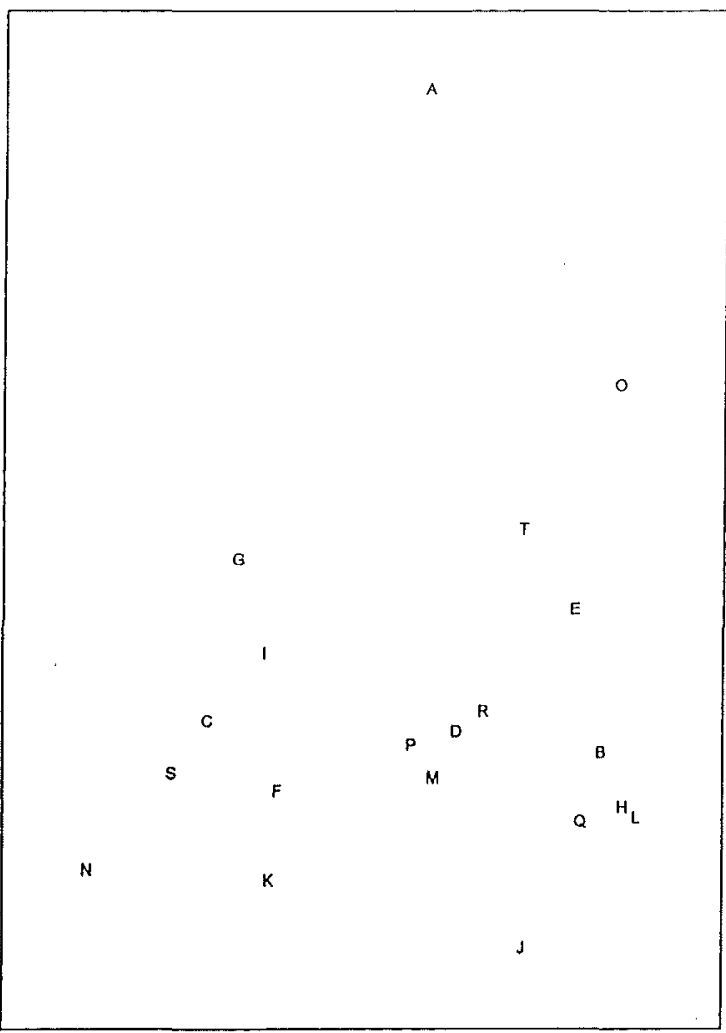

Figure 1. The position of 20 most abundant protein spots of rat liver cells labeled alphabetically.

nor the criteria of selection is essential. Because of the wide variability of experimental data on proteomics in general we focused on the most abundant proteins. Whether 20 most abundant proteins suffice to represent a map as a whole or one should select 50 , or 200 , remains yet to be better explored. Equally it remains to be seen if the selection of a subset of $\mathrm{N}$ proteins rather than all of them could be justified. If $\mathrm{N}$ is sufficiently large (and that may be 20,50 , or 200) one hopes that such subsets may capture the most features of a map that may suffice for comparison of different proteomics maps.

A proteomics map invariant is a quantity that is independent of the coordinates and the labels of the proteins in proteomics maps or 2-D gels. Why is it so important for mathematical descriptors to be independent of the adopted protein labels? A quantity independent of the arbitrary labeling of proteins characterizes a system as a whole and does not pertain to any of its local features or any particular protein. To be more correct we should say that such characterization pertains to a set of protein spots selected as the basis for numerical characterization of a map. Hence, map invariants are properties of the cell/tissue considered as represented by a group of proteins as a whole and not of any particular protein. This, however, in no way prevents one to compare protein expressions across different samples taken from the same species.

It is obvious that we need many invariants if we are to capture the majority of salient features of information-rich proteomics maps. Thus this paper represents a continuation of our recent efforts ${ }^{1-6}$ to develop mathematical characterization of proteomics maps. In particular, in this article we will consider sets of invariants that can be constructed by a procedure outlined in ref 4 which can be viewed as part I of this paper, in which an approach is outlined in which one associates an embedded graph to a proteomics map through partial ordering of spots with respect to their charge and mass.

Because the intensity of a spot in the 2D gel will depend not only on the concentration of proteins but also on the total amount of protein used in running the gel, reliability of experimental data should be known. We used experimental data from a laboratory known for following a rigorous protocol, ${ }^{7}$ and thus we have some confidence that the overall effects of various peroxisome proliferators resulted in reduced overall production or proteins.

\section{PARTIAL ORDERING BASED ON CHARGE AND MASS}

Partial ordering has not been widely used in chemistry despite the fact that it offers a useful tool for comparison of objects characterized by sequences. For example, partial ordering allows one to compare properties of isomers, ${ }^{8,9}$ and it can be used to search for pharmacophore in QSAR studies. ${ }^{10,11}$ For a recent review of use of partial orderings in chemistry see the special issue of MATCH. ${ }^{12}$ In this paper we will use partial order to generate a graph to be associated with a map. Once we have a graph, we can construct various graph matrices, and from such matrices we can construct graph invariants to serve as map descriptors.

To obtain a graph associated with protein spots listed in Table 1, we will order the 20 spots of Table 1 with respect to the charge and the mass. By ordering protein spots with respect to mass we obtain the sequence $A, O, T, G, E, I \ldots$ which gives an ordering in which spots appear when we list them as they appear in going from the top of the map to the bottom, beginning with the protein having the greatest mass and ending with the protein having the smallest mass. As we see at the top of Figure 1, the protein spot A has the largest mass and is followed by spot $\mathrm{O}$, the protein with the second largest mass, and so on. When we order the 20 protein spots with respect to the charge we obtain the sequence $L$, $\mathrm{H}, \mathrm{O}, \mathrm{B}, \mathrm{Q}, \mathrm{E}$... which reflects the order in which protein spots appear if we go from the right (the largest charge) to the left (the smallest charge). As we see from Figure 1 protein $\mathrm{L}$ has the largest charge, followed by protein $\mathrm{H}$, and so on.

Partial ordering is defined as a set of all sequential orderings given by subsets of the two sequences, the elements of which maintain the ordering for both sequences. To obtain all sequential orderings preserved in both sequences it is convenient to construct a diagram, as shown in Figure 2, in which the two sequences are listed, one above the other and lines are drawn that connect the same element in both sequences. Any set of noncrossing lines constitutes a subset of partial ordering, the subsequence the relative order of which is present in both sequences. For example, the lines $\mathrm{O}-\mathrm{O}, \mathrm{E}-\mathrm{E}, \mathrm{R}-\mathrm{R}, \mathrm{D}-\mathrm{D}, \mathrm{P}-\mathrm{P}, \mathrm{F}-\mathrm{F}$, and $\mathrm{N}-\mathrm{N}$ do not cross giving the subsequence $\mathrm{O}>\mathrm{E}>\mathrm{R}>\mathrm{D}>\mathrm{P}$ $>\mathrm{F}>\mathrm{N}$. In Table 2 we have listed all such fragmentary orderings that start with protein spot A. The totality of all fragmentary orderings makes the partial ordering for the selected 20 proteins of the map of Figure 1.

A complete list of all sequences of partial ordering is neither a necessary nor particularly useful representation of the partial ordering sought. It is customary to represent partial 


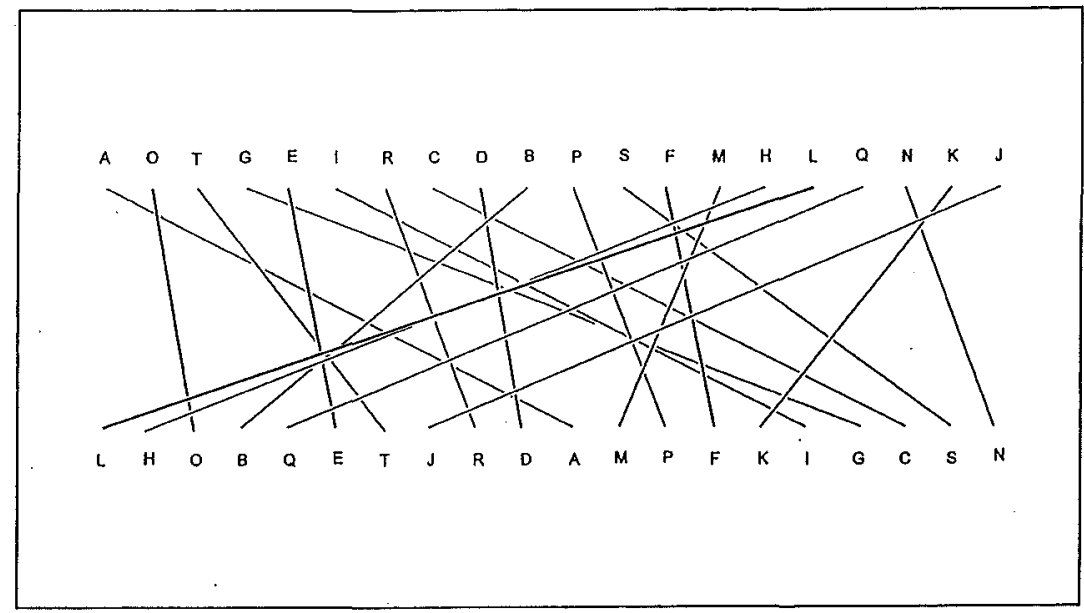

Figure 2. Ordering of proteins of Figure 1 relative to their mass (the top sequence) and their charge (the bottom sequence). The same labels in both sequences are connected by line to help to identify partial orderings.

Table 2. Fragmentary Orderings of Protein Spots Holding with Respect to Charge and Mass Respectively that Start with Protein A

\begin{tabular}{llllll}
\hline 1 & $\mathrm{~A} \rightarrow$ & $\mathrm{G} \Rightarrow$ & $\mathrm{C} \Rightarrow$ & $\mathrm{S} \Rightarrow$ & $\mathrm{N}$ \\
2 & $\mathrm{~A} \Rightarrow$ & $\mathrm{I} \Rightarrow$ & $\mathrm{C} \Rightarrow$ & $\mathrm{S} \Rightarrow$ & $\mathrm{N}$ \\
3 & $\mathrm{~A} \Rightarrow$ & $\mathrm{M} \Rightarrow$ & $\mathrm{K}$ & & \\
4 & $\mathrm{~A} \Rightarrow$ & $\mathrm{M} \Rightarrow$ & $\mathrm{N}$ & & \\
5 & $\mathrm{~A} \Rightarrow$ & $\mathrm{P} \Rightarrow$ & $\mathrm{F} \Rightarrow$ & $\mathrm{K}$ & \\
6 & $\mathrm{~A} \Rightarrow$ & $\mathrm{P} \Rightarrow$ & $\mathrm{F} \Rightarrow$ & $\mathrm{N}$ & \\
7 & $\mathrm{~A} \Rightarrow$ & $\mathrm{P} \Rightarrow$ & $\mathrm{S} \Rightarrow$ & $\mathrm{N}$ & \\
\hline
\end{tabular}

ordering by a directed graph or a hierarchical diagram shown in Figure 3 in which points at the left dominate points to the right. Dominance here means that an element at the left in Figure 3 is also at the left in both sequences of Figure 2. Figure 3 facilitates visual inspection of the partial ordering and immediately shows which protein dominates others by mass and charge or is dominated by others. In making Figure 3 one can place vertices (labels) in arbitrary positions as long as the dominance relation is preserved. Nevertheless, one tries to construct partial ordering diagrams that will have, for better visibility, as few crossings of lines as possible.

The next important step in analysis of proteomics map based on partial ordering is to embed the partial ordering diagram of Figure 3 directly over the map. This is a critical step that has been for the first time considered in relationship to partial ordering in general and proteomics maps in particular in ref 4. Embedding of graphs in 2-D space (plane) or 3-D space in Graph Theory implies that a fixed geometry is assigned to the graph that can no longer be viewed as only giving adjacency relationships between vertices but also giving the distances between linked elements. While Graph Theory ${ }^{13}$ is usually concerned with topological and combinatorial properties of mathematical objects, in the case of embedded graphs it is concerned solely with the geometry and the combinatorial properties of the same objects, while topology of objects is no longer of concern. In Figure 4 we have imposed the adjacency relations of Figure 3 directly over the map of Figure 1, and as a result we obtain a partial ordering graph embedded on the map. Because the protein spots have definite $(x, y)$ coordinates now proteins at the top dominate those below if connected by lines and proteins at the right dominate those at the left if connected by lines.

Observe an important and interesting feature of the embedded graph of Figure 4 in that all the lines (links) in the graph have positive slope. This is a consequence of the dominance relation and is a property which can be exploited for a direct construction of embedded graph for a given map, without a need for construction of Figure 2, listing the various fragmentary orderings, and depicting the hierarchical diagram of the partial ordering, such as Figure 3. To obtain Figure 4 directly from Figure 1 one can start with the top vertex (spot A in Figure 4) and coninect it to the next lower spot to the left of A which is spot G. One can continue the same with vertex $G$ and connect it to the next lower vertex below it and to the left, which is spot $\mathrm{C}$. We continue to connect $\mathrm{C}$ to $S$ and finally $S$ to $N$. By exhausting this particular trail we return to vertex $A$ and repeat the process: we connect $A$ to I and then I to $C$. In the next step, we connect $A$ to $P$ and finally $P$ to $S$. By backtracking we connect $P$ to $F$ and $F$ both to $K$ and $N$, since both $K$ and $N$ are below $P$ and at the left of $P$. Finally we backtrack again to protein $A$ and we connect it to $\mathrm{M}$, which is connected to $\mathrm{K}$ and $\mathrm{N}$. This has exhausted all the fragmentary orders starting with protein spot A, that have been listed in Table 2 . The process continues with spot $\mathrm{O}$, then $\mathrm{L}$ and $\mathrm{H}$, which completes construction of the embedded graph of partial order for the map considered.

\section{THE EUCLIDEAN-ADJACENCY MATRIX $E$ OF A MAP}

The adjacency matrix is a well-known binary matrix of Graph Theory. Its matrix elements are defined as

$$
\begin{gathered}
\mathrm{A}_{i j}=1 \quad \text { if vertices } i \text { and } j \text { are adjacent } \\
\mathrm{A}_{i j}=0 \text { otherwise }
\end{gathered}
$$

The Euclidean-adjacency matrix $E$ is a novel matrix only recently suggested, ${ }^{13}$ that is defined only for embedded graphs (because only embedded graph in 2-D or 3-D space have fixed geometry). Its matrix elements are defined by

$$
\begin{gathered}
\mathrm{E}_{i j}=\text { Euclidean distance if vertices } i \text { and } j \text { are adjacent } \\
\qquad \mathrm{E}_{i j}=0 \text { otherwise }
\end{gathered}
$$

Hence, the difference between A and $\mathrm{B}$ matrices is only in the magnitudes of the nonzero entries, which in the former 


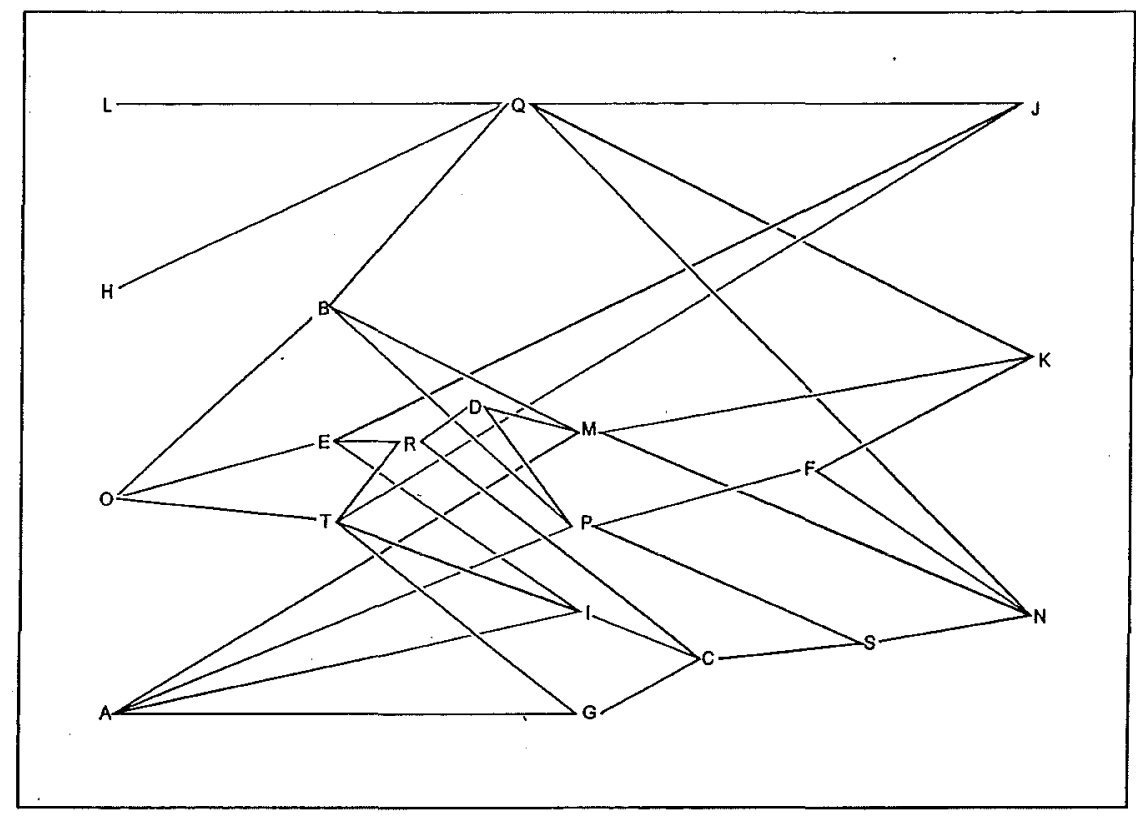

Figure 3. Graphical representation of the partial ordering of Figure 1.

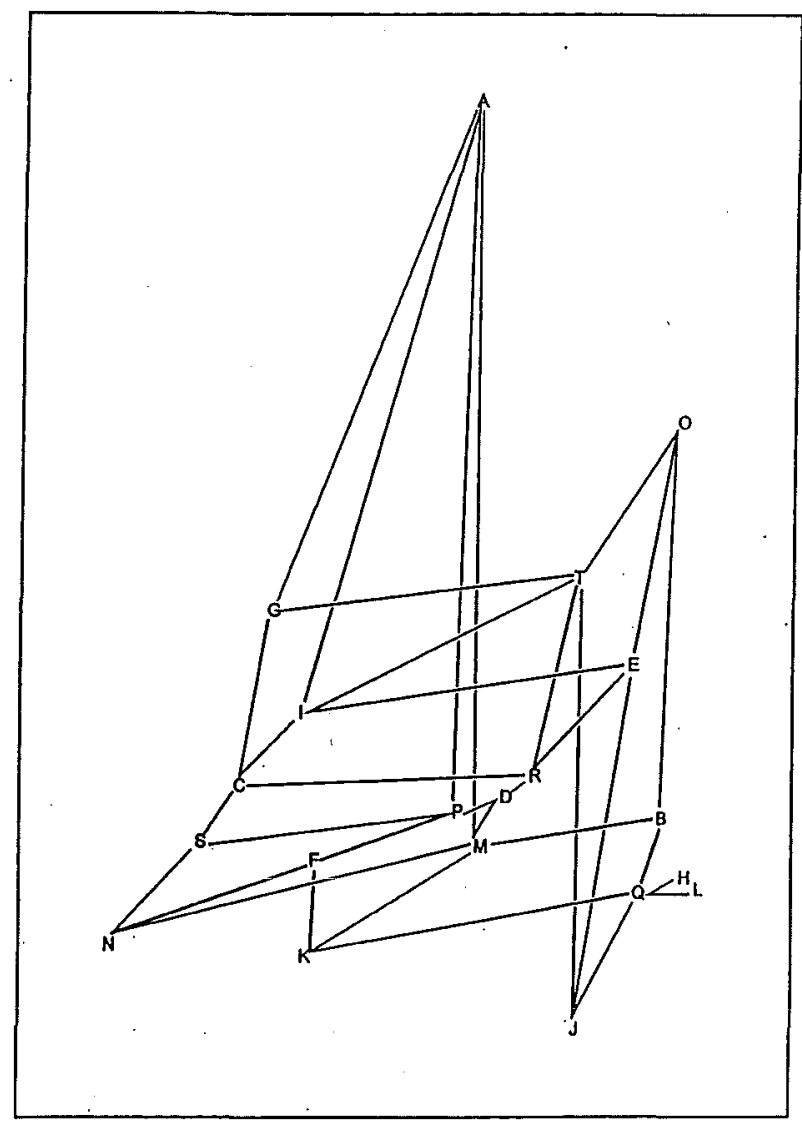

Figure 4. The adjacency relationships of Figure 3 embedded upon the proteomics map of Figure 1 that results in embedded graph of the partial order of proteins with respect to mass and charge.

take the value 1 and in the latter assume numerical values determined by the distance between points considered.

The embedded graph of Figure 4 is the starting point for our mathematical characterization of proteomics maps. First, in view of the fact that that $x, y$ coordinates represent
Table 3. Reported Distances, Normalized Distances between Adjacent Spots, as Defined by the Partial Order for the 20 Intensive Protein Spots of Normal Rat Liver Cells, as the Corresponding Matrix Elements of Euclidean-Adjacency Matrix

\begin{tabular}{llrrrl:rr}
\hline $\mathrm{E}_{i j}$ & line & distance & norm & \multicolumn{1}{c}{$\mathrm{E}_{i j}$} & line & distance & norm \\
\hline 1,7 & AG & 1201.7 & 1.6207 & 5,18 & ER & 411.4 & 0.5549 \\
1,9 & AI & 1358.1 & 1.8319 & 6,11 & FK & 169.2 & 0.2282 \\
1,13 & AM & 1456.1 & 1.9641 & 6,14 & FN & 899.3 & 1.2130 \\
1,16 & AP & 1378.1 & 1.8588 & 6,16 & FP & 510.7 & 0.6888 \\
2,13 & BM & 740.3 & 0.9986 & 7,20 & GT & 1173.6 & 1.5830 \\
2,15 & BO & 748.4 & 1.0095 & 8,18 & HQ & 116.5 & 0.1571 \\
2,16 & BP & 771.6 & 1.0408 & 9,20 & IT & 1140.4 & 1.5383 \\
2,17 & BQ & 147.3 & 0.1987 & 10,17 & JQ & 467.5 & 0.6306 \\
3,7 & CG & 425.0 & 0.5733 & 10,20 & JT & 959.2 & 1.2938 \\
3,9 & CI & 273.1 & 0.3684 & 11,13 & KM & 615.0 & 0.8296 \\
3,18 & CR & 1150.5 & 1.5519 & 11,17 & KQ & 1282.6 & 1.7301 \\
3,19 & CS & 161.4 & 0.2177 & 12,17 & LQ & 222.3 & 0.2999 \\
4,13 & DM & 155.3 & 0.2095 & 13,14 & MN & 1434.4 & 1.9348 \\
4,16 & DP & 151.7 & 0.2047 & 14,17 & NQ & 2112.7 & 2.8497 \\
4,18 & DR & 161.1 & 0.2173 & 14,19 & NS & 455.1 & 0.6138 \\
5,9 & EI & 1281.7 & 1.7288 & 15,20 & OT & 443.9 & 0.5988 \\
5,10 & EJ & 821.4 & 1.1079 & 16,19 & PS & 979.9 & 1.3217 \\
5,15 & EO & 486.4 & 0.6561 & 18,20 & RT & 426.1 & 0.5748 \\
\hline
\end{tabular}

physically distinct properties and are therefore measured in different units, we will follow a recommendation of Kowalski and Bender ${ }^{14}$ and will rescale the coordinate units. However, rather than using the scaling that reduces both coordinate values to an interval $(-1,+1)$, that Kowalski and Bender suggested, we will scale the distances between adjacent spots of Figure 4 so that the average distance becomes 1 . The reason for this is that then not only the entries in the Euclidean-adjacency matrix $E$ and the adjacency matrix $A$ are of comparable magnitude but also because their averages are the same, for both matrices the sum of all entries above the main diagonal is the same. ${ }^{20,21}$ In Table 3 in the column "norm" we show the rescaled Euclidean distances between the adjacent vertices of the embedded graph of Figure 4.

Adjacency and the Euclidean-adjacency matrices will generate a number of different topological or topographic indices. ${ }^{15}$ As we can see from Table 4 , where we have listed the row sums for the adjacency matrix and the Euclidean- 
Table 4. Row Sums for the Adjacency Matrix (A) and the Euclidean-Adjacency Matrix (E) of Graph of Figure 4

\begin{tabular}{ccccc}
\hline spot & spot & row sum for A & row sum for E & new label \\
\hline 1 & A & 4 & 7.2756 & 1 \\
2 & $\mathrm{~B}$ & 4 & 3.2476 & 10 \\
3 & $\mathrm{C}$ & 4 & 2.7113 & 14 \\
4 & $\mathrm{D}$ & 3 & 0.6315 & 18 \\
5 & $\mathrm{E}$ & 4 & 4.0477 & 8 \\
6 & $\mathrm{~F}$ & 3 & 2.1302 & 17 \\
7 & $\mathrm{G}$ & 3 & 3.7771 & 9 \\
8 & $\mathrm{H}$ & 1 & 0.1571 & 20 \\
9 & $\mathrm{I}$ & 4 & 5.4673 & 6 \\
10 & $\mathrm{~J}$ & 3 & 3.0323 & 11 \\
11 & $\mathrm{~K}$ & 3 & 2.7880 & 13 \\
12 & $\mathrm{~L}$ & 1 & 0.2999 & 29 \\
13 & $\mathrm{M}$ & 5 & 5.9476 & 3 \\
14 & $\mathrm{~N}$ & 4 & 6.6114 & 2 \\
15 & $\mathrm{O}$ & 3 & 2.2544 & 25 \\
16 & $\mathrm{P}$ & 5 & 5.1148 & 7 \\
17 & $\mathrm{Q}$ & 6 & 5.8661 & 5 \\
18 & $\mathrm{R}$ & 4 & 2.8989 & 12 \\
19 & $\mathrm{~S}$ & 3 & 2.1533 & 16 \\
20 & $\mathrm{~T}$ & 5 & 5.887 & 4 \\
\hline
\end{tabular}

adjacency matrix of the graph of Figure 4, already the two matrices have different row sums and therefore matrix invariants based on them will be distinct. In the case of the adjacency matrix of a graph row sum represents the valence (or the degree) of the vertex, hence, we can interpret the row sums of the Euclidean-adjacency matrix as $\mathrm{E}$ as the "distance valences" of vertices of the embedded graph. Vertices that have as neighbors more vertices at larger distances will have a larger value for their "distance valence," while vertices having fewer neighbors and at shorter distances will have lower values of their "distance" valence.

\section{ADDITIONAL MAP MATRICES}

An advantage of having a graph (and embedded graph) associated with a map as compared to the case of associating a map with a zigzag curve is that for such a graph we can construct a number of additional matrices, which subsequently will lead to additional alternative characterizations of the same map. We will briefly outline several of such additional matrices associated with the embedded graph of Figure 4 that may be of interest as a source of novel map invariants.

The Geometry Matrix G. In - contrast to the adjacency and the Euclidean-adjacency matrices which are sparse matrices (that is matrices having many zero elements) ${ }^{16}$ geometrical matrix $\mathrm{G}$ is a dense matrix having zero entries only on the main diagonal. Its matrix element $(i, j)$ represents the Euclidean distance between vertex $i$ and vertex $j$ regardless whether $i$ and $j$ are adjacent or not.

D/D Matrix. As is known, the elements of the Graph Theoretical Distance Matrix D express graph theoretical distances between a pair of vertices, which are defined by the smallest number of edges that separate two vertices. ${ }^{17}$ Once $\mathrm{D}$ is constructed, it can be combined with the $\mathrm{G}$ matrix for construction of the so-called D/D matrix, ${ }^{18}$ the elements of which are given as the quotient of the corresponding elements of $\mathrm{D}$ and $\mathrm{G}$ matrix.

The Map Connectivity Matrices $\mathbf{D}_{\mathrm{kx}}$. These new matrices are reported for the first time in this article. They are based on partitioning of the connectivity index ${ }^{19}$ and the higher order connectivity indice ${ }^{20}$ into contributions arising from paths of length $\mathrm{k}$.
Table 5. Nonzero Matrix Elements of the Map Connectivity Matrix $\mathrm{D}_{1 \chi}$

\begin{tabular}{llllrl}
\hline element & & element & element \\
\hline 1,7 & 0.19076 & 4,13 & 0.51599 & 9,20 & 0.17627 \\
1,9 & 0.15855 & 4,16 & 0.55642 & 10,17 & 0.23710 \\
1,13 & 0.15201 & 4,18 & 0.73909 & 10,20 & 0.23668 \\
1,16 & 0.16393 & 5,9 & 0.21257 & 11,13 & 0.24557 \\
2,13 & 0.22753 & 5,10 & 0.28544 & 11,17 & 0.24727 \\
2,15 & 0.36958 & 5,15 & 0.33104 & 12,17 & 0.75395 \\
2,16 & 0.24536 & 5,18 & 0.29193 & 13,14 & 0.15947 \\
2,17 & 0.22911 & 6,11 & 0.41034 & 14,17 & 0.16057 \\
3,7 & 0.31249 & 6,14 & 0.26647 & 14,19 & 0.26503 \\
3,9 & 0.25973 & 6,16 & 0.30295 & 15,20 & 0.27450 \\
3,18 & 0.35669 & 7,20 & 0.21207 & 16,19 & 0.30132 \\
3,19 & 0.41387 & 8,17 & 1.04169 & 18,20 & 0.24207 \\
\hline
\end{tabular}

Table 6. Nonzero Matrix Elements of the Map Connectivity Matrix $\mathrm{D}_{2 \chi}$

\begin{tabular}{cccccc}
\hline element & & element & element \\
\hline 1,2 & 0.06575 & 3,4 & 0.48660 & 7,18 & 0.13858 \\
1,3 & 0.22998 & 3,5 & 0.33215 & 8,10 & 0.64853 \\
1,4 & 0.45814 & 3,14 & 0.12049 & 8,12 & 2.06227 \\
1,5 & 0.08544 & 3,16 & 0.15350 & 8,14 & 0.43921 \\
1,6 & 0.10142 & 3,20 & 0.42577 & 9,10 & 0.11265 \\
1,11 & 0.09879 & 4,5 & 0.39825 & 9,15 & 0.13033 \\
1,14 & 0.06416 & 4,6 & 0.34423 & 9,18 & 0.11519 \\
1,19 & 0.00332 & 4,11 & 0.33532 & 10,12 & 0.46939 \\
1,20 & 0.16018 & 4,14 & 0.21775 & 10,14 & 0.09997 \\
2,3 & 0.12350 & 4,19 & 0.31807 & 10,15 & 0.17500 \\
2,4 & 0.22318 & 4,20 & 0.33893 & 10,18 & 0.15467 \\
2,5 & 0.14273 & 5,17 & 0.12776 & 11,12 & 0.48952 \\
2,8 & 0.45016 & 5,20 & 0.51371 & 11,14 & 0.20789 \\
2,10 & 0.10246 & 6,13 & 0.13572 & 12,14 & 0.31789 \\
2,11 & 0.23803 & 6,17 & 0.22519 & 13,17 & 0.07145 \\
2,12 & 0.32581 & 6,19 & 0.22979 & 13,19 & 0.08143 \\
2,14 & 0.30305 & 7,9 & 0.10091 & 14,16 & 0.09830 \\
2,16 & 0.20979 & 7,10 & 0.13550 & 15,18 & 0.17898 \\
2,20 & 0.12147 & 7,15 & 0.15680 & & \\
\hline
\end{tabular}

Table 7. Row Sums of the Map Connectivity Matrix $\mathrm{D}_{1 x}$ and the Row Sums of the Map Connectivity Matrix $D_{2 x}$

\begin{tabular}{rccccccc}
\hline row & sum & row & sum & row & sum & row & sum \\
\hline 1 & 0.7230 & 11 & 0.8422 & 1 & 1.2672 & 11 & 1.3696 \\
2 & 1.0381 & 12 & 0.7957 & 2 & 2.3059 & 12 & 3.6645 \\
3 & 1.2819 & 13 & 1.3061 & 3 & 1.8720 & 13 & 0.2886 \\
4 & 1.9829 & 14 & 0.7401 & 4 & 3.1205 & 14 & 1.6608 \\
5 & 1.1822 & 15 & 0.9248 & 5 & 1.6000 & 15 & 0.4661 \\
6 & 1.0115 & 16 & 1.3641 & 6 & 1.0363 & 16 & 0.4616 \\
7 & 0.7608 & 17 & 1.8465 & 7 & 0.5318 & 17 & 0.4244 \\
8 & 1.0993 & 18 & 1.7266 & 8 & 3.6002 & 18 & 0.5874 \\
9 & 0.8567 & 19 & 0.9388 & 9 & 0.4591 & 19 & 0.6312 \\
10 & 0.8078 & 20 & 1.2358 & 10 & 1.7855 & 20 & 1.5601 \\
\hline
\end{tabular}

In the case of the first order connectivity index (referred to as the Randic index of order 1 in CODESSA, the computer program of Katritzky, Lobanov, and Kerelson ${ }^{21}$ for computation of molecular descriptors) matrix elements represent contributions of paths of length one (bonds) to the ${ }^{1} \chi$. In Table 5 we give the nonzero matrix elements for the Map Connectivity Matrix $D_{1 x}$. Similarly, in Table 6 we give the nonzero matrix elements for the Map Connectivity Matrix $\mathrm{D}_{2 x}$, which is based on contributions from paths of length two. Finally in Table 7 we have listed the row sums for these two matrices. Tables 4-7 offer new possibilities for construction of additional numerical map descriptors. The situation is similar to that of the construction of novel topological indices from matrices associated with graphs, which produce novel chemodescriptors for QSAR. The difference is that descriptors obtained from matrices associ- 
Table 8. Normalized Abundance for the 20 Most Intensive Protein Spots of Rat Liver Cells of the Normal Cells and the Corresponding Abundance for Rat Liver Cells Exposed to Four Chemicals

\begin{tabular}{lccccc}
\hline & control & PFOA & PFDA & clofibrate & DEHP \\
\hline A & 1.44457 & 1.08788 & 0.95094 & 1.47183 & 1.66001 \\
B & 1.43730 & 1.55673 & 1.88713 & 1.60009 & 1.55162 \\
C & 1.36748 & 1.13938 & 1.50357 & 1.63758 & $\mathbf{0 . 0 8 1 1 6}$ \\
D & 1.27283 & 0.99229 & 0.73122 & 0.76695 & 1.12174 \\
E & 1.18663 & 1.12868 & $\mathbf{0 . 4 9 8 0 3}$ & 1.09932 & 1.38891 \\
F & 1.15009 & 1.62550 & $\mathbf{2 . 2 1 7 2 1}$ & 1.66195 & 1.80715 \\
G & $\mathbf{1 . 1 2 3 2 9}$ & 0.58710 & $\mathbf{0 . 3 8 9 4 2}$ & $\mathbf{0 . 7 3 2 1 0}$ & 0.77128 \\
H & 1.08958 & $\mathbf{0 . 2 6 1 2 3}$ & $\mathbf{0 . 5 0 7 7 0}$ & $\mathbf{0 . 4 5 9 2 3}$ & 1.16930 \\
I & 0.98292 & 0.91210 & 0.83020 & $\mathbf{0 . 1 9 6 1 4}$ & 0.93006 \\
J & 0.93666 & 0.83230 & $\mathbf{0 . 6 2 9 7 8}$ & 0.79925 & 1.09457 \\
K & 0.90066 & 1.29430 & 1.12439 & 1.12733 & 1.19485 \\
L & 0.86790 & 0.70795 & 0.78746 & 1.05833 & 1.16362 \\
M & 0.84901 & 0.73865 & 0.45514 & 0.71961 & 0.97512 \\
N & 0.82549 & 0.74025 & 0.74518 & 0.84762 & 0.88606 \\
O & 0.82022 & $\mathbf{0 . 1 6 1 4 8}$ & $\mathbf{0 . 1 6 5 1 2}$ & 0.60119 & $\mathbf{1 . 4 9 0 9 5}$ \\
P & 0.80070 & $\mathbf{0 . 7 7 3 6 8}$ & 0.80127 & 0.76080 & 1.00906 \\
Q & 0.79902 & $\mathbf{0 . 2 0 7 9 6}$ & $\mathbf{0 . 1 3 1 1 2}$ & $\mathbf{0 . 3 8 8 4 3}$ & 0.53867 \\
R & 0.72841 & 0.76422 & 0.52786 & 0.55638 & 0.77486 \\
S & 0.72223 & 0.78036 & 0.60418 & 0.46840 & 0.78175 \\
T & 0.69500 & 0.37864 & $\mathbf{0 . 1 6 1 4 0}$ & 0.57207 & 0.71323 \\
\hline
\end{tabular}

ated with proteomics maps serve as potential biodescriptors in an integrated QSAR, ${ }^{22}$ in which chemodescriptors (topological indices, 3-D descriptors, and quantum chemical indices) are used in combination with invariants of proteomics maps (graph theoretical indices derived from embedded graphs) to predict the bioactivity/toxicity of chemicals. Moreover, any of the new matrices can be algebraically manipulated to produce the so-called "higher order" matrices either by using the Kronecker multiplication of matrices or the standard matrix multiplication.

\section{AUGMENTED MAP MATRICES}

So far we have not used information on abundance of proteins as measured by the intensity of gel spots. This information is critical when considering the role of various drugs and xenobiotic agents that perturb the proteome. The information on abundance can be combined in two ways with the information of locations of spots to produce invariants that will characterize altered proteomics maps. One approach would be to introduce the numerical values of experimental abundances as the diagonal entries of the matrix in analogy with differentiation of heteroatoms in construction of variable connectivity indices. ${ }^{23-27}$ Another way to incorporate the abundance is to view it as the third coordinate to the $2-\mathrm{D}$ map based on the charge and the mass of proteins as separated by electrophoresis and chromatography. ${ }^{2}$ This approach has been outlined in the introductory papers on the mathematical characterization of proteomics maps based on 2-D zigzag pattern of proteomics map and its generalization to 3-D zigzag pattern. ${ }^{2}$ In both cases we will obtain novel matrices for proteomics maps of control cells as well as those derived from cells exposed to different chemicals.

Before one augments a map matrix by either including abundance as the diagonal entries or considers them as the third coordinate, one has to rescale the abundance values so that they are of the same order of magnitude as are the other two coordinates used for construction of the matrix. We have rescaled abundance values so that the average abundance equals one. In Table 8 we listed the rescaled abundance values for the 20 most intense protein spots of normal rat hepatocytes and the corresponding abundance values for rat hepatocytes exposed to peroxisome proliferators: perfluorooctanoic acid (PFOA), perfluorodecanoic acid (PFDA), clofibrate, and diethylhexyl phthalate (DEHP). As is known, the sites of the protein spots for the same protein always occur in the same locations on the gel (the same $x, y$ coordinates), so correspondence can be established without identifying proteins. The 20 most abundant spots of the control group determine the selection of the spots for the remaining four proteomics maps corresponding to PFOA, PFDA, clofibrate, and DEHP. As can be seen in the first numerical column of Table 8 , belonging to the control cells, the relative abundance values steadily decrease because spots were ordered by abundance. This, however, need not and is not the case with the remaining four columns, which show considerable oscillations in the relative magnitudes of the abundances when compared to the regular decrease of spots for the control group.

The most abundant spot appears to be the protein having label F for liver cells exposed to PFDA (2.217), and the least abundant is protein $\mathrm{C}$ for liver cells exposed to DEHP (0.081). From looking at Table 8 , the question to consider is the following: how significant are the changes in abundance and how reproducible would the values in Table 8 be if the experiments were repeated. These are questions directed to experimentalists to which we cannot answer, except that we may add that the reproducibility of our numerical analysis is guaranteed once the reliability of the experimental data is established. Our contribution is toward developing theoretical methodology for quantitative comparison of proteomics maps, and the questions of reproducibility/error of protein data, which may be a major unanswered question in experimental proteomics, will not affect our methodology. In other words, as experimental techniques improve, the proposed numerical analysis will gain more and more reliability, which currently depends solely on the reliability of the experimental data.

In Table 8 we have indicated in bold type abundances of proteins, which show considerable change in rats, treated with peroxisome proliferators. As a threshold we have taken $\pm 50 \%$ change in the abundance as compared with that of the control group. It is interesting to observe protein $O$, which in the case of PFOA and PFDA has decreased in abundance considerably; but in the case of DEHP, its abundance has increased. In many cases there appear to be considerable change in abundances in comparison with that of the control group even if the change is within the set bounds of $\pm 50 \%$. In some cases abundances of proteins increased slightly after exposure to chemicals. Similarly, for some proteins the abundance decreased, although often not evenly $(G, I$, and Q). Protein $N$ appears to have been the least affected by any of the four chemical agents.

Table 9 gives the degree of similarity/dissimilarity for pairwise comparisons of proteomics maps. The values in the table were computed by viewing each column in Table 8 as a 20-component vector. The Euclidean distance (in 20dimensional vector space) gives the distance between the corresponding end-points of vectors. The smaller is the distance the more similar are vectors (or alternatively the more similar are the corresponding proteomics maps). The first row gives the similarity with the map of the control group, which suggests that among the four peroxisome 
Table 9. Similarity/Dissimilarity among Perturbations of Abundances of Proteome Rat Liver Celis for the Control and the Four Peroxisome Proliferators Based on 20-Component Vectors of Table 8

\begin{tabular}{lccccc}
\hline & control & PFOA & PFDA & clofibrate & DEHP \\
\hline control & 0 & 1.606 & 2.190 & 1.499 & 1.769 \\
PFOA & & 0 & 1.217 & 1.261 & 2.198 \\
PFDA & & & 0 & 1.481 & 2.714 \\
Clofibrate & & & & 0 & 2.224 \\
DEHP & & & & & 0 \\
\hline
\end{tabular}

proliferators clofibrate is causing the least perturbation in the liver cell proteome. However, such comparisons may obscure important details on how each agent affects individual protein types. Thus, clofibrate may give an overall (average) least perturbation of the map but it introduces the largest change in abundance of protein $I$, while the other three chemicals hardly change the abundance of the protein I in proteome of liver rat cells exposed to them.

Clearly a single type of descriptor, such as are the components of vectors in 20-D space already mentioned, cannot capture the complexity of variations occurring even for relatively small maps such as a map considered here that is based on information on only 20 proteins. In the next section we will consider 3-D representation of the proteomics maps for control groups, PFOA, PFDA, clofibrate, and DEHP from which we will construct additional map descriptors.

\section{3-D MAP MATRICES}

By viewing normalized abundances shown in Table 8 as the third coordinate of a 3-D space, one can combine this information with the data on $(x, y)$ coordinates of Table 3 and compute Euclidean distances for a graph embedded in 3-D space, of which Figure 4 now represents projection on the $(x, y)$ plane. The nonzero matrix elements of the 3-D distance-adjacency graphs are listed in Table 10, where the alphabetic labels indicate line segments of the graph and the numerical labels indicate the corresponding matrix elements for graph considered. A comparison of Tables 3 and 10 for the control group shows some (but not large) changes for corresponding matrix elements. As expected, the new $(x, y$, $z$ ) distances between adjacent spots in 3-D map representation have somewhat increased compared to $(x, y)$ distances of 2-D map when the third dimension is added to the graph. The changes in the abundance between the proteins extracted from control cells and cells treated with the four peroxisome proliferators introduced uneven changes in the matrix elements for the four corresponding matrices.

\section{THE LEADING EIGENVALUE OF MAP MATRICES}

In the following we will consider the leading eigenvalues of the 3-D matrices as map descriptors. In Table 11 in the first numerical row we have listed the leading eigenvalue of the 3-D distance-adjacency matrices of proteomics maps for the normal (control) rat liver cells and those treated with the four peroxisome proliferators. To obtain additional map invariants we constructed the "higher order" map matrices, as outlined in ref 4 by considering the Kronecker products of each of the five matrices with itself. The leading eigenvalues of the matrices thus obtained were normalized using the reciprocal factorial $(1 / n !)$ as a normalization factor,
Table 10. Nonzero Matrix Elements of the Euclidean Distance-Adjacency Matrix for the 20 Most Intensive Protein Spots of Rat Liver Cells of the Normal Cells (Control) and the Corresponding Abundance for Rat Liver Cells Exposed to Four Chemicals

\begin{tabular}{lcccccc}
\hline line & & control & PFOA & PFDA & clofibrate & DEHP \\
\hline AG & 1,7 & 1.6516 & 1.6963 & 1.7152 & 1.7815 & 1.8484 \\
AI & 1,9 & 1.8892 & 1.8403 & 1.8359 & 2.2323 & 1.9720 \\
AM & 1,13 & 2.0524 & 1.9949 & 2.0257 & 2.1032 & 2.0801 \\
AP & 1,16 & 1.9672 & 1.8852 & 1.8648 & 1.9902 & 1.9687 \\
BM & 2,13 & 1.1590 & 1.2909 & 1.7458 & 1.3313 & 1.1531 \\
BO & 2,15 & 1.1832 & 1.7222 & 1.9961 & 1.4202 & 1.0113 \\
BP & 2,16 & 1.2201 & 1.3025 & 1.5041 & 1.3370 & 1.1737 \\
BQ & 2,17 & 0.6685 & 1.3633 & 1.7672 & 1.2278 & 1.0324 \\
CG & 3,7 & 0.6231 & 0.7960 & 1.2530 & 1.0717 & 0.8972 \\
CI & 3,9 & 0.5325 & 0.4329 & 0.7676 & 1.4878 & 0.9254 \\
CR & 3,18 & 1.6783 & 1.5966 & 1.8331 & 1.8914 & 1.0347 \\
CS & 3,19 & 0.6810 & 0.4199 & 0.9254 & 1.1893 & 0.7336 \\
DM & 4,13 & 0.4728 & 0.3290 & 0.3466 & 0.2148 & 0.2557 \\
DP & 4,16 & 0.5146 & 0.2995 & 0.2189 & 0.2048 & 0.2337 \\
DR & 4,18 & 0.5862 & 0.3150 & 0.2976 & 0.3026 & 0.4093 \\
EI & 5,9 & 1.7408 & 1.7423 & 1.7604 & 1.9505 & 1.7887 \\
EJ & 5,10 & 1.1357 & 1.1469 & 1.1157 & 1.1478 & 1.1463 \\
EO & 5,15 & 0.7515 & 1.1687 & 0.7357 & 0.8238 & 0.6640 \\
ER & 5,18 & 0.7196 & 0.6639 & 0.5557 & 0.7763 & 0.8276 \\
FK & 6,11 & 0.3381 & 0.4022 & 1.1164 & 0.5813 & 0.6534 \\
FN & 6,14 & 1.2557 & 1.5017 & 1.9074 & 1.4610 & 1.5231 \\
FP & 6,16 & 0.7723 & 1.0955 & 1.5746 & 1.1342 & 1.0542 \\
GT & 7,20 & 1.6399 & 1.5967 & 1.5993 & 1.5911 & 1.5841 \\
HQ & 8,17 & 0.3303 & 0.1659 & 0.4080 & 0.1723 & 0.6499 \\
IT & 9,20 & 1.5650 & 1.6282 & 1.6774 & 1.5836 & 1.5535 \\
JQ & 10,17 & 0.6454 & 0.8874 & 0.8039 & 0.7526 & 0.8406 \\
JT & 10,20 & 1.3162 & 1.3710 & 1.3760 & 1.3136 & 1.3488 \\
KM & 11,13 & 0.8312 & 0.9985 & 1.0659 & 0.9334 & 0.8582 \\
KQ & 11,17 & 1.7331 & 2.0429 & 1.9950 & 1.8813 & 1.8504 \\
LQ & 12,17 & 0.3077 & 0.5830 & 0.7216 & 0.7340 & 0.6932 \\
MN & 13,14 & 1.9349 & 1.9348 & 1.9564 & 1.9390 & 1.9368 \\
NQ & 14,17 & 2.8498 & 2.8990 & 2.9151 & 2.8865 & 2.8708 \\
NS & 14,19 & 0.6224 & 0.6151 & 0.6298 & 0.7215 & 0.6226 \\
OT & 15,20 & 0.6118 & 0.6370 & 0.5988 & 0.5995 & 0.9815 \\
PS & 16,19 & 1.3240 & 1.3217 & 1.3363 & 1.3537 & 1.3411 \\
RT & 18,20 & 0.5758 & 0.6921 & 0.6817 & 0.5750 & 0.5781 \\
\hline & & & & & &
\end{tabular}

Table 11. Leading Eigenvalue of the Euclidean Distance-Adjacency Matrix E and the Higher Order Matrices Obtained by Kronecker Product of a Matrix E by Itself

\begin{tabular}{cccccc}
\hline power & control & PFOA & PFDA & clofibrate & DEHP \\
\hline 1 & 5.1421 & 5.4972 & 6.0381 & 5.6998 & 5.4203 \\
2 & 5.0141 & 5.4730 & 6.0599 & 5.4770 & 5.2738 \\
3 & 4.1788 & 4.5492 & 4.8440 & 4.4267 & 4.3376 \\
4 & 2.8409 & 3.0998 & 3.2291 & 3.0112 & 2.9445 \\
5 & 1.5887 & 1.7483 & 1.8098 & 1.6985 & 1.6526 \\
6 & 0.7486 & 0.8340 & 0.8642 & 0.8093 & 0.7832 \\
7 & 0.3037 & 0.3433 & 0.3571 & 0.3323 & 0.3199 \\
8 & 0.1080 & 0.1241 & 0.1297 & 0.1197 & 0.1146 \\
9 & 0.0342 & 0.0399 & 0.0419 & 0.0384 & 0.0365 \\
10 & 0.0097 & 0.0116 & 0.0122 & 0.0111 & 0.0105 \\
\hline
\end{tabular}

where $n$ is the power to which matrices were raised. The normalized leading eigenvalues listed in Table 11 show a fast convergence. Already when $n=10$ they practically approach zero. We can view columns in Table 11 as vectors in 10-dimensional vector space. In Table 12 entries above the main diagonal represent the similarity/dissimilarity matrix between the five vectors corresponding to the control and the treated animals. Some similarities and differences can be observed when Table 12 is compared to Table 9 in which the similarity/dissimilarity between the five proteomics maps was based solely on the relative abundances of the leading 20 spots of the control map. 
Table 12. Similarity/Dissimilarity Table between the Control Data and Data for the Four Chemicals ${ }^{a}$

\begin{tabular}{lccccl}
\hline & control & PFOA & PFDA & clofibrate & DEHP \\
\hline control & 0 & 0.7586 & 0.8635 & 0.8318 & 0.3671 \\
PFOA & 25.88 & 0 & 1.5985 & 0.2591 & 0.3562 \\
PFDA & 85.69 & 52.86 & 0 & 0.8302 & 1.1705 \\
Clofibrate & 44.63 & 18.83 & 41.23 & 0 & 0.3671 \\
DEHP & 19.34 & 6.56 & 66.51 & 25.33 & 0
\end{tabular}

${ }^{a}$ Entries above the main diagonal: based on the vectors using the leading eigenvalue of the Euclidean distance-adjacency matrix and the higher order matrices obtained by Kronecker product of a matrix. Entries below the main diagonal: based on the vectors using the leading eigenvalue of the distance-adjacency matrix and the higher order matrices obtained by use of the standard product of a matrices.

Table 13. Leading Eigenvalue of the Euclidean-Adjacency Matrix and the Higher Order Matrices Obtained by Use of the Standard Product of a Matrix by Itself

\begin{tabular}{crrrrr}
\hline power & control & \multicolumn{1}{c}{ PFOA } & \multicolumn{1}{c}{ PFDA } & clofibrate & DEHP \\
\hline 1 & 5.1421 & 5.4972 & 6.0381 & 5.6998 & 5.4203 \\
2 & 13.2204 & 15.1094 & 18.2290 & 16.2437 & 14.6897 \\
3 & 22.6600 & 27.6864 & 36.6893 & 30.8618 & 26.5409 \\
4 & 29.1298 & 38.7149 & 55.3830 & 43.9762 & 35.9647 \\
5 & 29.9574 & 41.8326 & 66.8810 & 50.1309 & 38.9878 \\
6 & 25.6738 & 38.3268 & 67.3052 & 47.6224 & 35.2209 \\
7 & 18.8595 & 30.0984 & 58.0560 & 38.7767 & 27.2724 \\
8 & 12.1221 & 20.6820 & 43.8182 & 27.6273 & 18.4780 \\
9 & 6.9258 & 12.6325 & 29.3974 & 17.4966 & 11.1285 \\
10 & 3.5613 & 6.9433 & 17.7503 & 9.9726 & 6.0319 \\
11 & 1.6648 & 3.4704 & 9.7434 & 5.1674 & 2.9723 \\
12 & 0.7134 & 1.5898 & 4.9026 & 2.4544 & 1.3425 \\
13 & 0.2822 & 0.6722 & 2.2771 & 1.0761 & 0.5598 \\
14 & 0.1036 & 0.2640 & 0.9821 & 0.4381 & 0.2167 \\
15 & 0.0036 & 0.0967 & 0.3953 & 0.2665 & 0.0783 \\
\hline
\end{tabular}

In addition to the Kronecker product of matrices (with themselves), we have also considered the standard product of matrices (with themselves). In Table 13 we show the leading eigenvalue of the corresponding "higher order" matrices. The first row in both Tables 11 and 13 is the same, but as we raise matrices to the higher powers using the standard matrix product we obtain larger matrix elements and consequently larger leading eigenvalues. We can view the columns of Table 13 as components of vectors in 15dimensional vector space for which we can calculate the degree of mutual similarity/dissimilarity, which are shown as entries below the main diagonal in Table 12. The leading eigenvalue as well as other Kronecker and standard matrix manipulations were calculated using MATLAB software. ${ }^{28}$

\section{"FOX TRAIL" APPROACH}

The zigzag curve introduced in the seminal work on quantification of proteomics maps ${ }^{1-3}$ was based on selecting $\boldsymbol{n}$ most abundant spots and ordering them with respect to relative abundance. The resulting zigzag graph besides offering numerous invariants based on the leading eigenvalue of the D/D matrix as discussed in refs $1-3$ allows one to construct additional, so-called, "band invariants" that were recently introduced by Randić and Balaban. ${ }^{29} \mathrm{~A}$ possible disadvantage of the zigzag curve is that its form may be sensitive to fluctuations in the relative abundance as reported in different maps belonging to the same proteome. This shortcoming has been recently addressed by $\mathrm{us}^{6}$ in which we introduced canonical labels for protein spots that are based on 2-D map and do not require information on

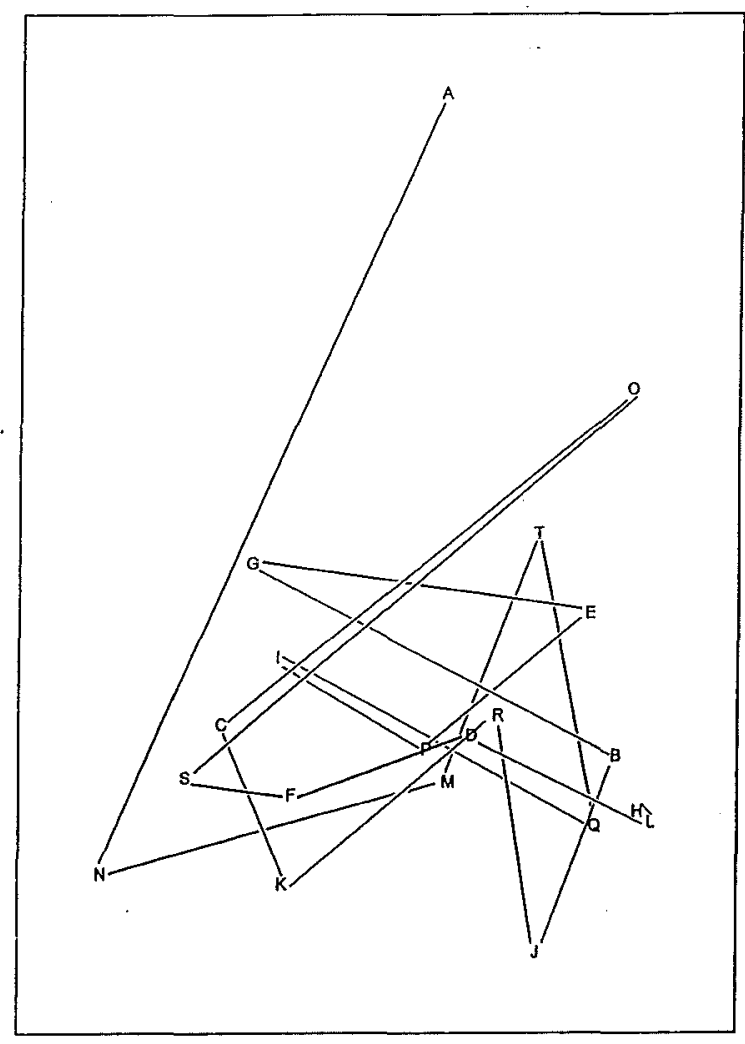

Figure 5. The "fox trail", the zigzag curve based on labels derived from two sums of the Euclidean-adjacency matrix $\mathrm{E}$.

abundance. After arriving at canonical labels for protein spots, one simply connects spots using canonical labels as guidance. To differentiate such zigzag curves from the zigzag curve based on relative abundance we referred to the novel zigzag curve as "fox trail", in view of a great similarity of zigzag curves and the experimentally observed paths of a wandering fox recorded at constant time intervals. ${ }^{30,31}$

We will here outline an alternative "fox trail" associated with the proteomics map of Figure 1 and illustrated in Figure 5. Each time we assign numerical labels to a proteomics map, we can derive a zigzag curve by connecting spots having neighboring labels. The problem is in formulating useful rules that lead toward a unique labeling. Advantage of the canonical labels based on the smallest binary code for the embedded graph of the partial ordering is not only that it is unique, but once a set of spots is selected as a basis for quantitative characterization of a map, it offers canonical labels for a map as a whole and allows cataloguing and ordering such maps in a systematic way. A disadvantage of canonical labeling is that it is associated with an nonpolynomial (NP) algorithm, that is, finding canonical labels represent an NP problem, ${ }^{32}$ which may become computationally intensive and even intractable as the number of spots grows larger.

We propose here to use row sums of the distanceadjacency matrix (listed in Table 4) as the criterion for labeling of vertices of Euclidean-adjacency graphs. In this way we bypassed the difficulties of NP associated with canonical labels and nevertheless obtain a "fox trail" graph that does not require relative abundance of proteomics spots for its construction, except for the selection of the 20 (or 
whatever number is selected) spots used as a basis of mathematical analysis of proteomics map. In Figure 5 we illustrated the "fox trail" graph for graph of Figure 4.

\section{DISCUSSION}

It is of interest to make a closer comparison between the three similarity/dissimilarity tables, Table 9, and both parts of Table 12. First, observe that there is more parallelism between the corresponding entries in Table 12 than those of Table 9, but even here there are a number of important differences. Both tables were based on the leading eigenvalues of the higher order map matrices, the difference being in the kind of matrix multiplication considered. Hence, it appears that computation of the Kronecker product of matrices and use of leading eigenvalues of the higher order Kronecker matrices as map descriptors, (which is simpler and faster), could not replace the standard matrix multiplication for construction of the higher order matrices, which offer somewhat different map descriptors.

The similarity/dissimilarity tables such as in Tables 9 and 12 do show some differences that illustrate the fact, that even though two approaches are based on similar methodologies, they capture somewhat different map information. For example, the smallest entry in Table 9 belongs to the pair (PFOA, PFDA), which is not the case with Table 12 where the smallest entry is associated with the pair (PFOA, clofibrate) and (PFOA, DEHP) for the 10-D and 15-D vectors, respectively. A relatively small value of the Euclidean distance in a similarity/dissimilarity table does not necessarily imply that the corresponding chemicals are the most similar, but it signifies that the effect of those chemicals on the 20 proteome spots of treated cells may be similar. The small amount of data presented may be merely anecdotal, and it would be premature to conclude that because of some parallelism Kronecker multiplication and standard matrix multiplication behave similarly in general. Future applications may clear this point; at this stage we are merely offering a tool for such applications.

Based on the entries in the first row of Table 9 one could conclude that clofibrate makes the least perturbation of the proteome of rat liver cells, but from the first row of Table 12 and the first column of Table 12 we see that DEHP apparently makes the least perturbation of the proteome of rat liver cells. The difference between these approaches is that in the first case, only changes in abundance are considered as a measure of perturbation; but in the other, the location of protein spots i.e., the charge and mass of proteins, makes an influence. Changes in abundance of protein spots which are in the vicinity (i.e., proteins having a similar mass and charge) will be more pronounced in the latter case.

It remains to be seen which graph invariants and what kind of weighting procedure may be more suitable when comparing different proteomics maps. At this stage it is more important to recognize the various possibilities, and it would be premature to suggest or to advocate one scheme over another. We should recollect that here we chose to consider a particular set of invariants, the leading eigenvalues of 3-D matrices as invariants, but other graph invariants ought to be examined. The main reason for the lack of definite conclusions with regard to one set of descriptors relative to another at this moment is the lack of suitable data for testing. The comparison of similarity/dissimilarity among proteomics maps with similarities/dissimilarities of chemical agents causing perturbations would be facilitated by having data on a set of chemical compounds showing closer structural features. If there is some parallelism between the biological descriptors of proteomics maps and chemodescriptors inducing changes in proteome, one could test the degree to which the paradigm that similar chemical structures induce similar properties, that holds in QSPR and QSAR, also applies to the proteome, even if proteomics data may point to drastic changes of the cell proteome under minor perturbations of biological systems. Another testing ground for the utility of biodescriptors derived from proteomics maps could be the study of perturbations of the cellular proteome induced by a gradual increase of concentrations of chemicals that produce visible perturbations of the proteome. Hopefully, as indicated in ref 22 for the first time, one may use a combination of chemodescriptors and biodescriptors derived from proteomics maps in an effort to arrive at an integrated QSAR.

\section{CONCLUDING REMARKS}

Here we have developed two measures of "proteomics similarity" of the chemicals, one based solely on the abundances of the spots and the other based on the Euclidean distance in the eigenvalue space. These two measures give different pictures of the intermolecular similarity of the chemicals analyzed in this paper. This brings us to the wellknown problem of similarity methods: Similarity methods are not uniquely defined, they are rather user-defined and context specific. ${ }^{33,34}$ In the area of molecular similarity for relatively small molecules, Basak et al. ${ }^{35-37}$ calculated and used various molecular similarity methods based on graph invariants as well as experimental properties to select analogues, cluster large libraries of chemicals, ${ }^{38}$ and estimate properties of chemicals from their selected neighbors ${ }^{39,40}$ in the selected spaces. It was concluded that no universal similarity method can handle all situations. It would be interesting to see how far map invariants are able to characterize various aspects of proteomics maps and their perturbation derived from the exposure of organisms/cells to drugs and xenobiotics.

\section{ACKNOWLEDGMENT}

This is contribution number 315 from the Center for Water and the Environment of the Natural Resources Research Institute. Research reported in this paper was supported in part by Grant F49620-02-1-013 from the United States Air Force Office of Scientific Research.

\section{REFERENCES AND NOTES}

(1) Randic, M. On Graphical Representation of Proteomics and Their Numerical Characterization. J. Chem. Inf. Comput. Sci. 2001, 4l, 1330-1338.

(2) Randic, M.; Zupan, J.; Novic, M. On 3-D Graphical Representation of Proteomics Maps. Chem. Inf. Comput. Sci. 2001, 41, 1339-1334.

(3) Randić, M.; Witzmann, F.; Vracko, M.; Basak, S. C. On Characterization of Proteomics Maps and Chemically Induced changes in Proteome Using Matrix Invariants: Application to Peroxisome Proliferators. Med. Chem. Rev. 2001, 10, 456-479.

(4) Randic, M. A Graph Theoretical Characterization of Proteomics Maps. Int. J. Quantum Chem. In press. 
(5) Randic, M.; Nović, M.; Vraćko, M. On Characterization of Dose Variations of 2-D Proteomics Maps by Matrix Invariants. J. Proreome Res. In press.

(6) Randic, M.; Basak, S. C. Canonical Labeling of Proteins and Proteomics Maps. J. Chem. Inf. Comput. Sci. Submitted for publication.

(7) Witzmann, F. Molecular Anatomy Laboratory, Department of Biology, Indiana University \& Purdue University, Columbus, IN

(8) Randic, M.; Wilkins, C. L. On a Graph Theoretical Basis for Ordering of Structures. Chem. Phys. Lett. 1979, 63, 332-336.

(9) Randić, M.; Wilkins, C. L. Graph Theoretical Ordering of Structures as a Basis for Systematic Searches for Regularities in Molecular Data. J. Phys. Chem. 1979, 83, 1525-1540.

(10) Randić, M.; Jerman-Blažic, B.; Rouvray, D. H.; Seybold, P. G.; Grossman, S. C. The Search for Active substructure in StructureActivity Studies. Int. J. Quantum Chem: Quantum Biol. Symp. 1987, $14,245-260$.

(11) Randic, M. Acta Chim. Slovenica 2000, 47, 143

(12) For a recent applications of partial order in chemistry, see: Partial Orderings in Chemistry. MATCH (Mathematical Chemistry Communication); Klein, D. J., Brickmann, J., guest Eds.; 2000; No. 42.

(13) Randic, M. Unpublished.

(14) Kowalski, B. R.; Bender, C. F. A Powerful Approach to Interpreting Chemical Data. J. Am. Chem. Soc. 1972, 94, 5632-5639.

(15) Randic, M. Topological Indices. In The Encyclopedia of Computational Chemistry; Schleyer, P. v. R., Allinger, N. L., Clark, T., Gasteiger, J., Kollman, P. A., Schaefer, H. F., III., Schreiner, P. R., Eds.; John Wiley \& Sons: Chichester, 1998; pp 3018-3032.

(16) Randic, M.; DeAlba, L. M. Dense Graphs and Sparse matrices. $J$. Chem. Inf. Comput. Sci. 1997, 37, 1078-1081.

(17) Harary, F. Graph Theory; Addison-Wesley: Reading, MA, 1969

(18) Randic, M.; Kleiner, A. F.; DeAlba, L. M. Distance/Distance matrices. J. Chem. Inf. Comput. Sci. 1994, 34, 277-286.

(19) Randic, M. On Characterization of Molecular Branching J. Am. Chem. Soc. 1975, 97, 6609-6615.

(20) Kier, L. B.; Murray, W. J.; Randic, M.; Hall, L. H. Molecular Connectivity V. Connectivity Series Concept Applied to Density. $J$. Pharm. Sci. 1976, 65, 1226-1230.

(21) Katritzky, A. R.; Lobanov, V. S.; Karelson, M. QSPR: The Correlation and Quantitative Prediction of Chemical and Physical Properties from Structure. Chem. Soc. Rev. 1995, 279-287.

(22) Basak, S. C.; Mills, D.; Gute, B. D.; Gnunwald, G. D.; Balaban, A. T Applications of Topological Indices in the Property/Bioactivity/ Toxicity Prediction of Chemicals. In Topology in Chemistry: Discrete Mathematics of Molecules; Rouvray, D. H., King, R. B., Eds. Horwood Publishing Ltd.: Chichester, West Sussex, United Kingdom, 2001; pp 113-184.

(23) Randić, M. Novel Graph Theoretical Approach to Heteroatom in Quantitative Structure-Activity Relationships. Chemometrics Intel. Lab. Systems 1991, 10, 213-227.

(24) Randić, M. On Computation of Optimal Parameters for Multivariate Analysis of Structure-Property Relationship. J. Comput. Chem. 1991, $12,970-980$.
(25) Randić, M.; Dobrowolski, J. C. Optimal Molecular Connectivity Descriptors for Nitrogen-Containing Molecules.Int. J. Quantum Chem. 1998, 70, 1209-1215.

(26) Randic, M.; Mills, D.; Basak. S. C. On Use of Variable Connectivity Index for Characterization of Amino Acids. Int. J. Quantum Chem. 2000, 80, 1199.

(27) Randic, M.; Basak, S. C. In Some Aspects of Mathematical Chemistry; Sinha, D. K., Basak, S. C., Mohanty, R. K., Basumallick, I. N., Eds.; Visva-Bharati University Press: In press.

(28) MATLAB (abbreviation for Matrix Laboratory) is a product of The MathWorks, Inc., 3 Apple Hill Drive, Natick, MA 01760.

(29) Randic, M.; Balaban, A. T. 4-Dimensional DNA Proc. Natl. Acad. Sci. U.S.A. Submitted for publication.

(30) Siniff, D. H; Jesson, C. R. Simulation Model of Animal Movement Patterns. Adv. Ecological Res. 1969, 6, 185-220.

(31) Hall, G. G. Modelling - A Philosophy for Applied Mathematicians. Bull. Institute Mathematics Applications 1972, 8, 226-228.

(32) Garey, M. R.; Johnson, D. S. Computers and Intractability (A Guide to the Theory of NP-Completeness); Freeman: San Francisco, 1979.

(33) Randic, M. Design of Molecules with Desired Properties. Molecular Similarity Approach to Property Optimization. In Concepts and Applications of Molecular Similarity; Johnson, M. A., Maggiora, G., Eds.; Wiley: New York, 1990; pp 77-145.

(34) Randic, M. Similarity Methods of Interest in Chemistry. In Mathematical Methods in Contemporary Chemistry; Kuchanov, S., Ed.; Gordon \& Breach Publ. Inc.: New York, 1995; pp 1-99.

(35) Basak, S. C.; Magnuson, V. R.; Niemi, G. J.; Regal, R. R. Determining Structural Similarity of Chemicals Using Graph-Theoretic Indices. Discrete Appl. Math. 1988, 19, 17-44.

(36) Basak, S. C.; Grunwald, G. D. Use of Topological Space and Property Space in Selecting Structural Analogues. Math. Modelling Sci. Computing 1994, 4, 464-469.

(37) Basak, S. C.; Gute, B. D.; Grunwald, G. D. Development and Applications of Molecular Similarity Methods Using Nonempirical Parameters. Math. Modelling Sci. Computing In press.

(38) Basak, S. C.; Mills, D.; Gute, B. D.; Balaban, A. T.; Basak, K. Grunwald, G. D. Use of Mathematical Structural Invariants in Analysis Combinatorial Libraries: A Case Study with Psoralen Derivatives. In Some Aspects of Mathematical Chemistry; Sinha, D. K., Basak, S. C., Mohanty, R. K., Basumallick, I. N., Eds.; Visva-Bharati University Press: In press.

(39) Gute, B, D.; Basak, S. C. Molecular Similarity-Based Estimation of Properties: A Comparison of Three Structure Spaces. J. Mol. Graphics 2001, 20,95-109.

(40) Basak, S. C.; Grunwald, G. D.; Host, G. E.; Niemi, G. J.; Bradbury, S. P. A Comparative Study of Molecular Similarity, Statistical, and Neural Methods for Predicting Toxic Modes of Action. Environ. Toxicol. Chem. 1998, 17, 1056-1064. 



\title{
NOVEL MATRIX INVARIANTS FOR CHARACTERIZATION OF CHANGES OF PROTEOMICS MAPS
}

\author{
M. RANDIĆ, ${ }^{1}$ J. ZUPAN, ${ }^{1}$ M. NOVIČ, ${ }^{1}$ B. D. GUTE, ${ }^{2}$ and S. C. BASAK ${ }^{2, \dagger}$ \\ ${ }^{1}$ National Institute of Chemistry, 1001 Ljubljana, Slovenia \\ ${ }^{2}$ Natural Resources Research Institute, University of Minnesota Duluth, \\ 5013 Miller Trunk Highway, Duluth, Minnesota 55811, USA
}

Previous studies on mathematical characterization of proteomics maps by sets of map invariants were based on construction of set of distance-related matrices obtained by matrix multiplication of a single matrix by itself. Here we consider an alternative characterization of proteomics maps based on set of matrices characterizing local features of embedded zigzag curve over the map. It is shown that novel invariants can well characterize proteomics maps. Advantages of the novel approach are discussed.

Keywords: Eigenvalue reduction; Graph spectra; Local matrices; Matrix invariants; Proteomics maps; Quantitative proteomics

\section{INTRODUCTION}

Numerical characterization of proteomics maps by invariants to allow for study of quantitative changes in the proteome has been initiated only recently. The first step in such studies is to associate a suitable mathematical object with a proteomics map. In the second stage, the selected mathematical object is represented by various matrices that record distances among various protein spots in 2-D map. In the third step, one constructs various matrix invariants to serve as map descriptors. Finally, one manipulates the matrices mathematically to derive other structurally related matrices, resulting in additional map descriptors. Suitable mathematical objects considered previously included the zigzag curve

\footnotetext{
${ }^{\dagger}$ Corresponding Author. e-mail: sbasak@nrri.umn.edu
} 
that connects spots of neighboring abundance [1-3], the embedded graph of partial ordering of protein spots with respect to dominance in mass and the charge [4-6], and embedded graph depicting clustering of spots at critical separation [7]. In all previous cases the numerical characterization of proteomics maps was based on the properties of the quotient matrix $Q$, the elements of which are obtained as the quotient of the direct (Euclidean) distance measured between the spots and the distance between the same spots measured along the lines of the zigzag curve, or along the shortest path of a graph. The leading eigenvalue of the Q matrix was taken as the leading map invariant, to be subsequently augmented by considering the higher order $\mathrm{Q}^{\mathrm{n}}$ matrices obtained either by use of the Kronecker (Hadammard) product or by the standard product of matrix $Q$ with itself.

In this contribution we introduce a novel approach to the mathematical characterization of proteomics maps based on local features of the zigzag curve used as the mathematical object for the characterization of such maps. As we will see, in this way one can formulate a set of map descriptors that characterize aspects of the structure of proteomics maps not accounted for by descriptors derived from the Q matrix approach.

\section{OUTLINE OF THE APPROACH}

We will illustrate the approach on the same proteomics data already considered in some of our earlier work, originating from the Molecular Anatomy Laboratory of F. Witzmann [8]. Table 1 lists the $x, y$ coordinates of the protein spots corresponding to charge and mass, respectively, as separated by electrophoresis and by chromatography. The $\mathrm{z}$ coordinate indicates the relative abundance of the proteins. The five columns in Table 1 belong to the control group, i.e. derived from rat liver of healthy animals, and the other four sets of data originating from liver of experimental animals exposed to four peroxisome proliferators: prefloroctanoic acid (PFOA), perflourodecanoic acid (PFDA), clofibrate, and di(ethylhexyl)phthalate (DEHP), respectively. The protein spots are ordered according to their control abundance, starting with the most abundant spot and ending with the least abundant one.

The zigzag curve is obtained by connecting the $(x, y)$ coordinates of the most abundant spot with the $(x, y)$ coordinates of the protein spot having the next lower abundance value, which in turn is connected with the spot having the next lower abundance value and so on until all of the 30 selected protein spots listed in Table 1 are connected. Figure 1 illustrates the zigzag curve resulting from the relative abundance of the control group, though the actual zigzag curve is in 3-D space with the abundance as the third coordinate. We use the same $(x, y)$ part of the zigzag curve for the construction of map invariants belonging to the remaining four proteomics maps obtained for the four peroxisome proliferators listed in Table 1. Observe the highly irregular pattern of lines in Figure 1, some of which are overlapping each 
B.D. GUTE, and S.C. BASAK

other, while others occur at almost perpendicular directions. The pattern of lines depends on the order in which spots are listed and for the same set of 30 spots alternative orderings based on alternative criteria can be considered. Instead of using the relative abundance for ordering spots, for instance, one can order protein spots according their relative charge, or relative mass. In such case one obtains different zigzag curves, seen in Figures 2 and 3, respectively. Hence, the mathematical approach based on the construction

TABLE I The $x, y$ coordinates of the selected proteomics spots. The next five columns give the abundance for proteomics maps of rat liver cells for the control group and the four peroxisome proliferators

\begin{tabular}{|c|c|c|c|c|c|c|c|}
\hline SID\# & $x$ & $y$ & control & PFOA & PFDA & Clofibrate & $D E H P$ \\
\hline 187 & 2111.7 & 2278.6 & 144,357 & 108,713 & 95,028 & 147,081 & 165,886 \\
\hline 77 & 2804.3 & 903.6 & 143,630 & 155,565 & 188,582 & 159,898 & 155,055 \\
\hline 22 & 1183.9 & 959.6 & 136,653 & 113,859 & 150,253 & 163,645 & 8,111 \\
\hline 52 & 2182.2 & 928.8 & 127,195 & 99,160 & 73,071 & 76,642 & 112,096 \\
\hline 134 & 2685.6 & 1196.1 & 118,581 & 112,790 & 49,769 & 109,856 & 138,795 \\
\hline 20 & 1527.9 & 825.5 & 114,929 & 192,437 & 221,567 & 166,080 & 180,590 \\
\hline 62 & 1346.0 & 1352.5 & 112,251 & 58,669 & 38,915 & 73,159 & 77,075 \\
\hline 67 & 2868.5 & 778.0 & 108,883 & 26,105 & 50,735 & 45,923 & 116,849 \\
\hline 48 & 1406.3 & 1118.1 & 98,224 & 91,147 & 82,963 & 84,196 & 92,942 \\
\hline 96 & 2450.2 & 409.2 & 93,601 & 83,172 & 62,934 & 79,870 & 109,381 \\
\hline 9 & 1474.0 & 665.1 & 90,004 & 129,340 & 112,361 & 112,655 & 119,402 \\
\hline 75 & 2974.9 & 772.8 & 86,730 & 70,746 & 78,691 & 105,760 & 116,821 \\
\hline 36 & 2068.4 & 823.1 & 84,842 & 73,814 & 45,482 & 71,911 & 97,444 \\
\hline 2 & 642.2 & 669.8 & 82,492 & 73,974 & 74,466 & 84,703 & 88,545 \\
\hline 250 & 2860.7 & 1649.9 & 81,965 & 16,137 & 16,501 & 60,077 & 148,992 \\
\hline 44 & 2032.7 & 902.8 & 80,015 & 77,314 & 80,072 & 76,027 & 100,836 \\
\hline 84 & 2752.7 & 765.6 & 79,847 & 20,782 & 13,103 & 38,816 & 53,830 \\
\hline 80 & 2334.2 & 982.2 & 72,791 & 76,369 & 52,749 & 55,599 & 77,432 \\
\hline 15 & 1053.6 & 864.3 & 72,173 & 77,982 & 60,376 & 46,808 & 78,121 \\
\hline 176 & 2519.5 & 1365.9 & 69,452 & 37,838 & 16,129 & 57,167 & 71,274 \\
\hline 309 & 2552.5 & 2409.4 & 67,772 & 75,767 & 98,740 & 135,758 & 124,008 \\
\hline 5 & 1214.3 & 620.0 & 64,684 & 63,511 & 38,075 & 58,364 & 75,760 \\
\hline 126 & 2651.1 & 1149.6 & 61,074 & 56,365 & 54,972 & 85,399 & 97,303 \\
\hline 74 & 2327.9 & 677.3 & 59,294 & 55,535 & 32,155 & 60,155 & 68,464 \\
\hline 45 & 2094.5 & 680.5 & 58,977 & 142,865 & 46,225 & 48,625 & 146,609 \\
\hline 1 & 1021.7 & 390.2 & 58,001 & 56,547 & 53,473 & 60,224 & 71,654 \\
\hline 296 & 1702.7 & 2138.3 & 57,400 & 51,760 & 20,272 & 49,295 & 52,226 \\
\hline 56 & 2070.4 & 929.6 & 55,402 & 46,146 & 33,152 & 59,438 & 69,031 \\
\hline 276 & 2771.7 & 1451.0 & 53,896 & 22,428 & 20,155 & 35,527 & 62,085 \\
\hline 180 & 2772.8 & 1326.9 & 51,347 & 29,115 & 40,392 & 45,713 & 74,507 \\
\hline
\end{tabular}




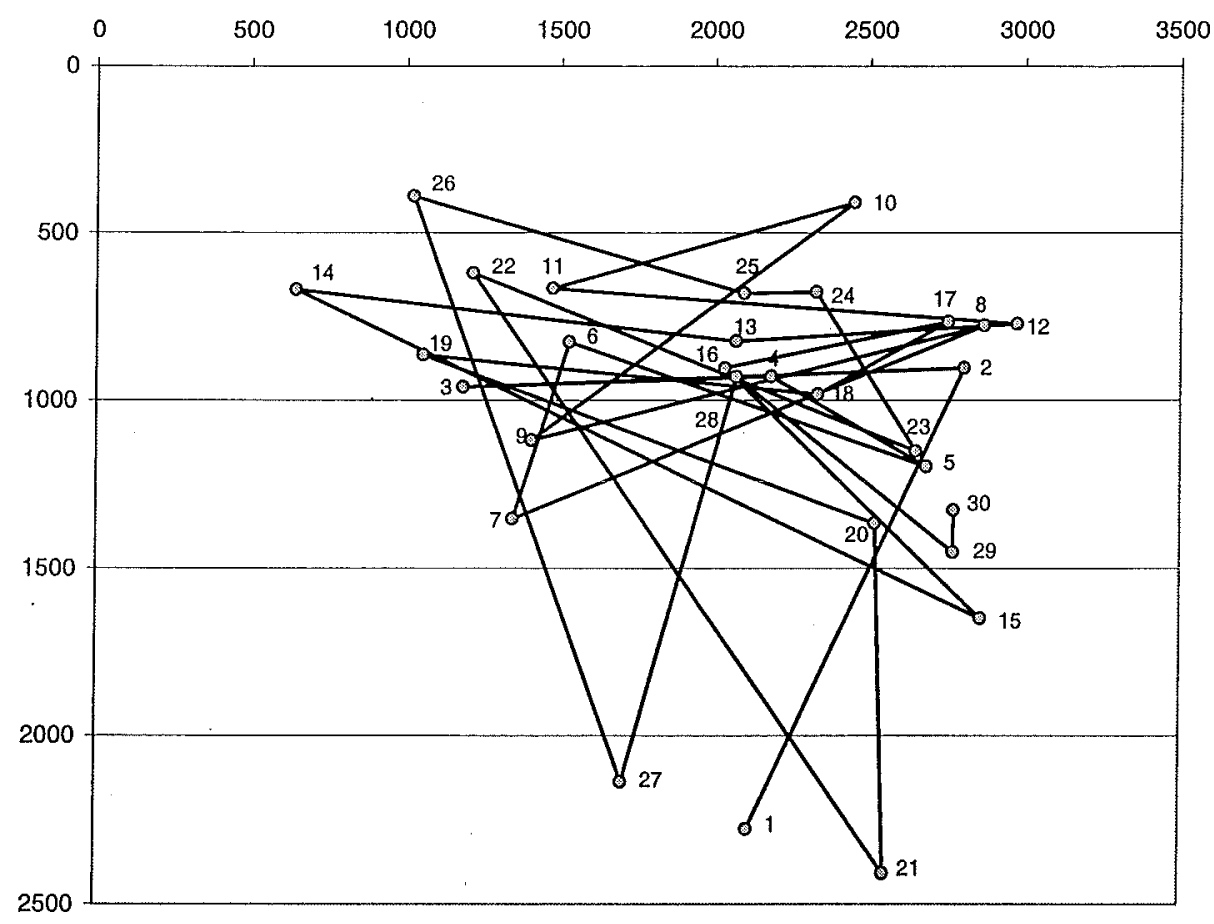

FIGURE 1 The zigzag curve based on the relative abundance of the protein spots of the control group.

of a zigzag curve has some flexibility, not only when considering which protein spots should be selected for the analysis of maps, but also when deciding on the criterion of construction for a zigzag curve. For example, in ref. [6] yet another zigzag curve, based on canonical labels for protein spots, was considered.

For the selected 3-D zigzag curve one first has to construct the following distance-related matrices: (1) The Euclidean Distance matrix, ED; (2) The Adjacency Distance Matrix, AD; and (3) The Quotient Matrix, Q. The matrices are briefly outlined below and are illustrated for the first five spots of Table 1 in Table 2.

\section{The Euclidean Distance Matrix ED}

The matrix elements $(i, j)$ are given by the Euclidean distance in the 3-D space in which the $(x, y)$ coordinates give the location of the protein spots on the 2-D gel, while the $\mathrm{z}$ coordinate gives the abundance of the corresponding spots:

$[E D]_{i j}=\sqrt{ }\left(x_{i}-x_{j}\right)^{2}+\left(y_{i}-y_{j}\right)^{2}+\left(z_{i}-z_{j}\right)^{2}$ 
M. RANDIĆ, J. ZUPAN, M. NOVIČ,

B.D. GUTE, and S.C. BASAK
MATRIX INVARIANTS FOR PROTEOMICS MAPS

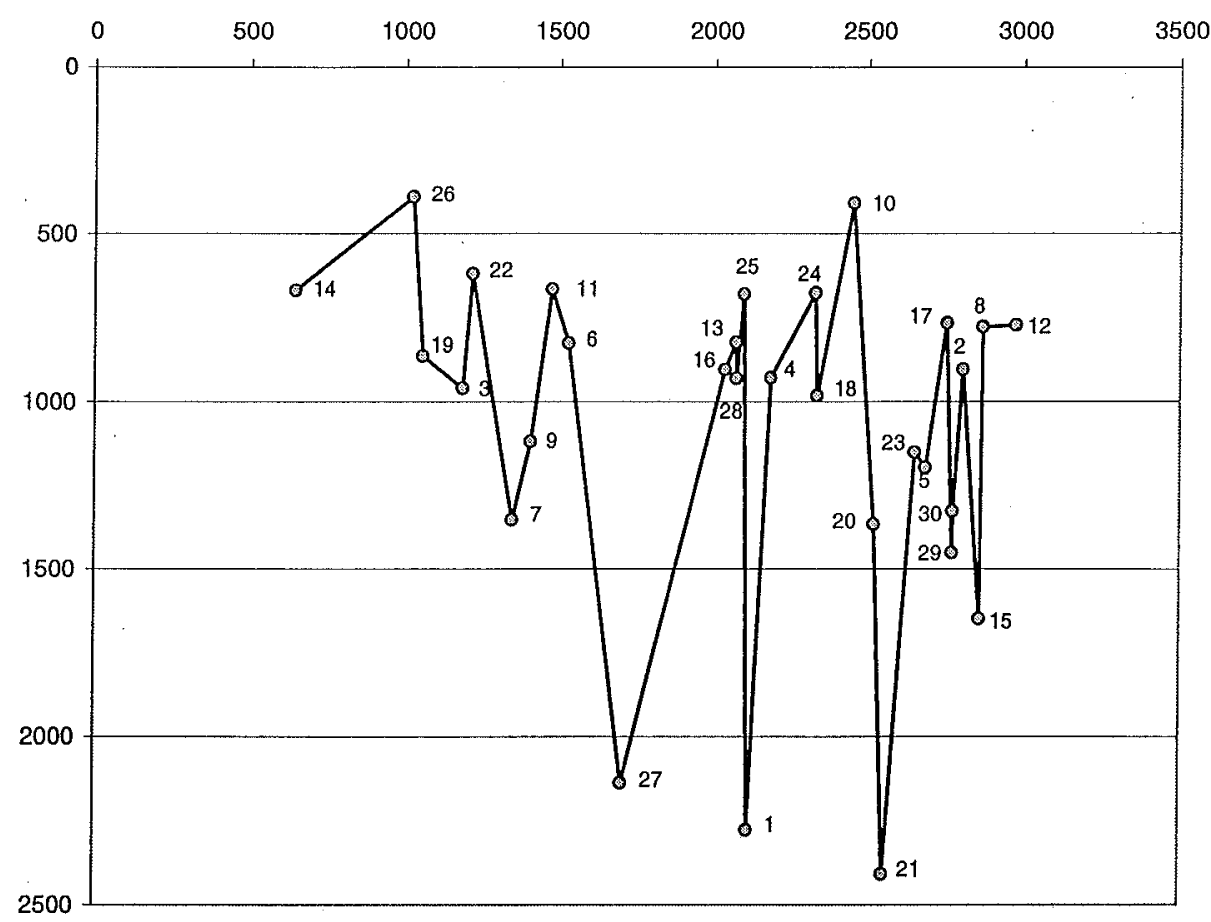

FIGURE 2 The zigzag curve based on the ordering of proteins spots relative to their charge.

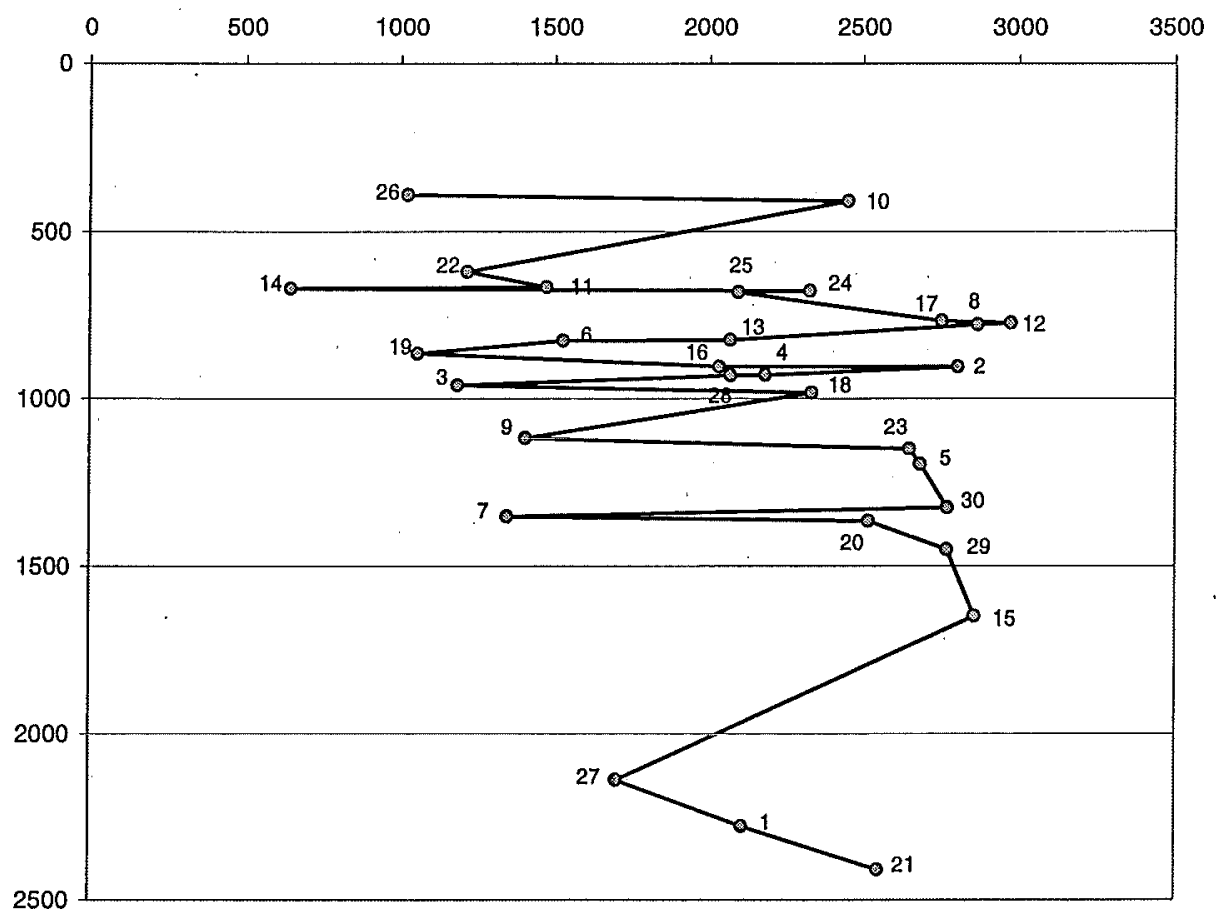

FIGURE 3 The zigzag curve based on the ordering of proteins spots relative to their mass. 
B.D. GUTE, and S.C. BASAK

TABLE II The $5 \times 5$ fragment of the Euclidean Distance matrix (ED), the Adjacency Distance matrix (AD) and the Quotient matrix ED/AD

\begin{tabular}{|c|c|c|c|c|c|}
\hline$E D$ & 1 & 2 & 3 & 4 & 5 \\
\hline 1 & 0 & 1702.60 & 7870.97 & 17215.14 & 25805.10 \\
\hline 2 & & 0 & 7162.92 & 16446.79 & 25050.99 \\
\hline 3 & & & 0 & 9510.59 & 18135.83 \\
\hline 4 & & & & 0 & 8632.84 \\
\hline 5 & & & & & 0 \\
\hline$A D$ & 1 & 2 & 3 & 4 & 5 \\
\hline$I$ & 0 & 1702.60 & 8865.527 & 18376.11 & 27008.95 \\
\hline 2 & & 0 & 7162.92 & 16673.51 & 25306.35 \\
\hline 3 & & & 0 & 9510.59 & 18143.43 \\
\hline 4 & & & & 0 & 8632.84 \\
\hline 5 & & & & & 0 \\
\hline$E D / A D$ & 1 & 2 & 3 & 4 & 5 \\
\hline 1 & 0 & 1 & 0.8879 & 0.9368 & 0.9554 \\
\hline 2 & & 0 & 1 & 0.9864 & 0.9899 \\
\hline 3 & & & 0 & 1 & 0.9996 \\
\hline 4 & & & & 0 & 1 \\
\hline 5 & & & & & 0 \\
\hline
\end{tabular}

\section{The Adjacency Distance Matrix AD}

The matrix elements $(i, j)$ of $A D$ differ from zero only if protein spots $i$ and $j$ are connected. The magnitude of non-zero elements are given by the Euclidean distance in 3-D space between the spots $i$ and $\mathrm{j}$, based on their $(x, y, z)$ coordinates (such as those of Table 1$)$ :

$$
[E D]_{i j} \quad \text { if } \quad[E D]_{i j} \leq D_{x}
$$

$[\mathrm{AD}]_{\mathrm{ij}}=$

$$
0 \quad \text { if } \quad[E D]_{i j}>D_{x}
$$




\section{The Quotient Matrix Q}

The matrix elements ( $i, j$ ) of the quotient matrix $Q$ are given by the quotient of the corresponding elements of the Euclidean distance matrix ED and the adjacency distance matrix $\mathrm{AD}$. The matrix elements for all adjacent pairs of spots are necessarily equal to one, and the matrix elements for the spots which are not adjacent elements are necessarily smaller than one, because three sides of a triangle in general define a triangular inequality $a+b>c$, except if it happens that spots are strictly collinear in which case one would have $a+b=c$, which is highly unlikely for a majority of spots in real proteomics maps.

$$
[Q]_{\mathrm{ij}}=E D_{\mathrm{ij}} / A D_{\mathrm{ij}}
$$

This matrix was introduced for the first time in ref. [1] and it represents a modification of the $\mathrm{D} / \mathrm{D}$ matrix considered for embedded molecular graphs [9]. In the case of molecular graphs embedded in 2-D or 3-D spaces, the quotient matrix has been based on the assumption that all bonds have the same length. Thus, the elements of the D/D matrix have been defined as the quotient of the Euclidean distance between vertices (atoms) superimposed on a graphite or diamond grid divided by the graph theoretical distance between vertices, which is given by the number of bonds (edges) between vertices [10]. In the case of linear chains, the leading eigenvalue of the D/D matrix has been interpreted as an index of the degree of bending or foldedness of a system [9].

The Q matrix combines information on the adjacency of and the distances between the selected spots into a single matrix. Since its elements are less than one (or at most equal to one) the elements of the $Q$ matrix do not require additional normalization. Such normalization is needed when one considers construction of the "higher order" matrices using the Kronecker matrix multiplication in order to ensure a convergence of matrix elements obtained by repeated multiplications. However, in this contribution we will not consider "higher order" quotient matrices. Instead we will consider local matrices and see that local matrices based on shorter segments of a zigzag curve offer a set of invariants that may serve as map descriptors.

In order to illustrate the differences between proteomics maps derived from tissues exposed to different peroxisome proliferators in Table 3 we illustrate the corresponding $5 \times 5 \mathrm{ED} / \mathrm{AD}$ quotient matrices obtained for the two to five columns of Table 1 with data on PFOA, PFDA, Clofibrate, and DEHP, respectively. As we can see, quotient matrices of widely different forms have emerged, suggesting that the approach based on local matrices is quite sensitive to changes in proteomics maps. Observe, however, that the changes that we see, as reflected in the magnitudes of the corresponding matrix elements of the quotient matrices belonging to different proliferators, are rather dramatic. 
M. RANDIĆ, J. ZUPAN, M. NOVIČ,

B.D. GUTE, and S.C. BASAK

TABLE III The local $5 \times 5$ matrices for the first five proteins spots of proteomics maps of rats exposed to PFOA, PFDA, Clofibrate, and DEHP

\begin{tabular}{c|ccccc}
$P F O A$ & 1 & 2 & 3 & 4 & 5 \\
\hline$I$ & 0 & 1 & 0.0609 & 0.0934 & 0.0364 \\
2 & & 0 & 1 & 0.9989 & 0.6101 \\
3 & & & 0 & 1 & 0.0655 \\
4 & & & & 0 & 1 \\
5 & & & & & 0
\end{tabular}

\begin{tabular}{c|ccccc}
$P F D A$ & 1 & 2 & 3 & 4 & 5 \\
\hline 1 & 0 & 1 & 0.4188 & 0.1052 & 0.1948 \\
2 & & 0 & 1 & 0.9997 & 0.9997 \\
3 & & & 0 & 1 & 1.0000 \\
4 & & & & 0 & 1 \\
5 & & & & & 0
\end{tabular}

\begin{tabular}{c|ccccc} 
Clofibrate & 1 & 2 & 3 & 4 & 5 \\
\hline 1 & 0 & 1 & 0.9794 & 0.6774 & 0.2714 \\
2 & & 0 & 1 & 0.9140 & 0.4026 \\
3 & & & 0 & 1 & 0.4476 \\
4 & & & & 0 & 1 \\
5 & & & & & 0
\end{tabular}

\begin{tabular}{c|ccccc}
$D E H P$ & 1 & 2 & 3 & 4 & 5 \\
\hline 1 & 0 & 1 & 0.9993 & 0.2055 & 0.0940 \\
2 & & 0 & 1 & 0.1712 & 0.0586 \\
3 & & & 0 & 1 & 1.0000 \\
4 & & & & 0 & 1 \\
5 & & & & & 0
\end{tabular}


M. RANDIĆ, J. ZUPAN, M. NOVIČ, MATRIX INVARIANTS FOR PROTEOMICS MAPS B.D. GUTE, and S.C. BASAK

TABLE IV The ordered list of eigenvalues of the initial six local $5 \times 5$ matrices for the control group (upper part) and six local $5 \times 5$ matrices for the animals exposed to PFOA

\begin{tabular}{cccccc} 
Control & \multicolumn{7}{c}{ (1-5 } & $2-6$ & $3-7$ & $4-8$ & $5-9$ & $6-10$ \\
\hline 3.9033 & 3.9734 & 3.9702 & 3.9260 & 3.9133 & 3.9257 \\
-0.8706 & -0.9789 & -0.9691 & -0.9220 & -0.9135 & -0.9340 \\
-0.9868 & -0.9844 & -0.9904 & -0.9787 & -0.9686 & -0.9666 \\
-1.0005 & -1.0043 & -1.0001 & -1.0098 & -1.0037 & -0.9993 \\
-1.0454 & -1.0058 & -1.0107 & -1.0155 & -1.0276 & -1.0259 \\
\hline PFOA & & & & \\
\hline $1-5$ & $2-6$ & $3-7$ & $4-8$ & $5-9$ & $6-10$ \\
\hline 2.5764 & 3.1119 & 2.7377 & 2.9094 & 2.7312 & 2.9496 \\
0.3272 & 0.1894 & 0.1122 & 0.4724 & 0.2316 & 0.2879 \\
-0.0703 & -0.4987 & -0.0779 & -0.9972 & -0.3822 & -0.9031 \\
-1.3373 & -1.2763 & -1.3281 & -1.0029 & -1.2680 & -1.0633 \\
-1.4960 & -1.5263 & -1.4439 & -1.3818 & -1.3126 & -1.2711 \\
\hline
\end{tabular}

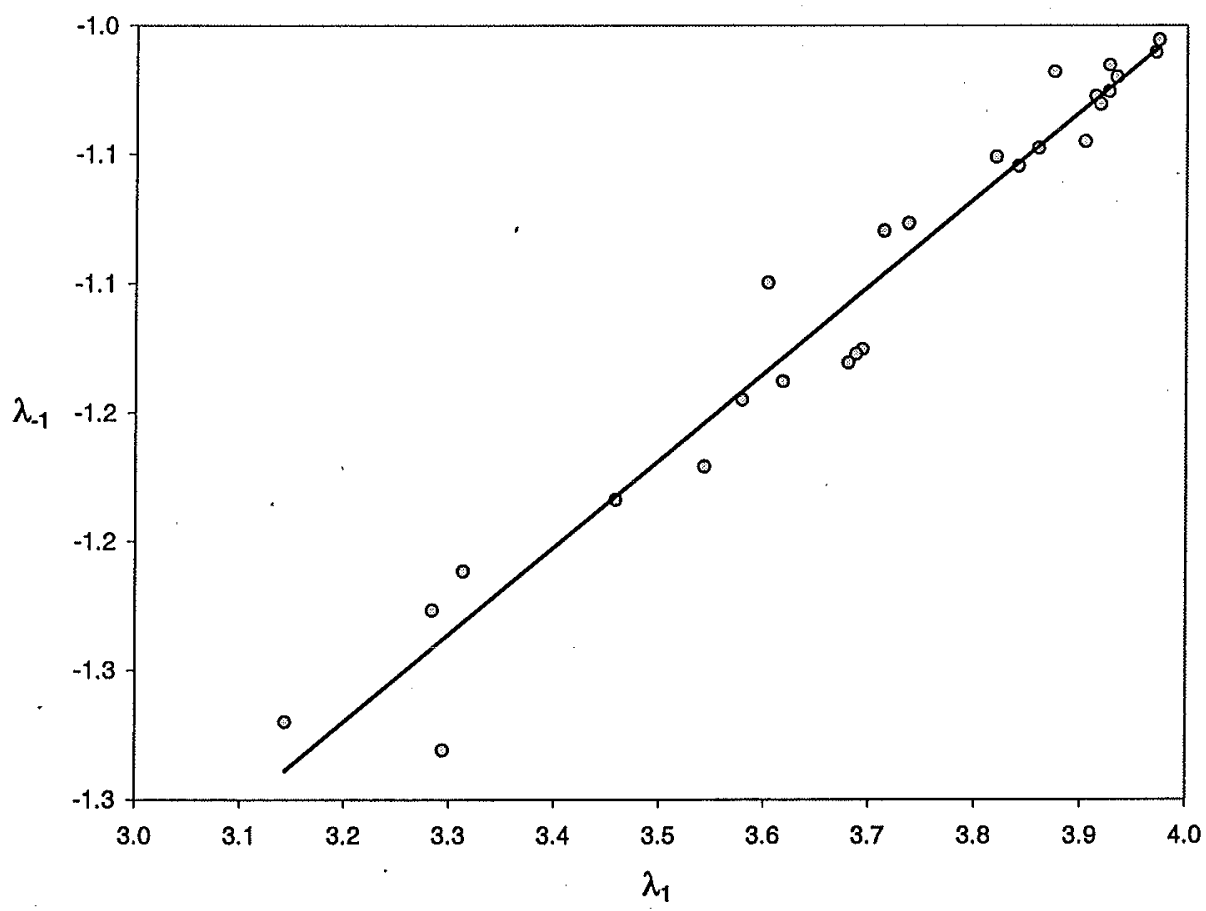

FIGURE 4 Plot of the smallest eigenvalue against the leading eigenvalue for the protein spots of the control group. 


\section{CHARACTERIZATION OF LOCAL FEATURES OF ZIGZAG CURVE.}

We will consider the $5 \times 5$ matrix of Table 2 as the first step in the construction of novel local map descriptors. The term local here does not represent a spatial (geometrical) localization but a sequential localization of a portion of the lengthy zigzag curve. Thus the zigzag curve is segmented into local groups of five spots, starting with spots $1-5$, and continuing with $2-6,3-7,4-8$, etc. We thus move gradually along the zigzag curve limiting attention each time to a small "window" of five spots for which we will extract local invariants.

Here we will confine our attention to the leading eigenvalues of the $5 \times 5$ local matrices and look upon them as the main map invariants. In the upper part of Table 4 we have listed the eigenvalues of the $5 \times 5$ quotient matrices for a few initial $5 \times 5$ matrices. An inspection of Table 4 shows a limited variation among the eigenvalues of the $5 \times 5$ matrices of the control group, which are all close to the values: $4,-1,-1,-1,-1$, which are the eigenvalues of $\mathrm{K}_{5}$, the complete graph with five vertices. In Table 5 we have listed the leading eigenvalue of all 26 local $5 \times 5$ matrices for the control group and local $5 \times 5$ matrices belonging to proteomics maps derived from animals exposed to the four peroxisome proliferators. As we see from the column of the "Control," the leading eigenvalues of the control group show rather limited variation in the magnitude of the leading eigenvalue. In part this is due to the dominant role of protein abundance. In contrast the eigenvalues of the $5 \times 5$ matrices belonging to maps of the four treatment groups show considerable variability, reflecting large perturbations of the proteome by the chemicals. If one is interested in a more balanced role of the three variables, charge, mass, and abundance, one should follow a recommendation of Kowalski and Bender [11] and re-scale the proteomics data accordingly. However, as our prime objective is to introduce novel invariants we will continue the analysis based on the nonscaled raw data.

Looking back to Table 4 where we listed all eigenvalues for the local $5 \times 5$ matrices we have to decide whether to use the leading eigenvalue or all eigenvalues for the construction of map invariants. In Figure 4 we have presented a plot of the last eigenvalue against the first (leading) eigenvalue for the control group in order to show the extent of correlation between the two. The correlation gave the following statistical parameters: the coefficient of correlation $r=0.979$; the standard error $s=0.017$; and the Fisher ratio $\mathrm{F}=546$. Although the residuals of the correlation are not negligible, which indicates that the two extreme eigenvalues do not fully duplicate each other, we decided to consider only the leading eigenvalue as the basic entity for construction of map invariants. Figure 5 presents a plot of the last eigenvalue against the first (leading) eigenvalue in the case of PFOA, which shows a similar overall relationship. The scatter of points in Figure 5, however, is greater, but there is still considerable parallelism between the extreme eigenvalues, as reflected by $\mathrm{r}=0.837, \mathrm{~s}=0.122$ and $\mathrm{F}=56$. 
M. RANDIĆ, J. ZUPAN, M. NOVIČ, MATRIX INVARIANTS FOR PROTEOMICS MAPS B.D. GUTE, and S.C. BASAK

In view of the observed parallelism between the extreme eigenvalues we will continue using only the leading eigenvalue as the basic matrix invariant. In Table 5 we have listed the leading eigenvalues for all five proteomics maps. Each column of Table 5 can be viewed as a 26-component vector characterizing each of the five proteomics maps. Hence, the similarities and dissimilarities among the five proteomics maps will be numerically represented by the differences among the five vectors.

TABLE V The leading eigenvalues of all 26 local $5 \times 5$ matrices based on zigzag curve of Fig. 1 for all five proteomics maps considered

\begin{tabular}{|c|c|c|c|c|c|}
\hline & Control & PFOA & $P F D A$ & Clofibrate & $D E H P$ \\
\hline 1 & 3.9033 & 2.5764 & 3.2362 & 3.1507 & 2.7307 \\
\hline 2 & 3.9734 & 3.1119 & 3.3506 & 2.8263 & 3.1429 \\
\hline 3 & 3.9702 & 2.7377 & 2.6996 & 2.5617 & 3.2769 \\
\hline 4 & 3.9260 & 2.9094 & 2.4484 & 2.8159 & 2.5997 \\
\hline 5 & 3.9133 & 2.7312 & 2.8567 & 2.7215 & 2.4564 \\
\hline 6 & 3.9257 & 2.9496 & 3.1507 & 2.9137 & 2.5042 \\
\hline 7 & 3.9332 & 3.0236 & 2.9362 & 2.9570 & 2.5989 \\
\hline 8 & 3.9178 & 2.8880 & 2.3705 & 3.3361 & 2.8545 \\
\hline 9 & 3.8591 & 2.5483 & 2.5315 & 2.9360 & 3.1889 \\
\hline 10 & 3.8402 & 2.9634 & 2.5554 & 2.8385 & 3.4237 \\
\hline 11 & 3.5428 & 3.7656 & 2.8135 & 3.0547 & 3.4209 \\
\hline 12 & 3.2941 & 2.9645 & 2.1947 & 2.4799 & 2.7120 \\
\hline 13 & 3.1434 & 2.3630 & 2.1012 & 2.4305 & 2.6499 \\
\hline 14 & 3.4579 & 1.9752 & 2.0482 & 2.4392 & 2.7158 \\
\hline 15 & 3.6933 & 2.3875 & 2.4741 & 3.1507 & 3.1717 \\
\hline 16 & 3.6800 & 2.7153 & 2.7255 & 2.4481 & 2.9840 \\
\hline 17 & 3.6878 & 2.7506 & 2.8027 & 3.0694 & 3.4465 \\
\hline 18 & 3.6033 & 2.4135 & 2.3861 & 2.6511 & 2.7437 \\
\hline 19 & 3.7134 & 2.6168 & 2.2526 & 2.4710 & 2.3154 \\
\hline 20 & 3.7368 & 3.3156 & 2.4563 & 2.2570 & 2.2612 \\
\hline 21 & 3.8193 & 3.7526 & 2.5347 & 2.7972 & 2.5245 \\
\hline 22 & 3.8740 & 2.9867 & 2.4442 & 2.5494 & 2.2715 \\
\hline 23 & 3.5787 & 2.5714 & 2.7391 & 2.6928 & 2.4788 \\
\hline 24 & 3.2839 & 3.0777 & 2.7321 & 2.0219 & 2.6027 \\
\hline 25 & 3.3132 & 3.9721 & 2.5721 & 2.3041 & 2.9137 \\
\hline 26 & 3.6180 & 3.5496 & 2.3849 & 2.3721 & 2.3774 \\
\hline
\end{tabular}




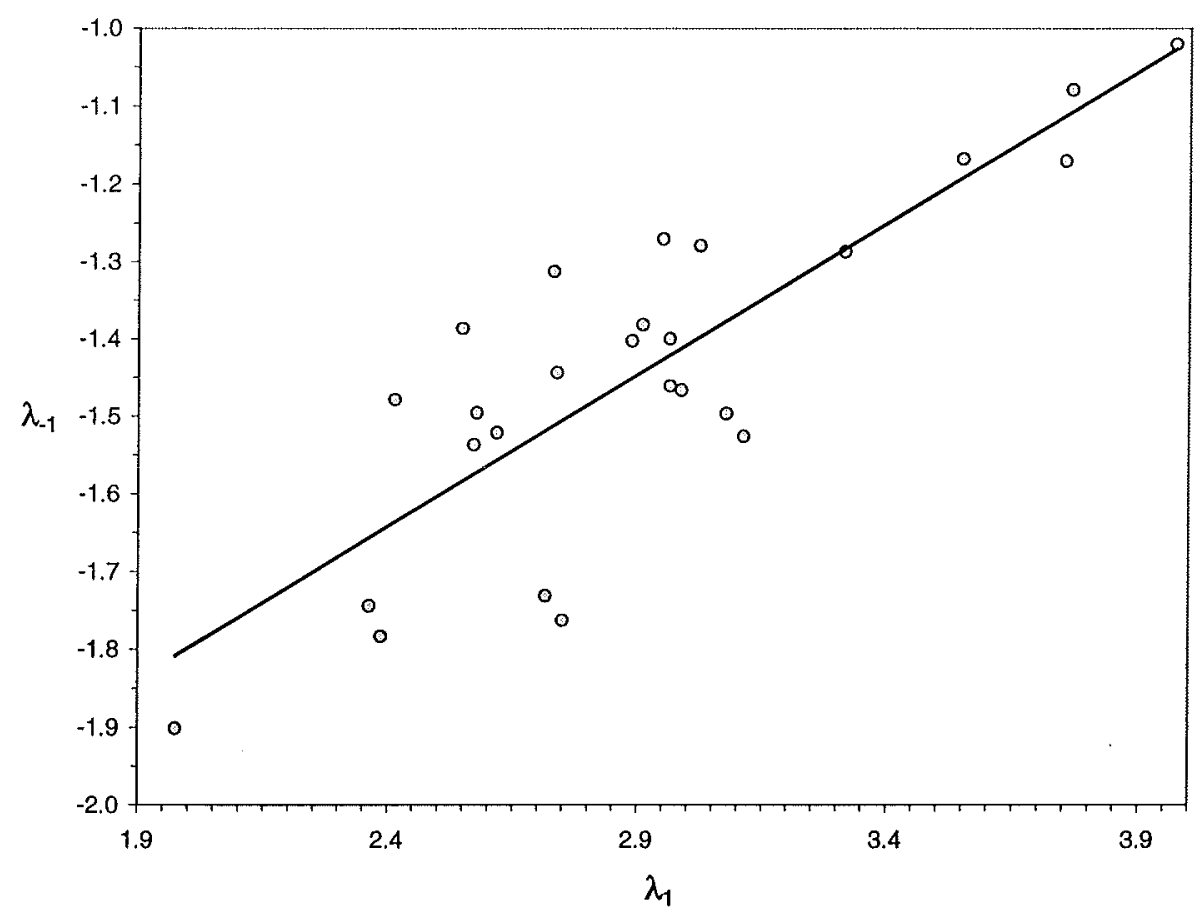

FIGURE 5 Plot of the smallest eigenvalue against the leading eigenvalue for the protein spots of the PFOA group.

\section{ON THE SIMILARITY/DISSIMILAIRTY OF PROTEOMICS MAPS}

We will use the 26-component vectors to represent proteomics maps and the Euclidean distance in the 26dimensional vector space as a measure of the degree of similarity/dissimilarity among the proteomics maps. Clearly, if two maps are very similar the corresponding 26-dimensional vectors will have similar components and the vector that has as components the difference between the corresponding components of the two vectors will have small magnitude. Consequently, the corresponding entry in the similarity/dissimilarity matrix will be small, close to zero. Conversely, large entries in the similarity/dissimilarity table would indicate vastly different proteomics maps. In Table 6 we show the corresponding values of similarity/dissimilarity for the five maps considered. In the first row of Table 6 are the similarities/dissimilarities with respect to the Control group. As we see all entries in the similarity/dissimilarity table are relatively large. The smallest entry in the first row of the table, which belongs to the PFOA proteomics map, indicates the greatest similarity to the control group. However, all the entries in the first row are of a similar, relatively large magnitude, suggesting little similarity to the control group. Equally, the four proteomics maps among themselves also show little similarity and 
considerable differences. Overall, the most similar pairs of are: PDFA and Clofibrate, and Clofibrate and DEHP.

TABLE VI The similarity/dissimilarity table based on map vectors having 26 components

\begin{tabular}{c|ccccc} 
& Control & PFOA & PFDA & Clofibrate & DEHP \\
\hline Control & 0 & 4.832 & 5.760 & 5.265 & 5.141 \\
PFOA & & 0 & 2.984 & 3.184 & 3.088 \\
PFDA & & & 0 & 1.874 & 2.349 \\
Clofibrate & & & & 0 & 1.877 \\
DEHP & & & & & 0
\end{tabular}

Just looking at Table 1 it is difficult to determine the degree of similarity among the different columns of the table. In contrast the columns of Table 5 are more informative. A similar magnitude of the leading eigenvalue in any of the 26 rows points to local similarity of the two maps with respect to the five protein spots considered. For example, in row 11 three of the four proteomics maps show close values for the leading eigenvalue suggesting minor variation in the respective abundance. More important, however, is the fact that the approach outlined here automatically gives a lower weight to the contributions of the protein spots that have changed very little by the treatment. This would not be the case if the approach considered a whole 30x30 matrix because the abundances of the spots which are marginally altered would contribute to the averaging of the abundances of the strongly perturbed spots and effectively decrease the signal to noise ratio.

This novel approach has additional flexibility that we will not consider here. This is associated with the liberty of selecting the width of the "window" for building local $n \times n$ matrices. In the case of $5 \times 5$ local matrices about half of the matrix entries are variable, while the diagonal zeros and adjacent one always remain constant.

\section{SPECTRAL CHARACTERISTICS OF PROTEOMICS MAPS}

In this section we will consider the use of all eigenvalues of local matrices for the characterization of proteomics maps, rather than limiting our attention solely to the leading eigenvalue. The complete set of eigenvalues of a graph is known in Graph Theory $[12,13]$ as "graph spectra," so we will refer to characterizations of proteomics maps based on information given by all eigenvalues as "spectral characteristics" of maps.

The extreme form for the $5 \times 5$ matrices that can occur corresponds, on the one hand, to the complete graph $\mathrm{K}_{5}$ and to the chain of length $\mathrm{L}_{5}$ on the other hand. The $5 \times 5$ matrices for both these extreme cases 
are shown in Table 7 together with their eigenvalues. Hence, any of the local $5 \times 5$ matrices that appear in our analysis (such as those shown in Table 3) can be viewed as representing a departure from the two extreme cases. It appears that the leading eigenvalue, which in the case of acyclic graphs has been interpreted by Lovasz and Pelikan [14] as an index of molecular branching, may have a somewhat modified, composite, interpretation. Branching is just one aspect of the departure of a structure from that of a linear model that has received considerable attention in Chemical Graph Theory [15-20]. The other factor which may influence the graph spectra, which has apparently not attracted attention and has, in fact, only recently been recognized [21], is the departure of a graph from the complete bipartite graph $\mathrm{K}_{1, \mathrm{n}}$ in the case of acylcic graphs, or a departure from the complete graph $\mathrm{K}_{\mathrm{n}}$ for more general graphs. This departure is reflected in the variable magnitudes (in the interval $[0,1]$ ) of the matrix elements of the local $5 \times 5$ matrices, which are then viewed as the edges of a weighted complete graph [21].

TABLE VII The $5 \times 5$ matrices belonging to the complete graph $\mathrm{K}_{5}$ and the linear chain $\mathrm{L}_{5}$ with their eigenvalues

\begin{tabular}{l|llllll} 
& 1 & 2 & 3 & 4 & 5 \\
\hline 1 & 0 & 1 & 1 & 1 & 1 \\
2 & & 0 & 1 & 1 & 1 \\
3 & & & 0 & 1 & 1 \\
4 & & & & 0 & 1
\end{tabular}

Eigenvalues: $4,-1,-1,-1,-1$

\begin{tabular}{l|lllll} 
& 1 & 2 & 3 & 4 & 5 \\
\hline 1 & 0 & 1 & 0 & 0 & 0 \\
2 & & 0 & 1 & 0 & 0 \\
3 & & & 0 & 1 & 0 \\
4 & & & 0 & 1 \\
5 & & & & & 0
\end{tabular}

Eigenvalues: $\sqrt{ } 3,1,0,-1,-\sqrt{ } 3$

That indeed, the eigenvalues of the novel local $5 \times 5$ matrices do represent the departure from "linearity" and "completeness" is supported by additional (unpublished) data [21], part of which is presented in Table 8. Here letters A, B, C, D, and E correspond to the control, PFOA, PFDA Clofibrate, and DEHP treatments, respectively, while subscripts $1-26$ correspond to local $5 \times 5$ matrices. Thus the label $A_{1}$ represent the local matrix based on spots $1-5$ of the zigzag curve of the control group, while the label $\mathrm{D}_{24}$ corresponds to zigzag curve of Clofibrate proteomics map using spots $24-28$. In Table 8 the labels $a_{K}$ and $a_{L}$ represent the coefficients of linear regressions based on the ordered sets of eigenvalues of 
the complete graph and eigenvalues of the chain of length five. The labels $r, s$, and $F$ stand for the correlation coefficient, the standard error, and the Fisher ratio in a correlation of eigenvalues of $5 \times 5$ matrices as the independent variable and the eigenvalues of $\mathrm{K}_{5}$ and $\mathrm{L}_{5}$ as the dependent variables. Thus the first numerical column abbreviates the regression equation shown below between the eigenvalues of the first local $5 \times 5$ matrix and the eigenvectors of the extreme $5 \times 5$ matrices of $\mathrm{K}_{5}$ and $\mathrm{L}_{5}$ :

\begin{tabular}{|l|}
\hline 3.9033 \\
-0.8706 \\
-0.9868 \\
-1.0005 \\
-1.0454
\end{tabular}
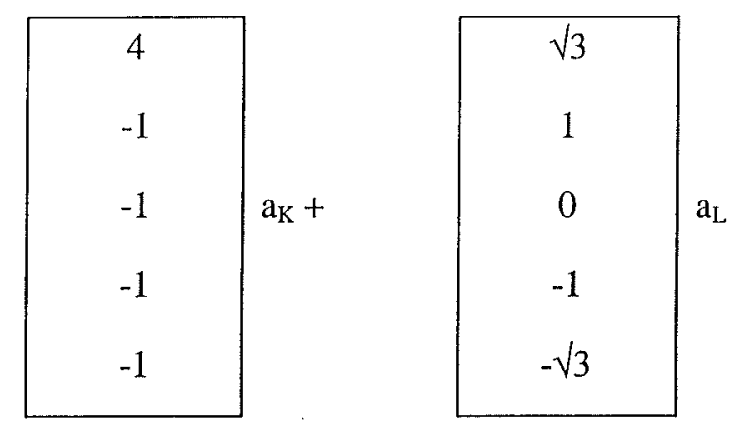

Observe that the correlation relates vectors built from all the five eigenvalues and that the constant term of the regression was not set to be zero, but was always found to be zero.

TABLE VIII The coefficients of the linear regression of the eigenvalues of a selection of $5 \times 5$ local matrices against the eigenvalues of $K_{5}$ and $L_{5}$. The statistical parameters $r, s$, and $F$ are included ( $r=$ regression coefficient, $s=$ standard error, $\mathrm{F}=$ Fisher ratio)

\begin{tabular}{l|llllll} 
& $A_{1}$ & \multicolumn{1}{c}{$A_{2}$} & \multicolumn{1}{c}{$A_{3}$} & $A_{4}$ & \multicolumn{1}{c}{$A_{5}$} & $A_{6}$ \\
\hline$K_{5}$ & 0.95031 & 0.98856 & 0.98619 & 0.96654 & 0.96046 & 0.96695 \\
$L_{5}$ & 0.05893 & 0.01104 & 0.01470 & 0.03453 & 0.04129 & 0.03346 \\
$r$ & 0.99995 & 1.00000 & 1.00000 & 0.99999 & 1.00000 & 1.00000 \\
$s$ & 0.0308 & 0.0048 & 0.0035 & 0.0149 & 0.0078 & 0.0012 \\
$F$ & 10072.2 & 431924.5 & 9796381.9 & 43388.5 & 158533.8 & 7309534.4
\end{tabular}

\begin{tabular}{l|llllll} 
& $B_{1}$ & $B_{2}$ & \multicolumn{1}{r}{$B_{3}$} & \multicolumn{1}{c}{$B_{4}$} & \multicolumn{1}{c}{$B_{5}$} \\
\hline$K_{5}$ & 0.32416 & 0.49658 & 0.40366 & 0.46155 & 0.41888 & 0.50555 \\
$L_{5}$ & 0.73885 & 0.64987 & 0.64840 & 0.61384 & 0.60952 & 0.53546 \\
$r$ & 0.9926 & 0.9990 & 0.9909 & 0.9834 & 0.9961 & 0.9898 \\
$s$ & 0.2820 & 0.1170 & 0.3215 & 0.4556 & 0.2082 & 0.3545 \\
$F$ & 66.8 & 507.8 & 54.0 & 29.3 & 125.8 & 48.1
\end{tabular}

\begin{tabular}{l|llllll} 
& $B_{14}$ & $C_{12}$ & \multicolumn{1}{c}{$C_{13}$} & \multicolumn{1}{c}{$C_{14}$} & \multicolumn{1}{c}{$C_{19}$} & \multicolumn{1}{c}{$D_{24}$} \\
\hline$K_{5}$ & 0.10779 & 0.19895 & 0.15997 & 0.13907 & 0.23165 & 0.12975 \\
$L_{5}$ & 0.89145 & 0.80763 & 0.84370 & 0.86134 & 0.76559 & 0.86760 \\
$r$ & 0.9895 & 0.9951 & 0.9973 & 0.9964 & 0.9900 & 0.9917 \\
$s$ & 0.2973 & 0.2080 & 0.1513 & 0.1754 & 0.2994 & 0.2638 \\
$F$ & 46.7 & 101.7 & 186.6 & 136.5 & 49.3 & 59.7
\end{tabular}


As we can see from the top part of Table 7, the eigenvalues of all $5 \times 5$ matrices of the control group show linear combinations dominated by the eigenvalues of $\mathrm{K}_{5}$ (from about $95 \%$ to $99 \%$ ), with a very small participation of $\mathrm{L}_{5}$ eigenvalues. On the other hand, from the middle part of Table 8 , which corresponds to proteomics maps of PFOA, we see a more balanced mixture of the roles of the eigenvalues of $\mathrm{K}_{5}$ and $\mathrm{L}_{5}$. In fact, in the case of $\mathrm{B}_{6}$ we see that an almost $50 / 50$ superposition of the eigenvalues of the complete graph and the linear chain give its eigenvalues. In the bottom part of Table 8 , we have collected six $5 \times 5$ matrices in which the eigenvalues of the linear graph appear to play the dominant role. In the latter cases, the eigenvalues of $5 \times 5$ matrices are approximately given as a linear combination of about 85 $\%$ of the eigenvalues of the linear model and about $15 \%$ of the eigenvalues of the complete graph $\mathrm{K}_{5}$.

TABLE IX The quotients $\mathrm{a}_{\mathrm{L}} / \mathrm{a}_{\mathrm{K}}$ as a single local map spectral descriptor

\begin{tabular}{lccccc}
\hline$L_{S} / K_{5}$ & Control & PFOA & PFDA & Clofibrate & DEHP \\
\hline 1 & $.0589 / .9503$ & $.7389 / .3242$ & $.5118 / .5875$ & $.4300 / .6015$ & $.7306 / .3663$ \\
2 & $.0110 / .9886$ & $.6499 / .4966$ & $.5034 / .6196$ & $.6759 / .4139$ & $.5786 / .5352$ \\
3 & $.0147 / .9862$ & $.6484 / .4037$ & $.7268 / .3602$ & $.7697 / .3071$ & $.4691 / .6161$ \\
4 & $.0345 / .9665$ & $.6138 / .4616$ & $.7802 / .2743$ & $.7708 / .3835$ & $.6786 / .3569$ \\
5 & $.0413 / .9605$ & $.6095 / .4189$ & $.6519 / .4319$ & $.6202 / .4118$ & $.6875 / .3164$ \\
6 & $.0335 / .9670$ & $.5355 / .5056$ & $.5030 / .5699$ & $.5974 / .4697$ & $.6652 / .3380$ \\
7 & $.0276 / .9714$ & $.4840 / .5463$ & $.5974 / .4754$ & $.5528 / .4983$ & $.7641 / .3189$ \\
8 & $.0394 / .9624$ & $.5730 / .4739$ & $.7407 / .2719$ & $.3146 / .6978$ & $.6465 / .4316$ \\
9 & $.0635 / .9373$ & $.7137 / .3281$ & $.7468 / .3095$ & $.5877 / .4795$ & $.5652 / .5525$ \\
10 & $.0798 / .9255$ & $.5936 / .4838$ & $.7087 / .3320$ & $.6207 / .4408$ & $.3469 / .7057$ \\
11 & $.2461 / .7792$ & $.1165 / .8910$ & $.6772 / .4101$ & $.5263 / .5358$ & $.3940 / .6846$ \\
12 & $.3615 / .6670$ & $.6329 / .4671$ & $.8076 / .1990$ & $.6984 / .3176$ & $.6880 / .3801$ \\
13 & $.3736 / .6241$ & $.8749 / .2119$ & $.8437 / .1600$ & $.7190 / .2963$ & $.7102 / .3550$ \\
14 & $.2738 / .7459$ & $.8915 / .1078$ & $.8613 / .1391$ & $.7105 / .3021$ & $.6508 / .3972$ \\
15 & $.4049 / .6872$ & $.8837 / .2142$ & $.7956 / .2740$ & $.7110 / .2895$ & $.4644 / .5918$ \\
16 & $.1594 / .8510$ & $.7789 / .3416$ & $.7081 / .3730$ & $.6860 / .3150$ & $.5071 / .5264$ \\
17 & $.1658 / .8502$ & $.7741 / .3525$ & $.6952 / .3996$ & $.6359 / .4920$ & $.3059 / .7292$ \\
18 & $.1751 / .8250$ & $.7868 / .2627$ & $.7366 / .2762$ & $.7617 / .3330$ & $.6588 / .4007$ \\
19 & $.1184 / .8771$ & $.7121 / .3459$ & $.7656 / .2317$ & $.7885 / .2763$ & $.7969 / .2338$ \\
20 & $.1165 / .8837$ & $.4161 / .6487$ & $.7119 / .3058$ & $.8056 / .2154$ & $.7840 / .2258$ \\
21 & $.0834 / .9187$ & $.1952 / .8536$ & $.7017 / .3298$ & $.7082 / .3927$ & $.6542 / .3479$ \\
22 & $.0598 / .9426$ & $.6330 / .4726$ & $.8064 / .2619$ & $.7369 / .3183$ & $.7883 / .2265$ \\
23 & $.2303 / .7949$ & $.8272 / .2847$ & $.6914 / .3854$ & $.7571 / .3454$ & $.7823 / .2810$ \\
24 & $.3459 / .6712$ & $.6274 / .4978$ & $.635 / .4080$ & $.8675 / .1298$ & $.7529 / .3246$ \\
25 & $.3158 / .6916$ & $.0206 / .9841$ & $.6827 / .3474$ & $.7692 / .2430$ & $.6456 / .4489$ \\
26 & $.1985 / .8185$ & $.2565 / .7763$ & $.7257 / .2820$ & $.7444 / .2707$ & $.7335 / .2767$ \\
\hline & & & & & \\
\hline & & & & &
\end{tabular}




\section{NOVEL SPECTRAL INDEX FOR MAPS}

The above description of linear regression of local matrices using the eigenvalues of $\mathrm{K}_{5}$ and $\mathrm{L}_{5}$ as dependent variables represents an important novelty in the area of topological indices [22-25], and may be of potential use in QSAR (quantitative structure-activity relationship) studies, QSPR (quantitative structure-property relationship) studies, and similarity studies of primary DNA sequences [26-33]. In particular this new way of combining data on eigenvalues among structurally related objects will open new directions in the investigation of eigenvalues as molecular descriptors [34]. Moreover, if one chooses, as we have done here, as extreme structures the linear chain and the complete bipartite graph $\mathrm{K}_{1, \mathrm{n}}$ (or alternatively $\mathrm{K}_{\mathrm{n}}$ ) as the two graphs to which others are compared one may consider, in an analogy with Kier's kappa shape indices [35], the coefficients of the linear regression as a new kind of shape descriptors [21]. As we have seen, it is possible to condense information on all eigenvalues of a system into a small number of parameters, such as the coefficients of regression $a_{K}$ and $a_{L}$. Relating the eigenvalues of $5 \times 5$ matrices in terms of the eigenvalues of the linear model and the complete graph is a striking novelty in view of the long and continuous interest in the eigenvalues and the eigenvectors of graphs [13]. Besides its important theoretical significance, the results allow us to construct novel map descriptors that condense the information on all five eigenvalues into a single entity! We can take the quotient of the coefficients of the regression $\left(a_{K} / a_{L}\right)$ or its reciprocal $\left(a_{L} / a_{K}\right)$, shown in Table 9 for all five proteomics maps, as novel condensed spectra map descriptors. For the data shown in Table 9 the quotients would range in the interval of about $(0.1,10)$. If we use the quotient $\left(a_{K} / a_{L}\right)$ we give somewhat greater weight to matrices approaching $K_{5}$, while if we use the quotient $\left(a_{L} / a_{K}\right)$ the opposite is the case, the matrices approaching $\mathrm{K}_{5}$ play a somewhat lesser role. The former case corresponds to protein spots that have been less perturbed, while in the latter case we give somewhat larger weight to protein spots that have undergone dramatic changes in their abundance compared to the control group. Both alternatives may be of interest. In the first case, we diminish to some extent the occurrence of observed changes, which may be fitting if one has some doubt in the accuracy of the reported abundance. In the second case, assuming that the reliability of data is satisfactory, we will improve somewhat the signal to noise ratio, as we give less weight to protein spots that have not changed much.

In Table 10 we show the similarity/dissimilarity table for the five proteomics maps based on the 26component vectors using the quotient $\left(a_{L} / a_{K}\right)$. Not surprisingly we obtain similar results to those of Table 5 when we used the leading eigenvalues of the 26 local matrices as the components of vectors. The

satisfaction is not even in the fact that the alternative approach allows for minor changes in the interpretation between results based on two 26-components vectors. The satisfaction is in the recognition 
B.D. GUTE, and S.C. BASAK

that we have used all the available spectral information, rather than restricting attention solely to the leading eigenvalues of the quotient matrices.

TABLE X The similarity/dissimilarity table based on vectors having 26 components obtained from spectral quotients $a_{\mathrm{L}} / \mathrm{a}_{\mathrm{K}}$

\begin{tabular}{l|ccccc} 
& Control & PFOA & PFDA & Clofibrate & DEHP \\
\hline Control & 0 & 11.7959 & 12.6593 & 11.3304 & 9.3268 \\
PFOA & & 0 & 6.8121 & 10.4034 & 9.9604 \\
PFDA & & & 0 & 8.1907 & 7.8770 \\
Clofibrate & & & & 0 & 10.0419 \\
DEHP & & & & & 0
\end{tabular}

\section{CONCLUDING REMARKS}

We continue our interest in developing biodescriptors for proteomics maps that could facilitate comparisons of proteomics maps from a single source, or even from different laboratories. In this contribution, we have come forward with two important novelties: (1) For the first time we present the concept of local map invariants; and (2) For the first time we have drawn attention to the possibility of a meaningful reduction of map invariants (here the complete spectrum of local $5 \times 5$ matrices) without any accompanying loss of essential information. All of these novelties can be combined with the already considered map descriptors based on alternative zigzag curves, embedded graphs based on partial ordering of protein spots, or cluster graphs based on embedded graph connecting spots below selected critical distance. It is expected that the group of local and global proteomics descriptors developed by our group will find applications in the quantitative characterization of proteomic patterns and the assessment of the effects of drugs and toxicants on the cellular proteome.

\section{Acknowledgments}

The authors acknowledge the financial support of this research by the United States Air Force Office of Scientific Research (grant F496200210138) and the Slovenian Ministry of Science (grants P104-507 and P104-508). This is contribution number 321 from the Center for Water and the Environment of the Natural Resources Research Institute. 


\section{References}

[1] Randić, M. (2001). On graphical and numerical characterization of proteomics maps. J. Chem. Inf. Comput. Sci. 41, 1330-1338.

[2] Randić, M., Zupan, J., and Novič, M. (2001). On 3-D graphical representation of proteomics maps and their numerical characterization. J. Chem. Inf. Comput. Sci. 41, 1339-1334.

[3] Randić, M., Witzmann, F., Vračko, M., and Basak, S.C. (2001). On characterization of proteomics maps and chemically induced changes in proteomics using matrix invariants: Application to peroxisome proliferators. Med. Chem. Res. 10, 456-479.

[4] Randić, M. (2002). A graph theoretical characterization of proteomics maps. Int. J. Quantum Chem. (in press).

[5] Randić, M. and Basak, S.C. (2002). A comparative study of proteomics maps using graph theoretical biodescriptors. J. Chem. Inf. Comput. Sci. (in press).

[6] Randić, M. and Basak, S.C. (2002). Canonical labeling for protein spots and proteomics maps. J. Chem. Inf. Comput. Sci. (submitted).

[7] Bajzer, Ž., Randić, M., Plavšić, D., and Basak, S.C. (2002). Novel matrix invariants for characterization of toxic effects on proteomics maps. J. Mol. Graphics and Modeling (submitted).

[8] Witzmann, F. Molecular Anatomy Laboratory, Department of Biology, Indiana University \& Purdue University, Columbus, IN 47203.

[9] Randić, M., Kleiner, A.F., and DeAlba, L.M. (1994). Distance/Distance matrices. J. Chem. Inf. Comput. Sci. 34, 277-286.

[10] Buckley, F. and Harary, F. (1989). Distance in Graphs. Addison-Wesley, Reading, MA.

[11] Kowalski, B.R. and Bender, C.F. (1972). A powerful approach to interpreting chemical data. J. Am. Chem. Soc. 94, 5632-5639.

[12] Harary, F. (1969). Graph Theory. Addison-Wesley, Reading, MA.

[13] Cvetković, D.M., Doob, M., and Sachs, H. (1995). Spectra of Graphs - Theory and Applications, $3^{\text {rd }}$ revised and enlarged edition. Johann Ambrosius Barth Verlag, Heidelberg-Leipzig.

[14] Lovasz, L. and Pelikan, J. (1973). On the eigenvalue of trees. Period. Math. Hung. 3, 175-182.

[15]. Randic, M. (1975). On characterization of molecular branching. J. Am. Chem. Soc. 97, 6609-6615.

[16] Cvetković, D. and Gutman, I. (1977). Note on branching. Croat. Chem. Acta 49, 115-121.

[17] Bonchev, D. and Trinajstić, N. (1978). On topological characterization of molecular branching. Int. J. Quantum Chem: Quantum Chem. Symp. 12, 293-303.

[18] Bonchev, D., Knop, J.V., and Trianjstic, N. (1979). Mathematical models of branching. MATCH 6, 21-47.

[19] Hasselbarth, W. (1984). Die verzweigheit von graphen. MATCH 16, 3-17.

[20] Randic, M. (1997). On molecular branching. Acta Chim. Slovenica 44, 57-77.

[21] Randić, M. (2002). MATH/CHEM/COMP 2002, June 23-29, Dubrovnik, Croatia.

[22] Randic, M. (1998). Topological indices. In The Encyclopedia of Computational Chemistry (P.v.R. Schleyer, N.L. Allinger, T. Clark, J. Gasteiger, P.A. Kollman, H.F. Schaefer III, and P.R. Schreiner, Eds.). John Wiley \& Sons, Chichester, pp. 3018-3032.

[23] Basak, S.C., D. Mills, B.D. Gute, G.D. Grunwald and A.T. Balaban. 2001. Applications of topological indices in the property / bioactivity/ toxicity prediction of chemicals, in: Topology in Chemistry: Discrete Mathematics of Molecules, D.H. Rouvray, R.B. King, Eds., Horwood Publishing Ltd.: Chichester, West Sussex, United Kingdom, pp. 113-184.

[24] Katritzky, A.R., Lobanov, V.S., and Karelson, M. (1995). QSPR: The correlation and quantitative prediction of chemical and physical properties from structure. Chem. Soc. Rev. 24, 279-287. 
B.D. GUTE, and S.C. BASAK

[25] Randić, M. and Basak, S.C. (2002). Variable molecular descriptors. In Some Aspects of Mathematical Chemistry (D.K. Sinha, S.C. Basak, R.K. Mohanty, and I.N. Basumallick, Eds.). Visva-Bharati University Press, Shantineketan, India (in press).

[26] Randic, M., Nandy, A., and Basak, S.C. (2002). On the numerical characterization of DNA primary sequences. J. Math. Chem. (submitted).

[27] Randić, M., Vračko, M., Nandy, A., and Basak, S.C. (2000). On 3-D graphical representation of DNA primary sequences and their numerical characterization. J. Chem. Inf. Comput. Sci. 40, 1235-1244.

[28] Randić, M. (2000). Condensed represenation of DNA primary sequences. J. Chem. Inf. Comput. Sci. 40, 5056.

[29] Randić, M. (2000). On characterization of DNA primary sequences by condensed matrix. Chem. Phys. Lett. 317, 29-34.

[30] Randić, M. and Vračko, M. (2000). On the similarity of DNA primary sequences. J. Chem. Inf. Comput. Sci. 40, 599-606.

[31] Randić, M. and Basak, S.C. (2001). Characterization of DNA primary sequences based on the average distance between bases. J. Chem. Inf. Comput. Sci. 41, 561-568.

[32] Randić, M., Guo, X., and Basak, S.C. (2000). On characterization of DNA primary sequences by triplet of nucleic acid bases. J. Chem. Inf. Comput. Sci. 41, 6191-626.

[33] Randić, M. and Balaban, A.T. (2002). 4-Dimensional DNA. DNA Sequence (submitted).

[34] Randić, M., Vračko, M., and Novic, M. (2001). Eigenvalues as molecular descriptors. In, QSPR/QSAR Studies by Molecular Descriptors (M.V. Diudea, Ed.). Nova Science Publishers, Huntington, NY, pp. 147211.

[35] Kier, L.B. (1985). A shape index from molecular graphs. Quant. Struct.- Act. Relat. 4, 109-116. 
. 


\title{
ON CANONICAL LABELING OF PROTEINS OF PROTEOMICS MAPS
}

\author{
MILAN RANDIĆ ${ }^{1,2}$ and SUBHASH C. BASAK ${ }^{2}$ \\ ${ }^{I}$ National Institute of Chemistry, POB 3430, 1001 Ljubljana, Slovenia; \\ ${ }^{2}$ Natural Resources Research Institute, Univ. of Minnesota at Duluth, Duluth, MN
}

\begin{abstract}
We propose a canonical labeling of protein spots in 2-D proteomics maps which will not only facilitate cataloging of 2-D gel maps but also to facilitate comparisons of gel maps originating from different laboratories. The canonical labels are based on labeling of vertices of an embedded graph derived from partial ordering of the protein spots of a 2-D gel that would result in adjacency matrix associated with the smallest binary number obtained by reading the matrix entries from the left to the right and from the top to the bottom.
\end{abstract}

\section{INTRODUCTION}

A 2-D proteomics map summarizes important information regarding a large number of proteins of a particular cell type, tissue or organ, which are separated electrophoretically by charge and by use of chromatography by mass. Typically proteomics gels may have 2000 or more protein spots and provide raw information on the make up of the totality of proteins in a particular cell type, tissue or organ under investigation. Besides a number of experimental challenges, a major challenge in proteomics research, from the theoretical point of view, is the "translation" of the information that proteomics map supplies into some numerical characterization independent of spot labels that would represent map invariants. In analogy with QSAR (quantitative structure-activity relationship) in which chemical structure is characterized by various mathematical graph invariants (topological ${ }^{1}$ and topographic ${ }^{2}$ descriptors like various connectivity indices and $\mathrm{such}^{3}$ derived from the representation of molecules by molecular graphs and 3-D models), we would like to characterize proteomics maps by mathematical invariants, to be referred as map invariants or biodescriptors, in view that they characterize a particular biological sample. Advantages of such characterization of proteomics maps are obvious: comparison of maps, instead of being visual, qualitative, and slow, can become computerized, fast, and quantitative. What is not obvious at all is how to accomplish this task.

Recently, however, research on the quantitative characterization of proteomics maps has been initiated, $^{4-8}$ and has been shown to offer promising novel tools for quantitative studies of map 
characterization and their perturbations by exposure of cells/tissue to chemical agents that cause of changes in protein patterns. ${ }^{9}$ Construction of biodescriptors for proteomics maps, which are numerical quantities of maps, is clearly of major importance. However, equally important is development of numerical representations of maps, which can then allow 2-D maps to be sorted and catalogued. We differentiate here characterizations of maps from representations of maps, in the former we associate with a map a set of descriptors, in the latter we associate with a map a set of codes (preferably those that can be directly manipulated by computer). Thus with characterization is associated some loss of information and a map can not be reconstructed from its descriptors, just as molecular structure in general can not be reconstructed from a set of numerical graph invariants (topological indices). In contrast representations of maps should lead to reconstruction, at least of the adjacency matrix of a graph associated with a map, if one know and follows the assumed convention on labeling of vertices, such as use of canonical labels. A full reconstruction of a map requires information on embedding of the graph which can be supplied by matrix the elements of which give the Euclidean distances between connected spots.

In this communication we propose the construction of a unique graph to be associated with a map using a particular canonical labeling of vertices of such graph. In this way, as will be seen, we can generate binary or decimal codes for proteomics maps, which can facilitate comparisons of gel maps either within a single laboratory or maps originating from different laboratories. The canonical labels that we have adopted are based on the labeling of vertices of an embedded graph (of the partial ordering) that would result in adjacency matrix associated with the smallest binary number obtained by reading the matrix entries from the left to the right and from the top to the bottom as a single number. Alternatively canonical labels associated with the smallest binary number can be interpreted a sequence of binary or decimal numbers, each corresponding to successive lines of the canonical matrix. We will outline the approach on a smaller hypothetical map that has twenty protein.

\section{PARTIAL ORDERING OF PROTEIN.SPOTS OF A 2-D MAP}

Partial ordering arises when a set of objects (here proteins of a gel map) are ranked with respect to at least two properties, e. g., the charge and the mass, to be considered here. As a results we obtain two sequences, each based on ordering of objects with respect to a single property. Partial ordering is the collection of all subsequences of the two sequences, which maintain the same relative ordering of objects with respect to both properties. If in both sequences element $X$ is ahead of $Y$, which is ahead of $Z$, we would say that $\mathrm{X}$ dominates $\mathrm{Y}$, which, in turn, dominates $\mathrm{Z}$. The subsequence $\mathrm{X}>\mathrm{Y}>\mathrm{Z}$ is a fragment of 
the partial ordering of objects considered. The totality of all such dominance relationships constitute the partial ordering.

We have used letters $\mathrm{A}-\mathrm{T}$ to label protein spots of the hypothetical proteomics map of Fig. 1 in which we will illustrate our canonical representation of proteomics maps. Proteins are separated by charge along the horizontal axis and are separated by mass along the vertical axis, such that the spots at the top belong to proteins having large mass and those at the bottom of Fig. 1 belongs to proteins having relatively small mass. Hence, the list $\mathrm{A}, B, C, \ldots R, S, T$ has protein spots already ordered according their mass, $A$ being the most "massive" protein. It is not difficult by a close look at Fig. 1 to find ordering of the spots according to the charge. The sequence starts with the protein $\mathrm{L}$, which is the most right (the highest charged) spot, followed by spot $\mathrm{T}$, which is the next most right spot, an so on.. Hence, the two sequences that will lead to partial ordering are:

\section{A B C D E FG H I J K L M N O P Q R S T$$
\text { L T D A G K N I S M E B H R J C P Q F O }
$$

A comparison of the two sequences reveals several subsets of elements of the two sequences that maintain their relative order in both sequences. For instance, in both sequences: $A>B>C>P>Q$ where we use sign $>$ to indicate the dominance. Similarly $\mathrm{A}>\mathrm{E}>\mathrm{H}>\mathrm{J}>\mathrm{O}$; or $\mathrm{A}>\mathrm{E}>\mathrm{F}>\mathrm{O}$; etc. There is an elegant graphical (pictorial) algorithm which outlines how one finds systematically all such possibilities which has been described in the literature. ${ }^{10}$ It is customary instead of listing all partial orderings to represent them pictorially by a hierarchical diagram (see ref. 7 for illustration). It is important to be aware that a hierarchical diagram does not lead to a unique pictorial representation of a partial ordering. Any diagram in which positioning of vertices from the left to the right preserves the dominance relationship offers an alternative illustration of the particular partial order. This allows one to redraw the diagram by shifting vertices at some length to the left and to the right, and of course, at any length up and down. One tries to construct diagrams with as little as possible crossing of lines merely for better visibility or different dominance relationships. So, a hierarchical diagram is essentially a directed graph, with all edges being directed from the left to the right. Clearly graphs and directed graphs have multitude of pictorial representations, hence are to be of limited use for our attempt to arrive at canonical representation of $2-\mathrm{D}$ maps.

\section{EMBEDDING OF PARTIAL ORDERING OF PROTEIN SPOTS OVER A 2-D MAP}

The critical step for canonical representation of maps is to arrive at a unique mathematical object to serve as the basis for such representation. We follow the procedure outlined in ref. 7 in which among 
continuum of alternative representation of the partial ordering of protein spots we select a single such representation which is defined by embedding of the partial order on the map. In Fig. 2 this is illustrated for the map of Fig. 1. As one can see from Fig. 2 charge dominance runs from the right to the left and the mass dominance runs from the top to the bottom. Observe an important property of the embedded graph representing the partial ordering: All line segments have positive slope! This can be easily understood: only spots linked by positive slope indicate dominance with respect to both charge and mass. We should also mention because of the transitivity of the ordering relationship that only lines connecting spots directly dominating one another are to be drawn. So, if $X>Y$ and $Y>Z$, then transitivity implies also $X>Z$, but we do not inscribe line $X Z$ because dominance of $X$ over $Z$ is depicted by lines indicating the dominance $X>Y$ and $Y>Z$.

Embedded graph of Fig. 2 represents a unique schematic diagram that captures some of the relationships for protein spots of the map. Observe that for the construction of this graph we only used the information on charge and mass and have not used information on abundance. Thus all maps that have the same set of proteins (which always come with the same $(x, y)$ coordinates, that is all maps originating from the same type of cell/tissue/organ/species) will have the same graphical representation by the same embedded graph. One way to differentiate such maps is to associate with individual protein spots canonical labels, in this case the labels $1-20$, and then list individual abundance. We will come later to this point but at this stage we want to introduce canonical labels so that maps belonging to different cell types will introduce different embedded graphs are associated with different binary matrices.

\section{CANONICAL LABELS}

Canonical labels have been used in chemical documentation and computer manipulations with chemical structures to resolve the isomorphism problem for graphs, ${ }^{11,12}$ the automorphism problem, ${ }^{13-15}$ and have also been used for an ordered construction of graphs. ${ }^{16}$ There are different schemes for deriving distinct canonical labels. Historically one of the earliest such scheme was due to Morgan, ${ }^{17}$ known as Morgan algorithm. In Morgan algorithm one considers extended connectivity which is obtained by adding in an iterative way valence of neighbors to replace valence of each vertex. Although computationally simple (or elegant) the algorithm may lead to oscillatory behavior and occasionally may not yielded the answer. ${ }^{18}$

We decided to opt for another approach, which is conceptually elegant but computationally is more involved and may occasionally require considerable computational time, particularly in the case of graphs in which all vertices are of the same degree. One should recall that graph isomorphism is inherently a NP problem, ${ }^{19}$ that is, a problem for which computational time increases exponentially with 
time. We will outline the approach on the embedded graph of the hypothetical proteomics map of Fig. 1, the graph of Fig. 2, and will show that in the case of proteomics maps canonical labels for protein spots can be found quite fast. The rule for canonical labels for graphs that we adopt has been described previously. ${ }^{12,14}$ We will choose a canonical labeling of vertices in a graph such that the accompanying adjacency matrix represents the smallest binary number when its rows are read from the left to the right and from the top to the bottom as a single number. What that means is that the smallest labels have adjacent the largest possible labels because then the corresponding row of the adjacency matrix will be the smallest possible number (subject to compliance wit the assignments of smaller labels already used).

In order to better see how we find canonical labels we will outline the search for canonical labels for the embedded graph of Fig. 2. Observe that spots $Q$ and $T$ are the only terminal vertices in this case. Hence we have to explore two possibilities: either assigning label 1 to spot $Q$ or to spot $T$. If we assign label 1 to spot $\mathrm{Q}$ that spot $\mathrm{P}$, which is adjacent, has to have label 20 because only then the first roe of the adjacency matrix will be

\section{1}

which is the smallest possible number for a $20 \times 20$ binary matrix. The same first row will also occur if we assign label 1 to spot $\mathrm{T}$ and label 20 to $\mathrm{L}$, which is adjacent to $\mathrm{T}$. Hence, we have to explore two alternatives for placing the labels 1 and 2 illustrated in Fig. 3. Next we search for the location of the next smallest label, label 3, which should have as few neighbors as possible, because that would assure the smallest entry for the second row of the adjacency matrix. Examination of Fig. 3 shows that there are two vertices that have only two neighbors: protein spots $D$ and $S$. This leads to the total of four possibilities illustrated in Fig. 4. We have assigned to the nearest neighbors of spot 3 labels 18/17 and those of label 4 as 16/15, because at this stage we know for sure that 3 has neighbors 18 and 17 and 4 has neighbors 16 and 15, but we cannot be sure which spots take labels 18 and 16 and which take labels 17 and 15 . We will see later that this ambiguity will be resolved satisfactorily without a need at this stage to multiplying all unresolved possibilities. Next we have to find location for label 5. After little of reflection we find that there are only two sites that 5 can take the sites of spots $\mathrm{A}$ and $\mathrm{K}$ respectively (Fig. 5). These are the only two sites in which there is only one neighbor spot that need assignment, leading to neighbors $(18,17$ and 14) for protein $\mathrm{A}$ and neighbors $(18,16,14)$ for protein $\mathrm{K}$. Any other spot will require at least two new labels, involving thus label 13, which would result in a larger binary number for the fifth row. Comparing neighbor labels for the sites $\mathrm{A}$ and $\mathrm{K}$ we see that $\mathrm{A}$ gives a smaller binary number, thus $\mathrm{A}$ takes number 5 as label. We still cannot assign 18 and 17 between spots $\mathrm{E}$ and $\mathrm{G}$, because $A$ is adjacent to both $A$ and $G$. At this point there are only two sites, which have three neighbors of which two have already, labels. These are: $\mathrm{K}$ with neighbors $(18 / 17,16 / 15)$ and $\mathrm{C}$ with neighbors (19, 
14). Clearly spot $\mathrm{K}$ is to be preferred as its smallest number for neighbor (16/15) is larger than the smaller number of spot $\mathrm{C}$ (which is 14). By assigning label 6 to spot $\mathrm{K}$ (Fig. 6) we can get the smallest number in row six of the adjacency matrix if we assign to its neighbors labels 18 and 16 (rather than 17 and 15).

We continue with assigning label 7 to protein spot $\mathrm{C}$, which leaves only four spots : $\mathrm{H}, \mathrm{J}, \mathrm{O}$, and $\mathrm{R}$ yet to be assigned labels from 8 to 11 . Label 8 immediately can be assigned to spot $\mathrm{R}$, which is the only sport that has already two neighbors assigned. Hence we introduce label 11 to spot $\mathrm{H}$; leaving only spots $\mathrm{J}$ and $\mathrm{O}$ for labels 9 and 10. It is not difficult to see that if we assign label 9 to spot $\mathrm{O}$ that makes the binary number smaller than the opposite assignment. Selecting spot $\mathbf{J}$ for label 10 completes the assignment. In Table 1 we listed the unique adjacency matrix for the graph of Fig. 2 as induced by the canonical labels. The canonical labels for all twenty vertices are shown in Fig. 7 and are listed below:

$$
\begin{array}{llllllllllllllllllll}
1 & 2 & 3 & 4 & 5 & 6 & 7 & 8 & 9 & 10 & 11 & 12 & 13 & 14 & 15 & 16 & 17 & 18 & 19 & 20 \\
\text { Q } & \text { T } & \text { D } & \text { S } & \text { A } & \text { K } & \text { C } & \text { R } & \text { O } & \text { J } & \text { H } & \text { F } & \text { M } & \text { B } & \text { I } & \text { N } & \text { E } & \text { G } & \text { L } & \text { P. }
\end{array}
$$

\section{DISCUSSION}

By arriving at the canonical adjacency matrix we in fact not only established unique labels for protein spots but also have arrived at unique label for the proteomics map. Instead of using a single binary number for label we can consider a sequence of 20 binary numbers, each representing one row of the adjacency matrix. The binary numbers representing each row of the adjacency matrix of Table 1 , when translated into decimal numbers, yield the sequence:

$$
1,2,12,48,76,148,321,656,1424,2593,5196, \ldots
$$

that is unique to the cell type of the considered proteomics map. Different maps will be represented by different sequences as it is unlikely that in different cells/tissues/organs/ species the same proteins will appear and be among those with the highest abundance.

Observe that the canonical labeling here proposed offers a relatively simple cataloguing of proteomics maps that can be ordered lexicographically. First we consider the leading number of the canonical sequence and order maps accordingly. If the leading number is the same we compare the next numbers in the sequence, and continue until we come upon two entries that are different, which then determines the ordering of maps.

It is possible, though it does not appear likely, that two different proteomics maps which will have different embedded graph nevertheless result the same graph when embedding is not considered. Such case would lead to the same canonical labeling for vertices of the graph and produce the same 
canonical adjacency matrix. For example, if in Fig. 1 we move the spot $\mathrm{A}$ a little up or a little right and do not by such "move" change the dominance relationship with the neighboring protein spots we will obtain different map but the same canonical labeling will hold. If such situation arises one would need to incorporate in the procedure additional information on maps, such as actual (suitably normalized) distances between connected vertices of embedded graphs.

\section{MAP SPECIFIC FACTORS}

As we have seen the process of arriving at canonical labels does not includes information of abundance. Thus the proteomics map of a healthy rat liver cell, the column indicated as $A_{0}$ in Table 2 and corresponding to the control group) as well as liver cells of mice exposed to various toxic substances will necessarily have the same canonical adjacency matrix. We believe that this is an advantage of the present scheme, rather than disadvantage, because it allows maps associated with the same species, the same organ, and the same tissue to be immediately grouped into the same class, under the same canonical label. We can nevertheless differentiate maps belonging to the same class by constructing a vector that has as components the abundance. Alternatively the abundance can be inserted as the diagonal elements of the augmented binary adjacency matrices.

A question to consider is how the canonical labeling of maps depends on the number of protein spots considered in constructing the partial ordering and how increase in the number of spots affect construction of the partial ordering. It is not difficult to recognize that canonical labeling of vertices of a graph is affected with addition of vertices or deletion of vertices. Because of this we have in advance to select the number of vertices to be used for construction of canonical labels for maps. For instance we can start with $\mathbf{n}=20$, that is select the twenty most abundant spots, as has been assumed in our hypothetical proteomics map of Fig. 1. If this is found somewhat restrictive we can increase $\mathrm{n}$ to 50, 100 or 250 . It is not very likely that among 2,000 to 10,000 that typically cells may contain the same twenty proteins will be among the most abundant. If this would happen there is not difficulty to increase the size of the graph of partial ordering to include 50,100 or 250 proteins, thus eliminating chance for fortuitous coincidence in protein distribution in different cells of different species and different organs.

The other question raised, that of construction of the partial ordering for maps that include larger number of spots, has been considered in the literature. ${ }^{7}$ In Fig. 8 we show embedded graph for the partial ordering of 70 most abundant protein spots (out of 1570 that have been given in a list supplied by the laboratory of Frank Witzmann ${ }^{20}$ ) corresponding to cells of normal rat liver. Observe that an increase in the number of protein spots from 20 to 70 has not been accompanied with a dramatic increase in the 
complexity of the embedded graph of the partial ordering. This need not be surprising, because partial ordering of protein spots is sensitive to local features of a map, and locally proteomics map having 20 , 200 or 2000 spots will be similar!

Finally, we may mention that the canonical labels introduced here can be combined with the approach that uses zigzag curve for arriving at $\mathrm{D} / \mathrm{D}$ matrices and set of invariants extracted from these matrices. Instead of using abundance as a "tool" for construction of zigzag curve we can use canonical labels and construct the zigzag curve by connecting spots having adjacent labels. In Fig. 9 we show so constructed zigzag curve for the hypothetical proteomics map of Fig. 1. What makes this construction rather interesting and "foxy" is that we have obtained zigzag curve without explicit reference to abundance of spots (which in the case of Fig. 1 have not even been specified). The basis of the quantitative characterization of protein maps reported in the literature has been use of abundance data to order spots and assigns them sequential labels. Here we may use abundance to select spots to be considered for analysis, but not to order them. Moreover, one can select spots using different criteria, besides abundance, such as functional role of proteins (if known). In order not to confuse this approach with the already established approach based on zigzag curve we may refer to curve of Fig. 9 as "fox trail." Indeed derived zigzag curves resemble very much the fox trail as reported by Siniff and Jessen, ${ }^{21}$ and depicted in a paper by George Hall $^{22}$ on mathematical modeling in chemistry.

\section{ILLUSTRATION}

We will illustrate the outlined approach on the five proteomics maps listed in Table 2 where $\mathrm{x}$ (charge) and $y$ (mass) give location of separated protein spots for the rat liver cells as reported by the Molecular Anatomy Laboratory of F. Witzmann. ${ }^{20}$ The first column gives the canonical labels, while the last column gives arbitrary labels used to identify individual protein spots. The abundance of the protein spots of the control group $\left(\mathrm{A}_{0}\right)$ are shown in bold, while the abundance of the corresponding protein spots for four peroxisome proliferators $\left(A_{x}\right)$ are given, following Anderson and collaborators ${ }^{23}$ as quotient $\mathrm{A}_{\mathbf{x}} / \mathrm{A}_{0}$. There is some advantage of such practice as we will see later when we consider map vectors (ordered sequence of proteins and their abundance).

\section{Partial ordering}

The first step in our analysis of proteomics map for documentation is to select the number $(\mathrm{N})$ of spots to be considered. We have taken data in Table 2 as reported in an article on characterization of proteomics maps and chemically induced changes in proteome using matrix invariants. ${ }^{6}$ This table includes data on 
$\mathrm{N}=29$ protein spots selected as those sots having the largest abundance. The (x,y) coordinates of these spots allow us to depict the locations of proteins (Fig. 10). We now proceed to find partial ordering of the 29 spots, recalling that the pictorial representation of the partial ordering as an embedded graph require all line segments (edges) in the embedded graph to have positive slope. For most pairs of spots inspection of Fig. 10 allows one to draw line connecting such spots, but one should first consider spots in the same neighborhood and only later connect spots at greater separation in order to avoid introducing redundant lines. For example, we can start at the top of the figure and connect spot 62 to spot 41 , then 48 to 35 and 41 , both 41 and 35 to spots 22 and 39, and so on. In few instances it may not be clearly visible from the Fig. 10 if two spots should be connected or not, as is the case with spots 15, 30, 29 and 39 or spots 36, 46 and 19 and 24. Each such case can be resolved by inspecting the (x,y) coordinates of Table 2. For instance the spots 15 and 30 should not be connected because the corresponding coordinates $(1053.6,864.3)$ and $(1122.7,863.0)$, respectively, show non-comparability: protein 15 has larger mass but protein 30 has larger charge. In contrast spots 29 and 39, having coordinates (1170.0, $862.2)$ and $(1278.8,981.6)$ respectively should be connected because 39 has larger charge and larger mass than 29, thus establishing the dominance in both properties. . In Fig.. 11 we show the graph of partial ordering embedded over the proteomics map of Table 2 with labels as shown in the last column of the Table 2.

\section{Canonical Labels}

Fig. 12 we show again the graph of partial ordering, however graph is no longer embedded over the map but is somewhat modified for better illustration of adjacency of connected protein spots. This is a graph for which we well seek canonical labels. Although it is not essential how we represent the graph, nevertheless we have drawn graph in a form that still preserves pictorial similarity with Fig. 11 in order to facilitate comparisons between the new and the old labels. In order to appreciate not only the complexity of the task but also a surprising efficiency of the algorithm associated with the smallest binary labels let us point out that we are searching for a unique labeling of 29 vertices of a graph which is one in 29 ! possibilities, a number between $10^{30}-10^{31}$. It took less than dozen trials to arrive at labels shown in Fig. 13. In fact since we have some experience with canonical labeling, that we previously considered as a tool for graph isomorphism problem, ordered construction of graphs, and determining symmetry properties of transitive graphs ${ }^{13-15,23-30}$ in this particular case we used only four templates of the graph to find canonical labels! We should add that search for canonical labels for transitive graphs is generally a more involved task, especially when degree of vertices of graph is higher, in view that all vertices have the same degree. Readers may consult this literature ant test their skills in trying to reproduce some of the canonical labeling of graphs reported in the literature. We should also mention that Brandt and Scholley 
to represent unique coding of chemical reactions.31 also used canonical labels based on the smallest binary number

Graph of Fig. 12, however, has vertices of different degree, which allows focusing attention first to vertices of the lowest degree to which one assigns the smallest labels. Clearly location of labels 1 and 2 (which also determines location for labels 29 and 28) are easy to find, because there is but a single terminal vertex and of the seven vertices of degree two only one site requires a single new label (label 28), all the other possibilities would demand two new labels (28 and 27) which correspond to a large binary number for row 2 .

When considering alternatives the guiding rule is to prefer locations which at each step introduce the smallest number of novel labels not previously introduced. Consider, for instance, the remaining six vertices of degree two, which received labels 3, 4, 5, 6, 7, and 10 in Fig. 13, which shows canonical labels. Where should we put label 3 and to it adjacent spots labels 27 and 26? We can immediately recognize that the three locations at the bottom left part of the graph should be preferred to other locations because if we assign to one of the three spots label 3 and to adjacent spots 27 and 26 in the next step we can assign label 4 without introducing novel labels, and again the same hold for label 5.

There is here an apparent novelty in assigning labels 3, 4, and 5 in that any of six possible alternative placing would result in the same adjacency matrix for the graph. This means that the three sites are equivalent. (with respect to adjacency) and that graph has some symmetry. The same again happens with labels 19 and 20, which can be interchanged without changing the form of the adjacency matrix. Hence, the order of the automorphism group of the graph of Fig. 12 is $(3 !) \times(2 !)=12$. That is, there are twelve different ways of placing labels (any labels, canonical or not) on a graph which produce the same adjacency matrix (and in the case of canonical labels all the twelve alternatives will give the same canonical matrix). Since we are interested in unique labels for protein spots of proteomics map we can resolve the ambiguities associated with automorphism group of a graph (which disappears when graph is embedded over the proteomics map) by giving preference to spots of proteins having a larger mass. Thus in our case we assigned labels $3,4,5$ to spots 18,14 and 12 respectively, and labels 19 and 20 to spots 57 and 44 respectively.

We have to decide on positions of labels 26 and 27. It is not difficult to see that the cut point vertex should have label 27 because in this way we will get smaller binary rows for vertices adjacent to the cut vertex (vertices 9,11 , and 13). The cut point vertex is defined in graph theory as a vertex which when deleted separates a graph into two or more disjoint fragments). Assignment of vertices 6 and seven requires some testing but it is not difficult to select the proper sites (shown in Fig. 13) as the case shown is optimally placing labels 25 and 24 to produce for subsequent labels minimal row binary numbers. In fact after assignment of 6 and to it adjacent 25 and 24 the locations of all subsequent labels is unique! 
Hence we are not continuing with describing justifications for the rest of labels, which readers can not only find by themselves but can find search for canonical labels even somewhat entertaining.

\section{Fox trail diagram}

We will end this section with construction of a zigzag curve based on the canonical labels to which we have referred as fox trail diagram (to differentiate this zigzag curve from zigzag curves based on ordering of spots as dictated by their relative abundance). In Fig. 14 we show fox trail diagram with canonical labels given for most spots (for clarity not all the canonical labels are shown). The fox trail diagram can be used in the same manner that zigzag curves based on the abundance have been used in building D/D matrices and construction of map invariants. An advantage of the fox trail diagram is that it does not utilize information on abundance, except in selecting the initial $\mathrm{N}$ spots, if not selected otherwise. This may in some circumstances allow construction of fox trail diagrams for proteomics maps of unknown origin if set of $\mathrm{N}$ proteins that will form a basis for analysis has been identified. Proteins can be selected if they are identified or if a list of coordinates for a set of "standard" proteins for comparative studies is composed.

\section{CANONICAL LABELS FOR PEROXISOME PROLIFERATORS}

In order to arrive at canonical representation of proteomics map we have first to get canonical labels for set of $\mathrm{N}$ pre-selected protein spots. In the procedure outlined here we selected 29 most abundant spots in the proteomics map for the control group of rat liver cells. The derived canonical labels lead to unique adjacency matrix which is the same for all proteomics maps derived for liver cells of rats, whether exposed to different chemicals, different diseases, possibly different diet, and so on. In Table 3 we show the first dozen rows of the adjacency matrix corresponding to the canonical labels found here. The corresponding decimal sequence is:

$$
1,3,12,12,12,48,80,144,391,1536,4098,7680, \ldots
$$

The occurrence of repetitions of a number in the sequence indicates the presence of symmetry equivalent vertices in the graph. Observe that the above sequence of numbers equally belong to all the five proteomics data (maps) of Table 2 because they differ only in relative abundance of individual spots, but protein spots (at least under the same experimental conditions) always appear at the same location. If we want to differentiate maps associated with different peroxisome proliferators we ought to incorporate information on abundance, because that is the only parameter that will vary when same cell types or the same tissue is considered. A way to do is to assign abundance values to the diagonal elements of the map 
graph or construct $\mathrm{N}$-component vector in which components are ordered according to the given canonical labels of spots with components representing abundance. For the five cases of Table 2 this is illustrated in Table 4.

We will refer to the common code for all five maps based on decimal interpretation of the rows of the canonical matrix as class code. Thus class code becomes a code for specific type of cell/organ/species. Within the class we differentiate individual maps by canonical abundance vectors. We define the lead map and the map of unperturbed cell/tissue/organ and such map will have numerical values of abundance explicitly given (preferably in some standardized unit scale). The rest map vectors (ordered sequence of proteins and their abundance) of maps belonging to the same cell/tissue/organ (that is to the same class) will have as entries quotients of relative abundance. In this way, when examining considers one can immediately distinguish the lead map from other maps of the same class.

\section{CONCLUDING REMARKS}

We have outlined a procedure for associating with proteomics maps a single binary code, which for convenience can be represented as numerical sequence based on decimal interpretation of individual rows of canonical adjacency matrix. The approach assigns to individual proteins unique labels, which may facilitate comparison of maps that may originate with different laboratories. Partial ordering is not very sensitive to minor variations between maps, as can happen with the maps made at somewhat different experimental conditions.

Alternative canonical scheme can be proposed. One such approach, elaborated by Hendrickson,

${ }^{25}$ represents a modification of our canonical scheme for molecules in that instead of searching for the minimal binary code for the adjacency matrix one is seeking the maximal possible binary number. Hendrickson and Toczko ${ }^{25}$ claimed that the maximal adjacency code is easier to find. We are skeptical about this claim that has not been substantiated at any great length, because one can construct graphs for which the smallest codes can be easily found, just as one can construct graphs for which the opposite is the case. In the case of graphs such as discussed here it is not difficult to see that finding the smallest binary matrix may be easier that finding the largest binary matrix. In the case of the smallest binary matrix one initiate labeling by looking for vertices with the smallest valence, while in the case of the largest binary matrix one initiate labeling by looking for vertices with the largest valence in order to have the largest possible entry in the first row of the adjacency matrix. In the case of the embedded graph of Fig. 1 vertices $Q$ and $T$ were given label 1 and 2 and to their neighbors we could immediately assign labels 20 and 19 respectively. Were we to follow Hendrickson and Toczko and search for the maximal 
binary numbers we would have to consider protein spots $M$ and $N$, each having six neighbors as candidates for labels 1 and 2 respectively. But to complete the assignment for the neighbors of each of the two vertices we would have to consider $6 !$ possibilities, that is $720^{2}$ possibilities or over half a million possibilities!

\section{REFERENCES}

1 Topological index is a misnomer as it actually represents a graph theoretical invariant of chemical structure (that reflects molecular topology to an extent, but also combinatorial content of a structure).

2 Topographic indices are analogous to topological indices but apply to graphs embedded in 2D or 3-D space (hence structures of fixed standard 3-D geometry)

3 Randic, M. Topological Indices, in: Encyclopedia of Computational Chemistry, Schleyer, P. v. R.; Allinger, N. L.; Clark, T.; Gasteiger, J.; Kollman, P.; Schaefer III, H. F. Schreiner, P. R. (Eds.); John Wiley \& Sons: Chichester, 1998, pp. 3018-3032.

4 Randić, M. On Graphical Representation of Proteomics and Their Numerical Characterization. J. Chem. Inf. Comput. Sci. 2001, 41, 1330-1338.

5 Randić, M. Zupan, J. Novič, M. On 3-D Graphical Representation of Proteomics Maps. Chem. Inf. Comput. Sci. 2001, 41, 1339-1334.

6 Randić, M. Witzmann, F. Vračko, M. Basak, S. C.; On Characterization of Proteomics Maps and Chemically Induced changes in Proteome Using Matrix Invariants: Application to Peroxisome Proliferators. Med. Chem. Rev. 2001, 10, 456-479

7 Randić, M. A Graph Theoretical Characterization of Proteomics Maps. Int. J. Quantum Chem. (in press)

8 Randić, M.; Basak, S. C. A Comparative Study of Proteomics Maps Using Graph Theoretical Descriptors. J. Chem. Inf. Comput. Sci. (submitted)

9 Randic, M., Novic, M.; Vračko, M. On Characterization of Dose Variations of 2-D Proteomics Maps by Matrix Invariants. J. Proteome Res. (in press).

10 Rouse Ball, W. W.; Coxeter, H. S. M. Mathematical Recreations \& Esseys, Dover Publ. 1987.

11 Read, R. C.; Corneil, D. G. The Graph Isomorphism Disease, J. Graph Theory, 1977, 1, 339-363.

12 Randic, M. On the recognition of identical graphs representing molecular topology. J. Chem. Phys. 1974, 60, 3920-3928. For additional clarifications see: Randić, M. On Rearrangement of the Connectivity Matrix of a Graph, J. Chem. Phys. 1975, 62, 309-310.

13 Randić, M. On Discerning Symmetry Properties of Graphs. Chem. Phys. Lett. 1976, 42, 283-287.

14 Randić, M. J. Systematic Study of Symmetry Properties of Graphs. I. Petersen Graph. Croat. Chem. Acta 1977, 49, 643-655.

15 Randić, M. Symmetry Properties of Graphs of Interest in Chemistry. II. Desargues-Levi Graph. Int. J. Quantum Chem. 1979, 15, 663-682.

16 Randić, M. A Graph Theoretical Basis for Structural Chemistry I. Structures Based on Trivalent Graphs with $\mathbf{n}$ $=10$ Vertices. Acta Chrystallogr. 1978, A34, 275-282.

17 Morgan, L. The Generation of a Unique Machine Description for Chemical Structures - A Technique Developed at Chemical Abstract Services. J. Chem. Documentation 1965, 5, 107-113.

18 Randic, M. On Unique Numbering of Atoms and Unique Codes for Molecular Graphs. J. Chem. Inf. Comput. Sci. 1975, 15, 105-108.

19 Garey, M. R.; Johnson, D. S. Computers and Intractability (A Guide to the Theory of NP-Completeness), Freeman: San Francisco, 1979.

20 Witzmann, F. Molecular Anatomy Laboratory, Dept. of Biology, Indiana University \& Purdue University, Columbus, IN.

21 Hall, G. G. Modelling - A Philosophy for Applied Mathematicians. Bulletin of the Institute of Mathematics and Its Applications, 1972, 8, 226-228.

22 Siniff, D. H.; Jesson, C. R. Simulation Model of Animal Movement Patterns, Advances in Ecological Research $1969,6,185-220$. 
23 Anderson, N. L.; Esquer-Blasco, R.; Richardson, F.; Foxworthy, P.; Eacho, P. The Effects of Peroxisome Proliferators on Protein Adundances in Mouse Liver. Toxicol. Appl. Pharacol. 1996, 137, 75-89.

24 Randić, M. Symmetry Properties of Graphs of Interest in Chemistry. III. Homotetrahedryl Rearrangement. Int. J. Quantum Chem. 1980, 14, 557-577.

25 Randić, M. Katović, Symmetry Properties of Chemical Graphs. IV. Rearrangement of Tetragonal Pyramidal Complexes. Int. J. Quantum Chem. 1982, 21, 647-663.

26 Randić, M. Symmetry Properties of Chemical Graphs. V. Internal Rotation in $X Y_{3} X Y_{2} X Y_{3}$. J. Comput. Chem. 1983, 4, 73-83.

27 Randić, M.; Davis M. I. Symmetry Properties of Chemical Graphs. VI. Isomerization of Octahedral Complexes. Int. J. Quantum Chem. 1984, 26, 69-89.

28 Randić, M.; Katović, V. Trinajstić, N. Symmetry Properties of Chemical Graphs. VII. Enatiomers of Tetragonal Pyramidal Rearrangements. In: Symmetry Properties of Non-Rigid Molecules: A Comprehensive Survey (Maruani, J; Serre, J. eds.) Studies in Phys. Theor. Chem. 1983, 23, 399-408.

29 Randić, M. Symmetry Properties of Chemical Graphs VIII. On Complementarity of Isomerization Modes. Theor. Chim. Acta 1985, 67, 137-155.

30 Randić, M.; Oakland, D. O.; Klein, D. J. Symmetry Properties of Chemical Graphs. IX. The Valence Tautomers in the Rearrangement of $\mathrm{P}_{7}{ }^{3-}$ Skeleton Comput. Chem. 1986, 7, 35-54.

31 Randić, M.; Klein, D. J.; Katović, V.; Oakland, D. O.; Seitz, W. A. Balaban, A. T. Symmetry Properties of Chemical Graphs. X. Rearrangement of Axially Distorted Octahedra. Graph Theory and Topology in Chemistry. (King, R. B; Rouvray, D. H., eds.) Elsevier: Amsterdam 1987 (Studies in Physical and Theoretical Chemistry, 1987, 51, 266-284).

32 Brandt, J.; Scholley, von A. Comput. \& Chem. 1983, 7, 51.

33 Hendrickson, J. B.; Toczko, A. G. J. Chem. Inf. Comput. Sci. 1983, 23, 171. 


\section{Figure Captions}

Fig. 1 Hypothetical proteomics map with twenty protein spots used to illustrate partial ordering of protein spots.

Fig. 2 The graph of partial ordering superimposed over the proteomics map. Observe that slopes of all line segments are positive.

Fig. 3 Initiation of the search for canonical labels: Assignment of labels 1 and 2 to terminal vertices.

Fig. 4 Four possible placement of labels 3 and 4 to divalent vertices D and S.

Fig. 5 The unique site for label 5 at spot $A$ forces labels $18 / 17$ for spots $E$ and $G$ and eliminates Fig. $4 \mathrm{~b}$ and Fig. $4 \mathrm{~d}$ from further considerations.

Fig. 6 The unique site for the label 6 at the spot $K$ forces label 18 for spot $G$ and label 16 for spot $\mathrm{N}$.

Fig. 7 Complete canonical labels for embedded graph of Fig. 2.

Fig. 8 Separation of 70 most abundant protein spots obtained by electrophoresis and chromatography of homogenized rat liver cells.

Fig. 9 Embedded graph of partial ordering for 70 protein spots of Fig. 8 showing that limited complexity of such graphs.

Fig. 10 The $x, y$ locations of 29 protein spots of Table 2.

Fig. 11 Embedded graph of the partial ordering for 29 protein spots of Table 2.

Fig. 12 A pictorial representation of the graph of the partial ordering for 29 protein spots of Table 2 (observe that no longer lines representing edges have to have positive slope)

Fig. 13 The canonical labeling of vertices for he graph of Fig. 12.

Fig. 14 The "fox trail" diagram based on the canonical labels for the proteomics maps of Fig. 11 
Table 1 Canonical Adjacency matrix of the Partial Ordering of the graph of Fig. 1

$$
\begin{array}{llllllllllllllllllll}
0 & 0 & 0 & 0 & 0 & 0 & 0 & 0 & 0 & 0 & 0 & 0 & 0 & 0 & 0 & 0 & 0 & 0 & 0 & 1 \\
0 & 0 & 0 & 0 & 0 & 0 & 0 & 0 & 0 & 0 & 0 & 0 & 0 & 0 & 0 & 0 & 0 & 0 & 1 & 0 \\
0 & 0 & 0 & 0 & 0 & 0 & 0 & 0 & 0 & 0 & 0 & 0 & 0 & 0 & 0 & 0 & 1 & 1 & 0 & 0 \\
0 & 0 & 0 & 0 & 0 & 0 & 0 & 0 & 0 & 0 & 0 & 0 & 0 & 0 & 1 & 1 & 0 & 0 & 0 & 0 \\
0 & 0 & 0 & 0 & 0 & 0 & 0 & 0 & 0 & 0 & 0 & 0 & 0 & 1 & 0 & 0 & 1 & 1 & 0 & 0 \\
0 & 0 & 0 & 0 & 0 & 0 & 0 & 0 & 0 & 0 & 0 & 0 & 1 & 0 & 0 & 1 & 0 & 1 & 0 & 0 \\
0 & 0 & 0 & 0 & 0 & 0 & 0 & 0 & 0 & 0 & 0 & 1 & 0 & 1 & 0 & 0 & 0 & 0 & 0 & 1 \\
0 & 0 & 0 & 0 & 0 & 0 & 0 & 0 & 0 & 0 & 1 & 0 & 1 & 0 & 0 & 1 & 0 & 0 & 0 & 0 \\
0 & 0 & 0 & 0 & 0 & 0 & 0 & 0 & 0 & 1 & 0 & 1 & 1 & 0 & 0 & 1 & 0 & 0 & 0 & 0 \\
0 & 0 & 0 & 0 & 0 & 0 & 0 & 0 & 1 & 0 & 1 & 0 & 0 & 0 & 1 & 0 & 0 & 0 & 0 & 1 \\
0 & 0 & 0 & 0 & 0 & 0 & 0 & 1 & 0 & 1 & 0 & 0 & 0 & 1 & 0 & 0 & 1 & 1 & 0 & 0 \\
0 & 0 & 0 & 0 & 0 & 0 & 1 & 0 & 1 & 0 & 0 & 0 & 0 & 0 & 0 & 0 & 1 & 0 & 0 & 0 \\
0 & 0 & 0 & 0 & 0 & 1 & 0 & 1 & 1 & 0 & 0 & 0 & 0 & 0 & 1 & 0 & 0 & 0 & 1 & 1 \\
0 & 0 & 0 & 0 & 1 & 0 & 1 & 0 & 0 & 0 & 1 & 0 & 0 & 0 & 0 & 0 & 0 & 0 & 0 & 0 \\
0 & 0 & 0 & 1 & 0 & 0 & 0 & 0 & 0 & 1 & 0 & 0 & 1 & 0 & 0 & 0 & 0 & 1 & 0 & 0 \\
0 & 0 & 0 & 1 & 0 & 1 & 0 & 1 & 1 & 0 & 0 & 0 & 0 & 0 & 0 & 0 & 0 & 0 & 1 & 1 \\
0 & 0 & 1 & 0 & 1 & 0 & 0 & 0 & 0 & 0 & 1 & 1 & 0 & 0 & 0 & 0 & 0 & 0 & 0 & 0 \\
0 & 0 & 1 & 0 & 1 & 1 & 0 & 0 & 0 & 0 & 1 & 0 & 0 & 0 & 1 & 0 & 0 & 0 & 0 & 0 \\
0 & 1 & 0 & 0 & 0 & 0 & 0 & 0 & 0 & 0 & 0 & 0 & 0 & 0 & 0 & 0 & 0 & 0 & 0 & 0 \\
1 & 0 & 0 & 0 & 0 & 0 & 0 & 0 & 0 & 0 & 0 & 0 & 0 & 0 & 0 & 0 & 0 & 0 & 0 & 0
\end{array}
$$


Table 2 The $\mathrm{x}, \mathrm{y}$ coordinates and the abundance for the control rat liver cells and when the animals were exposed to the four peroxisome proliferators.

\begin{tabular}{|l|l|l|l|l|l|l|l|l|}
\hline $\begin{array}{l}\text { Canoni- } \\
\text { cal } \\
\text { labels }\end{array}$ & $\mathrm{x}$ & $\mathrm{y}$ & $\mathrm{A}_{0}$ & $\mathrm{~A}_{1} / \mathrm{A}_{0}$ & $\mathrm{~A}_{2} / \mathrm{A}_{0}$ & $\mathrm{~A}_{3} / \mathrm{A}_{0}$ & $\mathrm{~A}_{4} / \mathrm{A}_{0}$ & $\begin{array}{l}\text { Old } \\
\text { labels }\end{array}$ \\
\hline 8 & & & & 0.8332 & 1.0995 & 1.1975 & 0.0712 & 22 \\
\hline 12 & & & & 0.7796 & 0.5745 & 0.6026 & 0.8813 & 52 \\
\hline 14 & & & & 1.6744 & 1.9279 & 1.4451 & 1.5713 & 20 \\
\hline 1 & & & & 0.5214 & 0.3467 & 0.6517 & 0.6866 & 62 \\
\hline 2 & & & & 0.9280 & 0.8446 & 0.8572 & 0.9462 & 48 \\
\hline 16 & & & & 1.4370 & 1.2484 & 1.2517 & 1.3266 & 9 \\
\hline 18 & & & & 0.8700 & 0.5361 & 0.8476 & 1.1485 & 36 \\
\hline 24 & & & & 0.8967 & 0.9027 & 1.0268 & 1.0734 & 2 \\
\hline 20 & & & & 0.9662 & 1.0007 & 0.9502 & 1.2602 & 44 \\
\hline 7 & & & & 1.0805 & 0.8365 & 0.6486 & 1.0824 & 15 \\
\hline 27 & & & & 0.9819 & 0.5886 & 0.9023 & 1.1712 & 5 \\
\hline 17 & & & & 2.4224 & 0.7838 & 0.8245 & 2.4859 & 45 \\
\hline 26 & & & & 0.9749 & 0.9219 & 1.0383 & 1.2354 & 1 \\
\hline 10 & & & & 0.8329 & 0.5984 & 1.0728 & 1.2460 & 56 \\
\hline 28 & & & & 0.8670 & 1.0634 & 0.9394 & 1.4118 & 35 \\
\hline 11 & & & & 1.6631 & 2.7300 & 1.3186 & 1.3471 & 19 \\
\hline 21 & & & & 0.8389 & 0.5016 & 0.9884 & 1.0873 & 47 \\
\hline 4 & & & & 1.1466 & 1.8036 & 1.0756 & 1.3869 & 14 \\
\hline 15 & & & & 1.4749 & 2.2973 & 1.9544 & 0.0938 & 26 \\
\hline 29 & & & & 0.8410 & 0.8676 & 0.9267 & 1.2267 & 41 \\
\hline 9 & & & & 0.8833 & 0.6073 & 0.8086 & 0.9181 & 39 \\
\hline 13 & & & & 1.3537 & 1.3300 & 1.3637 & 2.2165 & 24 \\
\hline 22 & & & & 0.9748 & 1.0090 & 0.8673 & 1.2393 & 29 \\
\hline 25 & & & & 1.0263 & 1.3494 & 1.3382 & 0.9409 & 33 \\
\hline 3 & & & & 0.9628 & 1.5907 & 0.8044 & 0.8859 & 18 \\
\hline 5 & & & & 0.8985 & 0.6803 & 0.8041 & 1.2152 & 12 \\
\hline 23 & & & 1.1912 & 1.5306 & 2.0926 & 1.9225 & 40 \\
\hline 6 & & & & 1.4279 & 0.8306 & 0.7795 & 0.8687 & 30 \\
\hline 19 & & & & & & & 57 \\
\hline & & & & & & & & \\
\hline
\end{tabular}


Table 3 Part of the canonical adjacency matrix for graph of Fig. 12

\begin{tabular}{|c|c|c|c|c|c|c|c|c|c|c|c|c|c|c|c|c|c|}
\hline & & 14 & 15 & 16 & 17 & 18 & 19 & 20 & 21 & 22 & 23 & 24 & 25 & 26 & 27 & 28 & 29 \\
\hline 1 & & 0 & 0 & 0 & 0 & 0 & 0 & 0 & 0 & 0 & 0 & 0 & 0 & 0 & 0 & 0 & 1 \\
\hline 2 &. & 0 & 0 & 0 & 0 & 0 & 0 & 0 & 0 & 0 & 0 & 0 & 0 & 0 & 0 & 1 & 1 \\
\hline 3 & . . & 0 & 0 & 0 & 0 & 0 & 0 & 0 & 0 & 0 & 0 & 0 & 0 & 1 & 1 & 0 & 0 \\
\hline 4 & . & 0 & 0 & 0 & 0 & 0 & 0 & 0 & 0 & 0 & 0 & 0 & 0 & 1 & 1 & 0 & 0 \\
\hline 5 & . . & 0 & 0 & 0 & 0 & 0 & 0 & 0 & 0 & 0 & 0 & 0 & 0 & 1 & 1 & 0 & 0 \\
\hline 6 & . . & 0 & 0 & 0 & 0 & 0 & 0 & 0 & 0 & 0 & 0 & 1 & 1 & 0 & 0 & 0 & 0 \\
\hline 7 & . & 0 & 0 & 0 & 0 & 0 & 0 & 0 & 0 & 0 & 1 & 0 & 1 & 0 & 0 & 0 & 0 \\
\hline 8 &. & 0 & 0 & 0 & 0 & 0 & 0 & 0 & 0 & 1 & 0 & 0 & 1 & 0 & 0 & 1 & 1 \\
\hline 9 & . . & 0 & 0 & 0 & 0 & 0 & 0 & 0 & 1 & 1 & 0 & 0 & 0 & 0 & 1 & 1 & 1 \\
\hline 10 & . . & 0 & 0 & 0 & 0 & 0 & 1 & 1 & 0 & 0 & 0 & 0 & 0 & 0 & 0 & 0 & 0 \\
\hline 11 & 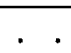 & 0 & 0 & 0 & 1 & 1 & 0 & 0 & 0 & 0 & 0 & 0 & 0 & 0 & 1 & 0 & 0 \\
\hline 12 & . & 0 & 0 & 0 & 1 & 1 & 1 & 1 & 0 & 0 & 0 & 0 & 0 & 0 & 0 & 0 & 0 \\
\hline 13 & . & 1 & 1 & 1 & 0 & 0 & 0 & 0 & 0 & 0 & 0 & 0 & 0 & 0 & 1 & 0 & 0 \\
\hline 14 & 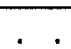 & 1 & 1 & 1 & 0 & 0 & 0 & 0 & 1 & 0 & 0 & 0 & 0 & 0 & 0 & 0 & 0 \\
\hline
\end{tabular}


Table 4

$1,3,12,12,12,48,80,144,391,1536,4098,7680, \ldots$

\begin{tabular}{|c|c|}
\hline Control & $1,3,12,12,12,48,80,144,391,1536,4098,7680, \ldots$ \\
\hline \multirow[t]{2}{*}{ PFOA } & $1,3,12,12,12,48,80,144,391,1536,4098,7680, \ldots$ \\
\hline & $0.521,0.928,0.963,1.147,1.428,1.081,0.833,0.883,0.833,1.663,0.780, \ldots$ \\
\hline \multirow[t]{2}{*}{ PFDA } & $1,3,12,12,12,48,80,144,391 ; 1536,4098,7680, \ldots$ \\
\hline & $0.347,0.845,1.591,1.804,0.680,0.831,0.837,1.100 ; 0.607,0.598,2.730, \ldots$ \\
\hline \multirow[t]{2}{*}{ Clofibrate } & $1,3,12,12,12,48,80,144,391,1536,4098,7680,$. \\
\hline & $0.652,0.857,0.804,1.076,0.804,0.780,0.649,1.198,0.809,1.073,1.319, \ldots$ \\
\hline \multirow[t]{2}{*}{ DEHP } & $1,3,12,12,12,48,80,144,391,1536,4098,7680, \ldots$ \\
\hline & $0.687,0.687,0.886,1.387,1.215,0.869,1.082,0.071,0.918,1.246,1.347$ \\
\hline
\end{tabular}




\section{Appendix 1.2}

Prediction of cellular toxicity of halocarbons from their computed chemodescriptors: A hierarchical QSAR approach

S.C. Basak, K. Balasubramanian, B.D. Gute, D. Mills, A. Gorczynska and S. Roszak

J. Chem. Inf. Comput. Sci., submitted 


\title{
PREDICTION OF CELLULAR TOXICITY OF HALOCARBONS FROM COMPUTED CHEMODESCRIPTORS: A HIERARCHICAL QSAR APPROACH
}

\author{
S. C. BASAK, ${ }^{1}$ K. BALASUBRAMANIAN, ${ }^{2 \cdot}$ B. D. GUTE, ${ }^{1}$ D. MILLS, ${ }^{1}$ \\ A. GORCZYNSKA, ${ }^{3}$ and S. ROSZAK ${ }^{3}$ \\ ${ }^{1}$ Natural Resources Research Institute, University of Minnesota Duluth, Duluth, MN 55811 \\ ${ }^{2}$ Department of Applied Sciences, University of California Davis, Hertz Hall, L-794 Livermore, CA 94550, \\ Chemistry \& Material Science Directorate, Lawrence Livermore National Laboratory, Livermore, CA 94550 and \\ Glenn T. Seaborg Center, Lawrence Berkeley National Laboratory, Berkeley CA 94720 \\ ${ }^{3}$ Institute of Physical and Theoretical Chemistry, Wroclaw University of Technology, Wyb. Wyspianskiego 27, 50- \\ 370 Wroclaw, Poland
}

\begin{abstract}
A hierarchical quantitative structure-activity relationship (HiQSAR) approach was used to estimate toxicity and genetic toxicity for a set of 55 halocarbons using computed chemodescriptors. The descriptors consisted of topostructural (TS), topochemical (TS), geometrical, semi-empirical (AM1) quantum chemical, and $a b$ initio (STO3G, 6-31G(d), 6-311G, 6-311G(d), and aug-cc-pVTZ) quantum chemical indices. For the two toxicity endpoints investigated, $\mathrm{ARR}$ and $\mathrm{D}_{37}$, the TC indices gave the best cross-validated $R^{2}$ values. The 3-D indices also performed either as well as, or slightly superior to the TC indices. For the four categories of quantum chemical indices used for the development of predictive models, the AM1 parameters gave the worst performance and the most advanced $a b$ initio (B3LYP/aug-CC-pVTZ) parameters gave the best results when used alone. This was also the case when the quantum chemical indices were used in the hierarchical QSAR approach for both of the toxicity endpoints, ARR and $\mathrm{D}_{37}$. The models resulting from HiQSAR are of sufficiently good quality to estimate toxicity of halocarbons from structure.
\end{abstract}

\section{INTRODUCTION}

An important objective of contemporary computational toxicology is the prediction of the potential toxic effects of chemicals from their computed molecular descriptors. ${ }^{1-20}$ Thousands of chemicals are already in the marketplace and their numbers are growing at an ever increasing rate. The TSCA Inventory of USEPA currently has over 81,000 entries and the list is increasing yearly. ${ }^{21}$ Most of these chemicals have none of the experimental data necessary for the estimation of their effects on human and environmental health. ${ }^{22}$ In the areas of chemical ecotoxicity and genotoxicity, the availability of data is even more appalling. Only $15 \%$ of TSCA chemicals have genotoxicity data; less than $6 \%$ have chronic toxicity or ecotoxicity data. ${ }^{22}$ Only a few hundred of the many thousands of candidate chemicals have 
been tested in the National Toxicology Program (NTP) for carcinogenicity in the two-year rodent bioassay program.

It is clear from the above that, in the foreseeable future, hazard assessment of chemicals must be carried out in a data-poor situation, while the number of candidate chemicals is increasing at a rate much faster than the availability of experimental physicochemical and toxicity data prerequisite to the proper hazard estimation of all these substances. A viable solution to this quagmire is the use of quantitative structureactivity relationship (QSAR) models which use algorithmically derived properties, i.e., properties which can be calculated directly from chemical structure without the input of any further experimental data.

Halocarbons constitute an important class of chemicals as solvents and useful intermediates in various synthetic processes. So, the potential of their release to the environment worldwide is substantial. ${ }^{23-25}$ Therefore, it was of interest to develop QSARs of halocarbons using descriptors that can be calculated from structure. Crebelli et al. determined various toxicity endpoints for a set of 55 halocarbons. ${ }^{26}$ They formulated QSAR models using a mixture of calculated and experimental properties, viz., molecular refractivity $(\mathrm{MR})$, octanol / water partition coefficient $(\log P)$, and quantum chemical indices such as HOMO energy, LUMO energy and the difference between HOMO and LUMO energies calculated by $a b$ initio methods. While MR characterized the generalized shape and size of molecules, the quantum chemical indices quantify the electrophilic nature of the molecules. In our hierarchical QSAR (HiQSAR) approach, we have used a combination of chemodescriptors, calculated directly from molecular structure, for the formulation of QSARs to predict various toxicity endpoints. ${ }^{5 \cdot 8,11-13,27-31}$ The HiQSAR method begins model building with parameters which can be computed most easily; parameters demanding more computational resources are added only if the easily calculable indices do not give satisfactory results. We have routinely used topostructural (TS), topochemical (TC), geometrical, and semi-empirical quantum chemical indices for the development of QSARs for toxicity estimation. Previous results with halocarbons by Crebelli et al showed that they achieved moderately good results in QSAR development using a combination calculated descriptors. ${ }^{26}$ So, in the current study we have attempted to develop QSARs for the set of 55 halocarbons using a combination of topological, geometrical, and quantum chemical inclices using the HiQSAR approach.

\section{METHOUS AND MATERIALS}

2.1. Halogenated Aliphatic Hydrocarbon Database. The database of halogenated aliphatic hydrocarbons used in this study was taken from the work of Crebelli et al. ${ }^{26}$ Crebelli and his co-workers present test data for mitotic chromosome malsegregation and lipid peroxidation tested in Aspergillus nidulans diploid strain P1. Results presented for mitotic chromosome malsegregation include lowest effective concentration affecting chromosome distribution (LEC), lowest concentration for arresting 
conidial development (ARR), and lowest concentration inducing one lethal hit per cell $\left(\mathrm{D}_{37}\right)$. Lipid peroxidation: study results were presented as lowest effective concentration resulting in lipid peroxidation $\left(\mathrm{LEC}_{\mathrm{LP}}\right)$, however not all of the chemicals were tested for lipid peroxidation. For the purposes of this study, we chose to model the two continuous biological endpoints ( $A R R$ and $D_{37}$ ) that were available for all 55 chemi ials. It should be noted that LEC data was available for all of the chemicals in the set as well, however, activity values were only available for twenty-four of the fifty-five chemicals, the remaining thirty-one chemicals tested negative. Chemical names and activity data ( $A R R$ and $D_{37}$ ) are presented in Table 1. Fi: ure 1 shows the optimized geometries of all 55 neutral halocarbons in the Crebelli set that we considered in this study. These geometries were optimized using the using the high-level cc-pVTZ basis set using the DFT/B3LYP method. The basis set and the technique are considered quite reliable for the geometry optimization. Subsequent to the geometry optimization of the neutral halocarbons we computed the energies of the anions at the optimized neutral geometries. The vertical electron affinities are taken as the difference of the geometries of the anion and neutral species at the optimized neutral geometries. For further details of the testing regimens, please refer to the original work of Crebelli and his co-workers. ${ }^{26,}$ 32, 33

2.2 Calculation of Topological Indices. The topological indices (TIs) used in this study were calculated using three main software programs: POLLY $2.3,{ }^{34}$ MolConn-Z 3.50, ${ }^{35}$ and Triplet. ${ }^{36}$ Included in the suite of 192 topological indices used in this study are: Wiener number, ${ }^{37}$ molecular connectivity indices as cilculated by Randic ${ }^{38}$ and Kier and Hall, ${ }^{39}$ frequency of path lengths of varying size, information theoretic indices defined on distance matrices of graphs using the methods of Bonchev and Trinajstić "' as well as those of Raychaudhury et al., ${ }^{41}$ parameters defined on the neighborhood complexity of vertices in hydrogen-filled molecular graphs, ${ }^{42.44}$ Balaban's $J$ indices, ${ }^{45-47}$ local orthogonal vertex invariants, ${ }^{36}$ and the electrotopological indices of Kier and Hall. ${ }^{48}$ More information on the topological indices calculated by POLLY has been reported in earlier studies. 4, 10, 13,49

2.3. Calculation of Geometrical Indices. The geometrical indices include van der Waals volume, ${ }^{50}$ the three-dimensional Wiener numbers for both the hydrogen-filled and hydrogen-suppressed molecular structures, ${ }^{51}$ and the Kappa shape descriptors of Kier and Hall. ${ }^{52,53}$ Van der Waals volume, $V_{W}$, was calculated using SYBYL 6.4 from Tripos Associates, Inc. ${ }^{54}$ The 3-D Wiener numbers were calculated in the SYBYL interface using an SPL (Sybyl Programming Language) program developed in our lab. Calculation of 3-D Wiener numbers consists of the sum entries in the upper triangular submatrix of the topographic Euclidean distance matrix for a molecule. The 3-D coordinates for the atoms were determined using CONCORD 3.2.1. ${ }^{55}$ Finally, the Kappa shape descriptors were calculated using MolConn-Z 3.50. ${ }^{35}$

2.4. Quantum Chemical Parameters. Semi-empirical quantum chemical parameters were calculated using the Austin Model version one (AM1) Hamiltonian. These parameters were calculated using 
MOPAC 6.00 in the SYBYL interface. ${ }^{56}$ Ab initio quantum chemical calculations were performed applying the second order Møller-Plesset perturbation theory ${ }^{57}$ for the sequence of standard Pople's atomic basis sets: STO-3G, ${ }^{58,} 59$ 6-31G(d), ${ }^{60-65} 6-311 \mathrm{G},{ }^{66,}, 67$ 6-311G(d), and aug-cc-pVTZ. ${ }^{68-72}$ Additional calculations were done within the density functional theory (DFT) approach. ${ }^{73}$ The DFT calculations utilized Becke's three-parameter functional ${ }^{74}$ with Vosco et al.'s ${ }^{75}$ local correlation part and Lee et al.'s ${ }^{76}$ nonlocal part (abbreviated as B3LYP). DFT studies were done applying extended aug-ccpVTZ basis set. ${ }^{57,73-76}$ This basis set consists of a set of diffuse functions to account for the negative electronic charge distribution on anions. The density functional theory has been shown as a reliable method for reproducing electron affinity properties. ${ }^{77}$

The calculations in the MP2/STO-3G approach were utilized as the lowest-level ab initio scheme, similar to semi-empirical quantum chemical approaches. All calculations applying Pople's basis sets were done at the MP2 level of theory and are denoted in the text by the name of the atomic basis sets. The DFT calculations are referred to as B3LYP/aug-cc-pVTZ. The results from the $a b$ initio calculations are presented in Tables 2-5. Table 5 provides additionally experimental dipole moments ${ }^{17}$ for the assessment of the quality of the charge distribution. All $a b$ initio quantum chemical calculations were conducted using Gaussian98W. ${ }^{78}$

2.5. Statistical Analysis. Initially, the majority of topological indices were transformed by the natural logarithm of the index plus one. This was done to scale the indices, since some may be several orders of magnitude greater than others, while other indices may equal zero. The geometric indices were transformed by the natural logarithm of the index for consistency, the addition of one was unnecessary. Other indices, including the majority of the quantum chemical indices were also transformed using natural logarithm, however, constants were chosen on a case-by-case basis to bring the minimal value for the descriptor above zero. Finally, the $a b$ initio values for Gap номо-имо their values were already distributed between negative one and one.

The set of 192 topological indices was then partitioned into the two distinct sets: topostructural indices (eighty-six) and topochemical indices (106). Each of the sets were then divided into subsets, or principal components (PCs), using the SAS principal components analysis (PCA) procedure (PRINCOMP) ${ }^{79}$ to further reduce the number of independent variables for use in model construction. This procedure results in the creation of a new set of completely orthogonal PCs derived from linear combinations of all indices. Only PCs with eigenvalues greater than or equal to one have been retained for this study. A more detailed explanation of this approach has been provided in a previous study by Basak et al ${ }^{49}$. After the PCA, a correlation analysis was conducted on the PCs to determine which TIs were most highly correlated with each of the PCs. The TI most-highly correlated with each PC was then selected for modeling, resulting in a greatly reduced set of indices. The PCA and selection of indices was performed independently for both 
the topostructural and topochemical indices and resulted in a set of five topostructural indices and a set of twelve topochemical indices.

As is well known, the smaller the data set, the greater the chance of spurious error when using a large number of independent variables (descriptors), so reducing the number of independent variables is crucial when modeling of data set of this size. Topliss and Edwards ${ }^{80}$ have thoroughly studied this issue of chance correlations. For a set with between fifty and sixty dependent variables (observations), to keep the probability of chance correlations less than 0.01 , at most about forty-five independent variables may be used (if a correlation coefficient of $R^{2} \geq 0.9$ is achieved). This number is dependent on the actual correlation achieved in the modeling process, a higher correlation results in a better chance of using more variables with the same limited probability of chance correlations. In this study we are well below the cutoff of forty-five independent variables. In fact, the total number of descriptors which will be considered for all models and estimation is twenty-three (this includes the topostructural, topochemical and geometrical indices), well within the bounds of the Topliss and Edwards criteria. ${ }^{80}$ Addition of all quantum chemical indices employed in hierarchical modeling takes the total up to sixty, beyond the allowable bounds of the Topliss and Edwards criteria. However, we consider the descriptors on the quantum chemical level to be exclusive, in other words we never use parameters from the four different quantum chemical approaches at the same time. So for any HiQSAR including quantum chemical parameters, the total number of indices considered in modeling never exceeds thirty, again well within the bounds of the Topliss and Edwards criteria.

Regression modeling was accomplished using the SAS procedure REG ${ }^{79}$ on nine distinct sets of indices. These sets were constructed as part of the HiQSAR approach to model development. The hierarchy begins with the simplest parameters, the TSIs. After using the TSIs to model the activity, the next level of parameters of higher complexity are added. To the indices included in the best TSI model, we add all of the TCIs and proceed to model the activity using these parameters. Likewise, the indices included in the best model from this procedure are combined with the indices from the next complexity level, the geometrical indices and modeling is conducted once again. Finally, the best model utilizing TSIs, TCIs and geometrical indices is combined with the various quantum chemical parameters to develop the highest level models in the hierarchy.

\section{RESULTS}

The PCA of the topostructural indices resulted in the retention of five PCs with eigenvalues greater than or equal to 1.0. These five PCs are presented in Table 6 in terms of their eigenvalues, the amount of data variance incorporated into each of the PCs, and the one topostructural index most-highly correlated with each of the PCs. The topostructural index most-highly correlated with each PC was chosen for use in 
HiQSAR modeling. Similarly, the PCA on the topochemical indices resulted in twelve PCs with eigenvalues greater than or equal to zero. These PCs and their attendant most-highly correlated topochemical index are presented in Table 7.

3.1. Results for Modeling ARR. For modeling ARR, all-subsets regression on the set of five topostructural indices resulted in the selection of a three-parameter model. This model used ${ }^{4} \chi_{\mathrm{PC}}, \mathrm{W}$ and $\mathrm{DS} 1_{3}$, in a poor regression with a regression coefficient $\left(R^{2}\right)$ of 0.3661 , a cross-validated regression coefficient $\left(R_{c \nu}^{2}\right)$ of 0.2213 , and a standard error (s.e.) of 1.27 . These three indices were then included in modeling with the topochemical indices, creating a beginning set of fifteen topological indices. Again, all-subsets regression was used and resulted in the selection of an eight-parameter model. This model retained $\mathrm{W}$ and $\mathrm{DS}_{3}$ and added the following topochemical indices: ${ }^{1} \chi, \mathrm{AZV}_{3}, \mathrm{H}_{\mathrm{MIN}}, \mathrm{SssssC}, \mathrm{SdCH}_{2}$, and SdsCH. This model showed great improvement $\left(R^{2}=0.8606, R_{c v}^{2}=0.7637\right.$, s.e. $\left.=0.63\right)$ over the topostructural model. The addition of the six geometrical indices to this set of eight topological indices result in further improvement in modeling $\mathrm{ARR}\left(R^{2}=0.9014, R_{c v}^{2}=0.8345\right.$, s.e. $\left.=0.52\right)$. The model selected eliminated all topostructural indices and two topochemical indices and added four geometrical parameters resulting in the following seven-parameter model: $\mathrm{H}_{\mathrm{MIN}}, \mathrm{SssssC}, \mathrm{SdCH}_{2}, \mathrm{City}_{\mathrm{H}}, \mathrm{V}_{\mathrm{w}}, \mathrm{Ka}_{3}$, and $\mathrm{Kp}_{3}$. Next, the addition of AM1, STO-3G, 6-31G(d) and 6-311G parameters to the topochemical and geometrical indices did not lead to any improvement in modeling. Modeling with the $6-311 \mathrm{G}(\mathrm{d})$ basis set resulted marginal improvement with an eight parameter model, showing a slight increase over the topochemical and geometrical model while using one more index. This model added the energy of the second-lowest unoccupied molecular orbital $\left(\mathrm{E}_{\mathrm{LUMO}+1}\right)$ to the existing hierarchical model. The model statistics are as follows: $R^{2}=0.9065, R_{c v}^{2}=0.8340$, and s.e. $=0.51$. Finally, the B31YP/aug-cc-pVTZ parameters were combined with the topochemical and geometrical parameters, resulting in the selection of an eight-parameter model incorporating the following indices: $\mathrm{H}_{\mathrm{MIN}}, \mathrm{SssssC}, \mathrm{SdCH}_{2}, \mathrm{City}_{\mathrm{H}}, \mathrm{V}_{\mathrm{w}}, \mathrm{Ka}_{3}$, $\mathrm{Kp}_{3}$, and $\mathrm{E}_{\mathrm{Lumo}}$. This model showed some improvement over the topochemical and geometrical model $\left(R^{2}\right.$ $=0.9120, R_{c v}^{2}=0.8511$, and s.e. $=0.50$ ). For the purposes of completeness, models were created using each of the levels of the hierarchy singly. These results, along with the results for the rest of the models can be found in Table 8.

3.2. Results for Modeling $D_{37}$. All-subsets regression on the set of five topostructural indices using the SAS procedure REG ${ }^{79}$ resulted in the selection of a two-parameter model. This model incorporated $\mathrm{W}$ and $\mathrm{DS}_{3}$, demonstrating a poor regression with a regression coefficient $\left(R^{2}\right)$ of 0.3659 , a cross-validated regression coefficient $\left(R_{c v}^{2}\right)$ of 0.2945 , and a standard error (s.e.) of 1.24 . These two indices were then included in modeling with the topochemical indices, creating a beginning set of fourteen topological indices. Again, all-subsets regression was used and resulted in the selection of a seven-parameter model. This model retained the Weiner index, $W$, and added the following topochemical indices: $A Z V_{3},{ }^{2} \chi^{b}, Q_{v}$, $\mathrm{SdCH}_{2}, \dot{\mathrm{H}}_{\mathrm{MIN}}$, and SsF. This model was much more satisfactory in terms of $R^{2}$ and s.e. $\left(R^{2}=0.8791, R_{c v}^{2}=\right.$ 
0.8074 , s.e. $=0.57)$. The addition of geometrical indices did not result in any improvement to modeling $\mathrm{D}_{37}$. The model selected during all-subsets regression was identical to the one selected during the previous step in modeling hierarchy. Next, the set of seven topostructural and topochemical parameters was supplemented with the various quantum chemical parameters. The use of AM1 semi-empirical descriptors led to the selection of a seven-parameter model showing slight improvement in both $R^{2}$ and s.e. $\left(R^{2}=\right.$ $0.8840, R_{c v}^{2}=0.8121$, s.e. $=0.56$ ). This model replaced the Triplet parameter, $\mathrm{AZV}_{3}$, with the energy of the second-lowest unoccupied molecular orbital $\left(\mathrm{E}_{\mathrm{LUMO+1}}\right)$. The addition of STO-3G and 6-31G(d) parameters to the topostructural and topochemical indices did not lead to any improvement in modeling. Next, the addition of 6-311G parameters to the seven topostructural and topochemical indices led to some improvement in modeling. A ten-parameter model, adding the energies of the lowest occupied $\left(\mathrm{E}_{\mathrm{Lumo}}\right)$ and second-lowest occupied $\left(\mathrm{E}_{\mathrm{LUMO+1}}\right)$ molecular orbitals and vertical electron affinity (VEA), was selected through all-subsets regression showing a reasonable increase in model performance over the topological model. This model resulted in an $R^{2}=0.9015$, a cross-validated $R^{2}=0.8283$, and s.e. $=0.53$. While this is a noticeable increase in the regression coefficient and the standard error, it is somewhat of a sacrifice with the inclusion of three additional indices. Modeling with the slightly higher 6-311G(d) basis set resulted in a more acceptable eight parameter model, showing a slight increase over the 6-311G model while using two fewer indices. This model removed $W$, but retained all five of the topochemical indices remaining at this point and added three $a b$ initio parameters: $\mathrm{E}_{\text {номо }}, \mathrm{E}_{\mathrm{LuMO}}$, and $\mathrm{E}_{\mathrm{LumO+1}}$. The model statistics are as follows: $R^{2}=0.9056, R_{c v}^{2}=0.8335$, and s.e. $=0.51$. Finally, the B3LYP/aug-cc-pVTZ parameters were combined with the topostructural and topochemical parameters, resulting in the selection of an eight-

parameter model incorporating the following indices: $\mathrm{W}, \mathrm{AZV}_{3},{ }^{2} \chi^{\mathrm{b}}, \mathrm{Q}_{\mathrm{V}}, \mathrm{SdCH}_{2}, \mathrm{H}_{\mathrm{MIN}}, \mathrm{SsF}$, and $\mathrm{E}_{\mathrm{Lumo}}$. This model was by far superior to all of the previous models $\left(R^{2}=0.9476, R_{c v}^{2}=0.9236\right.$, and s.e. $\left.=0.38\right)$. For the purposes of completeness, we created models using each of the levels of the hierarchy alone. These results, along with the results for the rest of the models can be found in Table 9.

\section{DISCUSSION}

The major objective of this study was to develop high quality QSAR models for the prediction of the toxicity of halocarbons from chemodescriptors calculated directly from their structure. To, this end we have developed HiQSARs for two toxicity endpoints, viz. ARR and $\mathrm{D}_{37}$, for which data was available for the entice set of 55 compounds.

It is clear from the HiQSAR models presented in Tables 8 and 9 that the simplest topostructural indices do not give very good predictive results. This is not surprising considering the fact that halocarbons are believed to act through a mechanism dominated by electron transfer to the molecule. The topostructural indices, being derived from the skeletal graphs of the molecule quantify the connectedness (adjacency and 
topological distances) of the atoms in the molecular structure. However, they are insensitive to such features as bonding pattern and atom type. The topochemical indices, on the other hand, contribute significantly to the predictive power of the models for both toxicity endpoints. These indices quantify both the topological and chemical aspects of atoms in the molecule, simultaneously being able to characterize the shape and size of molecules. It is interesting to note that Crebelli et $a l^{26}$ found MR to be well correlated with both $A R R$ and $D_{37}$ toxicity values for this set of halocarbons. MR is not only a shape and size descriptor, but it also encodes information about the electronic nature of the molecule.

The 3-D (geometrical) indices also performed surprisingly well for both properties, though more so in modeling ARR. This should be expected in the light of the fact that both the topochemical indices and MR did well in predicting these toxicity endpoints.

In the realm of quantum chemical indices we used both semi-empirical (AM1) and ab initio (STO-3G, 6-31G(d), 6-311G, 6-311G(d) and B3LYP/aug-cc-pVTZ) indices. In our model development using the HiQSAR method, we used each of these six classes of quantum chemical indices alone and in the hierarchical modeling with one type of quantum chemical indices being used at a time. As is clear from Table 8, there is little noticeable model improvement for ARR with the addition of quantum chemical parameters of any of the levels included in this study. Even at the high B3LYP/aug-cc-pVTZ level, there is only minor improvement over the existing topochemical and geometrical model. However, as can be seen in Table 9, there is significant improvement in the modeling of $D_{37}$ with the addition of the B3LYP/aug-cc-pVTZ parameters.

It is interesting to note that of the six classes of quantum chemical indices, the high level B3LYP/augcc-pVTZ indices were most useful in toxicity prediction either alone or as independent variables in the HiQSAR scheme. This vindicates the belief that the critical step in the toxic mode of action of halocarbons is the attachment of the electron to the halocarbon moiety. The best models after the full hierarchical modeling process yielded the following high quality QSARs:

$$
\begin{gathered}
\operatorname{Ln}(\mathrm{ARR})=39.902+0.898\left(\mathrm{H}_{\mathrm{MIN}}\right)-0.551(\mathrm{SssssC})-0.623\left(\mathrm{SdCH}_{2}\right)+1.859\left(\mathrm{City}_{\mathrm{H}}\right) \\
-10.726\left(\mathrm{~V}_{\mathrm{w}}\right)+7.417\left(\mathrm{Ka}_{3}\right)-8.097\left(\mathrm{Kp}_{3}\right)+0.582\left(\mathrm{E}_{\mathrm{LUMO}}\right) \\
R^{2}=0.9120, R_{c . v \mathrm{~V}}^{2}=0.8511, n=55, \text { s.e. }=0.50, F=59.59 \\
\operatorname{Ln}\left(\mathrm{D}_{37}\right)=4.016-5.282(\mathrm{~W})+10.771\left(\mathrm{AZV}_{3}\right)+2.010\left({ }^{2} \chi^{\mathrm{b}}\right)-0.745\left(\mathrm{Q}_{\mathrm{V}}\right) \\
-0.546\left(\mathrm{SdCH}_{2}\right)+0.926\left(\mathrm{H}_{\mathrm{MIN}}\right)+0.547(\mathrm{SsF})+1.329\left(\mathrm{E}_{\mathrm{LUMO}}\right) \\
R^{2}=0.9476, R_{c . v .}^{2}=0.9236, n=55, \text { s.e. }=0.38, F=103.98
\end{gathered}
$$


For comparative purposes, we examined the models initially developed by Crebelli et al. ${ }^{26}$ The following equations represent our re-analysis of the Crebelli models (using MR, $\log P$, and STO-3G $a b$ initio parameters) to calculate cross-validated regression coefficients:

$$
\begin{gathered}
\operatorname{Ln}(1 / \mathrm{ARR})=-0.085+0.186(\mathrm{MR})-10.962\left(\mathrm{Gap}_{\text {Lимо-номо }}\right) \\
R^{2}=0.6154, R_{\text {c.r. }}^{2}=0.5298, n=55, \text { s.e. }=0.98, F=41.60 \\
\operatorname{Ln}\left(1 / \mathrm{D}_{37}\right)=-0.330+0.203(\mathrm{MR})-11.249\left(\mathrm{Gap}_{\text {симономо }}\right) \\
R^{2}=0.7284, R_{\text {c.v. }}^{2}=0.6726, n=55, \text { s.e. }=0.81, F=69.73
\end{gathered}
$$

The greater success of the B3LYP/aug-cc-pVTZ indices as compared to the STO-3G, 6-31G(d), 6$311 \mathrm{G}, 6-311 \mathrm{G}(\mathrm{d})$, and AM1 parameters in predicting toxicity indicates that more sophisticated or higher level QC indices might better reflect the electronic basis of the toxicity of these halocarbons. To this end, we are currently calculating VEA for a subset of halocarbons at the $\operatorname{CCSD}(\mathrm{T})$ level, however only a small subset will be calculated at this level since is it prohibitively time consuming to do so for the entire set. We would like to investigate how far the success of the less expensive parameters can be vindicated using the higher level of parameters. Also, we will be doing calculations on solvated molecules as opposed to gas phase calculations to see whether such involved calculations give better models or shed some light in the toxic modes of action of halocarbons. Such studies are in progress and will be reported subsequently.

\section{ACKNOWLEDGEMENT}

The authors would like to acknowledge the financial support of this research by the Air Force Office of Scientific Research grant F49620-02-1-0138. This is contribution number XXX from the Center for Water and the Environment of the Natural Resources Research Institute. The research at UC Davis was supported by the US Department of Energy under Grant No. DEF03-00ER15116. The work at LLNL was performed under the auspices of the US Department of Energy by the University of California, LLNL under contract number W-7405-Eng-48. 


\section{REFERENCES}

(1) Benigni, R.; Andreoli, C.; Giuliani, A. QSAR Models for Both Mutagenic Potency and Activity: Application to Nitroarenes and Aromatic Amines. Environ. Mol. Mutagen. 1994, 24, $208-219$.

(2) Mekenyan, O.; Peitchev, D.; Bonchev, D.; Trinajstic, N.; Bangov, I. Modelling the Interaction of Small Organic Molecules with Biomacromolecules. I. Interaction of Substituted Pyridines with Anti-3Azopyridine Antibody. Arzneim.-Forsch./Drug Research 1986, 36, 176-183.

(3) Mekenyan, O.; Basak, S. C. In Graph Theoretic Approaches to Chemical Reactivity; Bonchev, D., Mekenyan, O., Eds.; Kluwer Academic Publishers: The Netherlands, 1994; pp 221-239.

(4) Basak, S. C.; Gute, B. D.; Grunwald, G. D. In Advances in Molecular Similarity, Vol. 2; Carbo-Dorca, R., Mezey, P. G., Eds.; JAI Press: Stamford, Connecticut, 1998; pp 171-185.

(5) Basak, S. C.; Gute, B. D.; Grunwald, G. D. In Quantitative Structure-Activity Relationships in Environmental Sciences Vii; Chen, F., Schuurmann, G., Eds.; SETAC Press: Pensacola, FL, 1998; pp 245261.

(6) Basak, S. C.; Gute, B. D.; Ghatak, S. Prediction of Complement Inhibitory Activity of Benzamidines Using Topological and Geometrical Parameters. J. Chem. Inf. Comput. Sci. 1999, 39, 255-260.

(7) Basak, S. C.; Gute, B. D.; Grunwald, G. D. Assessment of Mutagenicity of Chemicals from Theoretical - Structural Parameters: A Hierarchical Approach. SAR QSAR Environ. Res. 1999, 10, 117-129.

(8) Basak, S. C.; Gute, B. D.; Grunwald, G. D. In Topological Indices and Related Descriptors in QSAR and Qspr; Devillers, J., Balaban, A. T., Eds.; Gordon and Breach Science Publishers: The Netherlands, 1999; pp 675-696.

(9) Basak, S. C.; Grunwald, G. D.; Gute, B. D.; Balasubramanian, K.; Opitz, D. Use of Statistical and Neural Net Approaches in Predicting Toxicity of Chemicals. J. Chem. Inf. Comput. Sci. 2000, 40, 885-890.

(10) Basak, S. C.; Gute, B. D.; Grunwald, G. D. In Discrete Mathematical Chemistry; Hansen, P., Fowler, P., Zheng, M., Eds.; American Mathematical Society: Providence Rhode Island, 2000; pp 9-24.

(11) Basak, S. C.; Mills, D. R.; Balaban, A. T.; Gute, B. D. Prediction of Mutagenicity of Aromatic and Heteroaromatic Amines from Structure: A Hierarchical QSAR Approach. J. Chem. Inf. Comput. Sci. 2001, 41, 671-678.

(12) Basak, S. C.; Mills, D. Prediction of Mutagenicity Utilizing a Hierarchical Approach. SAR QSAR Environ. Res. 2001, 12, 481-496.

(13) Basak, S. C.; Mills, D.; Gute, B. D.; Grunwald, G. D.; Balaban, A. T. In Topology in Chemistry: Discrete Mathematics of Molecules; Rouvray, D. H., King, R. B., Eds.; Horwood Publishing Ltd.: Chichester, UK, 2001, pp. 113-184.

(14) Gute, B. D.; Basak, S. C. Predicting Acute Toxicity of Benzene Derivatives Using Theoretical Molecular Descriptors: A Hierarchical QSAR Approach. SAR QSAR Environ. Res. 1997, 7, 117-131.

(15) Gute, B. D.; Grunwald, G. D.; Basak, S. C. Prediction of the Dermal Penetration of Polycyclic Aromatic Hydrocarbons (Pahs): A Hierarchical QSAR Approach. SAR QSAR Environ. Res. 1999, 10, 1-15.

(16) Roszak, S.; Koski, W. S.; Kaufman, J. J.; Balasubramanian, K. Structures and Electron Attachment Properties of Halomethanes $\left(\mathrm{CX}_{n} Y_{m}, X=\mathrm{H}, \mathrm{F} ; Y=\mathrm{Cl}, \mathrm{Br}, \mathrm{I} ; N=0,4 ; M=4-N\right)$. SAR QSAR Environ. Res. 2001, $11,383-396$.

(17) CRC Handbook of Chemistry and Physics, CRC Press: Boca Raton, FL, 1996. 
(18) Basak, S. C.; Balasubramanian, K. Characterization of Isospectral Graphs Using Graph Invariants and Derived Orthogonal Parameters. J. Chem. Inf. Comput. Sci. 1998, 38, 367-373.

(19) Koski, W. S.; Roszak, S.; Kaufman, J. J.; Balasubramanian, K. Potential Toxicity of $\mathrm{Cf}_{3} \mathrm{x}$ Halocarbons. In Vitro Toxicol. 1998, 10, 455-457.

(20) Roszak, S.; Koski, W. S.; Kaufman, J. J.; Balasubramanian, K. Structure and Energetics of $\mathrm{Cf}_{3} \mathrm{cl}^{-}, \mathrm{Cf}_{3}$ br", $\mathrm{Cf}_{3}{ }^{\top}{ }^{\top}$ Radical Ions. J. Chem. Phys. 1997, 106, 7709-7713.

(21) Cash, G. G. Personal Communication, 2001.

(22) Auer, C. M.; Nabholz, J. V.; Baetcke, K. P. Mode of Action and the Assessment of Chemical Hazards in the Presence of Limited Data: Use of Structure-Activity Relationships (SAR) under TSCA, Section 5. Environ. Health Perspect. 1990, 87 183-197.

(23) Fishbein, L. In Environmental Carcinogens - Selected Methods of Analysis; Fishbein, L., O'Neill, I. K., Eds.; International Agency for Research on Cancer: Lyon, 1985; 7, pp 47-67.

(24) Pearson, C. R. In Handbook of Environmental Chemistry; Hutzinger, O., Ed.; Springer-Verlag: New York, 1982; 3, pp 69-88.

(25) Stephenson, M. E. An Approach to the Identification of Organic Compounds Hazardous to the Environment and Human Health. Ecotoxicol. Environ. Safety 1977, 1, 407-425.

(26) Crebelli, R.; Andreoli, C.; Carere, A.; Conti, L.; Crochi, B.; Cotta-Ramusino, M.; Benigni, R. Toxicology of Halogenated Aliphatic Hydrocarbons: Structural and Molecular Determinants for the Disturbance of Chromosome Segregation and the Induction of Lipid Peroxidation. Chem.-Biol. Interact. 1995, 98, 113129.

(27) Basak, S. C.; Grunwald, G. D. Predicting Mutagenicity of Chemicals Using Topological and Quantum Chemical Parameters: A Similarity Based Study. Chemosphere 1995, 31, 2529-2546.

(28) Basak, S. C.; Gute, B. D.; Grunwald, G. D. A Comparative Study of Topological and Geometrical Parameters in Estimating Normal Boiling Point and Octanol-Water Partition Coefficient. J. Chem. Inf. Comput. Sci. 1996, 36, 1054-1060.

(29) Basak, S. C.; Gute, B. D.; Grunwald, G. D. Use of Topostructural, Topochemical, and Geometric Parameters in the Prediction of Vapor Pressure: A Hierarchical Approach. J. Chem. Inf. Comput. Sci. 1997, $37,651-655$.

(30) Basak, S. C.; Grunwald, G. D.; Niemi, G. J. In From Chemical Topology to Three-Dimensional Geometry; Balaban, A. T., Ed. Plenum Press: New York, 1997; pp 73-116.

(31) Basak, S. C.; Mills, D. Quantitative Structure-Property Relationships (QSPRs) for the Estimation of Vapor Pressure: A Hierarchical Approach Using Mathematical Structural Descriptors. J. Chem. Inf. Comput. Sci. 2001, 41, 692-701.

(32) Crebelli, R.; Andreoli, C.; Carere, A.; Conti, G.; Conti, L.; Cotta-Ramusino, M.; Benigni, R. The Induction of Mitotic Chromosome Malsegregation in Aspergillus Nidulans. Quantitative Structure Activity Relationship (QSAR) Analysis with Chlorinated Aliphatic Hydrocarbons. Mutat. Res. 1992, 266, 117-134.

(33) Benigni, R.; Andreoli, C.; Conti, L.; Tafani, P.; Cotta-Ramusino, M.; Carere, A.; Crebelli, R. Quantitative Structure-Activity Relationship Models Correctly Predict the Toxic and Aneuploidizing Properties of Halogenated Methanes in Aspergillus Nidulans. Mutagenesis 1993, 8, 301-305.

Polly, Copyright of the University of Minnesota, 1988.

Molconn-Z, v 3.50, Hall Associates Consulting Quincy, MA, 2000. 
(36) Filip, P. A.; Balaban, T. S.; Balaban, A. T. A New Approach for Devising Local Graph Invariants: Derived Topological Indices with Low Degeneracy and Good Correlational Ability. J. Math. Chem. 1987, 1, 61-83.

(37) Wiener, H. Structural Determination of Paraffin Boiling Points. J. Am. Chem. Soc. 1947, 69, 17-20.

(38) Randic, M. On Characterization of Molecular Branching. J. Am. Chem. Soc. 1975, 97, 6609-6615.

(39) Kier, L. B.; Hall, L. H. Molecular Connectivity in Structure-Activity Analysis, Research Studies Press: Letchworth, Hertfordshire, U.K., 1986.

(40) Bonchev, D.; Trinajstic, N. Information Theory, Distance Matrix and Molecular Branching. J. Chem. Phys. $1977,67,4517-4533$.

(41) Raychaudhury, C.; Ray, S. K.; Ghosh, J. J.; Roy, A. B.; Basak, S. C. Discrimination of Isomeric Structures Using Information Theoretic Topological Indices. J. Comput. Chem. 1984, 5, 581-588.

(42) Basak, S. C.; Roy, A. B.; Ghosh, J. J. Study of the Structure-Function Relationship of Pharmacological and Toxicological Agents Using Information Theory, University of Missouri-Rolla: Rolla, Missouri, 1980.

(43) Basak, S. C.; Magnuson, V. R. Molecular Topology and Narcosis: A Quantitative Structure-Activity Relationship( QSAR) Study of Alcohols Using Complementary Information Content (Cic). Arzneim.Forsch./Drug Res. 1983, 33, 501-503.

(44) Roy, A. B.; Basak, S. C.; Harriss, D. K.; Magnuson, V. R. In Mathl. Modelling Sci. Tech.; Avula, X. J. R., Kalman, R. E., Liapis, A. I., Rodin, E. Y., Eds.; Pergamon Press: 1984; pp 745-750.

(45) Balaban, A. T. Highly Discriminating Distance-Based Topological Indices. Chem. Phys. Lett. 1982, 89, 399-404.

(46) Balaban, A. T. Topological Indices Based on Topological Distances in Molecular Graphs. Pure and Appl. Chem. 1983, 55, 199-206.

(47) Balaban, A. T. Chemical Graphs. Part 48. Topological Index $J$ for Heteroatom-Containing Molecules Taking into Account Periodicities of Element Properties. Math. Chem. (MATCH) 1986, 21, 115-122.

(48) Kier, L. B.; Hall, L. H. Molecular Structure Description: The Electrotopological State, Academic Press: San Diego, CA, 1999.

(49) Basak, S. C.; Magnuson, V. R.; Niemi, G. J.; Regal, R. R. Determining Structural Similarity of Chemicals Using Graph-Theoretic Indices. Discrete Appl. Math. 1988, 19, 17-44.

(50) Bondi, A. Van Der Waals Volumes and Radii. J. Phys. Chem. 1964, 68, 441-451.

(51) Bogdanov, B.; Nikolic, S.; Trinajstic, N. On the Three-Dimensional Wiener Number. J Math Chem. 1989, 3, 299-309.

(52) Kier, L. B. A Shape Index from Molecular Graphs. Quant. Struct.-Act. Relat. 1985, 4, 109-116.

(53) Kier, L. B.; Hall, L. H. In Topological Indices and Related Descriptors in QSAR and Qspr; Devillers, J., Bal:aban, A. T., Eds.; Gordon and Breach Science Publishers: Amsterdam, 1999; pp 455-489.

(54) Tripos Associates, Inc., St. Louis, MO, 1997.

(55) Tripos Associates, Inc., St. Louis, MO, 1997.

(56) Steivart, J. J. P. 1990, MOPAC Version 6.00, QCPE \#455, Frank J Seiler Research Laboratory, US Air Force Academy, CO. 
(57) Møller, C.; Plesset, M. Note on an Approximation Treatment for Many-Electron Systems. Phys. Rev. 1943, $46,618-622$.

(58) Hehre, W. J.; Stewart, R. F.; Pople, J. A. J. Chem. Phys. 1969, 51, 2657.

(59) Collins, J. B.; Schleyer, P. v. R.; Binkley, J. S.; Pople, J. A. Self-Consistent Molecular Orbital Methods. 17. Geometries and Binding Energies of Second-Row Molecules. A Comparison of Three Basis Sets. J. Chem. Phys. 1976, 64, 5142.

(60) Ditchfield, R.; Hehre, W. J.; Pople, J. A. J. Chem. Phys. 1971, 54, 724.

(61) Hehre, W. J.; Ditchfield, R.; Pople, J. A. J. Chem. Phys. 1972, 56, 2257.

(62) Hariharan, P. C.; Pople, J. A. Mol. Phys. 1974, 27, 209.

(63) Gordon, M. S. Chem. Phys. Lett. 1980, 76, 163.

(64) Hariharan, P. C.; Pople, J. A. Theo. Chim. Acta 1973, 28, 213.

(65) Binning Jr., J. R.; Curtiss, L. A. J. Comp. Chem. 1990, 11, 1206.

(66) McLean, A. D.; Chandler,G. S. J. Chem. Phys. 1980, 72, 5639.

(67) Krishnan, R.; Binkley, J. S.; Seeger, R.; Pople, J. A. J. Chem. Phys. 1980, 72, 650.

(68) Woon, D. E.; Dunning Jr., T. H. J. Chem. Phys. 1993, 98, 1358.

(69) Kendall, R. A.; Dunning Jr., T. H.; Harrison, R. J. J. Chem. Phys. 1992, 96, 6796.

(70) Dunning Jr., T. H. J. Chem. Phys. 1989, 90, 1007.

(71) Peterson, K. A.; Woon, D. E.; Dunning Jr., T. H. J. Chem. Phys. 1994, 100, 7410.

(72) Wilson, A.; van Mourik, T.; Dunning Jr., T. H. J. Mol. Struct. (Theochem) 1997, $388,339$.

(73) Parr, R. G.; Yang, W. Density Functional Theory of Atoms and Molecules, Oxford University Press: New York, 1989.

(74) Becke, A. D. Density Functional Theory Thermochemistry. 3. The Role of Exact Exchange. J. Chem. Phys. 1993, 98, 5648-5652.

(75) Vosko, S. H.; Wilk, L.; Nusiar, M. Accurate Spin-Dependent Electron Liquid Correlation Energies for Local Spin Density Calculations: A Critical Analysis. Can. J. Phys. 1980, 58, 1200-1211.

(76) Lee, C.; Yang, W.; Parr, R. G. Development of the Colle-Salvetti Correlation-Energy into a Functional Theory of the Electron Density. Phys. Rev. B 1988, 37, 785-789.

(77) Brown, S. T.; Rienstra-Kiracofe, J. C.; Schaefer, H. F. J. Phys. Chem. A 1999, 103, 4065.

(78) Gaussian 98(Revision A.11.2), Gaussian, Inc.: Pittsburgh, PA, 1998.

(79) Sas/Stat User's Guide, Release 6.03, SAS Institute Inc.: Cary, NC., 1988.

(80) Topliss, J. G.; Edwardș, R. P. Chance Factors in Studies of Quantitative Structure-Activity Relationships. $J$. Med. Chem. 1979, 22, 1238-1244. 
Figure Caption:

Figure 1. The optimized ground state geometries of 55 neutral halocarbons in the Crebelli set. The geometries were optimized at the cc-pVTZ/DFT/B3LYP level. 
Table 1. The 55 halocarbons and their experimental and estimated toxicity values for ARR and $\mathrm{D}_{37}$ in Aspergillus nidulans diploid strain $\mathrm{P} 1$.

\begin{tabular}{|c|c|c|c|c|c|}
\hline & Chemical Name & $\mathrm{D}_{37}$ & Est. $D_{37}{ }^{2}$ & ARR & Est. ARR \\
\hline 1 & Dichloromethane & 93.6 & 124.3 & 135.7 & 158.9 \\
\hline 2 & Chloroform & 24.8 & 22.9 & 23.5 & 18.1 \\
\hline 3 & Carbon tetrachloride & 3.1 & 1.9 & 2.9 & 2.5 \\
\hline 4 & 1,1-Dichloroethane & 47.6 & 47.9 & 38.1 & 37.2 \\
\hline 5 & 1,2-Dichloroethane & 50.8 & 32.7 & 44.4 & 33.4 \\
\hline 6 & 1,1,1-Trichloroethane & 10 & 8.4 & 11 & 10.1 \\
\hline 7 & 1,1,2-Trichloroethane & 10.7 & 9.9 & 11.8 & 7.5 \\
\hline 8 & 1,1,1,2-Tetrachloroethane & 2.8 & 2.9 & 2.4 & 2.5 \\
\hline 9 & 1,1,2,2-Tetrachloroethane & 2.8 & 4.3 & 3.3 & 5.0 \\
\hline 10 & Pentachloroethane & 1.7 & 1.6 & 1.2 & 4.9 \\
\hline 11 & Hexachloroethane & 0.8 & 1.5 & 16.9 & 4.8 \\
\hline 12 & 1,1,2-Trichloroethylene & 11.1 & 8.6 & 10.6 & 7.9 \\
\hline 13 & Tetrachloroethylene & 1.2 & 1.6 & 1.5 & 1.8 \\
\hline 14 & 1,2-Dichloroethylene & 29.9 & 25.9 & 25.5 & 34.5 \\
\hline 15 & 1,1-Dichloroethylene & 25 & 17.8 & 21.9 & 14.4 \\
\hline 16 & 1,2-Dichloropropane & 15.4 & 15.2 & 17.4 & 12.6 \\
\hline 17 & 2,2-Dichloropropane & 19.2 & 17.6 & 17.2 & 20.5 \\
\hline 18 & 1,3-Dichloropropane & 10.5 & 8.9 & 13.7 & 13.4 \\
\hline 19 & 1,2,3-Trichloropropane & 9.4 & 4.2 & 6.7 & 7.3 \\
\hline 20 & 1-Chlorobutane & 14.4 & 13.4 & 12.4 & 16.1 \\
\hline 21 & 2-Chlorobutane & 18.9 & 22.2 & 25.5 & 14.9 \\
\hline 22 & 1,3-Dichlorobutane & 8.8 & 6.5 & 8.3 & 8.3 \\
\hline 23 & 2,3-Dichlorobutane & 8.7 & 8.7 & 10.5 & 8.3 \\
\hline 24 & 1-Chloro-2-methylpropane & 14.3 & 20.7 & 14.3 & 13.2 \\
\hline 25 & 2-Chloro-2-methylpropane & 18.4 & 23.8 & 23.9 & 29.8 \\
\hline 26 & 1-Chloropentane & 5 & 5.1 & 5 & 5.0 \\
\hline 27 & 1-Chlorohexane & 1.5 & 2.3 & 1 & 2.1 \\
\hline 28 & 1-Chlorooctane & 1.5 & 0.7 & 1 & 0.5 \\
\hline 29 & 1,1-Dichloropropene & 6.6 & 11.6 & 5 & 11.1 \\
\hline 30 & 2,3-Dichloro-1-propene & 2.7 & 4.7 & 2.7 & 5.3 \\
\hline 31 & 1,3-Dichloropropene & 5.3 & 6.7 & 7.8 & 10.9 \\
\hline 32 & 1,1,3-Trichloropropene & 2.4 & 2.8 & 1.7 & 2.6 \\
\hline 33 & 3-Chloro-2-chloromethyl propene & 2.7 & 2.3 & 2.6 & 3.5 \\
\hline 34 & 1-Chloro-2-methylpropene & 12.7 & 17.4 & 9.7 & 16.4 \\
\hline 35 & 3-Chloro-2-methylpropene & 7.6 & 7.2 & 10.1 & 5.8 \\
\hline 36 & Chlorodibromofluoromethane & 3.1 & 3.1 & 2.6 & 2.7 \\
\hline 37 & Bromoform & 5.2 & 2.4 & 5.5 & 2.0 \\
\hline 38 & Bromochloromethane & 61.6 & 56.0 & 69.3 & 74.8 \\
\hline 39 & Bromotrichloromethane & 0.8 & 0.8 & 1 & 0.6 \\
\hline
\end{tabular}




\begin{tabular}{llrrrr}
40 & Bromodichloromethane & 10.6 & 10.5 & 11.1 & 8.0 \\
41 & Chlorodibromomethane & 2.9 & 5.1 & 3.3 & 3.4 \\
42 & 1-Bromo-2-chloroethane & 24 & 16.2 & 20.4 & 16.6 \\
43 & 1-Bromobutane & 5.6 & 8.5 & 4.9 & 9.6 \\
44 & 2-Bromobutane & 11.5 & 14.4 & 9.8 & 10.1 \\
45 & 1-Bromo-3-chloropropane & 7.5 & 5.2 & 8.1 & 7.2 \\
46 & 2-Bromo-1-chloropropane & 9.8 & 9.6 & 8.3 & 8.2 \\
47 & 1-Bromo-2-methylpropane & 18.4 & 12.8 & 14.7 & 9.0 \\
48 & 2-Bromo-2-methylpropane & 26 & 14.8 & 26 & 13.8 \\
49 & 1-Bromo-4-chlorobutane & 3.3 & 2.7 & 3.4 & 2.6 \\
50 & 1-Bromo-3-methylbutane & 3.1 & 4.9 & 3.6 & 2.9 \\
51 & Dibromodichloromethane & 0.2 & 0.3 & 0.1 & 0.2 \\
52 & Dibromomethane & 21.4 & 26.0 & 25.7 & 36.8 \\
53 & Tetrabromomethane & 0.01 & 0.0 & 0.01 & 0.0 \\
54 & 1,1,2,2-Tetrabromoethane & 0.4 & 0.6 & 0.7 & 1.1 \\
55 & 1,2-Dibromoethylene & 9.1 & 10.0 & 9.2 & 9.5 \\
\hline
\end{tabular}

${ }^{8}$ Estimated values for $\mathrm{D}_{37}$ are derived from the HiQSAR model combining topostructural, topochemical and aug-ccpVTZ ab initio parameters.

"Estimated values for ARR are derived from the HiQSAR model combining topostructural, topochemical, geometrical and aug-cc-pVTZ ab initio parameters.

Table 2. Ab initio parameters calculated using the MP2/ 6-31G(d) level of theory. Energies in eV, dipole moments in debye.

\begin{tabular}{lccccccc}
\hline & $\mathbf{E}_{\text {IOMM } \cdot 1}$ & $\mathbf{E}_{\text {IOMO }}$ & $\mathbf{E}_{\text {LUMO }}$ & $\mathbf{E}_{\text {LUMO+1 }}$ & $\mathbf{G A P}_{\text {LH }}$ & VEA & $\mu$ \\
\hline 1 & -12.2280 & -12.1594 & 4.1663 & 5.7209 & 16.3257 & -2.9518 & 2.0604 \\
2 & -12.7148 & -12.3504 & 3.2414 & 4.9435 & 15.5919 & -1.9258 & 1.4484 \\
3 & -12.6291 & -12.6291 & 2.5086 & 4.2164 & 15.1377 & -1.0713 & 0.0003 \\
4 & -12.0587 & -11.9823 & 4.3136 & 5.5887 & 16.2958 & -2.9718 & 2.3255 \\
5 & -12.1567 & -12.0038 & 4.8406 & 5.1389 & 16.8444 & -3.2024 & 0.0000 \\
6 & -12.4813 & -12.1474 & 3.4172 & 4.8216 & 15.5646 & -1.9919 & 2.0236 \\
7 & -12.3050 & -12.1526 & 4.0262 & 4.5742 & 16.1788 & -2.6031 & 1.7334 \\
8 & -12.4209 & -12.3200 & 3.2115 & 4.0412 & 15.5314 & -1.7687 & 1.8895 \\
9 & -12.4367 & -11.9959 & 3.8632 & 3.9612 & 15.8591 & -2.1399 & 0.0000 \\
10 & -12.5632 & -12.1853 & 3.1073 & 3.7010 & 15.2925 & -1.6118 & 1.3176 \\
11 & -12.5632 & -12.1853 & 3.1073 & 3.7010 & 15.2925 & -1.6118 & 1.3176 \\
12 & -12.5624 & -9.9637 & 3.2572 & 4.3721 & 13.2209 & -2.1823 & 1.2378 \\
13 & -12.3197 & -9.9539 & 2.8583 & 4.1413 & 12.8122 & -1.7918 & 0.0000 \\
14 & -12.6713 & -9.9466 & 3.6452 & 4.9087 & 13.5918 & -2.5886 & 0.0000 \\
15 & -12.3945 & -10.1033 & 3.7138 & 4.5941 & 13.8171 & -2.6501 & 1.6788 \\
16 & -11.8087 & -11.7485 & 4.7356 & 5.5639 & 16.4841 & -3.2712 & 3.4002 \\
17 & -11.9793 & -11.7795 & 4.4077 & 5.2970 & 16.1872 & -2.9447 & 2.4800
\end{tabular}




\begin{tabular}{|c|c|c|c|c|c|c|c|}
\hline 18 & -11.9273 & -11.6914 & 4.3119 & 6.0426 & 16.0033 & -2.8340 & 2.1247 \\
\hline 19 & -12.0546 & -11.9638 & 3.9726 & 5.0763 & 15.9364 & -2.4362 & 3.4644 \\
\hline 20 & -11.6367 & -11.5469 & 5.3481 & 6.2355 & 16.8950 & -4.0458 & 2.4272 \\
\hline 21 & -11.5485 & -11.5393 & 5.4801 & 6.2110 & 17.0194 & -4.0190 & 2.1906 \\
\hline 22 & -11.7423 & -11.7151 & 4.8738 & 5.5582 & 16.5889 & -3.3023 & 3.4267 \\
\hline 23 & -11.9463 & -11.6574 & 4.7223 & 5.3606 & 16.3796 & -2.9426 & 0.0000 \\
\hline 24 & -11.6087 & -11.5646 & 5.2649 & 6.4597 & 16.8294 & -4.0238 & 2.2074 \\
\hline 25 & -11.4971 & -11.4971 & 5.2695 & 5.9215 & 16.7666 & -3.8193 & 2.2666 \\
\hline 26 & $-11 .(269$ & -11.5080 & 5.3655 & 6.1269 & 16.8735 & -4.0381 & 2.4338 \\
\hline 27 & -11.6220 & -11.4721 & 5.3680 & 6.0529 & 16.8400 & -4.0292 & 2.4983 \\
\hline 28 & -11.6171 & -11.4160 & 5.3753 & 6.0262 & 16.7913 & -4.0248 & 2.5311 \\
\hline 29 & -12.2576 & -9.6622 & 3.8523 & 4.7541 & 13.5145 & -2.7223 & 2.1433 \\
\hline 30 & -11.9746 & -9.9256 & 3.7633 & 4.6836 & 13.6890 & -2.6546 & 3.0483 \\
\hline 31 & -12.1474 & -10.0152 & 3.6918 & 4.4934 & 13.7069 & -2.5717 & 1.9498 \\
\hline 32 & -12.2666 & -10.1284 & 3.3603 & 4.1857 & 13.4887 & -2.1945 & 0.5262 \\
\hline 33 & -11.9126 & -10.1811 & 4.2885 & 4.4395 & 14.4697 & -3.0517 & 2.1328 \\
\hline 34 & $-11.0 \$ 50$ & -9.1711 & 4.2406 & 5.8777 & 13.4117 & -3.1091 & 2.2321 \\
\hline 35 & $-11 . \$ 236$ & -9.6293 & 4.4357 & 5.3745 & 14.0650 & -3.2574 & 1.6584 \\
\hline 36 & $-11 . \$ 359$ & -11.4397 & 1.8868 & 3.8216 & 13.3265 & -0.6251 & 0.5398 \\
\hline 37 & $-11 . .9975$ & -10.9855 & 2.0438 & 4.0852 & 13.0294 & -0.7113 & 1.1128 \\
\hline 38 & -11.3140 & -11.2995 & 3.4681 & 5.3696 & 14.7676 & -2.2490 & 1.9306 \\
\hline 39 & -11.7725 & -11.7725 & 1.9647 & 3.6363 & 13.7371 & -0.7056 & 0.2805 \\
\hline 40 & -11.7224 & -11.5365 & 2.6490 & 4.4643 & 14.1856 & -1.3901 & 1.3435 \\
\hline 41 & -11.025 & -11.1629 & 2.3132 & 3.9908 & 13.4762 & -1.0163 & 1.2314 \\
\hline 42 & -11.1934 & -11.1659 & 3.9840 & 5.0202 & 15.1499 & -2.6013 & 0.0935 \\
\hline 43 & -10.5331 & -10.6092 & 4.5201 & 6.0986 & 15.1293 & -3.1173 & 2.4445 \\
\hline 44 & -10.5877 & -10.5520 & 4.5462 & 6.1351 & 15.0982 & -3.0473 & 2.2093 \\
\hline 45 & -10.9684 & -10.9292 & 3.7416 & 5.8377 & 14.6708 & -2.4781 & 2.0906 \\
\hline 46 & -10.9191 & -10.8065 & 4.0803 & 5.2396 & 14.8868 & -2.7061 & 3.3259 \\
\hline 47 & -10.6 .367 & -10.6122 & 4.4994 & 6.1852 & 15.1116 & -3.1047 & $2: 2147$ \\
\hline 48 & -10.5074 & -10.5074 & 4.3655 & 5.8319 & 14.8729 & -2.8626 & 2.3084 \\
\hline 49 & -10.5693 & -10.8467 & 4.2406 & 5.0619 & 15.0874 & -2.8356 & 0.0168 \\
\hline 50 & -10.609 & -10.5795 & 4.4529 & 6.0589 & 15.0324 & -3.0402 & 2.4962 \\
\hline 51 & -11.6 .503 & -11.3526 & 1.6577 & 3.2542 & 13.0103 & -0.3403 & 0.3071 \\
\hline 52 & -11.2127 & -10.9167 & 3.0928 & 4.7481 & 14.0095 & -1.8362 & 1.8031 \\
\hline 53 & $-11.1 \$ 09$ & -11.1809 & 1.1878 & 3.4833 & 12.3687 & -0.2475 & 0.0002 \\
\hline 54 & -11.4187 & -10.5292 & 2.6512 & 2.9663 & 13.1804 & -0.9331 & 0.0000 \\
\hline 55 & -11.5749 & -9.6502 & 3.4948 & 3.6953 & 13.1450 & -2.2764 & 0.0000 \\
\hline
\end{tabular}


Table 3. Ab initio parameters generated within the MP2/ 6-311G computational scheme. Energy in eV, dipole moments in delsye.

\begin{tabular}{|c|c|c|c|c|c|c|c|}
\hline & $\mathbf{E}_{110 \cdots \cdots-1}$ & $\mathrm{E}_{\text {tоOмแ }}$ & $E_{\text {tLM }}$ & $\mathrm{E}_{\mathrm{LUMO+1}}$ & $\mathbf{G A P}_{\text {L-H }}$ & VEA & $\mu$ \\
\hline 1 & -12.5910 & -12.4228 & 2.6191 & 4.0907 & 15.0419 & -1.6695 & 2.5911 \\
\hline 2 & -13.0432 & -12.8117 & 1.6863 & 3.5100 & 14.4980 & -0.6978 & 1.7400 \\
\hline 3 & -13.1605 & -13.1605 & 0.7396 & 2.8879 & 13.9001 & 0.2161 & 0.0001 \\
\hline 4 & -12.4253 & -12.1442 & 2.9089 & 3.9206 & 15.0531 & -1.7708 & 3.1867 \\
\hline 5 & -12.3932 & -12.2955 & 2.8455 & 3.5427 & 15.1410 & -1.5355 & 0.0000 \\
\hline 6 & $-12.7 ! 51$ & -12.5390 & 1.9004 & 3.4289 & 14.4395 & -0.7912 & 2.7738 \\
\hline 7 & -12.6626 & -12.4718 & 2.3108 & 2.9456 & 14.7826 & -1.2098 & 1.9151 \\
\hline 8 & -12.7747 & -12.6911 & 1.6362 & 2.2107 & 14.3273 & -0.5188 & 2.1691 \\
\hline 9 & -13.0 .465 & -12.4302 & 1.9402 & 2.1437 & 14.3703 & -0.6789 & 0.0000 \\
\hline 10 & -13.0296 & -12.6495 & 1.4999 & 1.8044 & 14.1494 & -0.3770 & 1.4106 \\
\hline 11 & -13.0296 & -12.6495 & 1.4999 & 1.8044 & 14.1494 & -0.3770 & 1.4106 \\
\hline 12 & -12.8770 & -10.8157 & 2.5954 & 2.6308 & 13.4111 & -1.7504 & 1.5650 \\
\hline 13 & -12.7953 & -10.9107 & 2.2931 & 2.3051 & 13.2038 & -0.8162 & 0.0000 \\
\hline 14 & -12.96 .30 & -10.6927 & 2.9296 & 2.9413 & 13.6223 & -2.1632 & 0.0000 \\
\hline 15 & -12.5728 & -10.7292 & 3.0488 & 3.0841 & 13.7779 & -2.2704 & 2.6089 \\
\hline 16 & $-12.0+\$ 44$ & -11.8944 & 3.1492 & 3.6999 & 15.0435 & -2.2592 & 4.5327 \\
\hline 17 & -12.2998 & -11.8868 & 3.0719 & 3.7734 & 14.9586 & -1.7795 & 3.5658 \\
\hline 18 & $-12.1+91$ & -11.9406 & 2.6058 & 3.9435 & 14.5464 & -1.4407 & 2.9276 \\
\hline 19 & -12.2979 & -12.2566 & 2.2999 & 3.2752 & 14.5565 & -1.1058 & 4.3320 \\
\hline 20 & -11.7393 & -11.6503 & 3.6246 & 4.2148 & 15.2748 & -2.6772 & 3.4043 \\
\hline 21 & -11.6016 & -11.5760 & 3.9282 & 4.1203 & 15.5042 & -2.7246 & 3.3348 \\
\hline 22 & -11.9907 & -11.8576 & 3.3326 & 3.6561 & 15.1902 & -2.3150 & 4.6201 \\
\hline 23 & $-12.1+39$ & -11.9597 & 2.8760 & 3.9076 & 14.8357 & -1.3815 & 0.0000 \\
\hline 24 & -11.7055 & -11.6721 & 3.5560 & 4.5868 . & 15.2280 & -2.6541 & 3.2249 \\
\hline 25 & $-11.4 S+6$ & -11.4846 & 3.7495 & 3.9435 & 15.2340 & -2.5521 & 3.5189 \\
\hline 26 & -11.7319 & -11.6179 & 3.6510 & 4.1797 & 15.2689 & -2.6827 & 3.4369 \\
\hline 27 & -11.7287 & -11.5855 & 3.6602 & 4.0959 & 15.2457 & -2.6678 & 3.4930 \\
\hline 28 & -11.7254 & -11.5276 & 3.6635 & 4.1089 & 15.1910 & -2.6620 & 3.5340 \\
\hline 29 & -12.4065 & -10.2737 & 3.2686 & 3.2983 & 13.5423 & -2.2046 & 3.2482 \\
\hline 30 & $\cdot-12.0813$ & -10.4481 & 3.0142 & 3.0768 & 13.4623 & -2.2311 & 4.2274 \\
\hline 31 & -12.3768 & -10.5972 & 2.8077 & 2.9543 & 13.4049 & -2.0280 & 2.6591 \\
\hline 32 & -12.5368 & -10.8560 & 2.6860 & 2.7070 & 13.5420 & -1.7526 & 0.6535 \\
\hline 33 & -12.1474 & -10.5180 & 2.7007 & 3.4605 & 13.2188 & -1.5870 & 2.1876 \\
\hline 34 & -12.0536 & -9.6100 & 3.6847 & 3.8896 & 13.2947 & -2.8000 & 3.2391 \\
\hline 35 & -11.9374 & -9.8750 & 3.6803 & 3.7367 & 13.5554 & -2.7111 & 2.5016 \\
\hline 36 & -12.3134 & -11.9932 & 0.6884 & 2.7807 & 12.6816 & 0.1121 & 1.6765 \\
\hline 37 & -11.5964 & $-11: 3169$ & 1.0501 & 3.1154 & 12.3670 & -0.2161 & 1.2637 \\
\hline 38 & $-11 .(\cdot 734$ & -11.5616 & 2.2743 & 3.9726 & 13.8359 & -1.4049 & 2.3641 \\
\hline 39 & -12.3273 & -12.3273 & 0.5287 & 2.5927 & 12.8560 & 0.3396 & 0.9380 \\
\hline
\end{tabular}




\begin{tabular}{llllllll}
40 & -12.1284 & -11.9959 & 1.3951 & 3.2964 & 13.3910 & -0.4991 & 1.7694 \\
41 & -11.9439 & -11.5847 & 1.2011 & 3.1070 & 12.7858 & -0.3453 & 1.6190 \\
42 & -11.4506 & -11.4304 & 2.6213 & 3.5059 & 14.0517 & -1.5996 & 0.6559 \\
43 & -10.7567 & -10.7216 & 3.3971 & 4.1429 & 14.1186 & -2.3824 & 2.8839 \\
44 & -10.6644 & -10.6367 & 3.6099 & 4.0276 & 14.2465 & -2.3921 & 2.8693 \\
45 & -11.1842 & -11.1447 & 2.4498 & 3.9633 & 13.5945 & -1.5114 & 2.7197 \\
46 & -11.1131 & -10.9953 & 2.9976 & 3.4907 & 13.9929 & -2.0144 & 4.1308 \\
47 & -10.7379 & -10.7295 & 3.3492 & 4.4428 & 14.0786 & -2.3666 & 2.7335 \\
48 & -10.5635 & -10.5635 & 3.4754 & 3.8578 & 14.0389 & -2.2455 & 3.0377 \\
49 & -11.0642 & -11.0334 & 3.0790 & 3.4052 & 14.1124 & -2.0769 & 0.5658 \\
50 & -10.7428 & -10.6900 & 3.3666 & 4.2017 & 14.0566 & -2.3266 & 2.9363 \\
51 & -12.1306 & -11.8941 & 0.3867 & 2.4213 & 12.2808 & 0.4507 & 1.0603 \\
52 & -11.4152 & -11.1382 & 2.0504 & 3.7835 & 13.1885 & -1.2116 & 1.9915 \\
53 & -11.5205 & -11.5205 & 0.1867 & 2.4474 & 11.7072 & 0.6307 & 0.0000 \\
54 & -11.6111 & -10.8511 & 1.5491 & 1.9266 & 12.4002 & -0.4372 & 0.0000 \\
55 & -11.7436 & -10.0015 & 2.5298 & 3.0479 & 12.5314 & -1.3099 & 0.0000 \\
\hline
\end{tabular}

Table 4. Ab initio parameters calculated within the MP2/ 6-311G(d) computational scheme. Energy in eV, dipole moments in debye.

\begin{tabular}{|c|c|c|c|c|c|c|c|}
\hline & $\mathbf{E}_{\text {110)10.1 }}$ & $\mathrm{E}_{\text {III)M }}$ & $\mathrm{E}_{\text {L. Unto }}$ & $\mathbf{E}_{\text {LUMO+1 }}$ & $\mathbf{G A P}_{\mathrm{L}-\mathrm{H}}$ & VEA & $\mu$ \\
\hline 1 & -12.3145 & -12.2432 & 3.4207 & 4.6330 & 15.6640 & -2.3414 & 2.0169 \\
\hline 2 & -12.7956 & -12.4231 & 2.8926 & 4.1829 & 15.3157 & -1.5352 & 1.3567 \\
\hline 3 & -12.6887 & -12.6887 & 2.2653 & 3.8349 & 14.9540 & -0.7347 & 0.0001 \\
\hline 4 & -12.1385 & -12.0661 & 3.7021 & 4.3296 & 15.7682 & -2.4713 & 2.4314 \\
\hline 5 & -12.2302 & -12.0838 & 3.8188 & 4.2888 & 15.9026 & -2.9407 & 0.0000 \\
\hline 6 & -12.5657 & -12.2117 & 3.1511 & 4.3239 & 15.3627 & -1.6131 & 2.1200 \\
\hline 7 & -12.3874 & -12.2332 & 3.5424 & 3.9187 & 15.7755 & -2.1506 & 1.5217 \\
\hline 8 & -12.4955 & -12.3910 & 2.9533 & 3.6512 & 15.3442 & -1.4192 & 1.6950 \\
\hline 9 & -12.5200 & -12.0775 & 3.4711 & 3.4975 & 15.5486 & -2.0442 & 0.0000 \\
\hline 10 & -12.6274 & -12.2568 & 2.8523 & 3.3462 & 15.1091 & -1.2735 & 1.1031 \\
\hline 11 & -12.6274 & -12.2568 & 2.8523 & 3.3462 & 15.1091 & -1.2735 & 1.1031 \\
\hline 12 & -12.6626 & -10.0435 & 2.9856 & 3.9369 & 13.0291 & -1.7801 & 1.1322 \\
\hline 13 & -12.4125 & -10.0258 & 2.6376 & 3.7209 & 12.6634 & -1.4436 & 0.0000 \\
\hline 14 & -12.7662 & -10.0375 & 3.3184 & 4.3429 & 13.3559 & -2.1237 & 0.0000 \\
\hline 15 & -12.4966 & -10.1877 & 3.3606 & 4.1446 & 13.5483 & -2.1711 & 1.8006 \\
\hline 16 & -11.8868 & -11.8220 & 3.6137 & 4.4736 & 15.4357 & -2.6913 & 3.4763 \\
\hline 17 & -12.0555 & -11.8557 & 4.0178 & 4.0945 & 15.8735 & -3.1157 & 2.6737 \\
\hline 18 & -12.0062 & -11.7602 & 3.3176 & $4.5394^{\circ}$ & 15.0778 & -2.2346 & 2.2087 \\
\hline 19 & -12.1287 & -12.0408 & 3.1805 & 4.0020 & 15.2212 & -1.9873 & 3.3222 \\
\hline 20 & -11.7134 & -11.6122 & 3.9114 & 4.4325 & 15.5236 & -2.9907 & 2.5987 \\
\hline
\end{tabular}




\begin{tabular}{|c|c|c|c|c|c|c|c|}
\hline 21 & -11.6269 & -11.6125 & 4.0580 & 4.6912 & 15.6705 & -3.2163 & 2.4815 \\
\hline 22 & -11.8168 & -11.7869 & 3.7554 & 4.3220 & 15.5423 & -2.8571 & 3.5707 \\
\hline 23 & -12.0285 & -11.7453 & 4.1182 & 4.2931 & 15.8634 & -3.1047 & 0.0000 \\
\hline 24 & -11.6832 & -11.6345 & 3.8469 & 4.8156 & 15.4814 & -2.8920 & 2.4380 \\
\hline 25 & -11.5746 & -11.5746 & 3.9233 & 4.5941 & 15.4980 & -3.0902 & 2.5560 \\
\hline 26 & -11.7031 & -11.5722 & 3.9263 & 4.3598 & 15.4985 & -2.9807 & 2.6117 \\
\hline 27 & -11.6979 & -11.5352 & 3.9440 & 4.2550 & 15.4792 & -2.9678 & 2.6700 \\
\hline 28 & -11.6927 & -11.4772 & 3.9478 & 4.2352 & 15.4251 & -2.9546 & 2.7021 \\
\hline 29 & -12.3621 & -9.7439 & 3.5671 & 4.0940 & 13.3110 & -2.2915 & 2.3368 \\
\hline 30 & -12.0800 & -10.0149 & 3.3361 & 3.8175 & 13.3510 & -2.1309 & 3.0929 \\
\hline 31 & -12.2310 & -10.1158 & 3.3032 & 3.6088 & 13.4190 & -2.0721 & 1.9999 \\
\hline 32 & -12.3472 & -10.2130 & 3.0795 & 3.8314 & 13.2925 & -1.7774 & 0.5362 \\
\hline 33 & -12.0029 & -10.3014 & 3.4194 & 3.7636 & 13.7208 & -2.3659 & 1.6886 \\
\hline 34 & $-12.0 \$ 40$ & -9.2619 & 3.8948 & 4.0393 & $13: 1567$ & -2.6302 & 2.3583 \\
\hline 35 & -11.9055 & -9.7262 & 3.9584 & 3.9870 & 13.6846 & -2.6881 & 1.8311 \\
\hline 36 & -11.9551 & -11.5774 & 1.5924 & 3.3530 & 13.1698 & -0.3506 & 0.7990 \\
\hline 37 & -11.5439 & -11.1159 & 1.6629 & 3.6354 & 12.7787 & -0.3872 & 1.1055 \\
\hline 38 & -11.4448 & -11.4405 & 2.8890 & 4.4638 & 14.3295 & -1.7754 & 1.9010 \\
\hline 39 & -11.8979 & -11.8979 & 1.6849 & 3.3168 & 13.5828 & -0.4090 & 0.2674 \\
\hline 40 & -11.8644 & -11.6726 & 2.2882 & 4.0276 & 13.9608 & -1.0451 & 1.2847 \\
\hline 41 & -11.7640 & -11.3140 & 1.9402 & 3.5884 & 13.2541 & -0.6800 & 1.2019 \\
\hline 42 & -11.3175 & -11.2884 & 3.3957 & 4.0131 & 14.6841 & -2.1363 & 0.1331 \\
\hline 43 & -10.7575 & -10.7289 & 3.6711 & 4.2638 & 14.4000 & -2.5361 & 2.5767 \\
\hline 44 & -10.7123 & -10.6832 & 3.9274 & 4.1799 & 14.6106 & -2.5691 & 2.5058 \\
\hline 45 & -11.0935 & -11.0481 & 3.0338 & 4.2959 & 14.0819 & -1.8860 & 2.1666 \\
\hline 46 & -11.0533 & -10.9490 & 3.4722 & 3.8814 & 14.4212 & -2.2523 & 3.3920 \\
\hline 47 & -10.7537 & -10.7363 & 3.6041 & 4.6839 & 14.3404 & -2.5200 & 2.4247 \\
\hline 48 & -10.6426 & -10.6426 & 3.8412 & 3.9372 & 14.4838 & -2.3910 & 2.6109 \\
\hline 49 & -10.9956 & -10.9686 & 3.4158 & 3.8692 & 14.3845 & -2.2843 & 0.0534 \\
\hline 50 & -10.7455 & -10.6976 & 3.6319 & 4.3527 & 14.3295 & -2.4794 & 2.6193 \\
\hline 51 & -11.7635 & -11.4955 & 1.3701 & 2.8852 & 12.8655 & -0.0607 & 0.2977 \\
\hline 52 & -11.3052 & -11.0712 & 2.5380 & 4.3027 & 13.6092 & -1.3889 & 1.7767 \\
\hline 53 & -11.2925 & -11.2925 & 0.9064 & 3.0265 & 12.1989 & 0.4890 & 0.0000 \\
\hline 54 & -11.5360 & -10.6658 & 2.3195 & 2.5034 & 12.9853 & -0.6484 & 0.0000 \\
\hline 55 & -11.6851 & -9.7292 & 3.1854 & 3.2874 & 12.9145 & -1.9003 & 0.0000 \\
\hline
\end{tabular}


Table 5. Ab initio parameters calculated using within the b3lyp/aug-cc-pVTZ approach. Energies in eV and dipole moment in debye. Experimental dipole moment values" are given in parenthesis.

\begin{tabular}{|c|c|c|c|c|}
\hline & $\mathrm{E}_{\mathrm{ILMK}}$ & $\mathrm{E}_{\text {tusto }}$ & VEA & $\mu$ \\
\hline 1 & -8.556 & -0.872 & 0.383 & $1.677(1.60)$ \\
\hline 2 & -8.685 & -1.492 & 0.263 & $1.085(1.04)$ \\
\hline 3 & -8.880 & -2.125 & -0.252 & 0.000 \\
\hline 4 & -8.420 & -0.676 & 0.404 & $2.163(2.06)$ \\
\hline 5 & -8.507 & -0.619 & 0.442 & 0.000 \\
\hline 6 & -8.486 & -1.373 & 0.247 & $1.878(1.755)$ \\
\hline 7 & -8.599 & -0.986 & 0.422 & 1.252 \\
\hline 8 & -8.662 & -1.549 & 0.171 & 1.405 \\
\hline 9 & -8.245 & -1.153 & 0.422 & 0.000 \\
\hline 10 & -8.563 & -1.618 & 0.117 & 0.869 \\
\hline 11 & -8.461 & -1.675 & -0.091 & 0.000 \\
\hline 12 & -7.260 & -1.159 & 0.453 & 0.859 \\
\hline 13 & -7.230 & -1.339 & 0.560 & 0.000 \\
\hline 14 & 7.260 & -0.998 & $0.537^{\circ}$ & 0.000 \\
\hline 15 & -7.462 & -1.000 & 0.452 & $1.373(1.34)$ \\
\hline 16 & -8.207 & -0.583 & 0.296 & 3.115 \\
\hline 17 & -8.223 & -0.618 & 0.354 & 2.422 \\
\hline 18 & -8.225 & -0.832 & 0.255 & $1.958(2.08)$ \\
\hline 19 & -8.429 & -1.005 & 0.186 & 2.880 \\
\hline 20 & -8.058 & -0.408 & 0.369 & 2.415 \\
\hline 21 & -8.017 & -0.357 & 0.383 & 2.307 \\
\hline 22 & -8.179 & -0.496 & 0.296 & 3.194 \\
\hline 23 & -8.184 & -0.602 & 0.435 & 0.000 \\
\hline 24 & -8.060 & -0.444 & 0.359 & 2.237 \\
\hline 25 & -7.984 & -0.431 & 0.354 & 2.415 \\
\hline 26 & -8.038 & -0.417 & 0.341 & 2.432 \\
\hline 27 & -8.020 & -0.408 & 0.326 & 2.497 \\
\hline 28 & -7.997 & -0.441 & 0.289 & 2.535 \\
\hline 29 & -7.048 & -0.692 & 0.379 & 1.935 \\
\hline 30 & -7.295 & -0.960 & 0.326 & 2.596 \\
\hline 31 & -7.369 & -0.968 & 0.354 & 1.787 \\
\hline 32 & -7.448 & -1.104 & 0.369 & 0.640 \\
\hline 33 & -7.472 & -0.695 & 0.341 & 1.900 \\
\hline 34 & -6.336 & -0.377 & 0.379 & 2.049 \\
\hline 35 & -7.102 & -0.467 & 0.392 & 1.537 \\
\hline 36 & -8.264 & -2.551 & -0.654 & 0.435 \\
\hline 37 & -7.924 & -2.369 & -0.403 & $0.865(0.99)$ \\
\hline 38 & -8.132 & -1.326 & 0.256 & 1.580 \\
\hline 39 & -8.427 & -2.498 & -0.632 & 0.083 \\
\hline
\end{tabular}




\begin{tabular}{lllll}
40 & -8.281 & -1.915 & -0.043 & 1.013 \\
41 & -8.067 & -2.169 & -0.327 & 0.939 \\
42 & -8.039 & -1.086 & 0.381 & 0.082 \\
43 & -7.562 & -0.598 & 0.339 & 2.456 \\
44 & -7.500 & -0.515 & 0.358 & 2.403 \\
45 & -7.850 & -1.148 & 0.159 & 1.923 \\
46 & -7.729 & -0.823 & 0.279 & 3.077 \\
47 & -7.547 & -0.649 & 0.313 & 2.292 \\
48 & -7.457 & -0.677 & 0.315 & 2.540 \\
49 & -7.785 & -0.789 & 0.270 & 0.022 \\
50 & -7.544 & -0.621 & 0.307 & 2.485 \\
51 & -8.174 & -2.726 & -0.900 & 0.102 \\
52 & -9.970 & -1.592 & 0.099 & $1.479(1.43)$ \\
53 & -8.044 & -3.058 & -1.305 & 0.000 \\
54 & -7.583 & -1.997 & -0.409 & 0.000 \\
55 & -7.117 & -1.229 & 0.297 & 0.000 \\
\hline
\end{tabular}

Table 6. Five principal components retained from the PCA of eighty-six topostructural indices.

\begin{tabular}{lrccc}
\hline PC & Eigenvalue & $\begin{array}{c}\text { Proportion of } \\
\text { Variance }\end{array}$ & $\begin{array}{c}\text { Cumulative } \\
\text { Variance }\end{array}$ & $\begin{array}{c}\text { Most-highly } \\
\text { Correlated TCI }\end{array}$ \\
\hline $\mathbf{1}$ & 54.2983 & 0.6314 & 0.6314 & $\mathrm{~W}$ \\
2 & 21.2275 & 0.2468 & 0.8782 & $\mathrm{DS} 1_{3}$ \\
3 & 5.4819 & 0.0637 & 0.9420 & ${ }^{4} \chi_{\mathrm{FC}}$ \\
4 & 2.2830 & 0.0265 & 0.9685 & ${ }^{6} \chi_{\mathrm{c}}$ \\
5 & 1.1342 & 0.0132 & 0.9817 & ${ }^{5} \chi_{\mathrm{FC}}$ \\
\hline
\end{tabular}

Table 7. Twelve principal components retained from the PCA of 106 topochemical indices.

\begin{tabular}{lrccc}
\hline PC & Eigenvalue & $\begin{array}{c}\text { Proportion of } \\
\text { Variance }\end{array}$ & $\begin{array}{c}\text { Cumulative } \\
\text { Variance }\end{array}$ & $\begin{array}{c}\text { Most-highly } \\
\text { Correlated TCI }\end{array}$ \\
\hline 1 & 37.9045 & 0.3384 & 0.3384 & $\mathrm{AZV}_{3}$ \\
2 & 20.7565 & 0.1853 & 0.5238 & ${ }^{2} \chi^{\mathrm{b}}$ \\
3 & 13.1183 & 0.1171 & 0.6409 & $\mathrm{Q}_{\mathrm{v}}$ \\
4 & 9.7944 & 0.0875 & 0.7283 & ${ }^{\mathrm{i}} \chi^{\mathrm{v}}$ \\
5 & 6.7769 & 0.0605 & 0.7888 & ${ }^{4} \chi_{\mathrm{PC}}^{\mathrm{v}}$ \\
6 & 4.9909 & 0.0446 & 0.8334 & $\mathrm{SdssC}$ \\
\hline
\end{tabular}




\begin{tabular}{llllc}
\hline 7 & 3.6443 & 0.0325 & 0.8659 & SdsCH \\
8 & 2.4341 & 0.0217 & 0.8877 & $\mathrm{SdCH}_{2}$ \\
9 & 2.1676 & 0.0194 & 0.9070 & ${ }^{5} \chi_{\mathrm{FC}}^{\mathrm{v}}$ \\
10 & 1.6602 & 0.0148 & 0.9219 & $\mathrm{Sssss}$ \\
11 & 1.4927 & 0.0133 & 0.9352 & $\mathrm{H}_{\mathrm{MIN}}$ \\
12 & 1.3051 & 0.0117 & 0.9468 & $\mathrm{SsF}$ \\
\hline
\end{tabular}

Table 8. Summary results for the HiQSAR modeling of ARR in Aspergillus nidulans for 55 halogenated aliphatic hydrocarbons.

\begin{tabular}{|c|c|c|c|c|c|}
\hline Model & $\begin{array}{l}\text { \#indep. } \\
\text { variables }\end{array}$ & $R^{2}$ & $R_{c v}^{2}$ & s.e. & $F$ \\
\hline TSI only & 3 & 0.3661 & 0.2213 & 1.273 & 9.82 \\
\hline TCI only & 8 & 0.8370 & 0.7272 & 0.6795 & 29.54 \\
\hline 3D only & 4 & 0.8326 & 0.7683 & 0.6605 & 62.19 \\
\hline AM1 only & 3 & 0.4285 & 0.2671 & 1.208 & 12.75 \\
\hline STO-3G only & 4 & 0.4240 & 0.2673 & 1.225 & 9.20 \\
\hline 6-31G(d) only & 4 & 0.3645 & 0.1899 & 1.287 & 7.17 \\
\hline 6-311G only & 4 & 0.4328 & 0.2790 & 1.216 & 9.54 \\
\hline $6-311 \mathrm{G}^{*}$ only & 4 & 0.5802 & 0.4337 & 1.046 & 17.28 \\
\hline cc-pVTZ only & 1 & 0.4880 & 0.4504 & 1.122 & 50.51 \\
\hline $\mathrm{TSI}+\mathrm{TCI}$ & 8 & 0.8606 & 0.7637 & 0.6285 & 35.50 \\
\hline $\mathrm{TSI}+\mathrm{TCI}+3 \mathrm{D}$ & 7 & 0.9014 & 0.8345 & 0.5228 & 61.41 \\
\hline $\mathrm{TSI}+\mathrm{TCI}+3 \mathrm{D}+\mathrm{AM} 1$ & \multicolumn{5}{|c|}{ Same as previous model } \\
\hline $\mathrm{TSI}+\mathrm{TCI}+3 \mathrm{D}+\mathrm{STO}-3 \mathrm{G}$ & \multicolumn{5}{|c|}{ Same as previous model } \\
\hline $\mathrm{TSI}+\mathrm{TCI}+3 \mathrm{D}+6-31 \mathrm{G}(\mathrm{d})$ & \multicolumn{5}{|c|}{ Same as previous model } \\
\hline $\mathrm{TSI}+\mathrm{TCI}+3 \mathrm{D}+6-311 \mathrm{G}$ & \multicolumn{5}{|c|}{ Same as previous model } \\
\hline $\mathrm{TSI}+\mathrm{TCI}+3 \mathrm{D}+6-311 \mathrm{G}(\mathrm{d})$ & 8 & 0.9065 & 0.8340 & 0.5146 & 55.77 \\
\hline $\mathrm{TSI}+\mathrm{TCI}+3 \mathrm{D}+$ aug-cc-pVTZ & 8 & 0.9120 & 0.8511 & 0.4994 & 59.59 \\
\hline
\end{tabular}


Table 9. Summary results for the HiQSAR modeling of $D_{37}$ in Aspergillus nidulans for 55 halogenated aliphatic hydrocarbons.

\begin{tabular}{lccccc}
\hline & $\begin{array}{c}\text { \# indep. } \\
\text { variables }\end{array}$ & $\boldsymbol{R}^{2}$ & $\boldsymbol{R}_{\boldsymbol{c} \boldsymbol{v}}^{2}$ & s.e. & $\boldsymbol{F}$ \\
\hline TSI only & 2 & 0.3659 & 0.2945 & 1.243 & 15.00 \\
TCI only & 8 & 0.8623 & 0.7749 & 0.6161 & 36.00 \\
3D only & 8 & 0.8838 & 0.6496 & 0.5660 & 43.72 \\
AM1 only & 3 & 0.4591 & 0.3008 & 1.159 & 14.43 \\
STO-3(i only & 3 & 0.3055 & 0.1624 & 1.314 & 7.48 \\
6-31G(d) only & 4 & 0.4111 & 0.2458 & 1.222 & 8.73 \\
6-311G only & 4 & 0.5140 & 0.3853 & 1.110 & 13.22 \\
6-311G* only & 4 & 0.6318 & 0.5053 & 0.9663 & 21.45 \\
cc-pVTZ only & 1 & 0.5099 & 0.4787 & 1.083 & 55.14 \\
TSI + TCI & 7 & 0.8791 & 0.8074 & 0.5710 & 48.83 \\
TSI + TCI + 3D & Same as previous model & & & \\
TSI + TCI + AM1 & 7 & 0.8840 & 0.8121 & 0.5594 & 51.17 \\
TSI + TCI + STO-3G & 7 & 0.8724 & 0.7923 & 0.5866 & 45.91 \\
TSI + TCI + 6-31G(d) & 7 & 0.8713 & 0.7926 & 0.5893 & 45.45 \\
TSI + TCI + 6-311G & 10 & 0.9015 & 0.8283 & 0.5328 & 40.26 \\
TSI + TCI + 6-311G(d) & 8 & 0.9056 & 0.8335 & 0.5100 & 55.17 \\
TSI + TCI + aug-cc-pVTZ & 8 & 0.9476 & 0.9236 & 0.3800 & 103.98 \\
\hline
\end{tabular}




\section{Appendix 1.3}

Manuscripts on tailored similarity (T-QMSA) 
Presented at the $10^{\text {th }}$ International Workshop on Quantitative Structure-Activity Relationships (QSARs) in Environmental Sciences (QSAR 2002), May 25-29, 2002, Ottawa, Canada

\title{
QUANTITATIVE MOLECULAR SIMILARITY ANALYSIS (QMSA) METHODS FOR PROPERTY ESTIMATION: A COMPARISON OF PROPERTY-BASED, ARBITRARY, AND TAILORED SIMILARITY SPACES
}

\author{
S. C. BASAK ${ }^{\dagger}$, B. D. GUTE and D. MILLS \\ Natural Resources Research Institute, University of Minnesota Duluth, \\ 5013 Miller Trunk Highway, Duluth, Minnesota 55811, USA
}

Three classes of arbitrary QMSA methods have been computed using atom pairs, topological indices, and physicochemical properties. Tailored QMSA models have been developed using selected number of TIs chosen by ridge regression. The methods have been applied to the $\mathrm{k}$ nearest neighbor (KNN) based estimation of $\log P$ of two sets of chemicals. Results show that the property-based and tailored QMSA methods are superior to the arbitrary similarity methods in estimating $\log P$ of both sets of chemicals

Keywords: Quantitative molecular similarity analysis (QMSA); Arbitrary QMSA method; Tailored QMSA method; Atom pairs; Topological indices; Physicochemical properties

\section{INTRODUCTION}

Quantitative molecular similarity analysis (QMSA) and quantitative structure-activity relationship (QSAR) models are important tools in modern drug design and the hazard assessment of chemicals [1-8]. QSARs attempt to develop relationships to estimate the physicochemical properties, biological activities, or toxicities of specific classes of chemicals from their experimentally-based properties or calculated molecular descriptors. The problem with the use of QSAR is that these models usually apply only to specific, narrowly defined structural classes of molecules. In many practical cases of hazard estimation an arbitrary candidate chemical cannot be unambiguously

\footnotetext{
${ }^{\dagger}$ Corresponding Author. e-mail: sbasak@nrri.umn.edu
} 
categorized into a particular class. For example, a molecule with five or ten different functional groups cannot be classified as belonging to any one particular structural class. QSARs based on experimental data, e.g., linear free / solvation energy related (LFER/ LSER) methods, suffer from the additional problem that such data are available for only a small fraction of candidate chemicals $[9,10]$.

When the applicability of QSAR is in doubt, the method of choice for property/activity/ toxicity estimation is molecular similarity. The tacit assumption underlying the use of molecular similarity is that "similar" molecules usually behave similarly in terms of physicochemical, biomedicinal, and toxicological processes. Similarity is also an intuitively understood concept so that given a set of chemicals one can pick the most similar pair of them based on one's own criteria of similarity. The problem with such intuitive criteria is that they are ill defined and can vary from time to time; for sets of very complex molecules they will be difficult to come up with. For the analysis of large databases such intuitive methods will either be inadequate or so slow as to be completely impractical.

Various molecular similarity methods have been formulated based on parameters computed directly from molecular structure. Quantum chemical indices [11], substructures, and topological indices $[1,4,5,12-30]$ have frequently been used for this purpose.

Most of the QMSA methods mentioned above can be considered arbitrary similarity methods because they are based on structure spaces, the elements of which are selected without having any necessary relationship with the property of interest. Designers of QMSA methods seem to fall into two major categories. Some have an intuitive notion of the structural attributes that will be useful for the property of interest, while others take a wide variety of structural attributes representing important and diverse aspects of molecular structure. In the latter case, because these arbitrary structural descriptors encompass such a broad range of important structural features, they are expected to be useful in estimating the property of interest through the selection of proper analogs.

To correct the arbitrariness in the QMSA methodology, we recently developed the idea of tailored similarity $[14,30]$. In this scheme we begin by selecting the elements of the structure space based on a specific property. The structure space thus created is then used for the selection of analogs and estimation of properties of chemicals from their analogs. It has been found that both for physicochemical and toxicological properties tailored similarity spaces outperformed arbitrary QMSA models.

As an expansion on a previously published study [29], in this paper we have compared three classes of QMSA methods: a) arbitrary QMSA methods based on calculated topological indices 
(TIs) and substructures called atom pairs (APs), b) arbitrary QMSA methods based on physicochemical properties, and c) tailored similarity methods based on calculated TIs. These method have been studied for two sets of chemicals: 1) a relatively homogeneous set of 166 hydrocarbons which are identified as constituents of the jet fuel JP-8 and 2) a structurally diverse subset of 76 chemicals.

\section{MATERIALS AND METHODS}

\section{Databases}

In this study, we are expanding on a study previously reported in this journal [29], using the same data sets as the original study. One is a set of 166 hydrocarbons, a subset of the constituents of JP-8 identified by GC/MS [31]. Of the 228 identified components, this subset represents the total group for which boiling point, vaporpressure, heat of vaporization, water solubility, adsorption coefficient, and $\log \mathrm{P}$ were available from the USEPA ASTER (ASsessment Tools for the Evaluation of Risk) system [32]. However, most of the data values available from ASTER were calculated, not experimental values.

The second set is a group of seventy-six diverse compounds that has been used in several earlier studies [17, 28, 29], we will refer to this set as the TSCA set hence forth. This set represents the intersection of a set of about 10,000 chemicals from the TSCA (Toxic Substances Control Act) Inventory [9], physicochemmical property data available from ASTER [32], and a database of solvatochromic parameters provided through personal communication with M. Kamlet

The chemical names and physicochemical property data for both sets has been reported previously in this journal [29] and thus will be omitted from the current report.

\section{Calculation of Molecular Descriptors}

The molecular descriptors used in this study were calculated using POLLY v.2.3 [33] and Molconn$\mathrm{Z}$ v.3.50 [34]. These descriptors include Wiener number [35], the molecular connectivity $(\chi)$ indices as calculated by Randić [36, 37] and Kier and Hall [38], information theoretic indices defined on distance matrices of graphs using the methods of Bonchev and Trinajstić [39] as well as those of Raychaudhury et al.[40], parameters defined on the neighborhood complexity of vertices in hydrogen-filled molecular graphs [41-43], Balaban's $J$ indices [44-46], the local orthogonal vertex 
invariants (LOVIs) [47], kappa shape descriptors [48, 49], and the electrotopological indices of Kier and Hall [50]. More information on the set of molecular descriptors calculated by POLLY has been reported in earlier studies [25, 51,52]. The two models using atom pairs were developed in our earlier work [29]. More details of the atom pair methodology can be found in that report and others $[4,5,51]$.

\section{STATISTICAL METHODS AND COMPUTATION OF INTERMOLECULAR SIMILARITY}

\section{Data Reduction}

Initially, all TIs were transformed by the natural logarithm of the index plus a constant. For all indices with minimum values greater than negative one, the constant used was one. In all other indices, the constant was determined on a case-by-case basis and was just large enough to make the minimum greater than zero. This rescaling was done since there may be several orders of magnitude difference between some of the molecular descriptors. After this step is completed, we begin data reduction. At this stage any descriptors with zero values for all compounds in the data set, or that are completely correlated with another descriptor, are removed from the descriptor set.

The primary purpose of this study was to compare several methods for selecting descriptors. To this end we have used both the original, limited set of topological indices from the first study and in the current study we have expanded the set to include the LOVI indices and molecular descriptors calculated using Molconn-Z. POLLY v2.3 alone calculates 102 molecular descriptors, far more descriptors than the number of dimensions we want in a similarity space. With the addition of the LOVI and Molconn-Z descriptor sets, our initial set of indices begins with 362 descriptors. Thus, some method is needed to reduce the number of descriptors available for modeling. In this study we make use of two such methods. The arbitrary similarity spaces make use of principal component analysis (PCA) for purposes of data reduction and selection of a small set of topological indices. The tailored similarity approach uses the ridge regression (RR) technique to select an "optimal" set of descriptors. Both of these approaches and their application to descriptor selection are described below.

Principal component analysis (PCA), conducted using the SAS PRINCOMP procedure [53], takes a large set of non-orthogonal variables (descriptors) and transforms them into a like number of completely orthogonal principal components. Each principal component is a linear combination of 
the complete set of initial variables. In this manner, we create a set of orthogonal descriptors for use a dimensions within a similarity space. Generally, only a small subset of the PCs is retained for modeling. Eigenvalues are reported for each of the PCs. The first PC explains the greatest amount of the variance in the data and has the largest eigenvalue. Each subsequent PC explains a smaller portion of the remaining data variance and has a likewise reduced eigenvalue. Routinely those PCs with eigenvalues greater than or equal to one are retained, leaving us with a small number of completely orthogonal descriptors that explain the bulk of the variance in our original descriptor set.

This technique can also be used for the selection of a small set of topological indices. In this approach, after construction of the set of PCs, a correlation analysis is used to determine which descriptors are most correlated with each of the principal components. In this manner, one or more descriptors are chosen for each PC with an eigenvalue greater than or equal to one. Reducing our set of hundreds of descriptors to a more manageable set of ten or fifteen. Both of these techniques were used in this study. For a more detailed explanation of this approach, please see a previous study by Basak et al [1].

As was mentioned previously, the tailored similarity method makes use of ridge regression (RR) for descriptor selection. RR is a method wherein modeling is conducted using the entire set of descriptors retained after initial data reduction as opposed to subset or principal component regression. This regression method is useful in cases where the descriptors are highly multicollinear and where the number of descriptors is substantially larger than the number of observations [54]. Conceptually, RR can be thought of as recasting the regression as one using the principal components of the predictor variables as new predictors. It differs in that in principal component regression the leading components are retained and used just as in ordinary least squares regression while the trailing components are dropped. RR retains all components, but weights each of them in accordance with the component's eigenvalue and the 'ridging constant' $k$. More details on the RR method can be found in some of our other papers $[14,55,56]$.

One of the by-products of the $R R$ is a ranking of the contribution of the descriptors. The absolute values of this ranking score were used to select the descriptors for development of the tailored similarity spaces. Separate RR studies were conducted for both the set of POLLY indices alone and the combined full set of descriptors for both data sets. 


\section{Similarity Measures}

Intermolecular similarity was measured using three methods. The first method used Euclidean distance $(E D)$ within an $n$-dimensional space to measure intermolecular similarity. $E D$ between molecules $i$ and $j$ is defined as:

$$
E D_{i j}=\left[\sum_{k=1}^{n}\left(D_{i k}-D_{j k}\right)^{2}\right]^{1 / 2}
$$

where $n$ equals the number of dimensions (descriptors) used. $\mathrm{D}_{i k}$ and $\mathrm{D}_{j k}$ are the data values of the $k^{\text {th }}$ dimension for molecules $i$ and $j$, respectively. This technique for measuring intermolecular similarity was used to develop similarity spaces from the PCs, the sets of descriptors selected from the PCs, and the sets. of descriptors selected using RR. The same technique was used for the development of the physicochemical property spaces as has been described previously [29]. The similarity spaces derived from atom pairs uses an associative measure described also described in detail in the previous study.

\section{Analog / K-Nearest Neighbor Selection}

Following the quantification of intermolecular similarity of the molecules, analogs or nearest neighbors are determined on the basis of $E D$ or the similarity score $(S)$ described previously. The $E D$ method measures the distance between molecules in the space, thus the lower the value of $E D$ the greater the similarity between two molecules. In the case of the AP method, two molecules are considered identical if $S=1$, while they have no atom pairs in common if $S=0$.

\section{Property Estimation}

Property estimation was carried out using the $K$-nearest neighbor (KNN) method. For each probe (or query) compound a number $(\mathrm{K})$ of similar chemicals, ranging from 1 to 25 , are selected and the property of interest is estimated based on the values of these nearest neighbors. In estimating the $\log P$ of the probe compound, the mean of the $\log P$ of the $K$-nearest neighbors was used as the estimate. 
TABLE I Principal component analysis results for POLLY TIs and the expanded set of descriptors for the $166 \mathrm{JP}-8$ constituents

\begin{tabular}{lccccc}
\hline \multicolumn{5}{c}{ POLLY Topological Indices only } \\
\hline PC & Eigenvalue & $\begin{array}{c}\text { \% data var. } \\
\text { explained }\end{array}$ & $\begin{array}{c}\text { Cumulative \% } \\
\text { var. explained }\end{array}$ & Most highly correlated index \\
\hline 1 & 31.4795 & 0.342 & 0.342 & $\mathrm{P}_{1}$ & 0.9433 \\
2 & 19.3298 & 0.210 & 0.552 & ${ }^{5} \chi_{\mathrm{PC}}^{\mathrm{b}}$ & 0.8311 \\
3 & 11.9527 & 0.130 & 0.682 & $\mathrm{ClC}_{0}$ & 0.8942 \\
4 & 8.5428 & 0.093 & 0.775 & $\mathrm{I}_{\mathrm{ORB}}$ & 0.6179 \\
5 & 6.2243 & 0.068 & 0.843 & ${ }^{6} \chi_{\mathrm{Ch}}^{\mathrm{b}}$ & 0.7149 \\
6 & 3.8318 & 0.042 & 0.884 & ${ }^{5} \chi_{\mathrm{C}}^{\mathrm{v}}$ & 0.5831 \\
7 & 2.4620 & 0.027 & 0.911 & ${ }^{3} \chi_{\mathrm{Ch}}$ & 0.4797 \\
8 & 1.7081 & 0.019 & 0.930 & $\dot{\mathrm{P}}_{9}$ & 0.4306 \\
9 & 1.2922 & 0.014 & 0.944 & $\mathrm{P}_{10}$ & 0.3508 \\
10 & 1.0024 & 0.011 & 0.955 & $\mathrm{O}_{\mathrm{ORB}}$ & 0.4648 \\
\hline
\end{tabular}

\begin{tabular}{lccccc}
\hline \multicolumn{5}{c}{ Expanded set of Topological Indices } \\
\hline$P C$ & Eigenvalue & $\begin{array}{c}\text { \% data var. } \\
\text { explained }\end{array}$ & $\begin{array}{c}\text { Cumulative \% } \\
\text { var. explained }\end{array}$ & Most highly correlated index \\
\hline 1 & 93.3837 & 0.421 & 0.421 & $\mathrm{DN}^{2} \mathrm{~N}_{4}$ & 0.9935 \\
2 & 45.8446 & 0.207 & 0.627 & $\mathrm{Phia}^{2}$ & -0.9796 \\
3 & 26.2411 & 0.118 & 0.745 & ${ }^{2} \chi^{\mathrm{b}}$ & 0.8424 \\
4 & 12.7416 & 0.057 & 0.803 & $\mathrm{IC}_{3}$ & 0.7474 \\
5 & 9.3209 & 0.042 & 0.845 & $\mathrm{~J}^{\mathrm{B}}$ & -0.6415 \\
6 & 6.3087 & 0.028 & 0.873 & ${ }^{10} \chi$ & 0.5502 \\
7 & 4.7517 & 0.021 & 0.895 & $\mathrm{SdsCH}$ & 0.5365 \\
8 & 3.9706 & 0.018 & 0.912 & ${ }^{5} \chi^{\mathrm{b}}$ & 0.4954 \\
9 & 2.6602 & 0.012 & 0.924 & ${ }^{3} \chi_{\mathrm{Ch}}$ & -0.5075 \\
10 & 2.3647 & 0.011 & 0.935 & ${ }^{9} \chi_{\mathrm{Ch}}^{\mathrm{v}}$ & -0.6410 \\
11 & 1.9136 & 0.009 & 0.944 & ${ }^{3} \chi_{\mathrm{Ch}}$ & 0.3557 \\
12 & 1.6762 & 0.008 & 0.951 & $\mathrm{SHvin}$ & -0.3997 \\
13 & 1.3773 & 0.006 & 0.958 & 0 & 0.3681 \\
14 & 1.1548 & 0.005 & 0.963 & ${ }^{10} \chi^{\mathrm{v}}$ & 0.4065 \\
15 & 1.0759 & 0.005 & 0.968 & ${ }^{6} \chi^{\mathrm{b}}$ & 0.3111 \\
\hline
\end{tabular}




\section{RESULTS}

\section{Development of QMSA Models for JP-8}

Initial data reduction on the set of POLLY TIs, resulted in the removal of ten TIs, leaving a set of ninety-two descriptors for the JP-8 set. These ninety-two TIs were then subjected to PCA and ten PCs were retained with eigenvalues greater than or equal to one. These PCs (and the index most highly correlated with each PC) are summarized in Table 1. This PCA and the correlation analysis of the PCs with the set of ninety-two TIs gave us two of the three descriptor sets using only the POLLY indices to be used in this study.

The tailored similarity model using only the ninety-two POLLY descriptors was developed using RR. The entire set of descriptors was run through RR and since we had ten PCs, we selected the ten descriptors that contributed the most to the RR based on their $t$ values. The $t$-values, indicated in Table 2, are model coefficients extracted from the RR procedure and used to rank the TIs from most to least influential based on the absolute value of the regression coefficient.

TABLE II Topological indices used in creating similarity spaces for the 166 JP-8 chemicals

\begin{tabular}{|c|c|c|c|c|}
\hline \multirow{2}{*}{$\begin{array}{l}T I \\
1\end{array}$} & \multicolumn{2}{|c|}{$\begin{array}{c}\text { TIs selected from Polly } R R \\
\text { (t-values) }\end{array}$} & \multicolumn{2}{|c|}{$\begin{array}{c}\text { TIs selected from Expanded RR } \\
(t \text {-values })\end{array}$} \\
\hline & ${ }^{0} \chi^{b}$ & $(9.23)$ & ${ }^{0} x^{b}$ & $(16.47)$ \\
\hline 2 & ${ }^{0} \chi^{\nu}$ & $(9.05)$ & ${ }^{0} \chi^{\mathrm{v}}$ & $(16.42)$ \\
\hline 3 & $P_{1}$ & $(-4.17)$ & Fw & $(14.77)$ \\
\hline 4 & $\mathrm{I}^{\mathrm{D}}$ & $(-4.07)$ & $\mathrm{AZS}_{1}$ & $(14.22)$ \\
\hline 5 & $\mathrm{SIC}_{0}$ & $(3.55)$ & W & $(14.03)$ \\
\hline 6 & $\mathrm{~J}^{\mathrm{B}}$ & (3.53) & $\mathrm{ANS}_{3}$ & $(14.00)$ \\
\hline 7 & ${ }^{0} x$ & (3.24) & $\mathrm{AZS}_{3}$ & $(13.31)$ \\
\hline 8 & $\mathbf{J}$ & $(-3.15)$ & $\mathrm{ANS}_{1}$ & $(12.19)$ \\
\hline 9 & ${ }^{3} \chi^{b}$ & $(3.10)$ & ${ }^{0} x$ & (11.71) \\
\hline 10 & $\mathrm{I}_{\mathrm{D}}^{\mathrm{W}}$ & $(-3.00)$ & $\mathrm{Ka}_{1}$ & $(11.42)$ \\
\hline 11 & & & $\mathrm{I}_{\mathrm{D}}{ }^{\mathrm{W}}$ & (11.31) \\
\hline 12 & & & $\mathrm{ANN}_{3}$ & (11.31) \\
\hline 13 & & & $\mathrm{DN}^{2} \mathrm{~S}_{3}$ & $(11.29)$ \\
\hline 14 & & & $\mathrm{ANN}_{5}$ & (11.14) \\
\hline 15 & & & $\mathrm{Q}_{\mathrm{v}}$ & $(11.08)$ \\
\hline
\end{tabular}


Data reduction on the expanded set of 369 descriptors resulted in the removal of 147 descriptors, leaving a set of 222. PCA on this set resulted in the retention of fifteen PCs and the one TI most correlated with each of the PCs (see Table 1). Following our protocol, a set of the fifteen descriptors with the highest $t$-values were selected by RR (Table 2). Additionally, to examine the importance of the size of the descriptor set versus the utility of the tailoring method, we decided to use the top ten TIs as determined by RR to see how this space would differ from the space using fifteen descriptors. Details of the atom pair and physicochemical modeling were already presented in the earlier study.

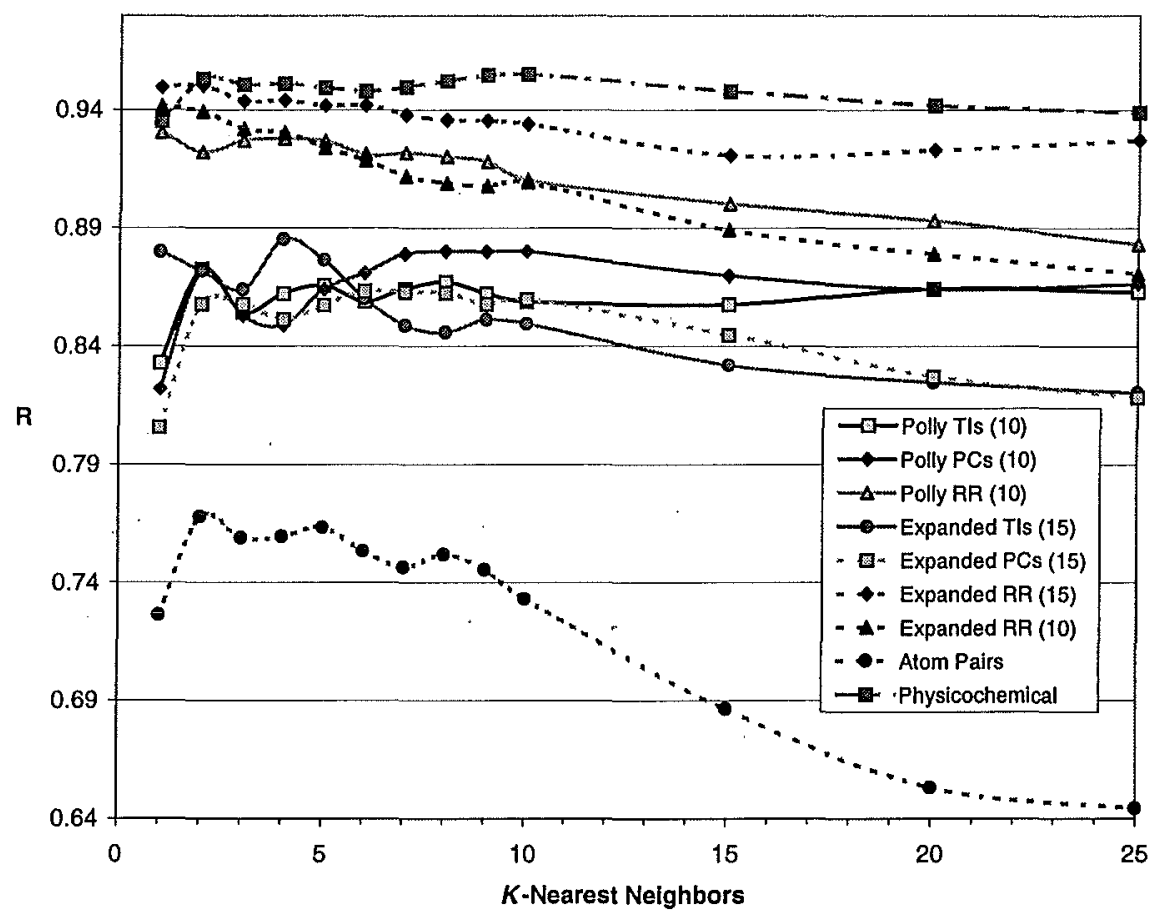

FIGURE 1 Plot of KNN estimation of $\log P$ for the JP-8 constituents demonstrating the variation in correlation between the nine methods over values of $\mathrm{K}$ ranging from 1 to 25 .

\section{K-Nearest Neighbor Property Estimation for JP-8}

Each similarity space developed above was used in K-nearest neighbor based property estimation for $\mathrm{K}=1-10,15,20$, and 25 . Correlation analyses were conducted on the property estimates to determine the strength of each of the similarity spaces in estimating $\log P$. Figure 1 presents a plot of the correlation coefficients ( $\mathrm{r}$ ) as they vary with $\mathrm{K}$ for all nine models. Correlation coefficients and standard errors for the POLLY-based spaces are presented in Table 3 and results for the expanded descriptor set models are summarized in Table 4. 
TABLE III K-Nearest Neighbor results for estimation of JP-8 $\log$ P for the models using only POLLY indices

\begin{tabular}{lcccccc} 
& \multicolumn{2}{c}{ PC Method } & \multicolumn{2}{c}{ TI method } & \multicolumn{2}{c}{ RR method } \\
\cline { 2 - 7 }$K$ & $r$ & se & $r$ & se & $r$ & se \\
\hline 1 & 0.8219 & 0.442 & 0.8330 & 0.425 & 0.9305 & 0.278 \\
2 & 0.8718 & 0.374 & 0.8726 & 0.372 & 0.9220 & 0.296 \\
3 & 0.8527 & 0.401 & 0.8542 & 0.401 & 0.9270 & 0.291 \\
4 & 0.8488 & 0.408 & 0.8622 & 0.399 & 0.9279 & 0.294 \\
5 & 0.8639 & 0.393 & 0.8655 & 0.400 & 0.9269 & 0.298 \\
6 & 0.8708 & 0.388 & 0.8588 & 0.409 & 0.9213 & 0.310 \\
7 & 0.8789 & 0.383 & 0.8641 & 0.409 & 0.9219 & 0.314 \\
8 & 0.8801 & 0.384 & 0.8671 & 0.411 & 0.9203 & 0.321 \\
9 & 0.8799 & 0.389 & 0.8622 & 0.421 & 0.9180 & 0.328 \\
10 & 0.8800 & 0.395 & 0.8587 & 0.429 & 0.9104 & 0.343 \\
15 & 0.8697 & 0.427 & 0.8576 & 0.464 & 0.9001 & 0.369 \\
20 & 0.8639 & 0.447 & 0.8642 & 0.486 & 0.8929 & 0.388 \\
25 & 0.8662 & 0.456 & 0.8629 & 0.508 & 0.8831 & 0.412 \\
\hline
\end{tabular}

TABLE IV $K$-Nearest Neighbor results for estimation of JP-8 $\operatorname{logP}$ for the models using the expanded set of indices

\begin{tabular}{lcccccc}
\hline & \multicolumn{2}{c}{ PC Method } & \multicolumn{2}{c}{ TI method } & \multicolumn{2}{c}{ RR method } \\
\cline { 2 - 7 }$K$ & $r$ & se & $r$ & se & $r$ & se \\
\hline 1 & 0.8058 & 0.459 & 0.8798 & 0.361 & 0.9497 & 0.238 \\
2 & 0.8578 & 0.393 & 0.8717 & 0.376 & 0.9504 & 0.239 \\
3 & 0.8575 & 0.399 & 0.8638 & 0.392 & 0.9438 & 0.255 \\
4 & 0.8513 & 0.409 & 0.8850 & 0.371 & 0.9441 & 0.260 \\
5 & 0.8573 & 0.403 & 0.8762 & 0.383 & 0.9419 & 0.268 \\
6 & 0.8632 & 0.401 & 0.8600 & 0.403 & 0.9420 & 0.271 \\
7 & 0.8628 & 0.404 & 0.8487 & 0.419 & 0.9379 & 0.278 \\
8 & 0.8625 & 0.408 & 0.8458 & 0.422 & 0.9358 & 0.284 \\
9 & 0.8578 & 0.413 & 0.8511 & 0.419 & 0.9356 & 0.285 \\
10 & 0.8598 & 0.413 & 0.8494 & 0.423 & 0.9340 & 0.290 \\
15 & 0.8448 & 0.436 & 0.8320 & 0.447 & 0.9208 & 0.325 \\
20 & 0.8272 & 0.461 & 0.8247 & 0.463 & 0.9230 & 0.341 \\
25 & 0.8184 & 0.479 & 0.8204 & 0.478 & 0.9272 & 0.360 \\
\hline
\end{tabular}


TABLE V Topological indices used in creating similarity spaces for the 76 industrial chemicals

\begin{tabular}{|c|c|c|c|c|c|}
\hline \multicolumn{6}{|c|}{ POLLY Topological Indices only } \\
\hline$P C$ & Eigenvalue & $\begin{array}{l}\text { \% data var. } \\
\text { explained }\end{array}$ & $\begin{array}{l}\text { Cumulative \% } \\
\text { var. explained }\end{array}$ & \multicolumn{2}{|c|}{ Most highly correlated index } \\
\hline 1 & 41.7583 & 0.469 & 0.469 & ${ }^{1} \chi$ & 0.9759 \\
\hline 2 & 14.0440 & 0.158 & 0.627 & $\mathrm{SIC}_{3}$ & 0.9673 \\
\hline 3 & 9.0400 & 0.102 & 0.729 & $\mathrm{CIC}_{1}$ & -0.7444 \\
\hline 4 & 6.2892 & 0.071 & 0.799 & $\mathrm{~J}$ & 0.8637 \\
\hline 5 & 3.8182 & 0.043 & 0.842 & ${ }^{4} \chi_{\mathrm{c}}$ & 0.7100 \\
\hline 6 & 2.9551 & 0.033 & 0.875 & $\mathbf{P}_{8}$ & 0.3656 \\
\hline \multicolumn{6}{|c|}{ Expanded set of Topological Indices } \\
\hline$P C$ & Eigenvalue & $\begin{array}{l}\% \text { data var. } \\
\text { explained }\end{array}$ & $\begin{array}{l}\text { Cumulative \% } \\
\text { var. explained }\end{array}$ & \multicolumn{2}{|c|}{ Most highly correlated index } \\
\hline 1 & 115.7516 & 0.469 & 0.469 & $\mathrm{DN}^{2} \mathrm{Z}_{4}$ & 0.9937 \\
\hline 2. & 30.3616 & 0.123 & 0.592 & $\mathrm{ASN}_{2}$ & 0.9080 \\
\hline 3 & 17.9402 & 0.073 & 0.664 & $\mathrm{~J}$ & 0.7789 \\
\hline 4 & 16.0951 & 0.065 & 0.729 & $\mathrm{SIC}_{1}$ & 0.8642 \\
\hline 5 & 9.9801 & 0.040 & 0.770 & ${ }^{8} \chi^{v}$ & 0.6077 \\
\hline 6 & 7.5558 & 0.031 & 0.800 & ${ }^{4} \chi_{c}$ & 0.6248 \\
\hline 7 & 5.4580 & 0.022 & 0.822 & $\mathrm{SdO}$ & -0.6203 \\
\hline 8 & 4.7299 & 0.019 & 0.842 & $\mathrm{SsCH}_{3}$ & 0.6015 \\
\hline 9 & 3.8975 & 0.016 & 0.857 & ${ }^{5} \chi_{\mathrm{Ch}}$ & 0.3578 \\
\hline 10 & 3.8378 & 0.016 & 0.873 & ${ }^{5} \chi^{\mathrm{b}}$ & 0.6295 \\
\hline 11 & 3.2325 & 0.013 & 0.886 & $\mathrm{H}_{\text {MAX }}$ & -0.3854 \\
\hline 12 & 2.6013 & 0.011 & 0.897 & $\mathrm{SHsNH}_{2}$ & 0.7109 \\
\hline 13 & 2.4524 & 0.010 & 0.907 & SHCsatu & -0.5985 \\
\hline 14 & 2.3494 & 0.010 & 0.916 & $\mathrm{SdssC}$ & -0.6674 \\
\hline 15 & 1.9808 & 0.008 & 0.924 & SsssN & 0.3948 \\
\hline 16 & 1.7841 & 0.007 & 0.931 & SsssCH & -0.4659 \\
\hline 17 & 1.7385 & 0.007 & 0.938 & StsC & 0.3934 \\
\hline 18 & 1.6391 & 0.007 & 0.945 & $\mathrm{SsBr}$ & 0.6731 \\
\hline 19 & 1.4685 & 0.006 & 0.951 & ${ }^{10} \chi_{\mathrm{Ch}}$ & -0.3286 \\
\hline 20 & 1.3449 & 0.005 & 0.956 & SHssNH & -0.5603 \\
\hline 21 & 1.2352 & 0.005 & 0.961 & SaaN & 0.6947 \\
\hline 22 & 1.1406 & 0.005 & 0.966 & SddsN & 0.4028 \\
\hline
\end{tabular}


TABLE VI Topological indices used in creating similarity spaces for the 76 industrial chemicals

\begin{tabular}{|c|c|c|c|c|}
\hline \multirow{2}{*}{$\frac{T I}{1}$} & \multicolumn{2}{|c|}{$\begin{array}{c}\text { TIs selected from Polly RR } \\
(t \text {-values })\end{array}$} & \multicolumn{2}{|c|}{$\begin{array}{l}\text { Tls selected from Expanded } R R \\
\text { (t-values })\end{array}$} \\
\hline & ${ }^{0} \chi^{v}$ & $(8.82)$ & $\mathrm{FW}_{\mathrm{W}}$ & $(13.92)$ \\
\hline 2 & ${ }^{1} \chi^{v}$ & $(6.73)$ & $\mathrm{AZV}_{4}$ & (13.49) \\
\hline 3 & ${ }^{2} \chi^{v}$ & $(6.51)$ & ${ }^{0} \chi^{v}$ & (12.52) \\
\hline 4 & ${ }^{3} \chi^{v}$ & $(5.76)$ & $\mathrm{ANZ}_{1}$ & $(12.12)$ \\
\hline 5 & ${ }^{3} \chi^{v}{ }_{C}$ & $(5.37)$ & $\mathrm{Ka}_{1}$ & (11.04) \\
\hline 6 & $\mathrm{IC}_{1}$ & $(-5.15)$ & $\mathrm{DN}^{2} 1_{4}$ & $(10.33)$ \\
\hline 7 & & & W & $(10.22)$ \\
\hline 8 & & & $\mathrm{DN}^{2} \mathrm{~N}_{4}$ & (9.89) \\
\hline 9 & & & $\mathrm{G}_{\max }$ & $(-9.70)$ \\
\hline 10 & & & $\mathrm{SsCl}$ & $(9.47)$ \\
\hline 11 & & & $\mathrm{ANS}_{1}$ & $(9.20)$ \\
\hline 12 & & & $\mathrm{AS1}_{4}$ & (9.14) \\
\hline 13 & & & $\mathrm{DN}^{2} \mathrm{Z}_{3}$ & (9.14) \\
\hline 14 & & & $\mathrm{DN}^{2} \mathrm{~S}_{3}$ & (8.98) \\
\hline 15 & & & $\mathrm{ASl}_{1}$ & $(-8.71)$ \\
\hline 16 & & & $\mathrm{P}_{0}$ & $(8.51)$ \\
\hline 17 & & & $\mathrm{ANN}_{3}$ & $(8.42)$ \\
\hline 18 & & & $\mathrm{AN}_{3}$ & (8.39) \\
\hline 19 & & & $\mathrm{DN}^{2} \mathrm{~S}_{1}$ & $(8.29)$ \\
\hline 20 & & & $\mathrm{AS}_{1}$ & $(-8.27)$ \\
\hline 21 & & & $\mathrm{DN}^{2} \mathrm{~S}_{5}$ & $(8.08)$ \\
\hline 22 & & & $P_{1}$ & $(8.07)$ \\
\hline
\end{tabular}

\section{Development of QMSA Models for the TSCA Chemicals}

Initial data reduction on the set of POLLY TIs, resulted in the removal of nine TIs, leaving a set of ninety-three descriptors for the TSCA set. PCA on these ninety-three TIs left us with six PCs. These PCs (and the index most highly correlated with each PC) are summarized in Table 5. The six most influential indices from $R R$ were also selected (Table 6).

Data reduction on the expanded set of 369 descriptors resulted in the removal of 122 descriptors, leaving a set of 247. In part, this demonstrates the greater diversity of this set vis-à-vis the JP-8 set. Many of the additional descriptors retained in this analysis were atom-type electrotopological indices that can be viewed as being similar to structural indicator variables. Since many of these structural features were not present in the JP-8 set, the descriptors were discarded. 
PCA on this set resulted in the retention of twenty-three PCs and the one TI most correlated with each of the PCs (see Table 5). Unfortunately, the twenty-third PC could not be meaningfully characterized as distinct from the other twenty-two through correlation with the TIs (the first five most correlated TIs were all the first most correlated with one of the other PCs) so it was dropped and we used only the first twenty-two. Following our protocol, a set of the twenty-two descriptors with the highest $t$-values were selected by RR. These indices and their $t$-values are presented in Table 6. Additionally, to examine the importance of the size of the descriptor set versus the utility of the tailoring method, we used the top six TIs as determined by RR to see how this space would differ from the space using twenty-two descriptors. Again, details of the atom pair and physicochemical modeling were already presented in the earlier study.

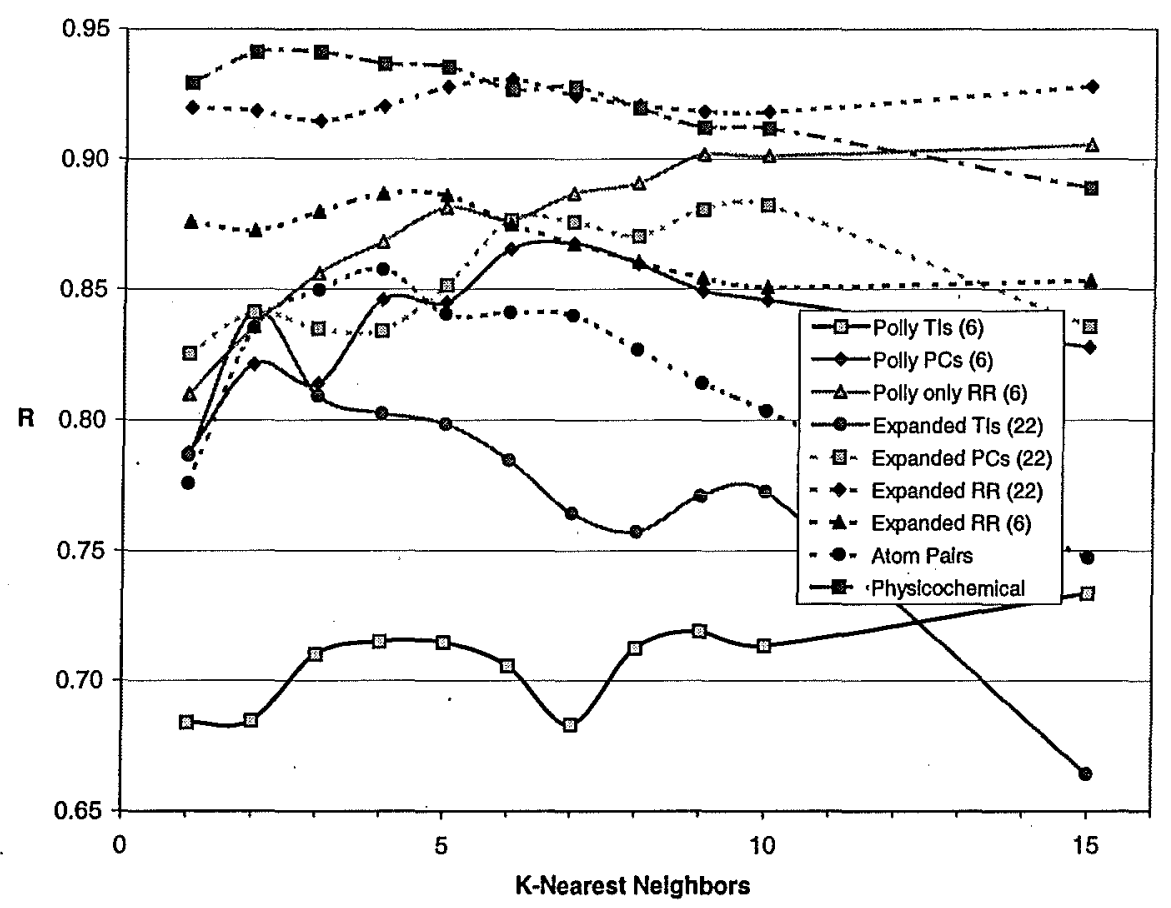

FIGURE 2 Plot of KNN estimation of $\log P$ for the TSCA chemicals demonstrating the variation in correlation between the nine methods over values of $\mathrm{K}$ ranging from 1 to 15 .

\section{K-Nearest Neighbor Property Estimation for the TSCA Set}

Each similarity space developed above was used in K-nearest neighbor based property estimation for $K=1-10$, and 15 . Due to the smaller size of the set, it was not deemed useful to examine $K$ nearest neighbors greater than fifteen. Correlation analyses were conducted on the property estimates to determine the strength of each of the similarity spaces in estimating $\log P$. Figure 2 presents a plot of the correlation coefficients ( $r$ ) as they vary with $\mathrm{K}$ for all nine models. Results for 
the POLLY only models are summarized in Table 7 and results for the expanded descriptor set models are summarized in Table 8.

TABLE VII $K$-Nearest Neighbor results for estimation of TSCA $\log P$ for the models using only POLLY indices

\begin{tabular}{lcccccc}
\hline & \multicolumn{2}{c}{ PC Method } & \multicolumn{2}{c}{ Tl method } & \multicolumn{2}{c}{ RR method } \\
\cline { 2 - 7 }$K$ & $r$ & se & $r$ & se & $r$ & se \\
\hline 1 & 0.7877 & 0.887 & 0.6840 & 1.066 & 0.8100 & 0.828 \\
2 & 0.8213 & 0.790 & 0.6846 & 1.012 & 0.8358 & 0.754 \\
3 & 0.8139 & 0.804 & 0.7102 & 0.962 & 0.8561 & 0.704 \\
4 & 0.8460 & 0.747 & 0.7151 & 0.949 & 0.8682 & 0.675 \\
5 & 0.8451 & 0.757 & 0.7148 & 0.948 & 0.8813 & 0.641 \\
6 & 0.8653 & 0.734 & 0.7056 & 0.961 & 0.8765 & 0.653 \\
7 & 0.8675 & 0.746 & 0.6832 & 0.990 & 0.8868 & 0.627 \\
8 & 0.8596 & 0.775 & 0.7125 & 0.953 & 0.8907 & 0.617 \\
9 & 0.8493 & 0.822 & 0.7191 & 0.944 & 0.9019 & 0.592 \\
10 & 0.8458 & 0.840 & 0.7135 & 0.954 & 0.9012 & 0.595 \\
15 & 0.8278 & 0.946 & 0.7336 & 0.939 & 0.9055 & 0.603 \\
\hline
\end{tabular}

TABLE VIII $K$-Nearest Neighbor results for estimation of TSCA $\log P$ for the models using the expanded set of indices

\begin{tabular}{lcccccc}
\hline & \multicolumn{2}{c}{ PCMethod } & \multicolumn{2}{c}{ TI method } & \multicolumn{2}{c}{ RR method } \\
\cline { 2 - 7 }$K$ & $r$ & se & $r$ & se & $r$ & se \\
\hline 1 & 0.8254 & 0.789 & 0.7865 & 0.846 & 0.9198 & 0.543 \\
2 & 0.8411 & 0.737 & 0.8413 & 0.736 & 0.9185 & 0.536 \\
3 & 0.8349 & 0.746 & 0.8091 & 0.803 & 0.9147 & 0.548 \\
4 & 0.8342 & 0.749 & 0.8025 & 0.820 & 0.9203 & 0.530 \\
5 & 0.8515 & 0.720 & 0.7985 & 0.824 & 0.9279 & 0.510 \\
6 & 0.8765 & 0.687 & 0.7847 & 0.846 & 0.9307 & 0.509 \\
7 & 0.8758 & 0.701 & 0.7644 & 0.879 & 0.9243 & 0.540 \\
8 & 0.8704 & 0.732 & 0.7573 & 0.887 & 0.9206 & 0.564 \\
9 & 0.8807 & 0.740 & 0.7711 & 0.867 & 0.9184 & 0.579 \\
10 & 0.8824 & 0.768 & 0.7727 & 0.877 & 0.9181 & 0.590 \\
15 & 0.8359 & 0.953 & 0.6641 & 1.053 & 0.9279 & 0.605 \\
\hline
\end{tabular}




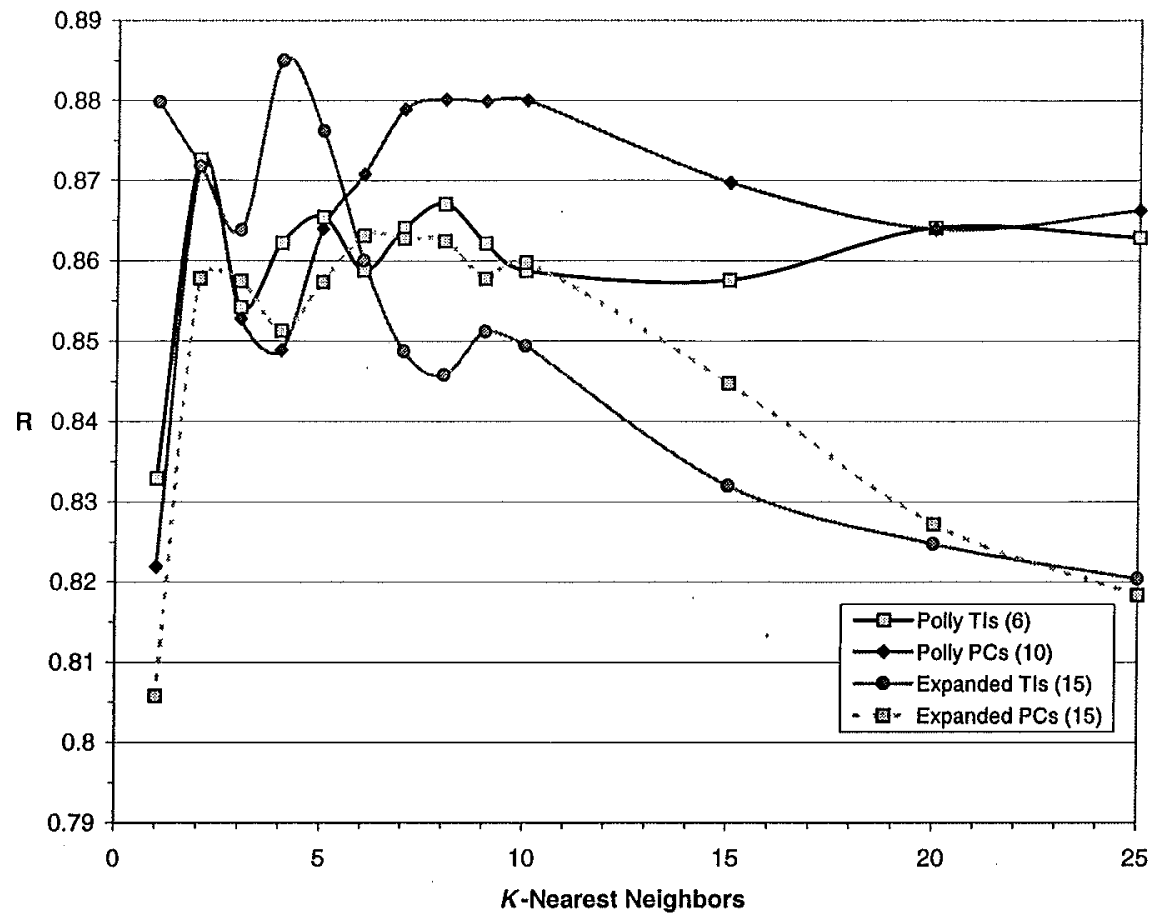

FIGURE 3 A closer examination of the overlap in the arbitrary similarity spaces for estimation of $\log P$ for JP-8 in terms of correlation coefficients.

\section{DISCUSSION}

The objective of this study was to evaluate the relative effectiveness of tailored versus arbitrary similarity methods in the estimation of properties of chemicals from their selected neighbors.

For the set of $166 \mathrm{JP}-8$ chemicals, we selected $\mathrm{K}(\mathrm{K}=1-25)$ nearest neighbors by nine different similarity methods used in this paper (Figure 1 and Tables 3 and 4) and used the K-nearest neighbor $(\mathrm{KNN})$ approach to estimate $\log P$. While the method based on atom pairs used the largest number of independent variables, that based on properties has the least number of such variables. Yet it is interesting to note that the AP method gives the weakest estimation of $\log P$ while the property method uses only five independent variables and gives the best estimates. This is probably because of the fact that many atom pair descriptors might not have any significant relationship with the property and their inclusion in the estimation method increases noise instead of information. On the other hand, one of the properties, viz., water solubility, is known to have strong relationship with hydrophobicity [57] while others may be indirectly related. When PCs are used to create the structure spaces, PCs derived from POLLY are lower in number (10) as compared to those derived 
from the expanded set of descriptors (15). Yet Figures 1 and 3 show that the $\mathrm{KNN} \log P$ estimations based on these two structure spaces are almost identical. This shows that the addition of more independent variables in the model does not necessarily increase the quality of the model. The same is true of the models developed from ten TIs selected from the POLLY indices versus the fifteen chosen from the expanded set.

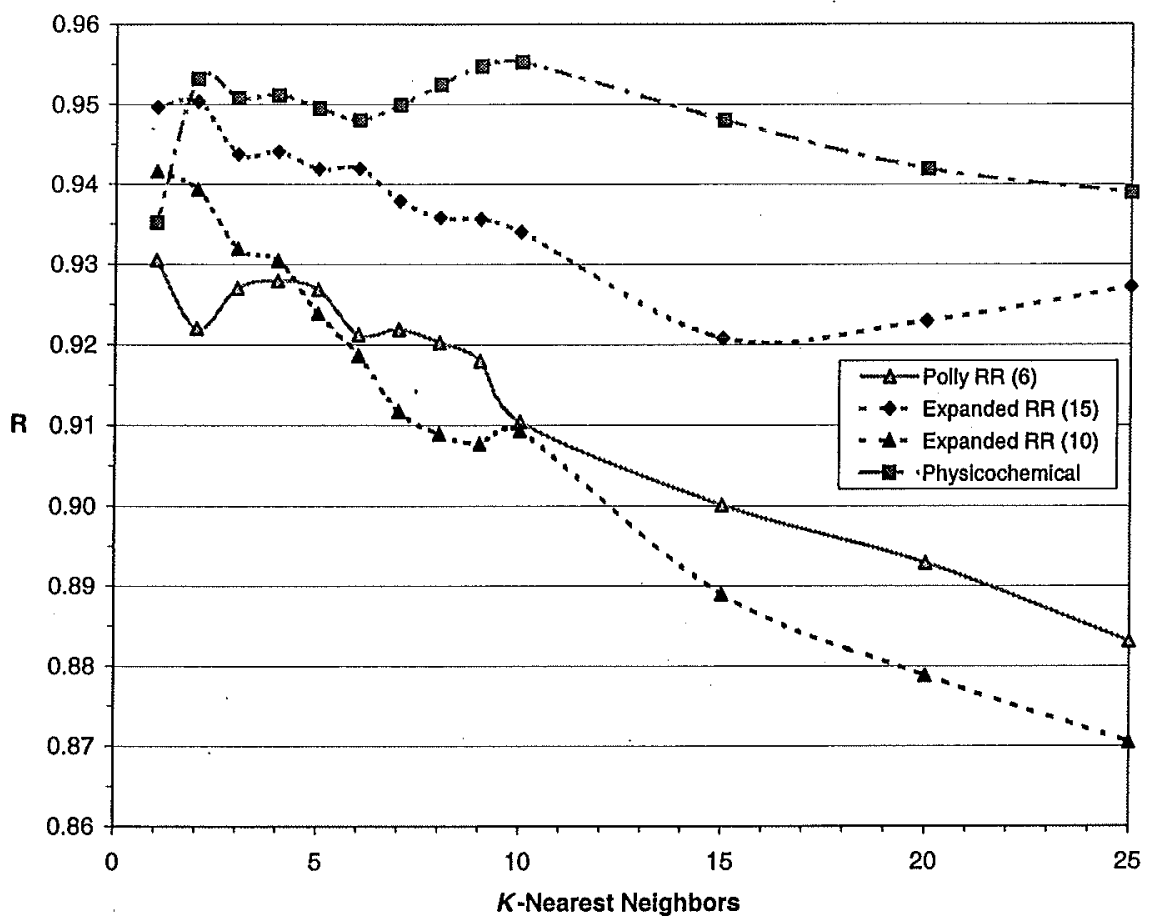

FIGURE 4 A closer examination of the overlap in the tailored and physicochemical property-derived similarity spaces for estimation of $\log P$ for JP-8 in terms of correlation coefficients.

The situation is different with the similarity spaces tailored to the $\log P$ using the $\mathrm{RR}$ method (Figures 1 and 4). The parameter space developed from ten selected POLLY indices outperforms the methods based on APs, PCs derived from POLLY, and the expanded set of indices. When we selected ten indices from the expanded set of independent variables selected by $R R$, there was not much improvement in model quality. However, an increase in the number of independent variables to fifteen for the expanded made significant improvement. In fact, the last model and the QMSA estimates using the experimental property data are of similar quality.

A perusal of results derived for the seventy-six diverse chemicals (Figure 2 and Tables 7 and 8) shows that QMSA models based on six PCs and TIs from POLLY, PCs and TIs from the expanded set of indices and APs do not perform as well as the tailored similarity methods. A comparison of 
estimates using six TIs in the arbitrary method and the RR method (Figure 5) shows that even at this level the RR method outperforms the arbitrary method substantially. The quality of the tailored method using the same number of TIs (6) goes up substantially when the indices are selected from the expanded set of independent variables. Presumably this is because the RR method selects the most relevant six indices related to the property. An increase in the number of independent variables from six to twenty-two descriptors makes another improvement in model quality (Figures 4 and 6). In fact, this model is almost as good as the property-based one. Using the same number of parameters (22) in the arbitrary QMSA techniques, the estimated $\log P$ values were inferior to the tailored similarity method.

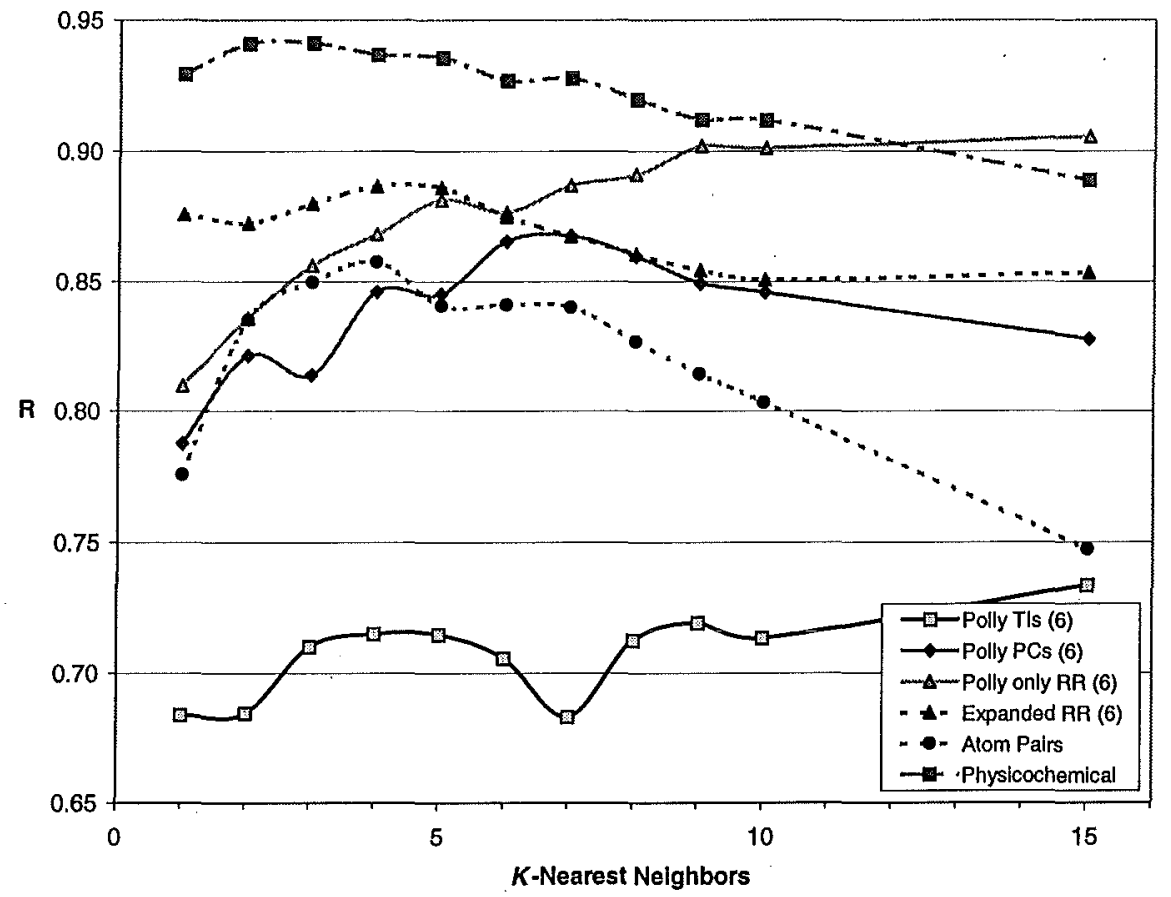

FIGURE 5 A closer examination of the overlap in the similarity spaces using a limited number of TIs and the property-based and atom pair based spaces for estimation of $\log P$ for TSCA chemicals in terms of correlation coefficients.

In conclusion, the results reported in this paper for one relatively homogeneous and one structurally diverse set of chemicals show that of the two classes of similarity methods, viz., arbitrary and tailored QMSA methods, the latter generally outperforms the former. Somewhat surprisingly, the tailored similarity spaces perform nearly as well as physicochemical spaces, results that until now have not been seen using molecular descriptors in the creation of similarity spaces. 
Further studies of the relative utility of these techniques are needed to determine their relative utility in property/ activity/ toxicity estimation and analog selection.

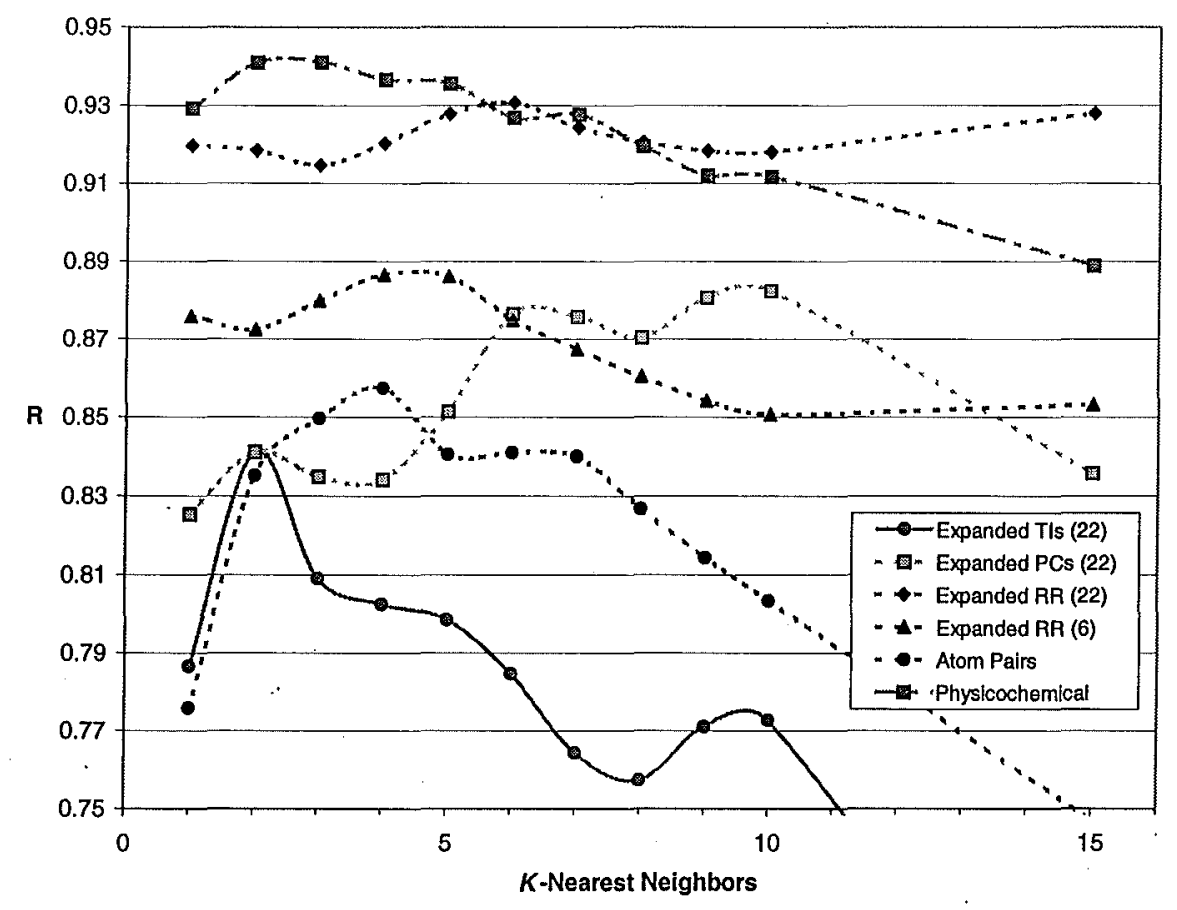

FIGURE 6 A closer examination of the overlap in the similarity spaces using the expanded set of TIs compared to the property-based and atom pair based spaces for estimation of $\log P$ for TSCA chemicals in terms of correlation coefficients.

\section{Acknowledgements}

The authors acknowledge the financial support of this research by grant F496200210138 from the United States Air Force Office of Scientific Research. This is contribution number 320 from the Center for Water and the Environment of the Natural Resources Research Institute.

\section{References}

[1] Basak, S.C., Magnuson, V.R., Niemi, G.J. and Regal, R.R. (1988). Determining structural similarity of chemicals using graph-theoretic indices. Discrete Appl. Math. 19, 17-44.

[2] Basak, S.C., Niemi, G.J. and Veith, G.D. (1990). Optimal characterization of structure for prediction of properties. J. Math. Chem. 4, 185-205.

[3] Basak, S.C., Gute, B.D. and Grunwald, G.D. (1999). A hierarchical approach to the development of QSAR models using topological, geometrical, and quantum chemical parameters. In, Topological Indices and Related Descriptors in QSAR and QSPR (J. Devillers and A.T. Balaban, Eds.). Gordon and Breach Science Publishers, The Netherlands, pp. 675-696. 
[4] Basak, S.C., Gute, B.D. and Grunwald, G.D. (2000). Use of graph invariants in QMSA and predictive toxicology, DIMACS Series 51. In, Discrete Mathematical Chemistry (P. Hansen, P. Fowler, and M. Zheng, Eds.). American Mathematical Society, Providence Rhode Island, pp. 9-24.

[5] Basak, S.C., Mills, D., Gute, B.D., Grunwald, G.D. and Balaban, A.T. (2001). Applications of topological indices in predicting property/ bioactivity/ toxicity of chemicals. In, Topology in Chemistry: Discrete Mathematics of Molecules (D.H. Rouvray and R.B. King, Eds.). Horwood Publishing Ltd., Chichester, UK, pp. 113-184.

[6] Hansch, C. and Leo, A. (1995). Exploring QSAR: Fundamentals and applications in Chemistry and Biology, American Chemical Society, Washington, D.C., p. 557.

[7] Kamlet, M.J., Abboud, J.-L.M., Abraham, M.H. and Taft, R.W. (1983). Linear solvation energy relationships. 23. A comprehensive collection of the solvatochromatic parameters, ${ }^{*}, a$ and $b$, and some methods for simplifying the general solvatochromatic equation. J. Org. Chem. 48, 2877-2887.

[8] Kamlet, M.J., Doherty, R.M., Abraham, M.H., Marcus, Y. and Taft, R.W. (1988). Linear solvation energy relationships. 46. An improved equation for correlation and prediction of octanol/water partition coefficients of organic nonelectrolytes (including strong hydrogen bond donor solutes). J. Phys. Chem. 92, 5244-5255.

[9] Auer, C.M., Nabholz, J.V. and Baetcke, K.P. (1990). Mode of action and the assessment of chemical hazards in the presence of limited data: use of structure-activity relationships (SAR) under TSCA, Section 5. Environ. Health Perspect. 87 183-197.

[10] Basak, S.C., Mills, D., Gute, B.D., Balaban, A.T., Basak, K. and Grunwald, G.D. (2002). Use of mathematical structural invariants in analyzing combinatorial libraries: A case study with Psoralen derivatives. In, Some Aspects of Mathematical Chemistry (D.K. Sinha, S.C. Basak, R.K. Mohanty, and I.N. Basumallick, Eds.). Visva-Bharati University, India, in press.

[11] Carbo-Dorca, R. and Mezey, P.G., Eds). (1998). Advances in Molecular Similarity. Vol. 2, JAI Press, Stamford, CN. p. 297.

[12] Willett, P. and Winterman, V. (1986). A comparison of some measures for the determination of intermolecular structural similarity. Quant. Struct.-Act. Relat. 5, 18-25.

[13] Johnson, M. and Maggiora, G.M. (1990). Concepts and Applications of Molecular Similarity, John Wiley \& Sons, Inc., New York, p. 393.

[14] Basak, S.C., Gute, B.D., Mills, D. and Hawkins, D.M. (2002). Quantitative molecular similarity methods in the property/toxicity estimation of chemicals: A comparison of arbitrary versus tailored similarity spaces. J. Mol. Struct. (Theochem), accepted.

[15] Basak, S.C., Bertelsen, S. and Grunwald, G. (1994). Application of graph theoretical parameters in quantifying molecular similarity and structure-activity studies. J. Chem. Inf. Comput. Sci. 34, 270-276.

[16] Basak, S.C. and Grunwald, G.D. (1994). Molecular similarity and risk assessment: analog selection and property estimation using graph invariants. SAR QSAR Environ. Res. 2, 289-307.

[17] Basak, S.C. and Grunwald, G.D. (1994). Use of topological space and property space in selecting structural analogs. Mathl. Model. Sci. Computing 4, 464-469.

[18] Basak, S.C. and Grunwald, G.D. (1995). Estimation of lipophilicity from molecular structural similarity. New J. Chem. 19, 231-237.

[19] Basak, S.C. and Grunwald, G.D. (1995). Molecular similarity and estimation of molecular properties. J. Chem. Inf. Comput. Sci. 35, 366-372.

[20] Basak, S.C. and Grunwald, G.D. (1995). Predicting mutagenicity of chemicals using topological and quantum chemical parameters: A similarity based study. Chemosphere 31, 2529-2546. 
[21] Basak, S.C. and Grunwald, G.D. (1995). Tolerance space and molecular similarity. SAR QSAR Environ. Res. 3, 265-277.

[22] Basak, S.C., Bertelsen, S. and Grunwald, G.D. (1995). Use of graph theoretic parameters in risk assessment of chemicals. Toxicol. Lett. 79, 239-250.

[23] Basak, S.C., Gute, B.D. and Grunwald, G.D. (1996). Estimation of normal boiling points of haloalkanes using molecular similarity. Croat. Chim. Acta 69, 1159-1173.

[24] Basak, S.C. and Gute, B.D. (1997). Use of graph theoretic parameters in predicting inhibition of microsomal hydroxylation of anilines by alcohols: a molecular similarity approach. In, Proceedings of the International Congress on Hazardous Waste: Impact on Human and Ecological Health (B.L. Johnson, C. Xintaras, and J.S. Andrews, Eds.). Princeton Scientific Publishing Co, Inc., pp. 492-504.

[25] Basak, S.C., Gute, B.D. and Grunwald, G.D. (1998). Characterization of the molecular similarity of chemicals using topological invariants. In, Advances in Molecular Similarity, vol. 2 (R. Carbo-Dorca and P.G. Mezey, Eds.). JAI Press, Stamford, Connecticut, pp. 171-185.

[26] Basak, S.C., Grunwald, G.D., Host, G.E., Niemi, G.J. and Bradbury, S.P. (1998). A comparative study of molecular similarity, statistical, and neural network methods for predicting toxic modes of action of chemicals. Environ. Toxicol. Chem. 17, 1056-1064.

[27] Basak, S.C., Gute, B.D. and Grunwald, G.D. (2002). Development and application of molecular similarity methods using nonempirical parameters. Mathl. Modelling Sci. Computing, in press.

[28] Basak, S.C., Gute, B.D. and Grunwald, G.D. (2002). Quantitative comparison of five molecular structure spaces in selecting analogs of chemicals. Mathl. Modelling Sci. Computing, in press.

[29] Gute, B.D., Grunwald, G.D., Mills, D. and Basak, S.C. (2001). Molecular similarity based estimation of properties: A comparison of structure spaces and property spaces. SAR QSAR Environ. Res. 11, 363-382.

[30] Gute, B.D., Basak, S.C., Mills, D. and Hawkins, D.M. (2002). Tailored similarity spaces for the prediction of physicochemical properties. Internet Electr. J. Mol. Design, accepted.

[31] AFOSR JP-8 Jet Fuel Workshop. (2000). University of Arizona: Tucson, AZ.

[32] Russom, C.L., Anderson, E.B., Greenwood, B.E. and Pilli, A. (1991). ASTER: An integration of the AQUIRE data base and the QSAR system for use in ecological risk assessments. Sci. Total Environ. 109/110, 667-670.

[33] Basak, S.C., Harriss, D.K. and Magnuson, V.R. (1988). POLLY.

[34] Molconn-Z (2000). Molconn-Z: v 3.50, Hall Associates Consulting Quincy, MA.

[35] Wiener, H. (1947). Structural determination of paraffin boiling points. J. Am. Chem. Soc. 69, 17-20.

[36] Randic, M. (1975). On characterization of molecular branching. J. Am. Chem. Soc. 97, 6609-6615.

[37] Kier, L.B., Murray, W.J., Randic, M. and Hall, L.H. (1976). Molecular connectivity. V. Connectivity series concept applied to diversity. J. Pharm. Sci. 65, 1226-1230.

[38] Kier, L.B. and Hall, L.H. (1986). Molecular Connectivity in Structure-Activity Analysis, Research Studies Press, Letchworth, Hertfordshire, U.K., p. 262.

[39] Bonchev, D. and Trinajstic, N. (1977). Information theory, distance matrix and molecular branching. $J$. Chem. Phys. 67, 4517-4533.

[40] Raychaudhury, C., Ray, S.K., Ghosh, J.J., Roy, A.B. and Basak, S.C. (1984). Discrimination of isomeric structures using information theoretic topological indices. J. Comput. Chem. 5, 581-588.

[41] Basak, S.C., Roy, A.B. and Ghosh, J.J., In, Proceedings of the 2nd International Conference on Mathematical Modelling (X.J.R. Avula, R. Bellman, Y.L. Luke, and A.K. Rigler, Eds.) Rolla, Missouri, 1980. University of Missouri-Rolla, City, Year, p. 851-856. 
[42] Basak, S.C. and Magnuson, V.R. (1983). Molecular topology and narcosis: A quantitative structureactivity relationship( QSAR) study of alcohols using complementary information content (CIC). Arzneim.-Forsch./Drug Res. 33, 501-503.

[43] Roy, A.B., Basak, S.C., Harriss, D.K. and Magnuson, V.R. (1984). Neighborhood complexities and symmetry of chemical graphs and their biological applications. In, Mathl. Modelling Sci. Tech. (X.J.R. Avula, R.E. Kalman, A.I. Liapis, and E.Y. Rodin, Eds.). Pergamon Press, pp. 745-750.

[44] Balaban, A.T. (1982). Highly discriminating distance-based topological indices. Chem. Phys. Lett. 89, 399-404.

[45] Balaban, A.T. (1983). Topological indices based on topological distances in molecular graphs. Pure and Appl. Chem. 55, 199-206.

[46] Balaban, A.T. (1986). Chemical graphs. Part 48. Topological index J for heteroatom-containing molecules taking into account periodicities of element properties. Math. Chem. (MATCH) 21, 115-122.

[47] Filip, P.A., Balaban, T.S. and Balaban, A.T. (1987). A new approach for devising local graph invariants: Derived topological indices with low degeneracy and good correlational ability. J. Math. Chem. 1, 61-83.

[48] Kier, L.B. (1985). A shape index from molecular graphs. Quant. Struct.-Act. Relat. 4, 109-116.

[49] Kier, L.B. and Hall, L.H. (1999). The kappa indices for modeling molecular shape and flexibility. In, Topological Indices and Related Descriptors in QSAR and QSPR (J. Devillers and A.T. Balaban, Eds.). Gordon and Breach Science Publishers, Amsterdam, pp. 455-489.

[50] Kier, L.B. and Hall, L.H. (1999). Molecular Structure Description: The Electrotopological State, Academic Press, San Diego, CA.

[51] Basak, S.C. and Gute, B.D. (1997). Characterization of molecular structures using topological indices. SAR QSAR Environ. Res. 7, 1-21.

[52] Basak, S.C. (1999). Information theoretic indices of neighborhood complexity and their applications. In, Topological Indices and Related Descriptors in QSAR and QSPR (J. Devillers and A.T. Balaban, Eds.). Gordon and Breach Science Publishers, The Netherlands, pp. 563-593.

[53] SAS Institute, Inc. (1988). SAS/STAT User's Guide, Release 6.03. SAS Institute Inc., Cary, NC.

[54] Hawkins, D., Basak, S. and Shi, X. (2001). QSAR with few compounds and many features. J. Chem. Inf. Comput. Sci., 41, 663-670.

[55] Basak, S.C., Hawkins, D.M. and Mills, D. (2002). Predicting blood:air partition coefficient of structurally diverse chemicals using theoretical molecular descriptors. In, Advances in Molecular Similarity (R. Carbo-Dorca and P.G. Mezey, Eds.). In press.

[56] Basak, S.C., El-Masri, H., Hawkins, D.M. and Mills, D. (2002). Exposure assessment of volatile organic chemicals (VOCs): Predicting blood:air partition coefficients of diverse chemicals using theoretical descriptors. J. Chem. Inf. Comput. Sci., submitted.

[57] Isnard, P. and Lambert, S. (1989). Aquaeous solubility and n-octanol/water pertition coefficient correlations. Chemosphere 18, 1837-1853. 


\title{
QUANTITATIVE MOLECULAR SIMILARITY METHODS IN THE PROPERTY/ TOXICITY ESTIMATION OF CHEMICALS: A COMPARISON OF ARBITRARY VERSUS TAILORED SIMILARITY SPACES
}

\author{
S. C. BASAK, ${ }^{a, \dagger}$ B. D. GUTE, ${ }^{a}$ D. MILLS ${ }^{a}$ and D. M. HAWKINS ${ }^{b}$ \\ ${ }^{a}$ Natural Resources Research Institute, University of Minnesota Duluth, \\ 5013 Miller Trunk Highway, Duluth, MN, 55811 \\ ${ }^{b}$ School of Statistics, University of Minnesota, \\ 224 Church St SE, Minneapolis, MN, 55455
}

Quantitative molecular similarity analysis (QMSA) methods based on structure spaces using a variety of calculated molecular descriptors and experimental properties have been used for selection of analogs and estimation of a variety of properties pertaining to chemistry, drug discovery, and environmental toxicology. The set of descriptors, orthogonalized or not, have been selected either arbitrarily or based on some intuitive notion of their association to the property of interest. In this study we have developed a method of 'tailoring' the QMSA method to a particular property of interest through the use of indices which are strongly correlated with the property instead of using an arbitrary set of parameters. Comparative studies of the tailored vis-à-vis two non-tailored (arbitrary) similarity spaces based on principal components (PCs) derived from topological indices show that the tailored method outperforms the two non-tailored techniques in the QMSA based estimation of hydrophobicity (logP, octanol/water) for a set of 213 compounds and Ames' mutagenicity of a group of 95 aromatic and heteroaromatic amines.

Keywords: Quantitative molecular similarity analysis (QMSA); Tailored QMSA method; Arbitrary QMSA method; Hydrophobicity; Mutagenicity

\section{INTRODUCTION}

The modern drug discovery process and the regulation of chemicals for the assessment of their potential deleterious effects on human and environmental health are both heavily dependent on the availability of a number of physicochemical, biochemical, and toxicological properties for the candidate chemicals. A partial list of such properties is presented in Table 1. Ideally, experimental data on bioactivity, various toxic effects, metabolic profile, environmental fate, and distribution should be available for all chemicals. However, such data are available only for a fraction of the candidate chemicals. In the area of

\footnotetext{
${ }^{\dagger}$ Corresponding Author. e-mail: sbasak@nrri.umn.edu
} 
ecotoxicology, the Toxic Substances Control Act (TSCA) Inventory currently includes over 81,000 chemicals and the list is growing by about 3,000 new submissions per year via the premanufacture notification (PMN) process. Few of the existing TSCA chemicals have any experimental toxicity, ecotoxicity, environmental fate, or physicochemical data necessary for hazard assessment. This data is also missing for many of the PMN chemicals. No physicochemical property data whatsoever is available for at least $50 \%$ of the chemicals currently listed in the TSCA Inventory. And, of the other $50 \%$ of the Inventory chemicals that do have some available data, fewer than $15 \%$ have available mutagenicity data, just over 12,000 out of 81,000 chemicals. And only about $6 \%$ of these chemicals, less than 5,000 , have ecotoxicological and environmental fate data [1]. In the realm of drug discovery, after a lead chemical is identified, large numbers of analogs are synthesized in order to find a compound with an acceptable therapeutic profile and few, if any, undesirable side effects. As a result, modern combinatorial chemistry is producing large real and virtual combinatorial libraries of chemicals, most of which do not contain any experimental data for their evaluation.

TABLE I Partial list of properties necessary for risk assessment of chemical

\begin{tabular}{cc}
\hline Physicochemical & Biological \\
\hline Molar Volume & Receptor Binding $\left(\mathrm{K}_{\mathrm{D}}\right)$ \\
Boiling Point & Michaelis Constant $\left(\mathrm{K}_{\mathrm{m}}\right)$ \\
Melting Point & Inhibitor Constant $\left(\mathrm{K}_{\mathrm{i}}\right)$ \\
Vapor Pressure & Biodegradation \\
Aqueous Solubility & Bioconcentration \\
Dissociation Constant (pKa) & Alkylation Profile \\
Reactivity (Electrophile) & Metabolic Profile \\
Partition Coefficient & Mutagenicity \\
Tissue-Air, Blood-Air & Carcinogenicity \\
& Chronic Toxicity \\
& Acute Toxicity \\
\hline
\end{tabular}

A perusal of the above conditions indicates that in the foreseeable future the regulation of chemicals for human and environmental health, as well as evaluation of chemicals for the discovery of life saving drugs, will need to be carried out with little or none of the experimental data necessary. A valid alternative under these circumstances has been the use of estimated property/ bioactivity/ toxicity values that are computed using parameters calculated directly from the chemical's structure. These theoretical descriptors of molecular structure can be used in both quantitative structure activity relationship (QSAR) and quantitative molecular similarity relationship (QMSA) models. 
QSAR is based on the idea that the structure of a molecule is the principal determinant of its physicochemical, toxicological, and biomedicinal properties [2-4]. Various types of calculated parameters, viz., topological [5-7], substructural [8, 9], geometrical [10], and quantum chemical parameters [11-16], have been used in the development of QSARs. Linear free energy related (LFER) and linear solvation energy related (LSER) parameters, as well as physicochemical properties such as the various types of partition coefficients, have been used in QSARs pertaining to drug discovery and environmental toxicology $[2,17]$. The problem with the use of LFER and LSER parameters and experimental physicochemical properties in QSARs for toxicity estimation is that such properties are not available for many chemicals. Another problem with the use of QSARs is that these models usually work most effectively for sets of molecules which are 'congeneric,' i.e., belong to a narrow class either by structural analogy or having the same biochemical mechanism of action. The United States Environmental Protection Agency (USEPA) currently has over 300 class-specific QSARs for various subsets of the TSCA Inventory [18]. But these models cover only a small fraction of the 81,000 substances in the Inventory.

Often it is difficult to unambiguously classify a molecule into a particular structural class. This happens when a molecule possesses a complex structure, e.g., contains many functional groups. In such circumstances, one practical alternative for hazard assessment of a chemical is to select a subset of chemicals that are structurally analogous to the candidate chemical and use the properties of these selected analogs in assessing the hazard posed by the chemical of interest. This can be done either intuitively, based on the subjective experience of the risk assessor, or it can be carried out using computational methods that are fast and objective. The intuitive selection of chemical analogs is generally based on a set of rules or unspoken notions developed through the practitioner's experience of structural attributes that have been found to be associated with some kind of activity or toxicity. On the other hand, the evaluation of a large number of chemicals through the use of their selected analogs necessitates the use of methods where the descriptors used in analog selection are rapidly computed directly from the molecular structure in an unambiguous manner.

Our research group has been involved in the development of QMSA methods based on computed theoretical molecular descriptors for the past two decades. These methods have used topological indices, atom pairs, and physicochemical properties in the creation of structure spaces and the selection of analogs. Specifically, we have used two major approaches to quantifying molecular similarity. The first method uses Euclidean distance (ED) within an $n$-dimensional space. For these studies we have used smaller sets of topological indices selected through methods such as variable clustering (VC) or principal component analysis (PCA). In using VC to select a set of indices we have taken the one TI most correlated with each cluster, while in using PCA for index selection we have taken the one TI most 
correlated with each principal component (PC) having an eigenvalue greater than or equal to zero [19-22]. Additionally, the clusters from VC method and the PCs from PCA can be used as new descriptors within a ED space. In this way we avoid some of the issues of intercorrelation between the individual TIs by creating completely orthogonal (PCs) [22-34] or nearly orthogonal (VCs) [19-21] descriptors derived from the TIs. ED has also been used in the construction of physicochemical property-based similarity spaces $[32,34,35]$. The second method employs a Tanimoto-type association coefficient to compare the atom pairs within two molecules as a measure of their similarity/dissimilarity [19-22, 24-27, 29, 31, 32, $34,36,37]$. We have carried out comparative studies on the relative utility of experimental property-based and molecular descriptor-based similarity spaces in the selection of analogs [25, 32] and the estimation of properties of chemicals from their selected analogs in these spaces [32, 34, 35]. The theoretical spaces have been found to behave in a manner analogous to those derived from experimental properties in some limited number of cases. The advantage of theoretical structure spaces is that they can be used for any arbitrary database of chemicals including libraries comprised of molecules that have never been synthesized.

In our earlier QMSA studies, we represented a molecule by a set of $n$ calculated descriptors, viz, topological indices and atom pairs. A chemical is then is represented as an object in the $n$-dimensional vector space. The pair-wise similarity/ dissimilarity of molecule is then quantified using some kind of distance function or association coefficient. These approaches have yielded reasonable results in the selection of analogs and prediction of properties of chemicals from their selected neighbors using the $k$ nearest neighbor (KNN) approach. In many of these approaches it is possible that the elements of the vector (the descriptors) are mutually correlated to some extent so that we are using redundant information. One approach to correct this problem has been to extract a set of orthogonal or minimally correlated variables derived from the set of original predictors using techniques such as principal component analysis or variable clustering. Such variables are orthogonal, or nearly so, but have no intrinsic relationship with the property that we are interested in estimating using the particular QMSA method. Such similarity spaces may be termed "arbitrary" similarity spaces. One may use the broadest category of descriptors in creating such spaces and the descriptors may be related to the property only by chance or intuitive association. On the other hand, our major interest in the selection of analogs is the prediction of properties.

In this paper we have developed a method for the design of similarity spaces from molecular descriptors which are "tailored" to a particular property. We have created such tailored spaces for two sets of molecules: a) a group of 213 compounds for which we are interested in estimating $\log P$ (octanollwater partition coefficient) and b) a collection of ninety-five aromatic and heteroaromatic amines for which the interest was to predict Ames' mutagenicity. In this paper we have presented the utility of our newly 
developed tailored QMSA method vis-à-vis arbitrary QMSA methods in the similarity-based estimation of $\log P$ and mutagenicity.

\section{MATERIALS AND METHODS}

\section{LogP Database}

The $\log P$ set consists of 213 diverse compounds used in several earlier studies [33, 38]. Measured values of $\log P$ were obtained from CLOGP [39], as this set is a subset of the STARLIST chemical database. In this study, as in the earlier studies, we have used only chemicals where $\mathrm{HB}_{1}$ was equal to zero $\left(\mathrm{HB}_{1}\right.$ is a measure of the hydrogen bonding potential of a chemical). Therefore, none of the chemicals have available hydrogen bonding centers, creating a more homogeneous group of chemicals and a more densely packed similarity space. Also, the chemicals were selected such that their $\log P$ values fall within the range of -2 to 5.5 , since actual measurements for $\log P$ beyond this range have been shown to be problematic [40]. It should be noted that six compounds included in the earliest study have been excluded from this study. All six of these compounds were excluded because they consist of only two nonhydrogen atoms and the triplet indices cannot be calculated for molecules with fewer than three nonhydrogen atoms. The chemicals used in this study and their $\log P$ values from CLOGP are reported in Table 2.

\section{Mutagenicity Database}

The mutagenicity data set, a set of 95 aromatic and heteroaromatic amines, was previously collected from the literature by Debnath et al. [41], and has been used to study mutagenic potency in a number of our earlier studies $[13,20,21,42,43]$. The mutagenic activities of these compounds in $S$. typhimurium TA98 + S9 microsomal preparation are expressed as the mutation rate, $\ln (\mathrm{R})$, in natural logarithm (revertants/nmol). Table 3 lists the compounds included in this study and their experimentally measured mutation rates.

\section{Calculation of Molecular Descriptors}

The first set of 102 TIs used in this study include the Wiener number [44], molecular connectivity indices as calculated by Randić [45] and Kier and Hall [5, 46], frequency of path lengths of varying size, information theoretic indices defined on distance matrices of graphs using the methods of Bonchev and Trinajstić [47] as well as those of Raychaudhury et al. [48], parameters defined on the neighborhood 

and D.M. HAWKINS

TABLE II List of the 213 STARLIST chemicals included in this study and their experimental and estimated values for $\log P$

\begin{tabular}{|c|c|c|c|c|c|}
\hline \multirow[b]{2}{*}{ ID\# } & \multirow[b]{2}{*}{ Compound name } & \multirow[b]{2}{*}{$\begin{array}{r}\log P \\
(\exp .)\end{array}$} & \multicolumn{3}{|c|}{ Estimated $\log P$} \\
\hline & & & $\begin{array}{c}\mathbf{P C} \\
(k=3)\end{array}$ & $\begin{array}{l}\text { TI/PC } \\
(k=8) \\
\end{array}$ & $\begin{array}{l}\text { TI/RR } \\
(k=2)\end{array}$ \\
\hline 1 & 1,4-dimethylnaphthalene & 4.37 & 4.35 & 4.41 & 4.32 \\
\hline 2 & cyclopropane & 1.72 & 2.75 & 2.57 & 1.61 \\
\hline 3 & 3,4-dimethylchlorobenzene & 3.82 & 3.65 & 3.50 & 3.60 \\
\hline 4 & 2,2-diphenyl-1,1,1-trichloroethane & 4.87 & 5.13 & 3.85 & 5.45 \\
\hline 5 & 2,6-dimethylnaphthalene & 4.31 & 4.38 & 4.41 & 4.42 \\
\hline 6 & hexafluoroethane & 2.00 & 3.06 & 2.26 & 3.10 \\
\hline 7 & 1-iodoheptane & 4.70 & 4.38 & 3.17 & 4.47 \\
\hline 8 & allylbromide & 1.79 & 2.14 & 2.85 & 2.50 \\
\hline 9 & 1,5-dimethylnaphthalene & 4.38 & 4.34 & 4.41 & 4.32 \\
\hline 10 & 1,8-dimethylnaphthalene & 4.26 & 4.38 & 4.42 & 4.38 \\
\hline 11 & 1,2,3-trichlorobenzene & 4.05 & 4.39 & 4.13 & 4.09 \\
\hline 12 & 2-ethylthiophene & 2.87 & 2.50 & 2.62 & 2.91 \\
\hline 14 & $\gamma$-phenylpropylfluoride & 2.95 & 3.59 & 3.35 & 3.69 \\
\hline 15 & iodobenzene & 3.25 & 3.55 & 3.28 & 2.93 \\
\hline 16 & 1-methylpentachlorocyclohexane & 4.04 & 3.73 & 3.14 & 3.63 \\
\hline 18 & $2,3^{\prime}-\mathrm{pcb}$ & 5.02 & 5.19 & 5.08 & 5.13 \\
\hline 19 & cyclopentane & 3.00 & 2.81 & 2.75 & 2.15 \\
\hline 20 & ethylchloride & 1.43 & 1.88 & 2.04 & 1.33 \\
\hline 21 & 2-phenylthiophene & 3.74 & 2.92 & 2.57 & 3.87 \\
\hline 22 & trichlorofluoromethane & 2.53 & 1.89 & 2.40 & 2.80 \\
\hline 23 & fluoroform & 0.64 & 1.13 & 1.37 & 0.92 \\
\hline 24 & dimethyldisulfide & 1.77 & 2.11 & 2.35 & 2.03 \\
\hline 25 & propane & 2.36 & 2.36 & 2.25 & 1.67 \\
\hline 26 & hexamethylbenzene & 5.11 & 4.22 & 3.98 & 4.79 \\
\hline 27 & butanethiol & 2.28 & 2.40 & 2.40 & 3.07 \\
\hline 28 & diethylsulfide & 1.95 & 2.68 & 2.44 & 2.03 \\
\hline 29 & cyclohexane & 3.44 & 2.66 & 3.50 & 2.60 \\
\hline 30 & diphenyldisulfide & 4.41 & 4.46 & 4.84 & 4.12 \\
\hline 31 & $m$-fluorobenzylchloride & 2.77 & 3.02 & 3.42 & 2.63 \\
\hline 32 & 1-chloropropane & 2.04 & 2.41 & 2.05 & 1.85 \\
\hline 33 & 2,4-dichlorobenzylchloride & 3.82 & 3.77 & 3.29 & 3.25 \\
\hline 34 & $m$-chlorotoluene & 3.28 & 3.66 & 3.59 & 3.38 \\
\hline 35 & butane & 2.89 & 3.09 & 2.35 & 2.10 \\
\hline 36 & 1,2,3-trimethylbenzene & 3.66 & 4.02 & 3.78 & 3.60 \\
\hline 37 & 1,1-difluoroethylene & 1.24 & 0.82 & 2.70 & 2.17 \\
\hline 38 & 1-chlorobutane & 2.64 & 2.32 & 2.31 & .2 .29 \\
\hline 39 & 2,3-dibromothiophene & 3.53 & 2.64 & 3.08 & 3.23 \\
\hline 40 & pentafluorethylbenzene & 3.36 & 2.61 & 2.82 & 3.85 \\
\hline 41 & 1,2,4,5-tetrabromobenzene & 5.13 & 3.98 & 3.49 & 4.87 \\
\hline 42 & $o$-dichlorobenzene & 3.38 & 3.50 & 3.41 & 3.51 \\
\hline 43 & 1,2,3,4-tetrachlorobenzene & 4.64 & 4.71 & 4.26 & 4.87 \\
\hline 44 & tribromoethene & 3.20 & 3.06 & 3.60 & 2.79 \\
\hline 45 & pentane & 3.39 & 3.13 & 2.37 & 2.52 \\
\hline
\end{tabular}




\begin{tabular}{|c|c|c|c|c|c|}
\hline \multirow[b]{2}{*}{ ID\# } & \multirow[b]{2}{*}{ Compound name } & \multirow[b]{2}{*}{$\begin{array}{l}\log P \\
(\exp .) \\
\end{array}$} & \multicolumn{3}{|c|}{ Estimated $\log P$} \\
\hline & & & $\begin{array}{c}\mathbf{P C} \\
(k=3) \\
\end{array}$ & $\begin{array}{l}\text { TI/PC } \\
(k=8) \\
\end{array}$ & $\begin{array}{l}\text { TI/RR } \\
(k=2)\end{array}$ \\
\hline 46 & isobutane & 2.76 & 2.88 & 3.18 & 2.24 \\
\hline 47 & mirex & 5.28 & 4.52 & 4.78 & 5.15 \\
\hline 48 & 1,3-dichlorobenzene & 3.60 & 3.42 & 3.38 & 3.45 \\
\hline 49 & 1,2-dimethylnaphthalene & 4.31 & 4.24 & 4.41 & 4.40 \\
\hline 50 & 2-ethylnaphthalene & 4.38 & 4.06 & 4.34 & 4.36 \\
\hline 51 & cycloheptatriene & 2.63 & 2.66 & 2.88 & 2.50 \\
\hline 52 & 3-chlorobiphenyl & 4.58 & 4.70 & 4.82 & 4.50 \\
\hline 53 & 3-ethylthiophene & 2.82 & 2.51 & 2.63 & 2.93 \\
\hline 54 & 1,3,5-tribromobenzene & 4.51 & 4.19 & 3.56 & 4.09 \\
\hline 55 & $\beta$-phenylethylchloride & 2.95 & 2.86 & 3.40 & 3.46 \\
\hline 56 & acenaphthene & 3.92 & 3.91 & 3.92 & 4.32 \\
\hline 57 & $m$-dibromobenzene & 3.75 & 3.18 & 3.66 & 3.72 \\
\hline 58 & dichlorodifluoromethane & 2.16 & 2.01 & 2.47 & 2.20 \\
\hline 59 & toluene & 2.73 & 3.16 & 3.36 & 2.68 \\
\hline 60 & anthracene & 4.45 & 4.28 & 4.03 & 4.36 \\
\hline 61 & hexachlorocyclopentadiene & 5.04 & 4.78 & 4.32 & 4.87 \\
\hline 62 & 3-phenyl-1-chloropropane & 3.55 & $\cdot 3.39$ & 3.50 & 3.39 \\
\hline 63 & bibenzyl & 4.79 & 4.49 & 3.97 & 4.96 \\
\hline 64 & 1-chloroheptane & 4.15 & 3.50 & 3.23 & 2.86 \\
\hline 65 & 2,4-dichlorotoluene & 4.24 & 3.66 & 3.47 & 4.22 \\
\hline 66 & 1,1-dichloroethane & 1.79 & 2.00 & 2.09 & 1.94 \\
\hline 67 & $(\beta)$-benzothiophene & 3.12 & 3.49 & 2.85 & 3.05 \\
\hline 68 & 2-bromothiophene & 2.75 & 2.90 & 2.72 & 2.58 \\
\hline 69 & chlorodifluoromethane & 1.08 & 0.98 & 1.31 & 1.15 \\
\hline 70 & pentachlorobenzene & 5.17 & 4.54 & 4.62 & 4.80 \\
\hline 71 & 9,10-dihydroanthracene & 4.25 & 4.64 & 4.07 & 4.46 \\
\hline 72 & 1,3-(bis-chloromethyl)benzene & 2.72 & 2.93 & 3.43 & 3.39 \\
\hline 73 & chlorobenzene & 2.84 & 3.08 & 3.26 & 2.63 \\
\hline 74 & 1,2,4-trichlorobenzene & 4.02 & 4.71 & 4.14 & 4.10 \\
\hline 75 & $2,2^{\prime}, 6-\mathrm{pcb}$ & 5.48 & 5.30 & 5.16 & 5.39 \\
\hline 76 & 2-butyne & 1.46 & 3.17 & 2.60 & 2.20 \\
\hline 77 & azulene & 3.20 & 3.33 & 3.82 & 3.16 \\
\hline 78 & trifluoromethylthiobenzene & 3.57 & 3.09 & 3.34 & 4.06 \\
\hline 79 & $2,5-\mathrm{pcb}$ & 5.16 & 5.15 & 5.07 & 4.96 \\
\hline 80 & 1,2,3-trichlorocyclohexene(34) & 2.84 & 3.56 & 3.06 & 2.77 \\
\hline 81 & biphenyl & 4.09 & 4.02 & 4.78 & 4.35 \\
\hline 82 & $p$-xylene & 3.15 & 3.25 & 3.58 & 3.27 \\
\hline 84 & thiophenol & 2.52 & 2.91 & 2.88 & 2.79 \\
\hline 85 & bromotrifluoromethane & 1.86 & 2.11 & 2.41 & 2.35 \\
\hline 86 & 9-methylanthracene & 5.07 & 4.37 & 4.25 & 4.95 \\
\hline 87 & trichloroethylene & 2.42 & 2.05 & 1.89 & 2.29 \\
\hline 88 & 1,4-dimethyltetrachlorocyclohexane & 4.40 & 3.61 & 3.02 & 4.01 \\
\hline 89 & propylene & 1.77 & 2.37 & 2.42 & 1.91 \\
\hline 90. & cyclohexene & 2.86 & 2.64 & 2.62 & 2.96 \\
\hline
\end{tabular}


TABLE II (Continued)

\begin{tabular}{|c|c|c|c|c|c|}
\hline \multirow[b]{2}{*}{ ID\# } & \multirow[b]{2}{*}{ Compound name } & \multirow[b]{2}{*}{$\begin{array}{l}\log P \\
\text { (exp.) }\end{array}$} & \multicolumn{3}{|c|}{ Estimated $\log P$} \\
\hline & & & $\begin{array}{c}\mathbf{P C} \\
(k=3) \\
\end{array}$ & $\begin{array}{l}\text { TI/PC } \\
(k=8)\end{array}$ & $\begin{array}{l}\mathbf{T} \mathbf{L} \mathbf{R} \mathbf{R} \\
(k=2)\end{array}$ \\
\hline 91 & methylthiobenzene & 2.74 & 3.30 & 3.38 & 3.06 \\
\hline 93 & $\gamma$-phenylpropyliodide & 3.90 & 3.89 & 3.51 & 3.99 \\
\hline 94 & $2,3,4^{\prime}-\mathrm{pcb}$ & 5.42 & 5.24 & 5.17 & 5.39 \\
\hline 95 & fluoropentachlorocyclohexane & 3.19 & 3.77 & 3.34 & 3.66 \\
\hline 96 & 1,2,3,5-tetrachlorobenzene & 4.92 & 4.62 & 4.23 & 4.73 \\
\hline 97 & $2,2^{\prime}-\mathrm{pcb}$ & 4.90 & 5.19 & 5.24 & 5.05 \\
\hline 98 & 1-butene & 2.40 & 2.62 & 3.10 & 2.18 \\
\hline 99 & 1,3-dimethylnaphthalene & 4.42 & 4.20 & 4.40 & 4.38 \\
\hline 100 & 1,7-dimethylnaphthalene & 4.44 & 4.37 & 4.40 & 4.37 \\
\hline 101 & 1-methylnaphthalene & 3.87 & 4.18 & 4.40 & 3.80 \\
\hline 102 & $2,6-p c b$ & 4.93 & 5.18 & 5.23 & 5.03 \\
\hline 103 & $\alpha$-bromotoluene & 2.92 & 3.28 & 3.59 & 3.56 \\
\hline 104 & $2,2^{\prime}, 3^{\prime}$-trichlorobiphenyl & 5.31 & 5.31 & 5.19 & 5.48 \\
\hline 105 & hexafluorobenzene & 2.22 & 3.00 & 4.48 & 3.47 \\
\hline 106 & 3-bromothiophene & 2.62 & 2.94 & 2.73 & 2.65 \\
\hline 107 & 1,2,3,5-tetramethylbenzene & 4.17 & 4.11 & 4.09 & 4.06 \\
\hline 108 & halothane & 2.30 & 2.33 & 2.58 & 2.36 \\
\hline 109 & $2,4,6-\mathrm{pcb}$ & 5.47 & 5.17 & 5.17 & 5.40 \\
\hline 110 & 1,1-dichloroethylene & 2.13 & 1.89 & 3.11 & 2.55 \\
\hline 111 & $o$-dibromobenzene & 3.64 & 4.02 & 3.67 & 3.77 \\
\hline 112 & 1,2,4,5-tetramethylbenzene & 4.00 & 3.56 & 4.11 & 4.14 \\
\hline 113 & 1-hexene & 3.39 & 3.65 & 3.83 & 3.61 \\
\hline 114 & neopentane & 3.11 & 3.02 & 3.23 & 2.51 \\
\hline 115 & chloroform & 1.97 & 1.75 & 1.43 & 1.67 \\
\hline 116 . & 1-fluorobutane & 2.58 & 2.34 & 2.32 & 2.32 \\
\hline 117 & pyrene & 4.88 & 4.66 & 4.12 & 5.28 \\
\hline 118 & 1,1-dichloro-2,2-diphenylethane & 4.51 & 4.84 & 3.52 & 5.15 \\
\hline 119 & isobutylene & 2.34 & 2.14 & 3.16 & 2.45 \\
\hline 120 & diphenylmethane & 4.14 & 4.56 & 4.00 & 4.60 \\
\hline 121 & isopropylbenzene & 3.66 & 3.48 & 3.47 & 3.63 \\
\hline 122 & naphthalene & 3.30 & 4.33 & 3.81 & 3.11 \\
\hline 123 & 1-heptene & 3.99 & 4.37 & 3.88 & 3.48 \\
\hline 124 & 2,2-dimethylbutane & 3.82 & 3.69 & 3.29 & 3.63 \\
\hline 125 & 1-fluoropentane & 2.33 & 3.12 & 2.53 & 2.85 \\
\hline 126 & $o$-xylene & 3.12 & 3.34 & 3.53 & 3.31 \\
\hline 127 & ethylbenzene & 3.15 & 3.37 & 3.44 & 3.16 \\
\hline 128 & trichloromethylthiobenzene & 3.78 & 4.21 & 4.42 & 4.81 \\
\hline 129 & thiophene & 1.81 & 2.40 & 2.75 & 2.74 \\
\hline 130 & bromochloromethane & 1.41 & 1.48 & 1.44 & 1.34 \\
\hline 131 & 1,2-dichlorotetrafluoroethane & 2.82 & 2.55 & 2.35 & 2.69 \\
\hline 132 & 2-chlorobiphenyl & 4.38 & 4.76 & 4.97 & 4.60 \\
\hline 133 & 2,4'-dichlorobiphenyl & 5.10 & 5.17 & 4.98 & 5.09 \\
\hline 134 & 1,3,5-trichlorobenzene & 4.15 & 3.91 & 4.12 & 4.04 \\
\hline 135 & 1-octene & 4.57 & 4.18 & 3.81 & 4.53 \\
\hline
\end{tabular}




\begin{tabular}{|c|c|c|c|c|c|}
\hline \multirow[b]{2}{*}{ ID\# } & \multirow[b]{2}{*}{ Compound name } & \multirow[b]{2}{*}{$\begin{array}{l}\log P \\
(\text { exp. })\end{array}$} & \multicolumn{3}{|c|}{ Estimated $\log P$} \\
\hline & & & $\begin{array}{c}\mathbf{P C} \\
(k=3) \\
\end{array}$ & $\begin{array}{l}\text { TI/PC } \\
(k=8) \\
\end{array}$ & $\begin{array}{l}\text { TI/RR } \\
(k=2) \\
\end{array}$ \\
\hline 137 & phenylethylsulfide & 3.20 & 3.40 & 3.52 & 3.26 \\
\hline 138 & 1-ethyl-2-methylbenzene & 3.53 & 3.75 & 3.53 & 3.54 \\
\hline 139 & propylbenzene & 3.72 & 3.69 & 3.42 & 3.45 \\
\hline 140 & indane & 3.18 & 3.67 & 3.55 & 3.02 \\
\hline 141 & 2-chloropropane & 1.90 & 2.17 & 2.37 & 1.92 \\
\hline 142 & phenylazide & 2.59 & 2.90 & 3.51 & 3.48 \\
\hline 143 & 2,4-dibromotetrachlorocyclohexane & 3.98 & 3.75 & 3.46 & 4.22 \\
\hline 144 & tetrachloroethylene & 3.40 & 4.00 & 4.07 & 3.84 \\
\hline 145 & 1-nonene & 5.15 & 3.98 & 3.60 & 4.31 \\
\hline 146 & 2,3-dimethylbutane & 3.85 & 3.01 & 3.09 & 3.61 \\
\hline 147 & dichlorofluoromethane & 1.55 & 1.27 & 1.48 & 1.73 \\
\hline 148 & 1,1,2,2-tetrachloroethane & 2.39 & 1.97 & 2.14 & 2.32 \\
\hline 149 & 1,2,4-trimethylbenzene & 3.78 & 3.79 & 3.76 & 3.54 \\
\hline 150 & fluorobenzene & 2.27 & 2.99 & 3.33 & 2.68 \\
\hline 151 & butylbenzene & 4.26 & 3.64 & 3.33 & 3.81 \\
\hline 152 & ethylbromide & 1.61 & 1.69 & 2.06 & 1.79 \\
\hline 153 & tetrafluoromethane & 1.18 & 1.89 & 2.28 & 1.91 \\
\hline 154 & p-cymene & 4.10 & 3.83 & 3.44 & 4.06 \\
\hline 155 & p-chlorotoluene & 3.33 & 3.80 & 3.45 & 3.40 \\
\hline 156 & 1-bromopropane & 2.10 & 2.72 & 2.86 & 2.34 \\
\hline 157 & bromocyclohexane & 3.20 & 3.00 & 3.55 & 3.04 \\
\hline 158 & 2-methylthiophene & 2.33 & 2.68 & 2.69 & 2.44 \\
\hline 159 & diphenylsulfide & 4.45 & 4.21 & 4.77 & 4.10 \\
\hline 160 & 1,2,4,5-tetrachlorobenzene & 4.82 & 4.10 & 4.24 & 4.78 \\
\hline 161 & 1,1,1-trichloroethane & 2.49 & 2.82 & 2.66 & 2.68 \\
\hline 162 & $p$-dichlorobenzene & 3.52 & 4.12 & 3.39 & 3.47 \\
\hline 163 & 1-bromobutane & 2.75 & 3.09 & 3.16 & 2.84 \\
\hline 164 & $p$-chlorobiphenyl & 4.61 & 4.62 & 4.82 & 4.48 \\
\hline 165 & cyclopropylbenzene & 3.27 & 2.98 & 3.40 & 3.05 \\
\hline 166 & 2,6-dichlorotoluene & 4.29 & 3.80 & 3.34 & 4.15 \\
\hline 167 & allene & 1.45 & 2.03 & 2.59 & 2.07 \\
\hline 168 & $\beta$-phenylethylbromide & 3.09 & 3.45 & 3.23 & 3.69 \\
\hline 169 & 1,3-butadiene & 1.99 & 2.61 & 2.45 & 1.93 \\
\hline 170 & 2-chlorothiophene & 2.54 & 2.57 & 2.66 & 2.34 \\
\hline 171 & 1-bromopentane & 3.37 & 3.64 & 3.21 & 3.40 \\
\hline 172 & $\gamma$-phenylpropylbromide & 3.72 & 3.42 & 3.15 & 4.08 \\
\hline 173 & 1,3-cyclohexadiene & 2.47 & 2.70 & 2.67 & 2.58 \\
\hline 174 & pentamethylbenzene & 4.56 & 3.98 & 4.04 & 5.11 \\
\hline 175 & $p$-dibromobenzene & 3.79 & 3.97 & 3.65 & 3.70 . \\
\hline 176 & 1,4-pentadiene & 2.48 & 2.59 & 2.70 & 2.84 \\
\hline 178 & 1,1-difluoroethane & 0.75 & 1.09 & 1.71 & 1.32 \\
\hline 179 & 1-bromohexane & 3.80 & 4.21 & 3.39 & 3.89 \\
\hline 180 & $m$-xylene & 3.20 & 3.31 & 3.57 & 3.14 \\
\hline 181 & dibenzothiophene & 4.38 & 3.74 & 2.93 & 4.28 \\
\hline
\end{tabular}




\begin{tabular}{|c|c|c|c|c|c|}
\hline \multirow[b]{2}{*}{ ID\# } & \multirow[b]{2}{*}{ Compound name } & \multirow[b]{2}{*}{$\begin{array}{l}\log P \\
\text { (exp.) }\end{array}$} & \multicolumn{3}{|c|}{ Estimated $\log P$} \\
\hline & & & $\begin{array}{c}\mathbf{P C} \\
(k=3)\end{array}$ & $\begin{array}{l}\mathbf{T I} / \mathbf{P C} \\
(k=8)\end{array}$ & $\begin{array}{l}\text { TI/RR } \\
(k=2)\end{array}$ \\
\hline 182 & ethyliodide & 2.00 & 1.71 & 2.68 & 1.59 \\
\hline 183 & trifluoromethylbenzene & 3.01 & 3.13 & 3.26 & 4.11 \\
\hline 184 & 2,3,6-trimethylnaphthalene & 4.73 & 4.39 & 4.36 & 4.48 \\
\hline 185 & difluoromethane & 0.20 & 1.03 & 1.27 & 1.34 \\
\hline 186 & 1,2,4-trifluorobenzene & 2.52 & 2.68 & 3.45 & 3.13 \\
\hline 187 & bromobenzene & 2.99 & 3.44 & 3.58 & 2.85 \\
\hline 188 & hexachloro-1,3-butadiene & 4.78 & 4.40 & 4.44 & 5.00 \\
\hline 189 & vinylbromide & 1.57 & 1.60 & 2.38 & 1.99 \\
\hline 190 & $o$-chlorotoluene & 3.42 & 3.78 & 3.57 & 3.33 \\
\hline 191 & $\alpha$-chlorotoluene & 2.30 & 3.29 & 3.14 & 2.86 \\
\hline 192 & 1,4-cyclohexadiene & 2.30 & 2.76 & 2.69 & 2.67 \\
\hline 193 & 1-bromoheptane & 4.36 & 4.02 & 3.39 & 4.64 \\
\hline 194 & styrene & 2.95 & 3.04 & 2.82 & 3.16 \\
\hline 195 & chlorotrifluoromethane & 1.65 & 2.18 & 2.32 & 2.01 \\
\hline 196 & (dimethyl)phenylphosphine & 2.57 & 3.35 & 3.36 & 4.20 \\
\hline 197 & cycloocta-1,5-diene & 3.16 & 2.54 & 2.72 & 3.24 \\
\hline 198 & tetrachlorocyclohexane & 2.82 & 4.14 & 2.85 & 2.78 \\
\hline 199 & 1-bromooctane & 4.89 & 3.84 & 3.50 & 4.53 \\
\hline 200 & 2-methylnaphthalene & 3.86 & 4.22 & 4.40 & 3.81 \\
\hline 201 & 3-methylthiophene & 2.34 & 2.67 & 2.69 & 2.44 \\
\hline 202 & methylenechloride & 1.25 & 1.44 & 1.39 & 1.42 \\
\hline 203 & hexachlorobenzene & 5.31 & 4.33 & 4.28 & 5.14 \\
\hline 204 & indene & 2.92 & 3.49 & 2.76 & 3.15 \\
\hline 205 & tert-butylbenzene & 4.11 & 3.85 & 3.41 & 4.05 \\
\hline 206 & 1,2-dichloroethane & 1.48 & 2.00 & 1.49 & 1.76 \\
\hline 207 & 1,3,5-trimethylbenzene & 3.42 & 3.42 & 3.81 & 3.72 \\
\hline 208 & phenanthrene & 4.46 & 4.27 & 4.03 & 4.35 \\
\hline 209 & benzene & 2.13 & 3.32 & 2.88 & 2.39 \\
\hline 210 & 3,3,3-trifluoropropylbenzene & 3.31 & 3.18 & 3.53 & 4.48 \\
\hline 211 & $\alpha$-(2,2,2-trichloroethyl)styrene & 4.56 & 3.96 & 3.59 & 4.92 \\
\hline 212 & 2,3-dimethylnaphthalene & 4.40 & 4.35 & 4.40 & 4.43 \\
\hline 213 & 1,3-dichloropropane & 2.00 & 2.05 & 2.02 & 2.50 \\
\hline 214 & 1,2,3,4-tetramethylbenzene & 4.11 & 4.13 & 4.10 & 4.09 \\
\hline 215 & stilbene-t & 4.81 & 4.34 & 3.57 & 4.95 \\
\hline 216 & fluorene & 4.18 & 3.83 & 3.90 & 4.38 \\
\hline 217 & 2-fluoro-3-bromotetrachlorocyclohexane & 3.28 & 3.74 & 3.37 & 3.62 \\
\hline 218 & allylbenzene & 3.23 & 3.05 & 3.02 & 3.16 \\
\hline 219 & carbontetrachloride & 2.83 & 2.71 & 3.27 & 2.51 \\
\hline
\end{tabular}


TABLE III Observed and estimated mutagenic potency [Ln(revertants/nmol)] for ninety-five aromatic and heteroaromatic amines

\begin{tabular}{|c|c|c|c|c|c|}
\hline \multirow[b]{2}{*}{ ID\# } & \multirow[b]{2}{*}{ Compound } & \multirow[b]{2}{*}{$\begin{array}{c}\mathbf{L n}(\mathbf{R})( \\
\text { exp. })\end{array}$} & \multicolumn{3}{|c|}{ Estimated Ln(R) } \\
\hline & & & $\begin{array}{c}\mathbf{P C} \\
(k=4)\end{array}$ & $\begin{array}{l}\text { TI/PC } \\
(k=4)\end{array}$ & $\begin{array}{l}\mathbf{T I} / \mathbf{R} \mathbf{R} \\
(k=3)\end{array}$ \\
\hline 1 & 2-bromo-7-aminofluorene & 2.62 & 2.09 & 0.17 & 0.59 \\
\hline 2 & 2-methoxy-5-methylaniline ( $p$-cresidine) & -2.05 & -2.11 & -1.54 & -2.35 \\
\hline 3 & 5-aminoquinoline & -2.00 & -1.89 & -1.74 & -2.32 \\
\hline 4 & 4-ethoxyaniline ( $p$-phenetidine) & -2.30 & -1.95 & -1.48 & -2.34 \\
\hline 5 & 1-aminonaphthalene & -0.60 & 1.93 & 1.93 & -1.03 \\
\hline 6 & 4-aminofluorene & 1.13 & 2.70 & 2.01 & 1.08 \\
\hline 7 & 2-aminoanthracene & 2.62 & 2.60 & 2.07 & 2.47 \\
\hline 8 & 7-aminofuoranthene & 2.88 & 3.34 & 2.95 & 3.46 \\
\hline 9 & 8-aminoquinoline & -1.14 & -2.10 & -1.96 & -2.60 \\
\hline 10 & 1,7-diaminophenazine & 0.75 & 0.45 & 0.44 & 1.14 \\
\hline 11 & 2-aminonaphthalene & -0.67 & 0.10 & -1.98 & -1.00 \\
\hline 12 & 4-aminopyrene & 3.16 & 1.83 & 2.13 & 2.97 \\
\hline 13 & 3-amino-3'-nitrobiphenyl & -0.55 & 0.78 & -0.38 & -0.94 \\
\hline 14 & 2,4,5-trimethylaniline & -1.32 & -1.81 & -1.81 & -1.74 \\
\hline 15 & 3-aminofluorene & 0.89 & 1.33 & 1.35 & 1.16 \\
\hline 16 & 3,3'-dichlorobenzidine & 0.81 & -0.49 & -0.14 & 0.30 \\
\hline 17 & 2,4-dimethylaniline (2,4-xylidine) & -2.22 & -1.59 & -1.59 & -1.73 \\
\hline 18 & 2,7-diaminofluorene & 0.48 & 0.96 & 0.47 & 0.35 \\
\hline 19 & 3-aminofluoranthene & 3.31 & 2.09 & 2.09 & 2.92 \\
\hline 20 & 2-aminofluorene & 1.93 & 1.07 & 1.09 & 0.82 \\
\hline 21 & 2-amino-4'-nitrobiphenyl & -0.62 & 0.22 & 0.22 & -0.79 \\
\hline 22 & 4-aminobiphenyl & -0.14 & -0.33 & -0.55 & -0.27 \\
\hline 23 & 3-methoxy-4-methylaniline (o-cresidine) & -1.96 & -1.95 & -1.65 & -2.38 \\
\hline 24 & 2-aminocarbazole & 0.60 & 1.00 & 1.42 & 1.19 \\
\hline 25 & 2-amino-5-nitrophenol & -2.52 & -1.45 & -1.54 & -2.44 \\
\hline 26 & 2,2'-diaminobiphenyl & -1.52 & 0.62 & 0.86 & -0.89 \\
\hline 27 & 2-hydroxy-7-aminofluorene & 0.41 & 1.04 & 0.52 & 1.32 \\
\hline 28 & 1-aminophenanthrene & 2.38 & 1.96 & 0.98 & 3.07 \\
\hline 29 & 2,5-dimethylaniline (2,5-xylidine) & -2.40 & -1.54 & -1.54 & -1.34 \\
\hline 30 & 4-amino-2'-nitrobiphenyl & -0.92 & -0.12 & -0.24 & -0.94 \\
\hline 31 & 2-amino-4-methylphenol & -2.10 & -2.40 & -2.30 & -2.58 \\
\hline 32 & 2-aminophenazine & 0.55 & 1.56 & -1.74 & 1.26 \\
\hline 33 & 4-aminophenylsulfide & 0.31 & 0.12 & 0.32 & 0.15 \\
\hline 34 & 2,4-dinitroaniline & -2.00 & -0.61 & -1.62 & -1.49 \\
\hline 35 & 2,4-diaminoisopropylbenzene & -3.00 & -1.94 & -1.30 & -2.41 \\
\hline 36 & 2,4-difluoroaniline & -2.70 & -1.71 & -2.31 & -2.70 \\
\hline 37 & 4,4'-methylenedianiline & -1.60 & -0.37 & -0.65 & -1.53 \\
\hline 38 & 3,3'-dimethylbenzidine & 0.01 & -1.42 & -1.55 & 1.45 \\
\hline 39 & 2-aminofluoranthene & 3.23 & 3.25 & 2.90 & 3.34 \\
\hline 40 & 2-amino-3'-nitrobiphenyl & -0.89 & -0.13 & 0.36 & -0.92 \\
\hline 41 & 1-aminofluoranthene & 3.35 & 3.22 & 2.84 & 3.30 \\
\hline 42 & 4,4'-ethylenebis (aniline) & -2.15 & -0.69 & -1.05 & -1.35 \\
\hline 43 & 4-chloroaniline & -2.52 & -2.29 & -2.41 & -3.01 \\
\hline
\end{tabular}




\begin{tabular}{|c|c|c|c|c|c|}
\hline \multirow[b]{2}{*}{ ID\# } & \multirow[b]{2}{*}{ Compound } & \multirow[b]{2}{*}{$\begin{array}{c}\operatorname{Ln}(\mathbf{R})( \\
\text { exp. })\end{array}$} & \multicolumn{3}{|c|}{ Estimated $\operatorname{Ln}(\mathrm{R})$} \\
\hline & & & $\begin{array}{c}\mathbf{P C} \\
(k=4)\end{array}$ & $\begin{array}{l}\text { TI/PC } \\
(k=4)\end{array}$ & $\begin{array}{l}\text { TI/RR } \\
(k=3)\end{array}$ \\
\hline 44 & 2-aminophenanthrene & 2.46 & 2.64 & 2.11 & 2.52 \\
\hline 45 & 4-fluoroaniline & -3.32 & -2.21 & -2.21 & -2.74 \\
\hline 46 & 9-aminophenanthrene & 2.98 & 2.51 & 2.07 & 2.47 \\
\hline 47 & 3,3'-diaminobiphenyl & -1.30 & -0.60 & -0.73 & -0.79 \\
\hline 48 & 2-aminopyrene & 3.50 & 1.78 & 2.84 & 2.82 \\
\hline 49 & 2,6-dichloro-1,4-phenylenediamine & -0.69 & -2.06 & -1.23 & -1.33 \\
\hline 50 & 2-amino-7-acetamidofluorene & 1.18 & 0.83 & -0.69 & 1.26 \\
\hline 51 & 2,8-diaminophenazine & 1.12 & 1.19 & 1.09 & 0.24 \\
\hline 52 & 6-aminoquinoline & -2.67 & -1.72 & -1.43 & -2.09 \\
\hline$\cdot 53$ & 4-methoxy-2-methylaniline ( $m$-Cresidine) & -3.00 & -1.87 & -1.30 & -2.04 \\
\hline 54 & 3-amino-2'-nitrobiphenyl & -1.30 & -0.03 & -0.09 & -0.79 \\
\hline 55 & 2,4'-diamino-biphenyl & -0.92 & -0.87 & -0.82 & -0.30 \\
\hline 56 & 1,6-diaminophenazine & 0.20 & 0.96 & 1.14 & 0.55 \\
\hline 57 & 4-aminophenyldisulfide & -1.03 & -0.71 & -0.75 & -1.63 \\
\hline 58 & 2-bromo-4,6-dinitroaniline & -0.54 & -1.23 & -0.84 & -2.09 \\
\hline 59 & 2,4-diamino- $n$-butylbenzene & -2.70 & -1.32 & -1.46 & -2.68 \\
\hline 60 & 4-aminophenylether & -1.14 & -0.14 & -0.73 & -1.59 \\
\hline 61 & 2-aminobiphenyl & -1.49 & 0.90 & 2.51 & 0.15 \\
\hline 62 & 1,9-diaminophenazine & 0.04 & 1.32 & 0.60 & 0.60 \\
\hline 63 & 1-aminofluorene & 0.43 & 2.88 & 2.19 & 1.32 \\
\hline 64 & 8-aminofluoranthene & 3.80 & 3.11 & 2.76 & 3.15 \\
\hline 65 & 2-chloroaniline & -3.00 & -1.58 & -2.29 & -1.90 \\
\hline 66 & 2-amino-aaa-trifluorotoluene & -0.80 & -0.45 & -1.85 & -2.35 \\
\hline 67 & 2-amino-1-nitronaphthalene & -1.17 & 0.29 & -0.85 & -1.33 \\
\hline 68 & 3-amino-4'-nitrobiphenyl & 0.69 & 0.06 & -0.03 & 0.47 \\
\hline 69 & 4-bromoaniline & -2.70 & -1.56 & -1.28 & -2.95 \\
\hline 70 & 2-amino-4-chlorophenol & -3.00 & -2.07 & -1.05 & -2.34 \\
\hline 71 & 3,3'-dimethoxybenzidine & 0.15 & -0.98 & -0.37 & 1.40 \\
\hline 72 & 4-cyclohexylaniline & -1.24 & -1.04 & -0.66 & -0.61 \\
\hline 73 & 4-phenoxyaniline & 0.38 & -0.23 & -2.12 & -0.44 \\
\hline 74 & 4,4'-methylenebis (o-ethylaniline) & -0.99 & -1.59 & -0.78 & -0.55 \\
\hline 75 & 2-amino-7-nitrofluorene & 3.00 & 0.82 & -0.12 & 0.96 \\
\hline 76 & Benzidine & -0.39 & -0.79 & -0.95 & -0.91 \\
\hline 77 & 1-amino-4-nitronaphthalene & -1.77 & -0.39 & -1.34 & -1.12 \\
\hline 78 & 4-amino-3'-nitrobiphenyl & 1.02 & -0.02 & -0.12 & 0.36 \\
\hline 79 & 4-amino-4'-nitrobiphenyl & 1.04 & -0.18 & -0.12 & -0.24 \\
\hline 80 & 1-aminophenazine & -0.01 & 2.03 & -1.60 & 0.70 \\
\hline 81 & $4,4^{\prime}$-methylenebis (o-fluoroaniline) & 0.23 & -0.75 & -0.47 & 0.28 \\
\hline 82 & 4-chloro-2-nitroaniline & -2.22 & -1.60 & -1.52 & -2.54 \\
\hline 83 & 3-aminoquinoline & -3.14 & -1.60 & -1.46 & -1.94 \\
\hline 84 & 3-aminocarbazole & -0.48 & 1.03 & -1.17 & -0.62 \\
\hline 85 & 4-chloro-1,2-phenylenediamine & -0.49 & -1.75 & -1.48 & -1.97 \\
\hline 86 & 3-aminophenanthrene & 3.77 & 2.31 & 1.87 & 2.09 \\
\hline
\end{tabular}




\begin{tabular}{llrrrr}
\hline & & & \multicolumn{3}{c}{ Estimated Ln(R) } \\
\cline { 5 - 7 } ID\# & Compound & Ln(R) & PC & TI/PC & TI/RR \\
& exp. & $(k=4)$ & $(k=4)$ & $(k=3)$ \\
\hline 87 & 3,4'-diaminobiphenyl & 0.20 & -0.12 & -0.88 & -0.68 \\
88 & 1-aminoanthracene & 1.18 & 2.96 & 2.43 & 2.95 \\
89 & 1-aminocarbazole & -1.04 & 1.17 & -1.03 & -0.43 \\
90 & 9-aminoanthracene & 0.87 & 2.86 & 2.60 & 2.26 \\
91 & 4-aminocarbazole & -1.42 & 1.27 & -0.94 & -0.31 \\
92 & 6-aminochrysene & 1.83 & 2.34 & 2.99 & 2.67 \\
93 & 1-aminopyrene & 1.43 & 2.37 & 3.35 & 3.30 \\
94 & 4-4'-methylenebis (o-isopropyl-aniline) & -1.77 & -1.40 & -0.59 & 0.39 \\
95 & 2,7-diaminophenazine & 3.97 & 0.34 & 0.47 & 0.13 \\
\hline
\end{tabular}

complexity of vertices defined by Basak and co-workers for hydrogen-filled molecular graphs [49-51], and Balaban's $J$ indices [52-54]. Ninety-eight of the TIs were calculated using POLLY 2.3 [55], while the remaining four $J$ indices were calculated using other in-house software. More information on the set of topological indices calculated by POLLY has been reported in earlier studies $[22,30,56]$. One hundred additional indices, the real-number local vertex invariants (LOVIs) [33, 57, 58], were added to this set of topological indices. The triplet indices, developed by Balaban and coworkers [57] result from a matrix, a main diagonal column vector, and a free term column vector, converting the matrix into a system. of linear equations whose solutions are the local vertex invariants. The notation used to represent the vectors and matrices is as follows:

$$
\begin{aligned}
& A=\text { Adjacency matrix } \\
& V=\text { Vertex degree } \\
& S=\text { Distance sum } \\
& N=\text { Total number of vertices in the graph } \\
& Z=\text { Atomic number } \\
& D=\text { Distance matrix } \\
& \text { I }=\text { Unity matrix. }
\end{aligned}
$$

After the system of $\mathrm{N}$ linear equations is solved, the local vertex invariants, $\mathrm{x}_{\mathrm{i}}$, are assembled into a triplet descriptor based on one of the following operations:

Summation, $\Sigma_{\mathrm{i}} \mathrm{x}_{\mathrm{i}}$

Summation of squares, $\Sigma_{\mathrm{i}} \mathrm{x}_{\mathrm{i}}^{2}$

Summation of square roots, $\Sigma_{\mathrm{i}} \mathrm{x}_{\mathrm{i}}^{1 / 2}$

Sum of inverse square root of cross-product over edges $i j, \Sigma_{\mathrm{ij}}\left(\mathrm{x}_{\mathrm{j}} \mathrm{x}_{\mathrm{j}}\right)^{-1 / 2}$

Product, $N\left(\Sigma_{\mathrm{i}} \mathrm{x}_{\mathrm{I}}\right)^{1 / N}$ 
TABLE IV Symbols and definitions of topological indices

\begin{tabular}{|c|c|}
\hline \multicolumn{2}{|r|}{ Topostructural Indices } \\
\hline $\mathrm{I}_{\mathrm{D}}^{\mathrm{W}}$ & $\begin{array}{l}\text { Information index for the magnitudes of distances between all possible pairs of vertices of a } \\
\text { graph }\end{array}$ \\
\hline $\mathrm{I}_{\mathrm{D}}^{\mathrm{W}}$ & Mean information index for the magnitude of distance \\
\hline W & Wiener index $=$ half-sum of the off-diagonal elements of the distance matrix of a graph \\
\hline $\mathrm{I}^{\mathrm{D}}$ & Degree complexity \\
\hline $\mathrm{H}^{\mathrm{V}}$ & Graph vertex complexity \\
\hline $\mathrm{H}^{\mathrm{D}}$ & Graph distance complexity \\
\hline $\mathrm{IC}$ & Information content of the distance matrix partitioned by frequency of occurrences of distance $h$ \\
\hline $\mathrm{M}_{1}$ & A Zagreb group parameter $=$ sum of square of degree over all vertices \\
\hline $\mathrm{M}_{2}$ & $\begin{array}{l}\text { A Zagreb group parameter = sum of cross-product of degrees over all neighboring (connected) } \\
\text { vertices }\end{array}$ \\
\hline${ }^{\mathrm{h}} \chi$ & Path connectivity index of order $h=0-6$ \\
\hline${ }^{\mathrm{h}} \chi_{\mathrm{c}}$ & Cluster connectivity index of order $h=3-6$ \\
\hline${ }^{\mathrm{h}} \chi_{\mathrm{Ch}}$ & Chain connectivity index of order $h=3-6$ \\
\hline${ }^{\mathrm{b}} \chi_{\mathrm{PC}}$ & Path-cluster connectivity index of order $h=4-6$ \\
\hline$P_{h}$ & Number of paths of length $h=0-10$ \\
\hline $\mathbf{J}$ & Balaban's J index based on distance \\
\hline Nrings & Number of rings in a graph \\
\hline Ncirc & Number of circuits in a graph \\
\hline $\mathrm{DN}^{2} \mathrm{~S}_{\mathrm{y}}$ & $\begin{array}{l}\text { Triplet index from distance matrix, square of graph order (\# of non-H atoms), and distance sum; } \\
\text { operation } y=1-5\end{array}$ \\
\hline $\mathrm{DN}^{2} 1_{\mathrm{y}}$ & Triplet index from distance matrix, square of graph order, and number 1 ; operation $y=1-5$ \\
\hline $\mathrm{AS}_{\mathrm{y}}$ & $\begin{array}{l}\text { Triplet index from adjacency matrix, distance sum, and number } 1 \text {; } \\
\text { operation } y=1-5\end{array}$ \\
\hline $\mathrm{DSl}_{\mathrm{y}}$ & $\begin{array}{l}\text { Triplet index from distance matrix, distance sum, and number } 1 \text {; } \\
\text { operation } y=1-5\end{array}$ \\
\hline $\mathrm{ASN}_{\mathrm{y}}$ & Triplet index from adjacency matrix, distance sum, and graph order; operation $y=1-5$ \\
\hline $\mathrm{DSN}_{\mathrm{y}}$ & $\begin{array}{l}\text { Triplet index from distance matrix, distance sum, and graph order; } \\
\text { operation } y=1-5\end{array}$ \\
\hline $\mathrm{DN}^{2} \mathrm{~N}_{\mathrm{y}}$ & Triplet index from distance matrix, square of graph order, and graph order; operation y $=1-5$ \\
\hline $\mathrm{ANS}_{\mathrm{y}}$ & Triplet index from adjacency matrix, graph order, and distance sum; operation $y=1-5$ \\
\hline $\mathrm{AN} 1_{\mathrm{y}}$ & $\begin{array}{l}\text { Triplet index from adjacency matrix, graph order, and number } 1 \text {; } \\
\text { operation } y=1-5\end{array}$ \\
\hline $\mathrm{ANN}_{\mathrm{y}}$ & Triplet index from adjacency matrix, graph order, and graph order again; operation y $=1-5$ \\
\hline $\mathrm{ASV}_{\mathrm{y}}$ & Triplet index from adjacency matrix, distance sum, and vertex degree; operation $y=1-5$. \\
\hline $\operatorname{DSV}_{\mathrm{y}}$ & Triplet index from distance matrix, distance sum, and vertex degree; operation $y=1-5$ \\
\hline $\mathrm{ANV}_{\mathrm{y}}$ & Triplet index from adjacency matrix, graph order, and vertex degree; operation $y=1-5$ \\
\hline \multicolumn{2}{|r|}{ Topochemical Indices } \\
\hline I ORB & $\begin{array}{l}\text { Information content or complexity of the hydrogen-suppressed graph at its maximum } \\
\text { neighborhood of vertices }\end{array}$ \\
\hline $\mathrm{O}$ & Order of neighborhood when $\mathrm{IC}_{\mathrm{r}}$ reaches its maximum value for the hydrogen-filled graph \\
\hline $\mathrm{O}_{\mathrm{ORB}}$ & Order of neighborhood when $\mathrm{IC}_{\mathrm{r}}$ reaches its maximum value for the hydrogen-suppressed graph \\
\hline
\end{tabular}


TABLE IV (Continued)

\begin{tabular}{|c|c|}
\hline $\mathrm{IC}_{\mathrm{r}}$ & $\begin{array}{l}\text { Mean information content or complexity of a graph based on the } \mathrm{r}^{\text {th }}(\mathrm{r}=0-6) \text { order neighborhood } \\
\text { of vertices in a hydrogen-filled graph }\end{array}$ \\
\hline $\mathrm{SIC}_{\mathrm{r}}$ & $\begin{array}{l}\text { Structural information content for } \mathrm{r}^{\text {th }}(\mathrm{r}=0-6) \text { order neighborhood of vertices in a hydrogen- } \\
\text { filled graph }\end{array}$ \\
\hline $\mathrm{CIC}_{\mathrm{r}}$ & $\begin{array}{l}\text { Complementary information content for } r^{\text {th }}(r=0-6) \text { order neighborhood of vertices in a } \\
\text { hydrogen-filled graph }\end{array}$ \\
\hline${ }^{\mathrm{h}} \chi^{\mathrm{b}}$ & Bond path connectivity index of order $h=0-6$ \\
\hline${ }^{\mathrm{b}} \chi_{\mathrm{C}}^{\mathrm{b}}$ & Bond cluster connectivity index of order $h=3-6$ \\
\hline${ }^{\mathrm{h}} \chi_{\text {Ch }}^{\mathrm{b}}$ & Bond chain connectivity index of order $h=3-6$ \\
\hline${ }^{\mathrm{b}} \chi_{\mathrm{PC}}^{\mathrm{b}}$ & Bond path-cluster connectivity index of order $h=4-6$ \\
\hline${ }^{\mathrm{h}} \chi^{\mathrm{v}}$ & Valence path connectivity index of order $h=0-6$ \\
\hline${ }^{\mathrm{h}} \chi_{\mathrm{C}}^{\mathrm{v}}$ & Valence cluster connectivity index of order $h=3-6$ \\
\hline${ }^{\mathrm{h}} \chi_{\mathrm{Ch}}^{\mathrm{v}}$ & Valence chain connectivity index of order $h=3-6$ \\
\hline${ }^{\mathrm{h}} \chi_{\mathrm{PC}}^{\mathrm{v}}$ & Valence path-cluster connectivity index of order $h=4-6$ \\
\hline $\mathbf{J}^{\mathbf{B}}$ & Balaban's J index based on bond types \\
\hline $\mathrm{J}^{\mathrm{X}}$ & Balaban's $\mathrm{J}$ index based on relative electronegativities \\
\hline$J^{Y}$ & Balaban's J index based on relative covalent radii \\
\hline$A Z V_{y}$ & Triplet index from adjacency matrix, atomic number, and vertex degree; operation $y=1-5$ \\
\hline $\mathrm{AZS}_{\mathrm{y}}$ & Triplet index from adjacency matrix, atomic number, and distance sum; operation $y=1-5$ \\
\hline $\mathrm{ASZ}_{\mathrm{y}}$ & Triplet index from adjacency matrix, distance sum, and atomic number; operation $y=1-5$ \\
\hline $\mathrm{AZN}_{\mathrm{y}}$ & Triplet index from adjacency matrix, atomic number, and graph order; operation y $=1-5$ \\
\hline $\mathrm{ANZ}_{\mathrm{y}}$ & Triplet index from adjacency matrix, graph order, and atomic number; operation $y=1-5$ \\
\hline $\mathrm{DSZ}_{\mathrm{y}}$ & Triplet index from distance matrix, distance sum, and atomic number; operation $y=1-5$ \\
\hline $\mathrm{DN}^{2} \mathrm{Z}_{\mathrm{y}}$ & Triplet index from distance matrix, square of graph order, and atomic number; operation $y=1-5$ \\
\hline Nvx & Number of non-hydrogen atoms in a molecule \\
\hline Nelem & Number of elements in a molecule \\
\hline Fw & Molecular weight \\
\hline${ }^{\mathrm{h}} \chi^{\mathrm{v}}$ & Valence path connectivity index of order $h=7-10$ \\
\hline${ }^{b} \chi_{C h}^{v}$ & Valence chain connectivity index of order $h=7-10$ \\
\hline Si & Shannon information index \\
\hline Totop & Total Topological Index $t$ \\
\hline SumI & Sum of the intrinsic state values I \\
\hline SumdelI & Sum of delta-I values \\
\hline Tets2 & Total topological state index based on electrotopological state indices \\
\hline Phia & Flexibility index $(\mathrm{kp} 1 * \mathrm{kp} 2 / \mathrm{nvx})$ \\
\hline $\mathrm{I}_{\mathrm{C}}^{\mathrm{D}}$ & Bonchev-Trinajstic mean information index \\
\hline $\mathrm{I}_{\mathrm{C}}^{\mathrm{D}}$ & Bonchev-Trinajstic information index \\
\hline$W_{p}$ & Wiener $p$ \\
\hline Pf & Platt $f$ \\
\hline $\mathrm{Wt}$ & Total Wiener number \\
\hline Knotp & Difference of chi-cluster-3 and path/cluster- 4 \\
\hline Knotpv & Valence difference of chi-cluster-3 and path/cluster- 4 \\
\hline Nclass & Number of classes of topologically (symmetry) equivalent graph vertices \\
\hline NumHBd & Number of hydrogen bond donors \\
\hline NumHBa & Number of hydrogen bond acceptors \\
\hline
\end{tabular}


TABLE IV (Continued)

\begin{tabular}{|c|c|}
\hline SHCsats & E-State of $\mathrm{C} \mathrm{sp}^{3}$ bonded to other saturated C atoms \\
\hline SHCsatu & E-State of $\mathrm{C} \mathrm{sp}{ }^{3}$ bonded to unsaturated $\mathrm{C}$ atoms \\
\hline Shvin & E-State of $\mathrm{C}$ atoms in the vinyl group, $=\mathrm{CH}-$ \\
\hline Shtvin & E-State of $\mathrm{C}$ atoms in the terminal vinyl group, $=\mathrm{CH}_{2}$ \\
\hline Shavin & E-State of $\mathrm{C}$ atoms in the vinyl group, $=\mathrm{CH}-$, bonded to an aromatic $\mathrm{C}$ \\
\hline Sharom & E-State of $\mathrm{C} \mathrm{sp}{ }^{2}$ which are part of an aromatic system \\
\hline SHHBd & $\begin{array}{l}\text { Hydrogen bond donor index, sum of Hydrogen E-State values for }-\mathrm{OH},=\mathrm{NH} \text {, } \\
-\mathrm{NH} 2,-\mathrm{NH}-,-\mathrm{SH} \text {, and } \# \mathrm{CH}\end{array}$ \\
\hline SHwHBd & $\begin{array}{l}\text { Weak hydrogen bond donor index, sum of } \mathrm{C}-\mathrm{H} \text { Hydrogen E-State values for hydrogen atoms on } \\
\text { a } \mathrm{C} \text { to which a } \mathrm{F} \text { and/or } \mathrm{Cl} \text { are also bonded }\end{array}$ \\
\hline SHHBa & $\begin{array}{l}\text { Hydrogen bond acceptor index, sum of the E-State values for }-\mathrm{OH},=\mathrm{NH} \text {, } \\
-\mathrm{NH} 2,-\mathrm{NH}-,>\mathrm{N}-,-\mathrm{O}-,-\mathrm{S}-\text {, along with }-\mathrm{F} \text { and }-\mathrm{Cl}\end{array}$ \\
\hline Qv & General Polarity descriptor \\
\hline NHBinty & Count of potential internal hydrogen bonders $(y=2-10)$ \\
\hline \multirow[t]{3}{*}{ SHBinty } & E-State descriptors of potential internal hydrogen bond strength $(y=2-10)$ \\
\hline & Electrotopological State index values for atoms types: \\
\hline & $\begin{array}{l}\text { SHsOH, SHdNH, SHsSH, SHsNH2, SHssNH, SHtCH, Shother, SHCHnX, Hmax Gmax, } \\
\text { Hmin, Gmin, Hmaxpos, Hminneg, SsLi, SssBe, Sssss,Bem, SssBH ,SsssB, SssssBm, } \\
\text { SsCH3, SdCH2, SssCH2, StCH, SdsCH, SaaCH, SsssCH, SddC,StsC, SdssC, SaasC, } \\
\text { SaaaC, SssssC, SsNH3p, SsNH2, SssNH2p, SdNH, SssNH, SaaNH, StN, SsssNHp, SdsN, } \\
\text { SaaN, SsssN, SddsN, SaasN, SssssNp, SsOH, SdO, SssO, SaaO, SsF, SsSiH3, SssSiH2, } \\
\text { SsssSiH, SssssSi, SsPH2, SssPH, SsssP, SdsssP, SsssssP, SsSH, SdS, SssS, SaaS, SdssS, } \\
\text { SddssS, SssssssS, SsC1, SsGeH3, SssGeH2, SsssGeH, SssssGe, SsAsH2, SssAsH, SsssAs, } \\
\text { SdsssAs, SsssssAs, SsSeH, SdSe, SssSe, SaaSe, SdssSe, SddssSe, SsBr, SsSnH3, SssSnH2, } \\
\text { SsssSnH, SssssSn, SsI, SsPbH3, SssPbH2, SsssPbH, SssssPb }\end{array}$ \\
\hline kp0 & Kappa zero \\
\hline kp1-kp3 & Kappa simple indices \\
\hline ka1-ka3 & Kappa alpha indices \\
\hline
\end{tabular}

The triplet indices were calculated using in-house software. Finally, Molconn- $Z$ v3.50 [59] was used to calculate an additional 167 topological and electrotopological indices. This brought the total number of indices used in this study to 369 . These sets of topological indices have recently been analyzed in detail to provide an overview of their utility [31,60]. Table 4 presents a comprehensive list of the topological indices included in this study.

\section{Data Reduction}

Initially, almost all of the TIs were transformed by the natural logarithm of the index plus one. Some TIs may be several orders of magnitude greater than others, so the scaling is conducted to minimize the effect of scale. However, minimal values for some of the Molconn-Z parameters were much less than zero. These indices were logarithmically scaled on a case by case basis. This was done using the natural logarithm of the index plus $x$, where $x$ was an integer large enough to make the minimal value of the 
index greater than zero. Next, correlation analysis was conducted on the indices. In all cases of a perfect correlation between several indices, only one of the indices was retained within the descriptor set.

\section{Statistical Analysis (PCA and Ridge Regression)}

Principal component analysis (PCA) was used on the transformed indices to minimize the intercorrelation of indices. The PCA was conducted using the SAS procedure PRINCOMP [61]. Only PCs with eigenvalues greater than or equal to one have been retained for this study. A more detailed explanation of this approach has been provided in a previous study by Basak et al [23]. These PCs were subsequently used as independent variables (in place of the TIs) to determine similarity scores in the Euclidean distance method described below. After the PCA, a correlation analysis was conducted on the PCs to determine which TIs were most highly correlated with each of the PCs. This not only allows us to select a small set of TIs for the creation of similarity spaces instead of using the PCs, but it can also provide some incite into the general nature of the principal components, i.e., what aspects of molecular structure are explained by the PCs $[22,62,63]$.

Additionally, ridge regression (RR) [64], a method wherein the entire set of descriptors is retained as opposed to subset regression was conducted on the sets of indices retained after the data reduction step. This regression method is useful in cases where the descriptors are highly multicollinear and where the number of descriptors is substantially larger than the number of observations [65]. Conceptually, RR can be thought of as recasting the regression as one using the principal components of the predictor variables as new predictors. It differs in that in principal component regression the leading components are retained and used just as in ordinary least squares regression while the trailing components are dropped. $\mathrm{RR}$ retains all components, but downweights each of them in accordance with the component's eigenvalue and the 'ridging constant' $k$.

The ridge parameter $k$ controls the amount of smoothing in ridge regression. If $k$ is large, then all regression coefficients are 'shrunk' towards zero. Smaller $k$ values shrink the directions of small eigenvalue substantially, but the directions with large eigenvalue less so. A suitable value for $k$ needs to be found when performing ridge regression. In the current study, the $k$ value was chosen to minimize the prediction sum of squares (PRESS), a cross-validation measure. As in jack-knifing, each compound in turn is temporarily omitted from the data set and the RR fitted to the remaining compounds. The resulting model is used to predict the compound that was held back. PRESS is the sum of squares of the differences between the actual values and these holdout predictions. The cross-validated $R^{2}$ is defined in terms of PRESS and provides an honest measure of the predictive power of the modeling approach. 


\section{Quantification of Molecular Similarity}

Intermolecular similarity was measured using Euclidean distance $(E D)$ within an $n$-dimensional space derived from TIs or PCs. The $E D$ between two molecules, $i$ and $j$, is defined as:

$$
E D_{i j}=\left[\sum_{k=1}^{n}\left(D_{i k}-D_{j k}\right)^{2}\right]^{1 / 2}
$$

where $n$ equals the number of dimensions utilized, whether those dimensions are derived from TIs or PCs. $\mathrm{D}_{i k}$ and $\mathrm{D}_{j k}$ are the data values of the $k^{\text {th }}$ dimension for molecules $i$ and $j$, respectively.

\section{Property Estimation}

Property estimation was carried out using the $k$-nearest neighbor (KNN) method. For each compound, a number of similar chemicals $(k=1-10,15,20,25)$ are selected and the property of interest is estimated based on the values of these nearest neighbors. For instance, in estimating the $\log P$ of the probe compound, the mean $\log P$ for the $k$-nearest neighbors was used as the estimate. KNN estimation was carried out for all chemicals in both of the data sets, resulting in a full cross-validation. Thus the correlation coefficients reported are the cross-validated correlation coefficients.

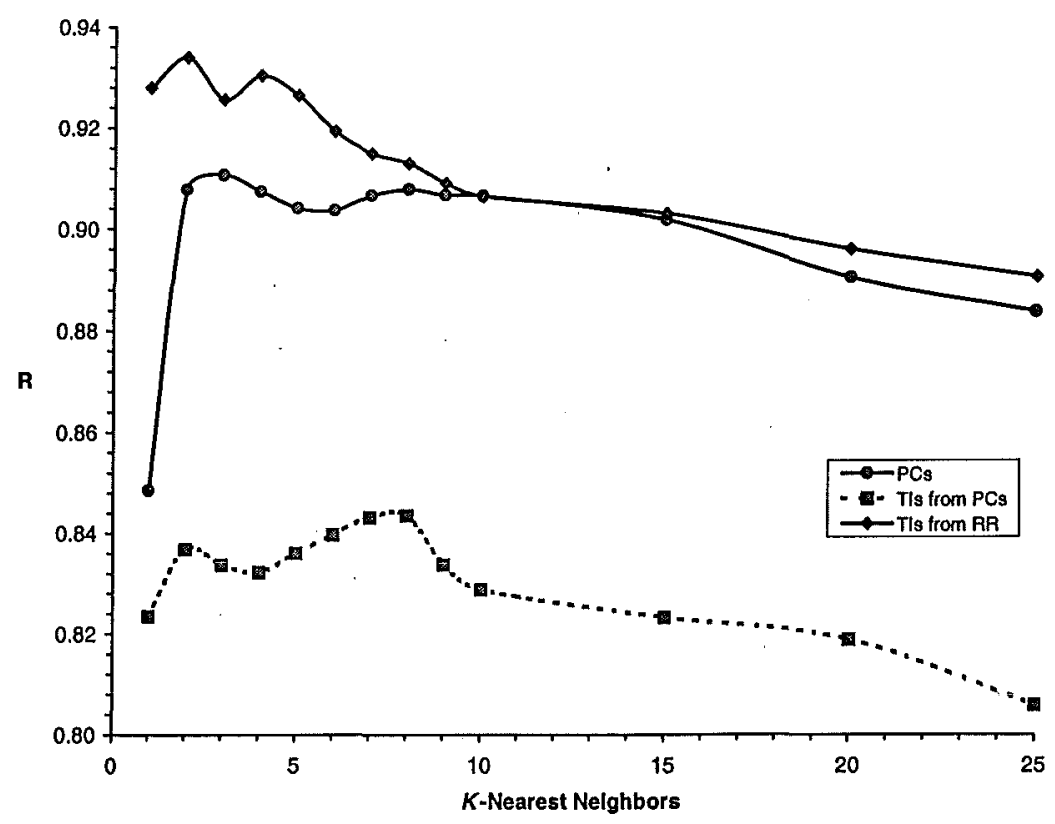

FIGURE 1 Pattern of correlation for the three similarity methods on the STARLIST set. 


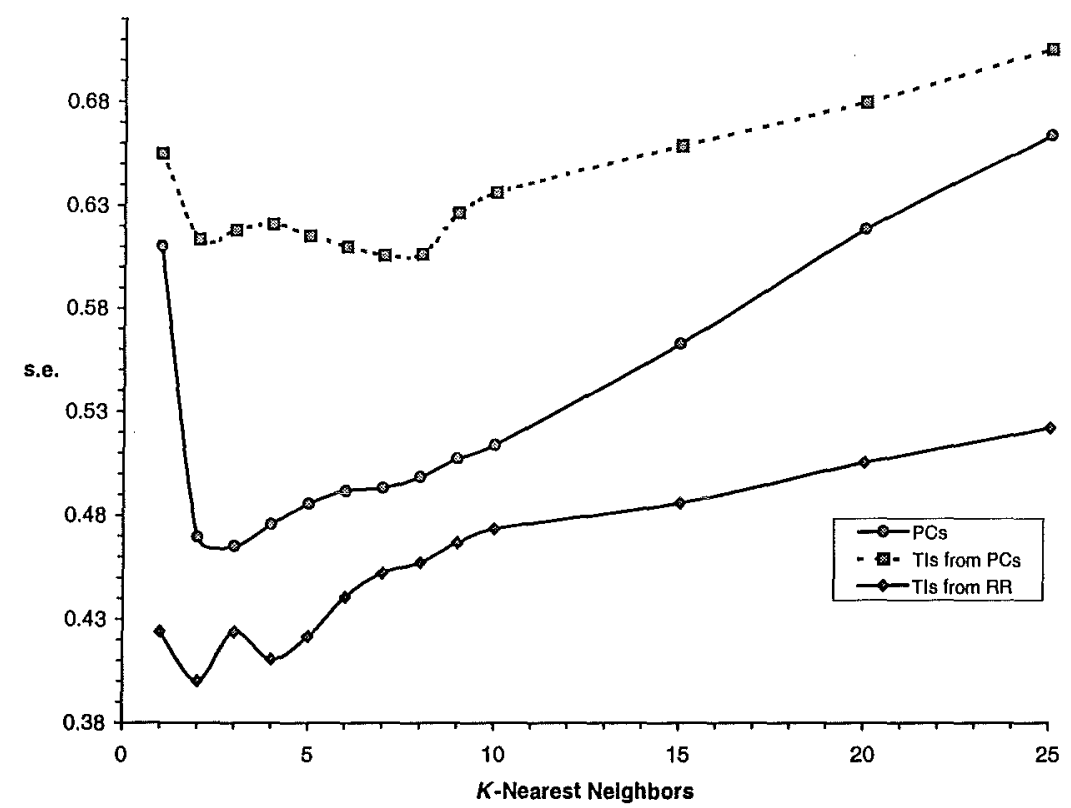

FIGURE 2 Pattern of standard error for the three similarity methods on the STARLIST set.

\section{RESULTS AND DISCUSSION}

The principal objective of this paper was to determine whether similarity spaces tailored to a specific property could be more useful in the prediction of that property from selected analogs, as compared to spaces which are constructed from orthogonal, or a minimally correlated subset of, descriptors derived from an arbitrary collection of indices. To this end, we used three spaces, viz., a principal component space, a topological index space based on the indices most highly correlated with each PC, and a topological index space based on the weighting of the indices as determined through ridge regression analysis.

For the STARLIST $\log P$ set, consisting of a group of 213 diverse structures, we selected analogs using three distinct similarity methods. These methods all began using the set of 252 calculated molecular descriptors (TIs) remaining after data reduction. PCA resulted in the selection of the first twenty-four principal components, all of which had eigenvalues greater than or equal to one (see Table 5). Also indicated on Table 5 are the two TIs most correlated with each of the PCs. Next, RR was carried out on the set of 252 TIs. Model coefficients were extracted and used to rank the TIs from most to least influential based on the absolute value of the regression coefficient. In this manner, a set of twenty-four topological indices were selected for creation of the "tailored" similarity space (see Table 6). Finally, three Euclidean distance-based similarity spaces were constructed based on: 1) twenty-four PCs derived from the set of 252 calculated TIs, b) twenty-four TIs, each one being the most correlated with one of the 
first twenty-four PCs, and c) the set of the twenty-four TIs having the most significant contribution ( $t$ value) in modeling $\log P$ using the RR method. To compare the methods, the $k$-nearest neighbors ( $k=1$ $10,15,20,25)$ for each chemical were chosen using each of the QMSA methods and the arithmetic mean of the $\log P$ values for the selected analogs was taken as the estimated $\log P$ of the compound under investigation. It is clear from the results (see Figures $1 \& 2$ ) that the tailored similarity space based on the indices selected by the RR method gives a much better estimate of $\log P$ as compared to the two nontailored methods.

TABLE V Summary of the first twenty-four principal components derived from a set of 252 topological indices calculated for a set of 213 STARLIST chemicals

\begin{tabular}{|c|c|c|c|c|c|c|c|}
\hline \multirow{2}{*}{$\frac{P C}{1}$} & \multirow{2}{*}{$\frac{\text { Eigenvalue }}{121.74}$} & \multirow{2}{*}{$\begin{array}{c}\begin{array}{c}\text { Proportion of } \\
\text { Variance } \\
\text { Explained }\end{array} \\
0.483\end{array}$} & \multirow{2}{*}{$\begin{array}{c}\begin{array}{c}\text { Cumulative } \\
\text { Variance } \\
\text { Explained }\end{array} \\
0.483\end{array}$} & \multicolumn{2}{|c|}{$\begin{array}{c}\text { First Most Correlated } \\
\text { TI } \\
\end{array}$} & \multicolumn{2}{|c|}{$\begin{array}{c}\text { Second Most } \\
\text { Correlated TI }\end{array}$} \\
\hline & & & & $\mathrm{DN}^{2} \mathrm{~N}_{3}$ & 0.993 & $\mathrm{P}_{0}$ & 0.993 \\
\hline 2 & 25.31 & 0.100 & 0.583 & SssssC & -0.748 & ${ }^{3} \chi^{b}{ }_{C}$ & 0.719 \\
\hline 3 & 17.23 & 0.068 & 0.651 & $\mathrm{IC}_{0}$ & 0.849 & $\mathrm{CIC}_{3}$ & -0.822 \\
\hline 4 & 14.37 & 0.057 & 0.708 & $\mathrm{~J}$ & -0.667 & ${ }^{5} \chi^{\mathrm{V}}{ }_{\mathrm{Ch}}$ & 0.598 \\
\hline 5 & 11.29 & 0.045 & 0.753 & Phia & 0.875 & $\mathrm{Ka}_{2}$ & 0.745 \\
\hline 6 & 7.38 & 0.029 & 0.782 & $\mathrm{~J}^{\mathrm{X}}$ & -0.532 & ${ }^{10} \chi_{\mathrm{PC}}$ & 0.494 \\
\hline 7 & 6.55 & 0.026 & 0.808 & Gmin & 0.552 & $\mathrm{SsF}$ & -0.533 \\
\hline 8 & 4.69 & 0.019 & 0.827 & SHwHBd & -0.590 & SHCHnX & -0.585 \\
\hline 9 & 4.03 & 0.016 & 0.843 & $\mathrm{SsCH}_{3}$ & 0.681 & Qv & 0.391 \\
\hline 10 & 3.22 & 0.013 & 0.856 & NumHBd & -0.589 & $\mathrm{SsSH}$ & -0.589 \\
\hline 11 & 2.98 & 0.012 & 0.868 & SHsSH & 0.724 & $\mathrm{SsSH}$ & 0.723 \\
\hline 12 & 2.60 & 0.010 & 0.878 & Shvin & 0.543 & $\mathrm{SdsCH}$ & 0.542 \\
\hline 13 & 2.50 & 0.010 & 0.888 & ${ }^{10} \chi_{\mathrm{Ch}}$ & -0.390 & ${ }^{10} \chi^{v} \mathrm{Ch}$ & -0.379 \\
\hline 14 & 2.14 & 0.008 & 0.896 & ${ }^{9} \chi_{\mathrm{Ch}}^{\mathrm{y}}$ & 0.672 & ${ }^{9} \chi_{\mathrm{Ch}}$ & 0.660 \\
\hline 15 & 1.91 & 0.008 & 0.904 & ${ }^{10} \chi_{\mathrm{Ch}}^{\mathrm{v}}$ & -0.382 & ${ }^{10} \chi_{\mathrm{Ch}}$ & -0.380 \\
\hline 16 & 1.81 & 0.007 & 0.911 & ${ }^{3} \chi_{\mathrm{Ch}}$ & -0.545 & ${ }^{4} \chi_{\mathrm{ch}}$ & -0.390 \\
\hline 17 & 1.67 & 0.007 & 0.918 & $\mathrm{SsBr}$ & 0.375 & $\mathrm{SsCl}$ & -0.355 \\
\hline 18 & 1.49 & 0.006 & 0.924 & ${ }^{8} \chi_{\mathrm{Ch}}$ & 0.569 & ${ }^{8} \chi^{\mathrm{v}} \mathrm{Ch}$ & 0.509 \\
\hline 19 & 1.42 & 0.006 & 0.930 & SsI & -0.298 & SdssC & 0.283 \\
\hline 20 & 1.23 & 0.005 & 0.935 & SaaS & 0.340 & SaasC & -0.355 \\
\hline 21 & 1.15 & 0.005 & 0.940 & SHCsatu & -0.394 & SddC & 0.265 \\
\hline 22 & 1.11 & 0.004 & 0.944 & SdssC & 0.583 & SsssP & -0.372 \\
\hline 23 & 1.08 & 0.004 & 0.948 & StsC & -0.521 & SsI & 0.489 \\
\hline 24 & 1.02 & 0.004 & 0.952 & SsssP & -0.598 & $\mathrm{SssNH}_{2} \mathrm{P}$ & 0.568 \\
\hline
\end{tabular}


TABLE VI Twenty-four TIs selected by PCA and RR for the 213 STARLIST chemicals. Bold typeface has been used to highlight the indices common to both sets of TIs

\begin{tabular}{|c|c|c|}
\hline PC & TIs from PCA & TIs from RR ( $t$-value) \\
\hline 1 & $\mathrm{DN}^{2} \mathrm{~N}_{3}$ & $\mathrm{ka}_{1} \quad(12.41)$ \\
\hline 2 & SssssC & W (11.95) \\
\hline 3 & $\mathrm{IC}_{0}$ & ${ }^{0} \chi^{v}(11.62)$ \\
\hline 4 & $\mathrm{~J}$ & $\mathrm{P}_{0}(11.32)$ \\
\hline 5 & Phia & $\mathrm{ANN}_{3}(11.31)$ \\
\hline 6 & $\mathrm{~J}^{\mathrm{X}}$ & $\mathrm{DN}^{2} \mathrm{~S}_{3}(11.12)$ \\
\hline 7 & Gmin & SHwHBd (-10.87) \\
\hline 8 & SHwHBd & $\mathrm{ANS}_{1}(10.66)$ \\
\hline 9 & $\mathrm{SsCH}_{3}$ & $\mathrm{ANN}_{1}(10.55)$ \\
\hline 10 & NumHBd & $\mathrm{AN}_{3}(10.47)$ \\
\hline 11 & SHsSH & $\mathrm{ANN}_{5}(10.39)$ \\
\hline 12 & Shvin & ${ }^{0} \chi(10.14)$ \\
\hline 13 & ${ }^{10} \chi_{C h}$ & $\mathrm{DN}^{2} 1_{4}(9.92)$ \\
\hline 14 & ${ }^{9} \chi_{\mathrm{Ch}}^{v}$ & SHCHnX $(-9.92)$ \\
\hline 15 & ${ }^{10} \chi^{v} \mathrm{Ch}$ & ${ }^{0} \chi^{b}(9.65)$ \\
\hline 16 & ${ }^{3} \chi_{\mathrm{Ch}}$ & $\mathrm{ASl}_{4}(9.32)$ \\
\hline 17 & $\mathrm{SsBr}$ & $\mathrm{DN}^{2} \mathrm{~S}_{1}$ \\
\hline 18 & ${ }^{8} \chi_{\mathrm{Ch}}$ & $\mathrm{DN}^{2} \mathrm{~N}_{4}(9.09)$ \\
\hline 19 & SsI & $\mathrm{DN}^{2} \mathrm{~N}_{3}(9.00)$ \\
\hline 20 & Saas & $\mathrm{DN}^{2} \mathrm{~S}_{5}(8.89)$ \\
\hline 21 & SHCsatu & SssS $(-8.74)$ \\
\hline 22 & SdssC & $\mathrm{ANN}_{2}(8.33)$ \\
\hline 23 & $\mathrm{StsC}$ & SaasC (8.16) \\
\hline 24 & SsssP & SsssP (-8.14) \\
\hline
\end{tabular}

An analogous situation is found with the set of ninety-five aromatic and heteroaromatic amines. 267 indices were retained after data reduction for this data set. PCA resulted in twenty principal components with eigenvalues greater than or equal to one (see Table 7). As with the STARLIST $\log P$ set, Table 7 also indicates the two TIs most correlated with each of the principal components. Table 8 lists the TIs selected from the RR analysis. As before, the number of TIs selected by RR (twenty) was selected to match the number of PCs with eigenvalues greater than or equal to one for creation of the "tailored" similarity space. Finally, the three Euclidean distance-based similarity spaces were constructed based on: 1) twenty PCs derived from the set of 267 calculated TIs, b) the twenty TIs most correlated with the first twenty PCs, and c) the set of the twenty TIs having the most significant contribution ( $t$ value) in modeling mutagenicity using the RR method. Here, the similarity method based on indices chosen by the RR method also gives a better estimate of mutagenicity as compared to the methods based either on the PCs or indices most correlated with the first twenty PCs with eigenvalues greater than or equal to one. (Figures $3 \& 4$ ). 


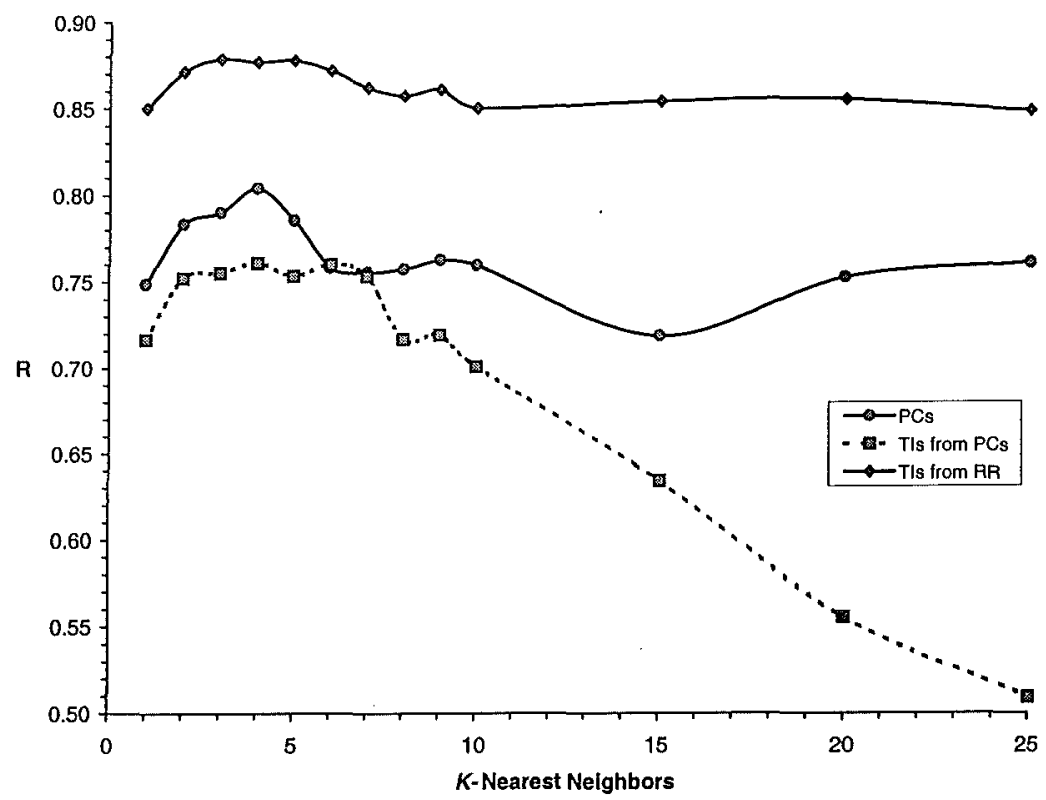

FIGURE 3 Pattern of correlation for the three similarity methods on the set of ninety-five aromatic and heteroaromatic amines.

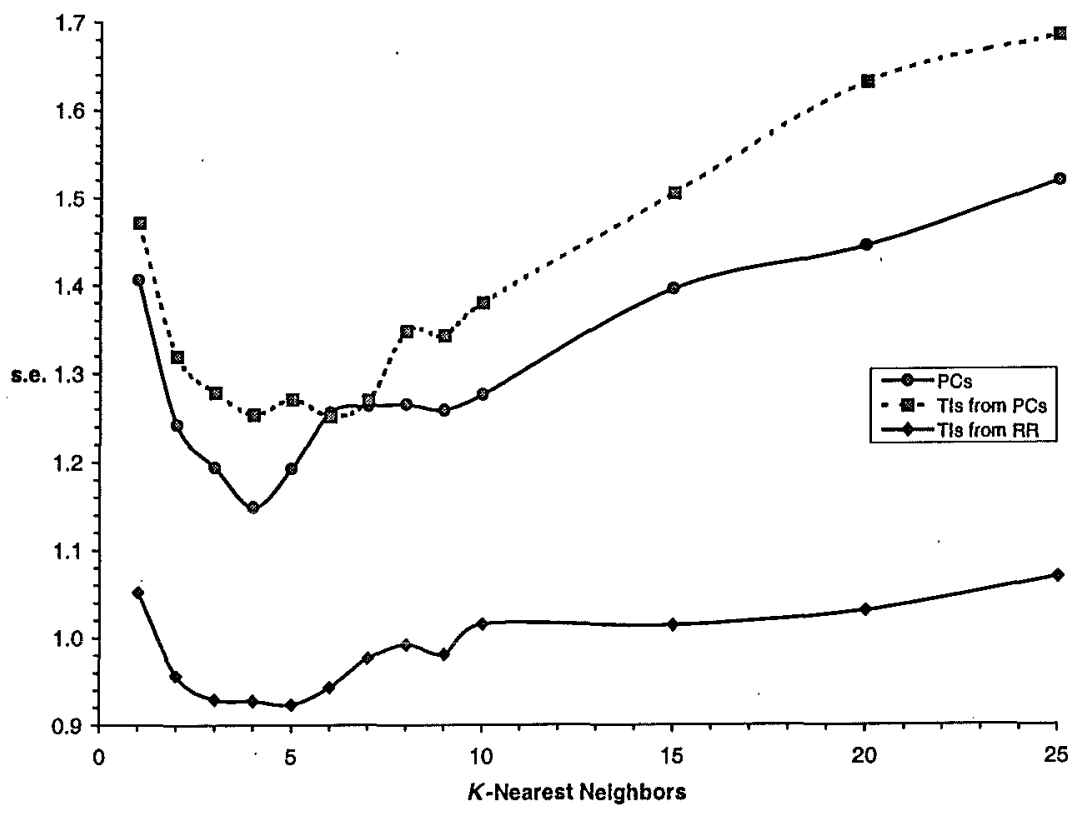

FIGURE 4 Pattern of standard error for the three similarity methods on the set of ninety-five aromatic and heteroaromatic amines. 
TABLE VII Summary of the first twenty principal components derived from a set of 267 TIs calculated for a set of 95 aromatic and heteroaromatic amines

\begin{tabular}{|c|c|c|c|c|c|c|c|}
\hline \multirow{2}{*}{$\frac{\mathbf{P C}}{1}$} & \multirow{2}{*}{$\begin{array}{c}\text { Eigenvalue } \\
141.47\end{array}$} & \multirow{2}{*}{$\begin{array}{c}\begin{array}{c}\text { Proportion of } \\
\text { Variance } \\
\text { Explained }\end{array} \\
0.532\end{array}$} & \multirow{2}{*}{$\begin{array}{c}\begin{array}{c}\text { Cumulative } \\
\text { Variance } \\
\text { Explained }\end{array} \\
0.532\end{array}$} & \multicolumn{2}{|c|}{$\begin{array}{c}\text { First Most Correlated } \\
\text { TI }\end{array}$} & \multicolumn{2}{|c|}{$\begin{array}{l}\text { Second Most } \\
\text { Correlated TI }\end{array}$} \\
\hline & & & & $\mathrm{AZV}_{3}$ & 0.994 & $A Z V_{1}$ & 0.993 \\
\hline 2 & 34.28 & 0.129 & 0.661 & $\mathrm{ASN}_{2}$ & 0.887 & $\mathrm{ka}_{3}$ & -0.879 \\
\hline 3 & 17.04 & 0.064 & 0.725 & SumdelI & 0.799 & SHHBa & 0.746 \\
\hline 4 & 10.41 & 0.039 & 0.764 & Knotpv & -0.539 & ${ }^{10} \chi^{\mathrm{v}}{ }_{\mathrm{PC}}$ & 0.528 \\
\hline 5 & 8.69 & 0.033 & 0.797 & Phia & 0.560 & Nvx & -0.556 \\
\hline 6 & 8.34 & 0.031 & 0.828 & Hmin & -0.666 & $\mathrm{IC}_{2}$ & 0.645 \\
\hline 7 & 6.06 & 0.023 & 0.851 & $\mathrm{ANZ}_{1}$ & 0.497 & $\mathrm{SsCH}_{3}$ & -0.476 \\
\hline 8 & 4.95 & 0.019 & 0.869 & SHHBd & 0.602 & NumHBd & 0.553 \\
\hline 9 & 3.85 & 0.015 & 0.884 & ${ }^{4} \chi_{\mathrm{c}}$ & -0.649 & $\mathrm{SsF}$ & -0.638 \\
\hline 10 & 3.11 & 0.012 & 0.896 & $\mathrm{ShsOH}$ & 0.487 & $\mathrm{SsOH}$ & 0.487 \\
\hline 11 & 2.76 & 0.010 & 0.906 & SaaN & 0.379 & $\mathrm{IC}_{1}$ & 0.340 \\
\hline 12 & 2.34 & 0.009 & 0.915 & SHssNH & -0.374 & SaaNH & -0.332 \\
\hline 13 & 2.10 & 0.008 & 0.923 & $\mathrm{SsOH}$ & -0.408 & ShsOH & -0.408 \\
\hline 14 & 1.82 & 0.007 & 0.929 & $\mathrm{NHBint}_{8}$ & 0.480 & SssNH & 0.398 \\
\hline 15 & 1.79 & 0.007 & 0.936 & $\mathrm{SsBr}$ & 0.427 & SssS & -0.384 \\
\hline 16 & 1.64 & 0.006 & 0.942 & SssNH & 0.510 & NHBint & -0.389 \\
\hline 17 & 1.56 & 0.006 & 0.948 & $\mathrm{SssO}$ & -0.633 & $\mathrm{NHBint}_{7}$ & 0.487 \\
\hline 18 & 1.38 & 0.005 & 0.953 & $\mathrm{NHBint}_{8}$ & 0.385 & SssS & 0.367 \\
\hline 19 & 1.21 & 0.005 & 0.958 & $\mathrm{SdsCH}$ & -0.582 & SssS & 0.391 \\
\hline 20 & 1.12 & 0.004 & 0.962 & NHBint $_{6}$ & 0.342 & $\mathrm{NHBint}_{7}$ & -0.336 \\
\hline
\end{tabular}

A comparison of the list of the twenty-four TIs most correlated with the twenty-four PCs and the twenty-four most important TIs necessary for the correlation of $\log P$ for the STARLIST set of 213 chemicals (Table 6) shows that they have only three TIs, viz., $\mathrm{DN}^{2} \mathrm{~N}_{3}, \mathrm{SHwHBd}$, and SsssP, in common. This shows that the set of minimally correlated TIs selected by the PCA process and the group of twentyfour TIs selected by the RR method are quite different. It is interesting to note that the group of twenty TIs chosen by the PCA procedure and those selected by the RR method for the data set of ninety-five aromatic amines have no TIs in common (Table 8).

It is interesting to note that for both types of QMSA methods, tailored and non-tailored, the value of $k$ $=3-8$ gives the best estimate of the properties, viz., $\log P$ and mutagenicity. This is in line with our earlier observation with various QMSA methods pertaining to different properties and diverse databases [19-37, 62]. It should also be noted that variable clustering was considered as a means of selecting indices or creating nearly orthogonal descriptors for the construction of ED spaces. However, this notion was discarded when we first attempted to cluster the sets of $250+$ descriptors. Unfortunately the implementation of variable clustering that we attempted to use required constant monitoring and had not finished clustering the variables even after two hours had elapsed. By comparison, the PCA took only a few seconds and produced a new set of descriptors that were completely orthogonal. 
TABLE VIII Twenty topological indices selected by PCA and RR for the ninety-five aromatic and heteroaromatic amines. There are no common TIs between the two descriptor sets

\begin{tabular}{lccc}
\hline PC & TIs from PCA & TIs from RR & t value \\
\hline 1 & $\mathrm{AZV}_{3}$ & $\mathrm{P}_{2}$ & 5.18 \\
2 & $\mathrm{ASN}_{2}$ & $\mathrm{M}_{1}$ & 4.82 \\
3 & SumdelI & $\mathrm{M}_{2}$ & 4.72 \\
4 & $\mathrm{Knotpv}$ & NHBint $_{9}$ & 4.39 \\
5 & Phia & $\mathrm{IC}_{1}$ & -4.20 \\
6 & $\mathrm{Hmin}$ & $\mathrm{SIC}_{2}$ & -4.17 \\
7 & $\mathrm{ANZ}$ & $\mathrm{AZN}_{4}$ & 3.97 \\
8 & $\mathrm{SHHBd}$ & $\mathrm{SIC}_{1}$ & -3.88 \\
9 & ${ }^{4} \chi_{\mathrm{C}}$ & $\mathrm{P}_{5}$ & 3.86 \\
10 & $\mathrm{ShsOH}$ & $\mathrm{P}_{6}$ & 3.84 \\
11 & $\mathrm{SaaN}$ & $\mathrm{IC}_{2}$ & -3.77 \\
12 & $\mathrm{SHssNH}$ & ${ }^{6}$ & 3.74 \\
13 & $\mathrm{SsOH}$ & ${ }^{9} \chi_{\mathrm{PC}}$ & 3.65 \\
14 & $\mathrm{NHBint}$ & $\mathrm{I}_{8}$ & 3.62 \\
15 & $\mathrm{SsBr}$ & $\mathrm{SaNH}$ & -3.55 \\
16 & $\mathrm{SssNH}$ & $\mathrm{CIC}_{2}$ & 3.52 \\
17 & $\mathrm{SssO}$ & $\mathrm{ANS}_{4}$ & 3.49 \\
18 & $\mathrm{SssS}$ & $\mathrm{AZV}_{2}$ & 3.42 \\
19 & $\mathrm{SdsCH}$ & $\mathrm{CIC}_{3}$ & 3.36 \\
20 & $\mathrm{NHBint}$ & $\mathrm{IC}_{5}$ & 3.19 \\
\hline
\end{tabular}

In conclusion, we have reported for the first time in this paper that one can construct appropriately tailored similarity spaces using adequately selected molecular descriptors: Such tailored spaces perform better in property/toxicity estimation than spaces constructed from an arbitrary collection of descriptors. We hope the idea of tailored similarity space will find application in analog selection, property/ toxicity estimation as well as in the property-specific clustering of databases for predictive toxicology research and drug design.

\section{Acknowledgments}

This is contribution number 308 from the Center for Water and the Environment of the Natural Resources Research Institute. Research reported in this paper was supported by grant F49620-01-1-0098 from the United States Air Force. 


\section{References}

[1] C.M. Auer, J.V. Nabholz and K.P. Baetcke, Environ. Health Perspect., 87 (1990) 183-197.

[2] C. Hansch and A. Leo, Exploring QSAR: Fundamentals and Applications in Chemistry and Biology, American Chemical Society, Washington, D.C., 1995.

[3] S.C. Basak, B.D. Gute and G.D. Grunwald, in J. Devillers and A.T. Balaban (Eds.), Topological Indices and Related Descriptors in QSAR and QSPR, Gordon and Breach Șcience Publishers, Amsterdam, 1999, Chapter 15.

[4] S.C. Basak, D. Mills, B.D. Gute, G.D. Grunwald and A.T. Balaban, in D.H. Rouvray and R.B. King (Eds.), Topology in Chemistry: Discrete Mathematics of Molecules, Horwood Publishing Ltd., 2001, Chapter 6.

[5] L.B. Kier and L.H. Hall, Molecular Connectivity in Structure-Activity Analysis, Research Studies Press, Letchworth, Hertfordshire, U.K., 1986.

[6] J. Devillers and A.T. Balaban (Eds), Topological Indices and Related Descriptors in QSAR and QSPR, Gordon \& Breach Science Publishers, Amsterdam, 1999.

[7] M. Randic and S.C. Basak, J. Chem. Inf. Comput. Sci., 41 (2001) 614-618.

[8] F.C. Smeeks and P.C. Jurs, Anal. Chim. Acta, 233 (1990) 111-119.

[9] D.V. Eldred, C.L. Weikel, P.C. Jurs and K.L.E. Kaiser, Chem. Res. Toxicol., 12 (1999) 670-678.

[10] A.T. Balaban (Ed.), From Chemical Topology to Three-Dimensional Geometry, Plenum Press, New York, 1997.

[11] S. Karabunarliev, O.G. Mekenyan, W. Karcher, C.L. Russom and S.P. Bradbury, Quant. Struct.-Act. Relat., 15 (1996) 302-310.

[12] J.J. Kaufman, W.S. Koski, S. Roszak and K. Balasubramanian, Chem. Phys., 204 (1996) 233-237.

[13] S.C. Basak, B.D. Gute and G.D. Grunwald, in F. Chen and G. Schuurmann (Eds.), Quantitative Structureactivity Relationships in Environmental Sciences VII, SETAC Press, Pensacola, FL, 1998, Chapter 17.

[14] T.W. Schultz and M.T.D. Cronin, J. Chem. Inf. Comput. Sci., 39 (1999) 304-309.

[15] S.C. Basak, D.R. Mills, A.T. Balaban and B.D. Gute, J. Chem. Inf. Comput. Sci., 41 (2001) 671-678.

[16] A.R. Katritzky, R. Petrukhin, D. Tatham, S.C. Basak, E. Benfenati, M. Karelson and U. Maran, J. Chem. Inf. Comput. Sci., 41 (2001) 679-685.

[17] M.J. Kamlet, R.M. Doherty, R.W. Taft, M.H. Abraham, G.D. Veith and D.J. Abraham, Environ. Sci. Technol., 21 (1987) 149-155.

[18] G. Cash, personal communication, 2001.

[19] S.C. Basak and G.D. Grunwald, SAR QSAR Environ. Res., 2 (1994) 289-307.

[20] S.C. Basak and G.D. Grunwald, J. Chem. Inf. Comput. Sci., 35 (1995) 366-372.

[21] S.C. Basak and G.D. Grunwald, SAR QSAR Environ. Res., 3 (1995) 265-277.

[22] S.C. Basak and B.D. Gute, SAR QSAR Environ. Res., 7 (1997) 1-21.

[23] S.C. Basak, V.R. Magnuson, G.J. Niemi and R.R. Regal, Discrete Appl. Math., 19 (1988) 17-44.

[24] S.C. Basak, S. Bertelsen and G.D. Grunwald, J. Chem. Inf. Comput. Sci., 34 (1994) 270-276.

[25] S.C. Basak, S. Bertelsen and G.D. Grunwald, Toxicol. Lett., 79 (1995) 239-250.

[26] S.C. Basak and G.D. Grunwald, New J. Chem., 19 (1995) 231-237.

[27] S.C. Basak and G.D. Grunwald, Chemosphere, 31 (1995) 2529-2546.

[28] S.C. Basak, B.D. Gute and G.D. Grunwald, Croat. Chem. Acta, 69 (1996) 1159-1173.

[29] S.C. Basak and B.D. Gute, in B.L. Johnson, C. Xintaras, and J.S. Andrews (Eds.), Hazardous Waste: Impacts on Human and Ecological Health: Proceedings of the International Congress on Hazardous Waste: Impact on Human and Ecological Health, Atlanta, USA, 5--8 June 1995. Princeton Scientific Publishing Co., Inc., Princeton, New Jersey, 1997, p. 492.

[30] S.C. Basak, B.D. Gute and G.D. Grunwald, in R. Carbo-Dorca and P. Mezey (Eds.), Advances in Molecular Similarity, JAI Press, Stanford, Connecticut, 1998, p.171.

[31] S.C. Basak and B.D. Gute, in P. Hansen, P. Fowler, and M. Zheng (Eds.), Discrete Mathematical Chemistry, American Mathematical Society, Providence, Rhode Island, 2000, p. 9.

[32] S.C. Basak, B.D. Gute and G.D. Grunwald, Mathl Model. Comput. Sci., (2002) in press.

[33] B.D. Gute and S.C. Basak, J. Mol. Graph. Model., 20 (2001) 95-109.

[34] B.D. Gute, G.D. Grunwald, D. Mills and S.C. Basak, SAR QSAR Environ. Res., 11 (2001) 363-382.

[35] S.C. Basak and G.D. Grunwald, Mathl. Model. Sci. Comput., 4 (1994) 464-469.

[36] S.C. Basak, G.D. Grunwald, G.E. Host, G.J. Niemi and S.P. Bradbury, Environ. Toxicol. Chem., 17 (1998) 1056-1064. 
[37] S.C. Basak, B.D. Gute and G.D. Grunwald, Mathl. Model. Sci. Comput., (2002) in press.

[38] S.C. Basak, B.D. Gute and G.D. Grunwald, J, Chem. Inf. Comput. Sci., 36 (1996) 1054-1060.

[39] A. Leo and D. Weininger, CLOGP Version 3.2 User Reference Manual, Medicinal Chemistry Project, Pomona College Claremont, CA, 1984.

[40] G.J. Niemi, S.C. Basak, G.D. Veith and G.D. Grunwald, Environ. Toxicol. Chem., 11 (1992) 893-898.

[41] A.K. Debnath, G. Debnath, A.J. Shusterman and C. Hansch, Environ. Mol. Mutagen., 19 (1992) 37-52.

[42] S.C. Basak, G.D. Grunwald and G.J. Niemi, in A.T. Balaban (Ed.), From Chemical Topology to ThreeDimensional Geometry, Plenum Press, New York, 1997, Chapter 4.

[43] S.C. Basak, D.R. Mills, A.T. Balaban and B.D. Gute, J. Chem. Inf. Comput. Sci., 41 (2001) 671-678.

[44] H. Wiener, J. Am. Chem. Soc., 69 (1947) 17-20.

[45] M. Randic, J. Am. Chem. Soc., 97 (1975) 6609-6615.

[46] L.B. Kier, W.J. Murray, M. Randic and L.H. Hall, J. Pharm. Sci., 65 (1975) 1226-1230.

[47] D. Bonchev and N. Trinajstic, J. Chem. Phys., 67 (1977) 4517-4533.

[48] C. Raychaudhury, S.K. Ray, J.J. Ghosh, A.B. Roy and S.C. Basak, J. Comput. Chem., 5 (1984) 581-588.

[49] S.C. Basak, A.B. Roy and J.J. Ghosh, in X.J.R. Avula, R. Bellman, Y.L. Luke, and A.K. Rigler (Eds.), Proceedings of the Second International Conference on Mathematical Modelling, University of MissouriRolla, Rolla, Missouri, 1980.

[50] S.C. Basak and V.R. Magnuson, Arzneim. Forsch., 33 (1983) 501-503.

[51] A.B. Roy, S.C. Basak, D.K. Harriss and V.R. Magnuson, in X.J.R. Avula, R.E. Kalman, A.I. Lapis, and E.Y. Rodin (Eds.), Mathematical Modelling in Science and Technology, Pergamon Press, New York, 1984.

[52] A.T. Balaban, Chem. Phys. Lett., 89 (1982) 399-404.

[53] A.T. Balaban, Pure and Appl. Chem., 55 (1983) 199-206.

[54] A.T. Balaban, Math. Chem. (MATCH), 21 (1986) 115-122.

[55] S.C. Basak, D.K. Harriss and V.R. Magnuson, POLLY v2.3, Copyright of the University of Minnesota, 1988.

[56] S.C. Basak, in J. Devillers and A.T. Balaban (Eds.), Topological Indices and Related Descriptors in QSAR and QSPR, Gordon \& Breach Science Publishers, Amsterdam, 1999, Chapter 12.

[57] P.A. Filip, T.S. Balaban and A.T. Balaban, J. Math. Chem., 1 (1987) 61-83.

[58] S.C. Basak, A.T. Balaban, G.D. Grunwald and B.D. Gute, J. Chem. Inf. Comput. Sci., 40 (2000) 891-898.

[59] Molconn-Z v3.50, Hall Associates Consulting: Quincy, MA, 2000.

[60] S.C. Basak, A.T. Balaban and B.D. Gute, J. Chem. Inf. Comput. Sci., (2002) submitted.

[61] SAS Institute Inc., in SAS/STAT User's Guide, Release 6.03 Edition, SAS Institute Inc., Cary, NC, 1988.

[62] S.C. Basak, G.J. Niemi and G.D. Veith, J. Math. Chem., 7 (1991) 243-272.

[63] S.C. Basak, D. Mills, B.D. Gute, A.T. Balaban, K. Basak and G.D. Grunwald, in D.K. Sinha, S.C. Basak, R.K. Mohanty, and I.N. Basumallick (Eds.), Some Aspects of Mathematical Chemistry, Visva-Bharati University, India, 2002, in press.

[64] A.E. Hoerl and R.W. Kennard, Technometrics, 8 (1970) 27-51.

[65] D. Hawkins, S. Basak and X. Shi, J. Chem. Inf. Comput. Sci., 41 (2001) 663-670. 


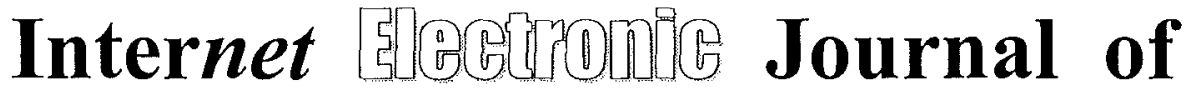 Molecular $\mathfrak{D e s i g n}$
}

August 2002, Volume 1, Number 8, Pages 374-387

Editor: Ovidiu Ivanciuc

Special issue dedicated to Professor Milan Randic on the occasion of the $70^{\text {th }}$ birthday Part 4

Guest Editor: Mircea V. Diudea

\section{Tailored Similarity Spaces for the Prediction of Physicochemical Properties}

\author{
Brian D. Gute, ${ }^{1}$ Subhash C. Basak, ${ }^{1}$ Denise Mills, ${ }^{1}$ and Douglas M. Hawkins ${ }^{2}$ \\ ${ }^{1}$ Center for Water and the Environment, Natural Resources Research Institute, 5013 Miller Trunk \\ Highway, Duluth, MN, 55811 \\ ${ }^{2}$ School of Statistics, University of Minnesota, 224 Church St SE, Minneapolis, MN, 55455
}

Received: June 27, 2002; Accepted: July 9, 2002; Published: August 31, 2002

Citation of the article:

B. D. Gute, S. C. Basak, D. Mills, and D. M. Hawkins, Tailored Similarity Spaces for the Prediction of Physicochemical Properties, Internet Electron. J. Mol. Des. 2002, 1, 374-387, http://www.biochempress.com. 


\title{
Tailored Similarity Spaces for the Prediction of Physicochemical Properties ${ }^{\#}$
}

\author{
Brian D. Gute, ${ }^{1}$ Subhash C. Basak, ${ }^{1, *}$ Denise Mills, ${ }^{1}$ and Douglas M. Hawkins ${ }^{2}$ \\ ${ }^{1}$ Center for Water and the Environment, Natural Resources Research Institute, 5013 Miller Trunk \\ Highway, Duluth, MN, 55811 \\ ${ }^{2}$ School of Statistics, University of Minnesota, 224 Church St SE, Minneapolis, MN, 55455
}

Received: June 27, 2002; Accepted: July 9, 2002; Published: August 31, 2002

\begin{abstract}
Internet Electron. J. Mol. Des. 2002, I (8), 374-387

Motivation. In the past, molecular similarity spaces have been developed from arbitrary sets of molecular properties or theoretical descriptors and the results of property estimation based on these methods have always been inferior to SAR and QSAR models. Tailored QMSA methods attempt to create similarity spaces specific for a property of interest, rather than being purely arbitrary spaces characterizing the general aspects of all chemicals within the space or intuitively selected structure spaces whose elements are chosen subjectively. To this end, we have created three similarity spaces, two tailored and one non-tailored, for a set of 166 chemicals for which we have both $\log P$ and normal boiling point (BP) data. The tailored spaces were each tailored to one of the properties, while the other similarity space was developed using standard QMSA methods.

Method. Ridge regression was used to determine which of the available molecular descriptors were most useful in modeling each of the available properties. Fifteen topological descriptors were selected for use as dimensions within each the tailored similarity spaces. The same number of principal components were developed using principal component analysis for the arbitrary similarity space.

Results. The $\log P$ tailored similarity space was superior to both the arbitrary structure space and the BP tailored space for the estimation of $\log P$. Also, the BP tailored similarity space was superior to the arbitrary structure space for the estimation of BP. Interestingly, the space tailored to model $\log P$ performed as well at modeling BP as did the BP tailored space. This unexpected result is explained by the degree of overlap between the indices used in both of the tailored spaces and in the presence of connectivity indices related to BP in the $\log P$ model.
\end{abstract}

Conclusions. The tailored similarity method presents a promising approach to creating property specific similarity spaces derived from structural descriptors based on the results of this study and from a previous study. Further work is necessary to determine to true utility of this method with large, diverse data sets.

Keywords. Quantitative molecular similarity analysis (QMSA); tailored QMSA; arbitrary QMSA; topological indices; lipophilicity; normal boiling point.

\begin{tabular}{ll}
\hline Abbreviations and notations & \\
\hline ASTER, Assessment Tools for the Evaluation of Risk & QMSA, quantitative molecular similarity analysis \\
BP, normal boiling point & QSAR, quantitative structure-activity relationship \\
ED, Euclidean distance & $R$, regression coefficient \\
JP-8, jet propellant formulation \#8 & RR, Ridge regression \\
$K N N, K$-nearest neighbor & s.e., standard error \\
$\log P$, lipophilicity & TI, topological index \\
PCs, principal components & USEPA, United States Environmental Protection Agency \\
PCA principal components analysis &
\end{tabular}

\# Dedicated to Professor Milan Randić on the occasion of the $70^{\text {th }}$ birthday.

* Correspondence author; phone: 00-218-720-4230; fax: 00-218-720-4328; E-mail: sbasak@nrri.umn.edu.

\section{BioCHEM Press}




\section{INTRODUCTION}

Quantitative molecular similarity analysis (QMSA) is an important computational tool both for the hazard assessment of environmental pollutants and pharmaceutical drug design. In the area of the hazard estimation of chemicals, QMSA methods are routinely used to assess the potential hazard of a chemical based on the toxicity profiles of analogous chemicals when little or no experimental toxicity data and toxicologically relevant property data are available for the chemical of interest [1-4]. This course of action is generally followed when the structure of the chemical is complex enough that it cannot be unambiguously classified into a particular structural category. If it could be categorized into a specific chemical class, class-specific quantitative structure-activity relationship (QSAR) models would instead be used for hazard assessment. In the area of drug discovery, QMSA techniques are useful for determining whether interesting lead compounds have structural analogs with similar pharmacological and toxicological profiles. The other side of similarity is dissimilarity. Dissimilarity-based clustering of large libraries of real or in silico (virtual libraries) of chemicals has been successfully used [5] and suggested [6] as possible methods in the management of combinatorial explosions in various drug design scenarios.

QMSA methods are based on the basic assumption that similar molecular structures usually have similar properties [7]. Two chemicals, X1 and X2, are said to be similar if they resemble each other with respect to some user-defined set of properties or structural attributes, or both. Substructural descriptors [8-17], experimental properties [12,17-19], and theoretical structural invariants [6,7,1117,19-32] have been widely used in the formulation of QMSA methods and ranking of chemical databases via such techniques.

Our research group has been involved in the development of novel QMSA techniques and their applications in analog selection and the $k$-nearest neighbor $(K N N)$ based estimation of properties, as well as the use of similarity spaces in the clustering of chemical databases. Our experience has shown that increasing the intrinsic dimensionality of similarity spaces by the progressive use of more diverse and mutually uncorrelated (or minimally correlated) indices leads to better analog selection as is evident from both a visual inspection of their structures and the predictive power of the selected analogs in property estimation for query chemicals using the $K \mathrm{NN}$ method.

The stepwise use of increasingly higher dimensional structure spaces, derived from collections of progressively more diverse and comprehensive indices, suffers from the fact that elements of the enhanced spaces do not have any intrinsic relationship to the property of interest that we are attempting to estimate from the chosen analogs. Rather, these spaces are simply a reflection of the chemical diversity within the selected data set. If there is an improvement in the usefulness of analogs selected, that is only by chance, not by design. This is why we have developed the idea of tailored QMSA methods where the structure space is constructed from parameters that are strongly associated with the property of interest [32]. The advantage of such directed spaces over blind or 
arbitrary spaces is that analogs selected by the former will be relevant with respect to the property to which they are tailored.

In a previous study, we reported for the first time the development of structure spaces tailored towards two properties, viz., $\log P$ (octanol/water) and Ames mutagenicity, based on calculated topological indices. We also showed that the analogs selected from the tailored similarity spaces gave much better results in $K \mathrm{NN}$-based estimation for both of the properties studied, as compared to our previous results using arbitrary similarity spaces. In the current study, we have used a set of 166 chemicals which represent a subset of the known constituents of jet propellant \#8 (JP-8), a jet fuel currently in use by the United States Armed Forces. This set of chemicals was of interest for this study since we have data for two physicochemical properties, $\log P$ and normal boiling point, for this set of chemicals. Three similarity spaces have been constructed for this study. Two of the similarity spaces are tailored spaces, one tailored towards $\log P$ and the other towards normal boiling point (hereafter simply referred to as BP). The third similarity space is a standard, arbitrary similarity space developed from the set of available molecular descriptors.

\section{MATERIALS AND METHODS}

Physicochemical property data used in this study represent property values extracted from the ASTER [33] system of the USEPA. These data are predominantly calculated values, rather than experimental values, reflecting the difficulty of obtaining simple physicochemical experimental data for common compounds.

\subsection{Chemical Data}

The set of chemicals used in this study represents a subset of the known constituents of JP-8 identified through GC/MS [34], a set of 166 hydrocarbons. This subset consisted of all of the chemicals in the full set of 228 chemicals for which $\log P$ and normal boiling point (BP) were both available from the ASTER database. However, even for the reduced set of 166 chemicals, most of the data values available from ASTER were calculated, not experimental values. This set of chemicals and the data obtained from ASTER are reported in Table 1.

\subsection{Calculation of Molecular Descriptors}

The topological indices (TIs) used in this study were calculated using three main software programs: POLLY 2.3 [35], MolConn-Z 3.50 [36], and Triplet [37]. Included in the suite of more than 220 indices in this study are: Wiener number [38], molecular connectivity indices as calculated by Randić [39] and Kier and Hall [40], frequency of path lengths of varying size, information theoretic indices defined on distance matrices of graphs using the methods of Bonchev and Trinajstić [41] as well as those of Raychaudhury et al. [42], parameters defined on the 
Tailored Similarity Spaces for the Prediction of Physicochemical Properties Internet Electronic Journal of Molecular Design 2002, 1, 374-387

neighborhood complexity of vertices in hydrogen-filled molecular graphs [43-45], Balaban's $J$ indices [46-48], local orthogonal vertex invariants [37], kappa shape descriptors [49,50], and the electrotopological indices of Kier and Hall [51]. More information on the topological indices calculated by POLLY has been reported in earlier studies [15,20,27,31].

Table 1. Chemicals and Their Physicochemical Property Data for the 166 Identified Components of JP-8

\begin{tabular}{|c|c|c|c|c|c|c|c|}
\hline No & Name & $\log P$ & $\mathrm{BP}$ & No & Name & $\log P$ & $\mathrm{BP}$ \\
\hline 1 & ISTD (d10-anthracene) & 4.49 & 300 & 50 & 3,3-dimethylheptane & 5.2 & 137 \\
\hline 2 & $2,2,3$-trimethylbutane & 4.01 & 81 & 51 & 2,4-dimethyl-3-ethylpentane & 5.07 & 137 \\
\hline 3 & 2,3,3-trimethyl-1-butene & 3.46 & 78 & 52 & 2,3,4-trimethylhexane & 5.07 & 139 \\
\hline 4 & 3,3-dimethylpentane & 4.14 & 86 & 53 & $2,2,3,3$-tetramethylpentane & 4.94 & 140 \\
\hline 5 & Benzene & 2.14 & 80 & 54 & 2,3,3,4-tetramethylpentane & 4.94 & 142 \\
\hline 6 & 2-methylhexane & 4.27 & 90 & 55 & 2,3-dimethylheptane & 5.2 & 141 \\
\hline 7 & 3-ethylpentane & 4.27 & 93.5 & 56 & 3,4-dimethylheptane & 5.2 & 141 \\
\hline 8 & $t-1,3-$ dimethylcyclopentane & 3.83 & 91 & 57 & 4-ethylheptane & 5.33 & 141 \\
\hline 9 & Iso-octane & 4.54 & 99 & 58 & Ethylbenzene & 3.32 & 136 \\
\hline 10 & 1-heptene & 3.85 & 94 & 59 & 4-methyloctane & 5.33 & 141 \\
\hline 11 & 3-heptene & 3.85 & 92.7 & 60 & $m$-xylene & 3.44 & 139 \\
\hline 12 & $n$-heptane & 4.4 & 98 & 61 & 3-methyloctane & 5.33 & 143 \\
\hline 13 & 2,2-dimethylhexane & 4.67 & 106 & 62 & $c-1,2,3$-trimethylcyclohexane & 4.91 & 144 \\
\hline 14 & 1,1,3-trimethylcyclopentane & 4.35 & 105 & 63 & 3,3-diethylpentane & 5.2 & 146 \\
\hline 15 & 2,3,3-trimethyl-1,4-pentadiene & 3.45 & 125 & 64 & 1,2,4-trimethylcyclohexane & 4.91 & 142 \\
\hline 16 & 2,4,4-trimethyl-2-pentene & 3.99 & 105 & 65 & $c, c, t-1,3,5$-trimethylcyclohexane & 4.91 & 144 \\
\hline 17 & 2,5-dimethylhexane & 4.67 & 109 & 66 & 1-nonene & 4.91 & 147 \\
\hline 18 & 2,4-dimethylhexane & 4.67 & 110 & 67 & $o-x y l e n e$ & 3.44 & 144 \\
\hline 19. & 3,3-dimethylhexane & 4.67 & 112 & 68 & 4-nonene & 4.91 & 145 \\
\hline 20 & 4-methylcyclohexene & 3.33 & 103 & 69 & n-nonane & 5.46 & 151 \\
\hline 21 & $c, t, c-1,2,3$-trimethylcyclopentane & 4.35 & 123 & 70 & $c, c, t-1,2,3$-trimethylcyclohexane & 4.91 & 144 \\
\hline 22 & 2,3,4-trimethylpentane & 4.54 & 114 & 71 & 3,3,5-trimethylheptane & 5.59 & 156 \\
\hline 23 & 2,3,3-trimethylpentane & 4.54 & 115 & 72 & 1-ethyl-1-methylcyclohexane & 4.92 & 144 \\
\hline 24 & t-3,4,4-trimethyl-2-pentene & 3.99 & 119 & 73 & $1,3,5,5$-tetramethyl-1,3-cyclohexadiene & 4.52 & 173 \\
\hline 25 & 1,1,3,3-tetramethylcyclopentane & 4.87 & 114 & 74 & $\mathrm{t}-1,1,3,5$-tetramethylcyclohexane & 5.43 & 166 \\
\hline 26 & 2-methylheptane & 4.8 & 118 & 75 & Isopropylcyclohexane & 4.8 & 155 \\
\hline 27 & 4-methylheptane & 4.8 & 118 & 76 & 3,5-dimethyloctane & 5.72 & 160 \\
\hline 28 & Toluene & 2.79 & 111 & 77 & Isopropylbenzene & 3.72 & 152 \\
\hline 29 & 3,4-dimethylhexane & 4.67 & 118 & 78 & 2,7-dimethyloctane & 5.72 & 160 \\
\hline 30 & 2,2,4,4-tetramethylpentane & 4.94 & 122 & 79 & $n$-propylcyclohexane & 4.93 & 157 \\
\hline 31 & 3-methylheptane & 4.8 & 119 & 80 & 2,6-dimethyloctane & 5.72 & 155 \\
\hline 32 & 3-ethylhexane & 4.8 & 119 & 81 & 3,4-diethylhexane & 5.72 & 162 \\
\hline 33 & $t-1,1,3,4$-tetramethylcyclopentane & 4.87 & 144 & 82 & 3,6-dimethyloctane & 5.72 & 160 \\
\hline 34 & 2-ethyl-1-hexene & 4.25 & 120 & 83 & 3-ethyl-2-methylheptane & 5.72 & 166 \\
\hline 35 & 2,2,4-trimẹthylhexane & 5.07 & 127 & 84 & 3,4,5-trimethylheptane & 5.59 & 164 \\
\hline 36 & 1-ethyl-1-methylcyclopentane & 4.36 & 122 & 85 & Propylbenzene & 3.85 & 159 \\
\hline 37 & $n$-octane & 4.93 & 126 & 86 & 2,3-dimethyloctane & 5.72 & 164 \\
\hline 38 & 2,4,4-trimethylhexane & 5.07 & 131 & 87 & 4-ethyloctane & 5.85 & 168 \\
\hline 39 & 2,4-dimethylheptane & 5.2 & 134 & 88 & 5-methylnonane & 5.85 & 165 \\
\hline 40 & 2,2,3-trimethylhexane & 5.07 & 134 & 89 & 4-methylnonane & 5.85 & 165 \\
\hline 41 & 4,4-dimethylheptane & 5.2 & 135 & 90 & 1-ethyl-3-methylbenzene & 3.97 & 161 \\
\hline 42 & 3,3,5-trimethylcyclohexene & 4.37 & 145 & 91 & 1-ethyl-4-methylbenzene & 3.97 & 162 \\
\hline 43 & 2,2,5,5-tetramethylhexane & 5.46 & 137 & 92 & 3-ethyloctane & 5.85 & 168 \\
\hline 44 & 2,6-dimethylheptane & 5.2 & 135 & 93 & 1,3,5-trimethylbenzene & 4.09 & 165 \\
\hline 45 & $c, c, c-1,3,5$-trimethylcyclohexane & 4.91 & 144 & 94 & 3-methylnonane & 5.85 & 167 \\
\hline 46 & Propylcyclopentane & 4.37 & 131 & 95 & 1-isopropyl-4-methylcyclohexane & 5.32 & 169 \\
\hline 47 & 1,3,5-trimethylcyclohexane & 4.91 & 144 & 96 & 1-ethyl-2-methylbenzene & 3.97 & 165 \\
\hline 48 & 3,5,5-trimethylcyclohexene & 4.37 & 145 & 97 & $2,2,4,6,6$-pentamethylheptane & 6.39 & 205 \\
\hline 49 & Ethylcyclohexane & 4.4 & 132 & 98 & $t$-butylbenzene & $4.12^{\circ}$ & 169 \\
\hline
\end{tabular}


B. D. Gute, S. C. Basak, D. Mills, and D. M. Hawkins

Table 1. (Continued)

\begin{tabular}{|c|c|c|c|c|c|c|c|}
\hline No & Name & $\log P$ & $\mathrm{BP}$ & No & Name & $\log P$ & $\mathrm{BP}$ \\
\hline 99 & 1,2,4-trimethylbenzene & 4.09 & 169 & 133 & 1,2-dimethyl-3-ethylbenzene & 4.62 & 194 \\
\hline 100 & $n$-decane & 5.98 & 174 & 134 & 1,2,4,5-tetramethylbenzene & 4.74 & 197 \\
\hline 101 & Isobutylbenzene & 4.25 & 173 & 135 & (2-methylbutyl-)-benzene & 4.78 & 205 \\
\hline 102 & sec-butylbenzene & 4.25 & 174 & 136 & i,2,3,5-tetramethylbenzene & 4.74 & 198 \\
\hline 103 & 3,7,7-trimethylbicyclo(4.1.0)-3-heptene & 4.12 & 170 & 137 & (3-methylbutyl-)-benzene & 4.78 & 199 \\
\hline 104 & 1-isopropyl-3-methylbenzene & 4.37 & 175 & 138 & 1,2-diisopropylbenzene & 5.3 & 204 \\
\hline 105 & 1,2,3-trimethylbenzene & 4.09 & 176 & 139 & $1,2,3,4$-tetramethylbenzene & 4.74 & 205 \\
\hline 106 & 1-ethyl-2,5-dimethylbenzene & 4.62 & 187 & 140 & $n$-pentylbenzene & 4.91 & 205 \\
\hline 107 & Dicyclopentadiene & 3.44 & 175 & 141 & 1,4-diisopropylbenzene & 5.3 & 203 \\
\hline 108 & Butylcyclohexane & 5.46 & 181 & 142 & 1-t-butyl-3,5-dimethylbenzene & 5.42 & 204 \\
\hline 109 & Indane $(2,3$-dihydro- $1 \mathrm{H}$-indene $)$ & 3.46 & 176 & 143 & Naphthalene & 3.32 & 218 \\
\hline 110 & 1-isopropyl-2-methylbenzene & 4.37 & 178 & 144 & 1-dodecene & 6.5 & 213 \\
\hline 111 & 1,3-diethylbenzene & 4.5 & 181 & 145 & 1,3,5-triethylbenzene & 5.68 & 215 \\
\hline 112 & 1-propyl-4-methylbenzene & 4.5 & 183 & 146 & $n$-hexylbenzene & 5.44 & 226 \\
\hline 113 & 1,4-diethylbenzene & 4.5 & 183 & 147 & (1,1-diethylpropyl-)-benzene & 5.71 & 243 \\
\hline 114 & Butylbenzene & 4.38 & 183 & 148 & 2-methylnaphthalene & 3.97 & 241 \\
\hline 115 & 1-ethyl-3,5-dimethylbenzene & 4.62 & 184 & 149 & 1-methylnaphthalene & 3.97 & 245 \\
\hline 116 & 4-methyldecane & 6.38 & 185 & 150 & Cyclohexylbenzene & 4.91 & 235 \\
\hline 117 & 1,2-diethylbenzene & 4.5 & 183 & 151 & 1-t-butyl-3,4,5-trimethylbenzene & 6.07 & 243 \\
\hline 118 & 2-methyldecane & 6.38 & 185 & 152 & $1,1,6$-trimethyltetralin & 5.7 & 247 \\
\hline 119 & Neopentylbenzene & 4.65 & 186 & 153 & $n$-heptylbenzene & 5.97 & 245 \\
\hline 120 & 1-propyl-2-methylbenzene & 4.5 & 185 & 154 & 1,1'-biphenyl & 4.03 & 254 \\
\hline 121 & 3-methyldecane & 6.38 & 185 & 155 & 2-ethylnaphthalene & 4.49 & 258 \\
\hline 122 & 1-isopropyl-4-methylbenzene & 4.37 & 177 & 156 & 1-ethylnaphthalene & 4.49 & 259 \\
\hline 123 & 1-ethyl-2,4-dimethylbenzene & 4.62 & 188 & 157 & 2,6-dimethylnaphthalene & 4.61 & 262 \\
\hline 124 & (1,2-dimethylpropyl-)-benzene & 4.65 & 188 & 158 & 2,3-dimethylnaphthalene & 4.61 & 268 \\
\hline 125 & 1-ethyl-3,4-dimethylbenzene & 4.62 & 190 & 159 & 1,4-dimethylnaphthalene & 4.61 & 268 \\
\hline 126 & 1-t-butyl-3-methylbenzene & 4.77 & 189 & 160 & 1,5-dimethylnaphthalene & 4.61 & 265 \\
\hline 127 & (1-ethylpropyl-)-benzene & 4.78 & 191 & 161 & 1,2-dimethylnaphthalene & 4.61 & 266 \\
\hline 128 & 1-undecene & 5.97 & 193 & 162 & $n$-octylbenzene & 6.49 & 262 \\
\hline 129 & 2-ethyl-1,3-dimethylbenzene & 4.62 & 190 & 163 & 1,8-dimethylnaphthalene & 4.61 & 270 \\
\hline 130 & $n$-undecane & 6.51 & 196 & 164 & Fluorene & 4.23 & 293 \\
\hline 131 & 1-ethyl-3-isopropylbenzene & 4.9 & 192 & 165 & 2,5-dimethylheptane & 5.2 & 136 \\
\hline 132 & sec-pentylbenzene & 4.78 & 193 & 166 & $p$-xylene & 3.44 & 138 \\
\hline
\end{tabular}

\subsubsection{Data reduction}

Initially, the TIs were transformed by the natural logarithm of the index plus one. Since the magnitude of some TIs is several orders greater than that of others, re-scaling is conducted to minimize the effect of scale. However, minimal values for some of the Molconn- $Z$ parameters were much less than zero. These indices were logarithmically scaled on a case-by-case basis using the natural logarithm of the index plus $x$, where $x$ was an integer large enough to make the minimal value of the index greater than zero. Next, correlation analysis was conducted on the indices. In all cases of a perfect correlation between several indices, only one of the indices was retained within the descriptor set. Additionally, a number of indices encoding features not present in the data set (having zero values for all compounds) were discarded. 


\subsubsection{Statistical analysis software}

Two statistical software packages were used for the construction of similarity spaces used in this study. For the development of the arbitrary similarity space, SAS [52] was used to conduct a principal component analysis (PCA) on the transformed indices to minimize the intercorrelation of indices. This was done using the SAS procedure PRINCOMP. For the tailored spaces, an in-house ridge regression (RR) [53] program was used to select a small set of descriptors for the development of each of the spaces.

\subsubsection{Construction of arbitrary similarity spaces}

A traditional (arbitrary) molecular similarity space was constructed for the set of $166 \mathrm{JP}-8$ constituents using the principal components created using the SAS PRINCOMP procedure. Only PCs with eigenvalues greater than or equal to one have been retained for this study. A more detailed explanation of this approach has been provided in a previous study by Basak et al. [20]. These PCs were subsequently used as independent variables (in place of the TIs) to determine similarity scores in the Euclidean distance method described later. After the PCA, a correlation analysis was conducted on the PCs to determine which TIs were most highly correlated with each of the PCs. This allows for the creation of similarity spaces based on a small set of TIs (as has been done previously), and also provides some insight into the general nature of the principal components, i.e., which aspects of molecular structure are explained by each of the PCs $[6,54,55]$.

\subsubsection{Construction of tailored similarity spaces}

Two tailored similarity spaces were constructed for use in this study. One of the spaces was tailored specifically to $\log P$ and the other for BP. As was mentioned earlier, the RR method was used in the development of these spaces. $\mathrm{RR}$ is a method wherein modeling is conducted using the entire set of descriptors retained after the data reduction step as opposed to subset regression. This regression method is useful in cases where the descriptors are highly multicollinear and where the number of descriptors is substantially larger than the number of observations [56]. Conceptually, $R R$ can be thought of as recasting the regression as one using the principal components of the predictor variables as new predictors. It differs in that in principal component regression the leading components are retained and used just as in ordinary least squares regression while the trailing components are dropped. RR retains all components, but weights each of them in accordance with the component's eigenvalue and the 'ridging constant' $k$. More details on the RR method can be found in some of our previous papers [32,57-58].

One of the by-products of the RR is a ranking of the contribution of the indices. The absolute values of this ranking score were used to select the descriptors for use in the development of tailored similarity spaces. Separate RR studies were conducted for $\log P$ and $\mathrm{BP}$, resulting in a selection of optimal descriptors for use in constructing the tailored similarity spaces. 
Table 2. Summary of the First Fifteen Principal Components Derived from a set of 222 Topological Indices Calculated for a Set of $166 \mathrm{JP}-8$ Constituents

\begin{tabular}{|c|c|c|c|c|c|c|c|}
\hline \multirow{2}{*}{$\frac{\mathrm{PC}}{1}$} & \multirow{2}{*}{$\begin{array}{c}\text { Eigenvalue } \\
93.38\end{array}$} & \multirow{2}{*}{$\begin{array}{c}\begin{array}{c}\text { Proportion of Explained } \\
\text { Variance }\end{array} \\
0.421\end{array}$} & \multirow{2}{*}{$\begin{array}{c}\begin{array}{c}\text { Cumulative Explained } \\
\text { Variance }\end{array} \\
0.421\end{array}$} & \multicolumn{2}{|c|}{$\begin{array}{c}\text { First Most Correlated } \\
\text { TI } \\
\end{array}$} & \multicolumn{2}{|c|}{$\begin{array}{c}\text { Second Most Correlated } \\
\text { TI }\end{array}$} \\
\hline & & & & $\mathrm{DN}^{2} \mathrm{~N}_{4}$ & 0.99349 & $\mathrm{DN}^{2} 1_{4}$ & 0.99136 \\
\hline 2 & 45.84 & 0.206 & 0.627 & Phia & -0.97965 & $\mathrm{ASN}_{2}$ & 0.96310 \\
\hline 3 & 26.24 & 0.118 & 0.745 & ${ }^{2} \chi^{b}$ & 0.84243 & ${ }^{2} \chi^{v}$ & 0.83715 \\
\hline 4 & 12.74 & 0.057 & 0.802 & $I_{3}$ & 0.74736 & $\mathrm{IC}_{4}$ & 0.73754 \\
\hline 5 & 9.32 & 0.042 & 0.844 & $\mathrm{~J}^{\mathrm{B}}$ & -0.64153 & ${ }^{4} x^{6}$ & 0.61874 \\
\hline 6 & 6.31 & 0.029 & 0.873 & ${ }^{10} \chi$ & 0.55023 & ${ }^{9} \chi^{v}$ & 0.54724 \\
\hline 7 & 4.75 & 0.021 & 0.894 & $\mathrm{SdsCH}$ & 0.53646 & Shvin & 0.52438 \\
\hline 8 & 3.97 & 0.018 & 0.912 & ${ }^{5} \chi^{b}{ }_{c}$ & 0.49541 & ${ }^{5} \chi^{v} \mathrm{c}$ & 0.49167 \\
\hline 9 & 2.66 & 0.012 & 0.924 & ${ }^{3} \chi_{\mathrm{Ch}}$ & -0.50749 & $\mathrm{G}_{\mathrm{MAX}}$ & 0.48248 \\
\hline 10 & 2.36 & 0.011 & 0.935 & ${ }^{9} \chi_{\mathrm{Ch}}^{\mathrm{v}}$ & -0.64096 & ${ }^{9} \chi_{\mathrm{Ch}}$ & -0.60901 \\
\hline 11 & 1.91 & 0.009 & 0.944 & $\mathrm{~J}^{\mathrm{B}}$ & 0.35572 & ${ }^{8} \chi_{\mathrm{Ch}}$ & 0.28873 \\
\hline 12 & 1.68 & 0.008 & 0.952 & Shvin & -0.39971 & $\mathrm{SdsCH}$ & -0.39780 \\
\hline 13 & 1.38 & 0.006 & 0.958 & $\mathrm{O}_{\mathrm{ORB}}$ & 0.36812 & $\mathrm{O}$ & 0.31193 \\
\hline 14 & 1.15 & 0.005 & 0.963 & ${ }^{10} \chi^{v}$ & 0.40648 & $\mathrm{SdsCH}$ & 0.26160 \\
\hline 15 & 1.07 & 0.005 & 0.968 & $x^{6}$ & 0.31114 & ${ }^{6} x^{v}$ & 0.30726 \\
\hline
\end{tabular}

Table 3. Fifteen TIs Selected by RR for the $166 \mathrm{JP}-8$ Chemicals. Indices Common to both RR Sets are Indicated in Bold

\begin{tabular}{|c|c|c|c|c|}
\hline \multirow{2}{*}{$\frac{\mathrm{PC}}{1}$} & \multicolumn{2}{|c|}{$\begin{array}{l}\text { TIs from RR for } \log P \\
\text { (t-value) }\end{array}$} & \multicolumn{2}{|c|}{$\begin{array}{l}\text { TIs from RR for } \mathrm{BP} \\
\text { (t-value) }\end{array}$} \\
\hline & ${ }^{0} \chi^{b}$ & $(16.47)$ & $\mathrm{ANN}_{5}$ & $(16.77)$ \\
\hline 2 & ${ }^{0} \chi^{\mathrm{v}}$ & $(16.42)$ & $\mathrm{ANN}_{3}$ & $(16.10)$ \\
\hline 3 & Fw & (14.77) & $\mathrm{ANl}_{3}$ & (15.81) \\
\hline 4 & $\mathrm{AZS}_{1}$ & $(14.22)$ & $\mathrm{ANN}_{1}$ & (15.51) \\
\hline 5 & W & $(14.03)$ & W & $(15.30)$ \\
\hline 6 & $\mathrm{ANS}_{3}$ & $(14.00)$ & $\mathrm{P}_{0}$ & $(15.07)$ \\
\hline 7 & $\mathbf{A Z S}_{\mathbf{3}}$ & (13.31) & $\mathbf{A N S}_{3}$ & (14.74) \\
\hline 8 & $\mathrm{ANS}_{1}$ & $(12.19)$ & $I^{W_{D}}$ & $(14.58)$ \\
\hline 9 & ${ }^{0} \chi$ & (11.71) & $\mathrm{DN}^{2} 1_{4}$ & $(14.10)$ \\
\hline 10 & $\mathrm{ka}_{1}$ & $(11.42)$ & $\mathbf{A Z S}_{\mathbf{3}}$ & (13.93) \\
\hline 11 & $I^{W_{p}}$ & (11.31) & $\mathrm{AZN}_{3}$ & (13.88) \\
\hline 12 & $\mathrm{ANN}_{3}$ & $(11.31)$ & $\mathrm{AZN}_{5}$ & (13.13) \\
\hline 13 & $\mathrm{DN}^{2} \mathrm{~S}_{3}$ & (11.29) & $A Z N_{1}$ & $(12.81)$ \\
\hline 14 & $\mathrm{ANN}_{5}$ & (11.14) & Fw & (12.65) \\
\hline 15 & $Q_{V}$ & $(11.08)$ & $\mathrm{DN}^{2} \mathrm{~N}_{3}$ & $(12.46)$ \\
\hline
\end{tabular}

\subsection{Quantification of Intermolecular Similarity}

Once the similarity spaces were constructed, it was possible to calculate similarity scores based on the intermolecular distances within the arbitrary and tailored molecular similarity spaces. Intermolecular similarity was measured using Euclidean distance (ED) within an $n$-dimensional space derived from TIs or PCs. The ED between two molecules, $i$ and $j$, is defined as:

$$
E D_{i !}=\left[\sum_{k=1}^{n}\left(D_{i k}-D_{j k}\right)^{2}\right]^{1 / 2}
$$

where $n$ is equal to the number dimensions (descriptors) used to define the similarity space, whether 
those dimensions are derived from TIs or PCs. $D_{i k}$ and $D_{j k}$ are the data values of the $k^{\text {th }}$ dimension for molecules $i$ and $j$, respectively.

Once distances between all molecules within the molecular similarity space have been calculated, these distance "scores" can then be used for analog selection or in $K \mathrm{NN}$-based property estimation. This type of quantifiable analog selection can be a powerful tool for finding chemicals that are similar to a chemical of interest, replacing the need for subjective assessment of molecular similarity. More often than not, we are interested in predicting a property of interest. In this case, $K \mathrm{NN}$-based similarity offers an alternative to standard linear regression approaches that works well for large, diverse data sets.

$K \mathrm{NN}$-based property estimation is carried out by selecting the $k$-nearest neighbors for each compound and using the average of the neighbor's properties as an estimate of the property of our chemical of interest. A number of similar chemicals $(k=1-10,15,20,25)$ are selected and the property of interest is estimated based on the values of these nearest neighbors. For instance, in estimating the $\log P$ of the probe compound, the mean $\log P$ for the $k$-nearest neighbors was used as the estimate. $K \mathrm{NN}$ estimation was carried out for all chemicals in all three of the similarity spaces, resulting in a full cross-validation. Thus the correlation coefficients reported are the crossvalidated correlation coefficients.

\section{RESULTS AND DISCUSSION}

The principal objective of this paper was to illustrate the utility of tailoring similarity spaces to a specific property as opposed to the standard method of constructing similarity spaces that are property independent. To this end, we used three spaces, viz., an arbitrary principal component space that would be used for the $K \mathrm{NN}$-based estimation of both $\log P$ and $\mathrm{BP}$, a topological index space based on the RR weighting of the indices for $\log P$, and a topological index space based on the RR weighting of the indices for BP.

From the initial set of 369 topological indices, 222 were retained for inclusion in the PCA and RR procedures after data reduction. From this set of 222 indices, 15 PCs were extracted with eigenvalues greater than or equal to one, resulting in the construction of a 15-dimensional arbitrary similarity space. Table 2 presents a summary of the two TIs most-highly correlated with each of the 15 PCs. For the sake of consistency, it was determined that we would then use the fifteen TIs with the highest rankings from the RR procedure. Table 3 presents the TIs selected for use in developing the similarity spaces tailored for $\log P$ and $\mathrm{BP}$.

The $t-v a l u e s$, indicated in Table 3, are model coefficients extracted from the RR procedure and used to rank the TIs from most to least influential based on the absolute value of the regression coefficient. On close examination of tables 2 and 3 we find that none of the TIs selected by RR are 
well represented in the PCs. Only one of the indices chosen for the tailored BP model, $\mathrm{DN}^{2} 1_{4}$, shows up as the second most-correlated $\mathrm{TI}$ in $\mathrm{PC}_{1}$. Otherwise, the tailored sets have little in common with the TIs selected by RR. Further analysis shows that, of the five TIs most correlated with each of the fifteen PCs, $\mathrm{DN}^{2} 1_{4}$, is still the only TI shared in common between the arbitrary similarity space and either of the tailored spaces. A much higher degree of overlap exists between the two tailored sets. These sets share a total of seven of the fifteen TIs in common (indicated in bold face in Table 3).

Interestingly, beyond the seven shared indices, each of the tailored sets show a marked difference in the types of indices selected. The set developed for modeling $\log P$ is skewed towards zero-order chi indices, while the BP set shows a strong tendency towards the AZN triplets. While there is significant overlap between the two tailored sets of descriptors, it is encouraging to see that they show distinct differences as well. It is also encouraging to see the low-degree of overlap between the indices prevalent in the arbitrary set versus those present in the tailored sets. The arbitrary set should be a general characterization of the structural diversity within the data set and while this is useful for property estimation, there is no intrinsic link to any particular property. The tailored sets are geared towards the prediction of a specific property and, as such, should be geared more strongly towards defining the property of interest than simply characterizing the structural diversity of the structure space.

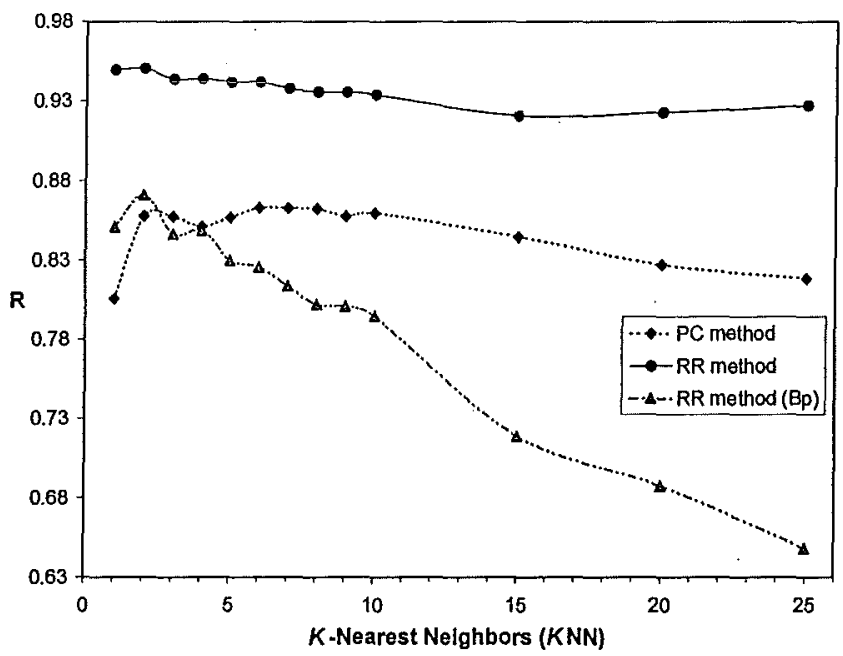

Figure 1. Plot of regression coefficient, $R$, for $K N N$-based estimation of $\log P$ in arbitrary and tailored similarity spaces at varying levels of $K(k=1-10,15,20$ and 25$)$.

Three Euclidean distance-based molecular similarity spaces were constructed from the PCs and TIs indicated in Tables 2 and 3: (a) an arbitrary molecular structure space using the fifteen PCs indicated in Table $2,(b)$ a space tailored for $\log P$ estimation based on the fifteen TIs presented in the second column of Table 3, and (c) a space tailored for BP estimation based on the TIs presented 
in the third column of Table 3.

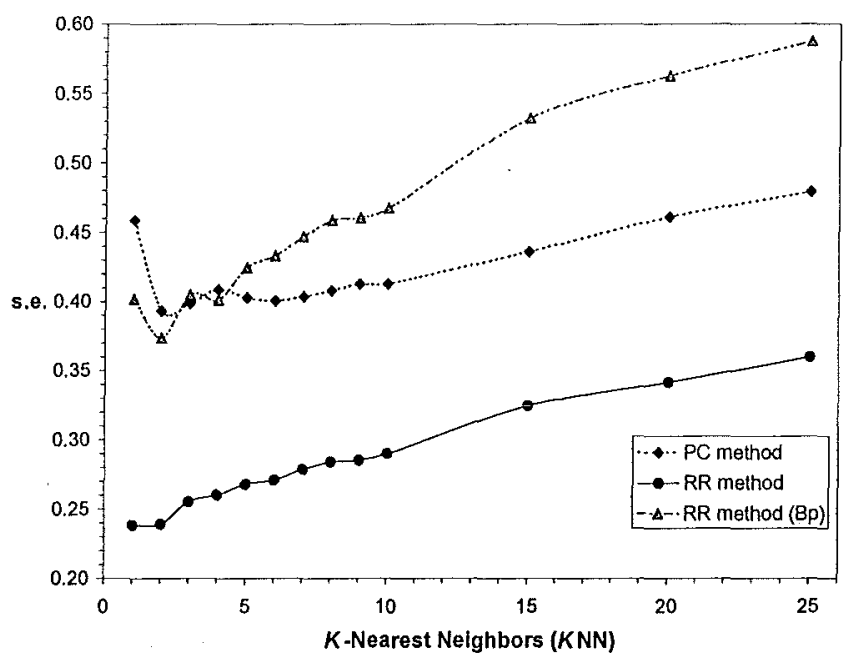

Figure 2. Plot of standard error, s.e., for $K N N$-based estimation of $\log P$ in arbitrary and tailored similarity spaces at varying levels of $K(k=1-10,15,20$ and 25$)$.

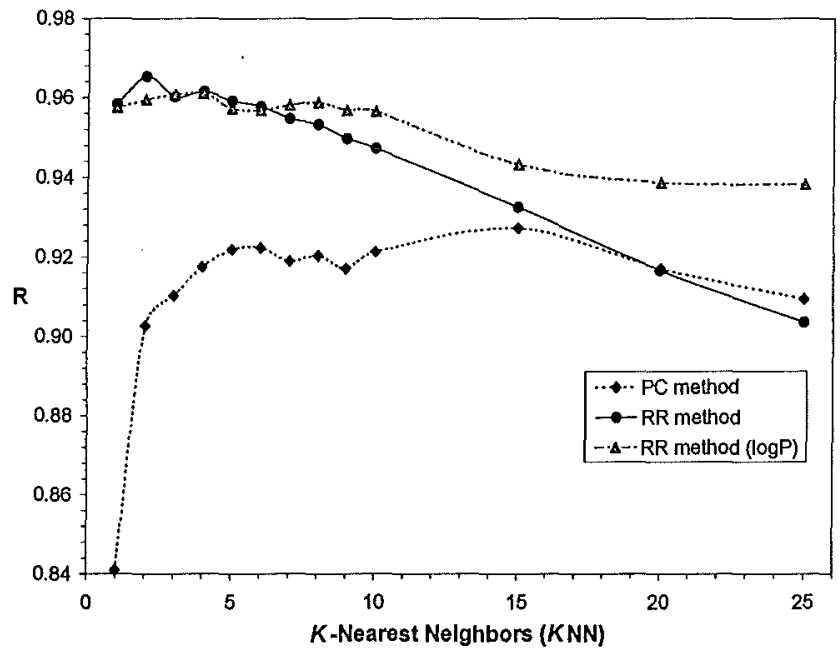

Figure 3. Plot of regression coefficient, $R$, for $K \mathrm{NN}$-based estimation of BP in arbitrary and tailored similarity spaces at varying levels of $K(k=1-10,15,20$ and 25).

Finally, $K N N$-based property estimation was carried out on the three similarity spaces. First we examined the ability of each of the three spaces to estimate $\log P$. In part this was done to verify that the tailored spaces are indeed fitted to the property of interest rather than simply another nonspecific structure space. The results of this analysis are depicted in Figures 1 and 2. Figure 1 presents the correlation coefficients for $\log P$ estimation in each of the similarity spaces for $K=1-$ 10, 15, 20 and 25. Likewise, Figure 2 presents the standard error of $\log P$ estimation for each of the similarity spaces. As can be seen from these figures, the space tailored to $\log P$ definitely outperforms both of the other spaces for the purposes of estimating $\log P$. As might be expected, the 
arbitrary structure space outperforms the BP tailored space in estimating $\log P$ except for when using the one and two nearest neighbors. So, for the purposes of $\log P$ prediction, our tailored similarity space meets our expectations in its performance versus the performance of other spaces.

The examination of these structure spaces for the estimation of BP was carried out in a manner identical to that for the estimation of $\log P$. Each of the three similarity spaces was used in $K \mathrm{NN}-$ based estimation of BP for the complete set of 166 chemicals. These results are summarized in Figures 3 and 4 . Figure 3 presents the correlation coefficients for BP estimation in each of the similarity spaces for $K=1-10,15,20$ and 25 . Likewise, Figure 4 presents the standard error of BP estimation for each of the similarity spaces. As can be seen from these figures, the space tailored to BP definitely out-performs the arbitrary structure space, though, somewhat surprisingly, the space tailored to $\log P$ performs about as well as the BP tailored space. The BP tailored space just slightly outperforms the $\log P$ tailored space through $K=1-6$. However, at higher values of $K$, the $\log P$ space actually outperforms the BP tailored space for the estimation of BP. While this is interesting, not too much weight should be given to the model's performance at higher values for $K$. As was shown in a recent study [30], loss of data variance is a real concern at the higher values of $K$. Thus we ideally want a model that has a high correlation, $R$, and low standard error, s.e., using a minimal number of neighbors. Taking this into consideration, the two tailored similarity spaces are still essentially identical with regards to the prediction of BP for this particular set of $166 \mathrm{JP}-8$ components.

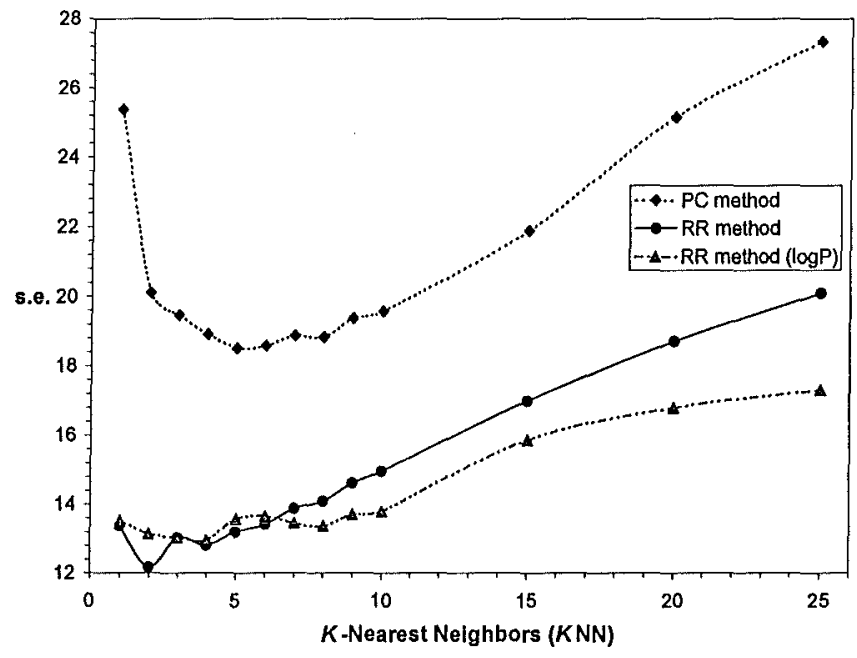

Figure 4. Plot of standard error, s.e., for $K N N$-based estimation of BP in arbitrary and tailored similarity spaces at varying levels of $K(k=1-10,15,20$ and 25).

It should be noted that while none of the molecular connectivity indices (chi indices) were selected by the RR method for modeling BP, they have been shown to be related to the modeling of normal boiling point in a number of studies [60-62]. Bearing this mind, we should not be terribly 
surprised by the performance of the $\log P$ tailored space in the estimation of BP. After all, seven of the fifteen indices were shared in common between the two tailored sets, and then if we consider the chi indices as also related to $\mathrm{BP}$, we now see that ten of the fifteen parameters in the $\log P$ set are also important for the prediction of BP.

\section{CONCLUSIONS}

As can be seen from the results presented in this study, tailored similarity spaces show definite promise in the development of property-specific similarity spaces, as opposed to standard structure-based similarity spaces. Further studies are needed to verify the general utility of this approach, specifically we need to examine the utility of spaces constructed from smaller "training sets" of chemicals when applied to large, diverse data sets. If these methods can be applied successfully to increase the predictive power of similarity measures for large, diverse data sets, this will become a powerful tool for both risk assessment and pharmaceutical design.

\section{Acknowledgment}

The authors acknowledge the financial support of this research by the Air Force Office of Scientific Research grant F496200210138. This is contribution number 318 from the Center for Water and the Environment of the Natural Resources Research Institute.

\section{REFERENCES}

[1] J. C. Arcos, Structure-Activity Relationships: Criteria for Predicting the Carcinogenic Activity of Chemical Compounds, Environ. Sci. Technol. 1987, 21, 743-745.

[2] C. M. Auer, J. V. Nabholz, and K. P. Baetcke, Environ. Health Perspect. 1990, 87, 183-197.

[3] D. M. Sanderson and C. G. Earnshaw, Computer Prediction of Possible Toxic Action from Chemical Structure: The DEREK System, Human Experimental Toxicol. 1991, 10, 261-273.

[4] J. Ashby and R. W. Tennant, Definitive Relationships among Chemical Structure, Carcinogenicity, and Mutagenicity for 301 Chemicals Tested by the U.S. NTP, Mutat. Res. 1991, 257, 229-306.

[5] M. Lajiness, Molecular Similarity-Based Methods for Selecting Compounds for Screening; in: Computational Chemical Graph Theory, Ed. D. H. Rouvray, Nova, New York, 1990, pp 299-316.

[6] S. C. Basak, D. Mills, B. D. Gute, A. T. Balaban, K. Basak, and G. D. Grunwald, Use of Mathematical Structural Invariants in Analyzing Combinatorial Libraries: A Case Study with Psoralen Derivatives, in: Some Aspects of Mathematical Chemistry, Eds. D. K. Sinha, S. C. Basak, R. K. Mohanty, and I. N. Basumallick, Visva-Bharati University, India, 2002, in press.

[7] M. Johnson, S. C. Basak, and G. Maggiora, A Characterization of Molecular Similarity Methods for a Property Prediction, Math. Comput. Modelling 1988, 11, 630-634.

[8] P. Willett and V. Winterman, A Comparison of Some Measures of Inter-Molecular Structural Similarity, Quant. Struct.-Act. Relat. 1986, 5, 18-25.

[9] P. Willett, J. M. Barnard, and G. M. Downs, Chemical Similarity Searching, J. Chem. Inf. Comput. Sci. 1998, 38, 983-996.

[10] R. E. Carhart, D. H. Smith, and R. Venkataraghavan, Atom Pairs as Molecular Features in Structure-Activity Studies: Definition and Applications, J. Chem. Inf. Comput. Sci. 1985, 25, 64-73.

[11] S. C. Basak and G. D. Grunwald, Molecular Similarity and Risk Assessment: Analog Selection and Property Estimation using Graph Invariants, SAR QSAR Environ. Res. 1994, 2, 289-307.

[12] S. C. Basak and G. D. Grunwald, Use of Topological Space and Property Space in Selecting Structural Analogs, Mathl. Modelling Sci. Computing 1994, 4, 464-469.

[13] S. C. Basak, S. Bertelsen, and G. Grunwald, Application of Graph Theoretical Parameters in Quantifying Molecular Similarity and Structure-Activity Studies, J. Chem. Inf. Comput. Sci. 1994, 34, 270-276. 
B. D. Gute, S. C. Basak, D. Mills, and D. M. Hawkins

Internet Electronic Journal of Molecular Design 2002, 1, 374-387

[14] S. C. Basak, S. Bertelsen, and G. D. Grunwald, Use of Graph Theoretic Parameters in Risk Assessment of Chemicals, Toxicol. Lett. 1995, 79, 239-250.

[15] S. C. Basak, B. D. Gute, and G. D. Grunwald, Use of graph invariants in QMSA and predictive toxicology, in: Discrete Mathematical Chemistry, Eds. P. Hansen, P. Fowler and M. Zheng, DIMACS Series 51, American Mathematical Society, Providence, Rhode Island, 2000, pp 9-24.

[16] S. C. Basak, B. D. Gute, and G. D. Grunwald, Development and Application of Molecular Similarity Methods using Nonempirical Parameters, Mathl. Modelling Sci. Computing 2002, in press.

[17] M. Randić, Similarity Methods of Interest in Chemistry; in: Mathematical Methods in Contemporary Chemistry, Ed. S. I. Kuchanov, Gordon and Breach, Amsterdam, 1996, pp 1-100.

[18] G. M. Downs, P. Willett, and W. Fisanick, Similarity Searching and Clustering of Chemical-Structure Databases using Molecular Property Data, J. Chem. Inf. Comput. Sci. 1994, 34, 1094-1102.

[19] B. D. Gute, G. D. Grunwald, D. Mills, and S. C. Basak, Molecular Similarity Based Estimation of Properties: A Comparison of Structure Spaces and Property Spaces, SAR QSAR Environ. Res, 2001, 11, 363-382.

[20] S. C. Basak, V. R. Magnuson, G. J. Niemi, and R. R. Regal, Determining Structural Similarity of Chemicals using Graph-Theoretic Indices, Discrete Appl. Math. 1988, 19, 17-44.

[21] S. C. Basak and G. D. Grunwald, Estimation of Lipophilicity from Molecular Structural Similarity, New J. Chem. 1995, 19, 231-237.

[22] S. C. Basak and G. D. Grunwald, Molecular similarity and estimation of molecular properties, J. Chem. Inf. Comput. Sci. 1995, 35, 366-372.

[23] S. C. Basak and G. D. Grunwald, Predicting Mutagenicity of Chemicals using Topological and Quantum Chemical Parameters: A Similarity Based Study, Chemosphere 1995, 31, 2529-2546.

[24] S. C. Basak and G. D. Grunwald, Tolerance Space and Molecular Similarity, SAR QSAR Environ. Res. 1995, 3, 265-277.

[25] S. C. Basak, B. D. Gute, and G. D. Grunwald, Estimation of Normal Boiling Points of Haloalkanes using Molecular Similarity, Croat. Chim. Acta 1996, 69, 1159-1173.

[26] S. C. Basak and B. D. Gute, Use of Graph Theoretic Parameters in Predicting Inhibition of Microsomal Hydroxylation of Anilines by Alcohols: A Molecular Similarity Approach, in Proceedings of the International Congress on Hazardous Waste: Impact on Human and Ecological Health, Eds. B. L. Johnson, C. Xintaras, and J. S. Andrews, Princeton Scientific Publishing Co, Inc, 1997, pp 492-504.

[27] S. C. Basak, B. D. Gute, and G. D. Grunwald, Characterization of the Molecular Similarity of Chemicals using Topological Invariants; in: Advances in Molecular Similarity, Eds. R, Carbo-Dorca and P. G. Mezey, JAI Press, Stanford, Connecticut, Vol. 2, 1998, pp. 171-185.

[28] S. C. Basak, G. D. Grunwald, G. E. Host, G. J. Niemi, and S. P. Bradbury, A Comparative Study of Molecular Similarity, Statistical and Neural Network Methods for Predicting Toxic Modes of Action of Chemicals, Environ. Toxicol. Chem. 1998, 17, 1056-1064.

[29] S. C. Basak, B. D. Gute, and G. D. Grunwald, Assessment of Mutagenicity of Chemicals from Theoretical Structural Parameters: A Hierarchical Approach, SAR QSAR Environ. Res. 1999, 10, 117-129.

[30] B. D. Gute and S. C. Basak, Molecular similarity-based estimation of properties: A comparison of three structure spaces, J. Mol. Graphics Modelling 2001, 20, 95-109.

[31] S. C. Basak, D. Mills, B. D. Gute, G. D. Grunwald, and A. T. Balaban, Applications of topological indices in predicting property/ bioactivity/ toxicity of chemicals; in: Topology in Chemistry: Discrete Mathematics of Molecules, Eds. D. H. Rouvray and R. B. King, Horwood Publishing Ltd., Chichester, United Kingdom, 2001.

[32] S. C. Basak, B. D. Gute, D. Mills, and D. M. Hawkins, Quantitative Molecular Similarity Methods in the Property/ Toxicity Estimation of Chemicals: A Comparison of Arbitrary versus Tailored Similarity Spaces, $J$. Mol. Struct. (Theochem) 2002, accepted.

[33] C. L. Russom, E. B. Anderson, B. E. Greenwood, and A. Pilli, ASTER: An Integration of the AQUIRE Data Base and the QSAR System for use in Ecological Risk Assessments. Sci. Total Environ. 1991, 109/1 10, 667-670.

[34] AFOSR JP-8 Jet Fuel Workshop, University of Arizona, Tucson, Arizona, Jan. 11-12, 2000.

[35] S. C. Basak, D. K. Harriss, and V. R. Magnuson, POLLY 2.3: Copyright of the University of Minnesota, 1988.

[36] Molconn-Z v3.50, Hall and Associates Consulting, Quincy, MA, 2000.

[37] P. A. Filip, T. S. Balaban, and A. T. Balaban, A New Approach for Devising Local Graph Invariants: Derived Topological Indices with Low Degeneracy and Good Correlation Ability, J. Math. Chem. 1987, 1, 61-83.

[38] H. Wiener, Structural Determination of Paraffin Boiling Points, J. Am. Chem. Soc. 1947, 69, 17-20.

[39] M. Randić, On Characterization of Molecular Branching, J. Am. Chem. Soc. 1975, 97, 6609-6615.

[40] L. B. Kier and L. H. Hall, Molecular Connectivity in Structure-Activity Analysis, Research Studies Press, Letchworth, 1986.

[41] D. Bonchev and N. Trinajstić, Information Theory, Distance Matrix and Molecular Branching, J. Chem. Phys. $1977,67,4517-4533$.

[42] C. Raychaudhury, S. K. Ray, J. J. Ghosh, A. B. Roy, and S. C. Basak, Discrimination of Isomeric Structures using 
Tailored Similarity Spaces for the Prediction of Physicochemical Properties Internet Electronic Journal of Molecular Design 2002, 1, 374-387

Information Theoretic Topological Indices, $J$. Comput. Chem. 1984, 5, 581-588.

[43] S. C. Basak, A. B. Roy, and J. J. Ghosh, Study of the Structure-Function Relationship of Pharmacological and Toxicological Agents using Information Theory; in: Proceedings of the 2nd International Conference on Mathematical Modelling, Eds. X. J. R. Avula, R. Bellman, Y. L. Luke, and A. K. Rigler, University of MissouriRolla, Rolla, Missouri, Vol. II, 1980, pp 851-856.

[44] S. C. Basak and V. R. Magnuson, Molecular Topology and Narcosis: A Quantitative Structure-Activity Relationship (QSAR) Study of Alcohols using Complementary Information Content (CIC), Arzneim. Forsch. 1983, 33, 501-503.

[45] A. B. Roy, S. C. Basak, D. K. Harriss, and V. R. Magnuson, Neighborhood Complexities and Symmetry of Chemical Graphs and Their Biological Applications; in: Mathematical Modelling in Science and Technology, Eds. X. J. R. Avula, R. E. Kalman, A. I. Lipais, and E. Y. Rodin, Pergamon Press, New York, 1984, pp 745-750.

[46] A. T. Balaban, Highly Discriminating Distance-Based Topological Index, Chem. Phys. Lett. 1982, 89, $399-404$.

[47] A. T. Balaban, Topological Indices based on Topological Distances in Molecular Graphs, Pure Appl. Chem. 1983, $55,199-206$.

[48] A. T. Balaban, Chemical Graphs. Part 48. Topological Index $\mathrm{J}$ for Heteroatom-Containing Molecules taking into Account Periodicities of Element Properties, MATCH (Commun. Math. Chem.) 1986, 21, 115-122.

[49] L. B. Kier, A Shape Index from Molecular Graphs, Quant. Struct.-Act. Relat. 1985, 4, 109-116.

[50] L. B. Kier and L. H. Hall, The Kappa Indices for Modeling Molecular Shape and Flexibility; in: Topological Indices and Related Descriptors in QSAR and QSPR, Eds. J. Devillers and A. T. Balaban, Gordon and Breach Science Publishers, Amsterdam, 1999, 455-489.

[51] L. B. Kier and L. H. Hall, The Electrotopological State: Structure Modeling Molecular for QSAR and Database Analysis; in: Topological Indices and Related Descriptors in QSAR and QSPR, Eds. J. Devillers and A. T. Balaban, Gordon and Breach Science Publishers, Amsterdam, 1999, 491-562.

[52] SAS Institute Inc., in SAS/STAT User's Guide, Release 6.03 Edition, SAS Institute Inc., Cary, NC, 1988.

[53] A. E. Hoerl and R. W. Kennard, Ridge Regression: Biased Estimation for Nonorthogonal Problems, Technometrics 1970, 8, 27-51.

[54] S. C. Basak, G. J. Niemi, and G. D. Veith, Optimal Characterization of Structure for Prediction of Properties, $J$. Math. Chem. 1991, 7, 243-272.

[55] S. C. Basak and B. D. Gute, Characterization of Molecular Structures using Topological Indices, SAR QSAR Environ. Res. 1997, 7, 1-21.

[56] D. Hawkins, S. Basak, and X. Shi, QSAR with Few Compounds and Many Features, J. Chem. Inf. Comput. Sci. 2001, 4I, 663-670.

[57] S. C. Basak, D. M. Hawkins, and D. Mills, Predicting Blood:Air Partition Coefficient of Structurally Diverse Chemicals using Theoretical Molecular Descriptors; in: Advances in Molecular Similarity, Eds. R. Carbo-Dorca and P.G. Mezey, Vol. 5, 2002, in press.

[58] S. C. Basak, H. El-Masri, D. M. Hawkins, and D. Mills, Exposure Assessment of Volatile Organic Chemicals (VOCs): Predicting Blood:Air Partition Coefficients of Diverse Chemicals using Theoretical Descriptors, $J$. Chem. Inf. Comput. Sci. 2002, submitted.

[59] S. C. Basak, D. Mills, D. M. Hawkins, and H. El-Masri, Prediction of Human Blood:Air Partition Coefficient: A Comparison of Structure-based and Property-based Methods, Risk Analysis 2002, submitted.

[60] L. B. Kier and L. H. Hall, Molecular Connectivity in Chemistry and Drug Research, Academic Press, New York, 1976.

[61] D. E. Needham, I.-C. Wei, and P. G. Seybold, Molecular Modeling of the Physical Properties of the Alkanes, $J$. Am. Chem. Soc. 1992, 110, 4186-4194.

[62] S. C. Basak, B. D. Gute, and G. D. Grunwald, A Comparative Study of Topological and Geometrical Parameters in Estimating Normal Boiling Point and Octanol/Water Partition Coefficient, J. Chem. Inf. Comput. Sci. 1996, 36, $1054-1060$. 
Appendix 1.4

Manuscripts on PBPK modeling 


\title{
PREDICTING BLOOD:AIR PARTITION COEFFICIENT OF STRUCTURALLY DIVERSE CHEMICALS USING THEORETICAL MOLECULAR DESCRIPTORS
}

\author{
S. C. BASAK, ${ }^{\mathrm{a}, \dagger}$ D. M. HAWKINS ${ }^{\mathrm{b}}$ and D. MLLS ${ }^{\mathrm{a}}$ \\ ${ }^{a}$ Natural Resources Research Institute, University of Minnesota, \\ 5013 Miller Trunk Highway, Duluth, Minnesota 55811, USA \\ ${ }^{b}$ School of Statistics, University of Minnesota, \\ 313 Ford Hall, 224 Church Street S.E., Minneapolis, Minnesota 55455, USA
}

\begin{abstract}
Quantitative structure-property relationship (QSPR) models for the estimation of rat blood:air partition coefficient were developed using ridge regression (RR), principal components regression (PCR), and partial least squares (PLS). A set of 24 compounds was analyzed, including 14 haloalkanes, 7 aromatic hydrocarbons, and 3 alkanes. Four classes of mathematical structural descriptors, viz., topostructural indices (TSI), topochemical indices (TCI), geometrical/shape indices (3DI), and quantum chemical indices (QCI), were used in the development of hierarchical and single-class models. The models developed using RR were superior to those developed using either PCR or PLS. The results indicate that the easily calculated TC descriptors give the best model.
\end{abstract}

Keywords: Blood:air partition coefficient; PBPK modeling; Mathematical molecular descriptors; Hierarchical QSPR; Ridge regression

\section{INTRODUCTION}

Partition coefficients of volatile organic chemicals are routinely used in the assessment of their hazard. In particular, partition coefficients are used in estimating uptake, distribution, metabolism and elimination of chemicals as well as the potential risk posed by such chemicals to human health as a result of exposure to them [1]. Therefore, there is an interest in estimating realistic blood:air and tissue:air partition coefficient (P) values of chemicals to which we are frequently exposed.

\footnotetext{
${ }^{\dagger}$ Corresponding author phone: (218) 720-4230; fax: (218) 720-4328; e-mail: sbasak@nrri.umn.edu
} 
Gargas et al. [1] determined various liquid:air and tissue:air partition coefficients for $0.9 \%$ saline, olive oil, blood, liver, muscle and fat tissue. Then they used regression models to predict the partition coefficient in one pair of media from the data for another pair. For example, human blood:air partition coefficient was estimated from the corresponding rat blood:air value; $\mathrm{P}$ for blood:air, liver:air, and muscle:air pairs were estimated from the $\mathrm{P}$ values of olive oil:air for various subsets of volatile organic chemicals (VOCs).

The blood:air partition coefficient (P, blood:air) is an important factor in determining the pulmonary uptake of VOCs through the inhalation route. $\mathrm{P}$ is routinely used in building physiologically based pharmacokinetic (PBPK) models for exposure assessment. Solubility of VOCs in blood is determined by two major factors: a) composition including the content of neutral lipid, phospholipid and water, and b) the extent of binding of these chemicals to specific blood components such as plasma proteins, hemoglobin, etc. Such physicochemical considerations have been used to develop physicochemically based estimation methods for blood:air P values of chemicals [2].

Methods for the estimation of $\mathrm{P}$ described above are essentially property-property correlations, which depend on the availability of relevant experimental and tissue composition data. In practical situations of hazard assessment of chemicals, the majority of them have very little or no available physicochemical data [3]. A viable alternative in such cases is the use of theoretical molecular descriptors, which can be calculated directly from molecular structure for the estimation of $P$. Quantitative structureproperty/activity/ toxicity relationships (QSPRs/ QSARs/QSTRs) based on such descriptors have been found to be useful in the estimation of diverse properties including boiling point [4-11], vapor pressure [12-17], octanol/water partition coefficient [7, 18], mutagenicity [19-23], aquatic toxicity [24-32], complement inhibitory activity [33], penetration through the blood-brain barrier [34], skin penetration [35], and toxic modes of action [36], to name just a few. In a recent paper, we found that computed molecular descriptors correlate well with blood:air partition coefficient of a congeneric set of 13 chlorocarbons [37]. In this paper, we have extended our study to estimate the blood:air P values for a large and structurally more diverse set of VOCs.

\section{METHODS}

\section{Database}

In a 1996 study, Poulin and Krishnan presented a table containing experimental blood:air partition coefficients for a set of 27 compounds including alkanes, haloalkanes, and aromatic hydrocarbons [2]; with the experimental values having been obtained from an earlier study performed by Gargas et al.using a modified version of the vial-equilibration technique [1]. Three of the 27 compounds were not included 
in the present study, as it was not possible to calculate all descriptors for one of these compounds, and two of the compounds were cis/trans isomers which are not distinguishable in terms of their descriptors. The 24 compounds used in the current study, along with their experimental blood:air partition coefficient values, are provided in Table I.

TABLE I Experimental rat blood:air partition coefficients (P, blood:air) for the set of 24 compounds

\begin{tabular}{lc}
\hline \multicolumn{1}{c}{ Chemicals } & Experimental $P^{a}$ \\
\hline Alkanes & \\
$n$-Hexane & $2.29 \pm 0.11$ \\
Cyclohexane & $1.39 \pm 0.09$ \\
$n$-Heptane & $4.75 \pm 0.15$ \\
Haloalkanes & \\
Dichloromethane & $19.4 \pm 0.8$ \\
Chloroform & $20.8 \pm 0.1$ \\
Carbon tetrachloride & $4.52 \pm 0.35$ \\
Bromochloromethane & $41.5 \pm 0.9$ \\
1,1-Dichloroethane & $11.2 \pm 0.1$ \\
1,2-Dichloroethane & $30.4 \pm 1.2$ \\
1,1,1-Trichloroethane & $5.76 \pm 0.5$ \\
1,1,2-Trichloroethane & $58.0 \pm 1.1$ \\
1,1,1,2-Tetrachloroethane & $41.7 \pm 1.0$ \\
1,1,2,2,-Tetrachloroethane & $142 \pm 6$ \\
Pentachloroethane & $104 \pm 3$ \\
1,2-Dichloropropane & $18.7 \pm 0.5$ \\
Trichloroethylene & $21.9 \pm 1.4$ \\
Tetrachloroethylene & $18.9 \pm 1.1$ \\
Aromatic hydrocarbons & \\
Benzene & $17.8 \pm 0.3$ \\
Chlorobenzene & $59.4 \pm 1.0$ \\
Toluene & $18.0 \pm 1.0$ \\
Styrene & $40.2 \pm 3.7$ \\
$o$-Xylene & $44.3 \pm 2.0$ \\
$m$-Xylene & $46.0 \pm 1.5$ \\
$p$-Xylene & $41.3 \pm 3.5$ \\
\hline a Vales represen &
\end{tabular}

${ }^{\mathrm{a}}$ Values represent means \pm standard error.

\section{Calculation of Molecular Descriptors}

A complete list of the 378 mathematical molecular descriptors calculated for use in the current study is provided in Table II. These parameters are partitioned into classes that are ordered hierarchically based on their level of complexity. At the lowest level of the hierarchy are the topostructural indices (TSI) which encode information strictly about the adjacency and topological distances of atoms in molecular structures. The topochemical indices (TCI) are more complex, encoding information not only about the topology but also chemical properties of the molecular structures. The geometrical indices (3DI) are more complex still, encoding information about the shape and 3-dimensional aspects of the structure. Finally, 
the quantum chemical indices (QCI) are at the upper end of the hierarchy, encoding electronic information. The software programs used for the calculation of these descriptors include POLLY [38], Triplet [39, 40], Molconn-Z [41], Sybyl [42], and MOPAC [43]. Together, POLLY and Triplet calculate a large number of topological, i.e. topostructural and topochemical, descriptors. Molconn- $Z$ provides additional topological descriptors as well as a small set of molecular shape indices. Sybyl and MOPAC calculate relatively small sets of 3-dimensional and quantum chemical descriptors, respectively.

TABLE II Descriptors calculated for use in the current study, along with brief descriptions and hierarchical classification

\begin{tabular}{|c|c|}
\hline \multicolumn{2}{|r|}{ Topostructural (TS) } \\
\hline$I_{\mathrm{D}}^{\mathrm{W}}$ & $\begin{array}{l}\text { Information index for the magnitudes of distances between all possible pairs of vertices of a } \\
\text { graph }\end{array}$ \\
\hline$\overline{I_{D}^{W}}$ & Mean information index for the magnitude of distance \\
\hline $\mathrm{W}$ & Wiener index $=$ half-sum of the off-diagonal elements of the distance matrix of a graph \\
\hline $\mathrm{I}^{\mathrm{D}}$ & Degree complexity \\
\hline $\mathrm{H}^{\mathrm{V}}$ & Graph vertex complexity \\
\hline $\mathrm{H}^{\mathrm{D}}$ & Graph distance complexity \\
\hline$\overline{\mathrm{IC}}$ & $\begin{array}{l}\text { Information content of the distance matrix partitioned by frequency of occurrences of distance } \\
\mathrm{h}\end{array}$ \\
\hline $\mathrm{M}_{1}$ & A Zagreb group parameter $=$ sum of square of degree over all vertices \\
\hline $\mathrm{M}_{2}$ & $\begin{array}{l}\text { A Zagreb group parameter = sum of cross-product of degrees over all neighboring (connected) } \\
\text { vertices }\end{array}$ \\
\hline${ }^{\mathrm{h}} \chi$ & Path connectivity index of order $h=0-10$ \\
\hline${ }^{\mathrm{h}} \chi_{\mathrm{C}}$ & Cluster connectivity index of order $h=3-6$ \\
\hline${ }^{\mathrm{h}} \chi_{\mathrm{PC}}$ & Path-cluster connectivity index of order $h=4-6$ \\
\hline${ }^{\mathrm{h}} \chi_{\mathrm{Ch}}$ & Chain connectivity index of order $h=3-10$ \\
\hline$P_{h}$ & Number of paths of length $h=0-10$ \\
\hline $\mathbf{J}$ & Balaban's J index based on topological distance \\
\hline nrings & Number of rings in a graph \\
\hline ncirc & Number of circuits in a graph \\
\hline $\mathrm{DN}^{2} \mathrm{~S}_{\mathrm{y}}$ & $\begin{array}{l}\text { Triplet index of distance matrix, square of graph order (\# of non-H atoms), and distance sum; } \\
\text { operation } y=1-5\end{array}$ \\
\hline $\mathrm{DN}^{2} 1_{y}$ & Triplet index of distance matrix, square of graph order, and number 1 ; operation $y=1-5$ \\
\hline $\mathrm{AS} 1_{\mathrm{y}}$ & Triplet index of adjacency matrix, distance sum, and number 1 ; operation $y=1-5$ \\
\hline DS1 $1_{y}$ & Triplet index of distance matrix, distance sum, and number 1 ; operation $y=1-5$ \\
\hline $\mathrm{ASN}_{\mathrm{y}}$ & Triplet index of adjacency matrix, distance sum, and graph order; operation $y=1-5$ \\
\hline $\operatorname{DSN}_{y}$ & Triplet index of distance matrix, distance sum, and graph order; operation $y=1-5$ \\
\hline $\mathrm{DN}^{2} \mathrm{~N}_{\mathrm{y}}$ & Triplet index of distance matrix, square of graph order, and graph order; operation $y=1-5$ \\
\hline $\mathrm{ANS}_{\mathrm{y}}$ & Triplet index of adjacency matrix, graph order, and distance sum; operation $y=1-5$ \\
\hline $\mathrm{AN} 1_{y}$ & Triplet index of adjacency matrix, graph order, and number 1 ; operation $y=1-5$ \\
\hline $\mathrm{ANN}_{\mathrm{y}}$ & Triplet index of adjacency matrix, graph order, and graph order again; operation $y=1-5$ \\
\hline $\mathrm{ASV}_{\mathrm{y}}$ & Triplet index of adjacency matrix, distance sum, and vertex degree; operation $y=1-5$ \\
\hline $\mathrm{DSV}_{\mathrm{y}}$ & Triplet index of distance matrix, distance sum, and vertex degree; operation $y=1-5$ \\
\hline $\mathrm{ANV}_{\mathrm{y}}$ & Triplet index of adjacency matrix, graph order, and vertex degree; operation y $=1-5$ \\
\hline
\end{tabular}




\begin{tabular}{|c|c|}
\hline \multicolumn{2}{|r|}{ Topochemical (TC) } \\
\hline $\mathrm{O}$ & Order of neighborhood when $\mathrm{IC}_{\mathrm{r}}$ reaches its maximum value for the hydrogen-filled graph \\
\hline $\mathrm{O}_{\text {orb }}$ & $\begin{array}{l}\text { Order of neighborhood when } \mathrm{IC}_{\mathrm{r}} \text { reaches its maximum value for the hydrogen-suppressed } \\
\text { graph }\end{array}$ \\
\hline$I_{\text {orb }}$ & $\begin{array}{l}\text { Information content or complexity of the hydrogen-suppressed graph at its maximum } \\
\text { neighborhood of vertices }\end{array}$ \\
\hline $\mathrm{IC}_{\mathrm{r}}$ & $\begin{array}{l}\text { Mean information content or complexity of a graph based on the } \mathrm{r}^{\text {th }}(\mathrm{r}=0-6) \text { order } \\
\text { neighborhood of vertices in a hydrogen-filled graph. }\end{array}$ \\
\hline $\mathrm{SIC}_{\mathrm{r}}$ & $\begin{array}{l}\text { Structural information content for } \mathrm{r}^{\mathrm{th}}(\mathrm{r}=0-6) \text { order neighborhood of vertices in a hydrogen- } \\
\text { filled graph }\end{array}$ \\
\hline $\mathrm{CIC}_{\mathrm{r}}$ & $\begin{array}{l}\text { Complementary information content for } \mathrm{r}^{\text {th }}(r=0-6) \text { order neighborhood of vertices in a } \\
\text { hydrogen-filled graph }\end{array}$ \\
\hline${ }^{\mathrm{h}} \chi^{\mathrm{b}}$ & Bond path connectivity index of order $h=0-6$ \\
\hline${ }^{\mathrm{h}} \chi_{\mathrm{c}}^{\mathrm{b}}$ & Bond cluster connectivity index of order $h=3-6$ \\
\hline${ }^{\mathrm{b}} \chi_{\mathrm{Ch}}^{\mathrm{b}}$ & Bond chain connectivity index of order $h=3-6$ \\
\hline${ }^{\mathrm{h}} \chi_{\mathrm{PC}}^{\mathrm{b}}$ & Bond path-cluster connectivity index of order $h=4-6$ \\
\hline${ }^{\mathrm{h}} \chi^{\mathrm{v}}$ & Valence path connectivity index of order $h=0-10$ \\
\hline${ }^{\mathrm{h}} \chi_{\mathrm{C}}^{v}$ & Valence cluster connectivity index of order $h=3-6$ \\
\hline${ }^{\mathrm{h}} \chi_{\mathrm{Ch}}^{\mathrm{v}}$ & Valence chain connectivity index of order $h=3-10$ \\
\hline${ }^{\mathrm{h}} \chi_{\mathrm{PC}}^{\mathrm{v}}$ & Valence path-cluster connectivity index of order $h=4-6$ \\
\hline $\mathrm{J}^{\mathrm{B}}$ & Balaban's J index based on bond types \\
\hline $\mathrm{J}^{\mathrm{X}}$ & Balaban's $\mathrm{J}$ index based on relative electronegativities \\
\hline $\mathrm{J}^{\mathrm{Y}}$ & Balaban's J index based on relative covalent radii \\
\hline$A Z V_{y}$ & Triplet index of adjacency matrix, atomic number, and vertex degree; operation $y=1-5$ \\
\hline $\mathrm{AZS}_{\mathrm{y}}$ & Triplet index of adjacency matrix, atomic number, and distance sum; operation $y=1-5$ \\
\hline $\mathrm{ASZ}_{\mathrm{y}}$ & Triplet index of adjacency matrix, distance sum, and atomic number; operation $y=1-5$ \\
\hline $\mathrm{AZN}_{\mathrm{y}}$ & Triplet index of adjacency matrix, atomic number, and graph order; operation y = 1-5 \\
\hline $\mathrm{ANZ}_{\mathrm{y}}$ & Triplet index of adjacency matrix, graph order, and atomic number; operation $y=1-5$ \\
\hline $\mathrm{DSZ}_{\mathrm{y}}$ & Triplet index of distance matrix, distance sum, and atomic number; operation $y=1-5$ \\
\hline $\mathrm{DN}^{2} \mathrm{Z}_{\mathrm{y}}$ & Triplet index of distance matrix, square of graph order, and atomic number; operation $y=1-5$ \\
\hline nvx & Number of non-hydrogen atoms in a molecule \\
\hline nelem & Number of elements in a molecule \\
\hline fw & Molecular weight \\
\hline${ }^{\mathrm{h}} \chi^{\mathrm{v}}$ & Valence path connectivity index of order $h=7-10$ \\
\hline${ }^{\mathrm{h}} \chi_{\mathrm{Ch}}^{\mathrm{v}}$ & Valence chain connectivity index of order $h=7-10$ \\
\hline si & Shannon information index \\
\hline totop & Total Topological Index $t$ \\
\hline sumI & Sum of the intrinsic state values I \\
\hline sumdell & Sum of delta-I values \\
\hline tets2 & Total topological state index based on electrotopological state indices \\
\hline phia & Flexibility index $\left(\mathrm{kp} 1^{*} \mathrm{kp} 2 / \mathrm{nvx}\right)$ \\
\hline IdCbar & Bonchev-Trinajstic information index \\
\hline IdC & Bonchev-Trinajstic information index \\
\hline Wp & Wienerp \\
\hline Pf & Plattf \\
\hline
\end{tabular}


TABLE II (Continued)

\begin{tabular}{|c|c|}
\hline Wt & Total Wiener number \\
\hline knotp & Difference of chi-cluster- 3 and path/cluster- 4 \\
\hline knotpv & Valence difference of chi-cluster-3 and path/cluster- 4 \\
\hline nclass & Number of classes of topologically (symmetry) equivalent graph vertices \\
\hline numHBd & Number of hydrogen bond donors \\
\hline numwHBd & Number of weak hydrogen bond donors \\
\hline numHBa & Number of hydrogen bond acceptors \\
\hline SHCsats & E-State of $\mathrm{C} \mathrm{sp}^{3}$ bonded to other saturated $\mathrm{C}$ atoms \\
\hline SHCsatu & E-State of $\mathrm{C} \mathrm{sp}{ }^{3}$ bonded to unsaturated $\mathrm{C}$ atoms \\
\hline SHvin & E-State of $\mathrm{C}$ atoms in the vinyl group, $=\mathrm{CH}-$ \\
\hline SHtvin & E-State of $\mathrm{C}$ atoms in the terminal vinyl group, $=\mathrm{CH}_{2}$ \\
\hline SHavin & E-State of $\mathrm{C}$ atoms in the vinyl group, $=\mathrm{CH}-$, bonded to an aromatic $\mathrm{C}$ \\
\hline SHarom & E-State of $\mathrm{C} \mathrm{sp}{ }^{2}$ which are part of an aromatic system \\
\hline SHHBd & $\begin{array}{l}\text { Hydrogen bond donor index, sum of Hydrogen E-State values for }-\mathrm{OH},=\mathrm{NH},-\mathrm{NH} 2,-\mathrm{NH}-\text {, } \\
\text {-SH, and \#CH }\end{array}$ \\
\hline SHwHBd & $\begin{array}{l}\text { Weak hydrogen bond donor index, sum of } \mathrm{C}-\mathrm{H} \text { Hydrogen } \mathrm{E} \text {-State values for hydrogen atoms } \\
\text { on a } \mathrm{C} \text { to which a } \mathrm{F} \text { and/or } \mathrm{Cl} \text { are also bonded }\end{array}$ \\
\hline $\mathrm{SHHBa}$ & $\begin{array}{l}\text { Hydrogen bond acceptor index, sum of the E-State values for }-\mathrm{OH},=\mathrm{NH} \text {, } \\
-\mathrm{NH} 2,-\mathrm{NH}-,>\mathrm{N}-,-\mathrm{O}-,-\mathrm{S}-\text {, along with }-\mathrm{F} \text { and }-\mathrm{Cl}\end{array}$ \\
\hline Qv & General Polarity descriptor \\
\hline NHBint $_{\mathrm{y}}$ & Count of potential internal hydrogen bonders $(y=2-10)$ \\
\hline \multirow[t]{4}{*}{ SHBint $_{\mathrm{y}}$} & E-State descriptors of potential internal hydrogen bond strength $(y=2-10)$ \\
\hline & Electrotopological State index values for atoms types: \\
\hline & $\begin{array}{l}\text { SHsOH, SHdNH, SHsSH, SHsNH2, SHssNH, SHtCH, SHother, SHCHnX, Hmax Gmax, } \\
\text { Hmin, Gmin, Hmaxpos, Hminneg, SsLi, SssBe, Sssss,Bem, SssBH, SsssB, SssssBm, SsCH3, } \\
\text { SdCH2, SssCH2, StCH, SdsCH, SaaCH, SsssCH, SddC,StsC, SdssC, SaasC, SaaaC, SssssC, } \\
\text { SsNH3p, SsNH2, SssNH2p, SdNH, SssNH, SaNH, StN, SsssNHp, SdsN, SaaN, SsssN, } \\
\text { SddsN, SaasN, SssssNp, SsOH, SdO, SssO, SaaO, SsF, SsSiH3, SssSiH2, SsssSiH, SssssSi, } \\
\text { SsPH2, SssPH, SsssP, SdsssP, SsssssP, SsSH, SdS, SssS, SaaS, Sdsss, SddssS, SssssssS, SsC1, } \\
\text { SsGeH3, SssGeH2, SsssGeH, SssssGe, SsAsH2, SssAsH, SsssAs, SdsssAs, SsssssAs, SsSeH, } \\
\text { SdSe, SssSe, SaaSe, SdssSe, SddssSe, SsBr, SsSnH3, SssSnH2, SsssSnH, SssssSn, SsI, } \\
\text { SsPbH3, SssPbH2, SsssPbH, SssssPb }\end{array}$ \\
\hline & Geometrical / Shape (3D) \\
\hline $\mathrm{kp} 0$ & Kappa zero \\
\hline kp1-kp3 & Kappa simple indices \\
\hline ka1-ka3 & Kappa alpha indices \\
\hline$V_{W}$ & Van der Waals volume \\
\hline${ }^{3 \mathrm{D}} \mathrm{W}_{\mathrm{H}}$ & 3D Wiener number based on the hydrogen-filled geometric distance matrix \\
\hline${ }^{3 \mathrm{D}} \mathrm{W}$ & 3D Wiener number based on the hydrogen-suppressed geometric distance matrix \\
\hline \multicolumn{2}{|r|}{ Quantum Chemical $(Q C)$} \\
\hline $\mathrm{E}_{\text {номо }}$ & Energy of the highest occupied molecular orbital \\
\hline $\mathrm{E}_{\text {Hомо-1 }}$ & Energy of the second highest occupied molecular orbital \\
\hline $\mathrm{E}_{\text {LUMO }}$ & Energy of the lowest unoccupied molecular orbital \\
\hline $\mathrm{E}_{\text {LUMO+1 }}$ & Energy of the second lowest unoccupied molecular orbital \\
\hline$\Delta \mathrm{H} f$ & Heat of formation \\
\hline$\mu$ & Dipole moment \\
\hline
\end{tabular}




\section{Statistical Analysis}

Prior to performing regression analysis, the number of independent variables was reduced as follows: (1) descriptors that had a value of 0 for all 24 compounds were eliminated, (2) descriptors which could not be calculated for all 24 compounds were eliminated, (3) perfectly correlated descriptors were eliminated. In the latter case, the CORR procedure of the SAS statistical package [44] was used to identify pairs of perfectly correlated descriptors, and only one of each of the correlated pairs was retained for use in the subsequent analysis. At this point, 219 descriptors remained. The independent variables were scaled by the natural logarithm prior to analysis, as their ranges differed in some cases by several orders of magnitude. A small constant was added prior to the transformation in order to allow for log scaling of descriptors with values less than or equal to zero. Likewise, the values of the dependent variable differed by more than one order of magnitude and therefore were also log scaled.

Three forms of regression analyses were used to analyze the data, namely, ridge regression (RR) [45], principal component regression (PCR) [46], and partial least squares (PLS) [47], all of which are methods wherein the entire set of descriptors is utilized in modeling as opposed to subset regression. These regression methods are also useful in cases where the number of descriptors is substantially larger than the number of observations [45]. Hierarchical models and single-class models were developed utilizing the TSI, TCI, 3DI, and QCI in order to illuminate the roles played by the various classes of descriptors. Statistical parameters reported include the following: 1) the ridging constant, $k$, which controls the amount of smoothing in ridge regression, 2) cross-validated $R^{2}$, which is obtained by temporarily removing each compound in turn from the data set and fitting the regression to the remaining $n-1$ compounds, and 3) the PRESS statistic, which is an accurate measure of model predictability.

TABLE III Summary statistics for ridge (RR), principal components (PCR), and partial least squares (PLS) regression models, $n=24$.

\begin{tabular}{|c|c|c|c|c|c|c|c|}
\hline \multirow[b]{2}{*}{ Model Type } & \multicolumn{3}{|c|}{$R R$} & \multicolumn{2}{|c|}{$P C R$} & \multicolumn{2}{|c|}{$P L S$} \\
\hline & $R_{c . v .}^{2}$ & $k$ & PRESS & $R_{c . v .}^{2}$ & PRESS & $R_{c . v .}^{2}$ & PRESS \\
\hline TS & 0.2975 & 11.44 & 21.391 & 0.2166 & 23.854 & -0.1100 & 33.797 \\
\hline $\mathrm{TS}+\mathrm{TC}$ & 0.6878 & 5.22 & 9.5059 & 0.6104 & 11.863 & 0.4480 & 16.809 \\
\hline $\mathrm{TS}+\mathrm{TC}+3 \mathrm{D}$ & 0.6758 & 6.88 & 9.8708 & 0.6090 & 11.905 & 0.4168 & 17.759 \\
\hline $\mathrm{TS}+\mathrm{TC}+3 \mathrm{D}+\mathrm{QC}$ & 0.7095 & 2.95 & 8.8467 & 0.6331 & 11.172 & 0.4812 & 15.795 \\
\hline TS & 0.2975 & 11.44 & 21.391 & 0.2166 & 23.854 & -0.1100 & 33.797 \\
\hline TC & 0.7732 & $0.1 \mathrm{E}-02$ & 6.9065 & 0.6905 & 9.4246 & 0.5345 & 14.175 \\
\hline $3 \mathrm{D}$ & 0.0812 & 0.522 & 27.976 & -0.1567 & 35.219 & -0.4600 & 44.456 \\
\hline QC & 0.2612 & 6.96 & 22.495 & -1.1381 & 65.103 & 0.0472 & 29.013 \\
\hline
\end{tabular}


TABLE IV Summary statistics for topological ridge regression models, $n=23$

\begin{tabular}{llll}
\hline & \multicolumn{3}{c}{$R R$} \\
\cline { 2 - 4 } \multicolumn{1}{c}{ Model Type } & $R_{\text {c.v. }}^{2}$ & $k$ & PRESS \\
\hline TS & 0.458 & 10.04 & 12.372 \\
TC & 0.888 & $0.112 \mathrm{E}-02$ & 2.565 \\
TS+TC & 0.885 & 0.888 & 2.6343 \\
\hline
\end{tabular}

TABLE V Fitted ridge regression for the TC model, $n=23$.

\begin{tabular}{|c|c|c|c|c|c|c|c|c|c|}
\hline Descriptor & Coeff. & s.e. & $t$ & $|t|$ & Descriptor & Coeff. & s.e. & $t$ & $|t|$ \\
\hline SHarom & 0.094 & 0.016 & 5.870 & 5.870 & ${ }^{2} \chi^{v}$ & -0.116 & 0.108 & -1.080 & 1.080 \\
\hline Wt & 0.033 & 0.006 & 5.760 & 5.760 & Gmin & 0.048 & 0.045 & 1.080 & 1.080 \\
\hline totop & 0.042 & 0.008 & 5.600 & 5.600 & $\mathrm{AZN}_{3}$ & 0.025 & 0.024 & 1.070 & 1.070 \\
\hline $\mathrm{SaaCH}$ & 0.070 & 0.013 & 5.550 & 5.550 & $\mathrm{SIC}_{4}$ & -0.222 & 0.211 & -1.050 & 1.050 \\
\hline${ }^{6} \chi^{\mathrm{b}} \mathrm{Ch}$ & 5.411 & 1.007 & 5.380 & 5.380 & $\mathrm{SIC}_{2}$ & -0.446 & 0.429 & -1.040 & 1.040 \\
\hline $\mathrm{AZV}_{3}$ & 0.085 & 0.020 & 4.380 & 4.380 & $\mathrm{IdC}$ & 0.017 & 0.017 & 1.020 & 1.020 \\
\hline $\mathrm{SsCH} 3$ & -0.165 & 0.044 & -3.770 & 3.770 & $\mathrm{SIC}_{0}$ & 0.231 & 0.230 & 1.010 & 1.010 \\
\hline${ }^{3} \chi^{v} \mathrm{c}$ & -0.124 & 0.034 & -3.670 & 3.670 & ${ }^{5} \chi_{\mathrm{PC}}^{\mathrm{v}}$ & 0.276 & 0.275 & 1.000 & 1.000 \\
\hline numwHBd & 0.281 & 0.078 & 3.590 & 3.590 & ${ }^{4} \chi_{\mathrm{PC}}^{\mathrm{b}}$ & 0.164 & 0.166 & 0.990 & 0.990 \\
\hline${ }^{3} \chi^{b} \mathrm{c}$ & -0.141 & 0.042 & -3.340 & 3.340 & ${ }^{4} \chi_{C}^{v}$ & -0.366 & 0.373 & -0.980 & 0.980 \\
\hline$A Z V_{1}$ & 0.097 & 0.031 & 3.160 & 3.160 & ${ }^{4} \chi_{\mathrm{PC}}^{\mathrm{v}}$ & 0.133 & 0.146 & 0.910 & 0.910 \\
\hline${ }^{3} \chi^{v}$ & 0.213 & 0.072 & 2.980 & 2.980 & $\mathrm{~J}^{\mathrm{X}}$ & -0.128 & 0.149 & -0.850 & 0.850 \\
\hline $\mathrm{DN}_{2} \mathrm{Z}_{4}$ & 0.041 & 0.014 & 2.910 & 2.910 & $H \max$ & 0.193 & 0.228 & 0.850 & 0.850 \\
\hline SHwHBd & 0.253 & 0.091 & 2.780 & 2.780 & $\mathrm{CIC}_{2}$ & 0.188 & 0.238 & 0.790 & 0.790 \\
\hline $\mathrm{ASZ}_{1}$ & 0.031 & 0.013 & 2.410 & 2.410 & $\mathrm{CIC}_{3}$ & 0.132 & 0.168 & 0.790 & 0.790 \\
\hline${ }^{3} \chi^{b}$ & 0.188 & 0.081 & 2.320 & 2.320 & SssssC & 0.047 & 0.063 & 0.750 & 0.750 \\
\hline $\mathrm{IC}_{0}$ & 0.809 & 0.354 & 2.290 & 2.290 & $\mathrm{AZN}_{1}$ & 0.020 & 0.028 & 0.710 & 0.710 \\
\hline nelem & 0.662 & 0.298 & 2.220 & 2.220 & $\mathrm{CIC}_{4}$ & 0.079 & 0.124 & 0.630 & 0.630 \\
\hline Qv & -0.421 & 0.199 & -2.110 & 2.110 & SHother & 0.020 & 0.033 & 0.610 & 0.610 \\
\hline fw & 0.181 & 0.087 & 2.070 & 2.070 & SHvin & -0.136 & 0.225 & -0.610 & 0.610 \\
\hline${ }^{0} x^{b}$ & -0.158 & 0.077 & -2.060 & 2.060 & $\mathrm{AZS}_{3}$ & 0.010 & 0.017 & 0.590 & 0.590 \\
\hline $\mathrm{AZN}_{4}$ & 0.814 & 0.397 & 2.050 & 2.050 & $\mathrm{IC}_{2}$ & -0.056 & 0.118 & -0.480 & 0.480 \\
\hline $\mathrm{ANZ}_{2}$ & 0.017 & 0.008 & 2.020 & 2.020 & $\mathrm{AZS}_{2}$ & -0.011 & 0.027 & -0.410 & 0.410 \\
\hline $\mathrm{SsBr}$ & 0.356 & 0.177 & 2.020 & 2.020 & ${ }^{0} \chi^{v}$ & -0.032 & 0.079 & -0.400 & 0.400 \\
\hline${ }^{2} \chi^{b}$ & -0.182 & 0.092 & -1.990 & 1.990 & $\mathrm{AZN}_{2}$ & 0.013 & 0.033 & 0.400 & 0.400 \\
\hline $\mathrm{AZV}_{5}$ & 0.058 & 0.030 & 1.960 & 1.960 & $\mathrm{CIC}_{1}$ & 0.031 & 0.083 & 0.370 & 0.370 \\
\hline $\mathrm{SssCH} 2$ & -0.169 & 0.087 & -1.950 & 1.950 & $\mathrm{O}_{\text {orb }}$ & -0.089 & 0.253 & -0.350 & 0.350 \\
\hline${ }^{1} \chi^{\mathrm{v}}$ & 0.277 & 0.143 & 1.940 & 1.940 & $\mathrm{AZN}_{5}$ & 0.009 & 0.026 & 0.340 & 0.340 \\
\hline sumdelI & 0.116 & 0.060 & 1.920 & 1.920 & IdCbar & 0.085 & 0.250 & 0.340 & 0.340 \\
\hline${ }^{6} \chi^{v}$ & 1.907 & 1.006 & 1.890 & 1.890 & tets2 & 0.024 & 0.080 & 0.290 & 0.290 \\
\hline SHCHnX & 0.357 & 0.189 & 1.890 & 1.890 & Hmin & 0.086 & 0.307 & 0.280 & 0.280 \\
\hline Gmax & 0.367 & 0.200 & 1.840 & 1.840 & $\mathrm{O}$ & -0.030 & 0.112 & -0.270 & 0.270 \\
\hline $\mathrm{IC}_{1}$ & 0.515 & 0.283 & 1.820 & .1 .820 & ${ }^{5} \chi^{b}$ & -0.128 & 0.551 & -0.230 & 0.230 \\
\hline $\mathbf{J}^{\mathbf{Y}}$ & -0.234 & 0.130 & -1.810 & 1.810 & $\mathrm{DN}_{2} \mathrm{Z}_{3}$ & -0.041 & 0.182 & -0.230 & 0.230 \\
\hline${ }^{6} x^{b}$ & 1.844 & 1.032 & 1.790 & 1.790 & $\mathrm{SIC}_{1}$ & 0.070 & 0.334 & 0.210 & 0.210 \\
\hline $\mathrm{DN}_{2} \mathrm{Z}_{2}$ & 0.055 & 0.032 & 1.700 & 1.700 & ${ }^{5} \chi^{v}$ & -0.114 & 0.544 & -0.210 & 0.210 \\
\hline SHHBA & 0.028 & 0.017 & 1.700 & 1.700 & SdssC & 0.280 & 1.335 & 0.210 & 0.210 \\
\hline
\end{tabular}


TABLE V (Continued)

\begin{tabular}{|c|c|c|c|c|c|c|c|c|c|}
\hline knotp & -0.248 & 0.154 & -1.610 & 1.610 & $I_{\text {orb }}$ & 0.030 & 0.170 & 0.180 & 0.180 \\
\hline $\mathrm{J}^{\mathrm{B}}$ & -0.217 & 0.143 & -1.520 & 1.520 & $\mathrm{CIC}_{0}$ & -0.013 & 0.071 & -0.180 & 0.180 \\
\hline $\mathrm{AZV}_{4}$ & 0.155 & 0.102 & 1.520 & 1.520 & $\mathrm{IC}_{3}$ & -0.017 & 0.108 & -0.160 & 0.160 \\
\hline${ }^{5} \chi^{\mathrm{v}} \mathrm{c}$ & 0.243 & 0.166 & 1.470 & 1.470 & ${ }^{4} \chi^{v}$ & 0.038 & 0.270 & 0.140 & 0.140 \\
\hline SaasC & 0.113 & 0.080 & 1.420 & 1.420 & $\mathrm{SdsCH}$ & -0.020 & 0.152 & -0.130 & 0.130 \\
\hline $\mathrm{DSZ}_{2}$ & 0.052 & 0.038 & 1.380 & 1.380 & ${ }^{4} \chi^{b}$ & 0.031 & 0.264 & 0.120 & 0.120 \\
\hline phia & -0.106 & 0.078 & -1.360 & 1.360 & $\mathrm{AZV}_{2}$ & 0.020 & 0.164 & 0.120 & 0.120 \\
\hline $\mathrm{DN}_{2} \mathrm{Z}_{1}$ & 0.030 & 0.023 & 1.350 & 1.350 & ${ }^{1} \chi^{b}$ & 0.012 & 0.104 & 0.110 & 0.110 \\
\hline $\mathrm{SdCH} 2$ & 0.203 & 0.151 & 1.350 & 1.350 & SHCsatu & 0.019 & 0.212 & 0.090 & 0.090 \\
\hline SsssCH & -0.389 & 0.291 & -1.340 & 1.340 & $\mathrm{AZS}_{5}$ & 0.002 & 0.020 & 0.080 & 0.080 \\
\hline${ }^{6} \chi^{\mathrm{b}}{ }_{\mathrm{PC}}$ & 0.398 & 0.302 & 1.320 & 1.320 & $\mathrm{IC}_{4}$ & 0.006 & 0.080 & 0.070 & 0.070 \\
\hline $\mathrm{ANZ}_{1}$ & 0.051 & 0.039 & 1.320 & 1.320 & $\mathrm{ASZ}_{2}$ & 0.001 & 0.022 & 0.050 & 0.050 \\
\hline $\mathrm{SIC}_{3}$ & -0.313 & 0.240 & -1.300 & 1.300 & $\mathrm{AZS}_{4}$ & 0.003 & 0.063 & 0.040 & 0.040 \\
\hline${ }^{5} \chi^{b} \mathrm{c}$ & 0.300 & 0.230 & 1.300 & 1.300 & $\mathrm{AZS}_{1}$ & 0.001 & 0.022 & 0.030 & 0.030 \\
\hline${ }^{6} \chi^{v}{ }_{P C}$ & 0.425 & 0.327 & 1.300 & 1.300 & ${ }^{7} \chi^{v}$ & -0.336 & 10.007 & -0.030 & 0.030 \\
\hline knotpv & -0.168 & 0.134 & -1.250 & 1.250 & SHCsats & 0.003 & 0.085 & 0.030 & 0.030 \\
\hline $\mathrm{DN}_{2} \mathrm{Z}_{5}$ & -0.182 & 0.146 & -1.240 & 1.240 & $\mathrm{DSZ}_{1}$ & 0.001 & 0.035 & 0.020 & 0.020 \\
\hline sumI & 0.097 & 0.078 & 1.240 & 1.240 & nclass & -0.002 & 0.127 & -0.010 & 0.010 \\
\hline${ }^{5} \chi^{b}{ }_{P C}$ & 0.432 & 0.373 & 1.160 & 1.160 & CONSTANT & -3.275 & & & \\
\hline numHBa & 0.020 & 0.018 & 1.110 & 1.110 & & & & & \\
\hline
\end{tabular}

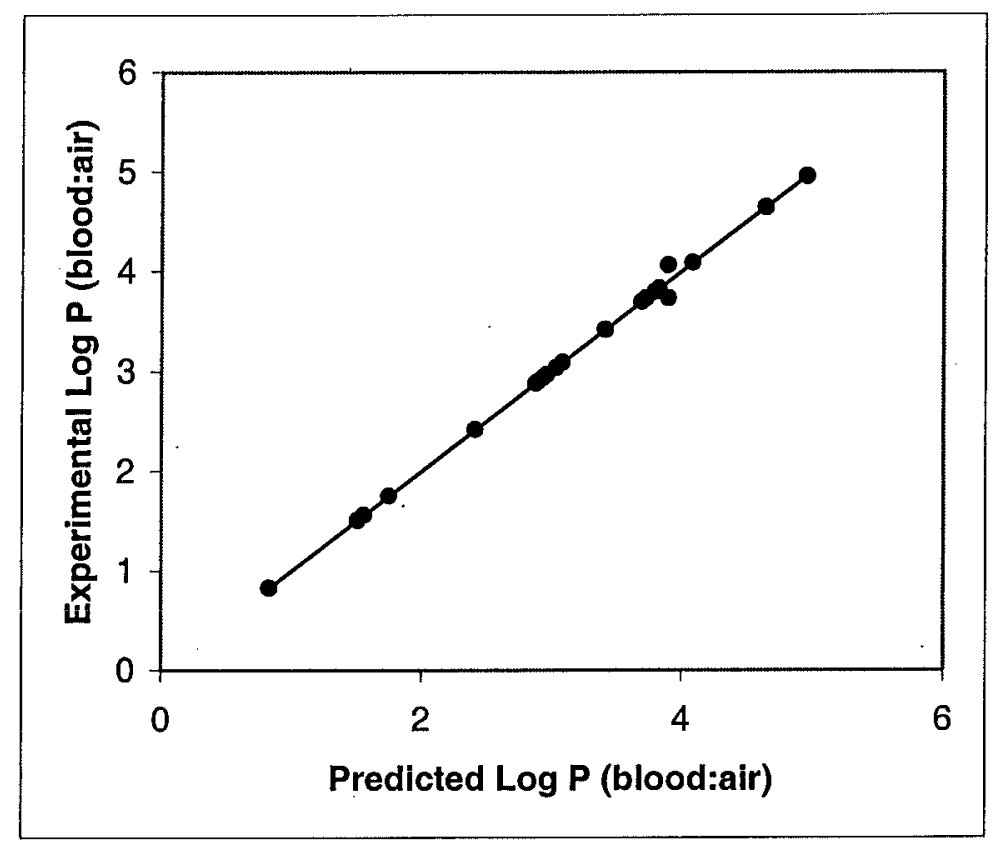

Figure 1 Experimental vs predicted $\log \mathrm{P}$ (blood:air) using the TC model, $n=23$. 
TABLE VI Experimental and predicted $\log \mathrm{P}$ (blood:air) using the TC model, $n=23$

\begin{tabular}{lccc}
\hline \multicolumn{1}{c}{ Chemicals } & $\begin{array}{c}\text { Experimental } \\
\log P\end{array}$ & $\begin{array}{c}\text { Predicted } \\
\log P\end{array}$ & Residual \\
\hline Alkanes & & & \\
$n$-Hexane & 0.82911 & 0.82855 & -0.00056 \\
$n$-Heptane & 1.55812 & 1.55814 & $2 \mathrm{E}-05$ \\
Haloalkanes & & & \\
Dichloromethane & 2.96563 & 2.96527 & -0.00036 \\
Chloroform & 3.03587 & 3.03495 & -0.00092 \\
Carbon tetrachloride & 1.50834 & 1.50851 & 0.00017 \\
Bromochloromethane & 3.72546 & 3.72569 & 0.00023 \\
1,1-Dichloroethane & 2.41493 & 2.41591 & 0.00098 \\
1,2-Dichloroethane & 3.4137 & 3.41444 & 0.00074 \\
1,1,1-Trichloroethane & 1.75148 & 1.75094 & -0.00054 \\
1,1,2-Trichloroethane & 3.8954 & 4.06044 & 0.16504 \\
1,1,1,2-Tetrachloroethane & 3.8954 & 3.7305 & -0.1649 \\
1,1,2,2,-Tetrachloroethane & 4.95521 & 4.95583 & 0.00062 \\
Pentachloroethane & 4.6445 & 4.64439 & -0.00011 \\
1,2-Dichloropropane & 2.92902 & 2.92852 & -0.0005 \\
Trichloroethylene & 3.08672 & 3.08649 & -0.00023 \\
Tetrachloroethylene & 2.93929 & 2.93916 & -0.00013 \\
Aromatic hydrocarbons & & & \\
Benzene & 2.87916 & 2.8792 & $4 \mathrm{E}-05$ \\
Chlorobenzene & 4.084 & 4.08429 & 0.00029 \\
Toluene & 2.89058 & 2.89037 & -0.00021 \\
Styrene & 3.69372 & 3.69387 & 0.00015 \\
$o$-Xylene & 3.79113 & 3.79098 & -0.00015 \\
$m$-Xylene & 3.82812 & 3.82864 & 0.00052 \\
$p$-Xylene & 3.72109 & 3.72086 & -0.00023 \\
\hline
\end{tabular}

\section{RESULTS}

Summary statistics for the models developed in this study based on the set of 24 structurally diverse compounds are provided in Table III. Overall, models developed using RR were superior to those developed using PCR or PLS. Therefore, the subsequent discussion will focus on RR results. Examining the hierarchically developed models, a significant increase in model quality is obtained upon the addition of TCI to the TSI set. The addition of 3DI and QCI yields little or no improvement in model quality. Of the single-class models, the TC is significantly better than any other descriptor class in modeling blood:air partition coefficient.

Further investigation showed that one compound in the set of 24, viz., cyclohexane, was an influential outlier. This is not altogether unexpected, as cyclohexane is the only cyclic non-aromatic compound in 
the data set. The omission of cyclohexane led to significant model improvement. Table IV provides summary results utilizing the data set with cyclohexane removed. Although cyclohexane was an outlier for all but one of the models developed in this study, i.e. the QC model, Table IV includes models developed with topological indices only.

Table $\mathrm{V}$ provides the fitted ridge regression for the TC model developed using the data set with cyclohexane omitted and includes the coefficient, standard error, and $t$ value for each of the descriptors in the model. The $t$ value is obtained by dividing the coefficient by the standard error. A large $|t|$ value indicates that the associated descriptor is important in the model; however, the reverse is not necessarily so. A plot of the experimental vs predicted $\log \mathrm{P}$ (blood:air) using the TC model is shown in Figure 1. The values of the $\log$-scaled experimental and predicted $\log \mathrm{P}$ values plotted in Figure 1 are provided in Table VI.

\section{DISCUSSION}

The goal of this study was to investigate the utility of four classes of calculated molecular descriptors, viz., topostructural indices, topochemical indices, 3-D descriptors, and quantum chemical indices, in estimating blood:air $\mathrm{P}$ values of a set of structurally diverse VOCs comprising alkanes, various haloalkanes and aromatics. In an earlier paper, Basak et al. [37] reported QSPRs for a congeneric subset of this set of compounds, viz., 13 chlorocarbons. A summary of the regression results from that study is provided in Table VII. Again, we find that the TC model obtained by ridge regression is superior to all other models developed. The TC model for the subset of chlorocarbons is nearly as good as the TC model developed for the set of 23 diverse compounds in terms of the cross-validated $R^{2}$ and the PRESS statistic. In many previous studies, we have found that either TSI or TSI+TCI provide acceptable QSAR models for both congeneric and diverse data sets $[13,21,23,31,37,48]$.

TABLE VII Summary statistics for ridge (RR), principal components (PCR), and partial least squares (PLS) regression models for the subset of 13 chlorocarbons.

\begin{tabular}{|c|c|c|c|c|c|c|c|}
\hline \multirow[b]{2}{*}{ Model Type } & \multicolumn{3}{|c|}{$R R$} & \multicolumn{2}{|c|}{$P C R$} & \multicolumn{2}{|c|}{$P L S$} \\
\hline & $R_{c, v .}^{2}$ & $k$ & PRESS & $R_{c . v .}^{2}$ & PRESS & $R_{\text {e.v. }}^{2}$ & PRESS \\
\hline TS & 0.452 & 9.212 & 6.60 & -1.300 & 27.71 & 0.252 & 8.89 \\
\hline $\mathrm{TS}+\mathrm{TC}$ & 0.886 & $0.10 \mathrm{E}-02$ & 1.37 & 0.790 & 2.53 & 0.807 & 2.33 \\
\hline $\mathrm{TS}+\mathrm{TC}+3 \mathrm{D}$ & 0.876 & 0.721 & 1.50 & 0.806 & 2.34 & 0.808 & 2.31 \\
\hline $\mathrm{TS}+\mathrm{TC}+3 \mathrm{D}+\mathrm{QC}$ & 0.883 & $0.43 \mathrm{E}-01$ & 1.40 & 0.819 & 2.18 & 0.821 & 2.16 \\
\hline TS & 0.452 & 9.212 & 6.60 & -1.300 & 27.71 & 0.262 & 8.89 \\
\hline $\mathrm{TC}$ & 0.916 & $0.10 \mathrm{E}-02$ & 1.02 & 0.843 & 1.89 & 0.848 & 1.83 \\
\hline $3 \mathrm{D}$ & 0.480 & $0.71 \mathrm{E}-01$ & 6.27 & -0.124 & 15.54 & -0.205 & 14.52 \\
\hline QC & 0.095 & 0.323 & 10.91 & -0.250 & 15.06 & -1.446 & 29.46 \\
\hline
\end{tabular}


TABLE VIII Ten model descriptors with highest $|t|$ values for the diverse data set and the chlorocarbon subset.

\begin{tabular}{|c|c|c|c|c|c|c|c|c|c|}
\hline \multicolumn{5}{|c|}{ Diverse data set $(n=23)$} & \multicolumn{5}{|c|}{ Chlorocarbons $(n=13)$} \\
\hline Descriptor & Coeff. & s.e. & $t$ & $|t|$ & Descriptor & Coeff. & s.e. & $t$ & $|t|$ \\
\hline SHarom & 0.094 & 0.016 & 5.870 & 5.870 & $\mathrm{AZV}_{3}$ & 0.130 & 0.022 & 6.010 & 6.01 \\
\hline $\mathrm{Wt}$ & 0.033 & 0.006 & 5.760 & 5.760 & $\mathrm{NoCl}$ & 0.027 & 0.005 & 4.990 & 4.99 \\
\hline totop & 0.042 & 0.008 & 5.600 & 5.600 & numHBa & 0.080 & 0.021 & 3.910 & 3.91 \\
\hline $\mathrm{SaaCH}$ & 0.070 & 0.013 & 5.550 & 5.550 & $\mathrm{SsssCH}$ & -0.280 & 0.080 & -3.510 & 3.51 \\
\hline${ }^{6} \chi_{\mathrm{Ch}}^{\mathrm{b}}$ & 5.411 & 1.007 & 5.380 & 5.380 & SHHBa & 0.061 & 0.018 & 3.340 & 3.34 \\
\hline $\mathrm{AZV}_{3}$ & 0.085 & 0.020 & 4.380 & 4.380 & ${ }^{3} \chi_{C}^{v}$ & -0.062 & 0.019 & -3.300 & 3.30 \\
\hline $\mathrm{SsCH} 3$ & -0.165 & 0.044 & -3.770 & 3.770 & $\mathrm{SsCH} 3$ & -0.124 & 0.038 & -3.280 & 3.28 \\
\hline${ }^{3} \chi_{\mathrm{c}}^{\mathrm{v}}$ & -0.124 & 0.034 & -3.670 & 3.670 & ${ }^{3} \chi_{C}^{b}$ & -0.073 & 0.023 & -3.170 & 3.17 \\
\hline numwHBd & 0.281 & 0.078 & 3.590 & 3.590 & $\mathrm{AZS}_{4}$ & -0.268 & 0.090 & -2.970 & 2.97 \\
\hline${ }^{3} \chi_{c}^{b}$ & -0.141 & 0.042 & -3.340 & 3.340 & SHother & -0.349 & 0.118 & -2.960 & 2.96 \\
\hline
\end{tabular}

It was of interest to examine which descriptors in the TC model developed in the current study had the greatest $|t|$ values, and thus held the greatest importance in the model. Comparing these with the important TC descriptors in the previous study involving only chlorocarbons (Table VIII) tells us something about which descriptors are important for the different sets of compounds. In doing so, we find that some descriptors are important for both data sets, including $\mathrm{AZV}_{3},{ }^{3} \chi_{\mathrm{c}}^{\mathrm{v}}$ and $\mathrm{SsCH}$. The hydrogen bond acceptor descriptors which were important in the chlorocarbon model, i.e. numHBa and SHHBa, have been replaced by the hydrogen donor index, numwHBd. Other descriptors important in the current model which were not important in the chlorocarbon model include totop, Wt, and SHarom, the latter of which is an E-state descriptor associated with aromatic systems. It is well known that QSPR models work best with homogeneous data sets, which represent some structural or mechanistic class. On the other hand, Basak et al. argued that a collection of diverse molecular descriptors should be able to predict properties of structurally diverse sets [26]. A comparison of QSPRs of the set of 23 diverse compounds and the congeneric set of 13 chlorocarbons (Table IV and Table VII) supports this notion. Further studies with different properties of various congeneric and structurally diverse sets and properties are needed to test the validity of this hypothesis.

\section{Acknowledgement}

This is contribution number XXX from the Center for Water and the Environment of the Natural Resources Research Institute. Research reported in this paper was supported in part by Grant F49620-011-0098 from the United States Airforce. 


\section{References}

[1] Gargas, M. L., Burgess, R. J., Voisard, D. E., Cason, G. H., and Andersen, M. E. (1989). Partition coefficients of low molecular weight volatile chemicals in various tissues and liquids. Toxicol. Appl. Pharmacol. 98, 87-99.

[2] Poulin, P. and Krishnan, K. (1996). A mechanistic algorithm for predicting blood:air partition coefficients of organic chemicals with the consideration of reversible binding in hemoglobin. Toxicol. Appl. Pharmacol. 136, 131-137.

[3] Auer, C. M., Nabholz, J. V., and Baetcke, K. P. (1990). Mode of action and the assessment of chemical hazards in the presence of limited data: use of structure-activity relationships (SAR) under TSCA, Section 5. Environ. Health Perspect. 87 183-197.

[4] Basak, S. C. and Grunwald, G. D. (1993). A comparative study of graph invariants, total surface area, and volume in predicting boiling points of alkanes. Mathl. Modelling Sci. Computing 2, 735-740.

[5] Balaban, A. T., Basak, S. C., Colburn, T. and Grunwald, G. D. (1994). Correlation between structure and normal boiling points of haloalkanes C1-C4 using neural networks. J. Chem. Inf. Comput. Sci. 34, 1118-1121.

[6] Basak, S. C., Gute, B. D., and Grunwald, G. D. (1996). Estimation of normal boiling points of haloalkanes using molecular similarity. Croat. Chim. Acta 69, 1159-1173.

[7] Basak, S. C., Gute, B. D., and Grunwald, G. D. (1996). A comparative study of topological and geometrical parameters in estimating normal boiling point and octanol-water partition coefficient. J. Chem. Inf. Comput. Sci. 36, 1054-1060.

[8] Balaban, A. T., Mills, D., and Basak, S. C. (1999). Correlation between structure and normal boiling points of acyclic carbonyl compounds. J. Chem. Inf. Comput. Sci. 39, 758-764.

[9] Randic, M. and Basak, S. C. (2000). Construction of high-quality structure-property-activity regressions: The boiling points of sulfides. J. Chem. Inf. Comput. Sci. 40, 899-905.

[10] Balaban, A. T., Basak, S. C., and Mills, D. (1999). Normal boiling points of 1, $\omega$-Alkanedinitriles: The highest increment in a homologous series. J. Chem. Inf. Comput. Sci. 39, 769-774.

[11] Hall, L. H. and Story, C. T. (1997). Boiling point of a set of alkanes, alcohols and chloroalkanes: QSAR with atom type electrotopological states indices using artificial neural networks. SAR QSAR Environ. Res. 6 139161.

[12] Basak, S. C., Gute, B. D., and Grunwald, G. D. (1997). Use of topostructural, topochemical, and geometric parameters in the prediction of vapor pressure: A hierarchical approach. J. Chem. Inf. Comput. Sci. 37, 651655.

[13] Basak, S. C. and Mills, D. (2001). Quantitative structure-property relationships (QSPRs) for the estimation of vapor pressure: A hierarchical approach using mathematical structural descriptors. J. Chem. Inf. Comput. Sci. 41, in press.

[14] McClelland, H. E. and Jurs, P. C. (2000). Quantitative Structure-Property Relationships for the Prediction of Vapor Pressures of Organic Compounds from Molecular Structures. J. Chem. Inf. Comput. Sci. 40, 967-975.

[15] Goll, E. S. and Jurs, P. C. (1999). Prediction of Vapor Pressures of Hydrocarbons and Halohydrocarbons from Molecular Structure with a Computational Neural Network Model. J. Chem. Inf. Comput. Sci. 39, 1081-1089.

[16] Liang, C. and Gallagher, D. A. (1998). QSPR Prediction of Vapor Pressure from Solely Theoretically-Derived Descriptors. J. Chem. Inf. Comput. Sci. 38, 321-324.

[17] Katritzky, A. R., Wang, Y., Sild, S., and Tamm, T. (1998). QSPR Studies on Vapor Pressure, Aqueous Solubility, and the Prediction of Water-Air Partition Coefficients. J. Chem. Inf. Comput. Sci. 38, 720-725.

[18] Niemi, G. J., Basak, S. C., Veith, G. D., and Grunwald, G. (1992). Prediction of octanol-water partition coefficient (Kow) using algorithmically-derived variables. Environ. Toxicol. Chem. 11, 891-898.

[19] Basak, S. C., Frane, C. M., Rosen, M. E., and Magnuson, V. R. (1986). Molecular topology and mutagenicity: A QSAR study of nitrosamines. IRCS Med. Sci. 14, 848-849.

[20] Basak, S. C., Gute, B. D., and Grunwald, G. D. (1998). Relative effectiveness of topological, geometrical, and quantum chemical parameters in estimating mutagenicity of chemicals. In, Quantitative Structure-activity Relationships in Environmental Sciences VII (F. Chen and G. Schuurmann, Eds.). SETAC Press, Pensacola, FL, pp. 245-261.

[21] Basak, S. C. and Mills, D. (2001). Prediction of mutagenicity utilizing a hierarchical approach. SAR QSAR Environ. Res., in press.

[22] Kier, L. B., Simons, R. J., and Hall, L. H. (1978). Structure activity studies on mutagenicity of nitrosamines using molecular connectivity. J. Pharm. Sci. 67, 725-726. 
[23] Basak, S. C., Mills, D. R., Balaban, A. T., and Gute, B. D. (2001). Prediction of mutagenicity of aromatic and heteroaromatic amines from structure: A hierarchical QSAR approach. J. Chem. Inf. Comput. Sci. 41, 671-678.

[24] Basak, S. C., Grunwald, G. D., Gute, B. D., Balasubramanian, K., and Opitz, D. (2000). Use of statistical and neural net approaches in predicting toxicity of chemicals. J. Chem. Inf. Comput. Sci. 40, 885-890.

[25] Gute, B. D. and Basak, S. C. (1997). Predicting acute toxicity of benzene derivatives using theoretical molecular descriptors: a hierarchical QSAR approach. SAR QSAR Environ. Res. 7, 117-131.

[26] Basak, S. C., Mills, D., Gute, B. D., Grunwald, G. D., and Balaban, A. T. (2001). Applications of topological indices in predicting property/bioactivity/toxicity of chemicals. In, Topology in Chemistry, in press.

[27] Cronin, M. T. D., Gregory, B. W., and Schultz, T. W. (1998). Quantitative structure-activity analyses of nitrobenzene toxicity to Tetrahymena pyriformis. Chem. Res. Toxicol. 11, 902-908.

[28] Dearden, J. C., Cronin, M. T. D., Schultz, T. W., and Lin, D. T. (1995). QSAR study of the toxicity of nitrobenzenes to Tetrahymena pyriformis. Quant. Struct.-Act. Relat. 14, 427-432.

[29] Estrada, E. and Uriarte, E. (2001). Quantitative structure-toxicity relationships using TOSS-MODE. 1. Nitrobenzene toxicity to Tetrahymena pyriformis. SAR QSAR Environ. Res., in press.

[30] Randic, M. and Basak, S. C. (2001). On use of the variable connectivity index in QSAR: Toxicity of aliphatic ethers. J. Chem. Inf. Comput. Sci. 41, 614-618.

[31] Basak, S. C. and Mills, D. (2001). Prediction of toxicity of nitrobenzenes to Tetrahymena pyriformis: A hieararchical QSAR approach. Chem. Res. Toxicol., submitted.

[32] Basak, S. C., Gieschen, D. P., and Magnuson, V. R. (1984). A quantitative correlation of the LC50 values of esters in Pimephales promelas using physicochemical and topological parameters. Environ Toxicol. Chem. 3, 191-199.

[33] Basak, S. C., Gute, B. D., and Ghatak, S. (1999). Prediction of complement inhibitory activity of benzamidines using topological and geometrical parameters. J. Chem. Inf. Comput. Sci. 39, 255-260.

[34] Basak, S. C., Gute, B. D., and Drewes, L. R. (1996). Predicting blood-brain transport of drugs: A computational approach. Pharm. Res. 13, 775-778.

[35] Gute, B. D., Grunwald, G. D., and Basak, S. C. (1999). Prediction of the dermal penetration of polycyclic aromatic hydrocarbons (PAHs): A hierarchical QSAR approach. SAR QSAR Environ. Res. 10, 1-15.

[36] Basak, S. C., Grunwald, G. D., Host, G. E., Niemi, G. J., and Bradbury, S. P. (1998). A comparative study of molecular similarity, statistical, and neural network methods for predicting toxic modes of action of chemicals. Environ. Toxicol. Chem. 17, 1056-1064.

[37] Basak, S. C., Hawkins, D. M., and Mills, D. (2001). Estimation of blood:air partition coefficient of chloroalkanes using QSPRs based on theoretical structural descriptors. Croat. Chim. Acta, submitted.

[38] Basak, S. C., Harriss, D. K., and Magnuson, V. R. (1988). POLLY, v. 2.3, Copyright of the University of Minnesota.

[39] Filip, P. A., Balaban, T. S., and Balaban, A. T. (1987). A new approach for devising local graph invariants: Derived topological indices with low degeneracy and good correlational ability. J. Math. Chem. 1, 61-83.

[40] Basak, S. C., Balaban, A. T., Grunwald, G. D., and Gute, B. D. (2000). Topological indices: Their nature and mutual relatedness. J. Chem. Inf. Comput. Sci. 40, 891-898.

[41] Molconn-Z (2000) v. 3.50, Hall Associates Consulting Quincy, MA.

[42] Tripos Associates (1994). SYBYL, v. 6.2, St. Louis, MO.

[43] Stewart, J. J. P. (1990). MOPAC v. 6.00, QCPE \#455, Frank J Seiler Research Laboratory, US Air Force Academy, CO.

[44] SAS Institute (1988). SAS/STAT User's Guide, Release 6.03, Cary, NC.

[45] Hoerl, A. E. and Kennard, R. W. (1970). Ridge regression: Biased estimation for nonorthogonal problems. Technometrics 8, 27-51.

[46] Massy, W. F. (1965). Principal components regression in exploratory statistical research. J. Am. Statistical Assoc, 60, 234-246.

[47] Wold, H. (1975). Soft modeling by latent variables: The nonlinear iterative partial least squares approach. In, Perspectives in Probability and Statistics, Papers in Honor of M. S. Bartlett (J. Gani, Ed.). Academic Press, London.

[48] Basak, S. C. and Mills, D. (2001). Use of mathematical structural invariants in the development of QSPR models. MATCH (Commun. Math. Comput. Chem.), in press. 



\title{
PREDICTION OF TISSUE:AIR PARTITION COEFFICIENTS: A COMPARISON OF STRUCTURE-BASED AND PROPERTY-BASED METHODS
}

\author{
S. C. BASAK, ${ }^{\mathrm{a}, \dagger}$ D. MILLS, ${ }^{\mathrm{a}}$ D. M. HAWKINS, ${ }^{\mathrm{b}}$ and H. A. EL-MASRI ${ }^{\mathrm{c}}$ \\ ${ }^{a}$ Natural Resources Research Institute, University of Minnesota Duluth, \\ 5013 Miller Trunk Highway, Duluth, MN 55811, USA \\ ${ }^{b}$ School of Statistics, University of Minnesota Minneapolis, \\ 313 Ford Hall, 224 Church Street S. E., MN 55455, USA \\ ${ }^{c}$ Computational Toxicology Laboratory, Division of Toxicology, \\ Agency for Toxic Substances and Disease Registry (ATSDR), \\ Executive Park Building 4, 1600 Clifton Road, E-29, Atlanta, GA 30333, USA
}

Three linear regression methods were used to develop models for the prediction of rat tissue:air partition coefficient (P). In general, ridge regression (RR) was found to be superior to principal component regression (PCR) and partial least squares (PLS). A set of 46 diverse low molecular-weight volatile chemicals was used to model fat:air, liver:air, and muscle:air partition coefficients for male Fischer 344 rats. Comparisons were made between models developed using descriptors based solely on molecular structure and those developed using experimental properties, including saline:air and olive oil:air partition coefficients, as independent variables, indicating that the structure-property correlations are comparable to the property-property correlations. Multiple structure-based models were developed utilizing various classes of structural descriptors based on level of complexity, i.e., topostructural (TS), topochemical (TC), 3-dimensional (3D), and calculated octanol:water partition coefficient. In most cases, the structure-based models developed using only the TC descriptors were found to be superior to those developed using other structural descriptor classes. Haloalkane subgroups were modeled separately for comparative purposes, and although models based on the congeneric compounds were superior, the models developed on the complete sets of diverse compounds were acceptable. Comparisons were also made with respect to the types of descriptors important for partitioning across the various media.

Key Words: Tissue:air partition coefficient; PBPK model; Theoretical molecular descriptors; Ridge regression; Hierarchical QSAR (HiQSAR)

\footnotetext{
${ }^{\dagger}$ Corresponding Author. e-mail: sbasak@nrri.umn.edu
} 


\section{INTRODUCTION}

Blood:air and tissue:air partition coefficients of volatile organic chemicals (VOCs) are important for their hazard assessment and estimation of bioavailability of such chemicals at the various tissues. Such properties are routinely used in the physiologically based pharmacokinetic (PBPK) models pertaining to risk assessment of chemicals and also extrapolation of toxicity of a chemical from one species to another.

Gargas et al.[1] determined blood:air and tissue:air partition coefficients for various tissues including fat, muscle and liver. While experimental determination of blood:fat, blood:liver, and blood:musle partition coefficients could be complicated and time consuming, these quantities can be computed as the ratio of blood:air and the various tissue:air partition coefficients. Such blood:tissue partition coefficients can be convenient input variables in PBPK models for hazard estimation.

In the practical hazard assessment of chemicals by regulatory agencies such as the United States Environmental Protection Agency (USEPA), Agency for Toxic Substances and Disease Registry (ATSDR), one must carry out rapid toxicity estimation of many chemicals. The Toxic Substances Control Act (TSCA) Inventory currently has over 75,000 chemicals [2]; the list of high production volume (HPV) chemicals contains over 2,800 substances [3]; and the 2001 Comprehensive Environmental Response, Compensation, and Liability Act (CERCLA) priority list [4] contains 275 substances, many of which are VOCs, that are most commonly found at facilities on the National Priorities List (NPL) and have been determined to pose the most significant threat to human health based on toxicity and potential exposure at the NPL sites. Experimental determination of the various blood:tissue partition coefficients of all these chemicals for hazard assessment and PBPK modeling could be a daunting task. The list would become considerably larger if we also include the probable metabolites of the VOCs under study. A viable alternative to this quagmire is the computation of these properties from molecular descriptors that can be computed directly from chemical structure without any further input of experimental data.

In one of our earlier studies, Basak et al.[5] used computed structural parameters to develop successful predictive models for the estimation of blood:air partition coefficient of VOCs. Calculated descriptors have also been used by us in the estimation of lipophilicity [6-9], vapor pressure [10, 11], boiling point [12, 13], toxicity including mutagenicity [14-17], and toxic modes of actions of various congeneric and structurally diverse groups of chemicals[18]. In this paper, we have used calculated structural descriptors to formulate models for the estimation of fat:air, liver:air and muscle:air partition coefficients for a set of 46 structurally diverse VOCs. 


\section{METHODS}

\section{Database}

Liquid:air and tissue:air partition coefficients were experimentally determined by Gargas et al. [1] using a modified version of the gas-phase vial equilibrium technique [19] and tissue homogenates prepared in $0.9 \%$ saline solution [20] for a set of low molecular-weight volatile chemicals. Table I includes experimentally determined male Fischer 344 rat tissue:air partition coefficients, including fat, liver, and muscle tissues, for a set of 46 chemicals including 26 haloalkanes, 2 nitroalkanes, 6 haloalkenes, 9 aromatics, and 3 additional compounds. Saline:air and olive oil:air partition coefficients, determined by Gargas et al., are also listed in Table I. All experimental values were obtained at $37^{\circ} \mathrm{C}$.

It should be noted that the data used in the current study represent a subset of that reported by Gargas et al. [1]. Two cis/trans isomers were eliminated because they are indistinguishable in terms of their calculated molecular descriptors based on SMILES input. Methyl chloride was also removed from the data set as it is not possible to calculate our entire set of theoretic descriptors on two-atom compounds. In addition, three compounds were reported without discrete values for $0.9 \%$ saline:air $P$, all of which were aliphatic hydrocarbons. Preliminary studies indicated that the remaining three aliphatic hydrocarbons were outliers thus the decision was made to eliminate those, as well.

TABLE I Experimental liquid:air, calculated $\log \mathrm{P}_{\mathrm{ow}}$, and experimental rat tissue:air partition coefficient data ${ }^{\mathrm{a}}$

\begin{tabular}{|c|c|c|c|c|c|c|c|}
\hline No. & Chemical & $\begin{array}{c}0.9 \% \\
\text { saline:air }\end{array}$ & Olive oil:air & $\log P_{O W}$ & Fat:air & Liver:air & Muscle:air \\
\hline \multicolumn{8}{|c|}{ Haloalkanes } \\
\hline 1 & Dichloromethane & $5.96 \pm 0.71$ & $131 \pm 7$ & 1.16 & $120 \pm 6$ & $14.2 \pm 1.2$ & $7.92 \pm 1.77$ \\
\hline 2 & Chloroform & $3.38 \pm 0.09$ & $402 \pm 12$ & 1.86 & $203 \pm 5$ & $21.1 \pm 1.5$ & $13.9 \pm 1.9$ \\
\hline 3 & Carbon tetrachloride & $0.35 \pm 0.03$ & $374 \pm 11$ & 3 & $359 \pm 11$ & $14.2 \pm 1.0$ & $4.57 \pm 0.59$ \\
\hline 4 & Difluoromethane & $1.31 \pm 0.05$ & $4.76 \pm 0.75$ & 0.56 & $1.43 \pm 0.31$ & $2.75 \pm 0.39$ & $1.44 \pm 0.25$ \\
\hline 5 & Fluorochloromethane & $3.08 \pm 0.07$ & $22.3 \pm 1.4$ & 0.68 & $15.4 \pm 1.0$ & $3.44 \pm 0.27$ & $2.46 \pm 0.52$ \\
\hline 6 & Bromochloromethane & $8.65 \pm 0.28$ & $361 \pm 9$ & 1.45 & $325 \pm 3$ & $29.2 \pm 0.5$ & $1 \cdot 1.1 \pm 1.8$ \\
\hline 7 & Dibromomethane & $14.4 \pm 0.4$ & $957 \pm 39$ & 1.95 & $792 \pm 14$ & $68.1 \pm 1.4$ & $40.5 \pm 2.0$ \\
\hline 8 & Chlorodibromomethane & $7.34 \pm 0.42$ & $2683 \pm 152$ & 1.77 & $1917 \pm 165$ & $126 \pm 7.1$ & $55.6 \pm 0.7$ \\
\hline 9 & Chloroethane & $1.09 \pm 0.06$ & $38.9 \pm 3.1$ & 1.47 & $38.6 \pm 0.7$ & $3.61 \pm 0.32$ & $3.22 \pm 0.68$ \\
\hline 10 & 1,1-Dichloroethane & $2.45 \pm 0.04$ & $186 \pm 7$ & 1.86 & $164 \pm 4$ & $10.8 \pm 0.5$ & $5.12 \pm 0.48$ \\
\hline 11 & 1,2-Dichloroethane & $11.4 \pm 0.1$ & $366 \pm 8$ & 1.6 & $344 \pm 5$ & $35.7 \pm 1.6$ & $23.4 \pm 1.4$ \\
\hline 12 & 1,1,1-Trichloroethane & $0.75 \pm 0.07$ & $295 \pm 22$ & 2.26 & $263 \pm 12$ & $8.6 \pm 0.6$ & $3.15 \pm 0.33$ \\
\hline 13 & 1,1,2-Trichloroethane & $13.3 \pm 0.3$ & $1776 \pm 26$ & 2.08 & $1438 \pm 58$ & $73.1 \pm 0.8$ & $22.9 \pm 0.8$ \\
\hline 14 & 1,1,1,2-Tetrachloroethane & $3.53 \pm 0.23$ & $2686 \pm 51$ & 2.64 & $2148 \pm 82$ & $88.2 \pm 1.8$ & $39.5 \pm 2.5$ \\
\hline 15 & 1,1,2,2-Tetrachloroethane & $23.4 \pm 2.0$ & $6358 \pm 402$ & 2.51 & $3767 \pm 93$ & $196 \pm 12$ & $101 \pm 10$ \\
\hline 16 & Pentachloroethane & $2.32 \pm 0.35$ & $6689 \pm 471$ & 3.07 & $4118 \pm 209$ & $260 \pm 11$ & $72.4 \pm 2.9$ \\
\hline 17 & Hexachloroethane & $0.66 \pm 0.21$ & $5015 \pm 318$ & 4.24 & $3321 \pm 193$ & $369 \pm 17.5$ & $75 \pm 0.9$ \\
\hline 18 & 1,2-Dibromoethane & $17.3 \pm 0.8$ & $1267 \pm 91$ & 2.13 & $1219 \pm 50$ & $119 \pm 4$ & $45.6 \pm 3.3$ \\
\hline 19 & 1-Bromo-2-chloroethane & $8.91 \pm 0.56$ & $569 \pm 23$ & 1.73 & $959 \pm 39$ & $42.8 \pm 3.3$ & $25.4 \pm 3.1$ \\
\hline
\end{tabular}


TABLE I (Continued)

\begin{tabular}{|c|c|c|c|c|c|c|c|}
\hline \\
\hline 20 & $\begin{array}{l}\text { 1,1,1-Trifluoro-2- } \\
\text { chloroethane }\end{array}$ & $0.42 \pm 0.04$ & $24.0 \pm 2.5$ & 1.7 & $21.2 \pm 0.6$ & $1.84 \pm 0.14$ & $1.23 \pm 0.14$ \\
\hline 21 & $\begin{array}{l}\text { 1,1,1-Trifluoro-2-bromo- } \\
\text { 2-chloroethane }\end{array}$ & $0.50 \pm 0.05$ & $198 \pm 4$ & 2.44 & $182 \pm 5$ & $7.62 \pm 1.20$ & $4.46 \pm 0.29$ \\
\hline 22 & 1-Chloropropane & $1.04 \pm 0.01$ & $105 \pm 2$ & 1.95 & $118 \pm 2$ & $5.18 \pm 0.38$ & $2.08 \pm 0.66$ \\
\hline 23 & 2-Chloropropane & $0.82 \pm 0.09$ & $69.9 \pm 3.5$ & 1.81 & $68.4 \pm 2.0$ & $3.15 \pm 0.24$ & $2.04 \pm 0.48$ \\
\hline 24 & 1,2-Dichloropropane & $2.75 \pm 0.11$ & $428 \pm 30$ & 2.18 & $499 \pm 30$ & $24.8 \pm 2.4$ & $12.0 \pm 1.1$ \\
\hline 25 & n-Propyl bromide & $1.44 \pm 0.12$ & $272 \pm 8$ & 2.13 & $236 \pm 6$ & $8.17 \pm 0.62$ & $4.21 \pm 0.32$ \\
\hline 26 & Isopropyl bromide & $1.08 \pm 0.04$ & $164 \pm 5$ & 1.63 & $158 \pm 5$ & $4.41 \pm 0.34$ & $4.12 \pm 0.35$ \\
\hline \multicolumn{8}{|c|}{ Nitroalkanes } \\
\hline 27 & 1-Nitropropane & $127 \pm 4$ & $1062 \pm 21$ & 0.8 & $506 \pm 33$ & $153 \pm 17$ & $28.9 \pm 6.1$ \\
\hline 28 & 2-Nitropropane & $98.3 \pm 5.4$ & $640 \pm 16$ & 0.61 & $155 \pm 4$ & $62.4 \pm 1.4$ & $29.1 \pm 3.3$ \\
\hline \multicolumn{8}{|c|}{ Haloalkenes } \\
\hline 29 & Vinyl chloride & $0.43 \pm 0.04$ & $24.4 \pm 3.7$ & 1.37 & $20.0 \pm 0.7$ & $1.60 \pm 0.17$ & $2.10 \pm 0.45$ \\
\hline 30 & 1,1-Dichloroethylene & $0.35 \pm 0.06$ & $64.3 \pm 3.4$ & 2.03 & $68.6 \pm 2.1$ & $4.42 \pm 0.30$ & $2.05 \pm 0.31$ \\
\hline 31 & Trichloroethylene & $0.83 \pm 0.30$ & $553 \pm 46$ & 2.36 & $554 \pm 21$ & $27.2 \pm 3.4$ & $10.1 \pm 2.7$ \\
\hline 32 & Tetrachloroethylene & $0.79 \pm 0.06$ & $2134 \pm 159$ & 3.47 & $1638 \pm 91$ & $70.3 \pm 9.0$ & $20.0 \pm 2.5$ \\
\hline 33 & Vinyl bromide & $0.44 \pm 0.06$ & $56.0 \pm 1.5$ & 1.61 & $49.2 \pm 1.3$ & $3.33 \pm 0.38$ & $2.26 \pm 0.13$ \\
\hline 34 & Allyl chloride & $2.75 \pm 0.10$ & $465 \pm 5$ & 2.04 & $101 \pm 2$ & $38.9 \pm 4.5$ & $11.0 \pm 0.2$ \\
\hline \multicolumn{8}{|c|}{ Aromatics } \\
\hline 35 & Benzene & $2.81 \pm 0.07$ & $2188 \pm 41$ & 2.64 & $499 \pm 12$ & $17.0 \pm 1.3$ & $10.3 \pm 0.9$ \\
\hline 36 & Chlorobenzene & $1.75 \pm 0.13$ & $1056 \pm 38$ & 2.66 & $1277 \pm 43$ & $86.1 \pm 3.0$ & $34.0 \pm 3.9$ \\
\hline 37 & Toluene & $1.41 \pm 0.47$ & $3548 \pm 269$ & 3.01 & $1021 \pm 11$ & $83.6 \pm 5.8$ & $27.7 \pm 4.0$ \\
\hline 38 & Styrene & $1.97 \pm 0.28$ & $14706 \pm 665$ & 3.73 & $3476 \pm 73$ & $139 \pm 7$ & $46.7 \pm 3.9$ \\
\hline 39 & m-Methlystyrene & $2.11 \pm 0.30$ & $13942 \pm 567$ & 3.75 & $11951 \pm 692$ & $327 \pm 23$ & $182 \pm 10$ \\
\hline 40 & p-Methylstyrene & $2.65 \pm 0.08$ & $3534 \pm 208$ & 3.15 & $11281 \pm 972$ & $324 \pm 17$ & $183 \pm 8$ \\
\hline 41 & o-Xylene & $1.92 \pm 0.12$ & $3245 \pm 116$ & 3.21 & $1877 \pm 132$ & $108 \pm 7$ & $51.5 \pm 6.7$ \\
\hline 42 & $\mathrm{~m}$-Xylene & $1.77 \pm 0.07$ & $3319 \pm 96$ & 3.2 & $1859 \pm 93$ & $90.9 \pm 4.4$ & $41.9 \pm 5.7$ \\
\hline 43 & $\mathrm{p}$-Xylene & $11.4 \pm 0.4$ & $55.6 \pm 1.4$ & 1.13 & $1748 \pm 65$ & $90.0 \pm 4.3$ & $38.4 \pm 4.1$ \\
\hline \multicolumn{8}{|l|}{ Other } \\
\hline 44 & Diethyl ether & $0.56 \pm 0.04$ & $78.9 \pm 9.1$ & 2.45 & $47.7 \pm 3.9$ & $6.82 \pm 0.54$ & $5.28 \pm 0.54$ \\
\hline 45 & Isoflurorane & $2.06 \pm 0.01$ & $109 \pm 5$ & 1.99 & $98.1 \pm 4.6$ & $4.07 \pm 0.20$ & $1.60 \pm 0.34$ \\
\hline 46 & Isoprene & $0.21 \pm 0.02$ & $8.81 \pm 0.15$ & 2.6 & $72.0 \pm 2.4$ & $3.12 \pm 0.87$ & $2.04 \pm 0.27$ \\
\hline
\end{tabular}

a Values represent mean \pm standard error.

\section{Molecular Descriptors}

Theoretical molecular descriptors may be divided into hierarchical classes based upon level of complexity and demand for computational resources for their calculation. Topostructural (TS) descriptors, which encode information strictly on the adjacency and connectedness of atoms within a molecule, are the simplest of the hierarchical classes. Topochemical (TC) descriptors encode information related to both molecular topology and the chemical nature atoms and bonds within a molecule, such as atomic valence and bond type. The 3-dimensional or shape descriptors (3D) are still more complex, encoding information about the 3-dimensional aspects of molecular structure. The topostructural and topochemical descriptors are collectively referred to as topological descriptors. With the hierarchical QSAR (HiQSAR) method, multiple models are developed, each time including an additional class of descriptors which are more 
complex and computationally demanding. Comparing the statistical parameters of the hierarchically developed models, we are able to see the relative contribution of each descriptor class.

Descriptors used in the present study were derived from molecular structure using software packages including POLLY [21], Triplet [22, 23], and Molconn-Z [24]. From POLLY and associated software developed by the authors, a set of 102 topological descriptors is available, including a large group of connectivity indices [25-28], path-length descriptors [25], Balaban's J indices [29-31], and information theoretic [32,33] and neighborhood complexity indices [33]. The Triplet program calculates a set of 100 topological parameters which are derived from a matrix, a main diagonal column vector, and a free term column vector, converting the matrix into a system of linear equations whose solutions are the local vertex invariants. These local vertex invariants are then used in the following mathematical operations in order to obtain the triplet descriptors:

1. Summation, $\mathrm{E}_{\mathrm{i}} \mathrm{x}_{\mathrm{i}}$

2. Summation of squares, $\mathrm{E}_{\mathrm{i}} \mathrm{x}_{\mathrm{i}}^{2}$

3. Summation of square roots, $\mathrm{E}_{\mathrm{i}} \mathrm{x}_{\mathrm{i}}^{1 / 2}$

4. Sum of inverse square root of cross-product over edges $i j, E_{i j}\left(x_{i} x_{j}\right)^{-1 / 2}$

5. Product, $\mathrm{N}\left(\mathrm{E}_{\mathrm{i}} \mathrm{X}_{\mathrm{i}}\right)^{1 / \mathrm{N}}$

We have also included 167 descriptors from Molconn-Z, including an extended set of connectivity indices, electrotopological indices $[34,35]$ and hydrogen bonding descriptors, as well as a small set of molecular shape descriptors.

$\mathrm{H}$-Bond, a software program developed by Basak [36], was used to calculate $\mathrm{HB}_{1}$, a measure of hydrogen bonding potential. $n$-octanol:water partition coefficients were calculated by the LogP program [37] and are included in Table I.

A brief description of the set of theoretical molecular descriptors used in the current study is provided in Table II. For the sake of brevity, the calculated values for these descriptors are not provided, though they may be obtained from the authors upon request.

\section{Statistical Analysis}

Independent and dependent variables were scaled by the natural logarithm, as their respective ranges differed by several orders of magnitude. The CORR procedure of the SAS statistical package [38] was used to identify perfectly correlated descriptors, i.e. $r=1.0$. In each case, only one descriptor of a perfectly correlated pair was retained for use in the subsequent analysis. Any descriptor that either had a value of zero for all compounds in the data set or could not be calculated for all compounds in the data set was removed. 
TABLE II Symbols, definitions and classification of calculated molecular descriptors

\begin{tabular}{|c|c|}
\hline \multicolumn{2}{|r|}{ Topostructural (TS) } \\
\hline$I_{D}^{W}$ & $\begin{array}{l}\text { Information index for the magnitudes of distances between all possible pairs of vertices of a } \\
\text { graph }\end{array}$ \\
\hline$\overline{I_{D}^{W}}$ & Mean information index for the magnitude of distance \\
\hline $\mathrm{W}$ & Wiener index $=$ half-sum of the off-diagonal elements of the distance matrix of a graph \\
\hline $\mathrm{I}^{\mathrm{D}}$ & Degree complexity \\
\hline $\mathrm{H}^{\mathrm{V}}$ & Graph vertex complexity \\
\hline $\mathrm{H}^{\mathrm{D}}$ & Graph distance complexity \\
\hline$\overline{\mathrm{IC}}$ & $\begin{array}{l}\text { Information content of the distance matrix partitioned by frequency of occurrences of distance } \\
h\end{array}$ \\
\hline $\mathrm{M}_{1}$ & A Zagreb group parameter $=$ sum of square of degree over all vertices \\
\hline $\mathrm{M}_{2}$ & $\begin{array}{l}\text { A Zagreb group parameter = sum of cross-product of degrees over all neighboring (connected) } \\
\text { vertices }\end{array}$ \\
\hline${ }^{\mathrm{b}} \chi$ & Path connectivity index of order $h=0-10$ \\
\hline${ }^{\mathrm{b}} \chi_{\mathrm{C}}$ & Cluster connectivity index of order $h=3-6$ \\
\hline${ }^{\mathrm{b}} \chi_{\mathrm{PC}}$ & Path-cluster connectivity index of order $h=4-6$ \\
\hline${ }^{\mathrm{h}} \chi_{\mathrm{Ch}}$ & Chain connectivity index of order $h=3-10$ \\
\hline$P_{h}$ & Number of paths of length $h=0-10$ \\
\hline $\mathbf{J}$ & Balaban's J index based on topological distance \\
\hline nrings & Number of rings in a graph \\
\hline ncirc & Number of circuits in a graph \\
\hline $\mathrm{DN}^{2} \mathrm{~S}_{\mathrm{y}}$ & $\begin{array}{l}\text { Triplet index of distance matrix, square of graph order (\# of non- } \mathrm{H} \text { atoms), and distance sum; } \\
\text { operation } y=1-5\end{array}$ \\
\hline $\mathrm{DN}^{2} 1_{\mathrm{y}}$ & Triplet index of distance matrix, square of graph order, and number 1 ; operation $y=1-5$ \\
\hline $\mathrm{AS} 1_{\mathrm{y}}$ & Triplet index of adjacency matrix, distance sum, and number 1 ; operation $y=1-5$ \\
\hline $\mathrm{DS} 1_{\mathrm{y}}$ & Triplet index of distance matrix, distance sum, and number 1 ; operation $y=1-5$ \\
\hline $\mathrm{ASN}_{\mathrm{y}}$ & Triplet index of adjacency matrix, distance sum, and graph order; operation $y=1-5$ \\
\hline $\mathrm{DSN}_{\mathrm{y}}$ & Triplet index of distance matrix, distance sum, and graph order; operation $y=1-5$ \\
\hline $\mathrm{DN}^{2} \mathrm{~N}_{\mathrm{y}}$ & Triplet index of distance matrix, square of graph order, and graph order; operation y $=1-5$ \\
\hline $\mathrm{ANS}_{\mathrm{y}}$ & Triplet index of adjacency matrix, graph order, and distance sum; operation $y=1-5$ \\
\hline$A N 1_{y}$ & Triplet index of adjacency matrix, graph order, and number 1 ; operation $y=1-5$ \\
\hline $\mathrm{ANN}_{\mathrm{y}}$ & Triplet index of adjacency matrix, graph order, and graph order again; operation $y=1-5$ \\
\hline $\mathrm{ASV}_{\mathrm{y}}$ & Triplet index of adjacency matrix, distance sum, and vertex degree; operation $y=1-5$ \\
\hline $\mathrm{DSV}_{\mathrm{y}}$ & Triplet index of distance matrix, distance sum, and vertex degree; operation $y=1-5$ \\
\hline $\mathrm{ANV}_{\mathrm{y}}$ & Triplet index of adjacency matrix, graph order, and vertex degree; operation $y=1-5$ \\
\hline
\end{tabular}

Topochemical (TC)

\begin{tabular}{|c|c|}
\hline $\mathrm{O}$ & Order of neighborhood when $\mathrm{IC}_{\mathrm{r}}$ reaches its maximum value for the hydrogen-filled graph \\
\hline $\mathrm{O}_{\text {orb }}$ & $\begin{array}{l}\text { Order of neighborhood when } \mathrm{IC}_{\mathrm{r}} \text { reaches its maximum value for the hydrogen-suppressed } \\
\text { graph }\end{array}$ \\
\hline $\mathrm{I}_{\text {orb }}$ & $\begin{array}{l}\text { Information content or complexity of the hydrogen-suppressed graph at its maximum } \\
\text { neighborhood of vertices }\end{array}$ \\
\hline $\mathrm{IC}_{\mathrm{r}}$ & $\begin{array}{l}\text { Mean information content or complexity of a graph based on the } r^{\text {th }}(r=0-6) \text { order } \\
\text { neighborhood of vertices in a hydrogen-filled graph }\end{array}$ \\
\hline $\mathrm{SIC}_{\mathrm{r}}$ & $\begin{array}{l}\text { Structural information content for } \mathrm{r}^{\text {th }}(\mathrm{r}=0-6) \text { order neighborhood of vertices in a hydrogen- } \\
\text { filled graph }\end{array}$ \\
\hline
\end{tabular}


TABLE II (Continued)

\begin{tabular}{|c|c|}
\hline $\mathrm{CIC}_{\mathrm{r}}$ & $\begin{array}{l}\text { Complementary information content for } \mathrm{r}^{\text {th }}(\mathrm{r}=0-6) \text { order neighborhood of vertices in a } \\
\text { hydrogen-filled graph }\end{array}$ \\
\hline${ }^{\mathrm{h}} \chi^{\mathrm{b}}$ & Bond path connectivity index of order $h=0-6$ \\
\hline${ }^{\mathrm{h}} \chi_{\mathrm{C}}^{\mathrm{b}}$ & Bond cluster connectivity index of order $h=3-6$ \\
\hline${ }^{\mathrm{h}} \chi_{\mathrm{Ch}}^{\mathrm{b}}$ & Bond chain connectivity index of order $h=3-6$ \\
\hline${ }^{\mathrm{h}} \chi_{\mathrm{pC}}^{\mathrm{b}}$ & Bond path-cluster connectivity index of order $h=4-6$ \\
\hline${ }^{\mathrm{h}} \chi^{\mathrm{v}}$ & Valence path connectivity index of order $h=0-10$ \\
\hline${ }^{\mathrm{b}} \chi_{\mathrm{C}}^{\mathrm{v}}$ & Valence cluster connectivity index of order $h=3-6$ \\
\hline${ }^{\mathrm{h}} \chi_{\mathrm{Ch}}^{\mathrm{v}}$ & Valence chain connectivity index of order $h=3-10$ \\
\hline${ }^{\mathrm{h}} \chi_{\mathrm{PC}}^{\mathrm{v}}$ & Valence path-cluster connectivity index of order $h=4-6$ \\
\hline $\mathrm{J}^{\mathrm{B}}$ & Balaban's J index based on bond types \\
\hline $\mathrm{J}^{\mathrm{X}}$ & Balaban's J index based on relative electronegativities \\
\hline $\mathrm{J}^{\mathrm{Y}}$ & Balaban's $\mathrm{J}$ index based on relative covalent radii \\
\hline $\mathrm{HB}_{1}$ & Hydrogen bonding parameter \\
\hline $\mathrm{AZV}_{\mathrm{y}}$ & Triplet index of adjacency matrix, atomic number, and vertex degree; operation $y=1-5$ \\
\hline $\mathrm{AZS}_{\mathrm{y}}$ & Triplet index of adjacency matrix, atomic number, and distance sum; operation $y=1-5$ \\
\hline $\mathrm{ASZ}_{\mathrm{y}}$ & Triplet index of adjacency matrix, distance sum, and atomic number; operation $y=1-5$ \\
\hline $\mathrm{AZN}_{\mathrm{y}}$ & Triplet index of adjacency matrix, atomic number, and graph order; operation $y=1-5$ \\
\hline $\mathrm{ANZ}_{\mathrm{y}}$ & Triplet index of adjacency matrix, graph order, and atomic number; operation $y=1-5$ \\
\hline $\mathrm{DSZ}_{\mathrm{y}}$ & Triplet index of distance matrix, distance sum, and atomic number; operation y $=1-5$ \\
\hline $\mathrm{DN}^{2} \mathrm{Z}_{\mathrm{y}}$ & Triplet index of distance matrix, square of graph order, and atomic number; operation y $=1-5$ \\
\hline nvx & Number of non-hydrogen atoms in a molecule \\
\hline nelem & Number of elements in a molecule \\
\hline fw & Molecular weight \\
\hline si & Shannon information index \\
\hline totop & Total Topological Index $t$ \\
\hline sumI & Sum of the intrinsic state values I \\
\hline sumdelI & Sum of delta-I values \\
\hline tets2 & Total topological state index based on electrotopological state indices \\
\hline phia & Flexibility index $(\mathrm{kp} 1 * \mathrm{kp} 2 / \mathrm{nvx})$ \\
\hline IdCbar & Bonchev-Trinajstic information index \\
\hline IdC & Bonchev-Trinajstić information index \\
\hline $\mathrm{Wp}$ & Wienerp \\
\hline Pf & Plattf \\
\hline Wt & Total Wiener number \\
\hline knotp & Difference of chi-cluster- 3 and path/cluster- 4 \\
\hline knotpv & Valence difference of chi-cluster-3 and path/cluster- 4 \\
\hline nclass & Number of classes of topologically (symmetry) equivalent graph vertices \\
\hline numHBd & Number of hydrogen bond donors \\
\hline numwHBd & Number of weak hydrogen bond donors \\
\hline numHBa & Number of hydrogen bond acceptors \\
\hline SHCsats & E-State of $\mathrm{C} \mathrm{sp}{ }^{3}$ bonded to other saturated $\mathrm{C}$ atoms \\
\hline SHCsatu & E-State of $\mathrm{C} \mathrm{sp}{ }^{3}$ bonded to unsaturated $\mathrm{C}$ atoms \\
\hline SHvin & E-State of $\mathrm{C}$ atoms in the vinyl group, $=\mathrm{CH}-$ \\
\hline SHtvin & E-State of $\mathrm{C}$ atoms in the terminal vinyl group, $=\mathrm{CH}_{2}$ \\
\hline
\end{tabular}


TABLE II (Continued)

\begin{tabular}{|c|c|}
\hline SHavin & E-State of $\mathrm{C}$ atoms in the vinyl group, $=\mathrm{CH}-$, bonded to an aromatic $\mathrm{C}$ \\
\hline SHarom & E-State of $\mathrm{C} \mathrm{sp}^{2}$ which are part of an aromatic system \\
\hline SHHBd & $\begin{array}{l}\text { Hydrogen bond donor index, sum of Hydrogen E-State values for }-\mathrm{OH},=\mathrm{NH} \text {, } \\
-\mathrm{NH} 2,-\mathrm{NH}-,-\mathrm{SH} \text {, and \#CH }\end{array}$ \\
\hline SHwHBd & $\begin{array}{l}\text { Weak hydrogen bond donor index, sum of } \mathrm{C}-\mathrm{H} \text { Hydrogen } \mathrm{E} \text {-State values for hydrogen atoms } \\
\text { on a } \mathrm{C} \text { to which a } \mathrm{F} \text { and/or } \mathrm{Cl} \text { are also bonded }\end{array}$ \\
\hline SHHBa & $\begin{array}{l}\text { Hydrogen bond acceptor index, sum of the E-State values for }-\mathrm{OH},=\mathrm{NH},-\mathrm{NH} 2,-\mathrm{NH}-,>\mathrm{N}-\text {, } \\
-\mathrm{O}-,-\mathrm{S}-\text {, along with }-\mathrm{F} \text { and }-\mathrm{Cl}\end{array}$ \\
\hline Qv & General Polarity descriptor \\
\hline NHBint $_{y}$ & Count of potential internal hydrogen bonders $(y=2-10)$ \\
\hline \multirow[t]{4}{*}{ SHBint $_{y}$} & E-State descriptors of potential internal hydrogen bond strength $(y=2-10)$ \\
\hline & Electrotopological State index values for atoms types: \\
\hline & $\begin{array}{l}\text { SHsOH, SHdNH, SHsSH, SHsNH2, SHssNH, SHtCH, SHother, SHCHnX, Hmax Gmax, } \\
\text { Hmin, Gmin, Hmaxpos, Hminneg, SsLi, SssBe, Sssss, Bem, SssBH, SsssB, SssssBm, SsCH3, } \\
\text { SdCH2, SssCH2, StCH, SdsCH, SaaCH, SsssCH, SddC,StsC, SdssC, SaasC, SaaaC, SssssC, } \\
\text { SsNH3p, SsNH2, SssNH2p, SdNH, SssNH, SaaNH, StN, SsssNHp, SdsN, SaaN, SsssN, } \\
\text { SddsN, SaasN, SssssNp, SsOH, SdO, SssO, SaaO, SsF, SsSiH3, SssSiH2, SsssSiH, SssssSi, } \\
\text { SsPH2, SssPH, SsssP, SdsssP, SsssssP, SsSH, SdS, SssS, SaaS, SdssS, SddssS, SssssssS, SsCl, } \\
\text { SsGeH3, SssGeH2, SsssGeH, SssssGe, SsAsH2, SssAsH, SsssAs, SdsssAs, SsssssAs, SsSeH, } \\
\text { SdSe, SssSe, SaaSe, SdssSe, SddssSe, SsBr, SsSnH3, SssSnH2, SsssSnH, SssssSn, SsI, } \\
\text { SsPbH3, SssPbH2, SsssPbH, SssssPb }\end{array}$ \\
\hline & Geometrical / Shape (3D) \\
\hline $\mathrm{kp0}$ & Kappa zero \\
\hline $\mathrm{kp} 1-\mathrm{kp} 3$ & Kappa simple indices \\
\hline ka1-ka3 & Kappa alpha indices \\
\hline
\end{tabular}

Models were developed using ridge regression (RR) [39], principal components regression (PCR) [40], and partial least squares (PLS) regression [41-43] methodologies, utilizing molecular descriptors in a hierarchical fashion. In addition, each class of descriptors was used independently to obtain single-class models. RR, PCR, and PLS are useful in cases wherein the number of descriptors is much greater than the number of observations, as well as in cases where the independent variables are highly intercorrelated. In addition, these regression methods make use of all independent variables as opposed to subset regression wherein it is possible that important parameters may be eliminated from the study. Statistical parameters reported include the cross-validated $\mathrm{R}^{2}$ value, which is obtained by removing each compound in turn from the data set and fitting the regression to the remaining $n-1$ compounds, and the PRESS statistic which is a reliable measure of model predictability. In addition, the $t$ values (coefficient estimate/std. err) can be examined in order to identify significant descriptors. Although a descriptor with a large $|t|$ indicates that the associated descriptor is important in the model, it should be cautioned that the reverse is not necessarily true. 
Honest assessment of the quality of a prediction model is seldom straightforward, but is particularly challenging in a situation such as this where the number of independent variables far exceeds the number of observations $[44,45]$. In these cases, conventional regression measures such as $\mathrm{R}^{2}$ are useless. The measure we use is the cross-validation (or jack-knife) sum of squares. For this measure, each compound in turn is omitted from the data set, and the coefficients of the regression model (RR, PLS or PCR) computed using the remaining n-1 cases. These coefficients are used to predict the hold-out case. The overall quality of the fit is measured by the prediction sum of squares PRESS - the sum of squares of the difference between the actual observed activity and that predicted from the regression. A cross-validation $\mathrm{R}^{2}$ can be defined by:

$$
R_{c v}^{2}=1-\frac{P R E S S}{S S T o t a l}
$$

Unlike $\mathrm{R}^{2}$, this $R_{c v}^{2}$ does not increase if irrelevant predictors are added to the model; rather it tends to decrease. And where $\mathrm{R}^{2}$ is necessarily non-negative, $R_{c \nu}^{2}$ may be negative. This non-uncommon situation is an indication that the model fitted is poor - worse, in fact, than making predictions by ignoring the predictors and using the mean activity as the prediction in all circumstances.

$R_{c v}^{2}$ mimics the results of applying the final regression to predicting a future case; large values can be interpreted unequivocally and without regard to either the number of cases or predictors as indicating that the fitted regression will accurately predict the activity of future compounds of the same chemical type as those used to calibrate the regression.

In an attempt to gain further insight into the types of descriptors that are important in the partitioning of chemicals across the various tissues, variable clustering was performed using the VARCLUS procedure of the SAS statistical package [38]. This procedure divides the set of descriptors into disjoint clusters such that each cluster is essentially uni-dimensional. Interpretation of the descriptors, in terms of what aspects of chemical structure they represent, may be aided by examining other descriptors that fall within the same cluster.

\section{RESULTS}

Studies were done on the complete set of 46 diverse compounds as well as the structurally homogeneous subset of 26 haloalkanes for the prediction of rat fat:air, liver:air, and muscle:air partition coefficients, the results of which are provided in Tables III-V, respectively. It was of interest to compare our models developed with theoretical structural descriptors to models developed with experimental properties used by Gargas et al. [1] as independent variables, namely, $\log \mathrm{P}_{\text {olive oilair }}$ and $\log \mathrm{P}_{\text {salineair. }}$ The results of these 
property-property correlations are also included in Tables III-V. It is important to note that the statistical results reported for the property-property correlations were not taken directly from the Gargas study. Rather, property data were taken from the Gargas study for the same sets of compounds utilized in the current study, and linear models were developed by our team using the SAS statistical package [38]. In general, the RR methodology outperformed either PCR or PLS, and either the TC or the TS+TC models were superior to those developed using other descriptor classes. It is not surprising that, for each type of partition coefficient, the model developed for the set of 26 haloalkanes is superior to the model developed for the more diverse set of 46 compounds. However, models developed for the larger, more diverse set are reasonable.

TABLE III Summary statistics of predictive models for rat fat:air partition coefficient based on experimental properties and theoretical structural descriptors

\begin{tabular}{|c|c|c|c|c|c|c|c|c|}
\hline \multicolumn{9}{|c|}{ A. 46 DIVERSE CHEMICALS } \\
\hline \multirow{2}{*}{$\begin{array}{c}\text { Independent } \\
\text { Variables }\end{array}$} & \multicolumn{2}{|c|}{$R R$} & \multicolumn{2}{|c|}{$P C R$} & \multicolumn{2}{|c|}{$P L S$} & \multicolumn{2}{|c|}{$L R$} \\
\hline & $R_{\text {c.v. }}^{2}$ & PRESS & $R_{\text {c.v. }}^{2}$ & PRESS & $R_{\text {c.p. }}^{2}$ & PRESS & $R_{\text {c.v. }}^{2}$ & PRESS \\
\hline \multicolumn{9}{|l|}{ Structural descriptors } \\
\hline $\mathrm{TS}$ & 0.325 & 106 & 0.239 & 119 & 0.328 & 106 & & \\
\hline $\mathrm{TS}+\mathrm{TC}$ & 0.947 & 8.35 & 0.701 & 46.8 & 0.886 & 17.8 & & \\
\hline $\mathrm{TS}+\mathrm{TC}+3 \mathrm{D}$ & 0.943 & 9.00 & 0.710 & 45.5 & 0.888 & 17.6 & & \\
\hline $\mathrm{TS}+\mathrm{TC}+3 \mathrm{D}+\log \mathrm{P}$ & 0.941 & 9.23 & 0.708 & 45.9 & 0.892 & 17.0 & & \\
\hline TS & 0.325 & 106 & 0.239 & 119 & 0.328 & 106 & & \\
\hline $\mathrm{TC}$ & 0.939 & 9.49 & 0.565 & 68.2 & 0.843 & 24.7 & & \\
\hline $3 \mathrm{D}$ & 0.253 & 117 & 0.015 & 154 & -0.023 & 160 & & \\
\hline \multicolumn{9}{|l|}{ Properties } \\
\hline $\log \mathrm{P}_{\text {olive oil:air }}$ & & & & & & & 0.927 & 11.5 \\
\hline $\log \mathrm{P}_{\text {olive oillair }}+\log \mathrm{P}_{\text {sali }}$ & & & & & & & 0.932 & 10.6 \\
\hline \multicolumn{9}{|c|}{ B. 26 HALOALKANES } \\
\hline \multirow{2}{*}{$\begin{array}{c}\text { Independent } \\
\text { Variables }\end{array}$} & \multicolumn{2}{|c|}{$R R$} & \multicolumn{2}{|c|}{$P C R$} & \multicolumn{2}{|c|}{$P L S$} & \multicolumn{2}{|c|}{$L R$} \\
\hline & $R_{\text {c.p. }}^{2}$ & PRESS & $R_{\text {c.v. }}^{2}$ & PRESS & $R_{\text {c.v. }}^{2}$ & PRESS & $R_{\text {c.V. }}^{2}$ & PRESS \\
\hline \multicolumn{9}{|l|}{ Structural descriptors } \\
\hline TS & 0.143 & 74.6 & -0.432 & 125 & -0.112 & 96.8 & & \\
\hline $\mathrm{TS}+\mathrm{TC}$ & 0.971 & 2.54 & -0.917 & 7.23 & 0.941 & 5.16 & & \\
\hline $\mathrm{TS}+\mathrm{TC}+3 \mathrm{D}$ & 0.969 & 2.68 & 0.894 & 9.21 & 0.930 & 6.06 & & \\
\hline $\mathrm{TS}+\mathrm{TC}+3 \mathrm{D}+\log \mathrm{P}$ & 0.969 & 2.74 & 0.898 & 8.91 & 0.930 & 6.09 & & \\
\hline TS & 0.143 & 74.6 & -0.432 & 125 & -0.112 & 96.8 & & \\
\hline $\mathrm{TC}$ & 0.972 & 2.46 & 0.952 & 4.19 & 0.945 & 4.81 & & \\
\hline $3 \mathrm{D}$ & 0.926 & 6.41 & 0.918 & 7.14 & 0.927 & 6.38 & & \\
\hline \multicolumn{9}{|l|}{ Properties } \\
\hline $\log \mathrm{P}_{\text {olive oiltair }}$ & & & & & & & 0.960 & 3.48 \\
\hline $\log \mathrm{P}_{\text {olive oiltair }}+\log \mathrm{P}_{\text {salin }}$ & & & & & & & 0.958 & 3.69 \\
\hline
\end{tabular}


TABLE IV Summary statistics of predictive models for rat liver:air partition coefficient based on experimental properties and theoretical structural descriptors.

\begin{tabular}{|c|c|c|c|c|c|c|c|c|}
\hline \multicolumn{9}{|c|}{ A. 46 DIVERSE CHEMICALS } \\
\hline \multirow{2}{*}{$\begin{array}{c}\text { Independent } \\
\text { Variables }\end{array}$} & \multicolumn{2}{|c|}{$R R$} & \multicolumn{2}{|c|}{$P C R$} & \multicolumn{2}{|c|}{$P L S$} & \multicolumn{2}{|c|}{$L R$} \\
\hline & $R_{\text {c.v. }}^{2}$ & PRESS & $R_{\text {c.v. }}^{2}$ & PRESS & $R_{c . y .}^{2}$ & PRESS & $R_{c . v .}^{2}$ & PRESS \\
\hline \multicolumn{9}{|l|}{ Structural descriptors } \\
\hline TS & 0.226 & 88.6 & -0.058 & 121 & 0.242 & 86.8 & & \\
\hline $\mathrm{TS}+\mathrm{TC}$ & 0.917 & 9.48 & 0.250 & 85.9 & 0.678 & 36.9 & & \\
\hline $\mathrm{TS}+\mathrm{TC}+3 \mathrm{D}$ & 0.916 & 9.56 & 0.259 & 84.9 & 0.746 & 29.1 & & \\
\hline $\mathrm{TS}+\mathrm{TC}+3 \mathrm{D}+\log \mathrm{P}$ & 0.920 & 9.20 & 0.243 & 86.7 & 0.743 & 29.4 & & \\
\hline TS & 0.226 & 88.6 & -0.058 & 121 & 0.242 & 86.8 & & \\
\hline $\mathrm{TC}$ & 0.942 & 6.62 & 0.064 & 107 & 0.678 & 36.8 & & \\
\hline $3 \mathrm{D}$ & 0.165 & 95.6 & 0.055 & 108 & 0.035 & 110 & & \\
\hline \multicolumn{9}{|l|}{ Properties } \\
\hline $\log \mathrm{P}_{\text {olive oil:air }}+\log \mathrm{P}_{\text {sal }}$ & & & & & & & 0.894 & 12.1 \\
\hline \multicolumn{9}{|c|}{ B. 26 HALOALKANES } \\
\hline Independent & \multicolumn{2}{|c|}{$R R$} & \multicolumn{2}{|c|}{$P C R$} & \multicolumn{2}{|c|}{ PLS } & \multicolumn{2}{|c|}{$L R$} \\
\hline Variables & $R_{c . v .}^{2}$ & PRESS & $R_{c, v,}^{2}$ & PRESS & $R_{\text {c.v. }}^{2}$ & PRESS & $R_{\text {c.v. }}^{2}$ & PRESS \\
\hline \multicolumn{9}{|l|}{ Structural descriptors } \\
\hline TS & 0.029 & 57.8 & -0.845 & 110 & -0.262 & 75.2 & & \\
\hline $\mathrm{TS}+\mathrm{TC}$ & 0.954 & 2.76 & 0.884 & 6.93 & 0.837 & 9.69 & & \\
\hline $\mathrm{TS}+\mathrm{TC}+3 \mathrm{D}$ & 0.950 & 2.95 & 0.870 & 7.74 & 0.779 & 13.1 & & \\
\hline $\mathrm{TS}+\mathrm{TC}+3 \mathrm{D}+\log \mathrm{P}$ & 0.957 & 2.54 & 0.871 & 7.70 & 0.813 & 11.1 & & \\
\hline TS & 0.029 & 57.8 & -0.845 & 110 & -0.262 & 75.2 & & \\
\hline $\mathrm{TC}$ & 0.964 & 2.16 & 0.896 & 6.17 & 0.837 & 9.70 & & \\
\hline $3 \mathrm{D}$ & 0.826 & 10.4 & 0.808 & 11.4 & 0.803 & 11.7 & & \\
\hline \multicolumn{9}{|l|}{ Properties } \\
\hline $\log \mathrm{P}_{\text {olive oil:air }}+\log \mathrm{P}$ & & & & & & & 0.851 & 8.89 \\
\hline
\end{tabular}

Ridge regression models developed using the TC or TS+TC theoretical descriptors were comparable or superior to models developed using experimental properties, i.e. $\log \mathrm{P}_{\text {olive oillair }}$ and/or $\log \mathrm{P}_{\text {saline:air }}$ (Tables III-V). For example, the cross-validated $\mathrm{R}^{2}$ and PRESS statistic for the rat muscle:air partition coefficient are 0.863 and 12.2 , respectively, for the TC model, based on the set of 46 diverse compounds and developed using theoretical descriptors; while the same statistical measures are 0.891 and 9.70, respectively for the model based on experimental $\log \mathrm{P}_{\text {olive oilair }}$ and $\log \mathrm{P}_{\text {saline:air }}$ (Table V). When the same study is done on the set of 26 haloalkanes, the cross-validated $R^{2}$ and PRESS are 0.906 and 4.30, respectively, for the TC model using theoretical descriptors; while the same statistical measures are 0.876 and 5.67 for the model based on experimental $\log \mathrm{P}_{\text {olive oil:air }}$ and $\log \mathrm{P}_{\text {saline:air }}$ (Table V). As would be expected, the PRESS statistic increases as the structurally different chemicals are added to the haloalkane subsets. 
Tables VI-VIII provide ridge regression coefficients, standard errors, and cluster membership for the top 20 descriptors based on $|t|$ values for the TC rat fat:air, liver:air, and muscle:air models, respectively, based on the set of 46 diverse chemicals. Examining only the 20 descriptors with highest $|t|$ values, descriptors from clusters 10 and 20 are present in both the muscle:air and liver:air models and are absent from the fat:air model. Cluster 10 is composed of descriptors representing polarity and hydrogen bonding and includes E-state descriptors for $\mathrm{O}$ and $\mathrm{N}$ atom types as well as a hydrogen bonding parameter. The descriptors from cluster 20 that are important here include Balaban's $\mathrm{J}$ indices, $\mathrm{J}^{\mathrm{B}}, \mathrm{J}^{\mathrm{X}}$, and $\mathrm{J}^{\mathrm{Y}}$. Descriptors from clusters 14 and 18 are present in all studies, the former including low-order valence connectivity indices and molecular weight, and the latter including descriptors of cyclicity and aromaticity.

TABLE V Summary statistics of predictive models for rat muscle:air partition coefficient based on experimental properties and theoretical structural descriptors

\begin{tabular}{|c|c|c|c|c|c|c|c|c|}
\hline \multicolumn{9}{|c|}{ A. 46 DIVERSE CHEMICALS } \\
\hline \multirow{2}{*}{$\begin{array}{c}\text { Independent } \\
\text { Variables }\end{array}$} & \multicolumn{2}{|c|}{$R R$} & \multicolumn{2}{|c|}{$P C R$} & \multicolumn{2}{|c|}{$P L S$} & \multicolumn{2}{|c|}{$L R$} \\
\hline & $R_{c . v .}^{2}$ & PRESS & $R_{\text {c.v. }}^{2}$ & PRESS & $R_{\text {c.v. }}^{2}$ & PRESS & $R_{\text {c.v. }}^{2}$ & PRESS \\
\hline \multicolumn{9}{|l|}{ Structural descriptors } \\
\hline TS & 0.203 & 70.8 & 0.004 & 88.7 & 0.169 & 73.8 & & \\
\hline $\mathrm{TS}+\mathrm{TC}$ & 0.849 & 13.4 & 0.222 & 69.0 & 0.624 & 33.4 & & \\
\hline$T S+T C+3 D$ & 0.855 & 12.9 & 0.232 & 68.2 & 0.589 & 36.4 & & \\
\hline $\mathrm{TS}+\mathrm{TC}+3 \mathrm{D}+\log \mathrm{P}$ & 0.855 & 12.9 & 0.216 & 69.6 & 0.599 & 35.6 & & \\
\hline TS & 0.203 & 70.8 & 0.004 & 88.7 & 0.169 & 73.8 & & \\
\hline $\mathrm{TC}$ & 0.863 & 12.2 & -0.010 & 89.7 & 0.576 & 37.6 & & \\
\hline $3 \mathrm{D}$ & 0.089 & 80.9 & -0.061 & 94.2 & -0.079 & 95.8 & & \\
\hline \multicolumn{9}{|l|}{ Properties } \\
\hline $\log \mathrm{P}_{\text {olive oiltair }}+\log \mathrm{P}_{\text {sali }}$ & & & & & & & 0.891 & 9.70 \\
\hline \multicolumn{9}{|c|}{ B. 26 HALOALKANES } \\
\hline \multirow{2}{*}{$\begin{array}{c}\text { Independent } \\
\text { Variables }\end{array}$} & \multicolumn{2}{|c|}{$R R$} & \multicolumn{2}{|c|}{$P C R$} & \multicolumn{2}{|c|}{$P L S$} & \multicolumn{2}{|c|}{$L R$} \\
\hline & $R_{\text {c.v. }}^{2}$ & PRESS & $R_{\text {c.p. }}^{2}$ & PRESS & $R_{\text {c.v. }}^{2}$ & PRESS & $R_{\text {c.v. }}^{2}$ & PRESS \\
\hline \multicolumn{9}{|l|}{ Structural descriptors } \\
\hline TS & -0.014 & 46.5 & -1.265 & 104 & -0.248 & 57.2 & & \\
\hline $\mathrm{TS}+\mathrm{TC}$ & 0.902 & 4.49 & 0.855 & 6.65 & 0.870 & 5.98 & & \\
\hline $\mathrm{TS}+\mathrm{TC}+3 \mathrm{D}$ & 0.900 & 4.60 & 0.855 & 6.64 & 0.826 & 7.96 & & \\
\hline $\mathrm{TS}+\mathrm{TC}+3 \mathrm{D}+\log \mathrm{P}$ & 0.899 & 4.63 & 0.855 & 6.64 & 0.826 & 7.99 & & \\
\hline TS & -0.014 & 46.5 & -1.265 & 104 & -0.248 & 57.2 & & \\
\hline $\mathrm{TC}$ & 0.906 & 4.30 & 0.871 & 5.91 & 0.848 & 6.98 & & \\
\hline $3 \mathrm{D}$ & 0.820 & 8.27 & 0.792 & 9.56 & 0.800 & 9.18 & & \\
\hline \multicolumn{9}{|l|}{ Properties } \\
\hline $\log \mathrm{P}_{\text {olive oil:air }}+\log \mathrm{P}_{\text {salin }}$ & & & & & & & 0.876 & 5.67 \\
\hline
\end{tabular}




\section{DISCUSSION}

The objective of the study reported in this paper was two-fold: a) estimation of fat:air, muscle:air, and liver:air partition coefficients of VOCs from their chemical structure and b) a comparative analysis of structure-based and property based descriptors in the estimation of tissue:air partition coefficients.

To this end, we developed HiQSAR models for a set of 46 diverse chemicals as well as a subset of 26 halocarbons for all three tissue:air partition coefficients. A perusal of the regression models presented in Table III shows that for the fat:air partition coefficient, either the TC indices or the combination of TS\&TC indices gave the best predictive models. The ridge regression method resulted in superior models compared to PLS and PCR techniques, in line with our earlier observations [5, 17, 46-48] It is interesting to note that attempts to estimate fat:air partition coefficients of the VOCs by property-property correlation using either olive oil:air or the combination of olive oil:air and saline:air partition coefficients resulted in models of comparable quality.

Models developed with the congeneric set of 26 haloalkanes for tissue:air partition coefficient show a similar trend. There is improvement in model quality for this smaller subset as compared to the total set of 46 VOCs. Here again either the TC or the TS plus TC indices gave the best result; these models also outperformed the property-property correlation models based on either olive oil:air partition coefficient or a combination of olive oil:air and saline:air partition coefficients.

Data depicted in Tables IV and V, dealing with regression models of liver:air and muscle:air partition coefficients, respectively, show the same trend as observed for the fat:air partition coefficient, viz., in the HiQSAR scheme, either the TC or TS+TC models performed the best; structure-based descriptors and experimental olive oil:air and saline:air partition coefficients resulted in QSARs of comparable quality; there was some improvement in the predictive models for the subset of 26 haloalkanes vis-a-vis the diverse set of 46 chemicals.

It is interesting to note the profile of molecular descriptors which play important role in the prediction of the three tissue:air partition coefficients. The RR routine provides as output the $t$ values of the individual indices which can be used to order the TIs according to their relative importance in estimating the property under investigation. The 20 TIs in the TC models with highest $|t|$ values are given in Tables VI-VIII for fat:air, liver:air, and muscle:air partition coefficients, respectively. A perusal of the data in Tabel VI for fat:air partition coefficient shows that the connectivity and triplet indices dominate the set of parameters. On the other hand, hydrogen-bonding parameters such as $\mathrm{HB}_{1}$ do not appear in the list of most important twenty indices. This makes sense in view of the fact that fatty tissues contain more hydrophobic materials such as triglycerides through which lipophilic chemicals will cross by passive 
transport mechanism, and this will be governed mainly by general shape, size, branching, etc, associate with the molecular architecture.

TABLE VI Cluster membership, ridge regression coefficient, and standard error for each of the top 20 descriptors, ranked by $|t|$, in the topochemical rat fat:air partition coefficient model, $n=46$

\begin{tabular}{|c|c|c|c|c|}
\hline Cluster \# & Descriptor & $R R$ coeff & s.e. & $t$ \\
\hline 14 & ${ }^{1} \chi^{v}$ & 1.223 & 0.073 & 16.66 \\
\hline 14 & ${ }^{0} \chi^{v}$ & 0.816 & 0.054 & 15.05 \\
\hline 14 & $\mathrm{AZV}_{4}$ & 0.421 & 0.029 & 14.44 \\
\hline 6 & $\dot{A} Z V_{1}$ & 0.227 & 0.025 & 8.97 \\
\hline 14 & $\mathrm{AZN}_{4}$ & 1.377 & 0.161 & 8.54 \\
\hline 6 & $\mathrm{AZV}_{3}$ & 0.172 & 0.021 & 8.14 \\
\hline 14 & ${ }^{2} \chi^{v}$ & 0.528 & 0.066 & 7.98 \\
\hline 13 & $\mathrm{SsF}$ & -0.200 & 0.027 & -7.32 \\
\hline 6 & $\mathrm{AZSS}_{5}$ & 0.073 & 0.010 & 6.95 \\
\hline 18 & $\mathrm{SaaCH}$ & 0.090 & 0.013 & 6.77 \\
\hline 18 & ${ }^{6} \chi_{\text {ch }}^{v}$ & 7.925 & 1.186 & 6.68 \\
\hline 6 & $\mathrm{AZS}_{3}$ & 0.086 & 0.013 & 6.65 \\
\hline 18 & SHarom & 0.121 & 0.018 & 6.65 \\
\hline 13 & Gmin & 0.432 & 0.066 & 6.59 \\
\hline 1 & ${ }^{1} \chi^{b}$ & 0.407 & 0.063 & 6.5 \\
\hline 17 & $\mathrm{ANZ}_{1}$ & 0.202 & 0.033 & 6.22 \\
\hline 6 & $\mathrm{Wt}$ & 0.063 & 0.010 & 6.09 \\
\hline 10 & $\mathrm{SdO}$ & 0.157 & 0.027 & 5.76 \\
\hline 14 & fw & 0.381 & 0.068 & 5.6 \\
\hline 18 & phia & 0.447 & 0.080 & 5.58 \\
\hline
\end{tabular}

A perusal of the TIs most important for the QSARs for muscle:air and liver:air partition coefficients (Tables VII and VIII) shows that parameters such as $\mathrm{HB}_{1}$, SdO and SddsN play an important role in predicting the property. The liver and muscle tissues, particularly their membranous components (which play a dominant role in the partitioning and uptake of chemicals) will contain higher amounts of polar lipids such as phospholipids, gangliosides, sulfolipids, etc. as compared to the fatty tissues. This may be the reason why parameters encoding information about hydrogen bonding and polarity assume dominant roles in the partitioning of chemicals between muscle/liver:air as opposed to fat:air. Independent variables belonging to clusters 14 and 18 are important in modeling all of the tissue:air partition coefficients. These two clusters contain the following parameters:

Cluster \#14: ${ }^{0} \chi^{v},{ }^{1} \chi^{v},{ }^{2} \chi^{v}, \mathrm{AZV}_{4}, \mathrm{AZN}_{4}, \mathrm{fw}, \mathrm{ka} 1$

Cluster \#18: ${ }^{6} \chi_{\mathrm{Ch}},{ }^{6} \chi_{\mathrm{Ch}}^{\mathrm{v}}$, nrings, ncirc, phia, SHother, SaaCH, Sharom 
TABLE VII Cluster membership, ridge regression coefficient, and standard error for each of the top 20 descriptors, ranked by $|t|$, in the topochemical rat liver:air partition coefficient model, $n=46$

\begin{tabular}{|c|c|c|c|c|}
\hline Cluster \# & Descriptor & $R R$ coeff & s.e. & $t$ \\
\hline 10 & $\mathrm{SdO}$ & 0.381 & 0.004 & 90.29 \\
\hline 20 & $\mathrm{~J}^{\mathrm{B}}$ & -1.628 & 0.022 & -75.08 \\
\hline 20 & $\mathrm{~J}^{\mathrm{Y}}$ & -1.620 & 0.022 & -73.83 \\
\hline 14 & fw & 1.372 & 0.022 & 63.6 \\
\hline 20 & $\mathrm{~J}^{\mathrm{X}}$ & -1.683 & 0.031 & -54.93 \\
\hline 10 & $\mathrm{HB}_{1}$ & 0.456 & 0.009 & 53.35 \\
\hline 18 & ${ }^{6} \chi^{\mathrm{v}} \mathrm{vh}$ & 19.137 & 0.432 & 44.26 \\
\hline 18 & SHarom & 0.309 & 0.007 & 43.3 \\
\hline 10 & SddsN & -2.341 & 0.055 & -42.72 \\
\hline 12 & $\mathrm{SdsCH}$ & 0.615 & 0.015 & 42.42 \\
\hline 6 & $A Z N_{1}$ & -0.149 & 0.004 & -41.07 \\
\hline 11 & $\mathrm{AZS}_{4}$ & -0.843 & 0.021 & -39.22 \\
\hline 16 & nelem & -1.999 & 0.051 & -39.21 \\
\hline 17 & $\mathrm{ASZ}_{2}$ & -0.146 & 0.004 & -39.13 \\
\hline 6 & totop & -0.174 & 0.004 & -39.06 \\
\hline 18 & $\mathrm{SaaCH}$ & 0.224 & 0.006 & 38.39 \\
\hline 6 & $\mathrm{AZN}_{5}$ & -0.129 & 0.004 & -35.05 \\
\hline 7 & ${ }^{4} \chi_{\mathrm{pc}}^{\mathrm{v}}$ & 0.707 & 0.020 & 35.01 \\
\hline 14 & ${ }^{2} \chi^{v}$ & 0.499 & 0.015 & 33.94 \\
\hline 14 & $\mathrm{AZV}_{4}$ & 0.501 & 0.015 & 33.5 \\
\hline
\end{tabular}

Clusters 14 and 18 have descriptors which encode information regarding general structural features; cluster 14 (e.g., ${ }^{0} \chi^{v},{ }^{1} \chi^{v},{ }^{2} \chi^{v}, \mathrm{fw}$ ) quantifying molecular size, and cluster 18 (e.g., ${ }^{6} \chi_{\mathrm{Ch}},{ }^{6} \chi^{v} \mathrm{Ch}$, nrings, ncirc, SHarom) containing information about cyclicity. It is important to note that 9 of the 46 compounds (Table I) analyzed in this paper are cyclic/aromatic, and cluster 18 represents information about molecular cyclicity/aromaticity. Therefore, it is clear that molecular features such as general shape and size, as well as special structural attributes of the particular set chosen (e.g., cyclicity) have dominant roles in determining the partitioning of the chemicals between air and nonpolar fatty tissue.

The picture is, however, quite different in the cases of liver:air and muscle:air partition coefficients. Whereas parameters belonging to clusters 14 and 18 play a less important but essential role, the dominant role is played by parameters belonging to clusters 10 and 20 . The latter two clusters contain the following independent variables:

Cluster \#10: SddsN, $\mathrm{SdO}, \mathrm{HB}_{1}$

Cluster \#20: $\mathrm{M}_{1}, \mathrm{M}_{2},{ }^{0} \chi,{ }^{2} \chi,{ }^{0} \chi^{\mathrm{b}}, \mathrm{P}_{2}, \mathrm{~J}^{\mathrm{B}}, \mathrm{J}^{\mathrm{X}}, \mathrm{J}^{\mathrm{Y}}, \mathrm{AZV}_{2}, \mathrm{DN}^{2} \mathrm{~S}_{4}, \mathrm{AS}_{3}, \mathrm{ASN}_{1}, \mathrm{ASN}_{3}, \mathrm{ASN}_{5}, \mathrm{DSN}_{3}, \mathrm{ANV}_{4}$, $\mathrm{AN1}_{1}, \mathrm{AN1}_{4}, \mathrm{ANN}_{2}, \mathrm{ANN}_{4}, \mathrm{Pf}$ 
TABLE VIII Cluster membership, ridge regression coefficient, and standard error for each of the top 20 descriptors, ranked by $|t|$, in the topochemical rat muscle:air partition coefficient model, $n=46$

\begin{tabular}{lcccc}
\hline Cluster \# & Descriptor & RR coeff & s.e. & $t$ \\
\hline 10 & $\mathrm{SdO}$ & 0.294 & 0.046 & 6.34 \\
10 & $\mathrm{SddsN}$ & -2.320 & 0.382 & -6.07 \\
10 & $\mathrm{HB}_{1}$ & 0.375 & 0.069 & 5.41 \\
14 & ${ }^{1} \chi^{\mathrm{v}}$ & 0.961 & 0.191 & 5.02 \\
20 & $\mathrm{~J}^{\mathrm{Y}}$ & -0.920 & 0.192 & -4.79 \\
2 & $\mathrm{SHCsatu}$ & 1.380 & 0.323 & 4.27 \\
18 & $\mathrm{SHarom}$ & 0.156 & 0.037 & 4.24 \\
20 & $\mathrm{~J}^{\mathrm{B}}$ & -0.908 & 0.221 & -4.1 \\
14 & $\mathrm{fw}$ & 0.640 & 0.163 & 3.94 \\
18 & $\mathrm{SaCH}^{6}$ & 0.108 & 0.027 & 3.94 \\
18 & ${ }^{\mathrm{v}} \chi_{\mathrm{ch}}^{\mathrm{v}}$ & 9.052 & 2.387 & 3.79 \\
20 & $\mathrm{~J}^{\mathrm{X}}$ & -0.980 & 0.262 & -3.74 \\
14 & $\mathrm{AZV}_{4}$ & 0.224 & 0.061 & 3.65 \\
14 & ${ }^{2} \chi^{\mathrm{v}}$ & 0.563 & 0.165 & 3.41 \\
9 & $\mathrm{IC}_{2}$ & -0.611 & 0.182 & -3.35 \\
21 & ${ }^{6} \chi^{\mathrm{b} c}$ & -0.714 & 0.216 & -3.31 \\
4 & $\mathrm{SIC}_{2}$ & -1.581 & 0.501 & -3.16 \\
16 & $\mathrm{IC}_{0}$ & 1.403 & 0.473 & 2.97 \\
7 & ${ }^{4} \chi_{\mathrm{pc}}^{\mathrm{v}}$ & 0.487 & 0.164 & 2.97 \\
16 & $\mathrm{SIC}_{0}$ & 1.010 & 0.346 & 2.92 \\
\hline
\end{tabular}

Whereas cluster 10 contains $\mathrm{HB}_{1}$, an explicit hydrogen-bonding parameter, as well as descriptors encoding information about nitrogen and oxygen atoms which are strong hydrogen bond acceptors, cluster 20 contains indices which are either TS parameters encoding molecular size or TC indices which quantify information both about size/shape and polarity of molecular structure. It is tempting to speculate that partitioning of chemicals between air and more polar tissues, viz., muscle and liver, are governed by: a) general size and shape, b) strength of hydrogen bonding, and c) dipolarity /polarizability. Such a conclusion is supported by Kamlet et al. [49] and our earlier studies on the prediction of $\log \mathrm{P}_{\text {octanol:water }}$ from molecular descriptors $[9,50,51]$.

In conclusion, results reported in this paper show that HiQSAR based on theoretical molecular descriptors can be useful in the estimation of tissue:air partition coefficients of VOCs. The theoretically based models are comparable or superior to those developed using experimental properties including olive oil:air and/or saline:air partition coefficients. The dominant parameters needed to develop the QSARs lead to some interpretation of the different biological situations involved in the partitioning of chemicals in the different types of tissues.

\section{Acknowledgements}

This is contribution number XXX from the Center for Water and the Environment of the Natural 
Resources Research Institute. Research reported in this paper was supported in part by Grant F49620-010098 from the United States Air Force.

\section{References}

[1] Gargas, M. L., Burgess, R. J., Voisard, D. E., Cason, G. H. and Andersen, M. E. (1989). Partition coefficients of low molecular weight volatile chemicals in various tissues and liquids. Toxicol. Appl. Pharmacol., 98, 8799.

[2] USEPA (2002). What is the TSCA Chemical Substance Inventory?, http://www.epa.gov/opptintr/newchems/invntory.htm.

[3] USEPA (2002). ChemRTK HPV Challenge Program Chemical List, http://www.epa.gov/opptintr/chemrtk/hpvchmlt.htm.

[4] ATSDR (2002). 2001 CERCLA Priority List of Hazardous Substances, http://www.atsdr.cdc.gov/clist.html.

[5] Basak, S. C., Hawkins, D. M. and Mills, D. (2002). Predicting blood:air partition coefficient of structurally diverse chemicals using theoretical molecular descriptors. In, Advances in Molecular Similarity (X. Girones, R. Carbo-Dorca, and P.G. Mezey, Eds.). Kluwer, in press.

[6] Basak, S. C., Harriss, D. K. and Magnuson, V. R. (1984). Comparative study of lipophilicity versus topological molecular descriptors in biological correlations. J. Pharm. Sci., 73, 429-437.

[7] Basak, S. C. and Grunwald, G. D. (1995). Estimation of lipophilicity from molecular structural similarity. New J. Chem., 19, 231-237.

[8] Basak, S. C., Monsrud, L. J., Rosen, M. E., Frane, C. M. and Magnuson, V. R. (1986). A comparative study of lipophilicity and topological indices in biological correlation. Acta Pharm.Yugosl., 36, 81-95.

[9] Niemi, G. J., Basak, S. C., Veith, G. D. and Grunwald, G. (1992). Prediction of octanol-water partition coefficient (Kow) using algorithmically-derived variables. Environ. Toxicol. Chem., 11, 891-898.

[10] Basak, S. C., Gute, B. D. and Grunwald, G. D. (1997). Use of topostructural, topochemical, and geometric parameters in the prediction of vapor pressure: A hierarchical approach. J. Chem. Inf. Comput. Sci., 37, 651655.

[11] Basak, S. C. and Mills, D. (2001). Quantitative structure-property relationships (QSPRs) for the estimation of vapor pressure: A hierarchical approach using mathematical structural descriptors. J. Chem. Inf. Comput. Sci., 41, 692-701.

[12] Basak, S. C., Gute, B. D. and Grunwald, G. D. (1996). A comparative study of topological and geometrical parameters in estimating normal boiling point and octanol-water partition coefficient. J. Chem. Inf. Comput. Sci., 36, 1054-1060.

[13] Basak, S. C. and Mills, D. (2001). Use of mathematical structural invariants in the development of QSPR models. MATCH (Commun. Math. Comput. Chem.), 44, 15-30.

[14] Basak, S. C., Gute, B. D. and Grunwald, G. D. (1999). Assessment of the mutagenicity of aromatic amines from theoretical structural parameters: A hierarchical approach. SAR QSAR Environ. Res., 10, 117-129.

[15] Basak, S. C., Mills, D. R., Balaban, A. T. and Gute, B. D. (2001). Prediction of mutagenicity of aromatic and heteroaromatic amines from structure: A hierarchical QSAR approach. J. Chem. Inf. Comput. Sci., 41, 671678.

[16] Basak, S. C. and Mills, D. (2001). Prediction of mutagenicity utilizing a hierarchical QSAR approach. SAR QSAR Environ. Res., 12, 481-496.

[17] Basak, S. C., Mills, D., Gute, B. D. and Hawkins, D. M. (2002). Predicting mutagenicity of congeneric and diverse sets of chemicals using computed molecular descriptors: A hierarchical approach. In, Quantitative Structure-Activity Relationship (QSAR) Models of Mutagens and Carcinogens (R. Benigni, Ed.). CRC.Press, Boca Raton, FL, in press.

[18] Basak, S. C., Grunwald, G. D., Host, G. E., Niemi, G. J. and Bradbury, S. P. (1998). A comparative study of molecular similarity, statistical, and neural network methods for predicting toxic modes of action of chemicals. Environ. Toxicol. Chem., 17, 1056-1064.

[19] Sato, A. and Nakajima, T. (1979). Partition coefficients of some aromatic hydrocarbons and ketones in water, blood and oil. Br. J. Ind. Med., 36, 231-234.

[20] Fiserova-Bergerova, V., Tichy, M. and DiCarlo, F. J. (1984). Effects of biosolubility on pulmonary uptake and disposition of gases and vapors for lipophilic chemicals. Drug Metab. Rev., 15, 1033-1070. 
[21] Basak, S. C., Harriss, D. K. and Magnuson, V. R. (1988). POLLY, Version 2.3, Copyright of the University of Minnesota.

[22] Filip, P. A., Balaban, T. S. and Balaban, A. T. (1987). A new approach for devising local graph invariants: Derived topological indices with low degeneracy and good correlational ability. J. Math. Chem., 1, 61-83.

[23] Basak, S. C., Balaban, A. T., Grunwald, G. D. and Gute, B. D. (2000). Topological indices: Their nature and mutual relatedness. J. Chem. Inf. Comput. Sci., 40, 891-898.

[24] Hall Associates Consulting, Molconn-Z Version 3.50, Quincy, MA, 2000.

[25] Kier, L. B. and Hall, L. H. (1986). Molecular Connectivity in Structure-Activity Analysis, Letchworth, Hertfordshire, U.K., Research Studies Press.

[26] Kier, L. B., Murray, W. J., Randic, M. and Hall, L. H. (1976). Molecular connectivity. V. Connectivity series concept applied to diversity. J. Pharm. Sci., 65, 1226-1230.

[27] Randic, M. (1975). On characterization of molecular branching. J. Am. Chem. Soc., 97, 6609-6615.

[28] Basak, S. C., Magnuson, V. R., Niemi, G. J. and Regal, R. R. (1988). Determining structural similarity of chemicals using graph-theoretic indices. Discrete Appl. Math., 19, 17-44.

[29] Balaban, A. T. (1982). Highly discriminating distance-based topological indices. Chem. Phys. Lett., 89, 399404.

[30] Balaban, A. T. (1983). Topological indices based on topological distances in molecular graphs. Pure and Appl. Chem., 55, 199-206.

[31] Balaban, A. T. (1985). Chemical graphs. Part 48. Topological index J for heteroatom-containing molecules taking into account periodicities of element properties. Math. Chem. (MATCH), 21, 115-122.

[32] Raychaudhury, C., Ray, S. K., Ghosh, J. J., Roy, A. B. and Basak, S. C. (1984). Discrimination of isomeric structures using information theoretic topological indices. J. Comput. Chem., 5, 581-588.

[33] Basak, S. C. (1999). Information theoretic indices of neighborhood complexity and their applications. In, Topological Indices and Related Descriptors in QSAR and QSPR (J. Devillers and A.T. Balaban, Eds.). Gordon and Breach Science Publishers, The Netherlands, pp. 563-593.

[34] Kier, L. B. and Hall, L. H. (1999). Molecular Structure Description: The Electrotopological State, San Diego, CA, Academic Press.

[35] Hall, L. H., Mohney, B. and Kier, L. B. (1991). The electrotopological state: Structure information at the atomic level for molecular graphs. J. Chem. Inf. Comput. Sci., 31, 76-82.

[36] Basak, S. C. (1988). H-Bond, Copyright of the University of Minnesota.

[37] Parham, M., Hall, L. H. and Kier, L. B. (2000). LogP. http://www.logP.com.

[38] SAS Institute, Inc. In SAS/STAT User Guide, Release 6.03 Edition, Cary, NC, 1988.

[39] Hoerl, A. E. and Kennard, R. W. (1970). Ridge regression: Biased estimation for nonorthogonal problems. Technometrics, 8, 27-51.

[40] Massy, W. F. (1965). Principal components regression in exploratory statistical research. J. Am. Statistical Assoc., 60, 234-246.

[41] Hoskuldsson, A. (1988). PLS regression methods. J. Chemometrics, 2, 211-228.

[42] Hoskuldsson, A. (1995). A combined theory for PCA and PLS. J. Chemometrics, 9, 91-123.

[43] Wold, H. (1975). Soft modeling by latent variables: The nonlinear iterative partial least squares approach. In, Perspectives in Probability and Statistics, Papers in Honor of M. S. Bartlett (J. Gani, Ed.). Academic Press, London.

[44] Miller, A. J. (1990). Subset selection in regression, New York, Chapman and Hall.

[45] Rencher, A. C. and Pun, F. C. (1980). Inflation of R2 in best subset regression. Technometrics, 22, 49-53.

[46] Hawkins, D., Basak, S. and Shi, X. (2001). QSAR with few compounds and many features. J. Chem. Inf. Comput. Sci., 41, 663-670.

[47] Basak, S. C., El-Masri, H., Hawkins, D. M. and Mills, D. (2001). Exposure assessment of volatile organic chemicals (VOCs): Predicting blood:air partition coefficients of diverse chemicals using theoretical descriptors. J. Chem. Inf. Comput. Sci., submitted.

[48] Basak, S., Mills, D., Hawkins, D. M. and El-Masri, H. (2002). Prediction of human blood:air partition coefficient: A comparison of structure-based and property-based methods. Risk Analysis, submitted.

[49] Kamlet, M. J., Abboud, J.-L. M., Abraham, M. H. and Taft, R. W. (1983). Linear solvation energy relationships. 23. A comprehensive collection of the solvatochromatic parameters, $\pi^{*}, \alpha$ and $\beta$, and some methods for simplifying the general solvatochromatic equation. J. Org. Chem., 48, 2877-2887. 
H.A. EL-MASRI

[50] Basak, S. C., Niemi, G. J. and Veith, G. D. (1990). Optimal characterization of structure for prediction of properties. J. Math. Chem., 4, 185-205.

[51] Basak, S. C., Niemi, G. J. and Veith, G. D. (1990). Recent developments in the characterization of chemical structure using graph-theoretic indices. In, Computational Chemical Graph Theory (D.H. Rouvray, Ed.). pp. 235-277. 


\title{
EXPOSURE ASSESSMENT OF VOLATILE ORGANIC CHEMICALS (VOCS): PREDICTING BLOOD:AIR PARTITION COEFFICIENTS OF DIVERSE CHEMICALS USING THEORETICAL DESCRIPTORS
}

\author{
S. C. BASAK, ${ }^{A}$ H. EL-MASRI, ${ }^{\mathrm{B}}$ D. M. HAWKINS, ${ }^{\mathrm{C}}$ and D. MILLS ${ }^{\mathrm{A}}$ \\ ${ }^{a}$ Natural Resources Research Institute, University of Minnesota Duluth \\ 5013 Miller Trunk Highway, Duluth, MN 55811, USA \\ ${ }^{b}$ Computational Toxicology Laboratory, Division of Toxicology Agency for Toxic Substances and Disease Registry \\ (ATSDR), Executive Park Building 4, 1600 Clifton Road, E-29, Atlanta, GA 30333, USA \\ ${ }^{c}$ School of Statistics, 313 Ford Hall, 224 Church Street S. E., University of Minnesota Minneapolis, Minnesota \\ 55455, USA
}

\begin{abstract}
Three regression methods, namely ridge regression (RR), partial least squares (PLS), and principal components regression (PCR), were used to develop models for the prediction of rat blood:air partition coefficient for increasingly diverse data sets. Initially, modeling was performed for a set of 13 chlorocarbons. To this set, 10 additional hydrophobic compounds were added, including aromatic and non-aromatic hydrocarbons. A set of 16 . hydrophilic compounds was also modeled. Finally, all 39 compounds were combined into one data set for which comprehensive models were developed. A large set of diverse, theoretical molecular descriptors, including topostructural (TS), topochemical (TC), and geometrical or 3-dimensional (3D) indices were used both hierarchically and independently in model development. In most cases, RR outperformed PLS and PCR, and the models developed using TC indices were superior to those developed using other combinations of descriptors.
\end{abstract}

\section{INTRODUCTION}

The quality of modern life is dependent upon the use of a multitude of man-made and natural chemicals. Collectively, we are exposed to thousands of these chemicals from their routine use as well as accidental spill to the environment. Risk assessment (RA) of chemicals is an important activity pertinent to sustainable human and ecological health. Important aspects of RA are: a) hazard identification, b) dose-response assessment, c) exposure assessment, and d) risk characterization ${ }^{1}$.

Health risk assessment of human exposure to environmental chemicals is usually based on experimental findings performed on test animals for single chemicals. Current assessment methods include two issues that are constantly debated in the scientific community. First, the use of these findings to estimate health risks to humans is routinely done by the use of default uncertainty factors, the purpose 
which is to extrapolate between experimental settings and real life situations among different doses, animal species and exposure scenarios. The acceptance of these uncertainty factors by the scientific community reflects the lack of understanding of physiological, and biochemical mechanisms underlying the toxicity of environmental chemicals. Secondly, in most cases, risks are only estimated for exposures to single chemicals in the environment. This also is an oversimplification of real world situations where people are exposed to multiple chemicals concurrently. Biologically based computational models can address both issues by linking tissue levels, pharmacokinetically, or mechanisms of actions, pharmacodynamically, with exposure levels. This linkage provides a numerical procedure which can be used to investigate the role of physiological (e.g., body weight, cardiac output), or biochemical factors (such as metabolism, or protein binding etc.) in determining risk estimates among different species, and exposure scenarios. Whenever possible, mechanisms of interactions, such as metabolic inhibitions or competition for protein binding sites, can also be introduced into the models to address the effect of combined chemicals exposure to human health.

Pharmacokinetics (PK) involves the study of the rates of absorption, distribution, excretion, and biotransformation of chemicals and their metabolites. PK models can be used to reconstruct extensive data sets based on a small number of kinetic parameters ${ }^{2}$. These models can be used to generate hypotheses, predict the results of new experiments, and integrate studies on kinetics, disposition and metabolism in various animal species ${ }^{3}$. In physiologically based pharmacokinetic (PBPK) models, compartments correspond more closely to actual anatomical structures, defined with respect to their volumes, blood flows, chemical binding (partitioning) characteristics, and the ability to metabolize or excrete the compounds of interest. Because the kinetic parameters of these models reflect tissue blood flows, and partitioning and other biochemical constants, these models are more readily scaled from one animal species to another ${ }^{4}$. Quantitative applications of PBPK models in risk assessment date from the development of a number of such models for methylene chloride in the mid $1980 \mathrm{~s}^{5}$. The use of PBPK models in toxicology research and chemical risk assessment situations today is primarily related to their ability to make more accurate predictions of target tissue dose for different exposure situations in different animal species, including humans. Such models constitute an important tool in improving the degree of accuracy of human health risk assessment for toxic substances in the environment ${ }^{6}$. One of the disadvantages of PBPK modeling is its heavy reliance on often experimentally determined parameters. However, advances in computational and mathematical chemistry can provide a methodology which in many cases is used to estimate model parameters such as tissue:air partition coefficients

Tissue-air partition coefficient values of volatile organic chemicals (VOCs) are used to estimate the uptake, distribution, metabolism, and elimination as well as potential hazard of xenobiotics to humans ${ }^{7}$. Gargas et al. ${ }^{7}$ experimentally determined various liquid solvent:air and tissue:air partition coefficients (P) 
for $.9 \%$ saline, olive oil, blood, liver, muscle, and fat tissue. Subsequently, they studied the relationship among the various partition coefficients using regression models. For example, $\mathrm{P}$ values for blood:air, liver:air and muscle:air pairs were found to be strongly corrrelated with P values for olive oil:air for various subsets of volatile organic chemicals (VOCs).

The blood:air partition coefficient (P, blood:air) is an important determinant of the pulmonary uptake of VOCs via the inhalation route. Solubility of VOCs in blood is primarily determined by two factors: a) tissue composition including the content of neutral lipid, phospholipid, and water, and b) the degree of binding of these chemicals to specific components of blood, e.g., hemoglobin, plasma proteins, etc. Such physiological considerations have been utilized by Poulin and Krishnan ${ }^{8}$ to estimate blood:air $\mathbf{P}$ values of different subsets of chemicals.

Attempts have been made by various authors to estimate PBPK parameters of chemicals from their physicochemical and calculated properties. de Jongh et al. ${ }^{9}$ estimated tissue: blood partition coefficients of a diverse set of chemicals from their octanol:water ( $\mathrm{P}$, octanol:water) partition coefficient. Poulin and Krishnan ${ }^{8}$, on the other hand, attempted to estimate tissue:air P values for hydrophobic and hydrophilic classes of chemicals separately. To this end, they developed an algorithm based the ratio of blood solubility to air solubility (saturable vapor concentration).

The estimation of various tissue:blood $\mathrm{P}$ values from other experimental properties (e.g. olive oil:water by Gargas et al. ${ }^{7}$ or calculated octanol:water P value by de Jongh et al. ${ }^{9}$ ) are examples of propertyproperty correlations where one property is used to estimate another property of interest. The basic paradigm of quantitative structure-activity relationship is that any property of a molecule can be estimated from the pertinent aspects of molecular structure which constitute the molecular and submolecular basis of that property ${ }^{10}$. Following that paradigm, attempts have been made to predict various properties, e.g., boiling point, ${ }^{11-17}$ vapor pressure, ${ }^{18-23} \log \mathrm{P}$ (octanol:water),${ }^{15,24-26}$ carcinogenicity, ${ }^{27,28}$ mutagenicity, ${ }^{28-37}$ blood-brain barrier transport, ${ }^{38}$ and skin penetration of chemicals ${ }^{38}$. Theoretical descriptors have been used to predict the so-called modes of actions (MOAs) of chemicals from their calculated theoretical descriptors. ${ }^{39}$ So, it is reasonable to attempt to estimate tissue:air P values of chemicals from their structure. Such an effort is also supported by the fact that calculated octanol:water $\mathrm{P}$ is primarily a structural parameter being calculated from fragment values of the molecule with some empirical corrections for structural peculiarities not accounted for by the fragmental constant ${ }^{40} 41$. The finding of de Jongh et al. ${ }^{9}$ that tissue:air $P$ can be estimated from octanol:water $P$ values is also an impetus for attempting to estimate blood:air P values directly from structural parameters.

In our previous studies, we first found a successful correlation between theoretical structural invariants and blood:air $P$ values for a congeneric set of chlorocarbons ${ }^{42}$. Subsequently, we added a few nonchlorocarbon hydrophobic chemicals to the set of chlorocarbons and found a satisfactory relationship 
Jetween the $\mathrm{P}$ values of the combined set and the calculated descriptors. A comparison of the models for the two data sets demonstrated that the best model for the combined set included parameters that reflected the structural diversity of the data set. This supports the belief that a diverse range of descriptors is required in order to model a diverse data set $^{43}$. In this paper, we have attempted to predict $\mathrm{P}$ (blood:air) values of a larger set of chemicals comprised of hydrophobic and hydrophilic molecules using a set of purely calculated molecular descriptors.

\section{METHODS}

2.1 Database. Rat blood:air partition coefficient data for set of 41 compounds was obtained from the literature ${ }^{7,44,45}$ by Poulin and Krishnan ${ }^{8}$, who divided the data into two subsets: a relatively hydrophobic set consisting of alkanes, haloalkanes, and aromatic hydrocarbons, and a set of relatively hydrophilic compounds made up of ketones, alcohols, acetate esters, and one ether. Four compounds included in the data set analyzed by Poulin and Krishnan are not included here, two of which were cis/trans isomers and therefore have identical values for theoretical descriptors based on SMILES input, and two for which it was not possible to calculate the entire set of descriptors used in the current study. The experimental blood:air partition coefficients are provided in Table 1.

2.2 Molecular Descriptors. The calculated molecular descriptors can be categorized into hierarchical groups based on level of complexity. At the lowest end of the hierarchy are the topostructural (TS) descriptors which encode information strictly on the adjacency and connectedness of atoms within a molecule. The topochemical (TC) descriptors also take into account the chemical nature of a molecule including bond type. The 3-dimensional or shape (3D) descriptors are even more complex, encoding information about the 3-dimensional aspects of a molecule. The topostructural and topochemical descriptors are known collectively as topological descriptors.

All descriptors used in the present study were derived strictly from molecular structure using software packages including POLLY, ${ }^{46}$ Triplet, ${ }^{47,48}$ and Molconn-Z. ${ }^{49}$ From POLLY, we obtain a set of topological descriptors, including a large group of connectivity indices, ${ }^{50-52}$ information theoretic ${ }^{53,54}$ and neighborhood complexity indices. ${ }^{54}$ The Triplet descriptors also constitute a large group of topological parameters. They are derived from a matrix, a main diagonal column vector, and a free term column vector, converting the matrix into a system of linear equations whose solutions are the local vertex invariants. These local vertex invariants are then used various mathematical operations in order to obtain the triplet descriptors. Molconn- $Z$ provides additional topological descriptors, including an extended set 
of connectivity indices, electrotopological indices, ${ }^{55,56}$ hydrogen bonding descriptors, as well as a small number of molecular shape descriptors.

For the analysis of one of the data sets analyzed in this study, namely the set of chlorocarbons, three additional 3-dimensional descriptors were obtained from Sybyl. ${ }^{57}$ These descriptors were not available for use at the time that the remaining data sets were analyzed. Also unique to the chlorocarbon analysis was a descriptor representing the number of chloride atoms.

A brief description of the complete set of molecular descriptors used in the current study is provided in Table 2.

2.3 Statistical Analysis. Prior to performing regression analysis, the descriptors were scaled by the natural logarithm as their scales differed by several orders of magnitude. The values of the dependent variable differed by more than one order of magnitude and therefore were also log scaled. The original set of independent variables was reduced by eliminating: a) descriptors which had a value of 0 for the entire set of compounds, b) descriptors which could not be calculated for all compounds in the data set, and c) all but one of a given set of perfectly correlated descriptors, i.e. $r=1.0$, as determined by the CORR procedure of the SAS statistical package. ${ }^{58}$ This procedure was performed independently for each of the following analyses:

1. A set of 13 chlorocarbons (a subset of the $\mathrm{N}=24$ hydrophobic data set)

2. A set of 24 hydrophobic compounds

3. A set of 17 hydrophilic compounds

4. A set of 41 hydrophobic + hydrophilic compounds

Ridge regression (RR), ${ }^{59}$ principal components regression (PCR) ${ }^{60}$, and partial least squares (PLS) regression ${ }^{61-63}$ models were developed using molecular descriptors in a hierarchical fashion. In addition, each class of descriptors was used independently to obtain single-class models. Each of these regression methods is useful in studies wherein the number of descriptors is much greater than the number of observations, as well as in cases where the independent variables are highly intercorrelated; and they make use of all independent variables as opposed to subset regression wherein it is possible that important parameters may be eliminated. Statistical parameters reported include the cross-validated $\mathrm{R}^{2}$ value, which is obtained by removing each compound in turn from the data set and fitting the regression to the remaining n-1 compounds, and the PRESS statistic which is a reliable measure of model predictability. In addition, the $t$ values can be examined in order to identify significant descriptors. Although a descriptor 
with a large $|t|$ indicates that the associated descriptor is important in the model, it should be cautioned that the reverse is not necessarily so.

\section{RESULTS}

A summary of the regression results for the various data sets analyzed in the present study are provided in Tables 3-6. The three statistical methods, viz., PCR, PLS and RR, were used for model development. Three classes of calculated descriptors, viz., topostructural (TS) indices, topochemical (TC) indices, and geometrical or 3-dimensional (3D) molecular descriptors, were used either alone or in various combinations for model building (Tables 3-6). Of the three statistical techniques, the RR method generally gave superior $\mathrm{R}_{\text {c.v. }}^{2}$ and PRESS values as compared to those derived by PCR or PLS methods. In addition, models derived either from TC descriptors alone or TS+TC descriptors were superior to those involving other combinations of descriptor classes. In some of our earlier studies involving the development of QSARs using TS, TC, 3D and quantum chemical (QC) indices, we found that the TS+TC combination explained most of the variance in the data and the addition of $3 \mathrm{D}$ or QC indices made only marginal or no improvement in the model quality. ${ }^{18,22,23,34,35,37,64-66} \mathrm{~A}$ reasonable model with a crossvalidated $R^{2}$ value 0.866 and a PRESS of 1.61 was obtained for the set of 13 chlorocarbons ${ }^{42}$, which may not be surprising in light of the homogenous nature of the data set (Table 3 ). When additional hydrophobic compounds are added to this set, we find that cyclohexane is an influential outlier. Cyclohexane is the only cyclic non-aromatic in the data set. With this compound removed, a crossvalidated $R^{2}$ of 0.873 is obtained with a PRESS of 2.90 (Table 4). The set of hydrophilic compounds is also well modeled, with a cross-validated $R^{2}$ of 0.964 and a PRESS of 0.92 . In this analysis, diethyl ether was found to be an outlier, which is not surprising since this compound is the only ether in the set (Table 5). A significant finding is the fact that when the data set is further diversified by combining the hydrophobic and hydrophilic compounds, an acceptable model is obtained, with a cross-validated $\mathrm{R}^{2}$ of 0.944 and a PRESS of 5.57. In the combined data set, we find that cyclohexane and bromochloromethane are influential outliers (Table 6).

For each of the four data sets analyzed, the descriptors in the TC ridge regression model were ordered according to their respective $|t|$ values. Approximately, the top $10 \%$ of the descriptors, based on $|t|$ values, are listed in Tables 7-10 for the four sets of chemicals for which models are developed in Tables 3-6, viz:, chlorocarbons, hydrophobic chemicals, hydrophilic chemicals, and the union of all three sets, along with the descriptor coefficient, standard error, and $t$ value. 
Table 11 provides the predicted $\log \mathrm{P}$ (blood:air) values and residuals for the $\mathrm{TC}$ ridge regression model developed for the combined set of hydrophobic and hydrophilic descriptors. The predicted values and residuals obtained by the solubility algorithm of Poulin and Krishnan ${ }^{8}$ are included for comparative purposes. A scatterplot of the experimental ws the predicted values given in Table 11 is shown in Figure 1.

\section{DISCUSSION}

The question we wanted to investigate in this study was three fold: a) Can we use theoretical structural descriptors instead of experimental physicochemical data to predict properties relevant to PBPK

modelling, b) Is it possible to predict relevant properties of not only congeneric sets, but also structurally diverse collections of molecules, and c) Can we interpret the resulting QSAR models for the congeneric vis-a-vis diverse sets in order to understand the basis of the roles of various types of descriptors in the predictive models?

Regarding the first point above, it is clear from results presented in Tables 3-6 that either the topochemical indices alone or the combination of $\mathrm{TS}+\mathrm{TC}$ indices gave reasonable regression equations for chlorocarbons, hydrophobics (chlorocarbons plus hydrocarbons), hydrophilics, and finally, a combined set of hydrophobic and hydrophilic compounds. There were some outliers which influenced the results for the smaller subsets as well as the final combined set. Such compounds, namely diethyl ether, cyclohexane and bromochloromethane, are but lone examples of their respective structural classes, which is probably the reason why the models could not account for them. It is interesting to note that of the three methods of modeling attempted, viz., PCR, PLS and RR, the ridge regression method gave the best predictive models in most cases. In an earlier paper, ${ }^{67}$ we showed with four different sets of physicochemical and toxicological properties that RR is superior to multiple linear regression (MLR) or PLS in the development of QSAR models.

There is some degradation of the PRESS statistic, an important measure of model quality, when we compare the class-specific models (Tables $4 \& 5$ ) with those derived from the combined set (Table 6 ) using the RR method. However, the difference between the measure for the combined set and the sum of those for the individual sets is not as large as one might expect, and the combined model is a reasonable one. This may be due to the large number of descriptors utilized, capable of characterizing many different structural features. Table 11 gives results of the relative effectiveness of our current approach, using the TC model developed with the ridge regression method for the combined set of hydrophobic and hydrophilic compounds, vis-a-vis a physicochemically-based approach derived by Poulin and Krishnan ${ }^{8}$ 
from mechanistic considerations in the prediction of blood:air partition coefficients of the VOCs analyzed in this paper.

It is instructive to look at the most important descriptors (in terms of $|t|$ values in the RR models) for the various subsets of chemicals. Some important indices for the subset of chlorocarbons are: the triplet indices $\mathrm{AZV}_{3}$ and $\mathrm{AZS}_{4}, \mathrm{NoCl}$ (the number of chlorine atoms), E-state indices encoding information about the electronic states of atoms (SHCsats and SsssCH), and both first and third order connectivity indices, the latter of which represents third order cluster subgraphs occurring frequently in the hydrogensuppressed graphs of the halocarbons.

The addition of the aromatic and nonaromatic hydrocarbons to the set of chlorocarbons results in an interesting change. It is clear from Table 8 that parameters such as SHarom (E-state aromatic indices) and sixth order cyclic connectivity index ${ }^{68}$ have become important in the regression equation. This is clearly a shift towards aromaticity and cyclicity, which are inherent structural features of the subset of compounds added to the group of chlorocarbons.

The predictors important for the hydrophilic subset (Table 9) show the importance of hydrogen bonding (SHHBd, numHBd) parameters, as well as indices related to atomic heterogeneity and symmetry, viz., nclass, SHCsatu, and molecular weight (representing molecular size). Kamlet et al. ${ }^{69}$ showed in their research with linear solvated energy related studies that molecular size, dipolarity, and hydrogen bonding are three important determinants of properties including water solubility, partition coefficients, etc.

Finally, in the RR model developed from the mixed set of chlorocarbons, aromatics and hydrophilics, we find that the following factors are important: a) Molecular size, encoded by zero order connectivity index $\left({ }^{0} \chi^{v}\right),{ }^{50}$ b) Hydrogen bonding represented by numHBd, SHHBd, SHHBa, and $\mathrm{HB}_{1}$, and c) Polarity (Qv). Results derived from the combined set clearly vindicate the proposition of Kamlet et al. ${ }^{69}$ that molecular size, dipolarity/polarizability, hydrogen bond donor acidity, and hydrogen bond acceptor basicity are the major determining factors for partitioning properties such as hydrophobicity. The importance of structural factors such as ${ }^{0} \chi^{\mathrm{v}}$ and van der Waals' volume (encoding molecular size), $\mathrm{IC}_{0}$, $\mathrm{CIC}_{1}, \mathrm{CIC}_{2}$, and $\mathrm{CIC}_{3}$ (representing atomic neighborhood heterogeneity), and $\mathrm{HB}_{1}$ (representing hydrogen bonding) in predicting octanol-water partition coefficient ( $\log \mathrm{P}$, octanol-water) was also evident in our previous studies of smaller subsets of $219^{15}, 139$ and 382 compounds $^{70,71}$, as well as larger diverse $\log \mathrm{P}$ sets of 4,111 and 4,000 chemicals. $^{24}$

In conclusion, results presented in this paper show that data relevant to toxicological and PBPK modeling of VOCs can be more accurately predicted using RR method and calculated molecular descriptors than by using the mechanistic approaches based on physicochemical and biological considerations. The wide spectrum of molecular descriptors considered in this paper afforded us the ability to model not only the particular structural types but also the combined set of different structural 
asses of chemicals without significant degradation in the model quality as compared to class-specific QSARs. Inspection of the parameters that play important roles in class-specific QSARs as well as the corresponding models for the combined set showed that parameters assuming important roles in the different predictive models reflect the nature and diversity of the chemicals under consideration. Further studies on the comparative QSARs of congeneric vis-a-vis structurally diverse sets using theoretical structural indices are needed to validate the utility of the approach outlined in this paper.

\section{ACKNOWLEDGEMENT}

This is contribution number XXX from the Center for Water and the Environment of the Natural Resources Research Institute. Research reported in this paper was supported in part by Grant F49620-010098 from the United States Airforce.

\section{REFERENCES AND NOTES}

(1) Becking, G. C. Use of mechanistic information in risk assessment for toxic chemicals. Toxicology Letters 1995, $77,15-24$.

(2) Andersen, M. E. Development of physiologically based pharmacokinetic and physiologically based pharmacodynamic models for applications in toxicology and risk assessment. Toxicol. Lett. 1995, 79, 35-44.

(3) Wagner, J. G. History of pharmacokinetics. Pharmacol. Ther. 1981, 12, 537-562.

(4) Dedrick, R. L. Animal scale-up. J. Pharmacokinet. Biopharm. 1973, 1, 435-461.

(5) Andersen, M. E.; Clewell, H. J., III; Gargas, M. L.; Smith, F. A.; Reitz, R. H. Physiologically based pharmacokinetics and the risk assessment process for methylene chloride. Toxicol. Appl. Pharmacol. 1987, 87, 185-205.

(6) Yang, R. S. H.; Thomas, R. S.; Gustafson, D. L.; Campain, J.; Benjamin, S. A.; Verhaar, H. J. M.; Mumtaz, M. M. Approaches to developing alternative and predictive toxicology based on PBPK/PD and QSAR modeling. Environ. Health Perspect. 1998, 106, 1385-1393.

(7) Gargas, M. L.; Burgess, R. J.; Voisard, D. E.; Cason, G. H.; Andersen, M. E. Partition coefficients of low molecular weight volatile chemicals in various tissues and liquids. Toxicol. Appl. Pharmacol. 1989, 98, 87-99.

(8) Poulin, P.; Krishnan, K. A mechanistic algorithm for predicting blood:air partition coefficients of organic chemicals with the consideration of reversible binding in hemoglobin. Toxicol. Appl. Pharmacol. 1996, 136, 131-137.

(9) de Jongh, J.; Verhaar, H. J. M.; Hermans, J. L. M. A quantitative structure-activity relationship approach to estimate in vitro tissue-blood partition coefficients of organic chemicals in rats and humans. Arch. Toxicol. 1997, 72, 17-25.

(10)Basak, S. C. Use of molecular complexity indices in predictive pharmacology and toxicology: A QSAR approach. Med. Sci. Res. 1987, 15, 605-609.

(11)Balaban, A. T.; Basak, S. C.; Colburn, T.; Grunwald, G. D. Correlation between structure and normal boiling points of haloalkanes C1-C4 using neural networks. J. Chem. Inf. Comput. Sci. 1994, 34, 1118-1121.

(12) Balaban, A. T.; Mills, D.; Basak, S. C. Correlation between structure and normal boiling points of acyclic carbonyl compounds. J. Chem. Inf. Comput. Sci. 1999, 39, 758-764.

(13) Basak, S. C.; Grunwald, G. D. A comparative study of graph invariants, total surface area, and volume in predicting boiling points of alkanes. Mathl. Modelling Sci. Computing 1993, 2, 735-740.

(14) Basak, S. C.; Gute, B. D.; Grunwald, G. D. Estimation of normal boiling points of haloalkanes using molecular similarity. Croat. Chim. Acta 1996, 69, 1159-1173.

(15) Basak, S. C.; Gute, B. D.; Grunwald, G. D. A comparative study of topological and geometrical parameters in estimating normal boiling point and octanol-water partition coefficient. J. Chem. Inf. Comput. Sci. 1996, 36, 1054-1060. 
(16) Hall, L. H.; Story, C. T. Boiling point of a set of alkanes, alcohols and chloroalkanes: QSAR with atom type electrotopological states indices using artificial neural networks. SAR QSAR Environ.Res. 1997, 6 139-161.

(17) Randic, M.; Basak, S. C. Construction of high-quality structure-property-activity regressions: The boiling points of sulfides. J. Chem. Inf. Comput. Sci. 2000, 40, 899-905.

(18) Basak, S. C.; Gute, B. D.; Grunwald, G. D. Use of topostructural, topochemical, and geometric parameters in the prediction of vapor pressure: A hierarchical approach. J. Chem. Inf. Comput. Sci. 1997, 37, 651-655.

(19) Goll, E. S.; Jurs, P. C. Prediction of Vapor Pressures of Hydrocarbons and Halohydrocarbons from Molecular Structure with a Computational Neural Network Model. J. Chem. Inf. Comput. Sci. 1999, 39, 1081-1089.

(20)Katritzky, A. R.; Wang, Y.; Sild, S.; Tamm, T. QSPR Studies on Vapor Pressure, Aqueous Solubility, and the Prediction of Water-Air Partition Coefficients. J. Chem. Inf. Comput. Sci. 1998, 38, 720-725.

(21) Liang, C.; Gallagher, D. A. QSPR Prediction of Vapor Pressure from Solely Theoretically-Derived Descriptors. J. Chem. Inf. Comput. Sci. 1998, 38, 321-324.

(22)McClelland, H. E.; Jurs, P. C. Quantitative Structure-Property Relationships for the Prediction of Vapor Pressures of Organic Compounds from Molecular Structures. J. Chem. Inf. Comput. Sci. 2000, 40, 967-975.

(23)Basak, S. C.; Mills, D. Quantitative structure-property relationships (QSPRs) for the estimation of vapor pressure: A hierarchical approach using mathematical structural descriptors. J. Chem. Inf. Comput. Sci. 2001, 41 , in press.

(24)Niemi, G. J.; Basak, S. C.; Veith, G. D.; Grunwald, G. Prediction of octanol-water partition coefficient (Kow) using algorithmically-derived variables. Environ. Toxicol. Chem. 1992, 11, 891-898.

(25)Klopman, G.; Wang, S. A computer automated structure evaluation (CASE) approach to calculation of partition coefficient. J. Comput. Chem. 1991, 12, 1025-1032.

(26)Kier, L. B.; Hall, L. H. Molecular Connectivity in Chemistry and Drug Research; Academic Press: New York, 1976.

(27) Arcos, J. C. Structure-activity relationships: criteria for predicting carcinogenic activity of chemical compounds. Environ. Sci. Technol. 1987, 21, 743-745.

(28) Benigni, R.; Giuliani, A. Quantitative structure-activity relationships (QSAR) of mutagens and carcinogens. Med. Res. Rev. 1996, 16, 267-.

(29) Benigni, R.; Andreoli, C.; Giuliani, A. QSAR models for both mutagenic potency and activity: Application to nitroarenes and aromatic amines. Environ. Mol. Mutagen. 1994, 24, 208-219.

(30)Cash, G. G. Prediction of the Genotoxicity of Aromatic and Heteroaromatic Amines Using Electrotopological State Indices. Mutation Res. Genet. Toxicol. Environ. Mutagen. 2001, 491, 31-37.

(31)Basak, S. C.; Frane, C. M.; Rosen, M. E.; Magnuson, V. R. Molecular topology and mutagenicity: A QSAR study of nitrosamines. IRCS Med. Sci. 1986, 14, 848-849.

(32) Basak, S. C.; Grunwald, G. D. Predicting mutagenicity of chemicals using topological and quantum chemical parameters: A similarity based study. Chemosphere 1995, 31, 2529-2546.

(33) Basak, S. C.; Gute, B. D.; Grunwald, G. D. Relative effectiveness of topological, geometrical, and quantum chemical parameters in estimating mutagenicity of chemicals. In Quantitative Structure-activity Relationships in Environmental Sciences VII; Chen, F., Schuurmann, G., Eds.; SETAC Press: Pensacola, FL, 1998, pp 245261.

(34)Basak, S. C.; Gute, B. D.; Grunwald, G. D. Assessment of mutagenicity of chemicals from theoretical structural parameters: A hierarchical approach. SAR QSAR Environ. Res. 1999, 10, 117-129.

(35)Basak, S. C.; Mills, D. Prediction of mutagenicity utilizing a hierarchical approach. SAR QSAR Environ. Res. 2001, submitted.

(36) Kier, L. B.; Simons, R. J.; Hall, L. H. Structure activity studies on mutagenicity of nitrosamines using molecular connectivity. J. Pharm. Sci. 1978, 67, 725-726.

(37) Basak, S. C.; Mills, D. R.; Balaban, A. T.; Gute, B. D. Prediction of mutagenicity of aromatic and heteroaromatic amines from structure: A hierarchical QSAR approach. J. Chem. Inf. Comput. Sci. 2001, 41, 671-678.

(38)Basak, S. C.; Gute, B. D.; Drewes, L. R. Predicting blood-brain transport of drugs: A computational approach. Pharm. Res. 1996, 13, 775-778.

(39) Basak, S. C.; Grunwald, G. D.; Host, G. E.; Niemi, G. J.; Bradbury, S. P. A comparative study of molecular similarity, statistical, and neural network methods for predicting toxic modes of action of chemicals. Environ. Toxicol. Chem. 1998, 17, 1056-1064.

(40) Hansch, C.; Leo, A. Exploring QSAR: Fundamentals and applications in Chemistry and Biology; American Chemical Society: Washington, D.C., 1995. 
(41) Franke, R.; Huebel, S.; Streich, W. J. Substructural QSAR approaches and topological phamacophores. Environ. Health Perspect. 1985, 61, 239-255.

(42)Basak, S. C.; Hawkins, D. M.; Mills, D. Estimation of blood:air partition coefficient of chloroalkanes using QSPRs based on theoretical structural descriptors. Croat. Chim. Acta 2001, submitted.

(43)Basak, S. C.; Mills, D.; Gute, B. D.; Grunwald, G. D.; Balaban, A. T. Applications of topological indices in predicting property/bioactivity/toxicity of chemicals. In Topology in Chemistry, 2001, in press.

(44) Kaneko, T.; Wang, P. Y.; Sato, A. Partition coefficients of some acetate esters and alcohols in water, blood, olive oil and tissues. Occup. Environ. Med. 1994, 51, 68-72.

(45)Madani, R.; Tardif, R.; Brodeur, J.; Krishnan, K. Estimation of rat tissue:blood partition coefficients for some aliphatic monoketonic solvents. 1996.

(46)Basak, S. C.; Harriss, D. K.; Magnuson, V. R. POLLY V 2.3, Copyright of the University of Minnesota, 1988.

(47)Filip, P. A.; Balaban, T. S.; Balaban, A. T. A new approach for devising local graph invariants: Derived topological indices with low degeneracy and good correlational ability. J. Math. Chem. 1987, 1, 61-83.

(48) Basak, S. C.; Balaban, A. T.; Grunwald, G. D.; Gute, B. D. Topological indices: Their nature and mutual relatedness. J. Chem. Inf. Comput. Sci. 2000, 40, 891-898.

(49) Molconn-Z V. 3.50, Hall Associates Consulting, Quincy, MA, 2000.

(50) Kier, L. B.; Hall, L. H. Molecular Connectivity in Structure-Activity Analysis; Research Studies Press: Letchworth, Hertfordshire, U.K., 1986.

(51)Kier, L. B.; Murray, W. J.; Randic, M.; Hall, L. H. Molecular connectivity. V. Connectivity series concept applied to diversity. J. Pharm. Sci. 1976, 65, 1226-1230.

(52)Randic, M. On characterization of molecular branching. J. Am. Chem. Soc. 1975, 97, 6609-6615.

(53) Raychaudhury, C.; Ray, S. K.; Ghosh, J. J.; Roy, A. B.; Basak, S. C. Discrimination of isomeric structures using information theoretic topological indices. J. Comput. Chem. 1984, 5, 581-588.

(54)Basak, S. C. Information theoretic indices of neighborhood complexity and their applications. In Topological Indices and Related Descriptors in QSAR and QSPR; Devillers, J., Balaban, A. T., Eds.; Gordon and Breach Science Publishers: The Netherlands, 1999, pp 563-593.

(55)Kier, L. B.; Hall, L. H. Molecular Structure Description: The Electrotopological State; Academic Press: San Diego, CA, 1999.

(56) Hall, L. H.; Mohney, B.; Kier, L. B. The electrotopological state: Structure information at the atomic level for molecular graphs. J. Chem. Inf. Comput. Sci. 1991, 31, 76-82.

(57) Tripos Associates, SYBYL, V. 6.2, St. Louis, MO, 1994.

(58) SAS Institute, Inc. SAS/STAT User's Guide, Release 6.03 Edition, Cary, NC., 1988.

(59)Hoerl, A. E.; Kennard, R. W. Ridge regression: Biased estimation for nonorthogonal problems. Technometrics $1970,8,27-51$.

(60) Massy, W. F. Principal components regression in exploratory statistical research. J. Am. Statistical Assoc. 1965, $60,234-246$.

(61) Hoskuldsson, A. A combined theory for PCA and PLS. J. Chemometrics 1988, 2, 211-228.

(62) Hoskuldsson, A. A combined theory for PCA and PLS. J. Chemometrics 1995, 9, 91-123.

(63) Wold, H. Soft modeling by latent variables: The nonlinear iterative partial least squares approach. In Perspectives in Probability and Statistics, Papers in Honor of M. S. Bartlett; Gani, J., Ed.; Academic Press: London, 1975.

(64) Gute, B. D.; Basak, S. C. Predicting acute toxicity of benzene derivatives using theoretical molecular descriptors: a hierarchical QSAR approach. SAR QSAR Environ. Res. 1997, 7, 117-131.

(65) Gute, B. D.; Grunwald, G. D.; Basak, S. C. Prediction of the dermal penetration of polycyclic aromatic hydrocarbons (PAHs): A hierarchical QSAR approach. SAR QSAR Environ. Res. 1999, 10, 1-15.

(66)Basak, S. C.; Gute, B. D.; Grunwald, G. D. A hierarchical approach to the development of QSAR models using topological, geometrical, and quantum chemical parameters. In Topological Indices and Related Descriptors in QSAR and QSPR; Devillers, J., Balaban, A. T., Eds.; Gordon and Breach Science Publishers: The Netherlands, 1999, pp 675-696.

(67)Hawkins, D.; Basak, S.; Shi, X. QSAR with few compounds and many features. J. Chem. Inf. Comput. Sci. 2001, 41, 663-670.

(68)Basak, S. C.; Magnuson, V. R.; Niemi, G. J.; Regal, R. R. Determining structural similarity of chemicals using graph-theoretic indices. Discrete Appl. Math. 1988, 19, 17-44.

(69)Kamlet, M. J.; Abboud, J.-L. M.; Abraham, M. H.; Taft, R. W. Linear solvation energy relationships. 23. A comprehensive collection of the solvatochromatic parameters, $\pi^{*}, \alpha$ and $\beta$, and some methods for simplifying the general solvatochromatic equation. J. Org. Chem. 1983, 48, 2877-2887. 
(70)Basak, S. C.; Niemi, G. J.; Veith, G. D. A graph-theoretic approach to predicting molecular properties. Mathl. Comput. Modelling 1990, 14, 511-516.

(71)Basak, S. C.; Niemi, G. J.; Veith, G. D. Recent developments in the characterization of chemical structure using graph-theoretic indices. In Computational Chemical Graph Theory; Rouvray, D. H., Ed., 1990, pp 235-277. 
Table 1. Experimental rat blood:air partition coefficients (P, blood:air)

\begin{tabular}{|c|c|}
\hline Chemicals & Experimental $P^{a}$ \\
\hline \multicolumn{2}{|l|}{ Alkanes ${ }^{b}$} \\
\hline 1. $n$-Hexane & $2.29 \pm 0.11$ \\
\hline 2. Cyclohexane & $1.39 \pm 0.09$ \\
\hline 3. n-Heptane & $4.75 \pm 0.15$ \\
\hline \multicolumn{2}{|l|}{ Haloalkanes $^{\mathrm{b}^{\mathrm{T}}}$} \\
\hline 4. Dichloromethane & $19.4 \pm 0.8$ \\
\hline 5. Chloroform & $20.8 \pm 0.1$ \\
\hline 6. Carbon tetrachloride & $4.52 \pm 0.35$ \\
\hline 7. Bromochloromethane & $41.5 \pm 0.9$ \\
\hline 8. 1,1-Dichloroethane & $11.2 \pm 0.1$ \\
\hline 9. 1,2-Dichloroethane & $30.4 \pm 1.2$ \\
\hline 10. 1,1,1-Trichloroethane & $5.76 \pm 0.5$ \\
\hline 11. 1,1,2-Trichloroethane & $58.0 \pm 1.1$ \\
\hline 12. 1,1,1,2-Tetrachloroethane & $41.7 \pm 1.0$ \\
\hline 13. 1,1,2,2,-Tetrachloroethane & $142 \pm 6$ \\
\hline 14. Pentachloroethane & $104 \pm 3$ \\
\hline 15. 1,2-Dichloropropane & $18.7 \pm 0.5$ \\
\hline 16. Trichloroethylene & $21.9 \pm 1.4$ \\
\hline 17. Tetrachloroethylene & $18.9 \pm 1.1$ \\
\hline \multicolumn{2}{|l|}{ Aromatic hydrocarbons $\mathrm{b}^{\mathrm{b}}$} \\
\hline 18. Benzene & $17.8 \pm 0.3$ \\
\hline 19. Chlorobenzene & $59.4 \pm 1.0$ \\
\hline 20. Toluene & $18.0 \pm 1.0$ \\
\hline 21. Styrene & $40.2 \pm 3.7$ \\
\hline 22. $o$-Xylene & $44.3 \pm 2.0$ \\
\hline 23. $m$-Xylene & $46.0 \pm 1.5$ \\
\hline 24. $p$-Xylene & $41.3 \pm 3.5$ \\
\hline \multicolumn{2}{|l|}{ Ketones $^{c}$} \\
\hline 25. Dimethyl ketone & $208 \pm 4$ \\
\hline 26. Methyl ethyl ketone & $191 \pm 2$ \\
\hline 27. Methyl propyl ketone & $127 \pm 3$ \\
\hline 28. Methyl isobutyl ketone & $79 \pm 6$ \\
\hline 29. Methyl pentyl ketone & $225 \pm 30$ \\
\hline \multicolumn{2}{|l|}{ Alcohols $^{\mathrm{c}}$} \\
\hline 30. Ethyl alcohol & $2140 \pm 80$ \\
\hline 31. $n$-Propyl alcohol & $1340 \pm 24$ \\
\hline 32. Isopropyl alcohol & $1290 \pm 21$ \\
\hline 33. $n$-Butyl alcohol & $1160 \pm 17$ \\
\hline 34. $n$-Pentyl alcohol & $829 \pm 25$ \\
\hline \multicolumn{2}{|l|}{ Acetate esters ${ }^{\mathrm{c}}$} \\
\hline 35. Methyl acetate ester & $100 \pm 2$ \\
\hline 36. Ethyl acetate ester & $81.7 \pm 2.1$ \\
\hline 37. $n$-Propyl acetate ester & $76.2 \pm 2.1$ \\
\hline 38. Isopropyl acetate ester & $35.1 \pm 1.3$ \\
\hline 39. $n$-Butyl acetate ester & $89.4 \pm 2.5$ \\
\hline 40. Isopentyl acetate ester & $64.7 \pm 2.0$ \\
\hline \multicolumn{2}{|l|}{ Ether $^{\circ}$} \\
\hline 41. Diethyl ether & $12.2 \pm 0.4$ \\
\hline
\end{tabular}

a Values represent means \pm standard error.

${ }^{b}$ Relatively hydrophobic compounds ${ }^{8}$

${ }^{c}$ Relatively hydrophilic compounds ${ }^{8}$ 
Table 2. Symbols, definitions and classification of calculated molecular descriptors

\begin{tabular}{|c|c|}
\hline & Topostructural (TS) \\
\hline $\mathrm{I}_{\mathrm{D}}^{\mathrm{W}}$ & $\begin{array}{l}\text { Information index for the magnitudes of distances between all possible pairs of vertices of a } \\
\text { graph }\end{array}$ \\
\hline $\mathrm{I}_{\mathrm{D}}^{\mathrm{W}}$ & Mean information index for the magnitude of distance \\
\hline W & Wiener index $=$ half-sum of the off-diagonal elements of the distance matrix of a graph \\
\hline $\mathrm{I}^{\mathrm{D}}$ & Degree complexity \\
\hline $\mathrm{H}^{\mathrm{V}}$ & Graph vertex complexity \\
\hline $\mathrm{H}^{\mathrm{D}}$ & Graph distance complexity \\
\hline IC & $\begin{array}{l}\text { Information content of the distance matrix partitioned by frequency of occurrences of distance } \\
\mathrm{h}\end{array}$ \\
\hline $\mathrm{M}_{1}$ & A Zagreb group parameter $=$ sum of square of degree over all vertices \\
\hline $\mathrm{M}_{2}$ & $\begin{array}{l}\text { A Zagreb group parameter = sum of cross-product of degrees over all neighboring (connected) } \\
\text { vertices }\end{array}$ \\
\hline${ }^{\mathrm{h}} x$ & Path connectivity index of order $h=0-10$ \\
\hline${ }^{\mathrm{h}} \chi_{\mathrm{C}}$ & Cluster connectivity index of order $h=3-6$ \\
\hline${ }^{\mathrm{h}} \chi_{\mathrm{PC}}$ & Path-cluster connectivity index of order $h=4-6$ \\
\hline${ }^{\mathrm{h}} \chi_{\mathrm{Ch}}$ & Chain connectivity index of order $h=3-10$ \\
\hline $\mathrm{P}_{\mathrm{h}}$ & Number of paths of length $h=0-10$ \\
\hline $\mathrm{J}$ & Balaban's J index based on topological distance \\
\hline nrings & Number of rings in a graph \\
\hline ncirc & Number of circuits in a graph \\
\hline $\mathrm{DN}^{2} \mathrm{~S}_{\mathrm{y}}$ & $\begin{array}{l}\text { Triplet index from distance matrix, square of graph order (\# of non- } \mathrm{H} \text { atoms), and distance } \\
\text { sum; operation } \mathrm{y}=1-5\end{array}$ \\
\hline $\mathrm{DN}^{2} 1_{\mathrm{y}}$ & Triplet index from distance matrix, square of graph order, and number 1 ; operation $y=1-5$ \\
\hline$A S 1_{y}$ & $\begin{array}{l}\text { Triplet index from adjacency matrix, distance sum, and number } 1 \text {; } \\
\text { operation } y=1-5\end{array}$ \\
\hline $\mathrm{DSl}_{\mathrm{y}}$ & $\begin{array}{l}\text { Triplet index from distance matrix, distance sum, and number } 1 \text {; } \\
\text { operation } y=1-5\end{array}$ \\
\hline $\mathrm{ASN}_{\mathrm{y}}$ & Triplet index from adjacency matrix, distance sum, and graph order; operation $y=1-5$ \\
\hline $\mathrm{DSN}_{\mathrm{y}}$ & $\begin{array}{l}\text { Triplet index from distance matrix, distance sum, and graph order; } \\
\text { operation } y=1-5\end{array}$ \\
\hline $\mathrm{DN}^{2} \mathrm{~N}_{\mathrm{y}}$ & Triplet index from distance matrix, square of graph order, and graph order; operation y $=1-5$ \\
\hline $\mathrm{ANS}_{\mathrm{y}}$ & Triplet index from adjacency matrix, graph order, and distance sum; operation $y=1-5$ \\
\hline $\mathrm{AN} 1_{\mathrm{y}}$ & $\begin{array}{l}\text { Triplet index from adjacency matrix, graph order, and number } 1 \text {; } \\
\text { operation } y=1-5\end{array}$ \\
\hline $\mathrm{ANN}_{\mathrm{y}}$ & Triplet index from adjacency matrix, graph order, and graph order again; operation y = 1-5 \\
\hline $\mathrm{ASV}_{\mathrm{y}}$ & Triplet index from adjacency matrix, distance sum, and vertex degree; operation $y=1-5$ \\
\hline $\mathrm{DSV}_{\mathrm{y}}$ & Triplet index from distance matrix, distance sum, and vertex degree; operation $y=1-5$ \\
\hline $\mathrm{ANV}_{\mathrm{y}}$ & Triplet index from adjacency matrix, graph order, and vertex degree; operation $y=1-5$ \\
\hline
\end{tabular}

$\begin{array}{ll}\mathrm{NoCl} & \text { Number of chlorine atoms } \\ \mathrm{HB}_{1} & \text { Hydrogen bonding parameter } \\ \mathrm{O} & \text { Order of neighborhood when } \mathrm{IC}_{\mathrm{r}} \text { reaches its maximum value for the hydrogen-filled graph } \\ \mathrm{O}_{\mathrm{orb}} & \begin{array}{l}\text { Order of neighborhood when } \mathrm{IC}_{\mathrm{r}} \text { reaches its maximum value for the hydrogen-suppressed } \\ \text { graph }\end{array} \\ \mathrm{I}_{\text {orb }} & \begin{array}{l}\text { Information content or complexity of the hydrogen-suppressed graph at its maximum } \\ \text { neighborhood of vertices }\end{array} \\ \mathrm{IC}_{\mathrm{r}} & \text { Mean information content or complexity of a graph based on the } \mathrm{r}^{\text {th }}(\mathrm{r}=0-6) \text { order }\end{array}$




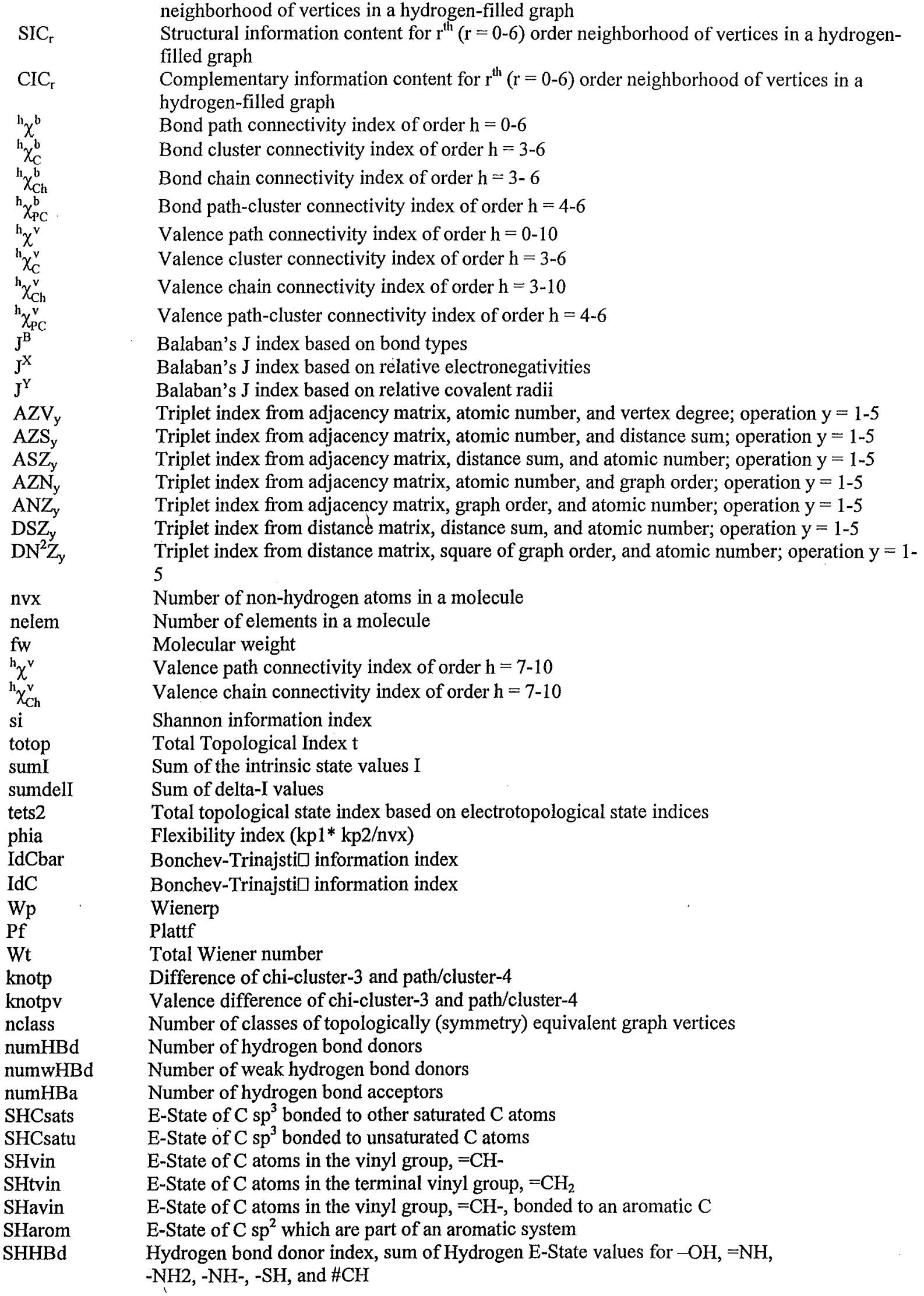


SHwHBd Weak hydrogen bond donor index, sum of C-H Hydrogen E-State values for hydrogen atoms on a $\mathrm{C}$ to which a $\mathrm{F}$ and/or $\mathrm{Cl}$ are also bonded

SHHBa Hydrogen bond acceptor index, sum of the E-State values for $-\mathrm{OH},=\mathrm{NH}$, $-\mathrm{NH} 2,-\mathrm{NH}-,>\mathrm{N}-,-\mathrm{O}-,-\mathrm{S}-$, along with $-\mathrm{F}$ and $-\mathrm{Cl}$

Qv General Polarity descriptor

NHBint $\quad$ Count of potential internal hydrogen bonders $(y=2-10)$

SHBinty E-State descriptors of potential internal hydrogen bond strength $(y=2-10)$

Electrotopological State index values for atoms types:

SHsOH, SHdNH, SHsSH, SHsNH2, SHssNH, SHtCH, SHother, SHCHnX, Hmax Gmax, Hmin, Gmin, Hmaxpos, Hminneg, SsLi, SssBe, Sssss,Bem, SssBH, SsssB, SssssBm, SsCH3, $\mathrm{SdCH} 2$, SssCH2, StCH, SdsCH, SaaCH, SsssCH, SddC, StsC, SdssC, SaasC, SaaaC, SssssC, SsNH3p, SsNH2, SssNH2p, SdNH, SssNH, SaaNH, StN, SsssNHp, SdsN, SaaN, SsssN, SddsN, SaasN, SssssNp, SsOH, SdO, SssO, SaaO, SsF, SsSiH3, SssSiH2, SsssSiH, SssssSi, SsPH2, SssPH, SsssP, SdsssP, SsssssP, SsSH, SdS, SssS, SaaS, SdssS, SddssS, SssssssS, SsCl, SsGeH3, SssGeH2, SsssGeH, SssssGe, SsAsH2, SssAsH, SsssAs, SdsssAs, SsssssAs, SsSeH, SdSe, SssSe, SaaSe, SdssSe, SddssSe, SsBr, SsSnH3, SssSnH2, SsssSnH, Ssssssn, SsI, $\mathrm{SsPbH} 3, \mathrm{SssPbH} 2$, $\mathrm{SsssPbH}, \mathrm{Sssss} \mathrm{Pb}$

\begin{tabular}{ll}
\hline & \\
\hline $\mathrm{kp} 0$ & Kappa zero \\
$\mathrm{kp} 1-\mathrm{kp} 3$ & Kappa simple indices \\
$\mathrm{kal-ka3}$ & Kappa alpha indices \\
$\mathrm{V}_{\mathrm{W}}$ & Van der Waals volume \\
${ }^{3 \mathrm{D}} \mathrm{W}_{\mathrm{H}}$ & 3D Wiener number based on the hydrogen-filled geometric distance matrix \\
${ }^{3 \mathrm{D}} \mathrm{W}$ & 3D Wiener number based on the hydrogen-suppressed geometric distance matrix \\
${ }^{\mathrm{a}} \mathrm{V}_{\mathrm{W}}{ }^{3 \mathrm{D}} \mathrm{W}_{\mathrm{H}, \text { and }}{ }^{3 \mathrm{D}} \mathrm{W}$ used in chlorocarbon analysis only. These descriptors were unavailable for subsequent analyses.
\end{tabular}

Table 3. Summary of regression results for the set of chlorocarbons ${ }^{\mathrm{a}}$

\begin{tabular}{|c|c|c|c|c|c|c|}
\hline \multirow[b]{2}{*}{ Model Type } & \multicolumn{2}{|c|}{ RR } & \multicolumn{2}{|c|}{ PCR } & \multicolumn{2}{|c|}{ PLS } \\
\hline & $\mathrm{R}_{\text {c.v. }}^{2}$ & PRESS & $\mathrm{R}_{\text {c.v }}^{2}$ & PRESS & $\mathrm{R}_{c, v .}^{2}$ & PRESS \\
\hline $\mathrm{TS}$ & 0.436 & 6.79 & -0.259 & 15.2 & -0.172 & 9.97 \\
\hline $\mathrm{TS}+\mathrm{TC}$ & 0.835 & 1.99 & 0.716 & 3.42 & 0.697 & 3.65 \\
\hline $\mathrm{TS}+\mathrm{TC}+3 \mathrm{D}$ & 0.827 & 2.08 & 0.762 & 2.87 & 0.726 & 3.31 \\
\hline TS & 0.436 & 6.79 & -0.259 & 15.2 & -0.172 & 9.97 \\
\hline $\mathrm{TC}$ & 0.866 & 1.61 & 0.732 & 3.23 & 0.800 & 2.41 \\
\hline $3 \mathrm{D}$ & 0.584 & 5.01 & 0.444 & 6.70 & 0.179 & 9.88 \\
\hline
\end{tabular}

${ }^{a}$ Subset of hydrophobic compounds $(\mathrm{N}=13)$

Table 4. Summary of regression results for the set of hydrophobic compounds ${ }^{\text {a }}$

\begin{tabular}{|c|c|c|c|c|c|c|}
\hline \multirow[b]{2}{*}{ Model Type } & \multicolumn{2}{|c|}{ RR } & \multicolumn{2}{|c|}{ PCR } & \multicolumn{2}{|c|}{ PLS } \\
\hline & $\mathrm{R}_{\text {c.v. }}^{2}$ & PRESS & $\mathrm{R}_{\text {c.y. }}^{2}$ & PRESS & $\mathrm{R}_{\text {c.v. }}^{2}$ & PRESS \\
\hline TS & 0.424 & 12.7 & 0.403 & 13.6 & 0.393 & 13.8 \\
\hline $\mathrm{TS}+\mathrm{TC}$ & 0.873 & 2.90 & 0.768 & 5.28 & 0.749 & 5.72 \\
\hline $\mathrm{TS}+\mathrm{TC}+3 \mathrm{D}$ & 0.870 & 2.98 & 0.768 & 5.30 & 0.717 & 6.45 \\
\hline TS & 0.424 & 12.7 & 0.403 & 13.6 & 0.393 & 13.8 \\
\hline $\mathrm{TC}$ & 0.856 & 3.30 & 0.757 & 5.54 & 0.708 & 6.68 \\
\hline $3 \mathrm{D}$ & 0.264 & 16.8 & 0.239 & 17.4 & -0.268 & 28.9 \\
\hline
\end{tabular}

\footnotetext{
${ }^{a}$ Cyclohexane removed from data set $(\mathrm{N}=23)$
} 
Table 5. Summary of regression results for the set of hydrophilic compounds ${ }^{\mathrm{a}}$

\begin{tabular}{|c|c|c|c|c|c|c|}
\hline \multirow[b]{2}{*}{ Model Type } & \multicolumn{2}{|c|}{$R R$} & \multicolumn{2}{|c|}{ PCR } & \multicolumn{2}{|c|}{ PLS } \\
\hline & $R_{c}^{2}$ & PRESS & $R^{2}$ & PRESS & $\mathrm{R}_{\text {c.v. }}^{2}$ & PRESS \\
\hline TS & 0.543 & 11.8 & -0.355 & 35.0 & 0.168 & 21.5 \\
\hline $\mathrm{TS}+\mathrm{TC}$ & 0.948 & 1.34 & 0.922 & 2.02 & 0.913 & 2.25 \\
\hline $\mathrm{TS}+\mathrm{TC}+3 \mathrm{D}$ & 0.945 & 1.42 & 0.917 & 2.15 & 0.906 & 2.43 \\
\hline TS & 0.543 & 11.8 & -0.355 & 35.0 & 0.168 & 21.5 \\
\hline TC & 0.964 & 0.92 & 0.924 & 1.95 & 0.919 & 2.10 \\
\hline $3 \mathrm{D}$ & 0.743 & 6.64 & -0.747 & 45.2 & -0.562 & 40.4 \\
\hline
\end{tabular}

${ }^{2}$ Diethyl ether removed from data set $(\mathrm{N}=16)$

Table 6. Summary of regression results for the set of hydrophobic + hydrophilic compounds ${ }^{\mathrm{a}}$

\begin{tabular}{|c|c|c|c|c|c|c|}
\hline \multirow[b]{2}{*}{ Model Type } & \multicolumn{2}{|c|}{$\mathrm{RR}$} & \multicolumn{2}{|c|}{ PCR } & \multicolumn{2}{|c|}{ PLS } \\
\hline & $\mathrm{R}_{c, y}^{2}$ & PRESS & $\mathrm{R}_{c, v}^{2}$ & PRESS & $\mathrm{R}_{c, v}^{2}$ & PRESS \\
\hline TS & -0.053 & 105 & -0.210 & 120 & -0.228 & 122 \\
\hline $\mathrm{TS}+\mathrm{TC}$ & 0.938 & 6.11 & 0.358 & 63.8 & 0.941 & 5.82 \\
\hline $\mathrm{TS}+\mathrm{TC}+3 \mathrm{D}$ & 0.939 & 6.06 & 0.349 & 64.6 & 0.943 & 5.69 \\
\hline TS & -0.053 & 105 & -0.210 & 120 & -0.228 & 122 \\
\hline TC & 0.944 & 5.57 & 0.396 & 60.0 & 0.942 & 5.80 \\
\hline 3D & 0.066 & 92.7 & -0.020 & 101 & 0.013 & 98.0 \\
\hline
\end{tabular}

${ }^{a}$ Cyclohexane and bromochloromethane removed from data set $(\mathrm{N}=39)$.

Table 7. Most significant $\sim 10 \%$ of TC model parameters based on $|t|$ value for the chlorocarbon data set $^{\mathrm{a}}$

\begin{tabular}{llcccc}
\hline \multicolumn{1}{c}{ Descriptor } & \multicolumn{1}{c}{ Descriptor type } & Coeff. & s.e. & $t$ & $|t|$ \\
\hline $\mathrm{AZV}_{3}$ & Triplet & 0.12127 & 0.01249 & 9.71 & 9.71 \\
$\mathrm{NoCl}$ & No. Cl atoms & 0.03012 & 0.00359 & 8.39 & 8.39 \\
numHBa & H-bond acceptor & 0.11561 & 0.01487 & 7.78 & 7.78 \\
$\mathrm{SHCsats}$ & Atom type E-state & 0.25081 & 0.03253 & 7.71 & 7.71 \\
${ }^{1} \chi^{\mathrm{b}}$ & $1^{\text {st }}$ order bond path connectivity & 0.29577 & 0.03846 & 7.69 & 7.69 \\
$\mathrm{AZS}_{4}$ & Triplet & -0.30389 & 0.03962 & -7.67 & 7.67 \\
${ }^{1} \chi^{\mathbf{v}}$ & $1^{\text {st }}$ order valence path connectivity & 0.30732 & 0.04019 & 7.65 & 7.65 \\
phia & Flexibility & 0.43651 & 0.05868 & 7.44 & 7.44 \\
SHHBa & H-bond acceptor & 0.09488 & 0.01280 & 7.41 & 7.41 \\
SsssCH & Atom type E-state & -0.30596 & 0.04188 & -7.31 & 7.31 \\
${ }^{3} \chi^{b}$ & $3^{\text {rd }}$ order bond cluster connectivity & -0.08884 & 0.01237 & -7.18 & 7.18 \\
idCbar & Information & 0.27432 & 0.03828 & 7.17 & 7.17 \\
\hline
\end{tabular}

${ }^{a}$ Subset of hydrophobic compounds $(\mathrm{N}=13)$ 
Table 8. Most significant $\sim 10 \%$ of TC model parameters based on $|t|$ value for the hydrophobic data set ${ }^{\mathrm{a}}$

\begin{tabular}{|c|c|c|c|c|c|}
\hline Descriptor & Descriptor type & Coeff. & s.e. & $t$ & $|t|$ \\
\hline Wt & Total Wiener number & 0.03421 & 0.00316 & 10.84 & 10.84 \\
\hline $\mathrm{AZV}_{3}$ & Triplet & 0.08093 & 0.00857 & 9.45 & 9.45 \\
\hline $\mathrm{SaaCH}$ & Atom type E-state & 0.06459 & 0.00714 & 9.05 & 9.05 \\
\hline SHarom & E-state, aromatic system & 0.08534 & 0.00958 & 8.90 & 8.90 \\
\hline${ }^{6} \chi^{v} \mathrm{Ch}$ & $6^{\text {th }}$ order valence cyclic connectivity & 5.01933 & 0.59190 & 8.48 & 8.48 \\
\hline totop & Total topological index & 0.03811 & 0.00464 & 8.21 & 8.21 \\
\hline$A Z V_{1}$ & Triplet & 0.09593 & 0.01175 & 8.17 & 8.17 \\
\hline Qv & Polarity & -0.48399 & 0.06541 & -7.40 & 7.40 \\
\hline $\mathrm{AZN}_{4}$ & Triplet & 0.92923 & 0.12848 & 7.23 & 7.23 \\
\hline${ }^{3} \chi^{\mathrm{v}}$ & $3^{\text {rd }}$ order valence path connectivity & 0.23300 & 0.03329 & 7.00 & 7.00 \\
\hline $\mathrm{SsCH} 3$ & Atom type E-state & -0.19602 & 0.03003 & -6.53 & 6.53 \\
\hline numwHBd & Hydrogen bond donor & 0.23978 & 0.03677 & 6.52 & 6.52 \\
\hline
\end{tabular}

${ }^{a}$ Cyclohexane removed from data set $(\mathrm{N}=23)$

Table 9. Most significant $\sim 10 \%$ of TC model parameters based on $|t|$ value for the hydrophilic data set ${ }^{\mathrm{a}}$

\begin{tabular}{llcccc}
\hline \multicolumn{1}{c}{ Descriptor } & \multicolumn{1}{c}{ Descriptor type } & Coeff. & s.e. & $t$ & $|t|$ \\
\hline totop & Total topological index & -0.094 & 0.003 & -30.47 & 30.47 \\
SumI & Sum of intrinsic state values & -0.152 & 0.006 & -24.44 & 24.44 \\
$\mathrm{ANZ}_{3}$ & Triplet & -0.117 & 0.005 & -23.80 & 23.80 \\
NumHBd & Hydrogen bond donor & 0.238 & 0.012 & 20.30 & 20.30 \\
SHHBd & Hydrogen bond donor & 0.139 & 0.007 & 20.11 & 20.11 \\
$\mathrm{Hmax}$ & Atom type E-state & 0.228 & 0.011 & 20.07 & 20.07 \\
$\mathrm{SdO}$ & Atom type E-state & -0.068 & 0.004 & -18.76 & 18.76 \\
$\mathrm{SHCsatu}^{2}$ & E-state, sp ${ }^{3}$ bonded to unsaturated C & -0.252 & 0.019 & -13.10 & 13.10 \\
$\mathrm{DN}^{2} \mathrm{Z}_{4}$ & Triplet & -0.017 & 0.001 & -12.31 & 12.31 \\
$\mathrm{AZV}_{4}$ & Triplet & -0.050 & 0.004 & -11.67 & 11.67 \\
$\mathrm{FW}$ & Molecular weight & -0.041 & 0.004 & -11.34 & 11.34 \\
nclass & No. classes of topologically equivalent & -0.079 & 0.007 & -10.53 & 10.53 \\
& graph vertices & & & & \\
\hline
\end{tabular}

${ }^{a}$ Diethyl ether removed from data set $(\mathrm{N}=16)$

Table 10. Most significant $\sim 10 \%$ of TC model parameters based on $|t|$ value for the hydrophobic + hydrophilic data set $^{\mathrm{a}}$

\begin{tabular}{llcccc}
\hline \multicolumn{1}{c}{ Descriptor } & \multicolumn{1}{c}{ Descriptor type } & Coeff. & s.e. & $t$ & $|t|$ \\
\hline numHBd & Hydrogen bond donor & 1.09032 & 0.04716 & 23.12 & 23.12 \\
SHHBd & Hydrogen bond donor & 0.63524 & 0.02806 & 22.64 & 22.64 \\
$\mathrm{SsOH}$ & Atom type E-state & 0.34518 & 0.01661 & 20.78 & 20.78 \\
$\mathrm{Hmax}$ & Atom type E-state & 0.71396 & 0.05969 & 11.96 & 11.96 \\
$\mathrm{HB}$ & Hydrogen bonding & 0.33321 & 0.02804 & 11.88 & 11.88 \\
sumI & Sum of intrinsic state values & 0.56713 & 0.05096 & 11.13 & 11.13 \\
$\mathrm{Qv}$ & General polarity & -1.50364 & 0.13949 & -10.78 & 10.78 \\
$\mathrm{Gmax}$ & Atom type E-state & 0.42754 & 0.04274 & 10.00 & 10.00 \\
${ }^{0} \chi^{\mathrm{v}}$ & Zero order valence connectivity & -0.38320 & 0.03854 & -9.94 & 9.94 \\
sumDELI & Sum of delta-I values & 0.30960 & 0.03158 & 9.80 & 9.80 \\
$\mathrm{SHHBa}_{\mathrm{ASZ}}$ & Hydrogen bond acceptor & 0.09410 & 0.01089 & 8.64 & 8.64 \\
$\mathrm{ASZ}_{1}$ & Triplet & -0.12561 & 0.01498 & -8.39 & 8.39 \\
\hline
\end{tabular}

${ }^{\text {a }}$ Cyclohexane and bromochloromethane removed from data set $(\mathrm{N}=39)$ 
Table 11. Log scaled experimental and predicted rat blood:air partition coefficients ( $P$, blood:air), as well as residuals, for the current study and the solubility algorithm derived by Poulin and Krishnan ${ }^{8}$

\begin{tabular}{|c|c|c|c|c|c|}
\hline \multirow[b]{2}{*}{ Chemicals } & \multirow[b]{2}{*}{ Exp. $\log \mathrm{P}^{\mathrm{a}}$} & \multicolumn{2}{|c|}{ Current study } & \multicolumn{2}{|c|}{ Solubility algorithm } \\
\hline & & Pred. $\log P$ & Residual & Pred. $\log \mathrm{P}^{\mathrm{c}}$ & Residual \\
\hline \multicolumn{6}{|l|}{ Alkanes } \\
\hline 1. n-Hexane & 0.828 & 0.999 & -0.170 & -1.204 & 2.032 \\
\hline 2. Cyclohexane & 0.329 & -- & -- & -0.511 & 0.840 \\
\hline 3. n-Heptane & 1.558 & 1.518 & 0.040 & -0.105 & 1.663 \\
\hline \multicolumn{6}{|l|}{ Haloalkanes } \\
\hline 4. Dichloromethane & 2.965 & 2.983 & -0.018 & 1.609 & 1.356 \\
\hline 5. Chloroform & 3.035 & 3.136 & -0.101 & 1.386 & 1.649 \\
\hline 6. Carbon tetrachloride & 1.508 & 1.513 & -0.005 & 0.000 & 1.508 \\
\hline 7. Bromochloromethane & 3.726 & - & -- & 2.079 & 1.647 \\
\hline 8. 1,1-Dichloroethane & 2.416 & 2.528 & -0.112 & 0.693 & 1.723 \\
\hline 9. 1,2-Dichloroethane & 3.414 & 3.390 & 0.024 & 2.303 & 1.111 \\
\hline 10. 1,1,1-Trichloroethane & 1.751 & 1.799 & -0.048 & 0.000 & 1.751 \\
\hline 11. 1,1,2-Trichloroethane & 4.060 & 3.749 & 0.312 & 2.708 & 1.352 \\
\hline 12. 1,1,1,2-Tetrachloroethane & 3.730 & 3.692 & 0.038 & 2.079 & 1.651 \\
\hline 13. 1,1,2,2,-Tetrachloroethane & 4.956 & 4.935 & 0.021 & 3.466 & 1.490 \\
\hline 14. Pentachloroethane & 4.644 & 4.640 & 0.005 & 2.708 & 1.936 \\
\hline 15. 1,2-Dichloropropane & 2.928 & 3.076 & -0.147 & 1.099 & 1.829 \\
\hline 16. Trichloroethylene & 3.086 & 3.179 & -0.092 & 0.693 & 2.393 \\
\hline 17. Tetrachloroethylene & 2.939 & 2.918 & 0.021 & 1.609 & 1.330 \\
\hline \multicolumn{6}{|l|}{ Aromatic hydrocarbons } \\
\hline 18. Benzene & 2.879 & 2.837 & 0.042 & 1.099 & 1.780 \\
\hline 19. Chlorobenzene & 4.084 & 4.120 & -0.036 & 1.946 & 2.138 \\
\hline 20. Toluene & 2.890 & 3.088 & -0.198 & 1.386 & 1.504 \\
\hline 21. Styrene & 3.694 & 3.655 & 0.039 & 2.079 & 1.615 \\
\hline 22. o-Xylene & 3.791 & 3.815 & -0.024 & 2.197 & 1.594 \\
\hline 23. $m$-Xylene & 3.829 & 3.672 & 0.157 & 2.079 & 1.750 \\
\hline 24. $p$-Xylene & 3.721 & 3.698 & 0.023 & 2.079 & 1.642 \\
\hline \multicolumn{6}{|l|}{ Ketones } \\
\hline 25. Dimethyl ketone & 5.337 & 5.233 & 0.104 & 5.215 & 0.123 \\
\hline 26. Methyl ethyl ketone & 5.252 & 5.269 & -0.016 & 5.170 & 0.082 \\
\hline 27. Methyl propyl ketone & 4.844 & 4.856 & -0.012 & 4.575 & 0.269 \\
\hline 28. Methyl isobutyl ketone & 4.369 & 4.372 & -0.002 & 3.989 & 0.380 \\
\hline 29. Methyl pentyl ketone & 5.416 & 5.300 & 0.117 & 5.193 & 0.223 \\
\hline \multicolumn{6}{|l|}{ Alcohols } \\
\hline 30. Ethyl alcohol & 7.669 & 7.585 & 0.084 & 7.493 & 0.175 \\
\hline 31. n-Propyl alcohol & 7.200 & 7.334 & -0.134 & 7.348 & -0.148 \\
\hline 32. Isopropyl alcohol & 7.162 & 6.993 & 0.169 & 7.138 & 0.024 \\
\hline 33. n-Butyl alcohol & 7.056 & 6.965 & 0.091 & 6.909 & 0.147 \\
\hline 34. n-Pentyl alcohol & 6.720 & 6.725 & -0.005 & 6.812 & -0.092 \\
\hline \multicolumn{6}{|l|}{ Acetate esters } \\
\hline 35. Methyl acetate ester & 4.605 & 4.691 & -0.085 & 4.511 & 0.094 \\
\hline 36. Ethyl acetate ester & 4.403 & 4.255 & 0.148 & 4.094 & 0.309 \\
\hline 37. $n$-Propyl acetate ester & 4.333 & 4.230 & 0.103 & 3.807 & 0.527 \\
\hline 38. Isopropyl acetate ester & 3.558 & 3.743 & -0.185 & 3.367 & 0.191 \\
\hline 39. $n$-Butyl acetate ester & 4.493 & 4.486 & 0.007 & 3.434 & 1.059 \\
\hline 40. Isopentyl acetate ester & 4.170 & 4.271 & -0.101 & 3.219 & 0.951 \\
\hline \multicolumn{6}{|l|}{ Ether } \\
\hline 41. Diethyl ether & 2.501 & 2.557 & -0.055 & 2.303 & 0.199 \\
\hline
\end{tabular}

alues represent $\log$ of mean $\mathrm{PC}$

$b$ TC model developed on combined hydrophobic and hydrophilic compounds using ridge regression

c Values have been log scaled for ease of comparison 


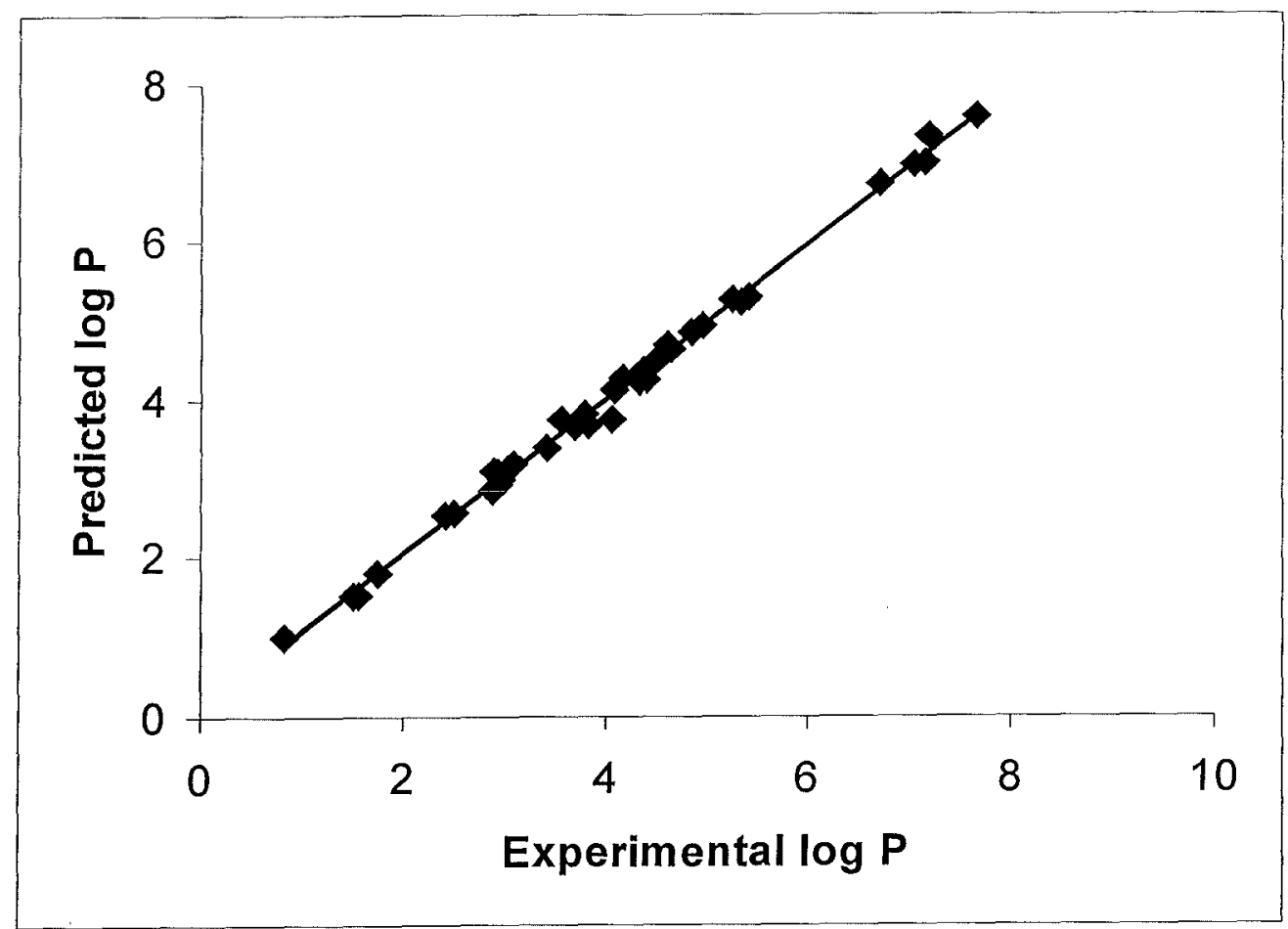

Figure 1. Scatterplot of the experimental vs predicted $\log \mathrm{P}$ (blood:air) values using the TC model developed with the ridge regression method for the combined set of hydrophobic and hydrophilic compounds $(N=39)$. 



\title{
PREDICTION OF HUMAN BLOOD:AIR PARTITION COEFFICIENT: A COMPARISON OF STRUCTURE-BASED AND PROPERTY-BASED METHODS
}

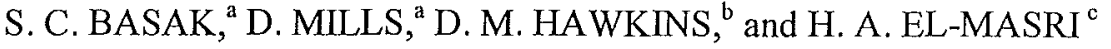 \\ ${ }^{a}$ Natural Resources Research Institute, University of Minnesota Duluth \\ 5013 Miller Trunk Highway, Duluth, MN 55811, USA \\ ${ }^{b}$ School of Statistics, 313 Ford Hall, 224 Church Street S. E., University of Minnesota Minneapolis, MN 55455, \\ USA \\ ${ }^{c}$ Computational Toxicology Laboratory, Division of Toxicology Agency for Toxic Substances and Disease Registry \\ (ATSDR), Executive Park Building 4, 1600 Clifton Road, E-29, Atlanta, GA 30333, USA
}

\begin{abstract}
In recent years, there has been increased interest in the development and use of quantitative structure activity/property relationship (QSAR/QSPR) models. For the most part, this is due to the fact that experimental data is sparse and obtaining such data is costly, while theoretical structural descriptors can be obtained quickly and inexpensively. In this study, three linear regression methods, viz. principal component regression (PCR), partial least squares (PLS), and ridge regression (RR), were used to develop QSPR models for the estimation of human blood:air partition coefficient ( $\log \mathrm{P}_{\text {blood:air }}$ ) for a group of 31 diverse low-molecular weight volatile chemicals from their computed molecular descriptors. In general, RR was found to be superior to PCR or PLS. Comparisons were made between models developed using parameters based solely on molecular structure and linear regression (LR) models developed using experimental properties, including saline:air partition coefficient $\left(\log \mathrm{P}_{\text {saline:air }}\right)$ and olive oil:air partition coefficient ( $\log \mathrm{P}_{\text {olive oil:air }}$ ), as independent variables, indicating that the structure-property correlations are comparable to the property-property correlations. The best models, however, were those which used rat $\log \mathrm{P}_{\text {blood:air }}$ as the independent variable. Haloalkane subgroups were modeled separately for comparative purposes, and although models based on the congeneric compounds were superior, the models developed on the complete set of diverse compounds were of acceptable quality. The structural descriptors were placed into one of three classes based on level of complexity: Topostructural (TS), topochemical (TC), or 3-dimensional / geometrical (3D). Modeling was performed using the structural descriptor classes both in a hierarchical fashion and separately. The results indicate that the highest quality structure-based models, in terms of descriptor classes, were those derived using TC or TS+TC descriptors.
\end{abstract}

Key Words: Blood:air partition coefficient; PBPK model; theoretical molecular descriptors; ridge regression; quantitative structure-property relationship (QSPR) model. 


\section{INTRODUCTION}

Modern lifestyle worldwide is based on the use of a large number of chemicals. Natural and synthetic chemicals are used as drugs, pesticides, herbicides, components of diagnostic tools, ingredients and solvents in industrial processes, to name just a few. The Toxic Substances Control Act (TSCA) Inventory maintained by the United States Environmental Protection Agency (USEPA) currently has over 81,000 entries and the list is growing every year. ${ }^{(1)}$ Many of these chemicals are used for various purposes and have the potential to be released in the environment. Therefore, it is natural that we need to carry out risk assessment of the TSCA chemicals, particularly for those that are used frequently and in large quantities. Volatile organic chemicals (VOCs) constitute a class of chemicals that are frequently used in various industrial processes. Therefore, there is an interest to predict the potential adverse effects of these chemicals on human and environmental health. The overall risk of a chemical is determined primarily by its intrinsic toxicity (hazard) and exposure potential.

The blood:air partition coefficient of VOCs is an important determinant of pulmonary uptake of such chemicals from inhaled air. Such parameters are routinely used in building physiologically-based pharmacokinetic (PBPK) models for exposure assessment of such chemicals. Solubility of VOCs in blood is determined by its composition including the content of neutral lipid, phospholipid, and water, as well as the extent of binding of these chemicals to specific components such as plasma proteins and hemoglobin. ${ }^{(2)}$ Such physicochemical considerations can be used to come up with physicochemicallybased methods for the estimation of partition coefficient values of chemicals. The other possibility is the use of molecular descriptors to estimate partition coefficient of chemicals directly from their structure. Such quantitative structure-activity/property relationship (QSAR/QSPR) methods derived using theoretical descriptors are based on the idea that observable physicochemical and biological properties of chemicals are determined by their molecular structure. In particular, QSPRs have been found to be useful in the estimation of physicochemical properties such as octanol:water partition coefficient of various groups of chemicals, ${ }^{(3,4)}$ as well as the degree of transport through the blood-brain barrier ${ }^{(5)}$ and skin ${ }^{(6)}$ of various congeneric and diverse sets.

While some quantitative models use experimental data per se as independent variables, it is important to note that experimental data does not exist for the majority of compounds, and obtaining such data is costly in terms of time and monetary resources. Computational modeling involving algorithmically calculated parameters based solely on molecular structure is an inexpensive alternative. In this paper, we have attempted to develop QSPR models to estimate human blood:air partition coefficients for a set of 31 VOCs using molecular descriptors which can be computed directly from molecular structure. 


\section{METHODS}

2.1 Database. Liquid:air partition coefficients were experimentally determined by Gargas et al. ${ }^{(7)}$ using a modified version of the gas-phase vial equilibrium technique ${ }^{(8)}$ for a set of low molecular-weight volatile chemicals. Table I includes experimentally determined human and male Fischer 344 rat blood:air partition coefficient data for a set of 31 chemicals including 18 haloalkanes, 2 nitroalkanes, 2 aliphatic hydrocarbons, 4 haloalkenes, and 5 aromatics compounds. The human blood:air partition coefficient values were determined on blood pretreated with diethyl maleate to inhibit an observed glutathione transferase reaction. Experimental saline:air and olive oil:air partition coefficients, determined by Gargas et al., are also listed in Table I. All experimental values were obtained at $37^{\circ} \mathrm{C}$.

It should be noted that the data used in the current study are a subset of that reported by Gargas et al. ${ }^{(7)}$ Two cis/trans isomers were eliminated because they are indistinguishable in terms of their calculated molecular descriptors based on SMILES input. Methyl chloride was also removed from the data set as it is not possible to calculate our entire set of theoretic descriptors on two-atom compounds. In addition, two compounds were reported without discrete values for $0.9 \%$ saline:air partition coefficient and thus were not included in this study.

2.2 Theoretical Molecular Descriptors. Theoretical molecular descriptors may be divided into hierarchical classes based upon level of complexity. Topostructural (TS) descriptors, which encode information strictly on the adjacency and connectedness of atoms within a molecule, make up the simplest of the hierarchical classes. Topochemical (TC) descriptors encode information related to the chemical nature of a molecule including bond type. The 3-dimensional or shape descriptors (3D) are still more complex, encoding information about the 3 -dimensional aspects of a molecule. Calculated $\log \mathrm{P}_{n \text {-octanol:water }}$ descriptors ${ }^{(9)}$ were included at the final stage of hierarchical model development. The topostructural and topochemical descriptors are collectively referred to as topological descriptors.

Descriptors used in the present study were derived from molecular structure using software packages including POLLY, ${ }^{(10)}$ Triplet, ${ }^{(11,12)}$ and Molconn-Z ${ }^{(13)}$ From POLLY, a set of topological descriptors is available, including a large group of connectivity indices, ${ }^{(14-17)}$ path-length descriptors, ${ }^{(14)}$ and information theoretic ${ }^{(18,19)}$ and neighborhood complexity indices. ${ }^{(19)}$ The Triplet descriptors also constitute a large group of topological parameters. They are derived from a matrix, a main diagonal column vector, and a free term column vector, converting the matrix into a system of linear equations whose solutions are the local vertex invariants. These local vertex invariants are then used in the following mathematical operations in order to obtain the triplet descriptors: 
1. Summation, $\mathrm{E}_{\mathrm{i}} \mathrm{x}_{\mathrm{i}}$

2. Summation of squares, $\mathrm{E}_{\mathrm{i}} \mathrm{x}_{\mathrm{i}}^{2}$

3. Summation of square roots, $\mathrm{E}_{\mathrm{i}} \mathrm{x}_{\mathrm{i}}^{1 / 2}$

4. Sum of inverse square root of cross-product over edges $\mathrm{ij}, \mathrm{E}_{\mathrm{ij}}\left(\mathrm{x}_{\mathrm{i}} \mathrm{x}_{\mathrm{j}}\right)^{-1 / 2}$

5. Product, $N\left(E_{i} x_{i}\right)^{1 / N}$

Molconn-Z provides additional topological descriptors, including an extended set of connectivity indices, electrotopological indices, ${ }^{(20,21)}$ and hydrogen bonding descriptors, as well as a small set of molecular shape descriptors.

$\mathrm{H}$-Bond, a software program developed by Basak, ${ }^{(22)}$ was used to calculate $\mathrm{HB}_{1}$, a measure of hydrogen bonding potential. Balaban's J indices were also calculated by software developed by the authors. ${ }^{(23-25)}$

$\log \mathrm{P}_{n-\text { octanol:water }}$ values were calculated by the $\operatorname{LogP}$ program $^{(9)}$ and are included in Table I. Table II provides a brief description of all other theoretical molecular descriptors used in the current study, though the calculated values for these descriptors are not included for the sake of brevity.

2.3 Statistical Analysis. Independent and dependent variables were scaled by the natural logarithm, as their respective ranges differed by several orders of magnitude. The CORR procedure of the SAS statistical package ${ }^{(26)}$ was used to identify perfectly correlated descriptors, i.e. $r=1.0$. In each case, only one descriptor of a perfectly correlated pair was retained for use in the subsequent analysis. Any descriptor that either had a value of zero for all compounds in the data set or could not be calculated for all compounds in the data set was removed.

The structure-property models were developed using ridge regression (RR), ${ }^{(27)}$ principal components regression (PCR), ${ }^{(28)}$ and partial least squares (PLS) regression ${ }^{(29-31)}$ methodologies, utilizing molecular descriptors in a hierarchical fashion. In addition, each class of descriptors was used independently to obtain single-class models. RR, PCR, and PLS are useful in cases wherein the number of descriptors is much greater than the number of observations, as well as in cases where the independent variables are highly intercorrelated. In addition, these regression methods make use of all independent variables as opposed to subset regression wherein it is possible that important parameters may be eliminated from the study. Linear regression (LR) was used to obtain the property-property models, which involve 1-2 independent variables. Statistical parameters reported include the cross-validated $R^{2}$ value and the PRESS statistic which are reliable measures of model predictability. In addition, the $t$ values can be examined in 
or ${ }^{\prime}$ s to identify significant descriptors. Although a descriptor with a large $|t|$ indicates that the sociated descriptor is important in the model, it should be cautioned that the reverse is not necessarily true.

Honest assessment of the quality of a prediction model is seldom straightforward, but is particularly challenging in a situation such as this where the number of independent variables far exceeds the number of observations. ${ }^{(32,33)}$ In these cases, conventional regression measures such as $\mathrm{R}^{2}$ are useless. The measure we use is the cross-validation (or jack-knife) sum of squares. For this measure, each compound in turn is omitted from the data set, and the coefficients of the regression model (RR, PLS or PCR) computed using the remaining $n-1$ cases. These coefficients are used to predict the hold-out case. The overall quality of the fit is measured by the prediction sum of squares PRESS - the sum of squares of the difference between the actual observed activity and that predicted from the regression. A cross-validation $\mathrm{R}^{2}$ can be defined by

$$
R_{c v}^{2}=1-\frac{P R E S S}{S S T o t a l}
$$

Unlike $\mathrm{R}^{2}$, this $R_{c v}^{2}$ does not increase if irrelevant predictors are added to the model; rather it tends to decrease. And where $\mathrm{R}^{2}$ is necessarily non-negative, $R_{c v}^{2}$ may be negative. This non-uncommon situation is an indication that the model fitted is poor - worse, in fact, than making predictions by ignoring the predictors and using the mean activity as the prediction in all circumstances.

$R_{c v}^{2}$ mimics the results of applying the final regression to predicting a future case; large values can be interpreted unequivocally and without regard to either the number of cases or predictors as indicating that the fitted regression will accurately predict the activity of future compounds of the same chemical type as those used to calibrate the regression.

\section{RESULTS AND DISCUSSION}

Table III provides results of studies done on the complete set of 31 diverse compounds as well as the subset of 18 haloalkanes for the prediction of human $\log \mathrm{P}_{\text {bloodair }}$. Examining the models developed using structural descriptors, we find that the RR methodology is generally superior to both PCR and PLS. This is supported by our earlier studies with various congeneric and diverse sets of chemicals. ${ }^{(34-36)}$ The model developed using TC descriptors as independent variables was superior to those developed with other structural descriptor classes in the analysis of the 31 diverse compounds, while the TS+TC model was superior in the analysis of the 18 haloalkanes. 
The results of QSPRs reported in this paper show that structure-property correlations are comparable or superior to property-property correlations involving experimental saline:air and olive oil:air partition coefficients in the prediction of human blood:air partition coefficient. For the set of 31 diverse chemicals, a cross-validated $\mathrm{R}^{2}$ of 0.874 and a PRESS of 7.79 is obtained for the TC model, while the propertyproperty model utilizing $\log \mathrm{P}_{\text {saline:air }}$ and $\log \mathrm{P}_{\text {olive:oil air }}$ yields a cross-validated $\mathrm{R}^{2}$ of 0.889 with a PRESS of 6.19 (Table III). For the set of 18 haloalkanes, the TS+TC models yields a cross-validated $\mathrm{R}^{2}$ of 0.897 with a PRESS of 3.02, while the property-property model utilizing $\log \mathrm{P}_{\text {saline:air }}$ and $\log \mathrm{P}_{\text {olive:oil air }}$ yields a cross-validated $\mathrm{R}^{2}$ of 0.846 with a PRESS of 4.50 . However, property-property models in which rat $\log \mathrm{P}_{\text {blood:air }}$ is used to predict human $\log \mathrm{P}_{\text {blood:air }}$ are superior to those in which either $\log \mathrm{P}_{\text {saline:air }}$ and $\log \mathrm{P}_{\text {olive:oil air }}$ or structural parameters are used as predictors; with a cross-validated $\mathrm{R}^{2}$ of 0.963 and PRESS of 2.25 for the full set of 31 compounds, and a cross-validated $R^{2}$ of 0.961 and PRESS of 1.16 for the subset of 18 haloalkanes.

It is clear from the results presented in Table III that experimental rat blood:air partition coefficient is the best predictor of human blood:air partition coefficient. Acquiring these data, however, is time consuming and requires laboratory testing resources along with the sacrifice of animals. Experimental determination of rat blood:air partition coefficient of hundreds or thousands of candidate chemicals would be a daunting task. The theoretical descriptor-based models, on the other hand, can provide reasonable estimates very quickly and at a low cost.

Ridge regression coefficients and standard errors for the top 10 descriptors based on $|t|$ values for the human $\log \mathrm{P}_{\text {bloodair }}$ TC model based on the set of 31 diverse chemicals are provided in Table IV. The indices most important for the prediction of human $\log \mathrm{P}_{\text {blood:air }}$ include: a) molecular weight (fw), quantifying molecular size, $\mathrm{b})$ triplet indices $\left(A Z V_{\mathrm{y}}\right)$, encoding information about the nature of atoms, $\left.\mathrm{c}\right)$ electrotopological state indices (SdO, SddSN, SSBr), which are numerical descriptors of the electronic states of atoms, $d)$ valence and bonding connectivity indices $\left({ }^{1} \chi^{b},{ }^{1} \chi^{v}\right)$, which quantify structural information regarding molecular size and shape, and e) a hydrogen bonding parameter $\left(\mathrm{HB}_{1}\right)$. The important role of molecular factors such as size, electronic interactions, and hydrogen bonding in determining partition coefficients of chemicals is evident from our earlier studies ${ }^{(3,37)}$ and those of Kamlet et al. ${ }^{(38)}$

It is important to reiterate that model predictability is best judged, not with a fitted model, but with a cross-validated model wherein each of the compounds, in turn, is omitted from the data set and its value then determined by the coefficients of the remaining $n-1$ compounds. In this way, we have an accurate, if not conservative, indication of how well the model will predict property values of new compounds which are similar to those used to create the model. Figure 1 illustrates the relationship between the fitted and experimental human $\log \mathrm{P}_{\text {blood:air }}$ values using the $\mathrm{TC}$ model for the set of 31 diverse compounds. All 
$\mathrm{s}^{\dagger}$ istical values reported in this paper, however, are based on cross-validated results. Accordingly, igure 2 illustrates the relationship between the cross-validated predicted and experimental human $\log \mathrm{P}_{\text {blood:air }}$ values using the TC model for the set of 31 diverse compounds.

In conclusion, the models based on rat $\log \mathrm{P}_{\text {bloodair }}$ are superior to any of the structure-based models. It is important to note, however, that experimental data are not currently available for the majority of compounds; and obtaining this data is costly in terms of time and monetary resources. In contrast, we are able to obtain reasonably good models using structural descriptors that can be calculated very quickly and inexpensively for both existing and unsynthesized chemicals. Modeling based on structural descriptors also promotes an understanding of the theoretical basis of properties and reduces the need for animal research, an area to which a growing aversion exists in our society.

\section{ACKNOWLEDGEMENT}

This is contribution number XXX from the Center for Water and the Environment of the Natural Resources Research Institute. Research reported in this paper was supported in part by Grant F49620-010098 from the United States Air Force.

\section{REFERENCES}

1. Cash, G. G. (2001). Personal communication.

2. Poulin, P. \& Krishnan, K. (1996). A mechanistic algorithm for predicting blood:air partition coefficients of organic chemicals with the consideration of reversible binding in hemoglobin. Toxicol. Appl. Pharmacol., 136, 131-137.

3. Niemi, G. J., Basak, S. C., Veith, G. D. \& Grunwald, G. (1992). Prediction of octanol-water partition coefficient (Kow) using algorithmically-derived variables. Environ. Toxicol. Chem., 11, 891-898.

4. Katritzky, A. R., Wang, Y., Sild, S. \& Tamm, T. (1998). QSPR studies on vapor pressure, aqueous solubility, and the prediction of water-air partition coefficients. J. Chem. Inf. Comput. Sci., 38, 720-725.

5. Basak, S. C., Gute, B. D. \& Drewes, L. R. (1996). Predicting blood-brain transport of drugs: A computational approach. Pharm. Res., 13, 775-778.

6. Gute, B. D., Grunwald, G. D. \& Basak, S. C. (1999). Prediction of the dermal penetration of polycyclic aromatic hydrocarbons (PAHs): A hierarchical QSAR approach. SAR QSAR Environ. Res., 10, 1-15.

7. Gargas, M. L., Burgess, R. J., Voisard, D. E., Cason, G. H. \& Andersen, M. E. (1989). Partition coefficients of low molecular weight volatile chemicals in various tissues and liquids. Toxicol. Appl. Pharmacol., 98, 87-99.

8. Sato, A. \& Nakajima, T. (1979). Partition coefficients of some aromatic hydrocarbons and ketones in water, blood and oil. Br. J. Ind. Med., 36, 231-234.

9. Parham, M., Hall, L. H. \& Kier, L. B. (2000). LogP. www.logP.com.

10. Basak, S. C., Harriss, D. K. \& Magnuson, V. R. (1988). POLLY, Version 2.3, Copyright of the University of Minnesota.

11. Filip, P. A., Balaban, T. S. \& Balaban, A. T. (1987). A new approach for devising local graph invariants: Derived topological indices with low degeneracy and good correlational ability. J. Math. Chem., 1, 61-83. 
12. Basak, S. C., Balaban, A. T., Grunwald, G. D. \& Gute, B. D. (2000). Topological indices: Their nature and mutual relatedness. J. Chem. Inf. Comput. Sci., 40, 891-898.

13. Hall Associates Consulting, Molconn-Z Version 3.50, Quincy, MA, 2000.

14. Kier, L. B. \& Hall, L. H. (1986). Molecular Connectivity in Structure-Activity Analysis, Research Studies Press, Letchworth, Hertfordshire, U.K.

15. Kier, L. B., Murray, W. J., Randić, M. \& Hall, L. H. (1976). Molecular connectivity. V. Connectivity series concept applied to diversity. J. Pharm. Sci., 65, 1226-1230.

16. Randić, M. (1975). On characterization of molecular branching. J. Am. Chem. Soc., 97, 6609-6615.

17. Basak, S. C., Magnuson, V. R., Niemi, G. J. \& Regal, R. R. (1988). Determining structural similarity of chemicals using graph-theoretic indices. Discrete Appl. Math., 19, 17-44.

18. Raychaudhury, C., Ray, S. K., Ghosh, J. J., Roy, A. B. \& Basak, S. C. (1984). Discrimination of isomeric structures using information theoretic topological indices. J. Comput. Chem., 5, 581-588.

19. Basak, S. C. (1999). Information theoretic indices of neighborhood complexity and their applications. In Topological Indices and Related Descriptors in QSAR and QSPR (Devillers, J. and Balaban, A.T., Eds.) pp. 563-593, Gordon and Breach Science Publishers, The Netherlands.

20. Kier, L. B. \& Hall, L. H. (1999). Molecular Structure Description: The Electrotopological State, Academic Press, San Diego, CA.

21. Hall, L. H., Mohney, B. \& Kier, L. B. (1991). The electrotopological state: Structure information at the atomic level for molecular graphs. J. Chem. Inf. Comput. Sci., 31, 76-82.

22. Basak, S. C. (1988). H-Bond, Copyright of the University of Minnesota.

23. Balaban, A. T. (1982). Highly discriminating distance-based topological indices. Chem. Phys. Lett., 89, 399404.

24. Balaban, A. T. (1983). Topological indices based on topological distances in molecular graphs. Pure and Appl. Chem., 55, 199-206.

25. Balaban, A. T. (1985). Chemical graphs. Part 48. Topological index $\mathrm{J}$ for heteroatom-containing molecules taking into account periodicities of element properties. Math. Chem. (MATCH), 21, 115-122.

26. SAS Institute, Inc. In SAS/STAT User Guide, Release 6.03 Edition; Cary, NC, 1988.

27. Hoerl, A. E. \& Kennard, R. W. (1970). Ridge regression: Biased estimation for nonorthogonal problems. Technometrics, 8, 27-51.

28. Massy, W. F. (1965). Principal components regression in exploratory statistical research. J. Am. Statistical Assoc., 60, 234-246.

29. Hoskuldsson, A. (1988). PLS regression methods. J. Chemometrics, 2, 211-228.

30. Hoskuldsson, A. (1995). A combined theory for PCA and PLS. J. Chemometrics, 9, 91-123.

31. Wold, H. (1975). Soft modeling by latent variables: The nonlinear iterative partial least squares approach. In Perspectives in Probability and Statistics, Papers in Honor of M. S. Bartlett (Gani, J., Ed.). Academic Press, London.

32. Miller, A. J. (1990). Subset selection in regression, Chapman and Hall, New York.

33. Rencher, A. C. \& Pun, F. C. (1980). Inflation of R2 in best subset regression. Technometrics, 22, 49-53.

34. Hawkins, D., Basak, S. \& Shi, X. (2001). QSAR with few compounds and many features. J. Chem. Inf. Comput. Sci. , 41, 663-670.

35. Basak, S. C., Hawkins, D. M. \& Mills, D. (2002). Predicting blood:air partition coefficient of structurally diverse chemicals using theoretical molecular descriptors. In Advances in Molecular Similarity; Girones, X., Carbo-Dorca, R., Mezey, P. G., Eds.; Kluwer, in press.

36. Basak, S. C., El-Masri, H., Hawkins, D. M. \& Mills, D. (2001). Exposure assessment of volatile organic chemicals(VOCs): Predicting blood:air partition coefficients of diverse chemicals using theoretical descriptors. J. Chem. Inf. Comput. Sci., submitted.

37. Basak, S. C., Niemi, G. J. \& Veith, G. D. (1990). Recent developments in the characterization of chemical structure using graph-theoretic indices. In Computational Chemical Graph Theory (Rouvray, D.H., Ed.). pp. 235-277.

38. Kamlet, M. J., Abboud, J.-L. M., Abraham, M. H. \& Taft, R. W. (1983). Linear solvation energy relationships. 23. A comprehensive collection of the solvatochromatic parameters, $\pi^{*}, \alpha$ and $\beta$, and some methods for simplifying the general solvatochromatic equation. J. Org. Chem., 48, 2877-2887. 
Table I. Experimental liquid:air partition coefficients ${ }^{\mathrm{a}}$ and calculated $\log \mathrm{P}_{n \text {-octanol:water }}$

\begin{tabular}{|c|c|c|c|c|c|c|}
\hline \multirow[b]{2}{*}{ No. } & \multirow[b]{2}{*}{ Chemical } & \multicolumn{4}{|c|}{ Experimental } & \multirow{2}{*}{$\begin{array}{c}\text { Calculated } \\
\log P(n \text {-octanol:water) }\end{array}$} \\
\hline & & $\mathrm{P}(0.9 \%$ saline:air $)$ & $\mathrm{P}$ (olive oil:air) & $\begin{array}{c}\text { Rat } \\
\text { P(blood:air) }\end{array}$ & $\begin{array}{c}\text { Human } \\
\text { P(blood:air) }\end{array}$ & \\
\hline \multicolumn{7}{|c|}{ Haloalkanes } \\
\hline 1 & Dichloromethane & $5.96 \pm 0.71$ & $131 \pm 7$ & $19.4 \pm 0.8$ & $8.94 \pm 0.13$ & 1.16 \\
\hline 2 & Chloroform & $3.38 \pm 0.09$ & $402 \pm 12$ & $20.8 \pm 0.1$ & $6.85 \pm 0.51$ & 1.86 \\
\hline 3 & Carbon tetrachloride & $0.35 \pm 0.03$ & $374 \pm 11$ & $4.52 \pm 0.35$ & $2.73 \pm 0.23$ & 3 \\
\hline 4 & Chlorodibromomethane & $7.34 \pm 0.42$ & $2683 \pm 152$ & $116 \pm 4$ & $52.7 \pm 1.2$ & 1.77 \\
\hline 5 & Chloroethane & $1.09 \pm 0.06$ & $38.9 \pm 3.1$ & $4.08 \pm 0.39$ & $2.69 \pm 0.20$ & 1.47 \\
\hline 6 & 1,1-Dichloroethane & $2.45 \pm 0.04$ & $186 \pm 7$ & $11.2 \pm 0.1$ & $4.94 \pm 0.24$ & 1.86 \\
\hline 7 & 1,2-Dichloroethane & $11.4 \pm 0.1$ & $366 \pm 8$ & $30.4 \pm 1.2$ & $19.5 \pm 0.7$ & 1.6 \\
\hline 8 & 1,1,1-Trichloroethane & $0.75 \pm 0.07$ & $295 \pm 22$ & $5.76 \pm 0.50$ & $2.53 \pm 0.13$ & 2.26 \\
\hline 9 & 1,1,2-Trichloroethane & $13.3 \pm 0.3$ & $1776 \pm 26$ & $58.0 \pm 1.1$ & $35.7 \pm 0.4$ & 2.08 \\
\hline 10 & 1,1,1,2-Tetrachloroethane & $3.53 \pm 0.23$ & $2686 \pm 51$ & $41.7 \pm 1.0$ & $30.2 \pm 1.3$ & 2.64 \\
\hline 11 & 1,1,2,2-Tetrachloroethane & $23.4 \pm 2.0$ & $6358 \pm 402$ & $142 \pm 6$ & $116 \pm 6$ & 2.51 \\
\hline 12 & Hexachloroethane & $0.66 \pm 0.21$ & $5015 \pm 318$ & $62.7 \pm 2.1$ & $52.4 \pm 1.4$ & 4.24 \\
\hline 13 & 1-Bromo-2-chloroethane & $8.91 \pm 0.56$ & $569 \pm 23$ & $52.7 \pm 3.5$ & $29.2 \pm 2.1$ & 1.73 \\
\hline 14 & 1-Chloropropane & $1.04 \pm 0.01$ & $105 \pm 2$ & $5.21 \pm 0.06$ & $2.85 \pm 0.06$ & 1.95 \\
\hline 15 & 2-Chloropropane & $0.82 \pm 0.09$ & $69.9 \pm 3.5$ & $3.10 \pm 0.17$ & $1.39 \pm 0.29$ & 1.81 \\
\hline 16 & 1,2-Dichloropropane & $2.75 \pm 0.11$ & $428 \pm 30$ & $18.7 \pm 0.5$ & $8.75 \pm 0.50$ & 2.18 \\
\hline 17 & $n$-Propyl bromide & $1.44 \pm 0.12$ & $272 \pm 8$ & $11.7 \pm 0.4$ & $7.08 \pm 0.40$ & 2.13 \\
\hline 18 & Isopropyl bromide & $1.08 \pm 0.04$ & $164 \pm 5$ & $5.95 \pm 0.14$ & $2.57 \pm 0.15$ & 1.63 \\
\hline 19 & 1-Nitropropane & $127 \pm 4$ & $1062 \pm 21$ & $223 \pm 10$ & $187 \pm 6$ & 0.8 \\
\hline 20 & 2-Nitropropane & $98.3 \pm 5.4$ & $640 \pm 16$ & $183 \pm 12$ & $154 \pm 17$ & 0.61 \\
\hline 21 & $n$-Heptane & $0.18 \pm 0.10$ & $405 \pm 3$ & $4.75 \pm 0.15$ & $8.19 \pm 0.10$ & 4.31 \\
\hline 22 & JP-10 (tricyclo $\left[5 \cdot 2 \cdot 1.0^{2,6}\right]$-decane) & $0.21 \pm 0.07$ & $12970 \pm 420$ & $62 \pm 4$ & $52.5 \pm 3.7$ & 3.75 \\
\hline 23 & Vinyl chloride & $0.43 \pm 0.04$ & $24.4 \pm 3.7$ & $1.68 \pm 0.18$ & $1.16 \pm 0.08$ & 1.37 \\
\hline 24 & Trichloroethylene & $0.83 \pm 0.30$ & $553 \pm 46$ & $21.9 \pm 1.4$ & $8.11 \pm 0.17$ & 2.36 \\
\hline 25 & Tetrachloroethylene & $0.79 \pm 0.06$ & $2134 \pm 159$ & $18.9 \pm 1.1$ & $10.3 \pm 1.1$ & 3.47 \\
\hline 26 & Vinyl bromide & $0.44 \pm 0.06$ & $56.0 \pm 1.5$ & $4.05 \pm 0.16$ & $2.27 \pm 0.16$ & 1.61 \\
\hline 27 & Benzene & $2.75 \pm 0.10$ & $465 \pm 5$ & $17.8 \pm 0.3$ & $8.19 \pm 0.10$ & 2.04 \\
\hline 28 & Chlorobenzene & $2.81 \pm 0.07$ & $2188 \pm 41$ & $59.4 \pm 1.0$ & $30.0 \pm 0.3$ & 2.64 \\
\hline 29 & $o$-Xylene & $2.65 \pm 0.08$ & $3534 \pm 208$ & $44.3 \pm 2.0$ & $34.9 \pm 1.7$ & 3.15 \\
\hline 30 & $m$-Xylene & $1.92 \pm 0.12$ & $3245 \pm 116$ & $46.0 \pm 1.5$ & $32.5 \pm 1.6$ & 3.21 \\
\hline 31 & $p$-Xylene & $1.77 \pm 0.07$ & $3319 \pm 96$ & $41.3 \pm 3.5$ & $44.7 \pm 1.9$ & 3.20 \\
\hline
\end{tabular}


Table II. Symbols, definitions and classification of calculated molecular descriptors

\begin{tabular}{|c|c|}
\hline & Topostructural (TS) \\
\hline$I_{D}^{W}$ & $\begin{array}{l}\text { Information index for the magnitudes of distances between all possible pairs of vertices of a } \\
\text { graph }\end{array}$ \\
\hline $\bar{I}_{\mathrm{D}}^{W}$ & Mean information index for the magnitude of distance \\
\hline W & Wiener index $=$ half-sum of the off-diagonal elements of the distance matrix of a graph \\
\hline $\mathrm{I}^{\mathrm{D}}$ & Degree complexity \\
\hline $\mathrm{H}^{\mathrm{V}}$ & Graph vertex complexity \\
\hline $\mathrm{H}^{\mathrm{D}}$ & Graph distance complexity \\
\hline $\mathrm{IC}$ & $\begin{array}{l}\text { Information content of the distance matrix partitioned by frequency of occurrences of distance } \\
h\end{array}$ \\
\hline $\mathrm{M}_{1}$ & A Zagreb group parameter $=$ sum of square of degree over all vertices \\
\hline $\mathrm{M}_{2}$ & $\begin{array}{l}\text { A Zagreb group parameter }=\text { sum of cross-product of degrees over all neighboring (connected) } \\
\text { vertices }\end{array}$ \\
\hline${ }^{\mathrm{h}} \chi$ & Path connectivity index of order $h=0-10$ \\
\hline${ }^{\mathrm{h}} \chi_{\mathrm{C}}$ & Cluster connectivity index of order $h=3-6$ \\
\hline${ }^{\mathrm{h}} \chi_{\mathrm{PC}}$ & Path-cluster connectivity index of order $h=4-6$ \\
\hline${ }^{\mathrm{h}} \chi_{\mathrm{Ch}}$ & Chain connectivity index of order $h=3-10$ \\
\hline $\mathrm{P}_{\mathrm{h}}$ & Number of paths of length $h=0-10$ \\
\hline J & Balaban's $J$ index based on topological distance \\
\hline nrings & Number of rings in a graph \\
\hline ncirc & Number of circuits in a graph \\
\hline $\mathrm{DN}^{2} \mathrm{~S}_{\mathrm{y}}$ & $\begin{array}{l}\text { Triplet index from distance matrix, square of graph order (\# of non-H atoms), and distance } \\
\text { sum; operation } y=1-5\end{array}$ \\
\hline $\mathrm{DN}^{2} 1_{\mathrm{y}}$ & Triplet index from distance matrix, square of graph order, and number 1 ; operation $y=1-5$ \\
\hline $\mathrm{ASl}_{y}$ & $\begin{array}{l}\text { Triplet index from adjacency matrix, distance sum, and number } 1 \text {; } \\
\text { operation } y=1-5\end{array}$ \\
\hline $\mathrm{DSl}_{\mathrm{y}}$ & $\begin{array}{l}\text { Triplet index from distance matrix, distance sum, and number } 1 \text {; } \\
\text { operation } y=1-5\end{array}$ \\
\hline $\mathrm{ASN}_{\mathrm{y}}$ & Triplet index from adjacency matrix, distance sum, and graph order; operation $y=1-5$ \\
\hline $\mathrm{DSN}_{\mathrm{y}}$ & $\begin{array}{l}\text { Triplet index from distance matrix, distance sum, and graph order; } \\
\text { operation } y=1-5\end{array}$ \\
\hline $\mathrm{DN}^{2} \mathrm{~N}_{\mathrm{y}}$ & Triplet index from distance matrix, square of graph order, and graph order; operation $y=1-5$ \\
\hline $\mathrm{ANS}_{\mathrm{y}}$ & Triplet index from adjacency matrix, graph order, and distance sum; operation $y=1-5$ \\
\hline $\mathrm{AN} 1_{\mathrm{y}}$ & $\begin{array}{l}\text { Triplet index from adjacency matrix, graph order, and number } 1 \text {; } \\
\text { operation } y=1-5\end{array}$ \\
\hline $\begin{array}{l}\mathrm{ANN}_{\mathrm{y}} \\
\mathrm{ASV}_{\mathrm{y}}\end{array}$ & $\begin{array}{l}\text { Triplet index from adjacency matrix, graph order, and graph order again; operation y }=1-5 \\
\text { Triplet index from adjacency matrix, distance sum, and vertex degree; operation } y=1-5\end{array}$ \\
\hline $\begin{array}{l}\text { Asvy } \\
\text { DSV }_{y}\end{array}$ & $\begin{array}{l}\text { Triplet index from adjacency matrix, distance sum, and vertex degree; operation } y=1-5 \\
\text { Triplet index from distance matrix, distance sum, and vertex degree; operation } y=1-5\end{array}$ \\
\hline $\mathrm{ANV}_{\mathrm{y}}$ & Triplet index from adjacency matrix, graph order, and vertex degree; operation $y=1-5$ \\
\hline
\end{tabular}

Topochemical (TC)

$\mathrm{O}$

$\mathrm{O}_{\text {orb }}$

$\mathrm{I}_{\text {orb }}$

$\mathrm{IC}_{\mathrm{r}}$

$\mathrm{SIC}_{\mathrm{r}}$
Order of neighborhood when $\mathrm{IC}_{\mathrm{r}}$ reaches its maximum value for the hydrogen-filled graph Order of neighborhood when $\mathrm{IC}_{\mathrm{r}}$ reaches its maximum value for the hydrogen-suppressed graph Information content or complexity of the hydrogen-suppressed graph at its maximum neighborhood of vertices Mean information content or complexity of a graph based on the $r^{\text {th }}(r=0-6)$ order neighborhood of vertices in a hydrogen-filled graph Structural information content for $\mathrm{r}^{\text {th }}(\mathrm{r}=0-6)$ order neighborhood of vertices in a hydrogen- 
$\mathrm{CIC}_{\mathrm{r}} \quad$ Complementary information content for $\mathrm{r}^{\text {th }}(r=0-6)$ order neighborhood of vertices in a

${ }^{\mathrm{h}} \chi^{\mathrm{b}} \quad$ Bond path connectivity index of order $\mathrm{h}=0-6$

Bond cluster connectivity index of order $h=3-6$

Bond chain connectivity index of order $h=3-6$

Bond path-cluster connectivity index of order $h=4-6$

Valence path connectivity index of order $h=0-10$

Valence cluster connectivity index of order $h=3-6$

Valence chain connectivity index of order $h=3-10$

Valence path-cluster connectivity index of order $h=4-6$

Balaban's J index based on bond types

Balaban's J index based on relative electronegativities

Balaban's J index based on relative covalent radii

Hydrogen bonding parameter

Triplet index from adjacency matrix, atomic number, and vertex degree; operation $y=1-5$

$\mathrm{AZV}_{\mathrm{y}}$

$\mathrm{AZS}_{\mathrm{y}}$

$\mathrm{ASZ}_{\mathrm{y}}$

$A Z N_{y}$

$A N Z_{y}$

$\mathrm{DSZ}_{\mathrm{y}}$

$\mathrm{DN}^{2} \mathrm{Z}_{\mathrm{y}}$

Triplet index from adjacency matrix, atomic number, and distance sum; operation $y=1-5$

Triplet index from adjacency matrix, distance sum, and atomic number; operation $y=1-5$

Triplet index from adjacency matrix, atomic number, and graph order; operation $y=1-5$

Triplet index from adjacency matrix, graph order, and atomic number; operation $y=1-5$

Triplet index from distance matrix, distance sum, and atomic number; operation $y=1-5$

Triplet index from distance matrix, square of graph order, and atomic number; operation $y=1$ -

nvx

nelem

fw

si

Number of non-hydrogen atoms in a molecule

Number of elements in a molecule

totop

Molecular weight

Shannon information index

sumI

Total Topological Index $t$

sumdelI

Sum of the intrinsic state values I

tets2

phia

IdCbar

Sum of delta-I values

Total topological state index based on electrotopological state indices

Flexibility index (kp1* $\mathrm{kp} 2 / \mathrm{nvx})$

$\mathrm{IdC}$

Bonchev-Trinajsti $\square$ information index

$\mathrm{Wp}$

Pf

Bonchev-Trinajsti $\square$ information index

Wienerp

Wt

Plattf

knotp

knotpv

nclass

numHBd

numwHBd

numHBa

Total Wiener number

SHCsats

SHCsatu

SHvin

Difference of chi-cluster-3 and path/cluster-4

Valence difference of chi-cluster- 3 and path/cluster-4

Number of classes of topologically (symmetry) equivalent graph vertices

Number of hydrogen bond donors

Number of weak hydrogen bond donors

Number of hydrogen bond acceptors

E-State of $\mathrm{C} \mathrm{sp}^{3}$ bonded to other saturated $\mathrm{C}$ atoms

E-State of $\mathrm{C} \mathrm{sp}^{3}$ bonded to unsaturated $\mathrm{C}$ atoms

SHtvin

E-State of $\mathrm{C}$ atoms in the vinyl group, $=\mathrm{CH}-$

SHavin

$\mathrm{E}$-State of $\mathrm{C}$ atoms in the terminal vinyl group, $=\mathrm{CH}_{2}$

SHarom

E-State of $\mathrm{C}$ atoms in the vinyl group, $=\mathrm{CH}-$, bonded to an aromatic $\mathrm{C}$

SHHBd

SHwHBd $\quad$ Weak hydrogen bond donor index, sum of $\mathrm{C}-\mathrm{H}$
on a $\mathrm{C}$ to which a $\mathrm{F}$ and/or $\mathrm{Cl}$ are also bonded

E-State of $\mathrm{C} \mathrm{sp}^{2}$ which are part of an aromatic system

Hydrogen bond donor index, sum of Hydrogen E-State values for $-\mathrm{OH},=\mathrm{NH}$,

$-\mathrm{NH} 2,-\mathrm{NH}-$, $-\mathrm{SH}$, and \#CH

SHHBa Hydrogen bond acceptor index, sum of the E-State values for $-\mathrm{OH},=\mathrm{NH}$, 
Qv

NHBinty SHBint
$-\mathrm{NH} 2,-\mathrm{NH}-,>\mathrm{N}-,-\mathrm{O}-,-\mathrm{S}-$, along with $-\mathrm{F}$ and $-\mathrm{Cl}$

General Polarity descriptor

Count of potential internal hydrogen bonders $(y=2-10)$

E-State descriptors of potential internal hydrogen bond strength $(y=2-10)$

Electrotopological State index values for atoms types:

SHsOH, SHdNH, SHsSH, SHsNH2, SHssNH, SHtCH, SHother, SHCHnX, Hmax Gmax,

Hmin, Gmin, Hmaxpos, Hminneg, SsLi, SssBe, Sssss, Bem, SssBH, SsssB, SssssBm, SsCH3,

$\mathrm{SdCH} 2$, SssCH2, StCH, SdsCH, SaaCH, SsssCH, SddC,StsC, SdssC, SaasC, SaaaC, SssssC, SsNH3p, SsNH2, SssNH2p, SdNH, SssNH, SaaNH, StN, SsssNHp, SdsN, SaaN, SsssN,

SddsN, SaasN, SssssNp, SsOH, SdO, SssO, SaaO, SsF, SsSiH3, SssSiH2, SsssSiH, Ssssssi,

SsPH2, SssPH, SsssP, SdsssP, SsssssP, SsSH, SdS, SssS, SaaS, SdssS, SddssS, SssssssS, SsCl,

SsGeH3, SssGeH2, SsssGeH, SssssGe, SsAsH2, SssAsH, SsssAs, SdsssAs, SsssssAs, SsSeH,

SdSe, SssSe, SaaSe, SdssSe, SddssSe, SsBr, SsSnH3, SssSnH2, SsssSnH, SssssSn, SsI, $\mathrm{SsPbH} 3, \mathrm{SssPbH} 2, \mathrm{SsssPbH}$, SssssPb

\begin{tabular}{ll}
\hline kp0 & Kappa zero \\
kp1-kp3 & Kappa simple indices \\
ka1-ka3 & Kappa alpha indices
\end{tabular}

Geometrical/ Shape (3D)

Kappa alpha indices 
Table III. Summary statistics of predictive models for human $\log \mathrm{P}_{\text {blod:air }}$ based on experimental properties and theoretical structural descriptors.

\section{A. 31 DIVERSE CHEMICALS}

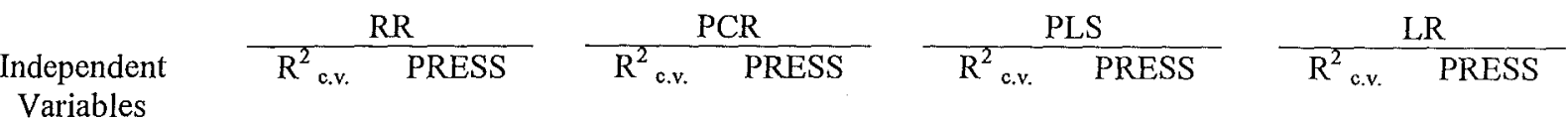

Variables

Structural descriptors

$\begin{array}{ccccccc}\text { TS } & 0.257 & 45.8 & -0.451 & 89.4 & 0.052 & 58.4 \\ \text { TS+TC } & 0.846 & 9.48 & 0.165 & 51.4 & 0.677 & 19.9 \\ \text { TS+TC+3D } & 0.827 & 10.6 & 0.140 & 53.0 & 0.620 & 23.4 \\ \text { TS+TC+3D+logP } & 0.835 & 10.2 & 0.112 & 54.7 & 0.652 & 21.4 \\ & & & & & & \\ \text { TS } & 0.257 & 45.8 & -0.451 & 89.4 & 0.052 & 58.4 \\ \text { TC } & 0.874 & 7.79 & 0.403 & 36.8 & 0.709 & 17.9 \\ \text { 3D } & 0.147 & 52.6 & -0.013 & 62.4 & -0.256 & 77.4\end{array}$

Properties

$\log P_{\text {olive oil:air }}+\log P_{\text {salinc:air }}$

Rat $\log \mathrm{P}_{\text {blood:air }}$

$0.899 \quad 6.19$

$0.963 \quad 2.25$

B. 18 HALOALKANES

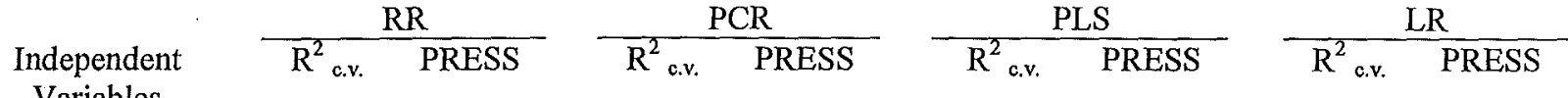

Variables

Structural descriptors

$\begin{array}{ccccccc}\text { TS } & 0.252 & 22.0 & -1.53 & 74.3 & -0.815 & 53.2 \\ \text { TS+TC } & 0.897 & 3.02 & 0.825 & 5.14 & 0.678 & 9.45 \\ \text { TS+TC+3D } & 0.892 & 3.16 & 0.856 & 4.22 & 0.702 & 8.74 \\ \text { TS+TC+3D+logP } & 0.892 & 3.18 & 0.856 & 4.23 & 0.704 & 8.69 \\ \text { TS } & & & & & & \\ \text { TC } & 0.252 & 22.0 & -1.53 & 74.3 & -0.815 & 53.2 \\ \text { 3D } & 0.891 & 3.21 & 0.853 & 4.32 & 0.616 & 11.3 \\ & 0.753 & 7.24 & 0.593 & 11.9 & 0.562 & 12.9\end{array}$

Properties

$\log \mathrm{P}_{\text {olive oil:air }}+\log \mathrm{P}_{\text {saline:air }}$

Rat $\log \mathrm{P}_{\text {blood:air }}$

$0.846 \quad 4.50$

$0.961 \quad 1.16$

${ }^{a}$ Calculated $\log \mathrm{P}_{n \text {-octanol:water; values included in Table I. }}$ 
Table IV. Ridge regression coefficient and standard error for each of the top 10 descriptors, ranked by $|t|$, in the topochemical model for the prediction of human $\log \mathrm{P}_{\text {blood:air }}, \mathrm{n}=31$.

\begin{tabular}{lccc}
\hline Descriptor & RR coeff & s.e. & t \\
\hline $\mathrm{SdO}$ & 0.227 & 0.021 & 10.690 \\
$\mathrm{HB}_{1}$ & 0.340 & 0.032 & 10.660 \\
$\mathrm{SddsN}$ & -1.694 & 0.159 & -10.640 \\
$\mathrm{AZV}_{3}$ & 0.130 & 0.016 & 8.000 \\
${ }^{1} \chi^{\mathrm{v}}$ & 0.345 & 0.052 & 6.670 \\
$\mathrm{AZV}_{4}$ & 0.224 & 0.034 & 6.580 \\
$\mathrm{AZV}$ & 0.133 & 0.024 & 5.640 \\
$\mathrm{SsBr}$ & 0.238 & 0.044 & 5.390 \\
$\mathrm{fw}$ & 0.287 & 0.054 & 5.310 \\
${ }^{1} \chi^{\mathrm{b}}$ & 0.139 & 0.028 & 5.060 \\
\hline
\end{tabular}




\section{FIGURE CAPTIONS}

Figure 1. Experimental vs fitted human $\log \mathrm{P}_{\text {blood:ar }}$ using the topochemical (TC) ridge regression (RR) model for the set.of 31 diverse compounds

Figure 2. Experimental $v s$ cross-validated predicted human $\log \mathrm{P}_{\text {blood:air }}$ using the topochemical (TC) ridge regression (RR) model for the set of 31 diverse compounds 
Figure 1.

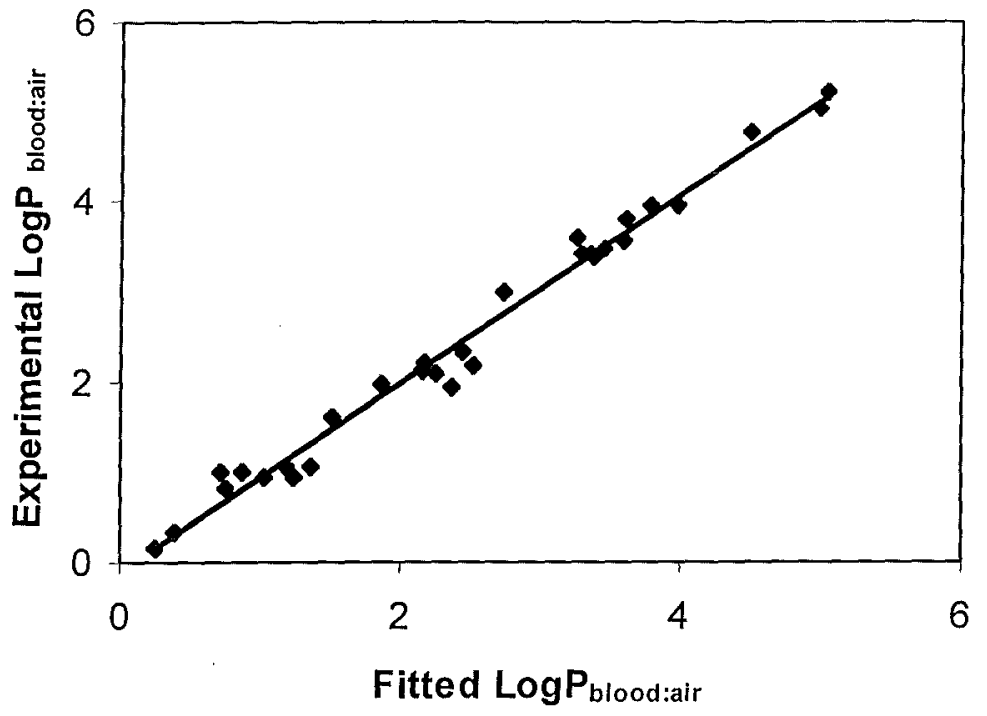


Figure 2.

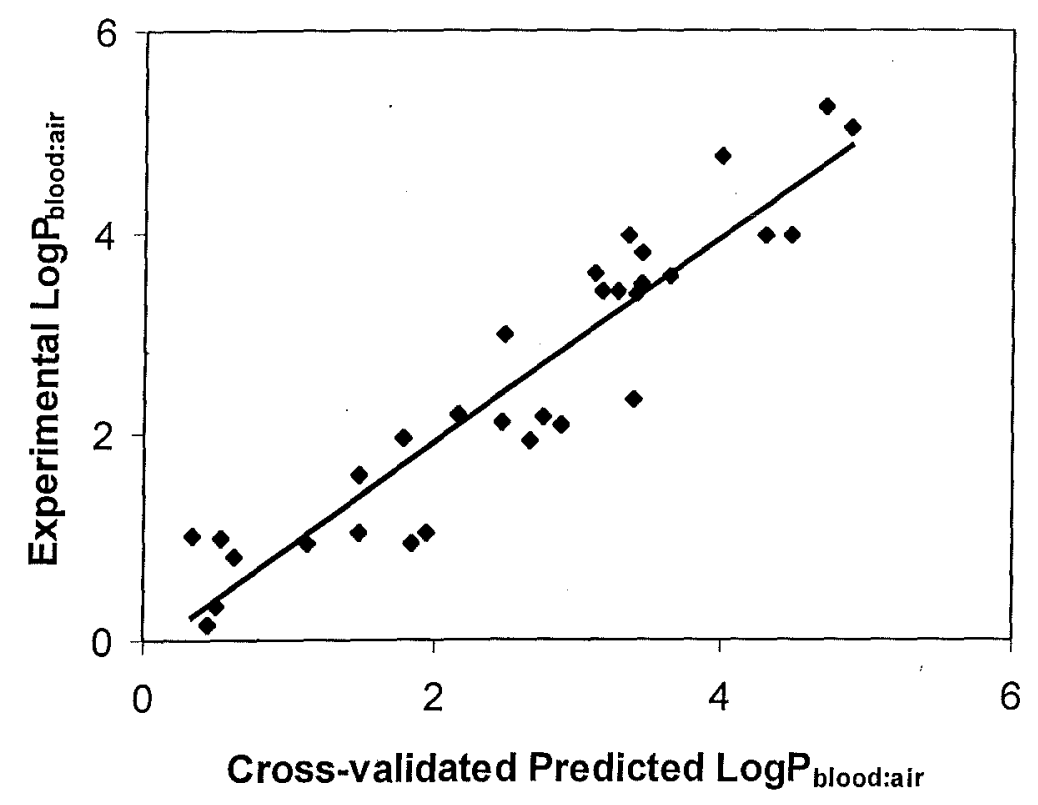




\section{Bibliography of papers on development of proteomics-based biodescriptors}

[1] Chemometrical treatment of proteomic maps, M. Vračko and S.C. Basak, J. Proteom. Res. (submitted).

[2] Novel matrix invariants for characterization of toxic effects on proteomics maps, Ž. Bajzer, $M$. Randić, D. Plavšić and S.C. Basak, J. Mol. Graphics and Modeling (submitted).

[3] On canonical labeling of proteins of proteomics maps, M. Randić and S.C. Basak, J. Chem. Inf. Comput. Sci. (submitted).

[4] A comparative study of proteomics maps using graph theoretical biodescriptors, M. Randić and S. C. Basak, J. Chem. Inf. Comput. Sci. (in press)

[5] A graph theoretical characterization of proteomics maps, M. Randić, Int. J. Quantum Chem. (in press)

[6] Novel matrix invariants for characterization of changes of proteomics maps, M. Randić, J. Zupan, M. Novič, B.D. Gute and S.C. Basak, SAR QSAR Environ. Res. (in press)

[7] On characterization of dose variations of 2-D proteomics maps by matrix invariants, $M$. Randić, M. Novič and M. Vračko, J. Proteome Res. (in press).

[8] On 3-D graphical representation of proteomics maps, M. Randić, J. Zupan and M. Novič, J. Chem. Inf. Comput. Sci. 41, 1339-1334 (2001).

[9] On characterization of proteomics maps and chemically induced changes in proteome using matrix invariants: Application to peroxisome proliferators, M. Randić, F. Witzmann, M. Vračko and S.C. Basak, Med. Chem. Res. 10, 456-479 (2001).

[10] On graphical and numerical characterization of proteomics maps, M. Randić, J. Chem. Inf. Comput. Sci. 41, 1330-1338 (2001).

[11] On graphical representation of proteomics and their numerical characterization, M. Randić, J. Chem. Inf. Comput. Sci. 41, 1330-1338 (2001). 